\title{
ZOOTAXA
}

1536

\section{Annotated checklist of weevils from the Papuan region (Coleoptera, Curculionoidea)}

\author{
GREGORY P. SETLIFF
}

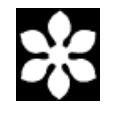

Magnolia Press

Auckland, New Zealand 
Gregory P. Setliff

Annotated checklist of weevils from the Papuan region (Coleoptera, Curculionoidea) (Zootaxa 1536)

296 pp.; $30 \mathrm{~cm}$.

30 July 2007

ISBN 978-1-86977-139-3 (paperback)

ISBN 978-1-86977-140-9 (Online edition)

FIRST PUBLISHED IN 2007 BY

Magnolia Press

P.O. Box 41-383

Auckland 1346

New Zealand

e-mail: zootaxa@mapress.com

http://www.mapress.com/zootaxa/

\section{(C) 2007 Magnolia Press}

All rights reserved.

No part of this publication may be reproduced, stored, transmitted or disseminated, in any form, or by any means, without prior written permission from the publisher, to whom all requests to reproduce copyright material should be directed in writing.

This authorization does not extend to any other kind of copying, by any means, in any form, and for any purpose other than private research use.

ISSN 1175-5326 (Print edition)

ISSN 1175-5334 (Online edition) 


\title{
Annotated checklist of weevils from the Papuan region (Coleoptera, Curculionoidea)
}

\author{
GREGORY P. SETLIFF \\ Department of Entomology, University of Minnesota, 219 Hodson, 1980 Folwell Avenue, St. Paul, Minnesota 55108 U.S.A. \& The New \\ Guinea Binatang Research Center, P. O. Box 604, Madang, Papua New Guinea.Email: setl0003@unm.edu
}

\section{Table of contents}

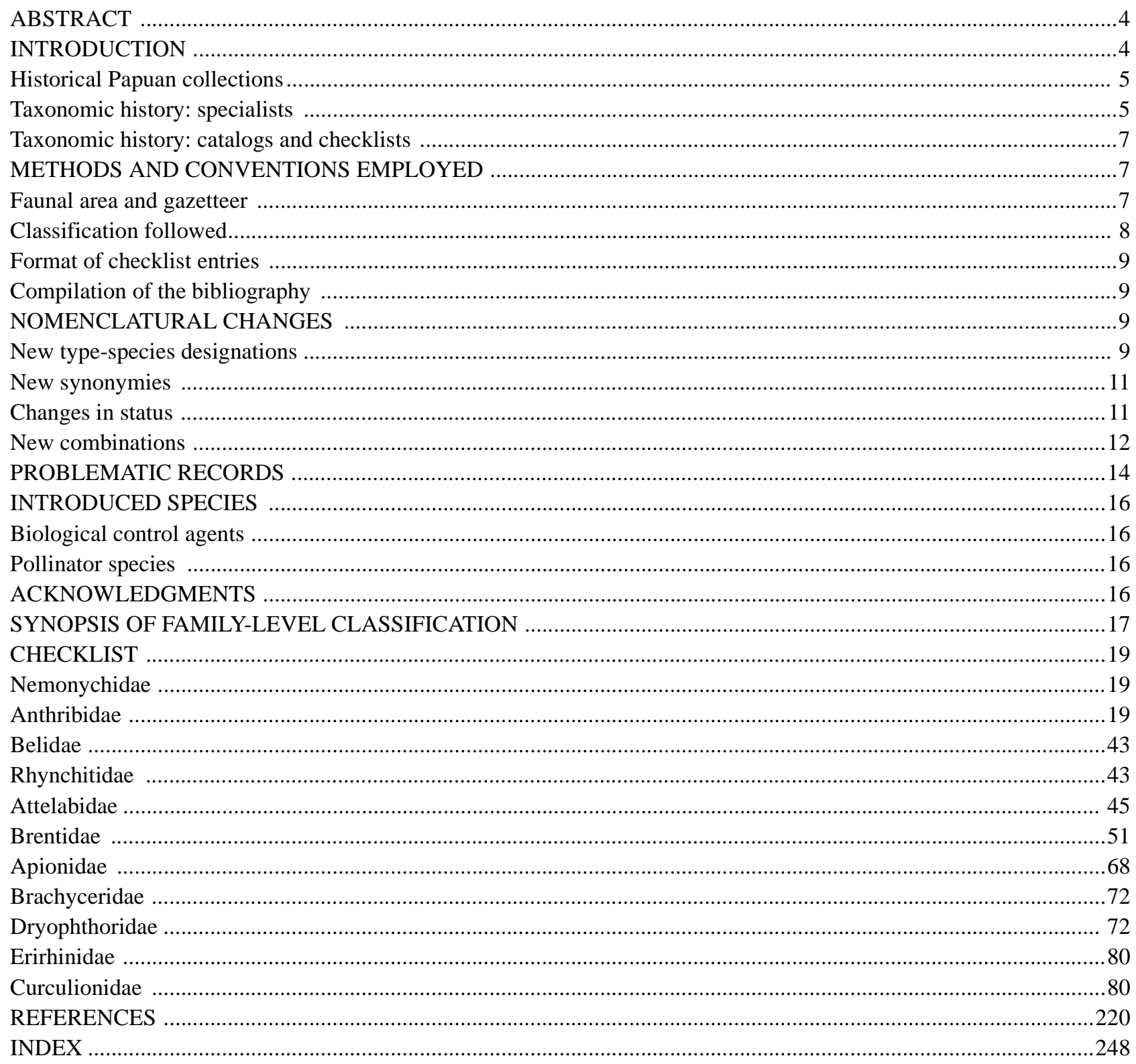




\begin{abstract}
The following checklist includes 2,955 species-group names and 553 genus-group names of weevils occurring in the Papuan region. Major islands treated are: Aru, Biak, Bougainville, Manus, Mysol, New Guinea, Salawatti, Trobriand, Waigeo, Woodlark, and Yapen Islands and the islands of the Admiralty, Bismarck, d'Entrecasteaux, and Louisaide Archipelagoes. Maps of the region with historically important collection localities are provided. Entomological expeditions to the region and collections containing significant weevil material are summarized. All available family-group, genusgroup and species-group names are arranged alphabetically for all families of Curculionoidea known from the region. All currently accepted species epithet are annotated with taxonomic references, notes on published distributions, past taxonomic changes, infrasubspecific names, and species-group synonymies. The following nomenclatural changes are proposed: the monotypic genus Neplaxa Casey is a new synonym of Pantoxystus Pascoe; its type species Neplaxa illustratus Casey is a new synonym of Pantoxystus rubricollis (Boisduval). Two subgenera of Neosynaptops, Neosynaptopsis Legalov and Pseudosynaptos Legalov are new synonymies of Euops (Neosynaptops) Voss revised status. Typespecies are designated for $\mathbf{2 5}$ genera, changes of rank or status are proposed for $\mathbf{1 9}$ taxa, and $\mathbf{8 8}$ new combinations are listed in "Nomenclatural changes."
\end{abstract}

Key words: checklist, Coleoptera, Curculionoidea, new combinations, new synonymies, new type-species designations, Papua New Guinea, Papuan region, weevils, West Papua

\title{
INTRODUCTION
}

Weevils are among the most important insect herbivores and their species diversity world-wide is well documented, particularly in the tropics (Anderson, 1993; 1995; Farrell, 1998; Basset \& Novotny, 1999). This remarkable diversity is acutely apparent to any student of the Papuan insect fauna. In 1994, noted weevil specialist, E. C. Zimmerman wrote, "New Guinea reveals its geologic youth with an island biota in an exuberant state of explosive speciation." The Papuan region, comprised of New Guinea and many satellite islands, hosts a fascinating weevil assemblage with 2,824 species and 131 subspecies described in 521 genera and 32 subgenera. Yet, weevils remain a poorly understood element of the Papuan biota. Life history and biology is all but unknown for most species save a handful of economically important pests. Insect collections throughout the world contain many specimens that cannot be referred to any described species. The majority of Papuan weevil genera are monotypic or contain few species. In most cases, this situation is a reflection of our ignorance rather than an accurate depiction of species diversity. For genera where a concerted effort has been made to study the taxonomy, the increase in the number of known species can be quite dramatic (e.g. Gressitt, 1966, 1967, 1970, \& 1977 on Gymnopholus; Gressitt, 1966 on Pantorhytes; Thompson 1977 \& 2006 on Apirocalus and 1996 on Phaenomerus; Riedel, 1999, 2000, 2001a, 2001b, 2002, \& 2006 on Euops). Recent works have added new records even at the family and subfamily levels, including the first reports of the families Caridae (see Wanat, 2001) and Nemonychidae (see Kuschel in Zimmerman, 1994a) and the curculionid subfamily Ceuthorhynchinae (see Colonnelli, 1979).

While a great deal of taxonomic work is needed, a review of existing literature is necessary to facilitate future studies of the fauna. Access to taxonomic information on Papuan weevils is impeded by the present state of the literature; much of which is antiquated and rare. Many incorrect citations of original descriptive works have been encountered, which hinder attempts to locate these critical documents. The purpose of this checklist is to make Papuan weevils more accessible for research by updating the incomplete and outdated regional checklists and catalogs currently available and to reconcile all available taxa with recent changes to 
the classification of Curculionoidea. To help orient readers, supplementary information on major collections, important collection localities, and past taxonomists who focused on the region is included.

\section{Historical Papuan collections}

For centuries naturalists have traveled to the Papuan region to study its unique biota. Early biological collections came solely from the coastal areas of New Guinea's Vokelkop peninsula and peripheral islands (e.g. expeditions of d'Urville in 1818-1824; Wallace 1858; Mountrouzier 1847-1852). Few ventured into the interior of New Guinea until d'Albertis traveled up the Fly River near the end of the 19th century (Frodin \& Gressitt, 1982). Papuan weevils have since attracted the attention of many collectors and are well represented in a number of collections. Large weevil collections were made by dozens of research expeditions to the region. A still greater amount of weevil material was amassed from permanent and semi-permanent research stations, such as the Wau Ecology Institute and National institutions such as the Department of Primary Industry and Forestry Research Institute in Papua New Guinea. A list of major expeditions, group research projects and stations, and individuals that have made significant weevil collections and the present day repositories for those collections is summarized in Appendix 1. Readers are referred to Froden \& Gressitt (1982), which provides a detailed account of the history of biological exploration in the Papuan region from 1700 to 1980. Parsons (1999), contains a superb chapter detailing the history of insect collecting in New Guinea with particular reference to butterflies. Information on the location of collections comes from Horn \& Kahle (1935) and Arnett et al. (1993).

\section{Taxonomic history: specialists}

Since Fabricius described the first weevils from the Papuan region in 1775, more than 140 authorities have contributed to weevil taxonomy of the region. The following eleven authorities were so influential to our understanding of Papuan weevils that special mention is made of their contributions here. The bibliography in Zimmerman (1993) should be consulted for detailed biographies of most of the authorities listed below as well as many others. The following biographical summaries are presented in chronological order.

\section{Francis P. Pascoe (1813-1893)}

Pascoe was one of the most influential weevil specialists to describe material from the region. He published many significant works on the fauna and proposed 112 genera and more than 280 species. $\mathrm{He}$ described material from the historically important weevil collections made by A.R. Wallace between 18571860 and L.M. d'Albertis and O. Beccari between 1872-1876. The Pascoe collection including most of Wallace's material is in the Natural History Museum in London (BMNH). The d'Albertis and Beccari collections are in the Museo Civico di Storia Naturale "Giacomo Doria" in Genoa, Italy (MCSN).

\section{Johannes K.E. Faust (1822-1903)}

Faust published extensively on the Papuan weevil fauna, describing 33 genera and 270 species. He described material collected by Loria et al. in Papua New Guinea (1888-1889) in an important publication Viaggo Di Lamberto Loria nella Papuasia orientale in 1889. Faust's collection, including types now resides mostly in the Museum für Tierkunde in Dresden, Germany (SMTD) and the Museum National d'Histoire Naturelle in Paris, France (MNHN).

\section{Karl M. Heller (1864-1945)}

Heller described 370 species from the Papuan region and he is second only to Pascoe with the 40 genera he proposed. His publications include many useful keys to genera and species; most of which are the only keys available for these groups. Heller was a major contributor to the Nova Guinea series, in which he described material from the Netherlands expeditions to New Guinea in 1907-1909. See Draeseke (1957) for 
an obituary. Heller's collection is in the Museum für Tierkunde in Dresden, Germany (SMTD).

Karl Jordan (1861-1959)

Jordan was a respected specialist on the Anthribidae and he described 11 genera and 69 species from New Guinea. He was employed by Lord Rothschild to curate the Lepidoptera and Coleoptera collections at Tring where he described material from New Guinean expeditions sponsored by Lord Rothschild as well as the Prince Leopold's Expedition in 1929. Jordan's types and determined material resided at the Walter Rothschild Zoological Museum in Tring, England, and were transferred to the Natural History Museum in London (BMNH).

Arthur Mills Lea (1868-1932)

Lea's major contributions were on Australian weevils, however much of his work is useful in understanding the Papuan fauna, particularly his series of publications on Australian Cryptorhynchinae (1898-1906; 1909; 1928). Lea also wrote an important work on the Baridinae of New Guinea (1931). His described material and collection is deposited at the South Australian Museum in Adelaide (SAMA).

Guy Alexander Knox Marshall (1871-1960)

Marshall described 27 genera and 73 species of many subfamilies of Papuan Curculionidae. His contributions on the Celeuthetini are essential tools in the study of Entiminae in the region. Marshall described material collected during the Wollaston expeditions (1909-1910; 1912-1913) and Prince Leopold expedition (1929). His types and associated material are in the Natural History Museum in London, U.K. (BMNH).

Richard Kleine (1874-1948)

Kleine was a brentid specialist who described 17 genera and 103 species from the Papuan region. His collection was assumed destroyed in Stettin during the Second World War. However, Sforzi \& Bartolozzi (2004) report that many of his types survived and are currently housed in the Museum and Institute of Zoology, Polish Academy of Science in Warsaw, Poland (ZMPA).

\section{Eduard Voss (1884-1974)}

Voss contributed a number of works on Papuan Rhynchitidae, Attelabidae, and Cossoninae and an important three part series entitled Die von Biró auf Neu Guinea aufgefudenen Rüsselkäfer (1956; 1958b; 1960). Osella (1976) gave an obituary and Weidner $(1976 ; 1979)$ listed Voss' surviving type material in the Zoologischen Institut und Zoologischen Museums, Universität Hamburg, Germany (ZMUH).

Karl Schedl (1898-1979)

Schedl was a prolific describer of bark and ambrosia beetle species, which is evident from the more than 400 Papuan species that he described. Wood \& Bright (1992: 2) provide enlightening commentary on the socalled "Schedl factor" in bark beetle taxonomy. Schedl's described material is in a separate collection in the Naturhistorisches Museum Wien, Vienna, Austria (NHMW).

\section{Elwood C. Zimmerman (1912-2004)}

Zimmerman was one of the most respected entomologists to study the Pacific fauna. His unfinished treatise on Australian weevils (Zimmerman, 1991; 1992; 1993; 1994a; 1994b), while not directly treating the Papuan fauna, is an indispensable guide to the faunistic region as a whole. It contains many illustrations, keys, taxonomic notes, and biographies of researchers that are pertinent to studies of Papuan weevils. The Zimmerman Weevil Research Laboratory has been established to house the Zimmerman collection at the Australian National Insect Collection in Canberra, Australia (ANIC) (Oberprieler in CURCULIO 51 newsletter, 2005 
available from www.coleopsoc.org/nwslttrs.shtml).

\section{J. Linsley Gressitt (1914-1982)}

Gressitt made a profound impact on biological and ecological research in the Papuan region. He established the Bishop Museum's biological research station at Wau in 1961, which became the Wau Ecology Institute (WEI) in 1971. This permanent scientific station marked the beginning of the modern era of research in New Guinea. He was a prolific writer, publishing on a variety of subjects including, beetle taxonomy, Pacific biogeography, and conservation in New Guinea. Gressitt described the epizoic symbiosis between weevils and the cryptogamic plants, mites, nematodes and microscopic organisms living on their specially modified elytra. He revised Gymnopholus and Pantorhytes, two of the entimine genera involved in this interesting relationship, and described 93 species in them. Most of Gressitt's material is in the Bernice P. Bishop Museum in Honolulu, Hawaii (BPBM), though many of his types are deposited in the National Museum of Natural History in Washington D.C., United States (USNM). The collections made by Gressitt and his many collaborators at WEI have made the Bishop Museum one of the most important Papuan insect collections in the world.

\section{Taxonomic history: catalogs and checklists}

There have been few attempts to synthesize the taxonomic literature for the Papuan region. The Coleopterorum Catologus (weevil parts 1910-1953; citations given in Appendix 2) remains the primary reference to the world weevil fauna at the species level, though it is outdated and contains numerous errors and omissions. Appendix 3 is a summary of major taxonomic works treating primarily Papuan weevils published after the relevant parts of the Coleopterum Catalogus. In 1888, Masters published the only comprehensive catalog to specifically treat Papuan Coleoptera. In this catalog, he cited 191 genera and 469 species of Curculionoidea. Nearly 100 years later, Bigger \& Schofield (1983), reported 98 additional weevil genera and more than double the number of species (1,744 species) in the only other beetle checklist for the region. The Bigger \& Schofield checklist focused primarily on beetles of importance to forestry and several families of Curculionoidea were not treated. In Table 1, the number of weevil genera, and species arranged by family included in these two regional works and this checklist are compared.

\section{METHODS AND CONVENTIONS EMPLOYED}

The World Catalogue of Families and Genera of Curculionoidea (Alonso-Zarazaga \& Lyal, 1999) served as the family and generic-level framework for this checklist. Initially, the Coleopterorum Catologus volumes on weevils (see Appendix 2) and recent world-wide catalogs treating specific weevil families or subfamilies were consulted and all relevant taxonomic references for the region extracted. All volumes of the Zoological Record from 1864-1978 were examined for additional records. Electronic searches of the Zoological Record were conducted for 1978-December 2006. Finally, the primary literature was consulted to locate original descriptions for all taxa presented in this list except for groups with a recent catalog. In these cases, all taxonomic references were extracted verbatim and crosschecked for errors and omissions. The following sources were utilized in this manner: Wood \& Bright (1992) and supplements (Bright \& Skidmore 1997; 2002) for Scolytinae and Platypodinae; Rheinheimer (2004a) for Anthribidae; Sforzi \& Bartolozzi (2004) for Brentidae; and Oberprieler (2004) for Eurhynchidae.

\section{Faunal area and gazetteer}

The Papuan region as treated in this checklist is delineated in Figure 1. At its center is the island of New Guinea, which is administratively divided along $141^{\circ}$ longitude between the country Papua New Guinea (PNG) and the Indonesian state of West Papua (formerly Irian Jaya). Also included in the region are the Aru, 
Baik, Batanta, Mysol, Salawatti, Waigeo, and Yapen Islands to the West and the Admiralty Islands, Bismarck Archipelago, Bougainville Island (a part of the North Solomon Islands), D'Entrecasteaux Islands, Louisaide Archipelago, Trobriand Islands and Woodlark Islands in the East. For convenience, specific DISTRIBUTION localities given for species in the checklist have been grouped into subdivisions based on the islands groups listed above; Figure 1 shows the limits of these subdivisions. The two political subdivisions of New Guinea island (West Papua and Papua New Guinea) are treated separately in this work. Maps (Figs 1-3) show major island groups, islands, towns, villages, and geographical features that represent historically important collection localities. Names that have subsequently changed or have fallen out of usage are listed beside their modern equivalents in the figure legends.

TABLE 1. Family representation of weevil genera and species in New Guinea in this work and the two previously published regional checklists. The classification follows Alonso-Zarazaga \& Lyal (1999) except for the bark and ambrosia beetles. Numbers of genus-group names are listed before the slash and species-group names after; an en-dash (-) indicates the family was not treated.

\begin{tabular}{llll}
\hline Family & Masters (1888) & Bigger \& Schofield (1983) & present work \\
\hline Nemonychidae & - & - & $1 / 3$ \\
Anthribidae & $15 / 31$ & - & $64 / 343$ \\
Belidae & $1 / 2$ & - & $1 / 6$ \\
Rhynchitidae & $1 / 1$ & $2 / 2$ & $8 / 11$ \\
Attelibidae & $1 / 5$ & $1 / 13$ & $12 / 84$ \\
Brentidae & $12 / 21$ & - & $58 / 202$ \\
Eurhynchidae & $1 / 1$ & - & $1 / 26$ \\
Apionidae & - & - & $19 / 29$ \\
Brachyceridae & - & $1 / 1$ & $1 / 2$ \\
Dryophthoridae & $11 / 34$ & $27 / 54$ & $33 / 76$ \\
Erirhinidae & $1 / 1$ & - & $2 / 2$ \\
Curculionidae auct. & $145 / 365$ & $205 / 1153$ & $292 / 1465$ \\
Scolytidae (-nae) & $1 / 1$ & $43 / 292$ & $50 / 375$ \\
Platypodidae (-nae) & $2 / 7$ & $9 / 229$ & $11 / 331$ \\
Total & $191 / 469$ & $288 / 1744$ & $553 / 2955$ \\
\hline
\end{tabular}

\section{Classification followed}

A stable, higher-level weevil classification does not exist. The composition and arrangement of even family-level taxa is unresolved and the subject of much debate (Morimoto, 1962; Thompson 1992; Zimmerman 1993; 1994a; 1994b; Kuschel 1995; Zerikhin \& Gratshev 1995; Maravaldi et al. 2002). In this checklist, the arrangement of all super-specific taxa follows Alonso-Zarazaga \& Lyal (1999) and its two addenda and corrigenda (Alonso-Zarazaga \& Lyal 2002; Lyal \& Alonso-Zarazaga 2006). One exception is that Eurhynchinae is not treated as a family, rather it has been included as a subfamily of Brentidae (following Oberpriler 2004 but see Oberpriler 2000). Scolytinae and Platypodinae were not treated in Alonso-Zarazaga \& Lyal's (1999) catalog. For these two groups, classification at all taxonomic levels follows Wood \& Bright (1992) and supplements (Bright \& Skidmore 1997; 2002) except that both groups are treated here as subfamilies of Curculionidae, not as families. All subordinate taxa are reduced in rank accordingly. Taxa described after Alonso-Zarazaga \& Lyal (1999) are arranged according to the placement proposed by the describing authority. Taxa are placed in "incertae sedis" at the end of the relevant tribe or subfamily when the describing authority did not specify supergeneric classification. 


\section{Format of checklist entries}

All subordinate ranks below family are arranged alphabetically. Family-group names are center justified, set in all-capital letters, and bold typeface except subtribes, which are in all-capital, regular typeface. Genusgroup names are typeset in small-capitals, bold typeface and center justified. Species-group epithets are left justified in bold typeface and are italicized. Author name and citation, consisting of the date of publication and starting page number of the original description for each taxon follows all generic and specific names. Readers are referred to Alonso-Zarazaga \& Lyal (1999) for generic synonyms and citations and supplementary information for all higher-level taxa. The page number in the citation does not necessarily represent the first appearance of a specific epithet in the case where it appears first in keys, abstracts, table of contents, illustrations, etc. Information on the type-species is given after the generic citation. Keys to Papuan species of a genus are cited after type information where applicable. For all available species-group names, the genus in which a species first appeared in combination is listed in parenthesis following the citation. Published distribution information is given for all available species-group names. As already mentioned above, specific distribution localities are grouped into subdivisions based on major archipelagoes and the political division of New Guinea island (Fig. 1). Subdivision names are listed first and are separated from specific localities within the region by a colon. Localities within a subdivision are separated from one another by a comma. Different subdivisions are separated from each other by a semi-colon. Only published localities are included in the checklist. No attempt was made to establish or verify ranges for species by examining material in collections. Localities outside of the Papuan region as defined above are given in square brackets. Museum and collection codons throughout follow Arnett et al. (1993).

Synonyms are italicized and typeset in a smaller font size than the rest of the text. Synonyms are arranged in chronological order below the currently accepted species name. Only synonyms occurring within the region covered by this checklist are included. Nomina nudua and incorrect subsequent spellings of valid names are not included. Infrasubspecific names are formatted the same as synonyms but are listed alphabetically after the valid specific name, before any synonyms. The following abbreviations are used to denote infrasubspecific names: v. =variety, ab. =aberration, f. $=$ form. All infrasubspecific names are listed in their most recent orthography in the literature. Some infrasubspecific names originally termed varieties described before 1961 may be considered valid subspecies under the conditions listed in Art. 45.6. of the Code (ICZN 1999). However, no attempt was made to determine the availability of infrasubspecific varieties in this work.

\section{Compilation of the bibliography}

All references cited in this work are taxonomic in nature. A number of resources were useful in compiling the bibliography presented here, namely Gressitt \& Szent-Ivany's Bibliography of New Guinea Entomology (1968) and its supplement (Miller 2004). Zimmerman (1993) and Horn \& Schenkling (1928-1929) were helpful for locating many older works. Alonso-Zarazaga \& Lyal (1999) provided citations of genus-group names.

\section{NOMENCLATURAL CHANGES}

While the purpose of this checklist is to report on the current state of the taxonomic literature, a number of situations were encountered during its collation that necessitate nomenclatural action. A summary of the changes proposed in this work are given here.

\section{New type-species designations}

The following species are designated as the type-species for 25 genera listed below. In each case, the authors of the genera failed to select a type-species and its identity could not be ascertained by monotypy. I follow the recommendation in Art. 69.A.8-10 in the Code (ICZN 1999); selecting the first species appearing 
after the generic description.

Dryophthoridae

Ganae Pascoe, 1885: Ganae pulchella Pascoe, 1885

Laogenia Pascoe, 1874: Laogenia sorex Pascoe, 1874

Curculionidae: Baridinae

Solenobaris Lea, 1906: Solenobaris decipiens Lea, 1906

Xenopsilus Faust, 1899: Xenopsilus fuscicornis Faust, 1899

Curculionidae: Conoderinae

Achirozetes Heller, 1924b: Achirozetes kleinei Heller, 1924b

Daedania Pascoe, 1871a: Daedania mesoleuca Pascoe, 1871a

Idopelma Faust, 1898: Idopelma bicolor Faust, 1898

Othippia Pascoe, 1874: Othippia distigma Pascoe, 1874

Panigena Pascoe, 1874: Panigena chalybea Pascoe, 1874

Curculionidae: Cossoninae

Aphyoda Pascoe, 1871: Aphyoda diura Pascoe, 1871

Gloedema Wollaston, 1873: Gloedema spatula Wollaston, 1873

Stereoderus Wollaston, 1873: Stereoderus barbatus Wollaston, 1873

Curculionidae: Cryptorhynchinae

Anchithyrus Pascoe, 1885: Anchithyrus obesus Pascoe, 1885

Asytesta Pascoe, 1865: Asytesta humeralis Pascoe, 1865

Coryssoglymma Faust, 1899: Coryssoglymma odiosa Faust, 1899

Glyptoporopterus Lea, 1912: Poropterus sharpi Faust, 1898a

Meroleptus Faust, 1898: Meroleptus gemmatus Faust, 1898

Microporopterus Lea, 1896: Poropterus tumulosus Pascoe, 1873b

Ptolycus Pascoe, 1885: Ptolycus trachypterus Pascoe, 1885

Semiathe Pascoe, 1871: Semiathe rufipennis Pascoe, 1871a

Syrichius Pascoe, 1871: Syrichius roridus Pascoe, 1871

Curculionidae: Curculioninae

Cenchrena Pascoe, 1874: Cenchrena fasciata Pascoe, 1874

Neomelanterius Lea, 1899: Neomelanterius carinicollis Pascoe, 1875

Curculionidae: Entiminae

Ittostira Heller, 1925: Ittostira elongatus Heller, 1925-73

Sphenomorpha Behrens, 1887: Sphenomorpha suturalis Behrens, 1887 


\section{New synonymies}

In all cases below, the junior synonym follows the valid senior name and is preceded by an equal sign (=).

Pantoxystus Pascoe, 1881 (Curculionidae: Molytinae)

$=$ Neplaxa Casey, 1922 syn. $\mathbf{n}$.

Casey erected the monobasic genus Neplaxa for a single specimen mistakenly attributed to the Brazilian fauna. The holotype in the USNH collection is conspecific with Pantoxystus rubricollis (Boisduval) =Neplaxa illustrus Casey syn. $\mathbf{n}$.

Euops (Neosynaptops) Voss, 1930 (Attelabidae: Euopini) stat. rev.

=Neosynaptops (Neosynaptopsis) Legalov, 2003: 372 syn. $\mathbf{n}$.

=Neosynaptops (Pseudosynaptos) Legalov, 2003: 372 syn. n.

Legalov (2003) gave generic status to Neosynaptops, formerly a subgenus of Euops Schönherr and described two subgenera of Neosynaptops. Riedel (2006) recommended that Legalov's Papuan genera from Euops be reduced to subgenera of Euops. Below I follow this recommendation and return Neosynaptops to a subgenus of Euops necessitating the synonymy of its two subgenera here.

\section{Changes in status}

Legalov (2003) erected many new taxa in Attelabidae: Euopini. In the Papuan region his genus-group taxa were largely based on species-groups defined by Riedel (2002c). Nine genera from Legalov (2003) and one subgenus of Euops (Paraeuops) Legalov, 2003 are reduced to subgenera of Euops following Riedel (2006).

Rank changed from genera of Euopini to subgenera of Euops:

Euops (Archeuops) Legalov, 2003: 360 stat. n.

Euops (Armeuops) Legalov, 2003: 375 stat. n.

Euops (Australoeuops) Legalov, 2003: 405 stat. n.

Euops (Euopsidius) Legalov, 2003: 370 stat. n.

Euops (Guineoeuops) Legalov, 2003: 370 stat. n.

Euops (Lijudmilinius) Legalov, 2003: 362 stat. n.

Euops (Metasynaptops) Legalov, 2003: 390 stat. n.

Euops (Paraeuops) Legalov, 2003: 394 stat. n.

Euops (Riedelinius) Legalov, 2003: 362 stat. n.

Rank changed from subgenus of Paraeuops to subgenus of Euops:

Euops (Paraeuopsis) Legalov, 2003: 396 stat. n.

Returned to subgenus of Euops from generic status in Legalov (2003):

Euops (Neosynaptops) Voss, 1930: 83 stat. rev.

Species returned to Euops (Neosynaptops) from Neosynaptops s.str. (sensu Legalov 2003):

E. (N.) viridiceps Voss, stat. rev. 
Species returned to Euops (Neosynaptops) from Neosynaptops (Neosynaptopsis) Legalov (synonymy above):

E. (N.) doertheae Riedel, stat. rev.

E. (N.) gladiator Riedel, stat. rev.

E. (N.) punctaticeps Riedel, stat. rev.

E. (N.) similis Riedel, stat. rev.

E. (N.) waigeoensis Riedel, stat. rev.

E. (N.) wapogae Riedel, stat. rev.

Species returned to Euops (Neosynaptops) from Neosynaptops (Pseudosynaptos) Legalov (synonymy above):

E. (N.) cupreosplendens Macleay, stat. rev.

\section{New combinations}

Species appearing for the first time in new combinations are listed below. All but one of the 88 new combinations reported here result from prior taxonomic actions by other authorities. Either synonymy of a junior generic name, resurrection of a senior generic name, or proposal of a replacement name for a preoccupied generic name has necessitated transferring all affected species. The remaining species transferred here, was overlooked by subsequent workers.

Arachnobas Boisduval, 1835 (Curculionidae: Conoderinae)

Alonso-Zarazaga and Lyal (1999) restored Arachnobas Boisduval over the junior name Arachnopus Guérin-Méneville. The type species A. striga (Guérin-Méneville) is assumed transferred by Alonso-Zarazaga and Lyal ibid. when they designated it as the type species of Arachnobas. The following 58 species-group names described in Arachnopus are transferred to Arachnobas:

A. acuminatus (Heller), comb. n.

A. acutipennis (Gestro), comb. $\mathbf{n}$.

A. affinis (Heller), comb. n.

A. alboscapulatus (Gestro), comb. n.

A. anthonyi (Faust), comb. n.

A. asignatus (Heller), comb. $\mathbf{n}$.

A. avus (Heller), comb. n.

A. basalis (Heller), comb. n.

A. biguttatus (Faust), comb. n.

A. binotatus (Pascoe), comb. $\mathbf{n}$.

A. caudatus (Heller), comb. n.

A. corpulentus (Heller), comb. n.

A. costulatus (Heller), comb. $\mathbf{n}$.

A. disparilis (Faust), comb. n.

A. effigies (Heller), comb. $\mathbf{n}$.

A. fenestratus (Faust), comb. n.

A. ferus (Heller), comb. n.

A. fimbriatus (Heller), comb. $\mathbf{n}$.

A. finschi (Heller), comb. $\mathbf{n}$.

A. fortis (Heller), comb. n.
A. fossulatus fossulatus (Faust), comb. n.

A. fossulatus nigrofimbriatus (Heller), comb. $\mathbf{n}$.

A. frenatus (Snellen), comb. $\mathbf{n}$.

A. furcillifer (Heller), comb. $\mathbf{n}$.

A. granulipennis (Heller), comb. n.

A. guttulifer (Gestro), comb. n.

A. imitator (Faust), comb. n.

A. incanus (Marshall), comb. n.

A. interruptus (Fairmaire), comb. n.

A. lacerta (Faust), comb. n.

A. lanceolatus (Marshall), comb. n.

A. loriai (Heller), comb. n.

A. melanospilus (Gestro), comb. n.

A. misoriensis (Gestro), comb. $\mathbf{n}$.

A. nepos (Heller), comb. n.

A. olivieri (Faust), comb. n.

A. papua (Heller), comb. n.

A. patronoides (Heller), comb. n.

A. patronus (Pascoe), comb. $\mathbf{n}$.

A. pauxillus (Heller), comb. $\mathbf{n}$. 
A. persona (Snellen), comb. n.

A. planatus (Marshall), comb. n.

A. praevius (Faust), comb. n.

A. quadraticollis (Heller), comb. n.

A. rugosus (Heller), comb. n.

A. sannio (Pascoe), comb. n.

A. scabiosus (Heller), comb. n.

A. sectator (Faust), comb. n.

A. semiruber (Heller), comb. n.

A. simius (Pascoe), comb. n.
A. stigmatus (Faust), comb. n.

A. striga denutatus (Heller), comb. n.

A. subcostatus (Heller), comb. n.

A. subfasciatus (Heller), comb. n.

A. tenuisignatus (Heller), comb. n.

A. tricolor (Heller), comb. n.

A. tristiculus (Heller), comb. n.

A. tristis (Heller), comb. n.

Bartolozziella Alonso-Zarazaga \& Lyal, 1999 (Curculionidae: Cryptorhynchinae)

Alonso-Zarazaga and Lyal (1999) proposed this replacement name for the preoccupied Endymia Pascoe (non Gistl), affecting two Papuan species. The authors of the replacement name listed the type species, Endymia vipio Pascoe under the new name and by doing so implied its transfer. The remaining species, Endymia effusa (Faust) comb. n. is transferred here to Bartolozziella.

Eupholus Boisduval 1835 (Curculionidae: Entiminae)

Alonso-Zarazaga \& Lyal (1999) showed that Eupholus Guérin-Méneville (1838) is both a junior homonym and a junior synonym of Eupholus Boisduval (1835). Riedel (2002) transferred eight species to Eupholus Boisduval. The remaining fifteen affected species are transferred here:

E. amalulu (Porion), comb. n.

E. clarki (Porion), comb. n.

E. detanii (Limoges \& Porion), comb. n.

E. fleurenti (Porion), comb. n.

E. helleri (Porion), comb. n.

E. hephaistos (Porion), comb. n.

E. hudsoni (Porion), comb. n.

E. kuntzmannorum (Limoges \& Porion), comb. $\mathbf{n}$.

E. lachaumei (Porion), comb. n.

E. lacordairei (Limoges \& Porion), comb. n.

E. leblanci (Limoges \& Porion), comb. n.

E. messagieri (Porion), comb. n.

E. rigouti (Porion), comb. n.

E. saugrenus (Porion), comb. n.

E. waigeuensis (Limoges \& Porion), comb. n.

Eutyrhinus Dejean, 1835 (Curculionidae: Cryptorhynchinae)

Six species were described in Euthyrhinus Schönherr, which Alonso-Zarazaga \& Lyal (1999) placed as a junior synonym of Eutyrhinus Dejean. The type-species, Curculio meditabundus Fabricius was listed for Eutyrhinus Dejean by Alonso-Zarazaga \& Lyal ibid. and is assumed to have been transferred. The five remaining species are transferred to Eutyrhinus Dejean:

E. brevispinosus (Fairmaire), comb. n.

E. dorsalis (Macleay), comb. n. 
E. iconicus (Pascoe), comb. n.

E. irroratus (Macleay), comb. n.

E. tessellatus (Blanchard), comb. n.

Hypsophorus Dejean in Boisduval, 1835a (Curculionidae: Cryptorhynchinae)

Alonso-Zarazaga \& Lyal, (1999) restored the senior name Hypsophorus Dejean over the junior name Protopalus Schönherr. One New Guinean species, H. alboguttatus (Chevrolat) comb. n. is transferred to Hypsophorus.

Aades Schönherr, 1823 (Curculionidae: Cyclominae)

The placement of Aterpus franklini Heller is unclear after Zimmerman, 1994a: 639 restored Aades and placed Atperpus in synonymy with it, but excluded this species from his list of newly combined species. It may belong in Aterpodes Zimmerman, though the name does not appear in the speceis list presented by Zimmerman for that genus. I provisionally place this species in new combination with the former; Aades franklini (Heller) comb. n. (sedis mutabilis).

Idiopsodes Thompson, 1977 (Curculionidae: Entiminae)

Thompson (1977) proposed Idiopsodes as a replacement name for the preoccupied Idiopsis Faust (non Brauer \& Bergenstamm) and transferred the type species Idiopsis griseus (Pascoe). The remaining six New Guinean species are here transferred from Idiopsis Faust to Idiopsodes Thompson:

I. coeruleus (Faust), comb. n.

I. kocki (Heller), comb. n.

I. maculosus (Faust), comb. n.

I. ornatus (Faust), comb. n.

I. perplexus (Faust), comb. n.

I. quinarius (Pascoe), comb. n.

Aporhina Boisduval, 1835 (Brentidae: Eurhynchinae)

Aporhina leai (Heller), 1908 comb. n. is transferred from Chalcocybebus Snellen van Vollenholven. This species was omitted from Oberprieler's (2004) catalog of the Eurhynchidae.

\section{PROBLEMATIC RECORDS}

Ten genera and one undescribed genus that are reported from New Guinea in the literature are based only on undescribed species, immature specimens identified only to genus, or species identifications that have not yet been published or require confirmation. Thus, these problematic taxa are not included in the checklist because they lack reference to a valid specific epithet. However, researchers working on the fauna should be aware of these reported range extensions as they represent the only records of one family, one subfamily, and two tribes from the Papuan region.

\section{Ancyttalia Zimmerman, 1994a (Curculionidae)}

Zimmerman (1994a) did not clearly state what species, described or otherwise, he was referring to when he gave the distribution of the genus as, "Australia and New Guinea (new record)." Once associated with a species, this will be the first record of this genus and the tribe Eugnomini from New Guinea. 
Basiliogeus Kuschel in Zimmerman, 1994a (Nemonychidae)

Larvae of an undetermined species belonging to this genus were reported from New Guinea. This is the only record of the tribe Rhinorhynchini from the region (Kuschel in Zimmerman, 1994a).

Lepropus Schönherr, 1823 (Curculionidae)

Ramamutrhy et al. (1998) reported a single specimen of the Indian species Lepropus (Lepropus) adamsoni Marshall, 1916 from New Guinea. This record should be substantiated by additional specimens before being included in a list of Papuan weevils.

Lissapion Zimmerman, 1994b (Apionidae)

Wanat (2001) reported 5-7 undescribed species from New Guinea. No other representatives of this genus are in the region.

Manilabaris Zimmerman, 1940b (Curculionidae: Baradinae)

Morimoto \& Yoshihara (1996) reported unnamed species in the Kyushu University collection.

Myrmacicelus Chevrolat, 1833 (Apionidae)

This genus is represented in New Guinea by 3 undescribed species (Wanat, 2001); these were the first records for the subfamily Myrmacicelinae from the region.

Myrmacyba Heller, 1929 (Apionidae)

Wanat (2001) stated that he examined, “.....approximately 50 undescribed species” from New Guinea and Moluccas. Only three of this number are from localities outside of the Papuan region. There are currently no described species from New Guinea.

Nanomyrmacyba Wanat, 1990 (Apionidae)

Wanat (2001) examined, “.....well over 30 undescribed New Guinean and Australian members....” There are no described species from New Guinea.

Stenommatus Wollaston, 1873b (Dryophthoridae)

One undescribed species is the only record of the genus for New Guinea (Zimmerman, 1993).

Sybulus Pascoe, 1871: 202 (Curculionidae)

Pascoe, 1871: 202 reported one undescribed species from West Papua in his collection. These species were never formally described.

Tomicoproctus Faust, 1898 (Curculionidae)

Zimmerman (1994a: 642), reported one new species from Bulolo, Papua New Guinea and illustrated this unnamed species in Zimmerman, 1992: plate 383. This is the first record of the genus from New Guinea.

Caridae Zimmerman, 1994a [=Carinae sensu Thompson, 1992]

In 2001, Wanat examined 3-4 undescribed species of an unknown genus related to Carodes Zimmerman from New Guinea in his assessment of phylogenetic relationships of Brentidae. This was the first published record of the family occurring in New Guinea. 


\section{INTRODUCED SPECIES}

\section{Biological control agents}

Five weevil species were introduced to Papua New Guinea by the Australian CSIRO as biological control agents of invasive plants. These species and their host plants are listed in Table 2 and are not included in the checklist. Cyrtobagous singularis Hustache was an unsuccessful control agent for Salvinia molesta leading Calder and Sands to investigate the reasons for the failure and discover a related species, $C$. salviniae which they described (Calder \& Sands 1985). This new species was introduced and rapidly controlled the weed (Zimerman, 1991, O’Brien 1995).

TABLE 2. Weevils introduced as biological control agents to the Papuan Region and the plants they control (from O’Brien \& Wibmer 1989 and Schneider 1999).

\begin{tabular}{ll}
\hline Biological control agent & Plant targeted \\
\hline Cyrtobagous salviniae Calder \& Sands & Salvinia molesta D. Mitch (Salviniaceae) \\
Cyrtobagous singularis Hustache & Salvinia molesta D. Mitch (Salviniaceae) \\
Microlarinus lypriformus (Wollaston) & Tribulus cistoides L. (Zygophyllaceae) \\
Neochetina bruchi Hustache & Eichhornia crassipes Solms (Pontederiaceae) \\
Neochetina eichhorniae Warner & Eichhornia crassipes Solms (Pontederiaceae) \\
Neohydronomus affinis Hustache & Pistia stratiotes L. (Araceae) \\
\hline
\end{tabular}

\section{Pollinator species}

The African species Elaeidobius kamerunicus (Faust) was introduced and is established in Papua New Guinea and the Bismarck Archipelago as a pollinator of the commercial oil palm, Elaeis guineensis Jacq. (Prior, 1983). This species is not included in the checklist.

\section{ACKNOWLEDGMENTS}

I thank Ralph Holzenthal, George Weiblen, and Susan Weller (all University of Minnesota), Scott Miller (USNH), Alexander Riedel (SMNK), and Charlie O'Brien for their suggestions and comments which greatly improved the manuscript. I am indebted to Steven Lingafelter, Norman Woodley, and Alexander Konstantinov (all USDA SEL), and the late E.C. Zimmerman for their encouragement and assistance in the early stages of this project. Julie Setliff was tireless in her support and assistance with editing, data entry, and proofreading. Jiri Hulcr (Michigan State University) provided valuable assistance with the bark beetles. I wish to acknowledge the considerable efforts of the librarians at the Entomology, Fisheries, and Wildlife Library and Wangenstein Library (both University of Minnesota) who endeavored to find many obscure references on my behalf. G.A. Samuelson (BPBM), Klaus Klass (SMTD) Hélène Perrin (MNHN), Patrick Grootaert (ISNB), and C.H.C. Lyal and M.V.L. Barclay (BMNH) provided information on and access to weevils in their respective collections for which I am grateful.

This checklist is based upon work supported by the National Science Foundation under grant number DEB-0211591 (G. Weiblen). Additional funding was provided by the Walter H. Judd International Graduate \& Professional Fellowship (University of Minnesota), Dayton and Wilkie Natural History Funds (Bell Museum, University of Minnesota), a Short Term Visitors Grant (Smithsonian Institution) (G. Setliff), and the University of Minnesota Experimentation Station Grant number MN17-022 (S. Weller). 
Genera and species that are incertae sedis, are arranged at the end of each relevant family-group taxon.

CURCULIONOIDEA

\section{NEMONYCHIDAE \\ RHINORHYNCHINAE \\ Mecomacerini}

\section{ANTHRIBIDAE}

ANTHRIBINAE

Basitropidini

Corrheccerini

Cratoparini

Discotenini

Ecelonerini

Mauiini

Mecocerini

Mycteini

Ozotomerini

Platystomini

Sintorini

Stenocerini

Tropiderini

Xenocerini

Xylinadini

Zygaenodini

CHORAGINAE

Apolectini

Araecerini

Choragini

\section{BELIDAE}

BELINAE

\section{RHYNCHITIDAE \\ RHYCHITINAE \\ Auletini \\ Auletobiina \\ Guineauletina \\ Auletorhinini \\ Deporaini \\ Rhynchitini}

ATTELABIDAE
ATTELABINAE

Euopini

BRENTIDAE

BRENTINAE

Arrhenodini

Eremoxenini

CYLADINAE

CYPHAGOGINAE

Cyphagogini

Hoplopisthiini

Stereodermini

EURHYNCHINAE

TRACHELIZINAE

Ithystenini

Microtrachelizini

Pseudoceocephalini

Trachelizini

APIONIDAE

APIONINAE

Ixapiini

RHADINOCYBINAE

Notapionini

\section{BRACHYCERIDAE}

OCLADIINAE

Desmidophorini

\section{DRYOPHTHORIDAE}

CRYPTODERMATINAE

DRYOPHTHORINAE

ORTHOGNATHINAE

Orthognathini

RHYNCHOPHORINAE

Diocalandrini

Litosomini

Polytini

Rhynchophorini

Sphenophorini 
STROMBOSCERINAE

\section{ERIRHINIDAE}

ERIRHININAE

TADIINAE

Tadiini

\section{CURCULIONIDAE}

BARIDINAE

Baridini

Baridina

Madarini

Leptoschoinina

Tonesiina

Madopterini

Madopterina

\section{CEUTHORHYNCHINAE}

Mecysmoderini

\section{CONODERINAE}

Arachnopodini

Campyloscelini

Phaenomerina

Coryssomerini

Lobotrachelini

Mecopini

Menemachini

Othippiini

Piazurini

Sphadasmini

COSSONINAE

Cossonini

Dryotribini

Onycholipini

Pentarthrini

Proecini

Rhyncolini

Rhyncolina

Phloeophagina

\section{CRYPTORHYNCHINAE}

Aedemonini

Camptorhinini

Cryptorhynchini

Cryptorhynchina

Mecistostylina

Tylodina

Gasterocercini

Psepholacini
CURCULIONINAE

Acalyptini

Cenchrenini

Ceratopodini

Curculionini

Curculionina

Pseudobalaninina

Rhamphini

Rhamphina

Storeini

Tychiini

Demimaeina

Ochyromerina

Tychiina

CYCLOMINAE

Aterpini

ENTIMINAE

Celeuthetini

Cyphicerini

Cyphicerina

Elytrurini

Eupholini

Naupactini

Ottistirini

Pachyrhynchini

Tanymecini

Piazomiina

HYPERINAE

Hyperini

Cepurini

LIXINAE

Lixini

MESOPTILIINAE

Laemosaccini

MOLYTINAE

Aminyopini

Cleogonini

Hylobiini

Hylobiina

Ithyporini

Ithyporina

Colobodina

Lithinini

Lithinina

Mecysolobini

Metatygini 
Molytini

Molytina

Paipalesomini

Pissodini

Orthorhinina

Pissodina

Trachodini

Trigonocolini

Trypetidini

PLATYPODINAE

Platypodini

Platypodina

Tesserocerini

Diapodina

Tesserocerina

SCOLYTIDINAE
Hylesinini

Diamerina

Hylesinina

Hyorrhynchina

Phloeosinina

Phloeotribina

Tomicina

Scolytini

Corthylina

Cryphalina

Dryocoetina

Ipina

Scolytoplatypodina

Xyleborina

Xyloctonina

\section{CHECKLIST}

\section{CURCULIONOIDEA}

\section{NEMONYCHIDAE}

\section{RHINORHYNCHINAE}

\section{MECOMACERINI}

ARAGOMACER Kuschel in Zimmerman, 1994a: 600

TYPE-SPECIES: Aragomacer leai Kuschel in Zimmerman, 1994a; original designation. KeY: Kushel in Zimmerman, 1994a: 601.

grayi Kuschel in Zimmerman, 1994a: 601. (Aragomacer)

DisTRIBUTION: Papua New Guinea: Wau.

munus Kuschel in Zimmerman, 1994a: 604. (Aragomacer)

DisTRIBUTION: Papua New Guinea.

papuae Kuschel in Zimmerman, 1994a: 606. (Aragomacer)

DisTRIBUTION: Papua New Guinea: Wau.

\section{ANTHRIBIDAE}

\section{ANTHRIBINAE}

\section{BASITROPINI}

BASITROPIS Jekel, 1855: 90

TYPE-SPECIES: Basitropis nitidicutis Jekel, 1855; original designation. 
diluta diluta Jordan, 1903: 434. (Basitropis)

Distribution: Papua New Guinea: Bulolo, Fly River, Garaina, Goilala, Lae, Kiunga, Owen Stanley Range, Port Moresby, Wau.

diluta rostralis Wolfrum, 1949: 146. (Basitropis)

DisTRIBUTION: Bismarck Archipelago: New Britain.

diluta ventralis Frieser, 1983: 54. (Basitropis)

DistRiBUtion: Papua New Guinea: Fly River, Kerema, Kiunga, Upper Jimmi Valley.

dolosa dolosa Jordan, 1924a: 243. (Basitropis)

Distribution: Papua New Guinea: Bulolo, Fly River, Kiunga, Upper Jimmi Valley, Wau.

dolosa quadrispinosus Frieser, 1983: 55. (Basitropis)

DistRIBUTION: Papua New Guinea: Fly River, Wau.

dolosa tenuiclava Frieser, 1983: 55. (Basitropis)

DisTRIBUTION: Bismarck Archipelago: New Britain.

humeralis Jordan, 1903: 433. (Basitropis)

DISTRIBUTION: Solomon Islands.

papuensis Jordan, 1924a: 243. (Basitropis)

Distribution: Papua New Guinea: Bulldog Trail, Bulolo, Fly River, Kiunga, Kokoda, Mt. Missim,

Popondetta, Torricelli Mountains, Wau; West Papua: Geelvink Bay, Mayuby-Benyas.

postica Frieser, 1983: 56. (Basitropis posticus)

DistRibution: Papua New Guinea: Fly River, Garaina, Kiunga, Port Moresby, Wau; West Papua: Mayuby-Benyas.

rara Frieser, 1983: 56. (Basitropis rarus)

DistRIBUtion: Papua New Guinea: Mt. Missim, Wau, Woitape.

relicta Blackburn, 1900: 154. (Basitropis)

DisTRIBUTION: Papua New Guinea.

Basitropis epipona Jordan, 1924a: 243. Distribution: Papua New Guinea: Garaina, Mt. Missim, Owen Stanley Range, Star Mountains, Wau.

sedlaceki Frieser, 1983: 53. (Basitropis)

DisTRIBUTION: Papua New Guinea: Fly River; West Papua: Batanta Island.

truncalis Jordan, 1937: 202. (Basitropis)

DISTRIBUTION: Solomon Islands.

Plesiobasis Jordan, 1939a: 427

TYPE-SPECIES: Plesiobasis monera Jordan, 1939a; original designation.

brunneovaria Frieser, 1992: 33. (Plesiobasis)

DistRIBUTION: West Papua: Cyclops Mountains.

multiguttata Frieser, 1992: 32. (Plesiobasis)

DistRIBUTION: West Papua: Mt. Meja.

\section{CORRHECERINI}

ANTIOXENUS Jordan, 1924a: 235

TYPE-SPECIES: Antioxenus bennigseni Jordan, 1924a; monobasic.

bennigseni Jordan, 1924a: 235. (Antioxenus)

DisTRIBUTION: Papua New Guinea. 
TYPE-SPECIES: Apatenia viduata Pascoe, 1859b; monobasic. KEY: Frieser, 1985: 96.

brevior Frieser, 1985: 92. (Apatenia)

DisTRIBUTION: Papua New Guinea: Madang.

cyclops Frieser, 1985: 88. (Apatenia)

DistRiBution: West Papua: Cyclops Mountains, Ifar, Mt. Meja.

festiva Jordan, 1932: 363. (Apatenia)

Distribution: Papua New Guinea: Lake Trist, Mt. Kaindi, Mt. Missim, Nami Creek, Salawakit Range, Wau.

gazellae Frieser, 1985: 95. (Apatenia)

DisTRIBUTION: Bismarck Archipelago: New Britain.

gracilis Jordan, 1903: 419. (Apatenia)

DISTRIBUTION: D'Entrecasteaux Islands.

grumosa Jordan, 1932: 375. (Apatenia)

DistRIBUTION: West Papua: Cyclops Mountains.

gularis Jordan, 1904: 86. (Apatenia)

Distribution: Papua New Guinea.

imprima Frieser, 1985: 90. (Apatenia)

DisTRIBUTION: Papua New Guinea.

indecorata Frieser, 1985: 92. (Apatenia)

DisTRIBUTION: Papua New Guinea: Kokoda.

irrorata Macleay, 1887: 196. (Apatenia)

DistRIBUTION: Papua New Guinea: Fly River.

milnei milnei Jordan, 1903: 417. (Apatenia)

Distribution: Papua New Guinea: Madang; West Papua: Dafo West of Jayapura.

milnei subgibbosa Frieser, 1985: 88. (Apatenia)

DistRIBUTION: Papua New Guinea: Fly River.

minor minor Jordan, 1894: 632. (Apatenia)

Distribution: Papua New Guinea: Lae, Mt. Wilhelm; West Papua.

minor signaticollis Frieser, 1985: 92. (Apatenia)

Distribution: Papua New Guinea: Nadzab, Torricelli Range; West Papua: Cyclops Mountains.

minor nigripennis Frieser, 1992: 29. (Apatenia)

DistRIBUTION: Papua New Guinea; West Papua; Biak Island.

nigroflava Frieser, 1985: 94. (Apatenia)

DISTRIBUTION: Bismarck Archipelago: New Britain.

olivacea Jordan, 1903: 418. (Apatenia)

Distribution: Papua New Guinea: Baiyer River, Bisianumu East of Pt. Moresby, Wau; Woodlark

Islands; West Papua: Cyclops Mountains.

pallidicepes Jordan, 1895d: 391. (Apatenia)

Distribution: Papua New Guinea.

phaeura Jordan, 1903: 418. (Apatenia)

DisTRIBUTION: Papua New Guinea.

plagifer Frieser, 1985: 93. (Apatenia)

DisTRIBUTION: West Papua: Nabire South of Geelvink Bay, Sentari, Waris South of Jayapura.

poecila Jordan, 1904: 85. (Apatenia)

Distribution: Papua New Guinea: Kokoda, Milne Bay; D’Entrecasteaux Islands: Normanby Island. 
raniceps raniceps Jordan, 1894: 631. (Apatenia)

Distribution: Papua New Guinea: Madang; West Papua: Bomberi, Cyclops Mountains, Vogelkop.

raniceps rectangula Frieser, 1985: 96. (Apatenia)

DistRIBUTION: Bismarck Archipelago: New Britain; West Papua: Cyclops Mountains.

raniceps rufovariegata Frieser, 1985: 96. (Apatenia)

DistRIBUTION: West Papua: Cyclops Mountains, Sabron.

subvittigera subvittigera Frieser, 1985: 93. (Apatenia)

DistRIBUTION: Papua New Guinea: Madang; Bismarck Archipelago: New Britain.

subvittigera suturata Frieser, 1985: 93. (Apatenia)

DisTRIBUTION: Papua New Guinea: Maprik.

unituberculata Macleay, 1887: 196. (Apatenia)

DistRIBUTION: Papua New Guinea: Fly River.

CaCePHatus Blackburn, 1900: 151

TYPE-SPECIES: Cacephatus serieus Blackburn, 1900; monobasic.

carinulatus Frieser, 1997: 56. (Caccephatus)

DisTRIBUTION: West Papua: Meja.

obscurus Frieser, 1997: 57. (Caccephatus)

Distribution: Papua New Guinea: Wau; West Papua: Sibil Valley, Star Mountains.

DiNOMELAENA Jordan, 1928: 113

TYPE-SPECIES: Apatenia scelesta Pascoe, 1862a; original designation. KEY: Frieser, 1985: 87.

bituberculata Frieser, 1993: 3. (Dinomelaena)

DistRIBUTION: Bismarck Archipelago: New Britain, New Ireland; West Papua: Cyclops Mountains, Nabire, Pemukiman.

immaculata immaculata (Jordan), 1894: 633. (Apatenia)

Distribution: Papua New Guinea: Fly River, Mt. Missim, Sepik River, Wau, Wewak; Bismarck Archipelago: New Ireland; West Papua: Cyclops Mountains, Humboldt Bay, Star Mountains; Aru Islands; Biak Island.

immaculata diversicollis Frieser, 1985: 85. (Dinomelaena)

Distribution: Papua New Guinea; Bismarck Archipelago: New Britain; West Papua: Lab Lab.

impressifrons Frieser, 1992: 28. (Dinomelaena)

DISTRIBUTION: West Papua: Kangime.

quadrituberculata (Montrouzier), 1855: 40. (Stenocerus)

Distribution: Papua New Guinea: Astrolabe Bay, Kuper Range, Milne Bay, Moroka, Sattelberg;

D’Entrecasteaux Islands: Fergusson Island, Normanby Island; Louisaide Archipelago: Sud-East Island;

Trobriand Islands; Woodlark Islands. NOTES: Transferred: Jordan, 1928: 113; Lectotype designation: Kuschel, 1998: 193.

scelesta scelesta (Pascoe), 1862a: 334. (Nessiara)

Distribution: Papua New Guinea: Redscar Bay; West Papua: Cyclops Mountains, Geelvink Bay, Mt. Meja.

scelesta angulicollis (Jordan), 1897: 177. (Apatenia)

Distribution: Papua New Guinea: Fly River, Mt. Missim, Sepik River, Wau, Wewak; Bismarck Archipelago: New Ireland; West Papua: Cyclops Mountains, Mt. Meja, Humboldt Bay, Star Mountains; Aru Islands, Biak Island. 
scelesta innotata Frieser, 1985: 84. (Dinomelaena)

DistRIBUTION: Papua New Guinea: Bulolo, Mt. Kaindi.

scripta Frieser, 1985: 86. (Dinomelaena)

DISTRIBUTION: Bismarck Archipelago: New Britain.

sedlaceki Frieser, 1985: 86. (Dinomelaena)

Distribution: Papua New Guinea: Mt. Kaindi, Wau.

testacea Frieser, 1992: 27. (Dinomelaena)

Distribution: West Papua: Mt. Meja.

tuberculosa (Jordan), 1894: 632. (Apatenia)

Distribution: Papua New Guinea: West Papua: Cyclops Mountains, Njau-limon South of Mt. Bougainville.

HABRISSUS Pascoe, 1859b: 432

TYPE-SPECIES: Habrissus pilicornis Pascoe, 1859b; monobasic.

pilicornis Pascoe, 1859b: 432. (Habrissus)

DISTRIBUTION: Aru Islands.

puncticollis Frieser, 1997: 59. (Habrissus)

DistRIBUTION: West Papua: Samboka, Upper Kolff River.

HyPSEUS Pascoe, 1860a: 39

TYPE-SPECIES: Hypseus fascicularis Pascoe, 1860a; monobasic. KeY: Frieser, 1997: 27.

aequabilis Frieser, 1997: 16. (Hypseus)

DisTRIBUTION: West Papua: Epomani.

amplicollis Frieser, 1997: 22. (Hypseus)

Distribution: Papua New Guinea: Fly River, Kiunga.

apicenotatus Frieser, 1997: 25. (Hypseus)

DisTRIBUTION: West Papua: Epomani.

axilaris axilaris Jordan, 1895d: 391. (Hypseus)

DISTRIBUTION: [Sumatra]; Papua New Guinea (?).

axillaris major Jordan, 1904: 87. (Hypseus)

DisTRIBUTION: Papua New Guinea.

batantae Frieser, 1997: 20. (Hypseus)

DISTRIBUTION: Batanta Island; Waigeo Island.

callosiventris Frieser, 1997: 23. (Hypseus)

DistRIBUTION: Papua New Guinea: Madang.

deplanatus Frieser, 1997: 15. (Hypseus)

DistRIBUtion: Papua New Guinea: Mt. Kaindi, Nami Creek, Wau.

formidatus Frieser, 1997: 23. (Hypseus)

DistRIBUTION: West Papua: Epomani.

maculiventris Frieser, 1997: 21. (Hypseus)

Distribution: West Papua: Nabire, Pemukiman.

mejai Frieser, 1997: 12. (Hypseus)

Distribution: West Papua: Mt. Meja.

nigriceps Frieser, 1997: 13. (Hypseus) 
Distribution: West Papua: Kwau, Hatam.

nigrosuturatus Frieser, 1997: 17. (Hypseus)

DistRIBUTION: West Papua: Testega-Meydoudga.

penicillus Frieser, 1997: 20. (Hypseus)

DiSTRIBUTION: West Papua: Angguruk-Tanggeam, Nabire, Wamena.

pustulifer Frieser, 1997: 16. (Hypseus)

DistRIBUTION: West Papua: Minyambou.

riedeli Frieser, 1992: 29. (Hypseus)

Distribution: Papua New Guinea: Bulolo; West Papua: Mt. Meja, Mayuby-Benyas.

salawattii salawattii Frieser, 1997: 24. (Hypseus)

DISTRIBUTION: West Papua: Salawatti Island.

salawattii robustior Frieser, 1997: 25. (Hypseus)

DisTRIBUTION: West Papua: Manokawari.

strigifer Frieser, 1997: 14. (Hypseus)

DistRIBUTION: West Papua: Sentani.

NeSSIARA Pascoe, 1860b: 60

TYPE-SPECIES: Nessia didyma Pascoe, 1859a; subsequent designation by Jordan, 1894: 630.

transversa Frieser, 1992: 26. (Nessiara)

DistRIBUTION: West Papua: Mt. Meja.

PANTORHaEnas Jordan, 1928: 126

TYPE-SPECIES: Pantorhaenas conspersus Jordan, 1928; original designation.

laevicollis Frieser, 1997: 39. (Pantorhaenas)

Distribution: West Papua: Epomani.

Phaulimia Pascoe, 1859b: 437

TYPE-SPECIES: Phaulimia ephippiata Pascoe, 1859b; monobasic.

inculta Frieser, 1997: 48. (Phaulimia)

Distribution: West Papua: Cyclops Mountains, Jayapura, Sentani.

punctata (Montrouzier), 1855: 40. (Stenocerus punctatus)

DistRIBUTION: Woodlark Islands. Notes: Transferred from Stenocerus to Hylopemon: Wolfrum, 1929:

40; Transferred from Hylopemon and Lectotype designation: Kuschel, 1998: 194.

\section{CRATOPARINI}

EUPARIUS Schönherr, 1823: 1135

TYPE-SPECIES: Anthribus lunatus Fabricius, 1801 [=Macrocephalus marmoreus Oliver, 1795]; monobasic. KeY: Zimmerman, 1994a: 119

lateripictus (Jordan), 1895a: 201. (Caccorhinus)

DisTRIBUTION: Papua New Guinea; West Papua: Kagime. NOTES: Transferred: Zimmerman, 1994a: 122. 


\section{DISCOTENINI}

ANCYLOTROPIS Jekel, 1855: 94

TYPE-SPECIES: Ancylotropis waterhousei Jekel, 1855; monobasic. KEY: Zimmerman, 1994a: 63.

scabratus (Motschulsky), 1875: 232. (Macrotrichus)

Distribution: Papua New Guinea; West Papua: Cyclops Mountains.

waterhousei Jekel, 1855: 96. (Ancylotropis)

DisTRIBUTION: Papua New Guinea.

Macrotrichus niveinasus Motschulsky, 1875: 232.

Genethila Pascoe, 1860a: 41

TYPE-SPECIES: Genethila retusa Pascoe, 1860a; monobasic.

instructa Frieser, 1989: 105. (Genethila)

Distribution: Papua New Guinea: Aroa River, Fly River, Kiunga, Redscar Bay, Wau.

\section{ECELONERINI}

DENDROPEMON Schönherr, 1839: 161

TYPE-SPECIES: Anthribus perfolicornis Fabricius, 1801; original designation. KEY: Zimmerman, $1994 \mathrm{a}$ : 84 (includes $D$. marmoratus only).

arciferus (Blanchard), 1853: 196. (Xenocerus)

Distribution: Papua New Guinea: Fly River. Notes: Transferred from Ecelonerus: Zimmerman, 1994a: 91.

hypocrita hypocrita (Jekel), 1855: 82. (Dendrotrogus)

DistRIBUTION: West Papua.

hypocrita levis (Jordan), 1929: 337. (Dendrotrogus)

DISTRIBUTION: West Papua: Biak Island.

marmoratus (Montrouzier), 1855: 45. (Eucorhinus)

DistRIBUTION: D’Entrecasteaux Islands; Woodlark Islands; West Papua: Andai, Mayuby. Notes: Transferred: Jordan, 1913: 211 and Zimmerman, 1994a: 90; Lectotype designation: Kuschel, 1998: 194; Illustrated in Zimmerman, 1994a: 86 under name D. papuanus.

Dendrotrogus colligens papuanus Jordan, 1904: 84. NOTES: Synonoymy: Valentine, 1972: 465; Frieser, 2002: 31 treats as valid.

molitor (Jordan), 1923b: 219. (Ecelonerus)

DisTRIBUTION: New Guinea; Aru Islands; Woodlark Island. NOTES: Transferred: Zimmerman, 1994a: 92.

EUCORYNUS Schönherr, 1823: 1135

TYPE-SPECIES: Anthribus crassicornus Fabricius, 1801; original designation.

crassicornis (Fabricius), 1801: 407. (Anthribus)

Distribution: Papua New Guinea; West Papua.

Eucorynus stevensi Pascoe, 1859b: 433. Distribution: West Papua: Cyclops Mountains, Dorey. NOTES: Synonoymy: Valentine, 1972: 462. Rheinheimer, 2004a and Frieser, 2002 treat as valid. 
HYLOPEMON Jekel, 1860: 238

TYPE-SPECIES: Anthribus garnotii Guérin-Méneville, [1833]; subsequent designation by Alonso-Zarazaga \& Lyal, 1999: 31.

garnotii (Guérin-Méneville), 1833: 108. (Anthribus)

DISTRIBUTION: New Guinea.

\section{JORDANTHRIBINI}

DINEMA Fairmaire, 1849b: 457

TYPE-SPECIES: Dinema filicorne Fairmaire, 1849b; monobasic.

aper (Jordan), 1939b: 441. (Proscopus)

DisTRIBUTION: Solomon Islands.

vellanum (Jordan), 1939b: 441. (Proscopus)

DISTRIBUTION: Solomon Islands.

\section{MAUIINI}

MAUIA Blackburn, 1885: 194

TYPE-SPECIES: Mauia satelles Blackburn, 1885 [=Araeocerus subnotatus Boheman, 1859]; monobasic.

colorata Frieser, 1992: 36. (Mauia)

DistRIBUtion: West Papua: Melanggama, Pass Valley.

ochra Wolfrum, 1956: 116. (Mauia)

DistRIBUTION: Papua New Guinea.

ovalis Frieser, 1992: 35. (Mauia)

DisTRIBUTION: West Papua: Mayuby.

pithekis Wolfrum, 1956: 116. (Mauia)

DisTRIBUTION: Papua New Guinea.

rotunda Frieser, 1992: 35. (Mauia)

DisTRIBUTION: West Papua: Mayuby.

squalens Jordan, 1923a: 185. (Mauia)

DisTRIBUTION: Papua New Guinea.

stephana Wolfrum, 1956: 116. (Mauia)

DisTRIBUTION: Papua New Guinea.

Protaedus Pascoe, 1860a: 39

TYPE-SPECIES: Protaedus moerens Pascoe, 1860a; monobasic. KeY: Frieser, 2001b: 57.

batantae batantae Frieser, 2001b: 47. (Protaedus)

DISTRIBUTION: Batanta Island.

batantae salawattii Frieser, 2001b: 48. (Protaedus)

Distribution: West Papua: Salawatti Island.

dentifer Frieser, 2001b: 48. (Protaedus) 
DISTRIBUTION: Batanta Island; Salawatti Island.

excisus Frieser, 1995a: 6. (Protaedus)

DisTRIBUTION: Papua New Guinea.

flavoangulatus Frieser, 2001b: 53. (Protaedus)

DistRIBUtion: West Papua: Borme to Omban.

flavus Frieser, 2001b: 51. (Protaedus)

Distribution: Papua New Guinea: Wau.

fuscatus Frieser, 2001b: 50. (Protaedus)

DisTRIBUTION: West Papua: Jayapura.

grandis Frieser, 2001b: 49. (Protaedus)

Distribution: Papua New Guinea: Angorum, Wewak; West Papua; Waigeo Island.

humeralis Jordan, 1923a: 183. (Protaedus)

DisTRIBUTION: D'Entrecasteaux Islands.

leptacinus Frieser, 1995a: 7. (Protaedus)

Distribution: Papua New Guinea: Mt. Missim.

leucomelas Jordan, 1923a: 184. (Protaedus)

DisTRIBUTION: Papua New Guinea.

litturatus Frieser, 2001b: 46. (Protaedus)

DistRibution: Papua New Guinea: Albert Mountains, Bodem, Wanama.

nigromarginatus Frieser, 2001b: 55. (Protaedus)

Distribution: Papua New Guinea: Wau.

pallidus Jordan, 1923a: 181. (Protaedus)

DisTRIBUTION: Papua New Guinea.

robusticornis Frieser, 1995a: 5. (Protaedus)

Distribution: West Papua.

seramis griseolus Frieser, 2001b: 57. (Protaedus)

DISTRIBUTION: Batanta Island.

signatellus Frieser, 2001b: 48. (Protaedus)

DistRIBUTION: West Papua: Lereh.

signatus Frieser, 1995a: 3. (Protaedus)

Distribution: Papua New Guinea; West Papua.

socius socius Frieser, 1992: 34. (Protaedus)

DistRIBUtion: Papua New Guinea: Brown River near Pt. Moresby; West Papua: Cyclops Mountains, Ifar,

Mt. Meja, Mayuby.

socius obtusus Frieser, 1992: 35. (Protaedus)

DisTRIBUTION: Bismarck Archipelago: New Britain.

trisulcatus Frieser, 2001b: 45. (Protaedus)

DisTRIBUTION: West Papua: Festega.

waigeoi Frieser, 2001b: 56. (Protaedus)

DisTRIBUTION: West Papua: Waigeo Island.

weigeli Frieser, 2001b: 49. (Protaedus)

DISTRIBUTION: West Papua: Nabire, Yamor Lake.

\section{MECOCERINI}


TYPE-SPECIES: Dolichocera childreni Gray, 1832; monobasic.

pupa pupa (Jordan), 1895a: 132. (Meganthribus)

DistRIBUTION: Papua New Guinea.

pupa papuanus (Jordan), 1928: 106. (Meganthribus)

DisTRIBUTION: West Papua: Geelvink Bay, Korido.

ECZESARIS Pascoe, 1859a: 330

TYPE-SPECIES: Eczesaris atomaria Pascoe, 1859a; monobasic. NOTES: Transferred from Cappadocini in Lyal \& AlonsoZarazaga, 2006.

atomaria atomaria Pascoe, 1859a: 331. (Eczesaris)

Distribution: Papua New Guinea: Fly River, Milne Bay, Kiunga; West Papua: Cyclops Mountains, Geelvink Bay; Aru Island; Biak Island.

atomaria aureomaculata Frieser, 1992: 23. (Eczesaris)

DISTRIBUTION: Biak Island.

atomaria cyclopis Frieser, 1992: 23. (Eczesaris)

DistRIBUTION: West Papua: Cyclops Mountains.

atomaria fuscata Frieser, 1999: 23. (Eczesaris)

DISTRIBUTION: Waigeo Island.

bolana Jordan, 1937: 200. (Eczesaris)

DisTRIBUTION: Papua New Guinea: Mt. Kainde.

brevis (Jordan), 1924a: 237. (Idiopus)

DistRiBution: Papua New Guinea: Bulolo, Mt. Missim, Wau.

franklini-muelleri (Wolfrum), 1925: 165. (Idiopus)

Distribution: Papua New Guinea: Mt. Missim, Wau; West Papua: Cyclops Mountains.

penicillus (Jordan), 1898: 363. (Idiopus)

Distribution: Papua New Guinea: Garina, Kokoda, Mailu Island, Popondetta.

striga (Lacordaire), 1866: 512. (Idiopus)

DisTRIBUTION: Papua New Guinea.

verruca verruca (Jordan), 1898: 364. (Idiopus verrucosus)

Distribution: Papua New Guinea: Fly River, Kiunga. West Papua: Cyclops Mountains, Ifar, Vogelkop. verruca circumornata Frieser, 1983: 52. (Eczesaris)

DistRIBUTION: Bismarck Archipelago: New Britain; West Papua: Mt. Meja.

MeCOTROPUS Lacordaire, 1866: 495

TYPE-SPECIES: Mecotropus bipunctata Lacordaire, 1866; original designation.

pantherinus Thomson, $1857 \mathrm{~b}$ : 436. (Mecocerus)

Distribution: Papua New Guinea; West Papua: Mt. Meja; Aru Island.

MeGAX Alonso-Zarazaga \& Lyal, 1999: 31

TYPE-SPECIES: Eugigas schönheri Thomson, 1857b; subsequent designation by Alonso-Zarazaga \& Lyal, 1999: 31. 
schoenherri (Thomson), 1857b: 435. (Eugigas schönheri)

DisTRIBUTION: New Guinea; Bismarck Archipelago; Aru Island.

\section{MYCTEINI}

\section{ALTIPECTUS Jordan, 1894: 605}

TYPE-SPECIES: Altipectus fasciatum Jordan, 1894; original designation.

fasciatum Jordan, 1894: 606. (Altipectus)

DistRIBUTION: Papua New Guinea.

SYMPAECTOR Kirsch, 1875: 52

TYPE-SPECIES: Sympaector vittifrons Kirsch, 1875; monobasic.

longicornis Jordan, 1894: 604. (Sympaector)

DisTRIBUTION: Papua New Guinea; West Papua.

\section{OZOTOMERINI}

Ozotomerus Perroud, 1853: 406

TYPE-SPECIES: Ozotomerus maculosus Perroud, 1853; monobasic.

bipunctatus (Montrouzier), 1855: 46. (EEdecerus)

DistRIBUTION: D’Entrecasteaux Islands; Woodlark Islands; West Papua: Cyclops Mountains, Mayuby;

Aru Islands. NOTES: Transferred: Lacordaire, 1866: 572; Lectotype designation: Kuschel, 1998: 194.

Ozotomerus waterhousei Pascoe, 1859a: 332.

\section{PLATYSTOMINI}

\section{DoEOThENA Pascoe, 1862a: 331}

TYPE-SPECIES: Doeothena platypoda Pascoe, 1862a; monobasic.

platypoda Pascoe, 1862a: 332. (Doeothena)

DisTRIBUTION: Papua New Guinea.

EXILlis Pascoe, 1860a: 43

TYPE-SPECIES: Exillis longicornis Pascoe, 1860a; monobasic. KEYS: Frieser, 1989: 121; Frieser, $1999: 35$.

aberrans Frieser, 1997: 70. (Exillis)

DisTRIBUTION: West Papua: Bilogay, Enarotadi.

aeruginosus Frieser, 1997: 69. (Exillis)

Distribution: West Papua: Anggi, between Kono and Piji, Wandanku.

distans Frieser, 1989: 120. (Exillis)

Distribution: Papua New Guinea: Busu River East of Lae; Bismarck Archipelago: New Britain; West

Papua: Geelvink Bay, Nabire. 
fasciger Frieser, 1989: 118. (Exillis)

DisTRIBUTION: Papua New Guinea: Enarotadi, Wisselmeren.

impressus impressus Wolfrum, 1956: 117. (Exillis)

Distribution: Papua New Guinea: Goilala, Kokoda, Wau; West Papua: Bomberi, Okaitadi, Vogelkop,

Wisselmeren.

impressus discoidalis Frieser, 1989: 118. (Exillis)

DistRIBUTION: Bismarck Archipelago: New Britain; West Papua: Jayapura.

papuensis papuensis Frieser, 1989: 119. (Exillis)

Distribution: Papua New Guinea: Brown River to Pt. Moresby, Daru Island, Mt. Missim, Wau.

papuensis subcarinatus Frieser, 1989: 120. (Exillis)

DistRIBUTION: Papua New Guinea: Goilala, Milne Bay.

rectangulus Frieser, 1989: 117. (Exillis)

DistRIBUTION: Papua New Guinea: Aiyura, Eastern Highlands, Hagen, Western Highlands.

sexcarinatus Frieser, 1989: 119. (Exillis)

DistRIBUTION: West Papua: Kebar Valley, Vogelkop.

subparallelus Frieser, 1989: 117. (Exillis)

DisTRIBUTION: Bismarck Archipelago: New Britain, New Ireland.

waigeoi Frieser, 1997: 69. (Exillis)

DISTRIBUTION: West Papua: Waigeo Island.

woodlarkianus Frieser, 1989: 118. (Exillis)

DISTRIBUTION: Woodlark Islands.

Penestica Pascoe, 1859a: 332

TYPE-SPECIES: Penestica inepta Pascoe, 1859a; monobasic.

inepta Pascoe, 1859a: 332. (Penestica)

DISTRIBUTION: Aru Islands.

samuelsoni Frieser, 2001a: 21. (Penestica)

DISTRIBUTION: Bismarck Archipelago: New Britain.

PHLOEOBIUS Schönherr, 1823: 1135

TYPE-SPECIES: Anthribus griseus Fabricius, 1792 [=Ptinus gigas Fabricius, 1775]; original designation.

gigas (Fabricius), 1775: 63. (Anthribus)

Distribution: Papua New Guinea; West Papua: Cyclops Mountains.

Anthribus griseus Fabricius, 1792: 377.

Anthribus longicornis Fabricius, 1798: 160.

papuanus Jordan, 1904: 89. (Phloeobius)

DistRIBUTION: Papua New Guinea; West Papua: Mayuby-Benyas.

Pioenia Pascoe, 1862a: 332

TYPE-SPECIES: Pioenia saginata Pascoe, 1862a; original designation.

ingentus (Frieser), 1992: 31. (Tropidobasis)

Distribution: Papua New Guinea: Busu River North East of Lae, Kokoda; West Papua: Mayuby, Potondetta; Morotai Island. 
TYPE-SPECIES: Pioenia divisa Jordan, 1923a; original designation.

albopicta Frieser, 2001a: 22. (Pioenidia)

Distribution: West Papua.

edentula Frieser, 2001a: 23. (Pioenidia)

DistRIBUTION: West Papua.

Platystomos Schneider, 1791: 21

TYPE-SPECIES: Curculio albinus Linnaeus, 1758 [see note in Alonso-Zarazaga \& Lyal, 1999: 33]; subsequent designation by Pierce, 1916: 463.

wallacei (Pascoe), 1860a: 47. (Anthribus)

DistRIBUTION: Papua New Guinea; West Papua; Aru Island.

TROPIDOBASIS Jordan, 1923a: 178

TYPE-SPECIES: Tropidobasis plasta Jordan, 1923a; original designation.

maculipes Frieser, 1993: 12. (Tropidobasis)

DistRIBUtion: Papua New Guinea: Fischhafen, Fly River, Kiunga; West Papua: Nabire, Pemukiman.

passer Frieser, 2001a: 19. (Tropidobasis)

DisTRIBUTION: Papua New Guinea.

rugulosa Frieser, 1993: 11. (Tropidobasis)

Distribution: West Papua: Bodem, Nabire, Pemukiman.

weigeli Frieser, 2001b: 43. (Tropidobasis)

DISTRIBUTION: West Papua: Nabire.

\section{SINTORINI}

Callanthribus Jordan, 1904: 82

TYPE-SPECIES: Callanthribus xanthomelas Jordan, 1904; original designation.

xanthomelas Jordan, 1904: 83. (Callanthribus)

DisTRIBUTION: Papua New Guinea.

EUSINTOR Jordan, 1904: 80

TYPE-SPECIES: Eusintor loriae Jordan, 1904; original designation.

cuprifer Frieser, 1983: 50. (Eusintor)

Distribution: Papua New Guinea: Mt. Gilum.

elegans Frieser, 1983: 49. (Eusintor)

Distribution: Papua New Guinea: Daulo Pass, Mt. Missim, Wau; West Papua: Vogelkop.

loriae Jordan, 1904: 81. (Eusintor)

DISTRIBUTION: Papua New Guinea. 
nigrorufus Frieser, 1983: 48. (Eusintor)

DisTRIBUTION: Papua New Guinea: Torricelli Mountains.

\section{STENOCERINI}

Esocus Pascoe, 1859b: 436

TYPE-SPECIES: Esocus lacrymans Pascoe, 1859b; monobasic

vittiger Frieser, 1992: 25. (Esocus)

DistRIBUTION: West Papua: Cyclops Mountains.

Plintheria Pascoe, 1859b: 435

TYPE-SPECIES: Plintheria luctuosa Pascoe, 1859b; monobasic.

biroi Wolfrum, 1956: 110. (Plintheria)

DisTRIBUTION: Papua New Guinea.

luctuosa Pascoe, 1859b: 436. (Plintheria)

DistRIBUTION: Papua New Guinea; West Papua: Mayuby.

morokana Jordan, 1898: 362. (Plintheria)

Distribution: Papua New Guinea; West Papua: Mayuby-Benyas.

paucis Frieser, 1992: 24. (Plintheria)

Distribution: West Papua: Belem, Enarotadi, Pass-Valley, Wisselmeren.

salomonis Frieser, 1983: 49. (Plintheria)

Distribution: Papua New Guinea.

woodlarkiana Jordan, 1898: 363. (Plintheria)

DISTRIBUTION: Woodlark Islands.

\section{TROPIDERINI}

ACORYNUS Schönherr, 1833: 123

TYPE-SPECIES: Acorynus sulcirostris Boehman in Schönherr, 1833; monobasic.

cruralis angustefasciatus Frieser, 1989: 106. (Acorynus)

DisTRIBUTION: Papua New Guinea; Bismarck Archipelago: New Britain.

eurous (Jordan), 1895d: 386. (Hucus)

DistRIBUTION: West Papua: Mayuby, Moroka. Notes: Transferred: Frieser, 1989: 106.

litigiosus (Pascoe), 1860a: 46. (Litocerus)

Distribution: West Papua: Cyclops Mountains, Dorey.

Hucus Pascoe, 1859b: 436

TYPE-SPECIES: Hucus melanostoma Pascoe, 1859b; monobasic.

parvulus (Macleay), 1887: 195. (Litocerus)

DistRIBUtION: Papua New Guinea: Fly River. NOTES: Transferred: Frieser, 1989: 107.

virgatus Jordan, 1911: 107. (Hucus)

Distribution: Papua New Guinea; Bismarck Archipelago: New Britain; West Papua: Mayuby. 
TYPE-SPECIES: Litocerus histrio Gyllenhal in Schönherr, 1833; original designation.

fasciatus Macleay, 1887: 195. (Litocerus)

DISTRIBUTION: Papua New Guinea: Fly River.

histrio fluviatilis Jordan, 1904: 85. (Litocerus)

DisTRIBUTION: Papua New Guinea; West Papua: Mt. Meja.

macrophtalmus (Montrouzier), 1855: 41. (Stenocerus)

Distribution: Papua New Guinea; Woodlark Island. NoTES: Transferred: Jordan, 1911: 106; see discussion in Kuschel, 1998: 194.

perplexus Pascoe, 1860a: 45. (Litocerus)

Distribution: Papua New Guinea; West Papua: Cyclops Mountains.

rhombicus Jordan, 1908: 351. (Litocerus)

DisTRIBUTION: West Papua: Waigeo Island.

striatus Jordan, 1894: 614. (Litocerus)

DistRIBUTION: West Papua: Cyclops Mountains.

striatus inflexus Frieser, 1992: 24. (Litocerus)

DisTRIBUTION: West Papua.

variegatus Jordan, 1894: 613. (Litocerus)

Distribution: Papua New Guinea; West Papua: Cyclops Mountains, Mayuby, Mt. Meja.

MeCOCERINA Jordan, 1895a: 164

TYPE-SPECIES: Mecocerina xenoceroides Jordan, 1895a; original designation.

nigropictus dissimilis Frieser, 1995b: 4. (Mecocerina)

DistRIBUTION: Papua New Guinea: Finisterre Range.

MECOCERINOPIS Zimmerman, 1994a: 139

TYPE-SPECIES: Litocerus balli Olliff, 1889; original designation.

amabilis amabilis (Pascoe), 1859a: 331. (Acorynus)

Distribution: Papua New Guinea: Bulolo, Fly River, Goilala, Kar Kar Island, Kiunga, Milne Bay, Mt.

Missim, Owen Stanley Range, Popondetta, Port Moresby, Wau; D’Entrecasteaux Islands: Normanby

Island; Aru Islands. NOTES: Transferred from Mecocerina: Zimmerman, 1994a: 141.

amabilis tarsalis Frieser, 1983: 48. (Mecocerinopis)

Distribution: Bismarck Archipelago: Gazelle Peninsula, New Britain, New Ireland; West Papua: Biak Island.

amabilis nigriventris Frieser, 1983: 48. (Mecocerinopis)

DISTRIBUTION: Admiralty Islands: Manus Island; Bismarck Archipelago.

MUCRONIANUS Jordan, 1894: 627

TYPE-SPECIES: Mucronianus rufipes Jordan, 1894; original designation. KeY: Frieser, 1989: 110.

cavicollis Frieser, 1989: 107. (Mucronianus)

Distribution: Papua New Guinea: Baier River, Bome, Bursa, Goilala, Mt. Wilhelm, Wau. 
cruceicollis Frieser, 2001b: 41. (Mucronianus)

DisTRIBUTION: Bismarck Archipelago: New Britain, Gazelle Peninsula.

latifrons Frieser, 1989: 109. (Mucronianus)

DistRiBUTION: Papua New Guinea: Bulolo, Bubia, Lae, Markham Valley.

procerus Frieser, 1989: 109. (Mucronianus)

Distribution: Papua New Guinea: Aseki, Mt. Missim, Wau.

rufipes Jordan, 1894: 627. (Mucronianus)

Distribution: Papua New Guinea: Bulolo, Fly River, Sattelberg, Sepik River; Bismarck Archipelago:

New Britain; Bacan Island.

Mucronianus tibioclaratus Wolfrum, 1925: 165.

subconvexus (Macleay), 1887: 195. (Litocerus)

Distribution: Papua New Guinea: Fly River; Bismarck Archipelago: New Britain; Solomon Islands.

NOTES: Transferred: Frieser, 1989: 107.

xenoceroides Frieser, 1989: 108. (Mucronianus)

Distribution: Papua New Guinea: Bulolo, Wau.

PHLOEOPS Lacordaire, 1866: 533

TYPE-SPECIES: Stenocerus platypennis Montrouzier, 1855; original designation.

platypennis (Montrouzier), 1855: 40. (Stenocerus)

Distribution: Papua New Guinea; Woodlark Islands; West Papua. NOTES: Transferred: Lacordaire,

1866: 533. See also Jordan, 1894: 630; Lectotype designation: Kuschel, 1998: 194.

Nessiara deplanata Fairmaire, 1883: 45.

Nessiara planata Pascoe, 1860b: 60.

\section{XENOCERINI}

EOTHAUMAS Jordan, 1945: 20

TYPE-SPECIES: Xenocerus bennigseni Jordan, 1906; original designation.

bennigseni (Jordan), 1906: 410. (Xenocerus)

DistRIBUTION: Papua New Guinea.

XenOCERUS Schönherr, 1833: 117

TYPE-SPECIES: Xenocerus saperdoides Gyllenhal in Schönherr, 1833; original designation.

albolineatus albolineatus Blanchard, 1853: 195. (Anthribus)

DistRIBUTION: West Papua: Rubi.

Anthribus arciferus Blanchard, 1853: 195.

anthriboides continens Jordan, 1937: 199. (Xenocerus)

DISTRIBUTION: North Solomon Islands: Bougainville.

barbicornis Gestro, 1875: 1018. (Xenocerus)

Distribution: Papua New Guinea; West Papua: Ramoi.

conjunctus Jordan, 1895d: 392. (Xenocerus)

Distribution: Papua New Guinea: Astrolabe Bay; West Papua: Cyclops Mountains.

corae corae Gestro, 1875: 1017. (Xenocerus)

DistRIBUTION: Papua New Guinea; West Papua: Andai, Mayuby, Ramoi; Mysori Islands. 
corae austrinus Jordan, 1945: 18. (Xenocerus)

DISTRIBUTION: New Guinea.

corae ypsilon Jordan, 1945: 18. (Xenocerus)

DISTRIBUTION: New Guinea.

corae singularis Jordan, 1945: 19. (Xenocerus)

DISTRIBUTION: New Guinea.

corae diversemaculatus Frieser, 1983: 58. (Xenocerus)

DISTRIBUTION: New Guinea.

dohertyi Jordan, 1894: 641. (Xenocerus)

DistRIBUTION: West Papua: Waigeo Island.

eichhorni Jordan, 1945: 17. (Xenocerus)

DiSTRIBUTION: Bismarck Archipelago: New Britain.

equestris equestris Pascoe, 1860a: 35. (Xenocerus)

Distribution: Papua New Guinea; West Papua; Aru Island.

equestris ancorninus Jordan, 1928: 107. (Xenocerus olivaceus ssp. ancorinus)

DISTRIBUTION: Bismarck Archipelago: New Hanover.

equestris olivaceus Motschulsky, 1875: 237. (Xenocerus)

DiSTRIBUTION: New Guinea.

equestris suadus Jordan, 1928: 107. (Xenocerus olivaceus ssp. suadus)

DISTRIBUTION: West Papua: Roon Island; Waigeou Island.

fastuosus fastuosus Gestro, 1875: 1012. (Xenocerus)

DistRIBUTION: West Papua: Geelvink Bay, Korido; Biak Island.

fastuosus albosignatus Frieser, 1983: 57. (Xenocerus)

Distribution: Papua New Guinea; West Papua: Biak Island.

fucatus Jordan, 1945: 14. (Xenocerus)

DisTRIBUTION: New Guinea.

humeralis Gestro, 1875: 1014. (Xenocerus)

DisTRIBUTION: West Papua: Geelvink Bay, Korido, Mayuby; Biak Island.

lacrymans lacrymans Thomson, 1857b: 438. (Xenocerus)

DisTRIBUTION: West Papua: Andai, Ansus, Mayuby, Ramoi, Rubi; Aru Islands.

lacrymans herbertus Jordan, 1906: 410. (Xenocerus)

DISTRIBUTION: Bismarck Archipelago: New Britain.

luctificus Fairmaire, 1883: 45. (Xenocerus)

DiSTRIBUTION: Bismarck Archipelago: Duke of York Island, New Britain, Kokopo; Umboi Island.

mesites Jordan, 1913: 270. (Xenocerus)

DISTRIBUTION: Aru Islands.

niveofasciatus Gestro, 1875: 1015. (Xenocerus)

DistRIBUTION: West Papua: Geelvink Bay, Mafor Island (?).

perfossus Wolfrum, 1938: 72. (Xenocerus)

DISTRIBUTION: North Solomon Islands: Bougainville.

perplexus perplexus Jordan, 1895a: 186. (Xenocerus)

Distribution: Papua New Guinea: Fly River; Aru Islands.

perplexus syndetus Jordan, 1945: 17. (Xenocerus)

DisTRIBUTION: Papua New Guinea.

speracerus speracerus Montrouzier, 1855: 44. (Xenocerus)

DISTRIBUTION: Woodlark Islands. 
speracerus mancus Frieser, 1985: 101. (Xenocerus)

DistRIBUTION: Papua New Guinea: Oriomo River, Kura, Ruka.

speracerus rosseliensis Jordan, 1945: 15. (Xenocerus)

DisTRIBUTION: Louisiade Archipelago.

speracerus sudestensis Jordan, 1903: 429. (Xenocerus)

DISTRIBUTION: Louisiade Archipelago.

suturalis suturalis Jordan, 1904: 89. (Xenocerus)

DisTRIBUTION: West Papua: Ansus, Roon Island.

suturalis tombarus Jordan, 1928: 107. (Xenocerus)

DISTRIBUTION: Bismarck Archipelago: New Ireland.

velutinus velutinus Gestro, 1875: 1012. (Xenocerus)

DistriBution: West Papua: Geelvink Bay, Korido; Biak Island.

velutinus signifer Frieser, 1983: 56. (Xenocerus)

DistRIBUTION: Papua New Guinea; West Papua: Biak Island.

websteri Jordan, 1898: 370. (Xenocerus)

DiSTRIBUTION: Bismarck Archipelago: New Hannover.

\section{XYLINADINI}

XYLINADA Berthold, 1827: 380

TYPE-SPECIES: Anthribus nodicornis Weber, 1801; subsequent designation by Alonso-Zarazaga \& Lyal, 1999: 36.

aruensis (Jordan), 1895b: 259. (Xylinades)

DisTRIBUTION: Aru Islands.

\section{ZYGAENODINI}

MALLORRHYNCHUS Jordan, 1925a: 254

TYPE-SPECIES: Mallorrhynchus hilaris Jordan, 1925a; original designation.

assilimis Jordan, 1925a: 254. (Mallorrhynchus)

DisTRIBUTION: Papua New Guinea.

hilaris Jordan, 1925a: 254. (Mallorrhynchus)

DistRIBUTION: Papua New Guinea.

RHAPHITROPHIS Reitter, 1916: 5

TYPE-SPECIES: Anthribus marchicus Herbst, 1797; subsequent designation by Jordan, 1925b: 257. KeY: Frieser, 1989: 115.

asterias Jordan, 1925a: 251. (Rhaphitrophis)

DISTRIBUTION: Woodlark Islands.

basiplaga basiplaga Frieser, 1989: 111. (Rhaphitrophis)

DisTRIBUTION: Papua New Guinea: Bulolo, Wau; West Papua: Jayapura.

basiplaga manca Frieser, 1989: 112. (Rhaphitrophis mancus)

Distribution: Papua New Guinea: Eddie Creek, Garaina, Jimmi Valley, Wau, Woitape. 
continua Frieser, 1989: 114. (Rhaphitrophis continuus)

DistRIBUTION: Papua New Guinea: Busu River East of Lae; West Papua: Cyclops Mountains, Jayapura. europus Wolfrum, 1956: 111. (Rhaphitrophis)

DisTRIBUTION: Papua New Guinea.

frater Frieser, 1989: 112. (Rhaphitrophis)

DistRIBUTION: Papua New Guinea: Mt. Kaindi; West Papua: Enarotadi, Wisselmeren.

limbalis papuensis Frieser, 1989: 111. (Rhaphitrophis)

DistRIBUTION: Papua New Guinea: Mt. Missim.

stephanus stephanus Jordan, 1925a: 251. (Rhaphitrophis)

Distribution: Papua New Guinea; West Papua: Cyclops Mountains.

stephanus gazellae Frieser, 1989: 114. (Rhaphitrophis)

DisTRIBUTION: Bismarck Archipelago: New Britain.

tricolor Frieser, 1989: 113. (Rhaphitrophis)

DISTRIBUTION: West Papua: Korido.

UNCIFER Jordan, 1904: 88

TYPE-SPECIES: Uncifer sticticus Jordan, 1904; original designation.

raphatus Wolfrum, 1956: 111. (Uncifer)

DISTRIBUTION: Papua New Guinea.

\section{INCERTAE SEDIS}

GARYUS Holloway, 1982: 82

TYPE-SPECIES: Anthribus altus Sharp, 1876; original designation. KeY: Frieser, 1997: 54.

acuticollis Frieser, 1997: 52. (Garyus)

Distribution: Papua New Guinea: Aseki, Mt. Kaindi, Woitape; West Papua: Enarotadi, Epomani, Wisselmeren.

carinifrons Frieser, 1997: 49. (Garyus)

DISTRIBUTION: West Papua: Waigeo Island.

hirtellus Frieser, 1997: 53. (Garyus)

DistRIBUTION: Papua New Guinea: Fly River, Kiunga; West Papua: Emdoman.

laevicollis Frieser, 1997: 50. (Garyus)

DistRIBUTION: Papua New Guinea: Mt. Sinnewitt.

laticollis Frieser, 1997: 51. (Garyus)

DISTRIBUTION: West Papua: Borme, Jayapura.

Papuatorhaenas Frieser, 1997: 40

TYPE-SPECIES: Papuatorhaenas riedeli Frieser, 1997: 40; original designation. KEY: Frieser, 1999: 29.

arduus Frieser, 1997: 42. (Papuatorhaenas)

Distribution: Papua New Guinea: Between Aseki and Menyamya, Mt. Kaindi. 
impressicollis Frieser, 1997: 41. (Papuatorhaenas)

Distribution: West Papua: Jayapura, Larye, Swart Valley.

riedeli Frieser, 1997: 40. (Papuatorhaenas)

DisTRIBUTION: West Papua: Borme, Jayapura, Membaham, Wamena.

subcarinatus Frieser, 1999: 28. (Papuatorhaenas)

Distribution: Papua New Guinea: Aseki.

\section{CHORAGINAE}

\section{APOLECTINI}

ApOLeCta Pascoe, 1859b: 431

TYPE-SPECIES: Mecocerus parvulus Thomson, 1857b; original designation.

angulicollis Frieser, 1983: 57. (Apolecta)

Distribution: Papua New Guinea: Kerema, Kokoda, Salamanga; West Papua: Cyclops Mountains, Ifar, Mt. Meja.

papuana papuana Jordan, 1898: 373. (Apolecta)

DistRIBUTION: Papua New Guinea: Fly River, Kiunga.

papuana virgata Jordan, 1916: 343. (Apolecta)

DistRIBUTION: Papua New Guinea.

parvula (Thomson), 1857b: 437. (Mecocerus parvulus)

DistRIBUTION: Papua New Guinea: Fly River; Aru Island.

transversa (Olivier), 1795: 10. (Macrocephalus)

DISTRIBUTION: West Papua.

\section{ARAECERINI}

ACHORAGUS Jordan, 1914: 265

TYPE-SPECIES: Achoragus tener Jordan, 1914; original designation.

papuanus Frieser, 1995b: 32. (Achoragus)

Distribution: West Papua: Anggi, Cyclops Mountains, Iranmeba, Mt. Kobrey, Membey, Ransiki, Tetaho, Wamena.

sparsutus Frieser, 1995b: 33. (Achoragus)

Distribution: West Papua: Anggi, Pinibut.

ARAECERUS Schönherr, 1823: 1135

TYPE-SPECIES: Araecerus coffeae Fabricius, 1801 [=Bruchus cacao? Fabricius, 1775]; original designation.

aethiops Frieser, 1995b: 10. (Araecerus)

DisTRIBUTION: West Papua: Jayapura.

atramentarius atramentarius Frieser, 1995b: 14. (Araecerus)

Distribution: Papua New Guinea: Bulolo, Kerema, Kiunga, Mt. Missim; West Papua: Geelvink Bay, Jayapura, Wisselmeren.

atramentarius latior Frieser, 2000: 30. (Araecerus) 
Distribution: Papua New Guinea: Upper Jimmi Valley, Wumi; West Papua: Nabire, Yamor Lake.

blandicaudatus Frieser, 1995b: 13. (Araecerus)

Distribution: Papua New Guinea: Aroa River, Brown River near Pt. Moresby, Bubia, Bulolo, Busu

River near Lae, Goroka, Kokoda, Milne Bay, Mt. Kaindi, Pt. Moresby, Wau; West Papua: Cyclops Mountains.

citius Frieser, 1995b: 19. (Araecerus)

Distribution: Papua New Guinea: Mt. Missim, Sattelberg, Wau; West Papua: Jayacoijaya, OklomaEndoman.

conformis Frieser, 1995b: 20. (Araecerus)

Distribution: Papua New Guinea: Finisterre Range, Saidor; West Papua: Bokondini North of Baliem Valley.

consobrinus Frieser, 2000: 29. (Araecerus)

DistRIBUTION: West Papua: Doorman Range.

cyrtus Jordan, 1924a: 249. (Araecerus)

DistRIBUTION: Papua New Guinea.

declivis Frieser, 1995b: 18. (Araecerus)

Distribution: Papua New Guinea: Port Moresby.

fasciculatus (De Geer), 1775: 276. (Curculio)

DisTRIBUTION: Sub-cosmopolitan: New Guinea.

hybus Wolfrum, 1956: 119. (Araecerus)

DISTRIBUTION: Papua New Guinea.

montanus Frieser, 2000: 30. (Araecerus)

Distribution: Papua New Guinea: Mt. Kaindi, Wau; West Papua: Yapen Island.

mopsus Frieser, 1995b: 15. (Araecerus)

Distribution: Papua New Guinea: Bubia near Lae, Bulolo, Pt. Moresby, Wau; Bismarck Archipelago:

New Britain; West Papua: Geelvink Bay.

rugiventrus Frieser, 1995b: 17. (Araecerus)

DistRIBUtion: Papua New Guinea: Brown River near Pt. Moresby, Goilala, Kainantu, Kokoda, Mt. Missim, Wau; West Papua: Cyclops Mountains, Jayapura.

silex Jordan, 1924a: 246. (Araecerus)

DisTRIBUTION: Papua New Guinea.

validus validus Frieser, 1995b: 7. (Araecerus)

Distribution: Papua New Guinea: Asaro Valley, Bome, Dalo Pass, Eddie Creek, Gararina, Kainantu, Mt.

Giluwe, Mt. Hagen, Mt. Kaindi, Mt. Otto, Mt. Wilhelm, Nami Creek near Wau, Okapa, Tari Gap, Wau.

validus capucinus Frieser, 1995b: 10. (Araecerus)

Distribution: Papua New Guinea: Eddie Creek, Finisterre Range, Kainantu, Mt. Kaindi, Lake Sirunki, Wau.

validus tripartitus Frieser, 1995b: 10. (Araecerus)

Distribution: Papua New Guinea: Gilume, Lake Sirunki.

venosus Frieser, 1995b: 11. (Araecerus)

Distribution: Papua New Guinea: Mt. Bosavi, Mt. Kaindi, Okapa, Urapura; West Papua: Itouda, Kamo,

Wamena, Wisselmeren.

zelusus Wolfrum, 1956: 118. (Araecerus)

DisTRIBUTION: Papua New Guinea; Bismarck Archipelago: New Britain, New Ireland. 
TYPE-SPECIES: Araeocorynus cummingii Jekel, 1855; original designation. NOTE: Treated as a synomym in Valentine, 1998: 252 and followed by Alonso-Zarazaga \& Lyal, 2002; generic status resumed in Reinheimer, 2004.

brachyurus brachyurus Jordan, 1924a: 245. (Araeocorynus)

DistRIBUtion: Papua New Guinea: Bulolo, Kokoda, Kundiawa, Wau.

brachyurus reductus Frieser, 1983: 60. (Araeocorynus)

DisTRIBUTION: Papua New Guinea: Wau.

brachyurus variabilis Frieser, 1983: 60. (Araeocorynus)

DistRIBUTION: Papua New Guinea: Finisterre Range, Saidor.

corismus corismus Jordan, 1924a: 245. (Araeocorynus)

Distribution: Papua New Guinea: Bulolo, Fly River, Kiunga, Milne Bay, Mt. Missim, Wau; Bismarck Archipelago: New Britain.

corysmus fuscopustulosus Frieser, 1983: 60. (Araeocorynus)

DisTRIBUTION: Papua New Guinea: Wau.

corysmus ornatipennis Frieser, 1983: 61. (Araeocorynus)

DisTRIBUTION: Papua New Guinea: Wau.

cummingii Jekel, 1855: 152. (Araeocorynus)

DistRIBUTION: Papua New Guinea: Garaina, Salamanga, Upper Jimmi Valley.

laticollis Wolfrum, 1957: 105. (Araeocorynus)

DISTRIBUTION: New Guinea.

multatus multatus Frieser, 1983: 58. (Araeocorynus)

Distribution: Papua New Guinea: Karimui, Mt. Missim, Wau.

multatus centralis Frieser, 1983: 59. (Araeocorynus)

Distribution: Papua New Guinea: Wau, Kundiawa, Karimui.

multatus nigroapicatus Frieser, 1983: 59. (Araeocorynus)

Distribution: Papua New Guinea: Wau.

planatus (Jordan), 1904: 91. (Doticus)

Distribution: Papua New Guinea: Milne Bay, Kokoda, Dogura.

ursus Frieser, 1983: 61. (Araeocorynus)

DistRIBUTION: Papua New Guinea: Milne Bay.

DEROPYGUS Sharp, 1891: 326

TYPE-SPECIES: Deropygus histrio Sharp, 1891; subsequent designation by Morimoto, 1978: 23.

acutus Jordan, 1924a: 254. (Deropygus)

DISTRIBUTION: Aru Islands.

alienus Frieser, 1992: 41. (Deropygus)

DistRiBution: West Papua: Bodem SE. of Oerberfarne, Cyclops Mountains, Mt. Meja.

continus Frieser, 1992: 42. (Deropygus)

DistRIBUTION: Papua New Guinea: Wau; West Papua: Umg. Pass Valley.

excisus Frieser, 1992: 42. (Deropygus)

DistRibution: West Papua: Mt. Meja.

hercules Jordan, 1924a: 254. (Deropygus)

DisTRIBUTION: West Papua: Humboldt Bay. 
TYPE-SPECIES: Doticus palmaris Pascoe, 1882a; monobasic.

planatus Jordan, 1904: 91. (Doticus)

Distribution: Papua New Guinea.

sentus sentus Jordan, 1939a: 431. (Doticus)

DisTRIBUTION: Papua New Guinea; West Papua.

sentus subornatus Frieser, 1995b: 7. (Doticus)

Distribution: Papua New Guinea: Eliptami Valley, Lufa, Mt. Kaindi; West Papua: Kosmena, Tetaho.

\section{Misthosima Pascoe, 1859b: 434}

TYPE-SPECIES: Misthosima mera Pascoe, 1859b; subsequent designation by Zimmerman, 1994a: 228.

aequalis Frieser, 1992: 38. (Misthosima)

Distribution: Papua New Guinea: Bulldog Road, Eddie Creek, Wau; West Papua: Pass Valley, Wamena.

biakensis Frieser, 1995a: 10. (Misthosima)

DISTRIBUTION: Biak Island.

callima Jordan, 1929: 339. (Misthosima)

Distribution: Papua New Guinea: Kiolo Creek West of Wau; West Papua: Biak Island.

comoda Frieser, 1992: 40. (Misthosima)

DistRIBUTION: Papua New Guinea: Wewak; West Papua: Cyclops Mountains.

crucifera Jordan, 1904: 90. (Misthosima)

Distribution: Papua New Guinea: Bulolo, Milne Bay; West Papua: Cyclops Mountains, Nabire South of

Geelvink Bay; Biak Island.

driesi Frieser, 2001a: 25. (Misthosima)

DisTRIBUTION: West Papua.

freudei Frieser, 2001a: 24. (Misthosima)

DistRIBUTION: Papua New Guinea; West Papua.

gracilipes Frieser, 1995a: 8. (Misthosima)

DistRIBUTION: Papua New Guinea; North Solomon Islands: Bougainville Island; West Papua.

griseovaria Frieser, 1992: 38. (Misthosima)

Distribution: Papua New Guinea: Busu River East of Lae; West Papua: Cyclops Mountains.

lata Pascoe, 1860b: 60. (Misthosima)

DistRIBUTION: Papua New Guinea.

mediocra Frieser, 1992: 40. (Misthosima)

DisTRIBUTION: West Papua: Kangime.

pedalis Wolfrum, 1956: 117. (Misthosima)

DisTRIBUTION: Papua New Guinea.

picturata Frieser, 1992: 41. (Misthosima)

Distribution: Papua New Guinea: Mt. Kaindi; West Papua: Baliem District.

rigua Frieser, 1992: 37. (Misthosima)

DistRIBUTION: Bismarck Archipelago: New Britain; West Papua: Bodem SE of Verberfaren, Cyclops

Mountains, Mayuby.

roppeli Frieser, 2001a: 26. (Misthosima)

Distribution: Papua New Guinea.

signatifrons Frieser, 1996: 36. (Misthosima) 
DistRIBUTION: West Papua: Taramlu.

subrubra Frieser, 1996: 37. (Misthosima)

DISTRIBUTION: West Papua: Epomani.

subsignata subsignata Frieser, 1995a: 7. (Misthosima)

DisTRIBUTION: Papua New Guinea; Bismarck Archipelago: New Britain.

subsignata striolata Frieser, 1995a: 8. (Misthosima)

DisTRIBUTION: New Guinea.

unifasciata Frieser, 1992: 39. (Misthosima)

Distribution: West Papua: Mt. Meja.

OXYCONUS Jordan, 1936: 325

TYPE-SPECIES: Oxyconus stipinus Jordan, 1936; monobasic.

stipinus Jordan, 1936: 325. (Oxyconus)

Distribution: Papua New Guinea: Busu River near Lae, Pt. Moresby, Upper Jimmi Valley, Watut, Wau;

West Papua: Fakfak, Vogelkop.

tangens Frieser, 1996: 34. (Oxyconus)

DisTRIBUTION: Bismarack Archipelago: New Britain, New Ireland; West Papua: Fakfak, Bomberai.

\section{CHORAGINI}

DYSNOS Pascoe, 1859b: 438

TYPE-SPECIES: Dysnos auricomus Pascoe, 1859b; monobasic.

auricomus Pascoe, 1859b: 438. (Dysnos)

DISTRIBUTION: Aru Islands.

MELANOPSACUS Jordan, 1924b: 608

TYPE-SPECIES: Melanopsacus fortis Jordan, 1924b; original designation.

atronitens Frieser, 1995b: 30. (Melanopsacus)

DisTRIBUTION: West Papua: Borme, Jayapura.

dispar Frieser, 1995b: 31. (Melanopsacus)

DistRIBUTION: West Papua: Borme, Jayapura, Membey, Ransiki.

imperialis Frieser, 1995b: 28. (Melanopsacus)

DistRiBution: West Papua: Angguruk-Tanggeam, Membahan, Wamena.

iniquus Frieser, 1992: 43. (Melanopsacus)

DistRibution: West Papua: Mt. Meja.

rugiceps Frieser, 1995b: 29. (Melanopsacus)

DisTRIBUTION: West Papua: Jayapura, Karimui.

variolosus Frieser, 1995b: 31. (Melanopsacus)

DistRIBUTION: West Papua: Borme. 


\section{BELIDAE \\ BELINAE}

RHINOTIA Kirby, 1819: 426

TYPE-SPECIES: Rhinotia haemoptera W. Kirby, 1819; monobasic.

biroi (Voss), 1956: 122. (Belus)

DistRIBUTION: Papua New Guinea: Sattelberg. NOTES: Transferred: Zimmerman, 1994a: 362.

corallimera (Heller), 1914a: 652. (Belus corallimerus)

DistRIBUTION: West Papua: Lorentz River; Biak Island. Notes: Transferred: Zimmerman, 1994a: 362.

divisa (Pascoe), 1885: 229. (Belus)

DistRIBUTION: [Australia: Somerset]; Papua New Guinea: Tala; West Papua. NotES: Transferred: Zimmerman, 1994a: 405.

inornata (Pascoe), 1874: 27. (Belus inornatus)

Distribution: Mysol Island. Notes: Transferred: Zimmerman, 1994a: 362.

viridimettalica (Heller), 1901: 16. (Belus)

Distribution: Papua New Guinea: Astrolabe Mountains. Notes: Transferred: Zimmerman, 1994a: 362. wallacei (Pascoe), 1874: 26. (Belus)

DisTRIBUTION: New Guinea: Kapaor; Aru Islands. NOTES: Transferred: Zimmerman, 1994a: 362.

\section{RHYNCHITIDAE}

\section{RHYNCHITINAE}

\section{AULETINI}

\section{AULETOBIINA}

AuletoBIUS Desbrochers des Loges, 1869: 396

TYPE-SPECIES: Auletes basilaris Gyllenhal in Schönherr, 1839 [=Involvulus sanguisorbae Schrank, 1798]; subsequent designation by Voss, 1934b: 118. KEY: Voss, 1937a: 205 (subgenera).

\section{Auletobius (Metopum) Agassiz, 1846: 232}

TYPE-SPECIES: Metopon suturalis Waterhouse, 1842; monobasic. NOTES: Status resumed: Alonso-Zarazaga \& Lyal, 1999: 42.

humboldti Voss, 1934b: 121. (Auletobius (Parauletes))

DisTRIBUTION: West Papua: Humboldt Bay.

\section{STictauletes Voss, 1934b: 135}

TYPE-SPECIES: Auletobius insularis Voss, 1934b; monobasic. NOTES: Status changed from subgenus of Auletobius in Legalov, 2003: 92.

insularis Voss, 1934b: 123. (Auletobius (Stictauletes))

DISTRIBUTION: Mysol Island. 
punctiger Voss, 1922a: 34. (Auletobius)

DisTRIBUTION: Mysol Island.

\section{GUINEAULETINA}

GUINEAULETES Legalov, 2003-110

TYPE-SPECIES: Guineauletes mirabilis Legalov, 2003; original designation.

mirabilis Legalov 2003: 110

Distribution: Papua New Guinea: Mt. Kaindi, Mt. Missim, Wau.

sculpturatus Legalov 2003: 111

DistRIBUTION: Papua New Guinea: Mt. Kaindi.

MaCroAuletes Legalov, 2003-111

TYPE-SPECIES: Auletes picticornis Pascoe, 1885; original designation.

picticornis (Pascoe), 1885: 234. (Auletes)

DistRIBUTION: Papua New Guinea: Sattelberg; West Papua: Andai.

NEAULETES Legalov, 2003-110

TYPE-SPECIES: Neauletes relictus Legalov, 2003; original designation.

relictus Legalov, 2003: 110

DisTRIBUTION: Papua New Guinea: Wau.

\section{AULETORHININI}

Auletanus Voss, 1922a: 20

TYPE-SPECIES: Auletobius ascendens Heller, 1915; subsequent designation by Voss, 1932: 101.

KEY: Voss, 1932: 101.

disparatus Voss, 1932: 103. (Auletanus)

DISTRIBUTION: New Guinea.

\section{DEPORAINI}

PSEUdOdePoraus Voss, 1922b: 388

TYPE-SPECIES: Deporaus periscelis Voss, 1922b; subsequent designation by Sawada, 1993: 85. Notes: Status changed from subgenus of Deporaus Samouelle, 1819 in Legalov, 2003. KEYs: Voss, 1938: 89 (subgenera of Deporaus); Voss, 1938: 90 (species-groups and species of Deporaus) 
kolbei Voss, 1942: 151. (Deporaus)

DisTRIBUTION: New Guinea.

mysolensis Voss, 1942: 153. (Deporaus)

DISTRIBUTION: Mysol Island.

\section{RHYNCHITINI}

MACULINVOLVULus Legalov, 2003: 282

TYPE-SPECIES: Rhynchites singularis Roelofs, 1874; original designation.

kaszabi Voss, 1956: 123. (Involvulus (Cartorhynchites))

DISTRIBUTION: New Guinea.

\section{ATTELABIDAE \\ ATTELABINAE}

\section{EUOPINI}

EUOPS Schönherr, 1839: 318

TYPE-SPECIES: Euops australasiae Fåhraeus in Schönherr, 1839 [=Attelabus falcatus Guérin-Méneville]; original designation. NoTES: Legalov (2003) elevated the subgenera of Euops to generic level and erected several genera, tribes, and subtribes, affecting all of the Papuan species listed below. His Papuan genera are based on species-groups of Euops previously defined by Riedel in 2002c. Here I follow Riedel's recommendation (Riedel, 2006: 5) by reducing Legalov's genera to subgenera and returning Neosynaptops to a subgenus of Euops. KeYs: Voss, 1924: 39; Voss, 1925: 293; Riedel, 1999: 85 (spinosus-group); Riedel, 2001 a: 146 (simulans-group); Riedel, 2001 b: 553 (quadrifasciculatus-group); Riedel, 2001c: 1180 (pygmaeus: group); Riedel, 2002a: 138 (subgenus Neosynaptops); Riedel, 2006: 10 (subgenus Metaeuops).

EUOPS (ARCHEUOPS) Legalov, 2003: 360 stat. n.

TYPE-SPECIES: Euops vossi Heller, 1929: 111; original designation. NOTES: Formerly Euops papua-group of Riedel, 2002c.

jekeli Pascoe, 1874: 29. (Euops jekelii)

Distribution: West Papua: Andai, Dorey; Aru Island; Salawatti Island, Waigeo Island.

papua Heller, 1914a: 653. (Euops)

DisTRIBUTION: West Papua: Lorentz River.

EUOPS (ARMEUOPS) Legalov, 2003: 375 stat. n.

TYPE-SPECIES: Euops armatipennis Voss, 1924; original designation. NOTES: Formerly Euops armatipennis-group of Riedel, 2002c.

armatipennis Voss, 1924: 51. (Euops)

Distribution: Papua New Guinea: Adelbert Mountains, Angoram, Lake Kutubu, Wewak; West Papua: Wapoga River, Waris. 
TYPE-SPECIES: Euops suturalis Lea, 1898b; original designation. NOTES: Formerly Euops eucalypti-group of Riedel, 2002c.

suturalis Lea, 1898b: 616. (Euops)

Distribution: Papua New Guinea: Port Moresby, Redscar Bay; Austraila.

EUOPS (EUOPSIDIUS) Legalov, 2003: 370 stat. n.

TYPE-SPECIES: Euops testaceus Voss, 1929b; original designation. NOTES: Formerly Euops testaceus-group of Riedel, 2002 c.

divisus Pascoe, 1874: 29. (Euops divisa)

DistRIBUTION: West Papua: Saylee; Mysol Island.

testaceus Voss, 1929b: 218. (Euops testacea)

DistRiBution: Papua New Guinea: Madang; West Papua: Nabire, Rouffaer Mountains, Testega, Wapoga River; Mysol Island; Salawatti Island.

EUOPS (GUINEOEUOPS) Legalov, 2003: 370 stat. n.

TYPE-SPECIES: Euops reticulatus Riedel, 2001a; original designation. NOTES: Formerly Euops simulans-group of Riedel, 2001a.

angulithorax Riedel, 2001a: 206. (Euops)

Distribution: Papua New Guinea: Torricelli Mountains; West Papua: Bime, Borme, Iba, Nabire, Testega; Mysol Island.

asekianus Riedel, 2001a: 204. (Euops)

Distribution: Papua New Guinea: Aseki.

balkei Riedel, 2001a: 159. (Euops)

DistRIBUTION: Papua New Guinea: Mt. Missim; D’Entrecasteaux Islands: Normanby Island; West Papua: Bommela.

batantae Riedel, 2001a: 186. (Euops)

DISTRIBUTION: Batanta Island.

breyniae Riedel, 2001a: 212. (Euops)

DistRIBUTION: West Papua: Ilugwa, Jiwika, Kwiyawagi.

buergersi Voss, 1924: 47. (Euops bügersi)

DistRibution: Papua New Guinea: Mäanderberg in the West Range, Mianmin.

cyclopensis Riedel, 2001a: 188. (Euops)

DistRIBUTION: West Papua: Cyclops Mountains.

deceptus Riedel, 2001a: 196. (Euops)

Distribution: West Papua: Bime, Diuremna, Nalca.

flyensis Riedel, 2001a: 208. (Euops)

Distribution: Papua New Guinea: Kinuga, Mt. Bosavi, Tekadu.

japensis Riedel, 2001a: 165. (Euops)

DISTRIBUTION: Yapen Island.

kukukuku Riedel, 2001a: 182. (Euops kukukuku)

DistRIBUTION: Papua New Guinea: Aseki, Wau. NOTES: Legalov, 2003: 371 proposed an unjustified eme- 
dation for this species.

kutubu Riedel, 2001a: 177. (Euops)

Distribution: Papua New Guinea: Lake Kutubu. Notes: Legalov, 2003: 371 proposed an unjustified emedation for this species.

lani Riedel, 2001a: 213. (Euops)

DistRIBUTION: West Papua: Bilogay, Ilugwa, Mulia, Sinak.

lobipes Riedel, 2001a: 172. (Euops)

DistRIBUTION: West Papua: Dabra, Wapoga River.

mysolensis Voss, 1924: 49. (Euops)

DisTRIBUTION: West Papua: Nabire; Gamang Island; Mysol Island; Salawatti Island.

Euops picipes Voss, 1929b: 219.

parangulithorax Riedel, 2001a: 209. (Euops)

DisTRIBUTION: West Papua: Arfak Mountains, Membey, Ransiki.

reticulatus Riedel, 2001a: 192. (Euops)

DisTRIBUTION: West Papua: Bime, Borme.

simulans Riedel, 2001a: 205. (Euops)

Distribution: Papua New Guinea: Eliptamin, Telefomin; West Papua: Angguruk, Bime, Borme, Emdoman, Kosarek, Okloma, Nalca.

vulgaris Riedel, 2001a: 199. (Euops)

DistriBution: West Papua: Bommela, Enarotadi, Kamo, Langda, Yalmabi.

wallacei Sharp, 1889: 55. (Euops)

DistRIBUTION: West Papua: Dorey Bay.

wei Riedel, 2001 a: 180. (Euops)

DistRIBUTION: Papua New Guinea: Bulolo, Wau.

zimmii Riedel, 2001a: 210. (Euops)

DistriBution: Papua New Guinea: Mianmin; West Papua: Cyclops Mountains.

EUOPS (LIJUDMILINIUS) Legalov, 2003: 362 stat. n.

TYPE-SPECIES: Euops femoralis Voss, 1924; original designation. Formerly Euops femoralis-group of Riedel, $2002 \mathrm{c}$.

femoralis Voss, 1924: 50. (Euops)

Distribution: Papua New Guinea: Karimui, Mianmin, Mt. Hunstein; West Papua: Bime, Okloma.

EUOPS (METAEUOPS) Legalov, 2003: 369

TYPE-SPECIES: Euops tibialis Voss, 1929b; original designation. NotES: Status changed from genus to subgenus: Riedel, 2006: 6. NOTES: Formerly Euops coelestinus-group of Riedel, 2002c.

arfakensis Riedel, 2006: 38. (Euops (Metaeuops))

DistRIBUTION: West Papua: Arfak Mountains, Membey, Ransiki.

biru Riedel, 2006: 46. (Euops (Metaeuops))

DISTRIBUTION: Yapen Island.

coelestinus Pascoe, 1874: 27. (Euops celestina)

Distribution: West Papua: Arfak Mountains, Epomani, Manokawari, Ransiki, Serui, Topo, Wandammen Bay.

coeruleus Riedel, 2006: 33. (Euops (Metaeuops))

DistRIBUTION: Papua New Guinea: Daradae Plain in the Central Provence. 
curvipes Riedel, 2006: 43. (Euops (Metaeuops))

Distribution: Papua New Guinea: Aseki, Karimui, Kiunga; West Papua: Angguruk, Bime Kosarek, Nabire, Nalca.

dintelmanni Riedel, 2006: 48. (Euops (Metaeuops))

DistRIBUTION: Papua New Guinea: Aseki, Wau.

illegalovi Riedel, 2006: 18. (Euops (Metaeuops)) DistriBution: West Papua: Angguruk, Borme, Elelim, Emdoman, Galbok, Okloma, Taramlu.

judithae Riedel, 2006: 28. (Euops (Metaeuops))

Distribution: Papua New Guinea: Mianmin.

ruficornis Voss, 1956: 124. (Euops femoralis f. ruficornis)

Distribution: Papua New Guinea: Brown River, Erima, Goilala, Madang, Salawaket Range, Torricelli Mountains, Wau.

oops Riedel, 2006: 24. (Euops (Metaeuops))

Distribution: West Papua: Cyclops Mountains.

paratibialis Riedel, 2006: 57. (Euops (Metaeuops))

Distribution: Papua New Guinea: Jimmi Valley, Mianimin; West Papua: Angguruk, Borme, Cyclops Mountains, Emdomen, Karubaga, Wapoga River.

piceus Riedel, 2006: 51. (Euops (Metaeuops))

Distribution: Papua New Guinea: Kiunga; West Papua: Holuwon, Samboka, Wandammen Bay.

ratcliffei Riedel, 2006: 35. (Euops (Metaeuops))

DISTRIBUTION: Biak Island; Yapen Island.

swartensis Riedel, 2006: 21. (Euops (Metaeuops))

DistRIBUTION: West Papua: Karubaga, Swart River Valley.

tenuiflagellaris Riedel, 2006: 30. (Euops (Metaeuops))

Distribution: Papua New Guinea: Aseki, Baiyer River in the Western Highlands, Mindik, Mt. Kaindi,

Pindiu, Wau.

tibialis Voss, 1929b: 220. (Euops)

Distribution: West Papua: Arfak Mountains, Epomani, Olsobip, Serui, Wandammen Bay, Wapoga

River; Mysol Island.

torricelliensis Riedel, 2006: 26. (Euops (Metaeuops))

DistRIBUTION: Papua New Guinea: Torricelli Mountains.

EUOPS (METASYNAPTOPS) Legalov, 2003: 390 stat. n.

TYPE-SPECIES: Euops micans Lea, 1930: 543; original designation. NOTES: Formerly Euops maculatus-group of Riedel, $2002 \mathrm{c}$.

maculatus Voss, 1924: 48. (Euops maculata)

DistRIBUTION: West Papua: Mysol Island.

trigeminatus Pascoe, 1874: 28. (Euops trigemmata)

DistRIBUTION: West Papua: Dorey.

EUOPS (NEOSYNAPTOPS) Voss, 1930: 83 stat. rev.

TYPE-SPECIES: Euops viridiceps Voss, 1930: 82; subsequent designation by Riedel, 1998: 100.

=Neosynaptops (Neosynaptopsis) Legalov, 2003: 372 syn. $\mathbf{n}$.

TYPE-SPECIES: Euops cupreosplendens Macleay, 1887; original designation.

=Neosynaptops (Pseudosynaptos) Legalov, 2003: 372 syn. $\mathbf{n}$.

TYPE-SPECIES: Euops viridiceps Voss, 1930; original designation. 
cupreosplendens Macleay, 1887: 186. (Euops) stat. rev.

Distribution: Papua New Guinea: Aseki, Finisterre Range, Fly River, Karimui, Kiunga, Lake Kutubu, May River, Mianmin, Saidor; West Papua: Cyclops Mountains, Emdoman, Samboka.

doertheae Riedel, 2002a: 150. (Euops (Neosynaptops)) stat. rev.

DistRIBUTION: West Papua: Samboka.

gladiator Riedel, 2002a: 153. (Euops (Neosynaptops)) stat. rev.

DisTRIBUTION: West Papua: Nabire.

paraviridiceps Riedel, 2002a: 142. (Euops (Neosynaptops))

Distribution: Papua New Guinea: Mianmin, Olsobip; Bismarck Archipelago: West Papua: Angguruk, Borme, Emdoman, Epomani, Kebar Valley, Lereh, Meydoudga, Hatam, Testega, Timika; Batanta Island; Salawatti Island.

punctaticeps Riedel, 2002a: 155. (Euops (Neosynaptops)) stat. rev.

DISTRIBUTION: Waigeo Island.

similis Riedel, 2002a: 159. (Euops (Neosynaptops)) stat. res.

DISTRIBUTION: Waigeo Island.

viridiceps Voss, 1930: 82. (Euops)

Distribution: Papua New Guinea: Bulolo, Sattelberg; Wau; West Papua: Waris S of Jayapura; Biak Island.

waigeoensis Riedel, 2002a: 160. (Euops (Neosynaptops)) stat. rev.

DISTRIBUTION: Waigeo Island.

wapogae Riedel, 2002a: 161. (Euops (Neosynaptops)) stat. rev.

DistRIBUTION: West Papua: Mamberamo, Rouffaer Mountains, Waropen.

EUOPS (PARAeUOPS) Legalov, 2003: 394 stat. n.

TYPE-SPECIES: Euops pygmaeus Riedel, 2001c; original designation. NOTES: Formerly Euops pygmaeus-group of Riedel, 2001c.

anggiensis Riedel, 2001c: 1225. (Euops)

DisTRIBUTION: West Papua: Anggi Lakes.

bicolor Riedel, 2001c: 1223. (Euops)

DisTRIBUTION: West Papua: Sinak.

convexus Riedel, 2001c: 1197. (Euops)

DistRIBUTION: West Papua: Anggi Lakes.

fraterculus Riedel, 2001c: 1207. (Euops)

Distribution: Papua New Guinea: Mt. Por.

ibelensis Riedel, 2001c: 1203. (Euops)

DisTRIBUTION: West Papua: Ibele Valley.

kurulu Riedel, 2001c: 1227. (Euops)

Distribution: West Papua: Baliem Valley, Jiwika, Nalca.

nothofagi Riedel, 2001c: 1217. (Euops)

DisTRIBUTION: West Papua: Sinak.

paraconvexus Riedel, 2001c: 1200. (Euops)

DisTRIBUTION: West Papua: Anggi.

parvus Riedel, 2001c: 1191. (Euops)

Distribution: West Papua: Bilogay, Epomani, Jiwika, Kamo Valley, Paniai Lakes.

platyrostris Riedel, 2001c: 1209. (Euops)

DisTRIBUTION: West Papua: Baliem Valley. 
porulosus Riedel, 2001c: 1195. (Euops)

DistRiBUtion: West Papua: Bilogay, Sinak.

pygmaeus Riedel, 2001c: 1229. (Euops)

DistRibution: Papua New Guinea: Mt. Albert Edward, Mt. Bosavi, Mt. Kaindi.

sedlaceki Riedel, 2001c: 1214. (Euops)

DistRIBUtion: Papua New Guinea: Chimbu Valley; West Papua: Baliem Valley, Bilogay, Jiwika. singularis Voss, 1924: 57. (Euops)

Distribution: Papua New Guinea: Telefomin; West Papua: Angguruk, Bime, Nalca, Taramulu.

EUOPS (PARAeUOPSIS) Legalov, 2003: 396 stat. n.

TYPE-SPECIES: Euops quadrifasciculatus Lea, 1929: 541; original designation. NOTES: Formerly Euops quadrifasciculatus-group of Riedel, 2001b.

goilala Riedel, 2001b: 580. (Euops)

DistRIBUTION: Papua New Guinea: Goilala.

lakekamuensis Riedel, 2001b: 579. (Euops)

DisTRIBUTION: Papua New Guinea: Lakekamu basin; Tekadu.

micros Riedel, 2001b: 577. (Euops)

DisTRIBUTION: West Papua: Testega.

EUOPS (RIEDELINIUS) Legalov, 2003: 362 stat. n.

TYPE-SPECIES: Euops armatus Riedel, 1999; original designation. NoTES: Formerly Euops spinosus-group of Riedel, 1999.

armatus Riedel, 1999: 99. (Euops)

Distribution: Papua New Guinea: Aseki; West Papua: Kosarek, Nalca, Taramlu.

aculeatus Riedel, 1999: 102. (Euops)

DistRIBUTION: Papua New Guinea: Kepilam South East of Laiagem in Enga Province.

gressitti Riedel, 1999: 89. (Euops)

Distribution: Papua New Guinea: Mt. Giluwe, Mt. Kaindi, Kerowagi; West Papua: Mulia, Nalca.

monstruosus Riedel, 1999: 95. (Euops)

DISTRIBUTION: Papua New Guinea: Okapa.

paniaiensis Riedel, 1999: 108. (Euops)

DisTRIBUTION: West Papua: Paniai Lakes.

paraspinosus Riedel, 1999: 94. (Euops)

DisTRIBUTION: West Papua: Anggi.

pseudomonstruosus Riedel, 1999: 99. (Euops)

DisTRIBUTION: Papua New Guinea: Wau.

spinosus Riedel, 1999: 92. (Euops)

Distribution: Papua New Guinea: Aseki, Mt. Hagen, Wau; West Papua: Bommela, Borme, Enrotadi, Epomani, Paniai Lakes.

yali Riedel, 1999a: 109. (Euops)

DistRIBUTION: West Papua: Angguruk, Kosarek.

zimmermanni Riedel, 1999: 105. (Euops)

Distribution: Papua New Guinea: Mt. Kaindi (?); West Papua: Bilogay. 


\section{BRENTIDAE}

\section{BRENTINAE}

\section{ARRHENODINI}

\section{BARYRHYNCHUS Lacordaire, 1866: 428}

TYPE-SPECIES: Arrhenodes latirostris Gyllenhal in Schönherr, 1833; subsequent designation by Kliene, 1938: 91. KEYS: Kleine, 1920d: 44; Kleine, 1926b: 266.

discolor Kleine, 1916d: 157. (Baryrrhynchus)

DISTRIBUTION: Aru Islands.

latirostris (Gyllenhal) in Schönherr, 1833: 323. (Arrhenodes)

DisTRIBUTION: New Guinea.

BARYRHYNCHUS (EUPSALOMIMUS) Kleine, 1916d: 151

TYPE-SPECIES: Baryrrhynchus lineicollis Power, 1879; subsequent designation by Zimmerman, $1994 \mathrm{~b}: 117$.

lineicollis Power, 1879: 297. (Baryrrhynchus)

Distribution: Papua New Guinea; Bismarck Archipelago: Duke of York Island, New Britain; West

Papua: Aru Islands; Mysol Island; Salawatti Island.

Baryrrhynchus compositus Kleine, 1923: 407. NoTES: Synonoymy: Damoiseau, 1972: 274.

Baryrrhynchus indocilis Fairmaire, 1883: 41. NOTES: Synonoymy: Damoiseau, 1972: 274.

Baryrrhynchus solidus Kleine, 1920d: 44. NoTES: Synonoymy: Damoiseau, 1972: 274.

Baryrhynchus linecollis kleinei Damoiseau, 1972: 274. NoTES: Synonoymy: Sforzi \& Bartolozzi, $2004: 178$.

\section{ECTOCEMUS Pascoe, 1862a: 388}

TYPE-SPECIES: Ectocemus wallacei Pascoe, 1862a; monobasic. KEY: Kleine, 1926b: 267.

decemmaculatus Montrouzier, 1855: 37. (Megacerus decemmaculatus)

Distribution: Papua New Guinea: Madang, Mimika River, Utakawa River; Bismarck Archipelago:

Duke of York Island, New Britain, New Ireland; North Solomon Islands: Bougainville; D’Entrecasteaux

Islands: Woodlark Islands; West Papua: Ansus; Aru Island; [Australia].

Ectocemus wallacei Pascoe, 1862b: 388. NoTES: Synonoymy: Senna, 1892c: 54.

Megacerus pulchellus Kirsch, 1875: 49. NOTES: Synonoymy: Schönfeltd, 1908: 44.

Ectocemus pterygorhinus Gestro, 1876: 519. NoTES: Synonoymy: Schönfeltd, 1908: 44.

Ectocemus ruficauda Bates, 1877: 156. NoTES: Synonoymy: Fairmaire, 1883: 42.

ELYTRACANTHA Kleine, 1915: 239

TYPE-SPECIES: Belopherus pogonocerus Montrouzier, 1855; original designation. KEY: Kleine, 1926b: 267.

cerbera Kleine, 1919a: 37. (Elytracantha cerberus)

DistRIBUTION: Papua New Guinea: Sattelberg; Bismarck Archipelago: New Britain; West Papua: Cyclops Mountains.

pogonocera (Montrouzier), 1855: 37. (Belopherus pogonocerus)

DistRIBUTION: Papua New Guinea; Bismarck Archipelago: Duke of York Island; North Solomon Islands: 
Bougainville; D’Entrecasteaux Islands: Woodlark Islands; West Papua: Cyclops Mountains, Mimika River, Utakwa River.

Ectocemus spinipennis Fairmaire, 1881a: 349. NOTES: Synonymy: Fairmaire, 1883: 43.

GYaLOSTOMA Kleine, 1914: 174

TYPE-SPECIES: Gyalostoma jucundum Kleine, 1914 [=Baryrrhynchus deyrollei Power, 1879]; original designation.

deyrollei (Power), 1879: 298. (Baryrrhynchus)

DisTRIBUTION: Aru Islands.

Gyalostoma jucundum Kleine, 1914: 176. NoTES: Synonoymy: Kleine, 1916e: 127.

elegans Kleine, 1916a: 184. (Gyalostoma)

DisTRIBUTION: New Guinea.

Baryrrhynchus ochraceus (Kleine), 1916d: 184. NOTES: Synonoymy: Damoiseau, 1989b: 52.

HENORYCHODES Kleine, 1921: 85

TYPE-SPECIES: Henorychodes pretiosus Kleine, 1921; original designation.

pretiosus Kleine, 1921: 87. (Henorychodes)

DISTRIBUTION: New Guinea.

OrFILAIA Haedo Rossi, 1955: 63

TYPE-SPECIES: Arrhenodes vulsellatus Gyllenhal in Schönherr, 1833; original designation.

testacea Kleine, 1917a: 131. (Eupsalis)

DisTRIBUTION: New Guinea; Bismarck Archipelago: New Britain.

ORYCHODES Pascoe, 1862b: 389

TYPE-SPECIES: Brentus serrirostris Lund, 1800; subsequent designation by Lacordaire, 1866: 433. KEY: Kleine, 1926b: 266 (for Caenorychodes Kleine).

digramma (Boisduval), 1835: 310. (Arrhenodes)

DistRIBUtion: Papua New Guinea: Yule Island; Bismarck Archipelago: New Britain; West Papua: Dorey; Aru Island.

nigerrimus (Kleine), 1921a: 95. (Caenorhychodes)

DISTRIBUTION: New Guinea.

Prophthalmus Lacordaire, 1866: 427

TYPE-SPECIES: Brentus tridentatus Lund, 1800; subsequent designation by Lucas, 1920b: 539. KeYs: Kleine, 1916b: 236; Kleine, 1926b: 266.

planipennis Pascoe, 1872c: 322. (Prophthalmus)

DISTRIBUTION: New Guinea.

tridentatus (Lund), 1800: 90. (Brenthus)

DisTRIBUTION: New Guinea. 
SCHIZOEUPSALIS Kleine, 1917a: 127

TYPE-SPECIES: Eupsalis promissa Pascoe, 1872c; subsequent designation by Zimmerman, 1994b: 124.

promissa (Pascoe), 1872c: 323. (Eupsalis)

DisTRIBUTION: New Guinea; Aru Islands.

STRATIORRHINA Pascoe, 1872c: 322

TYPE-SPECIES: Arrhenodes xiphias Westwood, 1848; original designation.

mirabilis Kleine, 1926a: 43. (Stratiorrhina)

DistRIBUTION: New Guinea; Aru Islands.

\section{EREMOXENINI}

ANKLEINEELLA Zimmerman, 1994b: 43

TYPE-SPECIES: Amorphocephalus sulcicollis Pascoe, 1872c; original designation. KeY: Zimmerman, 1994b: 43.

sulcicollis (Pascoe), 1872c: 321. (Amorphocephalus)

Distribution: Papua New Guinea: Milne Bay. NOTES: Transferred from Kleineella: Zimmerman, 1994b:

53.

CORDUS Schönherr, 1847: 10

TYPE-SPECIES: Cordus hospes Schönherr, 1847; original designation.

archboldi Damoiseau, 1980: 10. (Cordus)

DISTRIBUTION: New Guinea.

armaticeps Senna, 1894a: 559. (Cordus)

DisTRIBUTION: New Guinea.

cheesmanae (Kleine), 1939: 108. (Kleineella)

DistRIBUTION: West Papua: Cyclops Mountains.

elytrostriatus (Kleine), 1939: 108. (Kleineella)

DisTRIBUTION: West Papua: Cyclops Mountains.

KLEINELLA Kleine, 1917b: 276

TYPE-SPECIES: Kleinella compressicornis Kleine, 1917b: 276; monobasic. NOTES: See note in Sforzi \& Bartolozzi, 2004:

303 and 304 concerning Kleineella Strand, 1918: 167. KeY: Kleine, 1926b: 265.

bougainvillei Damoiseau, 1966b: 150. (Kleineella)

DISTRIBUTION: North Solomon Islands: Bougainville.

compressicornis Kleine, 1917b: 276. (Kleineella)

DisTRIBUTION: West Papua: Waigeo Island.

fraterna Kleine, 1924: 33. (Kleineella fraternus)

DISTRIBUTION: New Guinea.

novaeguineae (Senna), 1894a: 560. (Amorphocephalus)

DISTRIBUTION: New Guinea. 


\section{CYLADINAE}

CYLAS Latreille, 1802: 196

TYPE-SPECIES: Brentus brunneus Olivier, 1790; monobasic.

formicarius (Fabricius), 1798: 174. (Brenthus)

DistRIBUTION: New Guinea; Aru Islands.

Attelabus formicarius Fabricius, 1798: 163. NoTES: Synonymy: Fabricius, 1808: 349.

\section{CYPHAGOGINAE}

\section{CYPHAGOGINI}

AllaeOmetrus Senna, 1903: 157

TYPE-SPECIES: Allaeometrus brevicepes Senna, 1903; original designation.

bimaculatus Goossens, 2005: 46. (Allaeometrus)

DisTRIBUTION: Papua New Guinea: Baiteta.

brevicepes Senna, 1903: 158. (Allaeometrus)

Distribution: Papua New Guinea.

setosus (Schönfeldt), 1906: 35. (Zemioses)

DisTRIBUTION: Papua New Guinea.

Callipareius Senna, 1892b: 444

TYPE-SPECIES: Callipareius feae Senna, 1892b; monobasic.

planitarsus (Perroud \& Montrouzier), 1865: 142. (Diastrophus)

DisTRIBUTION: New Guinea.

Calliparsius flavolineatus Calabresi, 1922: 107. NOTES: Synonymy: Damoiseau, 1965: 19.

\section{Cyphagogus Parry, 1849: 182}

TYPE-SPECIES: Cyphagogus westwoodi Parry, 1849; subsequent designation by Lucas, 1920: 219. KeYs: Kleine, 1925:

17; Kleine, 1926b: 262; Damoiseau, 1989a: 117.

bimaculatus Damoiseau, 1989a: 134. (Cyphagogus)

DisTRIBUTION: Papua New Guinea.

cheesmanae Damoiseau, 1989a: 135. (Cyphagogus)

DISTRIBUTION: New Guinea.

crassicollis Damoiseau, 1989a: 137. (Cyphagogus)

DisTRIBUTION: New Guinea.

delicatus Lea, 1898b: 634. (Cyphagogus)

DisTRIBUTION: Papua New Guinea: Sattelberg.

Cyphagogus hauseri Kleine, 1924: 33. NOTES: Synonoymy: Damoiseau, 1989a: 122.

eichhorni Kirsch, 1875: 45. (Cyphagogus)

DistRIBUTION: Papua New Guinea.

elongatus Kleine, 1916c: 5. (Cyphagogus) 
DisTRIBUTION: Papua New Guinea: Bulolo.

hornabrooki Damoiseau, 1989a: 135. (Cyphagogus)

DISTRIBUTION: New Guinea.

modiglianii Senna, 1893b: 258. (Cyphagogus)

DistRIBUTION: West Papua: Cyclops Mountains.

nigraustralis Damoiseau, 1966c: 8. (Cyphagogus)

DisTRIBUTION: New Guinea.

odewahni Pascoe, 1864: 46. (Cyphagogus)

DISTRIBUTION: New Guinea.

Cyphagogus praecipuus Kleine, 1943: 138. Notes: Synonoymy: Damoiseau, 1964b: 478.

papuanus Damoiseau, 1989a: 135. (Cyphagogus)

DISTRIBUTION: New Guinea.

pilosus Damoiseau, 1989a: 136. (Cyphagogus)

DistRIBUTION: Papua New Guinea: Madang.

rugaticollis Damoiseau, 1989a: 135. (Cyphagogus)

DisTRIBUTION: New Guinea.

sarasini Senna, 1899: 302. (Cyphagogus)

DistRIBUTION: New Guinea; Mysol Island.

Cyphagogus frugalis Kleine, 1925: 16. NotES: Synonoymy: Damoiseau, 1964b: 493.

silvanus Senna, 1903: 154. (Cyphagogus)

Distribution: West Papua: Cyclops Mountains.

splendens Kleine, 1916c: 7. (Cyphagogus)

Distribution: Papua New Guinea; Bismarck Archipelago: New Britain; West Papua: Cyclops Mountains; Aru Island.

Cyphagogus mundus Kleine, 1941: 131. NOTES: Synonoymy: Damoiseau, 1989a: 141.

tristriatus Kleine, 1916c: 16. (Cyphagogus delicatus f. tristriatus)

DisTRIBUTION: New Guinea.

ETEROZEMUS Senna, 1903: 160

TYPE-SPECIES: Zemioses pubens Senna, 1892b; subsequent designation by Kleine, 1938: 43.

fucatus (Kleine), 1925: 17. (Mesoderes)

DisTRIBUTION: New Guinea.

Eterozemus rufomaculatus Damoiseau, 1963: 29. NOTES: Synonoymy: Damoiseau, 1979: 18.

papuanus Damoiseau, 1962: 109. (Eterozemus)

DisTRIBUTION: Papua New Guinea: Moroka.

ISOMORPHUS Kleine, 1916c: 55

TYPE-SPECIES: Isomorphus unicolor Kleine, 1916c [=Microsebus kerimi Senna, 1902]; original designation.

papuanus Damoiseau, 1964a: 407. (Isomorphus)

DisTRIBUTION: Papua New Guinea. 
TYPE-SPECIES: Mesoderes sexnotatus Senna, 1898a; subsequent designation by Lucas, 1920: 406.

KEY: Kleine, 1926b: 262.

maculatus Senna, 1898a: 67. (Mesoderes)

DiSTRIBUTION: New Guinea.

sexnotatus Senna, 1898a: 66. (Mesoderes)

DisTRIBUTION: West Papua: Humboldt Bay.

Dysmorphorhynchus amabilis Kleine, 1916c: 52.

Microsebus Kolbe, 1892: 168

TYPE-SPECIES: Microsebus adelphus Kolbe, 1892; subsequent designation by Lucas, 1920: 416.

biakianus Damoiseau, 1989a: 156. (Eusebus)

DISTRIBUTION: New Guinea. NOTES: Transferred: Sforzi \& Bartolozzi, 2004: 382.

loriae Senna, 1894a: 554. (Microsebus)

DisTRIBUTION: Papua New Guinea: Ighibirei.

NEOZEMIOSES Damoiseau, 1989a: 162

TYPE-SPECIES: Neozemioses humboldti Damoiseau, 1989a; original designation.

humboldti Damoiseau, 1989a: 163. (Neozemioses)

DISTRIBUTION: New Guinea.

\section{NESIDIOBRENTUS Damoiseau, 1964a: 415}

TYPE-SPECIES: Opisthenoxys samoanus Kleine, 1944; original designation.

bacchusi Damoiseau, 1989a: 163. (Nesidiobrentus)

DISTRIBUTION: New Guinea.

\section{OPISTHENOXYS Kleine, 1922a: 26}

TYPE-SPECIES: Opisthenoxys ochraceus Kleine, 1922a; original designation.

ochraceus Kleine, 1922a: 28. (Opisthenoxys)

DISTRIBUTION: New Guinea.

XeSTOTHORAX Damoiseau, 1989a: 169

TYPE-SPECIES: Xestothorax papuanus Damoiseau, 1989a; original designation. papuanus Damoiseau, 1989a: 169. (Xestothorax)

DisTRIBUTION: New Guinea.

\section{HOPLOPISTHIINI}

CARCINOPISTHIUS Kolbe, 1892: 174

TYPE-SPECIES: Carcinopisthius fruhstorferi Kolbe, 1892; subsequent designation by Lucas, 1920: 164. KEY: Kleine, 1926b: 265. 
doriae (Senna), 1893a: 254. (Hoplopisthius)

DISTRIBUTION: New Guinea.

forcipitiger Damoiseau, 1987: 41. (Carcinopisthius)

DISTRIBUTION: New Guinea.

fruhstorferi Kolbe, 1892: 174. (Carcinopisthius)

DISTRIBUTION: New Guinea.

Carcinopisthus interrupticosta Senna, 1898b: 224. NoTES: Synonymy: Damoiseau, 1987: 36.

kolbei Senna, 1893a: 255. (Carcinopisthius)

Distribution: Papua New Guinea: Fly River; West Papua: Andai, Salawatti Island.

Carcinopisthus papuanus Senna, 1894a: 555. NoTES: Synonymy: Damoiseau, 1987: 39.

Pseudotaphroderes forticatus Bolkay, 1911: 264. NOTES: Synonymy: Kleine, 1916c: 85.

Pseudotaphroderes papuanus Bolkay, 1911: 265. NoTES: Synonymy: Kleine, 1916c: 85.

Stratiopisthius forticula Arrow, 1940: 320. NoTES: Synonymy: Damoiseau, 1987: 39.

lamingtoni Damoiseau, 1987: 42. (Carcinopisthius)

Distribution: New Guinea.

loriae Senna \& Calabresi, 1919: 72. (Carcinopisthius)

DistRIBUTION: Papua New Guinea: Moroka.

ocularis Damoiseau, 1987: 42. (Carcinopisthius)

DisTRIBUTION: New Guinea.

\section{STEREODERMINI}

Cerobates Schönherr, 1840: 487

TYPE-SPECIES: Brentus tristriatus Lund, 1800; original designation. KEYS: Kleine, 1926b: 262; Zimmerman, 1994b: 158; Mantilleri, 2005: 623.

angustipennis Senna, 1895a: 182. (Cerobates)

DisTRIBUTION: West Papua: Dorey.

Cerobates adustus Senna, 1895a: 184. NoTES: Synonymy: Damoiseau, 1987: 70.

laevipennis Senna, 1896: 219. (Cerobates)

Distribution: Papua New Guinea.

Cerobates aequalis Kleine, 1922d: 203. Notes: Synonymy: Damoiseau, 1971b: 270.

sexsulcatus Motschulsky, 1858: 95. (Cerobates)

DistRIBUTION: Papua New Guinea; D’Entrecasteaux Islands: Fergusson Islands; North Solomon Islands;

West Papua: Cyclops Mountains; Dorey, Ighibirei.

tristriatus (Lund), 1800: 66. (Brenthus)

DistriBution: Papua New Guinea; West Papua: Cyclops Mountains, Dorey.

zazae Mantilleri, 2005: 614. (Cerobates)

Distribution: Papua New Guinea: Mailu Island, Mt. Alexander to Mt. Nisbet; West Papua: Dekai, Ormu Wari.

\section{Cerobates (IonTHocerus) Lacordaire, 1866: 415}

TYPE-SPECIES: Ionthocerus crematus Lacordiare, 1866; original designation.

cingulatus Kleine, 1939: 106. (Cerobates)

Distribution: West Papua. Notes: Transferred from Cerobates (Cerobates): Mantilleri, $2005: 619$.

Cerobates nigrothorax Damoiseau, 1987: 88. (Cerobates (Jonthocerus)) DISTRIBUTION: Papua New Guinea: 
Cromwell Range. NOTES: Synonymy: Mantilleri, 2005: 619.

ophthalmicus Pascoe, 1872c: 320. (Ionthocerus)

DistRIBUTION: New Guinea

papuensis Macleay, 1887: 194. (Cerobates (Jonthocerus))

DistRIBUTION: Papua New Guinea: Fly River.

STEREODERMUS Lacordaire, 1866: 419

TYPE-SPECIES: Arrhenodes pygmaeus Gyllenhal in Schönherr, 1833; original designation. NoTES: Synonymy of Metatrachelizus Kleine: Mantilleri, 2005: 424. KEYS: Kleine, 1925: 20; Kleine, 1926b: 263.

castigatus (Kleine), 1926a: 20. (Metatrachelizus)

DistRIBUTION: New Guinea; Salawatti Island. NOTES: Transferred: Mantilleri, 2005: 425.

confragosus (Kleine), 1926a: 20. (Metatrachelizus)

DisTRIBUTION: Mysol Island. NOTES: Transferred: Mantilleri, 2005: 425.

constans (Kleine), 1926a: 20. (Metatrachelizus)

DISTRIBUTION: Mysol Island. NOTES: Transferred: Mantilleri, 2005: 425.

\section{EURHYNCHINAE}

APORHINA Boisduval, 1835: 310

TYPE-SPECIES: Aporhina bispinosa Boisduval, 1835; monobasic. KEY: Wagner, 1912: 316.

alboguttata (Snellen van Vollenhoven), 1866: 226. (Chalcocybebus alboguttatus)

DistRIBUTION: West Papua: Salawatti Island; Waigeo Island. NoTES: Transferred: Oberprieler, 2004: 890.

aspericollis (Heller), 1910a: 27. (Chalcocybebus)

Distribution: Papua New Guinea: Torricelli Mountains.

assimilis (Györffy), 1917: 276. (Chalcocybebus)

DisTRIBUTION: Papua New Guinea: Kalo, SE of Port Moresby.

bennigseni (Wagner), 1912: 310. (Chalcocybebus)

Distribution: Papua New Guinea: Kani Mountains. NOTES: Transferred: Oberprieler, 2004: 891.

biroi (Györffy), 1917: 277. (Chalcocybebus)

DistRIBUTION: Papua New Guinea: Sattelberg. NOTES: Transferred: Oberprieler, 2004: 892.

bispinosa bispinosa Boisduval, 1835: 310. (Aporhina)

Distribution: Papua New Guinea: Bongu, Fly River, Paumomu River, Sattelberg; West Papua: Dorey,

Waigeo Island; Aru Island.

Aporhina spinosa Lacordaire, 1863: 528.

Eurhinus splendidus Blanchard, 1849: 144.

Eurhynchus superbus Heller, 1895b: 12.

bispinosa aruensis (Heller), 1896: 19. (Chalcocybebus)

DisTRIBUTION: Aru Islands.

bispinosa guttifera (Heller), 1896: 19. (Chalcocybebus)

DISTRIBUTION: West Papua: Andai.

bispinosa intermedia (Heller), 1896: 20. (Chalcocybebus)

Distribution: Papua New Guinea: Port Moresby.

bispinosa macrospilotus (Heller), 1925c: 287. (Chalcocybebus)

Distribution: Papua New Guinea: Bongu, Sattelberg. 
exarmata (Heller), 1905a: 73. (Chalcocybebus exarmatus)

Distribution: Papua New Guinea: Sattelberg.

granosispina (Heller), 1925c: 289. (Chalcocybebus)

DistRIBUTION: Papua New Guinea: Bongu, Sattelberg.

helleri (Wagner), 1912: 312. (Chalcocybebus)

DisTRIBUTION: Papua New Guinea: Kani Mountains. Notes: Transferred: Oberprieler, 2004: 894.

inermis (Heller), 1897b: 3. (Chalcocybebus)

Distribution: Papua New Guinea: Owen Stanley Range, between Alexander and Nisbet Mountains.

NOTES: Transferred: Oberprieler, 2004: 894.

insignis (Heller), 1905a: 72. (Chalcocybebus)

DisTRIBUTION: Papua New Guinea: Sattelberg. NOTES: Transferred: Oberprieler, 2004: 894.

leai (Heller), 1908a: 16. comb. nov. (Chalcocybebus)

DisTRIBUTION: West Papua: Arfak Mountains.

levigata (Heller), 1925c: 288. (Chalcocybebus levigatus)

DistRIBUtion: Papua New Guinea: Bongu, Sattelberg. NOTES: Transferred: Oberprieler, 2004: 895.

magdalenae (Györffy), 1917: 280. (Chalcocybebus)

DistRIBUTION: Papua New Guinea: Sattelberg. NOTES: Transferred: Oberprieler, 2004: 895.

massutei massutei (Heller), 1901: 16. (Chalcocybebus)

Distribution: Papua New Guinea: Aroa River. Notes: Transferred: Oberprieler, 2004: 895.

massutei interrupta (Heller), 1901: 16. (Chalcocybebus massutei v. interrupta)

DistRIBUTION: Papua New Guinea: Astrolabe Mountains. NOTES: Transferred: Oberprieler, 2004: 895.

mesopila (Györffy), 1917: 279. (Chalcocybebus mesopilus)

DistRIBUTION: Papua New Guinea: Sattelberg. NOTES: Transferred: Oberprieler, 2004: 896.

mutica (Heller), 1925c: 288. (Chalcocybebus muticus)

DistriBution: Papua New Guinea: Bongu, Sattelberg. NOTES: Transferred: Oberprieler, 2004: 896.

nitens nitens (Snellen van Vollenhoven), 1866: 225. (Chalcocybebus)

DistRIBUTION: West Papua: Waigeo Island. NOTES: Transferred: Oberprieler, 2004: 897.

nitens papuana (Heller), 1896: 20. (Chalcocybebus nitens papuanus)

DisTRIBUTION: Papua New Guinea: Bongu. NOTES: Transferred: Oberprieler, 2004: 897.

obtusispina (Heller), 1925c: 287. (Chalcocybebus)

DistRIBUtion: Papua New Guinea: Bongu, Sattelberg. NOTES: Transferred: Oberprieler, 2004: 897.

richteri (Faust), 1892b: 205. (Chalcocybebus)

DistRIBUTION: Papua New Guinea: Astrolabe Bay. Notes: Transferred: Oberprieler, 2004: 898.

\section{TRACHELIZINAE}

\section{ITHYSTENINI}

Ceocephalus Guérin-Méneville, 1833: 139

TYPE-SPECIES: Ceocephalus furcillatus Guérin-Méneville, 1833; monobasic.

griseus (Kleine), 1922b: 173. (Diurus)

DisTRIBUTION: New Guinea; Aru Islands.

philippinicus (Senna), 1911: 45. (Diurus)

DistRIBUTION: Papua New Guinea.

Diurus grootaerti Damoiseau, 1989b: 66. NOTES: Transferred: Sforzi \& Bartolozzi, 2004: 600. 
ISCHNOMERUS Labram \& Imhoff, 1838: fasc. 2

TYPE-SPECIES: Ischnomerus erythroderes Labram \& Imhoff, 1838; monobasic, combined description.

tristis (Senna), 1892a: 181. (Cediocera)

DisTRIBUTION: New Guinea.

ITHYSTENOMORPHUS Kleine, 1919b: 121

TYPE-SPECIES: Ithystenomorphus femoralis Klenie, 1919b; monobasic.

femoralis Kleine, 1919b: 123. (Ithystenomorphus)

DisTRIBUTION: New Guinea.

ITHYSTENUS Pascoe, 1862b: 390

TYPE-SPECIES: Brentus angustatus Guérin-Méneville, 1831; monobasic. KeYs: Kleine, 1919: 66; Kleine, 1925: 28; Kleine, 1926b: 268.

adoptivus Kleine, 1919b: 121. (Ithystenus)

Distribution: Papua New Guinea: Sattelberg.

alatus Kleine, 1919b: 102. (Ithystenus)

DistRIBUTION: West Papua: Cyclops Mountains.

angustatus (Guérin-Méneville), 1838: 111. (Brenthus)

DisTRIBUTION: West Papua: Dorey; Aru Islands.

appendiculatus Kleine, 1919b: 104. (Ithystenus)

DISTRIBUTION: Papua New Guinea: Mailu Island.

bicolor Sforzi \& Bartolozzi, 2004: 608. (Ithystenus)

Distribution: Papua New Guinea; Bismarck Archipelago: New Britain; West Papua: Dorey, Njau Limon

South of Mt. Bougainville.

bistriatus Kleine, 1919b: 107. (Ithystenus)

DISTRIBUTION: New Guinea.

caudatus Kleine, 1919b: 100. (Ithystenus)

DisTRIBUTION: Bismarck Archipelago: New Britain, Gazelle Peninsula.

cavicaudatus Goossens, 2005: 50. (Ithystenus)

DisTRIBUTION: Papua New Guinea: Wau.

chevrolatii (Boisduval), 1835: 313. (Brenthus)

DISTRIBUTION: Woodlark Islands.

confluens Kleine, 1919b: 119. (Ithystenus)

DisTRIBUTION: New Guinea; Bismarck Archipelago: New Britain.

cultellatus Kleine, 1919b: 96. (Ithystenus)

DisTRIBUTION: New Guinea.

cupreus Kleine, 1943: 140. (Ithystenus)

DistRIBUTION: Papua New Guinea: Sattelberg.

curvidens (Montrouzier), 1855: 38. (Leptorynchus)

Distribution: Papua New Guinea; Bismarck Archipelago: New Britain; Woodlark Island; West Papua;

Aru Islands.

cyaneiventris Kleine, 1926b: 250. (Ithystenus)

DISTRIBUTION: New Guinea. 
debilis Sharp, 1900: 387. (Ithystenus)

DistrIBUtION: Papua New Guinea; Bismarck Archipelago: New Britain.

decorus Kleine, 1919b: 117. (Ithystenus)

DISTRIBUTION: Papua New Guinea: Mailu Island.

densepunctatus Kleine, 1919b: 89. (Ithystenus)

DistRIBUTION: Papua New Guinea: Sattelberg.

diversicolor Kleine, 1930: 409. (Ithystenus)

DisTRIBUTION: Papua New Guinea: Finisterre Mountains.

forficulatus Kleine, 1931: 97. (Ithystenus)

DiSTRIBUTION: New Guinea.

francoisi (Desbrochers des Loges), 1892: 109. (Coptorhynchus)

DisTRIBUTION: New Guinea; Bismarck Archipelago: New Britain.

franklini Kleine in Kleine et al., 1925: 53. (Ithystenus)

DISTRIBUTION: New Guinea; Bismarck Archipelago.

franklinmuelleri Kleine in Kleine et al., 1925: 54. (Ithystenus franklin-mülleri)

Distribution: Papua New Guinea; West Papua: Cyclops Mountains, Njau Limon South of Mt. Bougainville.

frontalis Pascoe, 1862b: 391. (Ithystenus)

DistriBution: New Guinea; Bismarck Archipelago: New Britain; Aru Island.

hollandiae (Boisduval), 1835: 315. (Brenthus)

DisTRIBUTION: New Guinea.

impar Kleine, 1943: 141. (Ithystenus)

DisTRIBUTION: Papua New Guinea: Sattelberg.

linearis Pascoe, 1862b: 391. (Ithystenus)

DISTRIBUTION: New Guinea; Aru Islands.

muelleri Kleine in Kleine et al., 1925: 53. (Ithystenus mülleri)

DISTRIBUTION: New Guinea.

noonadani Damoiseau, 1966d: 451. (Ithystenus)

DISTRIBUTION: Papua New Guinea; Bismarck Archipelago.

ophiopsis Pascoe, 1862b: 391. (Ithystenus)

DistRIBUTION: West Papua: Njau Limon South of Mt. Bougainville; Aru Island.

perlongus Kleine, 1919b: 105. (Ithystenus)

DISTRIBUTION: New Guinea.

pumilus (Boisduval), 1835: 314. (Brenthus)

DistRIBUTION: Papua New Guinea; Bismarck Archipelago: New Britain.

punctifrons Kleine, 1919b: 113. (Ithystenus)

DISTRIBUTION: New Guinea.

sabulosus Kleine, 1919b: 92. (Ithystenus)

DISTRIBUTION: West Papua: Andai, Arfak; Aru Islands.

similis Kleine, 1919b: 98. (Ithystenus)

DISTRIBUTION: New Guinea.

spinosus Kleine, 1919b: 69. (Ithystenus)

Distribution: Papua New Guinea; Bismarck Archipelago: New Britain, New Ireland; West Papua: Aru

Islands; Mysol Island.

unicolor Kleine, 1919b: 74. (Ithystenus)

DISTRIBUTION: New Guinea; Aru Islands.

wallacei Pascoe, 1862b: 390. (Ithystenus)

DistRIBUTION: West Papua; Aru Island, Mysol Island. 
PSEUDOPHOCYLIDES Kleine, 1920d: 47

TYPE-SPECIES: Pseudophocylides insularis Kleine, 1920d; original designation. KEY: Kleine, 1926b: 268.

clarus Kleine, 1923: 406. (Pseudophocylides)

DisTRIBUTION: New Guinea.

insularis Kleine, 1920d: 48. (Pseudophocylides)

DiSTRIBUTION: New Guinea.

SYGGENITHYSTENUS Kleine, 1919b: 124

TYPE-SPECIES: Ithystenus nigronitens Kleine, 1919b [=Leptorynchus guerinii Mountrouzier, 1855]; monobasic.

guerinii (Montrouzier), 1855: 39. (Leptorynchus)

Distribution: Papua New Guinea: Bongu; D'Entrecasteaux Islands: Woodlark Islands. Ithystenus nigronitens Kleine, 1919b: 125. NOTES: Synonymy: Damoiseau, $1966 \mathrm{a}: 17$.

\section{MICROTRACHELIZINI}

Araiorrhinus Senna, 1893c: 325

TYPE-SPECIES: Araiorrhinus longirostris Senna, 1893c; subsequent designation by Kleine, 1937: 71.

armatus Damoiseau, 1987: 68. (Araiorrhinus)

DISTRIBUTION: Papua New Guinea.

exportatus Senna, 1893c: 327. (Araiorrhinus)

Distribution: Papua New Guinea.

Higonius Lewis, 1883: 299

TYPE-SPECIES: Higonius poweri Lewis, 1883; subsequent designation by Lucas, 1920: 329.

trisulcatus Damoiseau, 1987: 46. (Higonius)

DisTRIBUTION: New Guinea.

HigonODES Zimmerman, 1994b: 184

TYPE-SPECIES: Higonius novenarius Damoiseau, 1987; original designation. KEY: Damoiseau, 1987: 44.

novenarius (Damoiseau), 1987: 48. (Higonius)

Distribution: Papua New Guinea; Aru Islands. Notes: Transferred: Zimmerman, 1994b: 188.

MiCROTRACHELIZUS Senna, 1893c: 315

TYPE-SPECIES: Trachelizus lyratus Perroud, 1865; subsequent designation by Lucas, 1920: 417. KeYs: Kleine, 1926b: 265; Kleine, 1935: 308.

bhamoensis (Senna), 1892b: 456. (Trachelizus)

DistRIBUTION: Papua New Guinea: Kokoda.

Microtrachelizus occultus Kleine, 1935: 307. Notes: Synonymy: Damoiseau, 1987: 56.

brevisulcatus Senna, 1894a: 558. (Microtrachelizus) 
DistRIBUTION: Papua New Guinea: Ighibirei.

brevitibia (Senna), 1892b: 454. (Trachelizus)

DISTRIBUTION: New Guinea.

dubius Kleine, 1935: 307. (Microtrachelizus)

DisTRIBUTION: Papua New Guinea: Kokoda.

incertus Kleine, 1935: 307. (Microtrachelizus)

Distribution: Papua New Guinea: Kokoda.

incisus Kleine, 1935: 307. (Microtrachelizus)

DistRIBUTION: Papua New Guinea: Kokoda.

semistriatus Damoiseau, 1987: 56. (Microtrachelizus)

DISTRIBUTION: New Guinea.

tabaci Senna, 1893c: 322. (Microtrachelizus)

DisTRIBUTION: New Guinea.

targionii Senna, 1893c: 322. (Microtrachelizus)

DisTRIBUTION: New Guinea.

temporalis Damoiseau, 1987: 56. (Microtrachelizus)

DisTRIBUTION: New Guinea.

NeOHIGONIUS Goossens, 2005: 52

TYPE-SPECIES: Neohigonius longirostris Goossens, 2005; original designation.

longirostris Goossens, 2005: 52. (Neohigonius)

DisTRIBUTION: Papua New Guinea: Baiteta.

Parapisthius Kleine, 1935: 308

TYPE-SPECIES: Parapisthius intermedius Kleine, 1935; original designation.

intermedius Kleine, 1935: 309. (Parapisthius)

DistRIBUTION: Papua New Guinea: Kokoda.

PSEUdOHigonius Damoiseau, 1987: 53

TYPE-SPECIES: Microtrachelizus rugosisculpturatus Kleine, 1939; original designation.

rugosisculpturatus (Kleine), 1939: 107. (Microtrachelizus)

DisTRIBUTION: Papua New Guinea: Kokoda.

PSEUDOCEOCEPHALINI

AtenOphthalmus Kleine, 1920b: 4

TYPE-SPECIES: Atenophthalmus tenuicostatus Kleine, 1920b; original designation.

punctatus (Montrouzier), 1855: 40. (Arrhenodes)

DISTRIBUTION: Woodlark Islands.

tenuicostatus Kleine, 1920b: 6. (Atenophthalmus)

DISTRIBUTION: New Guinea. 
TYPE-SPECIES: Cacoschizus schmeltzii Fairmaire, 1881d; monobasic.

laevipennis Damoiseau, 1989b: 77. (Cacoschizus)

DISTRIBUTION: New Guinea.

schmeltzii (Fairmaire), 1881d: 421. (Schizotrachelus)

DistRIBUTION: Papua New Guinea; Bismarck Archipelago: Duke of York Island, New Britain.

Calyptulus Kleine, 1922c: 135

TYPE-SPECIES: Calyptulus irritans Kleine, 1922c; original designation. KEY: Kleine, 1926b: 271.

irritans Kleine, 1922c: 136. (Calyptulus)

DistRIBUTION: Papua New Guinea.

rostratus Kleine, 1922c: 137. (Calyptulus)

Distribution: Papua New Guinea: Finschhafen.

Chalybdicus Kleine, 1922d: 218

TYPE-SPECIES: Chalybdicus hahnei Kleine, 1922d; original designation.

cupronitens Kleine, 1935: 309. (Chalybdicus)

DisTRIBUTION: Papua New Guinea: Mt. Tafa.

EUBACTRUS Lacordaire, 1866: 456

TYPE-SPECIES: Eubactrus semiaeneus Lacordaire, 1866; subsequent designation by Kleine, 1938: 142. KEY: Kleine, 1926b: 270.

cavus Kleine, 1931: 97. (Eubactrus)

DISTRIBUTION: New Guinea.

spissicornis Fairmaire, 1881b: 373. (Eubactrus)

DISTRIBUTION: Bismarck Archipelago: Duke of York Island.

HORMOCERUS Schönherr, 1823: 1137

TYPE-SPECIES: Brentus reticulatus Lund, 1800; original designation. KEY: Zimmerman, 1994b: 174.

compressitarsis (Senna), 1895b: 61. (Apterorrhinus)

DisTRIBUTION: New Guinea. NOTES: Transferred: Zimmerman, 1994b: 174.

Apterorrhinus albatus Kleine, 1922e: 226. NOTES: Synonymy: Zimmerman, 1994b: 175.

reticulatus (Lund), 1800: 81. (Brentus)

Distribution: Papua New Guinea: Ighibirei.

Phocylides Pascoe, 1872c: 324

TYPE-SPECIES: Phocylides collaris Pascoe, 1872c [=Brentus bicolor Guérin-Méneville, 1831]; subsequent designation by

Kleine, 1938: 128. KEY: Kleine, 1926b: 268. 
bicolor Guérin-Méneville, 1831: pl. vi, fig. 11. (Brenthus)

DistRIBUTION: Papua New Guinea: Fly River.

Phocylides collaris Pascoe, 1872c: 325. NoteS: Synonymy: Damoiseau, 1972b: 276.

Phocylides ebenius Pascoe, 1872c: 325. NoTES: Synonymy: Damoiseau, 1972b: 276.

Phocylides pascoei Macleay, 1887: 193. NoteS: Synonymy: Damoiseau, 1972b: 276.

SCHIZOTRACHELUS Lacordaire, 1866: 454

TYPE-SPECIES: Schizotrachelus brevicaudatus Lacordaire, 1866; subsequent designation by Kleine, 1938: 148. KEY: Kleine, 1926b: 270.

cheesmani (Kleine), 1935: 304. (Hypomiolispa)

DisTRIBUTION: Papua New Guinea: Kokoda.

opulentus Kleine, 1943: 142. (Schizotrachelus)

DisTRIBUTION: New Guinea.

UROPTEROIDES Kleine, 1922d: 217

TYPE-SPECIES: Brentus douei Montrouzier, 1861; original designation.

douei (Montrouzier), 1861: 874. (Brenthus)

Distribution: Papua New Guinea; Bismarck Archipelago: New Britain; Aru Islands.

gestroi (Senna), 1894a: 562. (Uropterus)

Distribution: Papua New Guinea; Bismarck Archipelago: New Britain; West Papua: Cyclops Mountains; Aru Island.

\section{TRACHELIZINI}

ALLODAPINUS Hedicke, 1923: 72

TYPE-SPECIES: Allodapus hospiton Kleine, 1922c; original designation.

flavosignatus (Kleine), 1935: 306. (Hypomiolispa flavosignata)

Distribution: Papua New Guinea: Mafulu.

hospiton Kleine, 1922c: 147. (Allodapinus)

DISTRIBUTION: New Guinea.

longithorax Damoiseau, 1989b: 75. (Allodapinus)

DISTRIBUTION: Papua New Guinea.

opulentus (Kleine), 1935: 306. (Hypomiolispa opulenta)

DistRIBUTION: Papua New Guinea: Kokoda.

papuanus Damoiseau, 1989b: 72. (Allodapinus)

DistRIBUTION: Papua New Guinea.

BOTHRIORHINUS Fairmaire, 1881d: 421

TYPE-SPECIES: Bothriorhinus costulipennis Fairmaire, 1881d; monobasic.

costulipennis Fairmaire, 1881d: 421. (Bothriorhinus)

DISTRIBUTION: Bismarck Archipelago: Duke of York Island. 
TYPE-SPECIES: Miolispa ceylonica Desbrochers, 1890; subsequent designation by Kleine, 1918b: 164.

dissimilis Kleine, 1943: 139. (Hypomiolispa)

DisTRIBUTION: New Guinea.

exarata (Desbrochers des Loges), 1890: 223. (Miolispa)

DisTRIBUTION: New Guinea.

invisitata Kleine, 1935: 305. (Hypomiolispa)

DisTRIBUTION: Papua New Guinea: Kokoda; Bismarck Archipelago.

MiolisPa Pascoe, 1862b: 393

TYPE-SPECIES: Miolispa suturalis Pascoe, 1862b; monobasic. KeYS: Kleine, 1917c: 294 (table to seperate M. testacea and M. crassifemoralis); Kleine, 1919c: 214; Kleine, 1925: 23; Kleine, 1926b: 263; Zimmerman, 1994 b: 201 (separates $M$. australiana and $M$. novaguineensis).

aeneicollis Kleine, 1919c: 294. (Miolispa)

DisTRIBUTION: Papua New Guinea: Sattelberg; Aru Island.

affinis Kleine, 1919c: 278. (Miolispa)

DistRIBUTION: Papua New Guinea; N. Solomon Islands.

annae Kleine, 1919c: 263. (Miolispa)

DistRIBUTION: Papua New Guinea: Torricelli Range.

apicepilosa Damoiseau, 1972: 272. (Miolispa)

DisTRIBUTION: New Guinea.

aruensis Kleine, 1919c: 276. (Miolispa)

DISTRIBUTION: New Guinea; Aru Islands; Misol Island.

australiana Senna, 1897: 228. (Miolispa)

DISTRIBUTION: New Guinea (?).

coerulans Kleine, 1919c: 273. (Miolispa)

DISTRIBUTION: Papua New Guinea: Lordburg (?).

conjuncta Kleine, 1919c: 302. (Miolispa)

DISTRIBUTION: New Guinea.

cordiformis Macleay, 1887: 193. (Miolispa)

DistRIBUTION: Papua New Guinea: Fly River.

crassifemoralis Kleine, 1917c: 292. (Miolispa)

DISTRIBUTION: New Guinea.

credula Kleine, 1926c: 364. (Miolispa)

DisTRIBUTION: New Guinea.

ebenina Macleay, 1887: 194. (Miolispa)

DistRIBUTION: Papua New Guinea: Fly River; Sattelburg.

fraudatrix Kleine, 1919c: 249. (Miolispa)

DISTRIBUTION: New Guinea.

frivola Kleine, 1924: 33. (Miolispa)

DisTRIBUTION: Papua New Guinea: Wareo.

nonfriedi Senna, 1895c: 363. (Miolispa)

DisTRIBUTION: New Guinea.

novaguineensis novaguineensis (Guérin-Méneville), 1831: 109. (Brenthus)

Distribution: Papua New Guinea: Madang, Wewak; N. Solomon Islands: Bougainville; West Papua: 
Andai, Cyclops Mountains, Dorey, Humboldt Bay; Aru Island; Salawatti Island.

Brenthus puncticollis Boisduval, 1835: 312. NoTES: Synonymy: Gemminger \& Harold, 1872: 2710.

Miolispa novaeguineensis v. antennata Senna, 1897: 229. NOTES: Synonymy: Schönfeldt, 1908: 25.

novaguineensis pumilla (Montrouzier), 1855: 37. (Cephalobarbus pulmillus)

DiSTRIBUTION: Bismarck Archipelago: Duke of York I, New Britain, New Ireland; Woodlark Island.

papuana Kleine, 1919c: 274. (Miolispa)

Distribution: Papua New Guinea: Sattelberg, Wareo; Bismarck Archipelago; West Papua: Cyclops Mountains.

pygmaea Senna, 1895c: 360. (Miolispa)

DisTRIBUTION: New Guinea; Bismarck Archipelago: New Britain.

salomonensis Senna, 1894b: 11. (Miolispa)

Distribution: Papua New Guinea: Sattelberg; Bismarck Archipelago: New Britain; North Solomon Islands: Bougainville Island.

Cacotrachelus sculptipennis Sharp, 1900: 387. NOTES: Synonymy: Kleine, 1927a: 25.

simulans Senna, 1895c: 362. (Miolispa)

DistRIBUTION: New Guinea; Bismarck Archipelago; West Papua: Cyclops Mountains.

sororia Kleine, 1919c: 257. (Miolispa)

Distribution: Papua New Guinea: Torricelli Range; Bismarck Archipelago; West Papua: Cyclops Mountains.

splendida Kleine, 1917c: 289. (Miolispa)

DisTRIBUTION: New Guinea; Bismarck Archipelago.

strandi Kleine, 1919c: 297. (Miolispa)

DisTRIBUTION: Papua New Guinea; Bismarck Archipelago: New Britain.

sulcicollis Kleine, 1919c: 290. (Miolispa)

DisTRIBUTION: Papua New Guinea: Aroa River.

testacea Kleine, 1919c: 261. (Miolispa)

DistRIBUTION: Papua New Guinea: Sattelberg.

unilineata Kleine, 1944: 151. (Miolispa)

DisTRIBUTION: New Guinea.

variabilis Damoiseau, 1989b: 83. (Miolispa)

DISTRIBUTION: New Guinea.

TraChelizus Dejean, 1834: 243

TYPE-SPECIES: Brentus bisulcatus Lund, 1800; see note in Alonso-Zarazaga \& Lyal, 1999: 53]; subsequent designation by Schönherr, 1840: 490.

bisulcatus (Lund), 1800: 67. (Brenthus)

Distribution: Papua New Guinea; Bismarck Archipelago; West Papua: Cyclops Mountains, Dorey; Aru Island.

quaesitus Kleine, 1935: 304. (Trachelizus)

DisTRIBUTION: Papua New Guinea: Kokoda.

TraChelOSChIzUS Damoiseau, 1966c: 21

TYPE-SPECIES: Schizotrachelus dichrorus Lacordaire, 1866; original designation.

alienus (Kleine), 1922e: 226. (Schizotrachelus) 
DistRIBUTION: Aru Islands. NOTES: Transferred: Sforzi \& Bartolozzi, 2004: 548.

altilis (Kleine), 1922e: 226. (Schizotrachelus)

DisTRIBUTION: West Papua: Cyclops Mountains; Iffar.

bicaudatus Goossens, 2005: 47. (Tracheloschizus)

DisTRIBUTION: Papua New Guinea: Baiteta.

cameratus (Lacordaire), 1866: 456. (Schizotrachelus)

DistRIBUTION: New Guinea. NOTES: Transferred: Sforzi \& Bartolozzi, 2004: 550.

fortis (Kleine), 1935: 309. (Schizotrachelus)

Distribution: Papua New Guinea: Kokoda. NOTES: Transferred: Sforzi \& Bartolozzi, 2004: 552.

multipunctatus Damoiseau, 1989b: 92. (Tracheloschizus)

DISTRIBUTION: New Guinea.

neohibernicus Damoiseau, 1966d: 440. (Tracheloschizus)

DisTRIBUTION: Papua New Guinea; Bismarck Archipelago: New Ireland.

suspensus (Kleine), 1943: 143. (Schizotrachelus)

Distribution: Papua New Guinea: Erima, Astrolabe Bay. Notes: Transferred: Sforzi \& Bartolozzi, 2004: 558.

unicolor (Montrouzier), 1855: 39. (Arrhenodes)

DistRIBUTION: Papua New Guinea; Bismarck Archipelago: New Britain; Woodlark Island.

Schizotrachelus dentatus Kleine, 1944: 155. NoteS: Synonymy: Damoiseau, $1966 \mathrm{a}: 27$.

viridescens (Shaw), 1953: 330. (Schizotrachelus)

DistRIBUTION: New Guinea. Notes: Transferred: Sforzi \& Bartolozzi, 2004: 559.

\section{APIONIDAE}

APIONINAE

\section{IXAPIINI}

PAPUAPION Korotyaev, 1987: 108

TYPE-SPECIES: Conapion sculpturatum Faust, 1899; original designation.

sculpturatum Faust, 1899: 38. (Conapion)

DisTRIBUTION: New Guinea.

\section{RHADINOCYBINAE}

\section{NOTAPIONINI}

ALISSAPION Wanat, 2001: 302

TYPE-SPECIES: Alissapion zimmermani Wanat, 2001: 304; original designation.

ibaense Wanat, 2001: 306. (Alissapion)

DisTRIBUTION: West Papua: Iba. 
TYPE-SPECIES: Allonotapion subulirostre Wanat, 2001: 268; original designation.

bommelense Wanat, 2001: 271. (Allonotapion)

DistRiBUTION: West Papua: Bommela.

APIONOCYBUS Wanat, 2001: 212

TYPE-SPECIES: Rhadinocyba aenea Heller, 1901: 17; original designation. KeY: Wagner, 1912: 316.

aeneus (Heller), 1901: 17. (Rhadinocyba aenea)

DistRibution: Papua New Guinea: Aroa River. Notes: Transferred to Myrmacyba Heller by Kissinger, 1968: 18; Transferred from Myrmacyba: Wanat, 2001: 224.

bennigseni (Wagner), 1912: 314. (Rhadinocyba)

DistRIBUTION: Papua New Guinea: Sattelberg. NOTES: Transferred: Wanat, 2001: 232.

nigricollis (Heller), 1901: 18. (Rhadinocyba)

Distribution: Papua New Guinea: Mt. Kaindi, Aseki, Okapa, Marawaka. West Papua: Bommela.

NOTES: Transferred to Myrmacyba Heller by Kissinger, 1968: 18; Transferred from Myrmacyba: Wanat, 2001: 227.

BRACHYCYBUS Wanat, 2001: 246

TYPE-SPECIES: Brachycybus silvaticus Wanat, 2001; original designation.

silvaticus Wanat, 2001: 247. (Brachycybus)

DistribUTION: Papua New Guinea: Baiteta.

BRACHYCYBUS (NOTOCYBUS) Wanat, 2001: 249

TYPE-SPECIES: Brachycybus (Notocybus) tegminalis Wanat, 2001; original designation.

tegminalis Wanat, 2001: 249. (Brachycybus (Notocybus))

DISTRIBUTION: Papua New Guinea.

COELIAPION Wanat, 2001: 286

TYPE-SPECIES: Coeliapion bilocalis Wanat, 2001; original designation.

bilocalis Wanat, 2001: 288. (Coeliapion)

DisTRIBUTION: Papua New Guinea: Angoram, Baiteta.

GANONOTAPION Wanat, 2001: 281

TYPE-SPECIES: Ganonotapion hornabrooki Wanat, 2001; original designation.

hornabrooki Wanat, 2001: 283. (Ganonotapion)

DisTRIBUTION: Papua New Guinea: Owen Stanley Range.

HETEROCYBUS Wanat, 2001: 238

TYPE-SPECIES: Heterocybus oxystomoides Wanat, 2001; original designation. 
debilifrons Wanat, 2001: 243. (Heterocybus)

DistRIBUTION: Papua New Guinea: Madang.

oxystomoides Wanat, 2001: 241. (Heterocybus)

DistRIBUTION: Papua New Guinea: Milne Bay.

ITHYOCYBUS Wanat, 2001: 202

TYPE-SPECIES: Ithyocybus bilogayensis Wanat, 2001; original designation.

bilogayensis Wanat, 2001: 205. (Ithyocybus)

DisTRIBUTION: West Papua: Bilogay.

MICRONOTAPION Wanat, 2001: 184

TYPE-SPECIES: Micronotapion glibbiceps Wanat, 2001: 186; original designation.

magnicolle Wanat, 2001: 188. (Micronotapion)

Distribution: Papua New Guinea: Baiteta.

MYRMECOCYBUS Wanat, 2001: 200

TYPE-SPECIES: Myrmecocybus asekianus Wanat, 2001; original designation.

asekianus Wanat, 2001: 202. (Myrmecocybus)

Distribution: Papua New Guinea: Aseki.

NOTAPION Zimmerman, 1994b: 330

TYPE-SPECIES: Apion trilobicolle Lea, 1926; original designation.

riedeli Wanat, 2001: 262. (Notapion)

DisTRIBUTION: West Papua: Sentani.

OMmatocybus Wanat, 2001: 323

TYPE-SPECIES: Ommatocybus simplex Wanat, 2001; original designation.

simplex Wanat, 2001: 325. (Ommatocybus)

DISTRIBUTION: Papua New Guinea: Baiteta.

PlatyCybus Wanat, 2001: 252

TYPE-SPECIES: Platycybus altipetax Wanat, 2001; original designation.

altipetax Wanat, 2001: 253. (Platycybus)

Distribution: Papua New Guinea: Baiteta.

missai Wanat, 2001: 256. (Platycybus)

DISTRIBUTION: Papua New Guinea: Baiteta. 
SCHISMATAION Wanat, 2001: 308

TYPE-SPECIES: Schismataion tetahoense Wanat, 2001; original designation.

tetahoense Wanat, 2001: 310. (Schismataion)

DisTRIBUTION: West Papua: Kosemena.

SCHISMATAION (LEPTOTAPION) Wanat, 2001: 312

TYPE-SPECIES: Schismataion (Leptotapion) magnum Wanat, 2001; original designation.

magnum Wanat, 2001: 314. (Schismataion (Leptotapion))

Distribution: Papua New Guinea: Mt. Por.

TAPINOCYBUS Wanat, 2001: 234

TYPE-SPECIES: Tapinocybus sinakiensis Wanat, 2001; original designation.

sinakiensis Wanat, 2001: 236. (Tapinocybus)

DistRIBUTION: West Papua: Sinak.

TITANAPION Wanat, 2001: 293

TYPE-SPECIES: Rhadinocyba splendida Heller, 1901: 17; original designation.

splendidum (Heller), 1901: 17. (Rhadinocyba splendida)

Distribution: Papua New Guinea. NoTES: Transferred to Myrmacyba Heller by Kissinger, 1968: 18;

Transferred from Myrmacyba: Wanat, 2001: 295.

\section{INCERTAE SEDIS}

\section{[from Apion]}

albopictum Faust, 1899: 40. (Apion)

DisTRIBUTION: Papua New Guinea: Kapakapa.

gagatinum Motschulsky, 1858: 92. (Apion)

DistRIBUTION: Papua New Guinea. Hula, Kapakapa.

gestroi Faust, 1899: 39. (Apion)

DisTRIBUTION: Papua New Guinea: Kapakapa.

infaustum Faust, 1899: 40. (Apion)

DisTRIBUTION: D'Entrecasteaux Islands: Goodenough Island.

sculpturatum (Faust), 1899: 38. (Conapion)

DistRIBUTION: Papua New Guinea: Bujakori.

symbolum Faust, 1897a: 178. (Apion)

DISTRIBUTION: D’Entrecasteaux Islands: Fergusson Island. 


\section{BRACHYCERIDAE}

\section{OCLADIINAE}

\section{DESMIDOPHORINI}

\section{DESMIDOPHORUS Dejean, 1835: 296}

TYPE-SPECIES: Curculio hebes Fabricius, 1781; monobasic. KeY: Hubenthal, 1917b: 109.

funebris funebris (Pascoe), 1888: 416. (Desmidophorinus)

DistRiBution: Papua New Guinea: Astrolabe Bay, Bongu; Waigeo Island.

funebris fulvopilosus Hubenthal, 1917b: 35. (Desmidophorus)

DisTRIBUTION: Papua New Guinea: Sattelberg.

\section{DRYOPHTHORIDAE}

\section{CRYPTODERMATINAE}

CRYPTODERMA Ritsema, 1885: 54

TYPE-SPECIES: Calandra discors Fabricius, 1801; original designation for Oxyrhynchus Schönherr.

collare (Ritsema), 1882: 185. (Oxyrhynchus collaris)

DISTRIBUTION: New Guinea.

discors (Fabricius), 1801: 432. (Calandra)

Distribution: Papua New Guinea: Paumomu River.

\section{DRYOPHTHORINAE}

\section{DRYOPHTHORUS Germar, 1824: 302}

TYPE-SPECIES: Curculio corticalis Paykull, 1792 [see note in Alonso-Zarazaga \& Lyal, 1999: 63]; monobasic.

tricuspis Faust, 1899: 122. (Dryophthorus)

DisTRIBUTION: Papua New Guinea: Moroka.

PSILODRYOPHTHORUS Wollaston, 1873b: 441

TYPE-SPECIES: Psilodryophthorus costatus Wollaston, 1873b; monobasic.

costatus Wollaston, 1873b: 595. (Psilodryophthorus)

DistRIBUTION: West Papua: Saylee.

patruelis Voss, 1956: 132. (Psilodryophthorus)

Distribution: Papua New Guinea: Astrolabe Bay.

ORTHOGNATHINAE 


\section{ORTHOGNATHINI}

SIPALINUS Marshall, 1943: 119

TYPE-SPECIES: Calandra granulata Fabricius, 1801 [=Curculio gigas Fabricius, 1775; for Sipalus Schönherr]; original designation. KEY: Vaurie, 1971: 11.

gigas granulatus (Fabricius), 1801: 432. (Calandra)

DISTRIBUTION: Widely distributed throughout the Papuan subregion.

\section{RHYNCHOPHORINAE}

\section{DIOCALANDRINI}

DIOCALANDRA Faust, 1894: 353

TYPE-SPECIES: Calandra frumenti Fabricius, 1801; monobasic. KeY: Zimmerman, 1993: 101 (separates D. frumenti from D. taitense).

frumenti (Fabricius), 1801: 438. (Calandra)

DisTRIBUTION: West Papua: Ramoi; Aru Island.

Calandra punctigera Pascoe, 1885: 305. Notes: Synonymy: Heller, 1927a: 2.

Sitophilus frumenti v. subfasciata Boheman in Schönherr, 1838: 971.

Sitophilus stigmaticollis Gyllenhal in Schönherr, 1838: 972.

\section{MYOCALANDRA Faust, 1894: 354}

TYPE-SPECIES: Myocalandra discors Faust, 1894; monobasic.

exarata (Boheman) in Schönherr, 1838: 970. (Sitophilus exaratus)

DisTRIBUTION: Aru Islands.

\section{LITOSOMINI}

GaNAE Pascoe, 1885: 307

TYPE-SPECIES: Ganaë pulchella Pascoe, 1885; present designation

amoena Pascoe, 1885: 307. (Ganaë amcena)

DistRIBUTION: West Papua: Andai.

pulchella Pascoe, 1885: 307. (Ganä̈)

DistRIBUtION: Papua New Guinea: Paumomu River; Aru Island.

\section{LAOGENIA Pascoe, 1874: 75}

TYPE-SPECIES: Laogenia sorex Pascoe, 1874; present designation. KEY: Heller, 1927a: 10.

geminata Faust, 1898a: 212. (Laogenia)

Distribution: Papua New Guinea: Mailu Island, Milne Bay, Paumomu River. 
TYPE-SPECIES: Calandra fuliginosa Pascoe, 1885; original designation.

fuliginosa Pascoe, 1885: 306. (Calandra)

DistRIBUtion: Papua New Guinea: Fly River, Ighibirei, Irupara, Kelesi; West Papua: Hatam.

SITOPHILUS Schönherr, 1838: 967

TYPE-SPECIES: Curculio oryzae Linnaeus, 1763; original designation. KeY: Zimmerman, 1993: 110.

cribrosus Pascoe, 1885: 306. (Calandra cribosa)

DisTRIBUTION: West Papua: Sorong.

granarius (Linnaeus), 1758: 378. (Curculio)

DisTRIBUTION: Cosmopolitan: New Guinea; Yule Island.

oryzae (Linnaeus), 1763: 395. (Curculio)

Distribution: Cosmopolitan: Papua New Guinea: Dilo, Haveri, Ighibirei, Kapakapa, Moroka, Paumomou River, Rigo.

rugosulus Pascoe, 1885: 306. (Calandra rugosula)

DISTRIBUTION: West Papua: Ramoi.

\section{POLYTINI}

PolyTus Faust, 1894: 353

TYPE-SPECIES: Sitophilus mellerborgii Boheman in Schönherr, 1838; original designation.

mellerborgii (Boheman) in Schönherr, 1838: 976. (Sitophillus)

DISTRIBUTION: New Guinea.

\section{RHYNCHOPHORINI}

ABRACHIUS Roelofs, 1892: 210

TYPE-SPECIES: Abrachius insularis Roelofs, 1892; monobasic.

insularis Roelofs, 1892: 211. (Abrachius)

DISTRIBUTION: Aru Islands.

OTIDOGNATHUS Lacordaire, 1866: 273

TYPE-SPECIES: Litorhynchus westermannii Boheman in Schönherr, 1845; original designation. KeYs: Hartmann, 1901: 291; Günther, 1938: 52.

foersteri Hartmann, 1901: 290. (Otidognathus försteri)

Distribution: Papua New Guinea: Bongu.

papuanus Hartmann, 1901: 293. (Otidognathus)

DistRIBUTION: Papua New Guinea: Bongu.

purpuratus Hartmann, 1901: 291. (Otidognathus)

Distribution: Papua New Guinea: Bongu. 
rufescens Hartmann, 1901: 292. (Otidognathus)

DisTRIBUTION: Papua New Guinea: Bongu.

ursinus (Faust), 1899: 117. (Litorhynchus)

Distribution: Papua New Guinea: Moroka.

ursinus squamulosus Heller, 1926a: 291. (Otidognathus ursinus ab. or subsp.? squamulosus [sic])

DisTRIBUTION: Papua New Guinea.

velutinus Hartmann, 1901: 293. (Otidognathus)

Distribution: Papua New Guinea: Bongu.

RHYNCHOPHORUS Herbst, 1795: pl. LX

TYPE-SPECIES: Curculio palmarum Linnaeus, 1758; subsequent designation by Schönherr, 1826: 23. KEY: Wattanapongsiri, 1966: 53 (adults), 55 (larvae).

bilineatus (Montrouzier), 1855: 55. (Calandra bilineata)

Distribution: Papua New Guinea: Aitape, Bara-Bara, Bogia, Bololo, Finchhaven, Kikori, Kiunga, Lae, Madang, Maprik, Milne Bay; Admiralty Islands: Manus Island; Bismarck Archipelago: New Ireland; D'Entrecasteaux Islands: Woodlark Island; North Solomon Islands: Bougainville Island; West Papua: Jayapura, Maffin Bay, Sentani, Sorong, Tumelo, Utakawa River; Biak Island, Waigeo Islands (see also DISTRIBUTION data for synonyms below).

Sphenophorus palmarum Montrouzier, 1860: 911.

Rhynchophorus kaupi Schaufuss, 1864: 22. (Rhynchophorus) DistRIBUTION: Papua New Guinea: Madang, Mioko; Bismarck Archipelago: New Britain, Duke of York Island. NoTES: Synonoymy: Wattanapongsiri, 1966: 87.

Rhynchophorus velutinus Fairmaire, 1877: 185.

Rhynchophorus pascha v. papuanus Kirsch, 1877: 156. DisTRIBUTION: Papua New Guinea. NOTES: Synonoymy: Wattanapongsiri, 1966: 87.

Rhynchophorus montrouzieri Chevrolat, 1882: CXXXVIII. DISTRIBUTION: Aru Islands. NOTES: Synonoymy: Wattanapongsiri, 1966: 87.

Rhynchophorus rubrocinctus Chevrolat, 1883: 563. DISTRIBUTION: Papua New Guinea: Katau River. NotES: Synonoymy: Wattanapongsiri, 1966: 87.

ferrugineus (Olivier), 1790: 473. (Curculio)

DisTRIBUTION: Papua New Guinea: Port Moresby; Aru Islands.

Rhynchophorus vulneratus (Panzer) in Voet, 1798: 10. NoTES: Synonoymy: Hallet et. al, 2004: 2879.

Rhynchophorus pascha Schönherr, 1845: 218. DisTRIBUTION: West Papua: Sakoemi, Sorong. Notes: Synonoymy with Rhynchophorus vulneratus (Panzer): Wattanapongsiri, 1966: 164.

\section{SPHENOPHORINI}

ANAThymus Pascoe, 1885: 299

TYPE-SPECIES: Anathymus singularis Pascoe, 1885; monobasic.

singularis Pascoe, 1885: 299. (Anathymus)

DistRIBUTION: Papua New Guinea: Fly River.

APOROPHEMUS Günther, 1941: 50

TYPE-SPECIES: Aporophemus weiskei Günther, 1941; original designation. 
weiskei Günther, 1941: 50. (Aporophemus)

DistRIBUTION: Papua New Guinea: Astrolabe Range.

BARYSTETHUS Lacordaire, 1866: 287

TYPE-SPECIES: Calandra melanosoma Boisduval, 1835a; monobasic. KeY: Heller, 1914b: 138.

aberrans Günther, 1935: 216. (Barystethus)

DistRIBUTION: Papua New Guinea: Purari River.

ater Pascoe, 1874: 71. (Barystethus)

DistRIBUtion: Papua New Guinea: Fly River, Port Moresby; West Papua: Dorey, Sakoemi; Aru Islands. Barystethus chevrolati Faust, 1899: 119.

Barystethus ater v. basalis Faust, 1899: 119.

Barystethus ater v. dispar (Chevrolat), 1880b: 333. (Dialtates dispar).

Barystethus ater v. parvulus Heller, 1914b: 139.

Barystethus ater v. puncticollis Heller, 1914b: 139.

Barystethus ater v. rufus Faust, 1899: 119.

cletusi Heller, 1914b: 140. (Barystethus)

DisTRIBUTION: Papua New Guinea: Monumbo.

dispar dispar (Chevrolat), 1880b: 333. (Dialtates)

DistRIBUTION: Papua New Guinea: Fly River, Port Moresby.

Barystethus tropicus Pascoe, 1885: 303. NOTES: Synonoymy: Faust, 1899: 119.

dispar basalis Faust, 1899: 119. (Barystethus)

DISTRIBUTION: New Guinea.

globithorax Heller, 1914b: 142. (Barystethus)

DisTRIBUTION: Papua New Guinea.

imparatus (Pascoe), 1885: 304. (Diathetes)

DistRIBUTION: Papua New Guinea: Fly River.

imperalis Heller, 1914d: 313. (Barystethus)

DisTRIBUTION: Papua New Guinea: Sattelberg.

macilentus Heller, 1914b: 141. (Barystethus)

DISTRIBUTION: Papua New Guinea.

wahnesi Hartmann, 1901: 294. (Barystethus)

Distribution: Papua New Guinea: Bongu.

COSMOPOLITES Chevrolat, 1885: 289

TYPE-SPECIES: Calandra sordida Germar, 1824; original designation. KEY: Zimmerman, 1968: 296.

sordidus (Germar), 1824: 299. (Calandra sordida)

DistRIBUTION: Circumtropical: Papua New Guinea: Haveri, Ighibirei, Rigo; West Papua. Sphenophorus cribricollis Walker, 1859: 218.

DiATHETES Pascoe, 1874: 71

TYPE-SPECIES: Diathetes rufficollis Pascoe, 1874; subsequent designation by Zimmerman, 1993: 61. KEY: Zimmerman, 1993: 63 (subgenera).

amoenus Faust, 1898a: 210. (Diathetes)

DistRIBUTION: West Papua: Humboldt Bay. 
intrusus Faust, 1898a: 209. (Diathetes)

DISTRIBUTION: Trobriand Islands.

kukenthali Faust, 1895a: 104. (Diathetes kükenthali)

DistRIBUTION: New Guinea. NOTES: Heller, 1914a: 621 reports this species from New Guinea.

maculosus Günther, 1934: 443. (Diathetes)

DISTRIBUTION: New Guinea.

morio Pascoe, 1874: 73. (Diathetes)

DisTRIBUTION: New Guinea; Aru Islands.

pictus Pascoe, 1885: 304. (Diathetes)

DisTRIBUTION: Papua New Guinea: Fly River.

planus Heller, 1910a: 36. (Diathetes)

DISTRIBUTION: New Guinea.

pulchellus Günther, 1934: 444. (Diathetes)

DISTRIBUTION: New Guinea.

rufficollis Pascoe, 1874: 72. (Diathetes)

DisTRIBUTION: West Papua: Waigeo Island.

Diathetes rufficollis v. buergersi Günther, 1934: fig. 1.

DISTRIBUTION: New Guinea.

Diathetes rufficollis v. eremothocus Günther, 1934: 442.

DisTRIBUTION: Bismarck Archipelago: New Ireland.

sanguinosus Heller, 1915: 527. (Diathetes)

DisTRIBUTION: Papua New Guinea: Sattelberg.

sannio Pascoe, 1874: 72. (Diathetes)

DisTRIBUTION: New Guinea; Aru Islands.

strenuous Pascoe, 1874: 72. (Diathetes strenuus)

DISTRIBUTION: Aru Islands.

DiATHETES (MEGADIATHETES) Zimmerman, 1993: 73

TYPE-SPECIES: Sphenophorus schoenherri Gyllenhal, 1838; original designation.

schoenherri (Gyllenhal) in Schönherr, 1838: 875. (Sphenophorus)

DISTRIBUTION: New Guinea.

DIATHETES (LISTRODIATHES) Zimmerman, 1993: 72

TYPE-SPECIES: Diathetes signaticollis Faust, 1899; original designation.

signaticollis Faust, 1899: 120. (Diathetes)

DistRIBUtion: Papua New Guinea: Hula SE of Port Moresby.

IPHTHIMORHINUS Roelofs, 1892: 207

TYPE-SPECIES: Iphthimorhinus australasiae Roelofs, 1892; monobasic.

australasiae Roelofs, 1892: 208. (Iphthimorhinus)

DISTRIBUTION: 
weiskei (Heller), 1901: 19. (Flamingorhynchus)

DisTRIBUTION: Papua New Guinea: Astrolabe Mountains.

POTERIOPHORUS Schönherr, 1838: 845

TYPE-SPECIES: Poteriophorus niveus Gyllenhal in Schönherr, 1838; original designation.

angulicollis Heller, 1915: 525. (Poteriophorus)

DisTRIBUTION: New Guinea.

Prodioctes Pascoe, 1874: 66

TYPE-SPECIES: Prodioctes quinarius Pascoe, 1874; subsequent designation by Voss, 1958a: 120.

heydeni Heller, 1910a: 35. (Prodioctes)

DISTRIBUTION: New Guinea.

RHABDOSCELUS Marshall, 1943: 119

TYPE-SPECIES: Sphenophorus maculatus Gyllenhal in Schönherr, 1838; subsequent designation by Faust, $1894: 348$. KEY: Zimmerman, 1993: 80.

interstitialis (Boheman), 1859: 148. (Sphenophorus)

DisTRIBUTION: New Guinea.

obscurus (Boisduval), 1835: 448. (Calandra)

Distribution: Papua New Guinea: Port Moresby; Bismarck Archipelago: New Ireland; Aru Island;

Mysol Island. NOTES: Transferred: Marshall, 1943: 119.

Sphenophorus nudicollis Kirsch, 1877: 156. NOTES: Synonymy: Faust, 1894: 348.

Sphenophorus promissa Pascoe, 1885: 300. NOTES: Synonymy: Faust, 1893: 150.

Sphenophorus beccarii Pascoe, 1885: 301. NOTES: Synonymy: Faust, 1893: 150.

Sphenophorus interruptocostatus Schaufuss, 1885: 204. Distribution: Papua New Guinea: Hula, Ighibirei,

Irupara, Kamali, Kelesi, Paumomu River, Port Moresby, Rigo.

tricolor Heller, 1915: 527. (Rhabdoscelus)

DiSTRIBUTION: New Guinea.

SCHLAGinHaUfenia Heller, 1910a: 37

TYPE-SPECIES: Schlaginhaufenia valida Heller, 1910a; monobasic.

valida Heller, 1910a: 37. (Schlaginhaufenia)

DisTRIBUTION: New Guinea.

SPARGANOBASIS Marshall, 1915: 531

TYPE-SPECIES: Sparganobasis subcruciata Marshall, 1915; monobasic.

subcruciata Marshall, 1915: 532. (Sparganobasis subcruciatus)

DistriBution: West Papua: Andai, Mysol, Saylee, Utakwa River; Bacan Island. 
Type-species: Curculio quadripunctatus Weber, 1801 [non Goeze; =Rhynchophorus cinereus Illiger in Wiedeman, 1800: 115]; original designation. Key: Günther, 1936: 100.

pygidialis Chevrolat, 1883: 566. (Sphenocorynes)

DisTRIBUTION: Sumatra; New Guinea (?). Note: Range extension: Günther, 1936: 102.

SPHENOPHORUS Schönherr, 1838: 874

TYPE-SPECIES: Curculio abbreviatus Fabricius, 1787; original designation.

circumscriptus (Gemminger \& Harold), 1871: 2647. (Calandra)

DisTRIBUTION: Woodlark Islands. NOTES: Replacement name for Calandra cinctus Montrouzier, 1855: 55 .

nebulosa Macleay, 1887: 192. (Sphenophorus)

DistRIBUTION: Papua New Guinea: Fly River.

torrida (Pascoe), 1885: 302. (Sphenophorus torridus)

DISTRIBUTION: Aru Islands.

TROCHORHOPALUS Kirsch, 1877: 156

TYPE-SPECIES: Sphenophorus strangulatus Gyllenhal in Schönherr, 1838; monobasic.

strangulatus (Gyllenhal) in Schönherr, 1838: 963. (Sphenophorus)

Distribution: Papua New Guinea: Dilo, Fly River, Hula, Ighibirei, Irupara; West Papua: Katau, Ramoi;

Yapen Island.

\section{STROMBOSCERINAE}

\section{DRYOPHTHOROIDES Roelofs, 1879: LIV}

TYPE-SPECIES: Dryophthoroides sulcatus Roelofs, 1879; monobasic.

beccarii (Pascoe), 1885: 310. (Elatticus)

DisTRIBUTION: West Papua: Hatam.

seftoni (Zimmerman), 1944b: 203. (Pembertonia)

DistRIBUTION: Papua New Guinea: Koitaki.

\section{Parasynommatus Voss, 1956: 131}

TYPE-SPECIES: Parasynommatus pectoralis Voss, 1956; original designation.

pectoralis Voss, 1956: 131. (Parasynommatus)

DISTRIBUTION: New Guinea.

SYNOMMATUS Wollaston, 1873b: 444

TYPE-SPECIES: Synommatus confluens Wollaston, 1873b; monobasic.

confluens Wollaston, 1873b: 596. (Synommatus)

Distribution: Papua New Guinea: Moroka. 


\section{ERIRHINIDAE}

\section{ERIRHININAE}

\section{INCERTAE SEDIS}

\section{CTYLindra Pascoe, 1885: 227}

TYPE-SPECIES: Ctylindra rhomboidea Pascoe, 1885; monobasic.

rhomboidea Pascoe, 1885: 227. (Ctylindra)

Distribution: Papua New Guinea: Fly River.

\section{TADIINAE}

\section{TADIINI}

TAdiUS Pascoe, 1885: 253

TYPE-SPECIES: Tadius erirhinoides Pascoe, 1885; monobasic. KEY: Zimmerman, 1993: 138.

erirhinoides Pascoe, 1885: 253. (Tadius)

DISTRIBUTION: New Guinea.

\section{CURCULIONIDAE}

\section{BARIDINAE}

\section{BARIDINI}

\section{BARIDINA}

\section{AulaCOBARIDIA Hustache, 1938: 94}

TYPE-SPECIES: Aulacobaris pictipennis Lea, 1931b: 160; monobasic.

pictipennis (Lea), 1931b: 160. (Aulacobaris)

DistRIBUTION: Papua New Guinea: Mt. Lamington.

BARIS Germar, 1817: 340

TYPE-SPECIES: Curculio artemisiae Herbst, 1795; subsequent designation by Curtis, 1839: 766.

antennalis Lea, 1931b: 145. (Baris)

DiSTRIBUTION: Papua New Guinea: Mt. Lamington.

atropolita Lea, 1931b: 141. (Baris)

Distribution: Papua New Guinea: Bisiatabu near Pt. Moresby, Mt. Lamington, Wareo. bialbivittata Lea, 1931b: 150. (Baris) 
DistRIBUTION: Papua New Guinea: Mt. Lamington.

bimaculibasis Lea, 1931b: 152. (Baris)

Distribution: Papua New Guinea: Mt. Lamington.

castaneicornis Lea, 1931b: 146. (Baris)

DistRIBUTION: Papua New Guinea: Wareo.

convergens Lea, 1931b: 148. (Baris)

DistRIBUTION: Papua New Guinea: Mt. Lamington.

cordipennis Lea, 1931b: 143. (Baris)

DISTRIBUTION: New Guinea.

ebenina Pascoe, 1885: 292. (Baris)

Distribution: West Papua: Andai.

edentata Lea, 1931b: 144. (Baris)

Distribution: Papua New Guinea: Bisiatabu near Pt. Moresby.

eurysterna Lea, 1931b: 152. (Baris)

Distribution: Papua New Guinea: Mt. Lamington.

fulvicornis Pascoe, 1885: 292. (Baris)

DistRIBUtion: West Papua: Fly River; West Papua: Dorey.

gibbicollis Lea, 1931b: 139. (Baris)

Distribution: Papua New Guinea: Manumbo; Aru Island.

inusiata Lea, 1931b: 151. (Baris)

DistRIBUTION: Papua New Guinea: Manumbo.

lacteomaculata Heyden, 1911: 362. (Baris)

DISTRIBUTION: Aru Islands.

laevissima Lea, 1931b: 150. (Baris)

DisTRIBUTION: Papua New Guinea: Mt. Lamington, Wareo.

leucospila Pascoe, 1885: 291. (Baris)

Distribution: Papua New Guinea: Katau River.

liosoma Lea, 1931b: 141. (Baris)

Distribution: Papua New Guinea: Mt. Lamington.

longicollidis Hustache, 1938: 60. (Baris)

DistRIBUTION: New Guinea. NOTES: Replacement name for B. longicollis Lea, 1915: 687, non Faust. megalops Lea, 1931b: 146. (Baris)

DisTRIBUTION: Papua New Guinea: Mt. Lamington.

mesosternalis Lea, 1931b: 149. (Baris)

Distribution: Papua New Guinea: Finschhafen, Mt. Lamington.

obscuripes Lea, 1931b: 145. (Baris)

DisTRIBUTION: Papua New Guinea: Wareo.

parvidentipes Lea, 1931b: 144. (Baris)

Distribution: Papua New Guinea: Mt. Lamington.

porosterna Lea, 1931b: 140. (Baris)

DistRIBUTION: Papua New Guinea: Astrolabe Bay; Aru Island.

punctimedia Lea, 1931b: 146. (Baris)

Distribution: Papua New Guinea: Mt. Lamington.

punctivaria Lea, 1931b: 140. (Baris)

Distribution: Papua New Guinea: Bisiatabu near Pt. Moresby, Mt. Lamington, Wareo.

semipunctata Lea, 1931b: 148. (Baris)

Distribution: Papua New Guinea: Mt. Lamington. 
simplicipennis Lea, 1931b: 150. (Baris)

DisTRIBUTION: Papua New Guinea: Mt. Lamington.

stictoptera Lea, 1931b: 151. (Baris)

DisTRIBUTION: New Guinea: Caprivizipfel (?).

sororia Lea, 1906: 87. (Baris)

Distribution: Papua New Guinea: Port Moresby; Aru Island. Notes: Range extension into the Papua subregion: Lea, 1931b: 154.

tenuicornis Lea, 1931b: 144. (Baris)

DisTRIBUTION: Papua New Guinea: Mt. Lamington.

tenuipes Lea, 1931b: 147. (Baris)

DisTRIBUTION: Papua New Guinea: Hudewa (near Finschhafen?).

trichocnemis Lea, 1931b: 141. (Baris)

DISTRIBUTION: Aru Islands.

vigilans Lea, 1931b: 148. (Baris)

DistRIBUTION: Papua New Guinea: Finschhafen.

Ptyctonotus Faust, 1899: 112

TYPE-SPECIES: Ptyctonotus gibbus Faust, 1899; monobasic.

gibbus Faust, 1899: 112. (Ptyctonotus)

Distribution: Papua New Guinea: Paumomu River.

SCLERODONTUS Faust, 1899: 114

TYPE-SPECIES: Sclerodontus comma Faust, 1899; monobasic.

comma Faust, 1899: 114. (Sclerodontus)

DisTRIBUTION: Papua New Guinea: Paumomu River.

SOLENOBARIS Lea, 1906: 102

TYPE-SPECIES: Solenobaris decipens Lea, 1906: 102; present designation.

casuarinae Lea, 1931b: 158. (Solenobaris)

DistRIBUTION: Papua New Guinea: Finschhafen.

denticulata Lea, 1931b: 155. (Solenobaris)

DISTRIBUTION: Aru Islands.

hamata Lea, 1931b: 156. (Solenobaris)

DistRIBUTION: Papua New Guinea: Hudewa (near Finschhafen?).

inermis Lea, 1931b: 157. (Solenobaris)

DistRIBUTION: Papua New Guinea: Mt. Lamington.

insignirostris Lea, 1931b: 155. (Solenobaris)

DISTRIBUTION: Papua New Guinea: Wareo.

minor Lea, 1931b: 158. (Solenobaris)

DistRIBUTION: Papua New Guinea: Mt. Lamington.

orthorrhina Lea, 1931b: 156. (Solenobaris)

DisTRIBUTION: Papua New Guinea: Mt. Lamington. 


\section{MADARINI}

\section{LEPTOSCHOININA}

ACYTHOPEUS Pascoe, 1874: 61

TYPE-SPECIES: Acythopeus tristis Pascoe, 1874; subsequent designation by Heller, 1940: 106.

bigeminatus Pascoe, 1874: 63. (Acythopeus)

Distribution: Papua New Guinea: Mt. Lamington (?); Aru Island. Notes: Range extension into Papua

New Guinea: Lea, 1931b: 163.

binotatus Faust, 1899: 108. (Acythopeus)

Distribution: Papua New Guinea: Ighibirei.

insignis Lea, 1931b: 161. (Acythopeus)

DistRIBUTION: Papua New Guinea: Bisiatabu near Pt. Moresby.

leucomelas Lea, 1931b: 160. (Acythopeus)

Distribution: Papua New Guinea: Mt. Lamington, Wareo; Aru Island.

melas Lea, 1931b: 161. (Acythopeus)

DISTRIBUTION: D'Entrecasteaux Islands: Fergusson Island.

tristis Pascoe, 1874: 62. (Acythopeus)

DisTRIBUTION: West Papua: Saylee.

BARINOGYNA Faust, 1899: 115

TYPE-SPECIES: Barinogyna ebenina Faust, 1899; monobasic.

ebenina Faust, 1899: 115. (Barinogyna)

DistRIBUTION: Papua New Guinea: Moroka.

DEGIS Pascoe, 1885: 294

TYPE-SPECIES: Degis trigonopterus Pascoe, 1885; monobasic.

castaneus Faust, 1899: 109. (Degis)

DisTRIBUTION: Papua New Guinea: Dilo.

coxalis Lea, 1931b: 170. (Degis)

DisTRIBUTION: Papua New Guinea: Mt. Lamington, Wareo.

imitator Lea, 1931b: 171. (Degis)

DisTRIBUTION: Papua New Guinea: Mt. Lamington.

residuus Lea, 1931b: 171. (Degis)

DistRIBUtion: Papua New Guinea: Mt. Lamington.

politus Hartmann, 1901: 287. (Degis)

DisTRIBUTION: Papua New Guinea: Bongu.

trigonopterus Pascoe, 1885: 294. (Degis)

Distribution: Papua New Guinea: Finschhafen, Fly River, Madang, Mt. Lamington, and Pt. Moresby.

IPSICHORA Pascoe, 1874: 58

TYPE-SPECIES: Ipsichora cupido Pascoe, 1874; subsequent designation by Lea, 1931b: 167. 
carinicollis Lea, 1931b: 167. (Ipsichora)

DisTRIBUTION: New Guinea.

coeletais Pascoe, 1874: 59. (Ipsichora coletais)

Distribution: West Papua: Dorey, Saylee.

cupido Pascoe, 1874: 58. (Ipsichora)

DistRIBUTION: Papua New Guinea: Yule Island.

femorata Pascoe, 1874: 59. (Ipsichora)

DisTRIBUTION: Aru Islands.

longipes Lea, 1931b: 166. (Ipsichora)

DistRIBUTION: Papua New Guinea: Finschhafen.

piliventris Lea, 1931b: 168. (Ipsichora)

Distribution: Papua New Guinea: Mt. Lamington.

pulchella Pascoe, 1874: 59. (Ipsichora)

DISTRIBUTION: Salawatti Island.

tibilalis Lea, 1931b: 167. (Ipsichora)

DistRIBUTION: Papua New Guinea: Mt. Lamington.

Metanthia Pascoe, 1874: 57

TYPE-SPECIES: Metanthia pyritosa Pascoe, 1874; subsequent designation by Lea, 1931b: 167.

apina Lea, 1931b: 165. (Metanthia)

DisTRIBUTION: Papua New Guinea: Wareo.

cyanea Pascoe, 1874: 58. (Metanthia)

DisTRIBUTION: Waigeo Island.

ebenina Pascoe, 1874: 57. (Metanthia)

DisTRIBUTION: New Guinea; Batchian.

gagatina Lea, 1931b: 165. (Metanthia)

Distribution: Papua New Guinea: Wareo.

granulipes Lea, 1931b: 164. (Metanthia)

DisTRIBUTION: Papua New Guinea: Mt. Lamington, Wareo.

pyritosa Pascoe, 1874: 57. (Metanthia)

DistRibution: Papua New Guinea: Fly River, Madang, Mt. Lamington; West Papua: Dorey, Saylee; Aru

Islands.

regularis Lea, 1931b: 164. (Metanthia)

DisTRIBUTION: Aru Islands.

scutellaris Lea, 1931b: 165. (Metanthia)

Distribution: Papua New Guinea: Mt. Lamington.

PSEUDOCHOLUS Lacordaire, 1866: 253

TYPE-SPECIES: Pseudocholus decipiens Lacordaire, 1886; original designation. KEY: Heller, 1908a: 32.

bivittatus Faust, 1892b: 224. (Pseudocholus)

DisTRIBUTION: New Guinea.

cinctus Pascoe, 1874: 56. (Pseudocholus)

DistribUtion: Papua New Guinea: Finschhafen (?); West Papua: Saylee, Ramoi. Notes: Range exten- 
sion into Papua New Guinea: Lea, 1931b: 169.

combinatus Heller, 1908a: 31. (Pseudocholus)

DISTRIBUTION: Bismarck Archipelago: New Britain.

decipiens Lacordaire, 1886: 254. (Pseudocholus)

DistRIBUTION: Papua New Guinea: Ighibirei.

fimbritarsis Lea, 1931b: 168. (Pseudocholus)

DistRIBUTION: Papua New Guinea: Mt. Lamington.

lacordairei Faust, 1892b: 225. (Pseudocholus)

DISTRIBUTION: New Guinea.

longimanus Heller, 1908a: 32. (Pseudocholus)

DistRIBUTION: Papua New Guinea: Astrolabe Mountains.

querulus Pascoe, 1885: 297. (Pseudocholus)

DistRIBUTION: Papua New Guinea: Mt. Lamington, Yule Island; Aru Island (?).

rutilicollis Heller, 1935: 202. (Pseudocholus)

DisTRIBUTION: New Guinea.

ZENA Pascoe, 1885: 295

TYPE-SPECIES: Zena cynethioides Pascoe, 1885; original designation.

megaphola Lea, 1931b: 154. (Zena)

DISTRIBUTION: New Guinea.

virgata (Boheman) in Schönherr, 1845: 176. (Baris)

DistRIBUTION: Papua New Guinea: Dilo, Fly River, Ighibirei.

Zena cynethioides Pascoe, 1885: 296.

vittipennis Lea, 1931b: 154. (Zena)

Distribution: Papua New Guinea: Finschhafen, Manumbo, Mt. Lamington.

\section{TONESIINA}

\section{MYCTIDES Pascoe, 1874: 59}

TYPE-SPECIES: Myctides barbatus Pascoe, 1874; monobasic.

barbatus Pascoe, 1874: 60. (Myctides)

Distribution: Papua New Guinea: Finschhafen, Pt. Moresby; Aru Island. Notes: Range extension into Aru and New Guinea: Lea, 1931b: 163.

filirostris Lea, 1931b: 163. (Myctides)

DiSTRIBUTION: New Guinea.

nitidulus Pascoe, 1885: 293. (Myctides)

DistRIBUTION: Papua New Guinea: Fly River.

\section{MADOPTERINI}

\section{MADOPTERINA}

CENTRINOPSIS Roelofs, 1875: 185 
TYPE-SPECIES: Centrinopsis nitens Roelofs, 1875; monobasic.

croceicornis (Pascoe), 1885: 287. (Idogenia)

Distribution: Papua New Guinea: Ighibirei. Notes: Transferred: Alonso-Zarazaga, 1999: 96; Range extention; Faust, 1899: 83.

XENOPSILUS Faust, 1899: 110

TYPE-SPECIES: Xenopsilus fuscicornis Faust, 1899; present designation.

bicolor Faust, 1899: 111. (Xenopsilus)

DistRIBUTION: Papua New Guinea: Paumomu River.

fuscicornis Faust, 1899: 110. (Xenopsilus)

DisTRIBUTION: Papua New Guinea: Moroka.

\section{CEUTHORHYNCHINAE}

\section{MECYSMODERINI}

\section{COELIOSOMUS Motschulsky, 1858: 70}

TYPE-SPECIES: Coeliosomus nigrorufus Motschulsky, 1858; subsequent designation by Colonnelli, 1992: 399.

australis (Colonnelli), 1979: 27. (Mecysmoderes)

Distribution: Papua New Guinea: Mt. Missim, Wau.

\section{CONODERINAE}

\section{ARACHNOPODINI}

ARaChNOBas Boisduval, 1835: 434

TYPE-SPECIES: Cryptorhynchus striga Guérin-Méneville, 1831; subsequent designation by Alonso-Zarazaga \& Lyal, 1999: 109. KeYs: Heller, 1908a: 25; Heller, 1933a: 20.

acuminatus (Heller), 1926a: 289. comb. nov. (Arachnopus)

Distribution: Papua New Guinea.

acutipennis (Gestro), 1879: 564. comb. nov. (Arachnopus)

Distribution: Papua New Guinea: Fly River.

affinis (Heller), 1933a: 24. comb. nov. (Arachnopus)

Distribution: Papua New Guinea: Finisterre Mountains, Komba.

alboscapulatus (Gestro), 1875: 1009. comb. nov. (Arachnopus)

DisTRIBUTION: West Papua: Geelvink Bay, Korido.

anthonyi (Faust), 1898a: 194. comb. nov. (Arachnopus)

DISTRIBUTION: Papua New Guinea: Mailu Island.

asignatus (Heller), 1933a: 25. comb. nov. (Arachnopus)

DistRIBUtION: Papua New Guinea: Astrolabe Mountains.

avus (Heller), 1933a: 23. comb. nov. (Arachnopus) 
DisTRIBUTION: Papua New Guinea: Bongu.

basalis (Heller), 1910a: 32. comb. nov. (Arachnopus)

DISTRIBUTION: New Guinea.

biguttatus (Faust), 1899: 98. comb. nov. (Arachnopus)

DisTRIBUTION: Papua New Guinea: Haveri.

binotatus (Pascoe), 1871a: 258. comb. nov. (Arachnopus)

DistRIBUTION: Papua New Guinea: Fly River; West Papua: Aru Islands.

caudatus (Heller), 1915: 522. comb. nov. (Arachnopus)

DistRIBUTION: Papua New Guinea: Huon Gulf.

corpulentus (Heller), 1926a: 288. comb. nov. (Arachnopus)

DisTRIBUTION: New Guinea.

costulatus (Heller), 1926a: 290. comb. nov. (Arachnopus)

DistRIBUTION: Papua New Guinea.

disparilis (Faust), 1898a: 191. comb. nov. (Arachnopus)

DistribUtion: Papua New Guinea: Mailu Island, Paumomou River.

effigies (Heller), 1910a: 33. comb. nov. (Arachnopus)

DiSTRIBUTION: New Guinea.

fenestratus (Faust), 1892b: 224. comb. nov. (Arachnopus)

DisTRIBUTION: New Guinea.

Arachnopus fenestratus v. demissus (Faust), 1898a: 192. Distribution: New Guinea: Moroka Bay, Paumomou River.

ferus (Heller), 1908a: 29. comb. nov. (Arachnopus)

DisTRIBUTION: Papua New Guinea: Aroa River.

fimbriatus (Heller), 1914a: 656. comb. nov. (Arachnopus)

DISTRIBUTION: Biak Island.

Arachnopus biplagiatus Marshall, 1915: 526. NOTES: Synonymy: Heller, 1933a: 19; see also Marshall, 1915: 541.

finschi (Heller), 1937a: 280. comb. nov. (Arachnopus)

DISTRIBUTION: New Guinea.

fortis (Heller), 1908a: 27. comb. nov. (Arachnopus)

Distribution: Papua New Guinea: Aroa River, Paumomu River.

fossulatus fossulatus (Faust), 1892b: 223. comb. nov. (Arachnopus)

DisTRIBUTION: New Guinea.

fossulatus nigrofimbriatus (Heller), 1937a: 281. comb. nov. (Arachnopus)

DISTRIBUTION: New Guinea.

frenatus (Snellen van Vollenhoven), 1866: 228. comb. nov. (Arachnopus)

DistRIBUTION: West Papua: Ramoi; Salawatti Island.

furcillifer (Heller), 1933a: 23. comb. nov. (Arachnopus)

DistribUtion: Papua New Guinea: Finisterre Mountains, Komba.

gazella Boisduval, 1835: 436. (Arachnobas)

Distribution: Papua New Guinea: Fly River; West Papua: Andai, Dorey; Waigeo Island; Aru Island.

Arachnopus gazella v. vitticollis Heller, 1908: 27. NOTES: Range extension: Heyden, 1911: 362.

granulipennis (Heller), 1937a: 280. comb. nov. (Arachnopus)

DISTRIBUTION: New Guinea.

guttulifer (Gestro), 1875: 1009. comb. nov. (Arachnopus)

Distribution: West Papua: Geelvink Bay, Korido.

imitator (Faust), 1898a: 190. comb. nov. (Arachnopus) 
Distribution: West Papua: Humboldt Bay.

incanus (Marshall), 1915: 530. comb. nov. (Arachnopus)

DisTRIBUTION: West Papua: Utakwa River.

interruptus (Fairmaire), 1883: 40. comb. nov. (Arachnopus)

DISTRIBUTION: Bismarck Archipelago: Duke of York Island.

lacerta (Faust), 1898a: 192. comb. nov. (Arachnopus)

Distribution: Papua New Guinea: Mailu Island, Moroka.

lanceolatus (Marshall), 1915: 528. comb. nov. (Arachnopus)

DisTRIBUTION: West Papua: Utakwa River.

loriai (Heller), 1933a: 22. comb. nov. (Arachnopus)

DistRIBUTION: Papua New Guinea: Paumomu River.

melanospilus (Gestro), 1879: 563. comb. nov. (Arachnopus)

DistRIBUTION: Papua New Guinea: Fly River.

misoriensis (Gestro), 1875: 1010. comb. nov. (Arachnopus)

Distribution: West Papua: Geelvink Bay, Korido.

nepos (Heller), 1933a: 22. comb. nov. (Arachnopus)

DISTRIBUTION: Waigeo Island.

olivieri (Faust), 1898a: 191. comb. nov. (Arachnopus)

Distribution: Papua New Guinea: Haveri, Mailu Island, Moroka, Paumomu River.

papua (Heller), 1910a: 34. comb. nov. (Arachnopus)

DISTRIBUTION: New Guinea.

patronoides (Heller), 1903: 313. comb. nov. (Arachnopus)

DistRIBUTION: Papua New Guinea: Sattelberg.

Arachnopus patronoides v. bicolor (Heller), 1910a: 34. DisTRIBUTION: New Guinea.

patronus (Pascoe), 1885: 281. comb. nov. (Arachnopus)

DistRIBUTION: West Papua: Fly River; West Papua: Sorong.

pauxillus (Heller), 1915: 521. comb. nov. (Arachnopus)

DisTRIBUTION: Papua New Guinea.

persona (Snellen van Vollenhoven), 1866: 226. comb. nov. (Arachnopus)

DISTRIBUTION: Waigeo Island.

phaleratus (Pascoe), 1871a: 259. comb. nov. (Arachnopus)

DistRIBUTION: [Ceram]; New Guinea (variety).

Arachnopus phaleratus v. frater (Heller), 1908a: 25. DisTRIBUTION: New Guinea.

planatus (Marshall), 1915: 529. comb. nov. (Arachnopus)

DistRIBUTION: West Papua: Utakwa River.

praevius (Faust), 1898a: 189. comb. nov. (Arachnopus)

Distribution: Papua New Guinea.

quadraticollis (Heller), 1933a: 24. comb. nov. (Arachnopus)

DisTRIBUTION: Papua New Guinea: Torricelli Mountains.

rugosus (Heller), 1908a: 29. comb. nov. (Arachnopus)

Distribution: Papua New Guinea: Aroa River.

sannio (Pascoe), 1871a: 259. comb. nov. (Arachnopus)

DisTRIBUTION: Papua New Guinea: Fly River; West Papua: Aru Islands.

scabiosus (Heller), 1910a: 33. comb. nov. (Arachnopus)

DisTRIBUTION: New Guinea.

sectator (Faust), 1899: 99. comb. nov. (Arachnopus)

DistRIBUTION: Papua New Guinea: Moroka. 
semiruber (Heller), 1937a: 279. comb. nov. (Arachnopus)

DisTRIBUTION: New Guinea.

simius (Pascoe), 1871a: 259. comb. nov. (Arachnopus)

DistRibution: West Papua: Mysol Island.

stigmatus (Faust), 1892b: 223. comb. nov. (Arachnopus)

DISTRIBUTION: New Guinea.

striga striga (Guérin-Méneville), 1833: 128. (Cryptorhynchus)

DisTRIBUTION: West Papua: Dorey, Hatam.

striga denutatus (Heller), 1942: 214. comb. nov. (Arachnopus)

DisTRIBUTION: West Papua: Idenburg River.

subcostatus (Heller), 1908a: 28. comb. nov. (Arachnopus)

DistRIBUTION: Papua New Guinea: Astrolabe Mountains, Moroka.

subfasciatus (Heller), 1926a: 289. comb. nov. (Arachnopus)

DISTRIBUTION: Papua New Guinea.

tenuisignatus (Heller), 1908a: 30. comb. nov. (Arachnopus)

DisTRIBUTION: Papua New Guinea: Sattelberg.

tricolor (Heller), 1926a: 290. comb. nov. (Arachnopus)

Distribution: Papua New Guinea: Huon Gulf.

tristiculus (Heller), 1933a: 22. comb. nov. (Arachnopus)

DisTRIBUTION: Papua New Guinea: Aroa River.

tristis (Heller), 1908a: 26. comb. nov. (Arachnopus)

DistRIBUTION: Papua New Guinea: Aroa River.

Caenochira Pascoe, 1885: 289

TYPE-SPECIES: Caenochira doriae Pascoe, 1885; monobasic.

doriae Pascoe, 1885: 290. (Caenochira)

DistRIBUTION: Papua New Guinea: Fly River.

\section{CAMPYLOSCELINI}

\section{PHAENOMERINA}

Phaenomerus Schönherr, 1836: 632

TYPE-SPECIES: Phaenomerus sundewalli Boheman in Schönherr, 1836; original designation. KEY: Thompson, 1996: 947.

auricomus Thompson, 1996: 970. (Phaenomerus)

Distribution: Papua New Guinea: Bulolo, Kainantu, Okapa, Wau; West Papua: Enarotadi, Membey,

Ransiki, Sibil Valley, Star Mountains, Wisselmeeren.

ater Thompson, 1996: 983. (Phaenomerus)

DistRIBUTION: West Papua: Mysol Island.

canalipectus Thompson, 1996: 978. (Phaenomerus)

Distribution: Papua New Guinea: Garaina, Wau; West Papua: Mt. Meja, Ramoi.

cavipectus Thompson, 1996: 958. (Phaenomerus)

Distribution: Papua New Guinea: Mt. Lamington, Port Moresby; Bismarck Archipelago: New Britain;

Louisaide Archipelago: Misima Island. 
contrarius Thompson, 1996: 968. (Phaenomerus)

DistRIBUTION: Papua New Guinea: Okapa, Wau.

conturbator Thompson, 1996: 963. (Phaenomerus)

Distribution: Papua New Guinea: Iongai, Wau; West Papua: Membey, Ransiki, Sibil Valley, Star Mountains.

densleonis Thompson, 1996: 962. (Phaenomerus)

Distribution: Papua New Guinea: Bulolo, Mt. Suckling, Wau.

excelsior Thompson, 1996: 977. (Phaenomerus)

Distribution: Papua New Guinea: Kassem Pass, Okapa, Wau.

exilis Pascoe, 1872d: 490. (Phaenomerus)

Distribution: Papua New Guinea: Mt. Lamington, Port Moresby; West Papua: Merauke.

fuliginosus Thompson, 1996: 988. (Phaenomerus)

Distribution: Papua New Guinea: Bulolo, Mt. Lamington, Mt. Missim; West Papua: Nabire.

hornabrooki Thompson, 1996: 964. (Phaenomerus)

DistRIBUTION: Papua New Guinea: Okapa.

laminifer Thompson, 1996: 973. (Phaenomerus)

Distribution: Papua New Guinea: Fly River, Kiunga, Lake Murray, Mt. Lamington, Mt. Missim, Popondetta, Port Moresby, Vanimo, Wau; West Papua: Brazza River, Cyclops Mountains, Mayuby, Ransiki.

lineatus Pascoe, 1885: 324. (Phaenomerus)

Distribution: Papua New Guinea: Bulolo, Fly River, Kunga, Pt. Moresby, Tabubil; West Papua: Manokwari, Membey, Ransiki.

notatus Pascoe, 1872d: 490. (Phaenomerus)

Distribution: Papua New Guinea: Finschhafen, Haveri, Ighibirei, Markham River, Mt. Lamington,

Wau; West Papua: Vogelkop, Manokwari, Andai, Brazza River, Geelvink Bay, Hatam, Mayuby, Ramoi, Ramsiki, Saylee.

occultus Thompson, 1996: 972. (Phaenomerus)

Distribution: Papua New Guinea: Dreikikir, Mt. Lamington; West Papua: Bodem 11 km SE of Oerberfaren, Membey, Ransiki, Star Mountains.

okapicus Thompson, 1996: 964. (Phaenomerus)

DistRIBUTION: Papua New Guinea: Okapa.

peregrinator peregrinator Thompson, 1996: 979. (Phaenomerus)

Distribution: Papua New Guinea: Bulolo, Finischhaven, Garina, Lae, Mt. Lamington, Mt. Missim, Popondetta, Port Moresby, Wau; West Papua: Maffin Bay, Manokwari, Mayuby, Ramoi, Ramsiki; Mysol Island; Yapen Island.

peregrinator salomonicus Thompson, 1996: 981. (Phaenomerus)

DisTRIBUTION: Papua New Guinea; Bismarck Archipelago: New Britain; North Solomon Islands: Bougainville Island; West Papua: Biak Island.

pertenuis Thompson, 1996: 969. (Phaenomerus)

Distribution: Papua New Guinea: Bulolo, Gumi.

riedeli Thompson, 1996: 966. (Phaenomerus)

DistRIBUTION: West Papua: Testega.

teretirostris Thompson, 1996: 951. (Phaenomerus)

Distribution: Papua New Guinea: Mt. Lamington, Port Moresby; West Papua: Mayuby, Nabire, Ransiki; Aru Island; Biak Island. 


\section{CORYSSOMERINI}

Metialma Pascoe, 1871a: 216

TYPE-SPECIES: Metialma scenica Pascoe, 1871a; subsequent designation by Marshall, 1939b: 10 .

novata Pascoe, 1871a: 218. (Metialma)

Distribution: West Papua: Andai, Hatam; Aru Islands.

OSPHILIA Pascoe, 1871a: 219

TYPE-SPECIES: Osphilia apicalis Pascoe, 1871a; subsequent designation by Marshall, 1939b: 10.

flavirostris Pascoe, 1871a: 220. (Osphilia)

DistRIBUTION: Papua New Guinea: Astrolabe Mountains; West Papua: Mysol Island.

\section{LOBOTRACHELINI}

Metetra Pascoe, 1874: 46

TYPE-SPECIES: Metetra suturalis Pascoe, 1874; monobasic.

suturalis Pascoe, 1874: 47. (Metetra)

DisTRIBUTION: West Papua: Waigeo Island.

\section{MECOPINI}

ACHIROzeTES Heller, 1924b: 288

TYPE-SPECIES: Achirozetes kleinei Heller, 1924b; present designation.

validipes (Heller), 1894b: 33. (Chirozetes)

DistRIBUTION: New Guinea: Wandessi. NOTES: Transferred: Heller, 1924b.

Agametis Pascoe, 1870d: 473

TYPE-SPECIES: Agametis festiva Pascoe, 1870d; monobasic.

aeruginosa Faust, 1899: 104. (Agametis)

DisTRIBUTION: Papua New Guinea: Moroka.

deleta Pascoe, 1871a: 208. (Agametis)

DisTRIBUTION: West Papua: Saylee.

festiva Pascoe, 1870d: 474. (Agametis)

DisTRIBUTION: West Papua: Andai.

ortyx Pascoe, 1871a: 208. (Agametis)

Distribution: West Papua: Mysol Island.

Chirozetes Pascoe, 1870c: 447

TYPE-SPECIES: Chirozetes pectorosus Pascoe, 1870c [=Rhynchaenus sphaerops Wiedemann, 1823]; monobasic. KEYS:

Pascoe, 1871a: 210; Heller, 1894a: 7. 
auguralis Pascoe, 1871a: 211. (Chirozetes)

DISTRIBUTION: Aru Islands.

grammicus Pascoe, 1871a: 212. (Chirozetes)

DistRIBUTION: West Papua: Ramoi; Mysol Island.

luteopictus Heller, 1937a: 278. (Chirozetes)

DISTRIBUTION: New Guinea.

junix Pascoe, 1871a: 211. (Chirozetes)

Distribution: West Papua: Mysol Island.

marmoreus Pascoe, 1885: 282. (Chirozetes)

DistRIBUTION: Papua New Guinea: Fly River.

DAEDANIA Pascoe, 1871a: 212

TYPE-SPECIES: Daedania mesoleuca Pascoe, 1871a: 213; present designation. KeY: Heller, 1894a: 10.

meleagris Pascoe, 1871a: 213. (Daedania)

DISTRIBUTION: Sarawak, Singapore.

Daedania meleagris v. papuana Heller, 1894a: 11.

DisTRIBUTION: New Guinea.

mesoleuca Pascoe, 1871a: 213. (Daedania)

Distribution: West Papua: Mysol Island.

onca (Pascoe), 1885: 280. (Mecopus)

DistRIBUtION: Papua New Guinea: Fly River.

MECOPUS Schönherr, 1825: 586

TYPE-SPECIES: Rhynchaenus bispinosus Fabricius, 1801 [=Curculio bispinosus Weber, 1801]; original designation. KEY: Heller, 1894a: 16.

bispinosus bispinosus Weber, 1801: 94. (Rhynchaenus bispinosus)

Distribution: Papua New Guinea: Madang, Paumomu River; West Papua: Andai, Korido.

bispinosus moluccarum Kirsch, 1875: 43. (Mecopus moluccarum)

DisTRIBUTION: [Moluccas]; West Papua: Mysol Island, Waigeo Island; Aru Islands.

doryphorus Quoy \& Gaimard, 1824: 82. (Mecopus)

Distribution: Papua New Guinea: Haveri, Moroka, Paumomou River; West Papua: Mysol, Salawatti;

Aru Islands; Kei Island; [Australia: Queensland].

Mecopus trilineatus Rosenschöld in Schönherr, 1838: 688.

Mecopus tenuipes Pascoe, 1871a: 205.

helleri Marshall, 1930: 576. (Mecopus)

DistRibution: New Guinea. NOTES: Replacement name for Mecopus serrirostris Heller, non Pascoe.

ludovici Heller, 1894a: 23. (Mecopus)

DisTRIBUTION: New Guinea, Waigeo Island.

serrirostris Pascoe, 1871a: 206. (Mecopus)

Distribution: West Papua: Andai, Dorey, Ramoi, Sylee.

trilineatus Guérin-Méneville, 1838: 126. (Mecopus)

DisTRIBUTION: New Guinea; Bismarck Archipelago: New Ireland. 
TYPE-SPECIES: Pempherulus affinis Faust, 1898b; original designation.

confusa (Pascoe), 1885: 282. (Phylaitis)

DistRIBUTION: Papua New Guinea: Fly River. Notes: Transfered: Marshall, 1941 b: 194.

lineata (Pascoe), 1871a: 214. (Phylaitis)

Distribution: Papua New Guinea: Astrolabe Mountains; West Papua: Mysol Island. Notes: Transfered:

Marshall, 1941b: 194.

Phylaitis Pascoe, 1871a: 213

TYPE-SPECIES: Phylaitis v: alba Pascoe, 1871a; subsequent designation by Hustache, 1925: 436.

v-alba Pascoe, 1871a: 214. (Phylaitis)

Distribution: Papua New Guinea: Paumomou River; West Papua: Dorey; Mysol Island.

Talanthia Pascoe, 1871a: 206

TYPE-SPECIES: Talanthia phalangium Pascoe, 1871a; monobasic. KeY: Heller, 1894a: 31.

macropus Heller, 1894a: 32. (Talanthia)

DISTRIBUTION: New Guinea.

papuana Heller, 1903: 312. (Talanthia)

DisTRIBUTION: Papua New Guinea: Sattelberg.

\section{MENEMACHINI}

IDOPELMA Faust, 1898a: 201

TYPE-SPECIES: Idopelma bicolor Faust, 1898a; present designation

bicolor Faust, 1898a: 202. (Idopelma)

DisTRIBUTION: Papua New Guinea: Mailu Island.

unicolor Faust, 1898a: 203. (Idopelma)

DistRIBUTION: Papua New Guinea: Astrolabe Mountains, Kelesi.

TelePhaE Pascoe, 1870d: 487

TYPE-SPECIES: Telephaë laticollis Pascoe, 1870d; monobasic.

denticollis Pascoe, 1874: 48. (Telephä̈)

DisTRIBUTION: West Papua: Dorey.

multicollis Faust, 1899: 107. (Telephä̈)

DistRIBUTION: Papua New Guinea: Ighibirei.

\section{OTHIPPIINI}

Oebrius Pascoe, 1874: 54

TYPE-SPECIES: Ebrius luteicornis Pascoe, 1874; monobasic. 
luteicornis Pascoe, 1874: 54. (Ebrius)

DisTRIBUTION: West Papua: Mysol Island, Waigeo Island.

OTHIPPIA Pascoe, 1874: 49

TYPE-SPECIES: Othippia distigma Pascoe, 1874; present designation.

podagrica Pascoe, 1874: 51. (Othippia)

DistRIBUTION: West Papua: Mysol Island.

Panigena Pascoe, 1874: 52

TYPE-SPECIES: Panigena chalybea Pascoe, 1874; present designation.

cyanoptera Pascoe, 1874: 53. (Panigena)

DisTRIBUTION: West Papua: Saylee.

pedestris Pascoe, 1874: 53. (Panigena)

DistRIBUTION: West Papua: Mysol Island, Salawatti Island.

PSEniclea Pascoe, 1874: 51

TYPE-SPECIES: Pseniclea puellaris Pascoe, 1874; monobasic.

puellaris Pascoe, 1874: 52. (Pseniclea)

DisTRIBUTION: West Papua: Dorey.

\section{PIAZURINI}

GUIOMATUS Faust, 1899: 100

TYPE-SPECIES: Guiomatus quadrinodosus Faust, 1899; monobasic.

amoenus Voss, 1958b: 219. (Guiomatus)

Distribution: Papua New Guinea: Astrolabe Bay, Sattelberg.

quadrinodosus Faust, 1899: 101. (Guiomatus)

DistribUtion: Papua New Guinea: Paumomu River.

\section{SPHADASMINI}

ElASSOPHILUS Faust, 1899: 102

TYPE-SPECIES: Elassophilus albopictus Faust, 1899; monobasic.

albopictus Faust, 1899: 103. (Elassophilus)

DISTRIBUTION: Rigo.

\section{INCERTAE SEDIS}

\section{[from Copturus]}


boisduvalii (Boisduval), 1835: 439. (Zygops)

DistRIBUTION: New Guinea. NOTES: Heller, 1895a: 65 writes that the description for this species agrees with that of Enteles vigorsi Gyllenhal.

\section{COSSONINAE}

\section{COSSONINI}

Cossonus Clairville, 1798: 58

TYPE-SPECIES: Curculio linearis Fabricius, 1775; subsequent designation by Latreille, 1810: 431.

anxius Pascoe, 1885: 315. (Cossonus)

DisTRIBUTION: Papua New Guinea: Kapakapa; West Papua: Dorey

basalis Pascoe, 1885: 317. (Cossonus)

DistRIBUTION: Papua New Guinea: Goodenough Island; West Papua: Andai, Dorey.

bilineatus (Pascoe), 1885: 318. (Isotrogus)

DisTRIBUTION: Papua New Guinea: Kapakapa, Rigo.

coeloderes Chevrolat, 1877: 189. (Cossonus coeloderes)

DisTRIBUTION: New Guinea.

ephippiger ephippiger Boheman in Schönherr, 1838: 1007. (Cossonus (Dyscocossonus))

DISTRIBUTION: West Papua: Hatam; Waigeou.

ephippiger centromaculatus Voss, 1956: 132. (Cossonus (Dyscocossonus))

DistRIBUTION: Papua New Guinea: Erima Bay, Astrolabe Bay; West Papua: Geelvink Bay.

illigeri Champion, 1909: 68. (Cossonus)

DistRIBUTION: Papua New Guinea: Rigo; West Papua: Hatam.

Cossonus canaliculatus Fabricius, 1801: 496. NOTES: non Fabricius, 1792.

Cossonus incisus Pascoe, 1885: 317. NOTES: Voss, 1956: 133 treats as valid.

macer Pascoe, 1885: 316. (Cossonus)

DisTRIBUTION: West Papua: Hatam.

novaguineensis Csiki, 1936b: 168. (Cossonus)

DistRIBUTION: Papua New Guinea: Fly River. NOTES: Replacement name for Cossonus thoracicus Pascoe 1885: 318, non Boheman.

papuanus Faust, 1898a: 213. (Cossonus)

DISTRIBUTION: D’Entrecasteaux Islands: Fergusson Island.

COSSONUS (HETEROPHASIS) Wollaston, 1873b: 483

TYPE-SPECIES: Cossonus (Heterophasis) ruficollis Wollaston, 1873b; subsequent designation by Voss, 1974: 475.

concolor Wollaston, 1873b: 626. (Cossonus)

DistRIBUTION: Papua New Guinea: Fly River; West Papua: Aru Island.

ruficollis Wollaston, 1873b: 625. (Cossonus)

DisTRIBUTION: West Papua: Dorey, Ramoi.

GLOEDEMA Wollaston, 1873b: 477

TYPE-SPECIES: Gloedema spatula Wollaston, 1873b: 619; present designation.

KEYs: Voss, 1951: 82 (key to varieties of G. spatula Wollaston); Rheinheimer, 2002: 203. 
ruficollis Wollaston, 1873b: 620. (Gloedema)

DistRIBUTION: New Guinea. NOTES: Treated as the female of G. spatula by Pascoe, 1885; Species status restored: Rheinheimer, 2002: 203.

spatula Wollaston, 1873b: 619. (Gloedema)

Distribution: Papua New Guinea: Finisterre Range, Fly River, Sattelberg; West Papua: Dorey, Hatam, Saylee.

Gloedema spatula v. bispatulata Roelofs, 1875: CVI.

Gloedema spatula v. dimidiata Voss, 1951: 82.

Gloedema spatula v. penicillata Voss, 1951: 82.

HETEROPHASEOLUS Voss, 1957: 39

TYPE-SPECIES: Phloeophagosoma glaberrimum Wollaston, 1873b; original designation.

sinuaticollis (Wollaston), 1873b: 609. (Phloeophagosoma (Amorphorhynchus) sinuaticolle)

DisTRIBUTION: New Guinea.

HomalotroguS Wollaston, 1873b: 482

TYPE-SPECIES: Homalotrogus angustifrons Wollaston, 1873b; original designation.

angustifrons Wollaston, 1873b: 624. (Homalotrogus)

DisTRIBUTION: New Guinea.

LISSOPIS Wollaston, 1873b: 495

TYPE-SPECIES: Lissopis speculifrons Wollaston, 1873b; monobasic.

speculifrons Wollaston, 1873b: 643. (Lissopis)

DisTRIBUTION: New Guinea.

OXYDEMA Wollaston, 1873b: 488

TYPE-SPECIES: Oxydema fusiformis Wollaston, 1873b [=Catolethrus subcaudatus Fairmaire, 1849]; subsequent designation by Zimmerman, 1940a: 286.

attenuatum Wollaston, 1873b: 632. (Oxydema)

DiSTRIBUTION: New Guinea.

elongata Pascoe, 1885: 321. (Oxydema)

Distribution: Papua New Guinea: Paumomu River.

naso Pascoe, 1885: 321. (Oxydema)

DistRIBUTION: West Papua: Hatam.

Phloeophagosoma Wollaston, 1873a: 23

TYPE-SPECIES: Phloeophagosoma minutum Wollaston, 1873a; original designation.

Phloeophagosoma (AmORPhORHYNChUS) Wollaston, 1873b: 464

TYPE-SPECIES: Phloeophagosoma rotundicolle Wollaston, 1873b; subsequent designation by Alonso-Zarazaga \& Lyal, 1999: 116. 
fusirostre Wollaston, 1873b: 610. (Phloeophagosoma (Amorphorhynchus))

DISTRIBUTION: New Guinea.

rotundicolle Wollaston, 1873b: 610. (Phloeophagosoma (Amorphorhynchus))

DISTRIBUTION: New Guinea.

PSEUdOCOSSONUS Wollaston, 1873a: 27

TYPE-SPECIES: Pseudocossonus brevitarsus Wollaston, 1873a; subsequent designation by Marshall, 1954b: 232. KEYS: Marshall, 1954b: 232; Zimmerman, 1957; Omar et al., 2006: 60.

dimidiatus Wollaston, 1873b: 621. (Pseudocossonus)

DisTRIBUTION: West Papua: Takar.

Psilotrogus Pascoe, 1885: 318

TYPE-SPECIES: Psilotrogus extensus Pascoe, 1885; monobasic.

extensus Pascoe, 1885: 318. (Psilotrogus)

Distribution: Papua New Guinea: Bujakori, Ighibirei; West Papua: Ansus, Hatam.

STENOTRUPIS Wollaston, 1873b: 447

TYPE-SPECIES: Stenotrupis crassifrons Wollaston, 1873b; subsequent designation by Champion, 1914: 465. KEY: Voss, 1956: 136 (separates S. curvirostris and S. lemienensis).

curvirostris Voss, 1956: 136. (Stenotrupis)

DistRIBUTION: Papua New Guinea: Berlinhafen, Lemien.

depressicollis (Faust), 1899: 124. (Dioedimorpha)

Distribution: Papua New Guinea: Ighibirei.

lemienensis Voss, 1956: 136. (Stenotrupis)

Distribution: Papua New Guinea: Berlinhafen, Lemien.

STENOTRUPIS (PSEUdAPHIODA) Voss, 1956: 137

TYPE-SPECIES: Pseudaphioda cercidoptera Voss, 1956; monobasic.

cercidoptera Voss, 1956: 137. (Stenotrupis (Pseudaphioda))

Distribution: Papua New Guinea: Berlinhafen, Lemien.

STEREOBORUS Wollaston, 1873b: 485

TYPE-SPECIES: Stereoborus robustus Wollaston, 1873b; subsequent designation by Voss, 1956: 133. KEY: Voss, $1951: 78$. affinis Wollaston, 1873b: 628. (Stereoborus)

DisTRIBUTION: West Papua: Hatam.

binodifrons Marshall, 1921: 598. (Stereoborus)

DisTRIBUTION: Samoa.

Stereoborus binodifrons v. testaceiventris Voss, 1956: 134.

DisTRIBUTION: Papua New Guinea: Sattelberg.

punctirostris Wollaston, 1873b: 628. (Stereoborus)

DistriBUTION: Papua New Guinea: Yule Island (?); D’Entrecasteaux Islands: Goodenough Island. 
Stereoborus punctirostris v. obliteratus Wollaston, 1873b: 629. DistRIBUTION: New Guinea; [Kei Island]. robustus Wollaston, 1873b: 628. (Stereoborus)

DistRIBUTION: Papua New Guinea: Yule Island; West Papua: Dorey; Aru Islands.

STEREODERUS Wollaston, 1873b: 487

TYPE-SPECIES: Stereoderus barbatus Wollaston, 1873; present designation.

fronto Faust, 1899: 128. (Stereoderus)

Distribution: Papua New Guinea: Moroka.

hospes Faust, 1899: 127. (Stereoderus)

DistRIBUTION: Papua New Guinea: Moroka.

longipennis Pascoe, 1885: 320. (Stereoderus)

DistRibution: West Papua: Hatam.

STEREONOTUS Faust, 1899: 128

TYPE-SPECIES: Stereonotus doriai Faust, 1899; monobasic.

doriai Faust, 1899: 129. (Stereonotus)

DistRIBUTION: Papua New Guinea: Moroka.

TeTracoptus Wollaston, 1873b: 494

TYPE-SPECIES: Tetracoptus reductus Wollaston, 1873b; original designation.

reductus Wollaston, 1873b: 641. (Tetracoptus)

DisTRIBUTION: New Guinea.

\section{DRYOTRIBINI}

OChronanus Pascoe, 1885: 313

TYPE-SPECIES: Ochronanus pygmaeus Pascoe, 1885; monobasic. KEY: Marshall, 1958: 99.

pygmaeus Pascoe, 1885: 314. (Ochronanus)

DistRIBUtion: Papua New Guinea: Moroka; D’Entrecasteaux Islands: Fergusson Island.

subreflexus Faust, 1899: 126. (Ochronanus)

DisTRIBUTION: Papua New Guinea: Moroka.

vicinus Faust, 1899: 125. (Ochronanus)

DisTRIBUTION: Papua New Guinea: Moroka; Deslacs Island.

\section{ONYCHOLIPINI}

PSEUdostenosCelis Wollaston, 1877: 84

TYPE-SPECIES: Pseudostenoscelis sculpturata Wollaston, 1877; subsequent designation by Decelle \& Voss, $1972: 495$.

araucariae Voss, 1972: 198. (Pseudostenoscelis)

Distribution: Papua New Guinea: Bulolo. 


\section{PENTARTHRINI}

Microcossonus Wollaston, 1873b: 448

TYPE-SPECIES: Microcossonus wallacei Wollaston, 1873b; monobasic.

wallacei Wollaston, 1873b: 603. (Microcossonus)

DistRIBUTION: Papua New Guinea: Astrolabe Bay

Pentarthrum Wollaston, 1854: 129

TYPE-SPECIES: Pentarthrum huttoni Wollaston, 1854; monobasic.

rugosum Wollaston, 1873b: 600. (Pentarthrum)

DisTRIBUTION: New Guinea.

Promicrocossonus Voss, 1971: 196

TYPE-SPECIES: Promicrocossonus schedli Voss, 1971; original designation.

schedli Voss, 1971: 197. (Promicrocossonus)

DistRIBUTION: Papua New Guinea: Watut.

PROECINI

APHANOCORYNES Wollaston, 1873b: 489

TYPE-SPECIES: Aphanocorynes depressus Wollaston, 1873b; monobasic.

APHANOCORYNES (SUBAPHANOCORYNUS) Voss, 1956: 139

TYPE-SPECIES: Aphanocorynes tectus Voss, 1956; original designation.

tectus Voss, 1956: 139. (Aphanocorynes (Subaphanocorynus))

DisTRIBUTION: Papua New Guinea: Sattelberg.

CONARTHRUS Wollaston, 1873b: 491

TYPE-SPECIES: Conarthrus tarsalis Wollaston, 1873b; subsequent designation by Zimmerman, 1956: 56.

ferrugineus (Wollaston), 1873b: 638. (Eutornus)

Distribution: West Papua.

praeustus (Boheman) in Schönherr, 1838: 1069. (Conarthrus)

DISTRIBUTION: New Guinea.

ORTHOTEMNUS Wollaston, 1873b: 489

TYPE-SPECIES: Orthotemnus reflexus Wollaston, 1873b; original designation.

disparilis Pascoe, 1885: 322. (Orthotemnus) 
DISTRIBUTION: New Guinea; Asutralia.

extensus (Pascoe), 1885: 318. (Psilotrogus? [sic])

DistRibution: West Papua: Hatam, Ansus.

reflexus Wollaston, 1873b: 634. (Orthotemnus)

DISTRIBUTION: New Guinea.

\section{RHYNCOLINI}

\section{RHYNCOLINA}

COPTUS Wollaston, 1873b: 492

TYPE-SPECIES: Coptus oculatus Wollaston, 1873b; subsequent designation by Voss, 1956: 141.

minor Wollaston, 1873b: 639. (Coptus)

DistRIBUTION: Papua New Guinea: Ighibirei; Aru Island.

Stenotrupis exilis Pascoe, 1885: 313.

Himatium Wollaston, 1873b: 461

TYPE-SPECIES: Himatium pubescens Wollaston, 1873b; monobasic.

pilosus (Faust), 1899: 123. (Pholidonotus)

DistRIBUTION: Papua New Guinea: Moroka.

ProtoplatyPus Wood, 1973: 81

TYPE-SPECIES: Protoplatypus vetulus Wood, 1973; original designation.

vetulus Wood, 1973: 82. (Protoplatypus)

DisTRIBUTION: New Guinea.

RHYNCOLUS Germar, 1817: 340

TYPE-SPECIES: Curculio ater Linnaeus, 1758; subsequent designation by ICZN, 1991b: 268.

castaneipennis Voss, 1956: 141. (Rhyncolus)

DistRIBUTION: Papua New Guinea: Sattelberg.

\section{PHLOEOPHAGINA}

XeNOTRUPIS Wollaston, 1873b: 496

TYPE-SPECIES: Xenotrupis fusiformis Wollaston, 1873b; monobasic.

fusiformis Wollaston, 1873b: 643. (Xenotrupis)

DISTRIBUTION: New Guinea; Bismarck Archipelago: New Britain.

INCERTAE SEDIS 
TYPE-SPECIES: Aphyoda diura Pascoe, 1871c; present designation.

brenthoides Pascoe, 1871c: 215. (Aphyoda)

DISTRIBUTION: Waigeo Island.

diura Pascoe, 1871c: 215. (Aphyoda)

DisTRIBUTION: West Papua: Dorey, Saylee.

Coptonus Kuschel, 2000: 788

TYPE-SPECIES: Coptonus fijianus Kuschel, 2000: 792; original designation. KEY: Kuschel, et al., $2000: 791$.

papuanus Kuschel, 2000: 793. (Coptonus)

DistRIBUTION: Papua New Guinea: Wau.

Dissostomus Kuschel, 2000: 793

TYPE-SPECIES: Dissostomus hornabrooki Kuschel, 2000; original designation.

hornabrooki Kuschel, 2000: 795. (Dissostomus)

DistRIBUTION: Papua New Guinea: Awande, Okapa.

\section{CRYPTORHYNCHINAE}

\section{AEDEMONINI}

ARISTOXENUS Zimmerman, 1941: 194

TYPE-SPECIES: Deretiosus lateripennis Lea, 1931c; original designation.

lateripennis (Lea), 1931c: 391. (Deretiosus)

DisTRIBUTION: New Guinea. Notes: Transferred: Zimmerman, 1941: 196.

Diatassa Pascoe, 1871c: 192

TYPE-SPECIES: Diatassa phalerata Pascoe, 1871c; monobasic. KEY: Heller, 1932: 108.

divisa Faust, 1898a: 150. (Diatassa)

DistribUtion: Papua New Guinea: Mailu Island., Milne Bay; Fergusson Island.

phalerata Pascoe, 1871c: 193. (Diatassa)

DisTRIBUTION: West Papua: Mysol Island.

picta Pascoe, 1885: 269. (Diatassa)

DistRIBUTION: Papua New Guinea: Fly River, Moroka.

venosa Faust, 1898a: 148. (Diatassa)

DisTRIBUTION: Papua New Guinea: Moroka.

DYSTROPICUS Pascoe, 1885: 252 
TYPE-SPECIES: Dystropicus squalidus Pascoe, 1885; monobasic.

bifasciata (Pascoe), 1885: 253. (Theoclia)

DistRIBUTION: Papua New Guinea: Yule Island.

calidris (Pascoe), 1885: 267. (Berosiris)

Distribution: Papua New Guinea: Fly River, Paumomu River; D’Entrecasteaux Islands: Fergusson

Island. West Papua: Andai.

Mechistocerus compositus Faust, 1898a: 143.

Mechistocerus compositus Lea, 1907: 409

incertus (Pascoe), 1885: 268. (Berosiris)

DisTRIBUTION: Aru Islands.

squalidus Pascoe, 1885: 252. (Dystropicus)

DisTRIBUTION: Papua New Guinea: Yule Island.

Berosiris mixta Lea, 1907: 416.

DISTRIBUTION: Queensland.

tristis (Pascoe), 1885: 267. (Berosiris)

DisTRIBUTION: Papua New Guinea: Yule Island.

MeChISTOCERUS Fauvel, 1862: 42

TYPE-SPECIES: Coleosternus impressus Montrouzier, 1860; monobasic.

affinis (Faust), 1898a: 147. (Isotocerus)

Distribution: Papua New Guinea: Mailu Island, Moroka, Paumomu River.

albovarius (Heller), 1937a: 267. (Isotocerus)

DISTRIBUTION: Bismarck Archipelago: New Britain.

atronitidus Lea, 1931c: 405. (Mechistocerus)

DISTRIBUTION: New Guinea.

bivittipennis Lea, 1931c: 397. (Mechistocerus)

DISTRIBUTION: New Guinea.

comptus (Heller), 1937a: 266. (Mecistocerus)

DisTRIBUTION: New Guinea.

exiguus (Heller), 1937a: 269. (Isotocerus)

Distribution: Papua New Guinea.

fallax (Faust), 1899: 69. (Isotocerus)

DistRIBUTION: Papua New Guinea: Dilo.

granibasis Lea, 1931c: 396. (Mechistocerus)

DISTRIBUTION: New Guinea.

ingenuus Faust, 1898a: 144. (Mechistocerus)

Distribution: Papua New Guinea: Milne Bay, Moroka; D’Entrecasteaux Islands: Fergusson Island; Trobriand Island.

maculibasis Lea, 1931c: 397. (Mechistocerus)

DISTRIBUTION: New Guinea.

maculosus Lea, 1931c: 404. (Mechistocerus)

DisTRIBUTION: New Guinea.

multimaculatus Lea, 1928a: 49. (Mechistocerus)

Distribution: Papua New Guinea: Bongu. 
parvicollis Lea, 1931c: 403. (Mechistocerus)

DISTRIBUTION: New Guinea.

petax (Faust), 1898a: 145. (Isotocerus)

DISTRIBUTION: North Solomon Islands: Shortland Island.

setiger (Faust), 1899: 70. (Isotocerus)

DistRIBUTION: Papua New Guinea: Ighibirei, Paumomu River.

sordidus Lea, 1931c: 396. (Mechistocerus)

DISTRIBUTION: New Guinea.

tenuipes (Faust), 1898a: 147. (Isotocerus)

DISTRIBUTION: New Guinea.

uniformis Lea, 1931c: 403. (Mechistocerus)

DISTRIBUTION: New Guinea.

PARENDYMia Kirsch, 1877: 154

TYPE-SPECIES: Parendymia pilipes Kirsch, 1877; monobasic.

pilipes Kirsch, 1877: 154. (Parendymia)

DistribUtion: Papua New Guinea; West Papua; Yapen Island.

Cyambolus atomosparsus Fairmaire, 1878: 282.

Mechistocerus languidus Lea, 1907: 413.

RHADINOMERUS Faust, 1892b: 215

TYPE-SPECIES: Mechistocerus mastersi Pascoe, 1870b; monobasic.

maestus Faust, 1892b: 216. (Rhadinomerus)

DISTRIBUTION: New Guinea.

\section{CAMPTORHININI}

CAMPTORHINUS Schönherr, 1825: 585

TYPE-SPECIES: Curculio statua Fabricius, 1792 [=Curculio statua Rossi, 1790]; original designation.

doriae Pascoe, 1885: 254. (Camptorhinus)

Distribution: Papua New Guinea: Paumomu River.

dorsalis (Boisduval), 1835a: 434. (Cryptorhynchus)

DISTRIBUTION: Papua New Guinea: Dilo, Ighibirei, Kapakapa, Moroka.

uniformis Fairmaire, 1893: 38. (Camptorhinus)

DistriBution: Papua New Guinea: Astrolabe Bay; Bismarck Archipelago: New Britain.

DYSCAMPTORHINUS Heller, 1926a: 278

TYPE-SPECIES: Dyscamptorhinus flexuosocarinatus Heller, 1926a; monobasic.

flexuosocarinatus Heller, 1926a: 278. (Dyscamptorhinus)

DisTRIBUTION: Papua New Guinea. 
TYPE-SPECIES: Pachyonyx affaber Boheman in Schönherr, 1837; original designation.

lineatus Hartmann, 1901: 283. (Pachyonyx)

DistriBUTION: Papua New Guinea: Bongu.

\section{CRYPTORHYNCHINI}

\section{CRYPTORHYNCHINA}

AsYTeSTA Pascoe, 1865: 426

TYPE-SPECIES: Asytesta humeralis Pascoe, 1865; present designation. KEY: Faust, 1898a: 162.

albifrons Voss, 1960: 331. (Asytesta)

DisTRIBUTION: Papua New Guinea: Sattelberg.

arachnopus Heller, 1895b: 16. (Asytesta)

DisTRIBUTION: Papua New Guinea: Sattelberg.

aucta Faust, 1898a: 168. (Asytesta)

DistRIBUTION: Papua New Guinea: Ighibirei, Astrolabe Mountains.

bivirgata Pascoe, 1885: 259. (Asytesta)

DisTRIBUTION: West Papua: Hatam.

brevipennis Faust, 1898a: 173. (Asytesta)

DisTRIBUTION: Papua New Guinea: Mailu Island.

circulifera Lea, 1928a: 74. (Asytesta)

DistriBUTION: Papua New Guinea: Manumbo.

compressipes (Chevrolat), 1877: 189 (Arachnopus)

DistRIBUTION: New Guinea. Notes: Transferred: Heller, 1908a: 23, Heller, 1933: 19 also mentions that this species belongs in Asytesta. This species was omitted from the Coleopterorum Catalogus (Hustache, 1934; 1936).

Asytesta trivittata (Pascoe), 1885: 274. (Cyamobolus trivittatus). NoTES: Synonymy: Heller, 1908: 23.

dorsalis Faust, 1898a: 170. (Asytesta)

DistRIBUTION: Papua New Guinea: between Alexander and Nisbet Mountains.

eudyasmoides Heller, 1914a: 655. (Asytesta)

DISTRIBUTION: Biak Island.

gazella (Olivier), 1807: 175. (Rhynchaenus)

Distribution: New Guinea. Notes: Considerable confusion surrounds the taxonomy of this species. It has been considered a valid species, a species of Tragopus, and a synonym of Asytesta vittata Pascoe under the mistaken identity of Tragopus gazella Lacordaire, 1866: 160.

Arachnopus rotundipennis Chevrolat, 1877: 189. NoTES: Synonymy: Heller, 1908a: 23, Heller, 1933 : 19 also mentions that this species belongs in Asytesta. This species was omitted from the Coleopterorum Catalogus (Hustache, 1934; 1936).

gestroi Heller, 1895b: 15. (Asytesta)

DisTRIBUTION: Papua New Guinea: Sattelberg.

granulifera Lea, 1928a: 75. (Asytesta)

DisTRIBUTION: Papua New Guinea: Astrolabe Mountains.

lugubris lugubris Heller, 1895b: 15. (Asytesta) 
DistRIBUTION: Papua New Guinea: Bongu.

lugubris bidentatus Voss, 1958b: 219. (Asytesta)

DISTRIBUTION: New Guinea; Bismark Archipelago: Deslacs Islands.

propinqua Faust, 1898a: 170. (Asytesta)

DISTRIBUTION: D'Entrecasteaux Islands: Fergusson Island, Goodenough Island.

rata Heller, 1910a: 29. (Asytesta)

DisTRIBUTION: Papua New Guinea.

sejuncta Faust, 1898a: 167. (Asytesta)

DistRIBUTION: Papua New Guinea: Moroka.

setipes Lea, 1928a: 76. (Asytesta)

DisTRIBUTION: Papua New Guinea: Bulolo, Wau.

signata Faust, 1898a: 171. (Asytesta)

DisTRIBUTION: D’Entrecasteaux Islands: Fergusson Island.

verecunda Faust, 1898a: 169. (Asytesta)

Distribution: Papua New Guinea: Mailu Island, Milne Bay.

versuta Faust, 1898a: 166. (Asytesta)

DisTRIBUTION: New Guinea.

vittata Pascoe, 1865: 431. (Asytesta)

DisTRIBUTION: [Moluccas]; Aru Islands.

ypsilon Heller, 1903: 312. (Asytesta)

DistRIBUTION: Papua New Guinea: Sattelberg.

ATHANASIUS Zimmerman, 1994a: 651

TYPE-SPECIES: Athanasius latipennis Lea, 1915; original designation.

madangensis (Lea), 1928a: 82. (Neomystocis)

DistRIBUtION: Papua New Guinea: Manumbo, Madang. Notes: Transferred: Zimmerman, 1994a: 652.

Autillia Heller, 1903: 309

TYPE-SPECIES: Autillia horridipes Heller, 1903; monobasic.

horridipes Heller, 1903: 310. (Autillia)

DistRIBUTION: Papua New Guinea: Sattelberg.

Berosicus Pascoe, 1885: 269

TYPE-SPECIES: Berosicus persona Pascoe, 1885; monobasic.

persona Pascoe, 1885: 269. (Berosicus)

DistRIBUTION: Papua New Guinea: Fly River.

BRACHYPEZICHUS Heller, 1935: 200

TYPE-SPECIES: Brachypezichus nodulosus Heller, 1935; monobasic.

nodulosus Heller, 1935: 200. (Brachypezichus)

DISTRIBUTION: Papua New Guinea. 
TYPE-SPECIES: Chaetoctesius albovertex Heller, 1937a; original designation.

albovertex Heller, 1937a: 277. (Chaetoctesius)

DisTRIBUTION: New Guinea.

CONOMALTHUS Heller, 1908a: 20

TYPE-SPECIES: Conomalthus balanophorus Heller, 1908a; monobasic.

balanophorus Heller, 1908a: 21. (Conomalthus)

DistRIBUTION: Papua New Guinea: Aroa River.

intermedius Heller, 1926a: 282. (Conomalthus)

DisTRIBUTION: Papua New Guinea.

lophonotus Heller, 1926a: 281. (Conomalthus)

DiSTRIBUTION: Papua New Guinea.

Critomerus Lea, 1903: 663

TYPE-SPECIES: Critomerus emblematicus Lea, 1903 [=Perissops iliacus Pascoe, 1871c]; monobasic.

iliacus (Pascoe), 1871c: 194. (Perissops)

Distribution: Papua New Guinea: Ighibirei, Rigo, Yule Island, West Papua: Dorey, Mysol; Aru Island. Critomerus emblematicus Lea, 1903: 663.

СуамовоLus Schönherr, 1837: 177

TYPE-SPECIES: Cyamobolus dehaani Mannerheim in Schönherr, 1837; original designation.

adumbratus Faust, 1898a: 176. (Cyamobolus)

Distribution: Papua New Guinea: Moroka Bay, Paumomu River.

duplicatus Pascoe, 1874: 37. (Cyamobolus)

DisTRIBUTION: West Papua: Saylee.

funereus Pascoe, 1885: 274. (Cyamobolus)

DistRibution: Papua New Guinea: Fly River.

ludiosus Pascoe, 1885: 274. (Cyamobolus)

DistRIBUTION: Papua New Guinea: Fly River.

mimicus Pascoe, 1885: 273. (Cyamobolus)

DistRIBUtION: Papua New Guinea: Fly River.

obscurus Hartmann, 1901: 285. (Cyamobolus)

DisTRIBUTION: Papua New Guinea: Bongu.

scutellaris Hartmann, 1901: 286. (Cyamobolus)

DistRIBUTION: Papua New Guinea: Bongu.

subbicristatus (Heller), 1941a: 216. (Cyambolus)

DISTRIBUTION: Bismarck Archipelago: New Britain, Gazelle Peninsula.

subsellatus subsellatus Pascoe, 1874: 36. (Cyamobolus)

DisTRIBUTION: West Papua: Saylee.

subsellatus humeralis (Heller), 1941a: 216. (Cyambolus) 
DistRIBUTION: Bismarck Archipelago: New Britain, Gazelle Peninsula.

Cyamotrox Heller, 1914d: 311

TYPE-SPECIES: Cyamotrox yuliensis Heller, 1914d; original designation.

solarii Heller, 1914d: 312. (Cyamotrox)

Distribution: West Papua: Geelvink Bay, Roon Island.

obliquatus (Heller), 1910a: 30. (Cyamobolus)

DisTRIBUTION: New Guinea.

yuliensis Heller, 1914d: 312. (Cyamotrox)

DistRIBUTION: Papua New Guinea: Yule Island.

\section{DYSOPIRHINUS Roelofs, 1880: XLIV}

TYPE-SPECIES: Dysopirhinus gestroi Roelofs, 1880; monobasic.

albosparsus Heller, 1895b: 16. (Dysopirhinus)

DistRIBUTION: Papua New Guinea: Sattelberg.

Dysopirhinus albosparsus v. ochreatus Faust, 1898a: 175. DiSTRIBUTION: Papua New Guinea: Mailu Island, Moroka.

costatus Marshall, 1915: 522. (Dysopirhinus)

DisTRIBUTION: West Papua: Utakwa River.

gestroi Roelofs, 1880: XLIV. (Dysopirhinus)

Distribution: West Papua: Dorey, Saylee, Ramoi.

Dysopirhinus gestroi v. aeruginosus Faust, 1898a: 175. Distribution: Papua New Guinea: Moroka, Paumomu River.

EMYDICA Pascoe, 1885: 255

TYPE-SPECIES: Emydica platynota Pascoe, 1885; monobasic.

platynota Pascoe, 1885: 255. (Emydica)

Distribution: West Papua: Ansus.

EUCRYPTORHYNCHUS Heller, 1937b: 71

TYPE-SPECIES: Cryptorhynchus scrobbiculatus Motschulsky, 1854 [=Curculio chinensis Olivier, 1790]; original designation.

papuanus (Heller), 1915: 518. (Cryptorhynchus)

DistRIBUtION: Papua New Guinea; Bismarck Archipelago: New Britain.

EudYASMUS Pascoe, 1885: 275

TYPE-SPECIES: Eudyasmus albertisii Pascoe, 1885; monobasic. KEY: Heller, 1935: 199.

albertisii Pascoe, 1885: 275. (Eudyasmus)

DistRIBUTION: Papua New Guinea: Fly River, Paumomu River.

planidorsis Heller, 1908a: 19. (Eudyasmus) 
DistRIBUTION: Papua New Guinea: Aroa River.

praecox Faust, 1898a: 177. (Eudyasmus)

DisTRIBUTION: Papua New Guinea: Mailu Island.

simplex Heller, 1935: 198. (Eudyasmus)

DisTRIBUTION: Papua New Guinea: Finschhafen.

GaSterosaga Heller, 1937a: 275

TYPE-SPECIES: Gasterosaga schneideri Heller, 1937a; original designation.

schneideri Heller, 1937a: 275. (Gasterosaga)

DisTRIBUTION: Bismarck Archipelago: New Britain.

HyPARINUS Pascoe, 1885: 275

TYPE-SPECIES: Hyparinus dispar, Pascoe, 1885; monobasic.

dispar Pascoe, 1885: 276. (Hyparinus)

Distribution: Papua New Guinea: Fly River; West Papua: Aru Island.

Miotus Pascoe, 1885: 219

TYPE-SPECIES: Miotus stypholoides Pascoe, 1885; monobasic.

stypholoides Pascoe, 1885: 220. (Miotus)

DisTRIBUTION: West Papua: Hatam.

MitRASTETHUS Redtenbacher, 1868: 167

TYPE-SPECIES: Mitrastethus baridioides Redtenbacher, 1868; monobasic.

australiae Lea, 1908a: 177. (Mitrastethus)

DistRIBUTION: New Guinea. NOTES: Range extension: Schneider 1999: 190.

NOTOCRYPTORHYNCHUS Lea, 1903: 675

TYPE-SPECIES: Notocryptorhynchus sinuatus Lea, 1903; monobasic. KeY: Heller, 1926a: 281.

carinulatus Heller, 1926a: 280. (Notocryptorhynchus)

Distribution: Papua New Guinea.

punctatocarinulatus Heller, 1926a: 280. (Notocryptorhynchus)

DisTRIBUTION: Papua New Guinea.

ORPHANYASMUS Heller, 1908a: 19

TYPE-SPECIES: Orphanyasmus lectus Heller, 1908a; monobasic.

lectus Heller, 1908a: 20. (Orphanyasmus)

DisTRIBUTION: New Guinea. 
tuberosus Heller, 1926a: 282. (Orphanyasmus)

DISTRIBUTION: New Guinea.

OXYMELUS Faust, 1899: 74

TYPE-SPECIES: Oxymelus concretus Faust, 1899; monobasic.

concretus Faust, 1899: 74. (Oxymelus)

DisTRIBUTION: Papua New Guinea: Ighibirei, Kapakapa.

Pelephicus Pascoe, 1874: 42

TYPE-SPECIES: Pelephicus stigmaticus Pascoe, 1874; monobasic.

stigmaticus Pascoe, 1874: 42. (Pelephicus)

DistRIBUTION: West Papua: Saylee.

PerISSOPS Pascoe, 1871c: 193

TYPE-SPECIES: Enteles ocellatus Redtenbacher, 1868; original designation.

apicalis Heller, 1926a: 287. (Perissops)

Distribution: Papua New Guinea: Bongu.

maculosus Faust, 1890: 75. (Perissops)

DISTRIBUTION: New Guinea.

pavonius Chevrolat, 1877: 189. (Perissops)

Distribution: Papua New Guinea.

Perissops sobrinus Faust, 1890: 76. DISTRIBUTION: Papua New Guinea: Kamali. NoTES: Synonoymy: Heller, 1926a: 287.

Perissops weidenbachi Lea, 1928b: 149. DISTRIBUTION: Papua New Guinea: Wau. NotES: Synonoymy: Marshall, 1946: 96.

sagax Faust, 1898a: 188. (Perissops)

DistRIBUTION: Papua New Guinea: Huon Gulf, Milne Bay; D’Entrecasteaux Islands: Fergusson Island. stellata Heller, 1926a: 286. (Perissops)

DistRIBUTION: Papua New Guinea: Astrolabe Mountains.

Platytenes Pascoe, 1870d: 466

TYPE-SPECIES: Platytenes varius Pascoe, 1870d; monobasic.

varius Pascoe, 1870d: 467. (Platytenes)

Distribution: Papua New Guinea: Dilo, Fly River, Paumomu River; North Solomon Islands: Bougainville Island; West Papua: Andai; Aru Island; Waigeo Island.

PSEUDOPERISSOPS Heller, 1926a: 285

TYPE-SPECIES: Pseudoperissops albicollis Heller, 1926a; original designation.

albicollis Heller, 1926a: 285. (Pseudoperissops)

DistRIBUTION: Papua New Guinea: Astrolabe Mountains. 
TYPE-SPECIES: Syrichius roridus Pascoe, 1871c; present designation.

dispar Faust, 1898a: 178. (Syrichius)

DistRIBUTION: Papua New Guinea: Mailu Island.

rusticus Pascoe, 1885: 271. (Syrichius)

DisTRIBUTION: Papua New Guinea: Yule Island.

servulus Pascoe, 1871c: 208. (Syrichius)

DistRIBUTION: West Papua: Dorey.

Xola Heller, 1910a: 31

TYPE-SPECIES: Xola notabilis Heller, 1910a; monobasic.

notabilis Heller, 1910a: 31. (Xola)

DISTRIBUTION: New Guinea.

\section{INCERTAE SEDIS}

[from Cryptorhynchus] NOTES: These species are placed here in anticipation of the forthcoming treatment on Montrouzier's type material by Dr. Kuschel (in litt.).

ficus Montrouzier, 1855: 53. (Cryptorhynchus)

DISTRIBUTION: Woodlark Islands.

guerini Montrouzier, 1855: 53. (Cryptorhynchus)

DISTRIBUTION: Woodlark Islands.

gyllenhali Montrouzier, 1855: 53. (Cryptorhynchus)

DISTRIBUTION: Woodlark Islands.

woodlarkianus Montrouzier, 1855: 54. (Cryptorhynchus)

DISTRIBUTION: Woodlark Islands.

\section{MECISTOSTYLINA}

Amalthus Pascoe, 1871c: 211

TYPE-SPECIES: Amalthus insignis Pascoe, 1871c; monobasic.

alboguttatus Heller, 1935: 198. (Amalthus)

DisTRIBUTION: New Guinea.

insignis Pascoe, 1871c: 211. (Amalthus)

DistRIBUTION: West Papua: Mysol Island; Morty.

sulcifrons Faust, 1899: 71. (Amalthus)

DistRIBUTION: Papua New Guinea: Moroka.

BARTOLOzZIELla Alonso-Zarazaga \& Lyal, 1999: 129

TYPE-SPECIES: Endymia vipio Pascoe, 1871c; monobasic. 
effusa (Faust), 1891: 190. comb. nov. (Endymia Pascoe, non Gistl)

Distribution: Aru Islands.

vipio (Pascoe), 1871c: 200. (Endymia Pascoe, non Gistl)

DistRIBUTION: West Papua: Dorey. NOTES: Transferred: Alonso-Zarazaga \& Lyal, 1999: 129.

BLEPIARDA Pascoe, 1865: 430

TYPE-SPECIES: Blepiarda undulata Pascoe, 1865; subsequent designation by Zimmerman, 1939: 325.

bacillifer (Heller), 1934: 27. (Trichogonus)

DISTRIBUTION: North Solomon Islands: Bougainville.

marmorata Kirsch, 1877: 155. (Blepiarda)

Distribution: Papua New Guinea; West Papua: Rubi.

neophyta Pascoe, 1871c: 211. (Blepiarda)

DisTRIBUTION: West Papua: Dorey.

simulator Pascoe, 1885: 279. (Blepiarda)

DistRIBUTION: Papua New Guinea: Katau River.

vitiata Pascoe, 1871c: 210. (Blepiarda)

Distribution: Aru Islands.

voluta Pascoe, 1871c: 210. (Blepiarda)

DistRIBUTION: West Papua: Dorey.

BOBANDERSONIA Alonso-Zarazaga \& Lyal, 1999: 129

TYPE-SPECIES: Eurhopala piazuroides Faust, 1898a; monobasic.

piazuroides (Faust), 1898a: 186. (Eurhopala Faust, non Agassiz)

DistriBution: Papua New Guinea: Moroka. Notes: Transferred: Alonso-Zarazaga \& Lyal, 1999: 129.

HYPSOPHORUS Dejean in Boisduval, 1835a: 292

TYPE-SPECIES: Cryptorhynchus dromedarius Boisduval, 1835a; monobasic.

alboguttatus (Chevrolat), 1877: 189. comb. nov. (Protopalus)

DisTRIBUTION: New Guinea.

\section{TYLODINA}

ACALLES Schönherr, 1825: 586

TYPE-SPECIES: Curculio camelus Fabricius, 1792; original designation.

ater (Montrouzier), 1855: 51. (Tylodes)

DISTRIBUTION: Woodlark Islands.

cinctus (Montrouzier), 1855: 52. (Tylodes)

DisTRIBUTION: Woodlark Islands.

griseus (Montrouzier), 1855: 50. (Tylodes) 
DISTRIBUTION: Woodlark Islands.

imhoffi (Montrouzier), 1855: 52. (Tylodes)

DISTRIBUTION: Woodlark Islands.

megapoda (Montrouzier), 1855: 51. (Tylodes)

DISTRIBUTION: Woodlark Islands.

oedothorax (Montrouzier), 1855: 52. (Tylodes æedothorax)

DISTRIBUTION: Woodlark Islands.

ACalles (MilichaCalles) Voss, 1960: 329

TYPE-SPECIES: Acalles flavomaculatus Voss, 1960; original designation.

flavomaculatus Voss, 1960: 329. (Acalles (Milichacalles))

DistRIBUTION: Papua New Guinea: Sattelberg.

ACalles (Trichacalles) Voss, 1960: 330

TYPE-SPECIES: Acalles longipilis Voss, 1960; original designation.

longipilis Voss, 1960: 330. (Acalles (Trichacalles))

DistRIBUTION: Papua New Guinea: Sattelberg.

AMbONODIRAS Heller, 1935: 191

TYPE-SPECIES: Ambonodiras scapha Heller, 1935; monobasic.

scapha Heller, 1935: 191. (Ambonodiras)

Distribution: Papua New Guinea: Astrolabe Bay.

Anchithyrus Pascoe, 1885: 257

TYPE-SPECIES: Anchithyrus obesus Pascoe, 1885: 257; present designation.

gestroi Pascoe, 1885: 258. (Anchithyrus)

DistRIBUTION: Papua New Guinea: Fly River.

obesus Pascoe, 1885: 257. (Anchithyrus)

DisTRIBUTION: Papua New Guinea: Yule Island.

quadripunctatus Faust, 1899: 65. (Anchithyrus)

Distribution: Papua New Guinea: Kamali.

truncatus Heller, 1910a: 29. (Anchithyrus)

DisTRIBUTION: New Guinea.

ANCHYPTOLYCUS Heller, 1926a: 279

TYPE-SPECIES: Anchyptolycus marginalis Heller, 1926a; monobasic.

marginalis Heller, 1926a: 280. (Anchyptolycus)

DISTRIBUTION: New Guinea. 
TYPE-SPECIES: Aporonotus simplex Pascoe, 1885; monobasic.

simplex Pascoe, 1885: 264. (Aporonotus)

DISTRIBUTION: Papua New Guinea: Yule Island.

AulaCocnemus Faust, 1899: 61

TYPE-SPECIES: Aulacocnemus solitarius Faust, 1899; monobasic.

solitarius Faust, 1899: 62. (Aulacocnemus)

DistRIBUTION: Papua New Guinea: Ighibirei, Paumomu River, Yule Island.

CAMIA Pascoe, 1885: 278

TYPE-SPECIES: Camia superciliaris Pascoe, 1885; monobasic.

semiopaca Faust, 1898a: 205. (Camia)

DISTRIBUTION: New Guinea.

superciliaris Pascoe, 1885: 288. (Camia)

DistRIBUTION: Papua New Guinea: Fly River.

Chirogonia Pascoe, 1885: 260

TYPE-SPECIES: Chirogonia opatroides Pascoe, 1885; monobasic.

opatroidea Pascoe, 1885: 260. (Chirogonia opatroides)

DistRIBUTION: Papua New Guinea: Fly River.

Cleodus Marshall, 1939a: 583

TYPE-SPECIES: Cleobis gemmatus Pascoe, 1885; monobasic.

gemmatus (Pascoe), 1885: 266. (Cleobis)

Distribution: Papua New Guinea: Fly River, Yule Island.

CORYSSOGLYMMA Faust, 1899: 66

TYPE-SPECIES: Coryssoglymma odiosa Faust, 1899; present designation.

enodata Faust, 1899: 68. (Coryssoglymma)

DistRIBUTION: Papua New Guinea: Dilo, Ighibirei, Kapakapa, Paumomu River.

odiosa Faust, 1899: 67. (Coryssoglymma)

Distribution: Papua New Guinea: Dilo, Ighibirei.

\section{DiATHRYPTUS Pascoe, 1885: 225}

TYPE-SPECIES: Diathryptus asper Pascoe, 1885; monobasic.

asper Pascoe, 1885: 256. (Diathryptus) 
DistRIBUTION: Papua New Guinea: Fly River.

DOUTTIA Zimmerman, 1944a: 193

TYPE-SPECIES: Douttia bicolor Zimmerman, 1944a; original designation.

basimaculata Voss, 1960: 343. (Douttia)

DisTRIBUTION: Papua New Guinea: Sattelberg.

ECTATOCYBA Faust, 1899: 59

TYPE-SPECIES: Ectatocyba tuberosa Faust, 1899; monobasic.

gibbosa Heller, 1908a: 17. (Ectatocyba)

DisTRIBUTION: Papua New Guinea: Aroa River.

permutata Heller, 1914d: 308. (Ectatocyba)

Distribution: Papua New Guinea: Sattelberg.

tuberosa Faust, 1899: 60. (Ectatocyba)

DistRIBUTION: Papua New Guinea: Haveri.

verrucosa Marshall, 1915: 523. (Ectatocyba)

DistRIBUTION: West Papua: Utakwa River.

EREBACES Pascoe, 1871c: 187

TYPE-SPECIES: Erebaces angulatus Pascoe, 1871c; subsequent designation: Zimmerman, 1974: 61. KEY: Zimmerman, 1974: 64.

ater Pascoe, 1885: 265. (Erebaces)

DisTRIBUTION: West Papua: Geelvink Bay, Korido; Biak Island.

Erebaces beccarii Pascoe, 1885: 266. DistriBution: West Papua: Geelvink Bay, Korido; Biak Island. NOTES: Synonymy: Zimmerman, 1974: 64.

EUDYASMODES Heller, 1942: 213

TYPE-SPECIES: Eudyasmodes heurni Heller, 1942; original designation.

heurni Heller, 1942: 213. (Eudyasmodes)

DisTRIBUTION: West Papua: Idenburg River.

GLYPTOPOROPTERUS Lea, 1912: 90

TYPE-SPECIES: Poropterus sharpi Faust, 1898a; present designation.

cucullatus (Heller), 1895b: 13. (Poropterus)

DistRIBUTION: Papua New Guinea: Sattelberg.

episternalis Lea, 1928a: 80. (Glyptoporopterus)

Distribution: Papua New Guinea: Manumbo, Madang.

insignicollis Lea, 1928a: 81. (Glyptoporopterus)

DistRIBUTION: Papua New Guinea: Wau Creek. 
sharpi (Faust), 1898a: 151. (Poropterus)

Distribution: Papua New Guinea: Mailu Island, Moroka; Redscar Bay; Trobriand Isands; Fergusson Island.

GYGAEUS Pascoe, 1885: 273

TYPE-SPECIES: Gygaeus prodigus Pascoe, 1885; monobasic.

prodigus Pascoe, 1885: 273. (Gygaeus)

DISTRIBUTION: Papua New Guinea: Fly River; Hughibagu; West Papua: Aru Islands.

HYBICUS Pascoe, 1885: 260

TYPE-SPECIES: Hybicus rotundatus Pascoe, 1885; monobasic.

rotundatus Pascoe, 1885: 260. (Hybicus)

DisTRIBUTION: Papua New Guinea: Yule Island.

ISOLEPTUS Faust, 1898a: 180

TYPE-SPECIES: Isoleptus variegatus Faust, 1898a [=Tylodes pulverulentus Montrouzier, 1855]; subsequent designation by Thompson, 1984: 207. KEY: Thompson, 1984: 220.

inflatipennis Thompson, 1984: 209. (Isoleptus)

DISTRIBUTION: North Solomon Islands: Bougainville.

obesus (Boisduval), 1835: 438. (Tylodes)

Distribution: Papua New Guinea: Madang; Bismarck Archipelago: Duke of York Island, New Britain,

New Ireland; Admiralty Islands: Manus Island; D’Entrecasteaux Islands: Fergusson Island, Trobriand

Islands; North Solomon Islands: Bougainville Island; West Papua: Geelvink Bay; Aru Islands.

Tylodes obesus Dejean, 1835: 297.

Acalles obesus Schönherr, 1837: 329.

Anaballus crassus Fairmaire, 1883: 39.

Acalles pallens Blanchard, 1853: 251.

Anaballus turbatus Faust, 1898a: 183.

Anaballus uniformis Faust, 1898a: 184.

pulverulentus (Montrouzier), 1855: 52. (Tylodes)

Distribution: Papua New Guinea: Dogura; D’Entrecasteaux Islands: Fergusson Island; Trobriand

Islands; Woodlark Islands.

Isoleptus variegatus Faust, 1898a: 181.

Isoleptus brevipennis Faust, 1898a: 182.

rubigineus (Fairmaire), 1883: 39. (Anaballus)

DISTRIBUTION: Bismarck Archipelago: Duke of York Island.

MerolePTUS Faust, 1898a: 157

TYPE-SPECIES: Meroleptus gemmatus Faust, 1898a; present designation. KeY: Heller, 1935: 189.

adspersus Faust, 1898a: 159. (Meroleptus)

DISTRIBUTION: New Guinea. 
bacatus Heller, 1935: 188. (Meroleptus)

DistRIBUTION: Papua New Guinea: Aroa River.

cinctor Marshall, 1959b: 45. (Meroleptus)

Distribution: Papua New Guinea: Goroka; West Papua: Jayapura.

foveifrons Heller, 1935: 189. (Meroleptus)

DISTRIBUTION: New Guinea.

gemmatus Faust, 1898a: 158. (Meroleptus)

DISTRIBUTION: New Guinea.

granifrons Heller, 1935: 188. (Meroleptus)

DistRIBUTION: Papua New Guinea: Aroa River.

laterosignatus Marshall, 1915: 518. (Meroleptus)

DistRIBUTION: West Papua: Mimika River; Aru Islands (?).

mus Heller, 1914d: 310. (Meroleptus)

DISTRIBUTION: New Guinea.

planiventris Heller, 1935: 189. (Meroleptus)

DistRIBUTION: Papua New Guinea: Aroa River.

squalidus Marshall, 1915: 520. (Meroleptus)

DisTRIBUTION: West Papua: Setakwa River.

tuberculosus Faust, 1899: 64. (Meroleptus)

DistRIBUTION: Papua New Guinea: Paumomu River.

velutinus Heller, 1914d: 309. (Meroleptus)

DistRIBUTION: Papua New Guinea: Sattelberg.

MiCROGYMNAPTERUS Voss, 1960: 327

TYPE-SPECIES: Microgymnapterus minutus Voss, 1960; original designation.

minutus Voss, 1960: 327. (Microgymnapterus)

DisTRIBUTION: Papua New Guinea: Sattelberg.

MicroporopteruS Lea, 1898a: 182

TYPE-SPECIES: Poropterus tumulosus Pascoe, 1873b: 198; present designation. KeY: Voss, 1960: 322.

curvipes Voss, 1960: 323. (Microporopterus)

DistRIBUTION: Papua New Guinea: Erima, Astrolabe Bay.

interruptus interruptus Voss, 1960: 326. (Microporopterus)

DistRIBUTION: Papua New Guinea: Sattelberg.

interruptus ornatus Voss, 1960: 327. (Microporopterus)

DistRIBUTION: Papua New Guinea: Sattelberg.

obsoletus Voss, 1960: 324. (Microporopterus)

Distribution: Papua New Guinea: Sattelberg.

ovatulus Voss, 1960: 325. (Microporopterus)

DistRIBUTION: Papua New Guinea: Sattelberg.

setosus Voss, 1960: 323. (Microporopterus)

Distribution: Papua New Guinea: Sattelberg.

Mimidotasia Voss, 1960: 328 
TYPE-SPECIES: Mimidotasia submetallica Voss, 1960; original designation.

submetallica Voss, 1960: 328. (Mimidotasia)

Distribution: Papua New Guinea: Berlinhafen, Tamara.

Miocalles Pascoe, 1883: 97

TYPE-SPECIES: Miocalles notatus Pascoe, 1883; monobasic.

notatus Pascoe, 1883: 97. (Miocalles)

DistRIBUTION: West Papua: Aru Islands; Mysol Island.

MORMOSINTES Pascoe, 1865: 429

TYPE-SPECIES: Mormosintes rubus Pascoe, 1865; monobasic. KEY: Heller, 1935: 181.

collaris Heller, 1935: 185. (Mormosintes)

DisTRIBUTION: New Guinea.

horrendus Heller, 1935: 184. (Mormosintes)

DISTRIBUTION: Papua New Guinea.

nodosus Pascoe, 1885: 265. (Mormosintes)

Distribution: Papua New Guinea: Fly River, Haveri.

ottonis Heller, 1935: 184. (Mormosintes)

DistRIBUTION: Papua New Guinea: Torricelli Range.

MORRISELLA Alonso-Zarazaga \& Lyal, 1999

TYPE-SPECIES: Pyroderes violaceipes Faust, 1899; monobasic.

violaceipes (Faust), 1899: 81. (Pyroderes)

DistRIBUtion: Papua New Guinea: Moroka.

NeChYRUS Pascoe, 1871c: 203

TYPE-SPECIES: Nechyrus lemur Pascoe, 1871c; subsequent designation by Heller, 1916: 325.

ater Lea, 1928a: 79. (Nechyrus)

Distribution: Papua New Guinea: Manumbo, Madang.

concussus Faust, 1899: 79. (Nechyrus)

DistRIBUtion: Papua New Guinea: Paumomu River.

cristulatus Faust, 1898a: 178. (Nechyrus)

Distribution: Papua New Guinea: Haveri, Mailu Island, Paumomu River.

decisus Pascoe, 1885: 271. (Nechyrus)

DistRIBUTION: New Guinea.

geniculatus Pascoe, 1871c: 205. (Nechyrus)

Distribution: West Papua: Mysol Island.

humeralis Faust, 1899: 77. (Nechyrus)

Distribution: Papua New Guinea: Paumomu River. 
indignus Pascoe, 1885: 270. (Nechyrus)

DisTRIBUTION: Papua New Guinea: Yule Island.

laticollis Heller, 1935: 195. (Nechyrus) DistRIBUTION: Papua New Guinea: Torricelli Mountains. Notes:

Heller used two names to refer to the same species in the same work, N. laticollatus 1935: 156 \& N. laticollis idem. Hustache, 1936: 175 used N. laticollis in the Catalogus and is considered the first reviser, thus making the former a lapsus name.

notatus Pascoe, 1871c: 206. (Nechyrus)

DistRIBUTION: West Papua: Saylee.

puncticollis Pascoe, 1871c: 204. (Nechyrus)

DISTRIBUTION: West Papua: Saylee; Aru Island.

restrictus Pascoe, 1885: 270. (Nechyrus)

DISTRIBUTION: Aru Islands.

satanus Heller, 1926a: 284. (Nechyrus)

DistRIBUTION: Papua New Guinea.

scutellatus Lea, 1928a: 78. (Nechyrus)

DISTRIBUTION: Aru Islands.

similis Heller, 1935: 197. (Nechyrus)

DistRIBUTION: Papua New Guinea: Aroa River.

ventralis Heller, 1935: 196. (Nechyrus)

Distribution: Papua New Guinea.

Oemethylus Pascoe, 1870b: 482

TYPE-SPECIES: Oemethylus lumbaris Pascoe, 1870b; monobasic.

lumbaris Pascoe, 1870b: 482. (Emethylus)

Distribution: [Australia]; Papua New Guinea: Moroka.

ORPHANISTES Pascoe, 1870c: 454

TYPE-SPECIES: Orphanistes eustictus Pascoe, 1870c; monobasic.

grandis Macleay, 1887: 190. (Orphanistes)

DisTRIBUTION: Papua New Guinea: Fly River.

Pachytragopus Heller, 1941a: 215

TYPE-SPECIES: Tragopus anaballoides Heller, 1908a; original designation.

anaballoides (Heller), 1908a: 17. (Tragopus)

DISTRIBUTION: Solomon Islands.

steinwehri Heller, 1941a: 215. (Pachytragopus)

DISTRIBUTION: Bismarck Archipelago: New Britain.

Paleticus Pascoe, 1870b: 462

TYPE-SPECIES: Paleticus pedestris Pascoe, 1870b; subsequent designation by Lea, 1898a: 212.

nigritarsis (Chevrolat), 1877: 189. (Petomis) 
DisTRIBUTION: New Guinea.

Pantiala Pascoe, 1885: 284

TYPE-SPECIES: Pantiala illusa Pascoe, 1885; monobasic.

germari Faust, 1898a: 205. (Pantiala)

DisTRIBUTION: New Guinea.

illusa Pascoe, 1885: 284. (Pantiala)

Distribution: Papua New Guinea: Fly River.

Perichius Pascoe, 1871c: 186

TYPE-SPECIES: Perichius verrucosus Pascoe, 1871c; monobasic.

verrucosus Pascoe, 1871c: 186. (Perichius)

DISTRIBUTION: Waigeo Island.

POROPTEROPIS Heller, 1937a: 270

TYPE-SPECIES: Poropteropis pan Heller, 1935; original designation. KEY: Heller, 1935: 182.

aroanus Heller, 1935: 186. (Poropteropis)

DistRIBUTION: Papua New Guinea: Aroa River.

biconifer Heller, 1935: 187. (Poropteropis)

DISTRIBUTION: New Guinea.

demissus Heller, 1935: 186. (Poropteropis)

DisTRIBUTION: Papua New Guinea.

dicyphus Heller, 1937a: 270. (Poropteropis)

Distribution: Papua New Guinea.

extorris Heller, 1937a: 271. (Poropteropis)

DistRIBUTION: Papua New Guinea.

faunus Heller, 1935: 185. (Poropteropis)

DistRIBUTION: Papua New Guinea: Aroa River.

ferox (Faust), 1898a: 154. (Poropterus)

DisTRIBUTION: New Guinea.

magicus Heller, 1935: 187. (Poropteropis)

DisTRIBUTION: New Guinea.

pan Heller, 1935: 185. (Poropteropis)

Distribution: Papua New Guinea.

papillosus (Heller), 1895b: 13. (Poropterus)

Distribution: Papua New Guinea: Sattelberg.

silenus Heller, 1935: 186. (Poropteropis)

DisTRIBUTION: New Guinea.

solidus solidus (Faust), 1898a: 155. (Poropterus)

Distribution: Papua New Guinea: Moroka, Paumomu River.

solidus prodigiosus Heller, 1935: 185. (Poropteropis)

DistRIBUTION: Papua New Guinea: Finnesterre Mountains. 
torricellianus Heller, 1935: 186. (Poropteropis)

DistRIBUTION: Papua New Guinea: Torricelli Mountains.

tristis Heller, 1937a: 272. (Poropteropis)

DisTRIBUTION: New Guinea.

POROPTERUS Schönherr, 1844: 431

TYPE-SPECIES: Poropterus antiquus Boheman in Schönherr, 1844; original designation.

afflictus Pascoe, 1888: 417. (Poropterus)

DistRIBUTION: West Papua: Saylee.

bituberosus Faust, 1898a: 152. (Poropterus)

DistRIBUTION: Papua New Guinea: between Alexander and Nisbet Mountains.

decapitatus Heller, 1895b: 14. (Poropterus)

DistRIBUTION: Papua New Guinea: Sattelberg.

echymis Pascoe, 1885: 262. (Poropterus)

DistRiBution: Papua New Guinea: Yule Island.

glanis Pascoe, 1885: 261. (Poropterus)

DistRIBUTION: Papua New Guinea: Fly River.

ordinarius Pascoe, 1885: 264. (Poropterus)

DisTRIBUTION: New Guinea: Faor Island.

pervicax Faust, 1898a: 153. (Poropterus)

DistRIBUTION: Papua New Guinea: Mailu Island.

sciureus Pascoe, 1885: 263. (Poropterus)

Distribution: Papua New Guinea: Yule Island.

PSEUdACAlles Fairmaire, 1883: 38

TYPE-SPECIES: Pseudacalles nuchalis Fairmaire, 1883; monobasic.

lateritius Fairmaire, 1883: 38. (Pseudacalles)

DisTRIBUTION: Bismarck Archipelago: Duke of York Island.

PSEUdOPOROPTERUS Lea, 1898a: 186

TYPE-SPECIES: Poropterus lemur, Pascoe, 1881; monobasic.

archaicus (Pascoe), 1885: 263. (Poropterus)

DisTRIBUTION: New Guinea; Aru Islands.

bivittatus (Faust), 1898a: 156. (Poropterus)

Distribution: Papua New Guinea.

concretus (Pascoe), 1885: 262. (Poropterus)

DistRIBUTION: Papua New Guinea: Fly River.

gemmifer (Pascoe), 1885: 261. (Poropterus)

DistRIBUTION: Papua New Guinea: Fly River.

karnyi Heller, 1935: 187. (Pseudoporopterus)

DISTRIBUTION: New Guinea.

leai Faust, 1899: 55. (Pseudoporopterus) 
Distribution: Papua New Guinea: Ighibirei.

mitratus (Pascoe), 1885: 262. (Poropterus)

Distribution: Papua New Guinea: Fly River.

pertinax (Pascoe), 1885: 263. (Poropterus)

Distribution: Papua New Guinea: Fly River, Paumomu River.

socius (Pascoe), 1885: 262. (Poropterus)

DistRIBUTION: Papua New Guinea: Paumomu River; Yule Island.

solus Heller, 1935: 187. (Pseudoporopterus)

DistRIBUTION: Papua New Guinea.

sulcicollis Faust, 1899: 58. (Pseudoporopterus)

DistRIBUtion: Papua New Guinea: Paumomu River.

vicarius (Pascoe), 1885: 263. (Poropterus)

DistRIBUTION: Papua New Guinea: Fly River.

Ptolycus Pascoe, 1885: 258

TYPE-SPECIES: Ptolycus trachypterus Pascoe, 1885; present designation. KeY: Heller, 1935: 195.

carinatus Heller, 1935: 193. (Ptolycus)

DISTRIBUTION: New Guinea.

carinirostris Pascoe, 1885: 259. (Ptolycus)

DistRIBUTION: Papua New Guinea: Fly River, Yule Island.

frater Heller, 1935: 194. (Ptolycus)

DISTRIBUTION: New Guinea.

fuligineus Pascoe, 1885: 259. (Ptolycus)

DISTRIBUTION: New Guinea.

multigranulatus Heller, 1935: 194. (Ptolycus)

DistRIBUTION: Papua New Guinea.

nodosus Marshall, 1915: 521. (Ptolycus)

Distribution: West Papua: Setakwa River.

subnodosus Heller, 1937a: 273. (Ptolycus)

DisTRIBUTION: New Guinea.

trachypterus Pascoe, 1885: 258. (Ptolycus)

DisTRIBUTION: Papua New Guinea: Fly River.

trimastus (Heller), 1910a: 29. (Anchithyrus)

DISTRIBUTION: New Guinea.

SALCUS Pascoe, 1870c: 447

TYPE-SPECIES: Salcus globosus Pascoe, 1870c; monobasic.

granulatus Pascoe, 1885: 256. (Salcus)

DistRIBUTION: Papua New Guinea: Yule Island.

SEMIATHE Pascoe, 1871a: 262

TYPE-SPECIES: Semiathe rufipennis Pascoe, 1871a: 263; present designation. KeY: Heller, 1915: 525. 
croceodiscus Heller, 1915: 523. (Semiathe)

Distribution: Papua New Guinea: Rawlinson Mountain.

linnei Faust, 1898a: 203. (Semiathe)

DISTRIBUTION: D'Entrecasteaux Islands: Fergusson Island; Trobriand Island.

ophthalmica Pascoe, 1871a: 263. (Semiathe)

DisTRIBUTION: West Papua: Mysol Island.

puncticollis Faust, 1898a: 204. (Semiathe)

DISTRIBUTION: New Guinea.

rufipennis Pascoe, 1871a: 263. (Semiathe)

DisTRIBUTION: West Papua: Dorey.

semirufa Heller, 1915: 524. (Semiathe)

Distribution: Papua New Guinea.

TAMPHILUS Zimmerman, 1994: 659

TYPE-SPECIES: Acalles amplicollis Fairmaire, 1849a; original designation.

amplicollis (Fairmaire), 1849a: 36. (Acalles)

DistRIBUTION: Papua New Guinea: Dilo, Yule Island; Bismarck Archipelago: Duke of York Island, New

Britain. NOTES: Transferred from Anaballus: Zimmerman, 1994: 660.

Anaballus amplicollis (Fairmaire), 1849a: 36.

Anaballus scabrosus Pascoe, 1885: 261. DistRiBution: Papua New Guinea: Yule Island.

Telaugia Pascoe, 1871: 203

TYPE-SPECIES: Telaugia coccosa Pascoe, 1871; subsequently monobasic: Pascoe, 1871a: 261. KeY: Heller, $1905 \mathrm{a}$ : 76.

affinis Faust, 1898a: 197. (Telaugia)

DistRIBUTION: Papua New Guinea: Milne Bay.

ampliata Faust, 1898a: 195. (Telaugia)

DISTRIBUTION: Trobriand Islands.

assimilis Pascoe, 1885: 283. (Telaugia)

DisTRIBUTION: Papua New Guinea: Fly River.

corpulenta Faust, 1899: 82. (Telaugia)

DistribUtion: Papua New Guinea: Paumomu River.

dimidiata Heller, 1910a: 34. (Telaugia)

DisTRIBUTION: New Guinea.

keysseri Heller, 1905a: 74. (Telaugia)

DisTRIBUTION: Papua New Guinea: Sattelberg.

subtilis Heller, 1905a: 75. (Telaugia)

DisTRIBUTION: Papua New Guinea: Sattelberg.

Thyestetha Pascoe, 1865: 426

TYPE-SPECIES: Thyestetha nitida Pascoe, 1865; monobasic.

carbonaria Faust, 1898a: 194. (Thyestetha)

DistriBution: Papua New Guinea: Milne Bay; D’Entrecasteaux Islands: Fergusson Island, Trobriand 
Islands.

nitida Pascoe, 1865: 427. (Thyestetha)

Distribution: Papua New Guinea: Fly River; West Papua: Dorey; Aru Island.

TragoPUS Schönherr, 1837: 356

TYPE-SPECIES: Tragopus asper, Boheman in Schönherr, 1837; original designation.

unicolor (Montrouzier), 1861: 905. (Acalles)

DISTRIBUTION: Aru Islands.

TrichoxyMus Heller, 1908a: 18

TYPE-SPECIES: Trichoxymus turpis Heller, 1908a; monobasic.

turpis Heller, 1908a: 19. (Trichoxymus)

DisTRIBUTION: New Guinea.

TrigONOPTERUS Fauvel, 1862: 157

TYPE-SPECIES: Trigonopterus insignis Fauvel, 1862; monobasic. KeYS: Pascoe, 1871a: 262; Pascoe, 1871: 23; Voss, 1960: 334.

amitinus (Voss), 1960: 337. (Idotasia)

Distribution: Papua New Guinea: Astrolabe Mountains, Sattelberg.

ampliatus (Pascoe), 1885: 286. (Idotasia ampliata)

DistRIBUTION: Papua New Guinea: Fly River.

anthracinus (Voss), 1960: 339. (Idotasia anthracina)

DisTRIBUTION: Papua New Guinea: Sattelberg.

binotatus Marshall, 1921: 591. (Trigonopterus)

DistRIBUTION: Papua New Guinea: Sattelberg. Notes: Range extension in Voss 1958b: 219.

convexus (Faust), 1898a: 199. (Idotasia convexa)

DISTRIBUTION: D'Entrecasteaux Islands: Fergusson Island.

cribratus (Faust), 1899: 86. (Idotasia cribrata)

DistRIBUTION: Papua New Guinea: Paumomu River.

cribricollis (Lea), 1928b: 156. (Idotasia)

Distribution: [Fiji]; Papua New Guinea (?).

cuneatus (Faust), 1899: 91. (Idotasia cuneata)

DistRIBUTION: Papua New Guinea: Dilo, Hughibagu, Ighibirei.

curtus (Voss), 1960: 336. (Idotasia curta)

DistRIBUTION: Papua New Guinea: Astrolabe Mountains, Sattelberg.

densatus (Faust), 1899: 84. (Idotasia densatus)

DistRIBUTION: Papua New Guinea: Ighibirei, Moroka.

difficilis (Faust), 1899: 85. (Idotasia)

DistriBution: Papua New Guinea: Bujakori, Hughibagu, Paumomu River.

dilaticollis (Faust), 1898a: 197. (Idotasia)

DistRibution: Papua New Guinea.

ebriosus (Pascoe), 1871a: 261. (Idotasia)

DisTRIBUTION: Salawatti Island. 
ephippiatus (Faust), 1899: 93. (Idotasia ephippiata)

DisTRIBUTION: Papua New Guinea.

femoralis (Faust), 1898a: 200. (Idotasia)

DisTRIBUTION: Papua New Guinea: Bujakori, Hughibagu, Ighibirei, Mailu Island, Moroka.

flavomaculatus (Voss), 1960: 342. (Idotasia flavomaculata)

DisTRIBUTION: Papua New Guinea: Sattelberg.

gibbirostris (Faust), 1899: 91. (Idotasia)

Distribution: Papua New Guinea: Bujakori, Dilo, Hughibagu, Ighibirei, Kelesi, Paumomu River, Moroka.

globatus (Voss), 1960: 340. (Idotasia globata)

DISTRIBUTION: New Guinea.

illex (Faust), 1899: 87. (Idotasia)

DistribUtion: Papua New Guinea: Paumomu River.

illitus (Faust), 1899: 89. (Idotasia illitus)

Distribution: Papua New Guinea: Bujakori.

impar (Faust), 1898a: 198. (Idotasia)

DistRIBUTION: Papua New Guinea: Mailu Island.

inclusus (Pascoe), 1871: 226. (Idotasia inclusa)

Distribution: West Papua: Mysol Island.

interpositus (Voss), 1960: 340. (Idotasia interposita)

DistRIBUTION: Papua New Guinea: Sattelberg.

melas (Faust), 1899: 96. (Idotasia)

Distribution: Papua New Guinea: Ighibirei.

minutus (Voss), 1960: 340. (Idotasia minuta)

DistRIBUTION: Papua New Guinea: Astrolabe Bay, Astrolabe Mountains, Erima, Huon Gulf, Sattelberg,

Stephansort.

morokensis (Faust), 1899: 85. (Idotasia)

Distribution: Papua New Guinea: Moroka.

nasutus (Pascoe), 1871a: 261. (Idotasia nasuta)

Distribution: Papua New Guinea: Dilo, Fly River, Ighibirei, Kapakapa, Kelesi; West Papua: Dorey;

Waigou.

neglectus (Faust), 1899: 96. (Idotasia neglecta)

Distribution: Papua New Guinea: Paumomu River.

oblongus (Pascoe), 1885: 287. (Idotasia oblonga)

DistribUtiOn: Papua New Guinea: Bujakori, Ighibirei, Paumomu River; D’Entrecasteaux Islands: Goodenough Island; Aru Islands.

obnixus (Faust), 1899: 94. (Idotasia obnixa)

Distribution: Papua New Guinea: Dilo, Ighibirei, Paumomu River; West Papua: Humboldt Bay.

pembertoni (Zimmerman), 1938: 148. (Idotasia)

DisTRIBUTION: Bismarck Archipelago: Kandan, New Ireland.

politus (Faust), 1899: 93. (Idotasia polita)

DisTRIBUTION: Papua New Guinea: Ighibirei.

proximus (Voss), 1960: 337. (Idotasia proxima)

Distribution: Papua New Guinea: Sattelberg.

pulchellus (Pascoe), 1885: 287. (Idotasia pulchella)

DISTRIBUTION: West Papua: Hatam.

pulicaris (Pascoe), 1885: 286. (Idotasia) 
DistRIBUTION: West Papua: Fly River; West Papua: Hatam.

pullus (Voss), 1960: 339. (Idotasia pulla)

DisTRIBUTION: Papua New Guinea: Sattelberg.

pusillus (Faust), 1899: 88. (Idotasia pusilla)

Distribution: Papua New Guinea: Moroka.

rufipennis (Pascoe), 1885: 286. (Idotasia)

Distribution: Papua New Guinea: Fly River.

salubris (Faust), 1898a: 200. (Idotasia)

DistRIBUTION: Papua New Guinea: Mailu Island.

scaphioides (Pascoe), 1871a: 262. (Idotasia)

Distribution: West Papua: Saylee.

seclusus (Faust), 1899: 97. (Idotasia seclusa)

DistRIBUTION: Papua New Guinea: Moroka.

sejunctus (Faust), 1899: 95. (Idotasia sejuncta)

DistRIBUTION: Papua New Guinea.

sellatus (Faust), 1899: 92. (Idotasia sellatus)

DisTRIBUTION: Papua New Guinea: Moroka.

similis (Heller), 1910a: 35. (Idotasia)

DISTRIBUTION: New Guinea.

solidus (Faust), 1899: 88. (Idotasia solida)

DistRIBUTION: Papua New Guinea: Ighibirei.

vanus (Faust), 1899: 95. (Idotasia vana)

DistRIBUTION: Papua New Guinea: Moroka.

TrigonOSOMUS Faust, 1892b: 220

TYPE-SPECIES: Trigonosomus draco Faust, 1892; original designation.

draco draco Faust, 1892b: 220. (Trigonosomus)

DISTRIBUTION: New Guinea.

draco papuanus Heller, 1926a: 284. (Trigonosomus)

DistRIBUTION: Papua New Guinea: Astrolobe mountains.

TyRTaEOSUS Pascoe, 1870b: 479

TYPE-SPECIES: Tyrtaeosus microthorax Pascoe, 1870b; subsequent designation by Faust, 1899: 75.

aversandus Pascoe, 1885: 272. (Tyrtaeosus)

DistRIBUTION: Papua New Guinea: Fly River.

coelosternoides Heller, 1926a: 283. (Tyrtaeosus)

DistRIBUTION: Papua New Guinea.

discrepans Pascoe, 1885: 272. (Tyrtaeosus)

DisTRIBUTION: Papua New Guinea: Yule Island.

ingens Faust, 1899: 75. (Tyrtaeosus)

DistRIBUTION: Papua New Guinea: Moroka.

plebejus Faust, 1899: 76. (Tyrtaeosus)

DisTRIBUTION: Papua New Guinea: Haveri.

suturalis Heller, 1937a: 274. (Tyrtaeosus) 
DisTRIBUTION: New Guinea.

XYChUSA Pascoe, 1871a: 227

TYPE-SPECIES: Xychusa larvata Pascoe, 1871a; monobasic.

larvata Pascoe, 1871a: 228. (Xychusa)

DistRIBUtion: Papua New Guinea: Dilo, Fly River, Kelesi, Yule Island; Aru Islands.

ZYGARA Pascoe, 1885: 288

TYPE-SPECIES: Asytesta doriae, Kirsch, 1879; original designation.

doriae (Kirsch), 1879: 19. (Asytesta)

DistRIBUTION: Papua New Guinea: Yule Island.

\section{GASTEROCERCINI}

Chaetectetorus Schönherr, 1844: 383

TYPE-SPECIES: Chaetectetorus bifasciatus Boheman in Schönherr, 1837; original designation.

cinereus Heller, 1941a: 217. (Chaetectetorus)

DISTRIBUTION: Bismarck Archipelago: New Britain.

histrio Pascoe, 1885: 277. (Chaetectetorus)

DisTRIBUTION: Papua New Guinea: Yule Island.

EUTYRHINUS Dejean, 1835: 292

TYPE-SPECIES: Curculio meditabundus Fabricius, 1775; monobasic.

brevispinosus (Fairmaire), 1883: 37. comb. nov. (Euthyrhinus)

DISTRIBUTION: Bismarck Archipelago: Duke of York Island, New Britain.

dorsalis (Macleay), 1887: 189. comb. nov. (Euthyrhinus)

DisTRIBUTION: New Guinea.

frontalis Kirsch, 1877: 152. (Eutyrhinus)

DisTRIBUTION: West Papua: Dorey.

iconicus (Pascoe), 1872d: 477. comb. nov. (Euthyrhinus)

Distribution: West Papua: Mysol Island.

irroratus (Macleay), 1887: 189. comb. nov. (Euthyrhinus)

DISTRIBUTION: New Guinea.

meditabundus (Fabricius), 1775: 139. (Curculio)

DistRIBUTION: Papua New Guinea: Dilo, Haveri, Ighibirei; Aru Island.

Cryptorhynchus monachus Boisduval, 1835: 430.

tessellatus (Blanchard), 1853: 249. comb. nov. (Euthyrhinus)

Distribution: West Papua: Fly River; West Papua: Dorey; Aru Island.

LOPHOCHEIRUS Marshall, 1915: 524 
TYPE-SPECIES: Odosyllis gemmata Pascoe, 1885; original designation. NOTES: Transferred to Gasterocercini: Lyal et al., 2006: 241; KEY: Heller, 1928: 104.

apicallis (Heller), 1915: 520. (Odosyllis)

DisTRIBUTION: Papua New Guinea.

Lophocheirus apicallis v. divisa Heller, 1928: 109.

aruensis Heller, 1928: 105. (Lophocheirus)

DISTRIBUTION: Aru Islands.

circumscriptus Heller, 1928: 104. (Lophocheirus)

DisTRIBUTION: New Guinea.

crucigera (Pascoe), 1885: 277. (Odosyllis)

DistRIBUTION: Papua New Guinea: Fly River, Ighibirei.

Lophocheirus cruciger v. fuscotriangularis Lea, 1910b: 635.

gemmatus (Pascoe), 1885: 277. (Odosyllis gemmata)

DisTRIBUTION: Papua New Guinea: Yule Island.

ingens (Pascoe), 1885: 276. (Odosyllis)

DistRIBUTION: Papua New Guinea: Fly River.

lactospretus Heller, 1935: 199. (Lophocheirus)

Distribution: Papua New Guinea: Finnesterre Mountains.

maior (Heller), 1908a: 21. (Odosyllis)

DisTRIBUTION: Papua New Guinea: Sattelberg.

Lophocheirus maior ab. lateralis (Heller), 1915: 519.

novaeguineae Heller, 1928: 106. (Lophocheirus)

DistRIBUTION: New Guinea.

oppositus (Faust), 1899: 80. (Odosyllis opposita)

DisTRIBUTION: Papua New Guinea: Irupara.

papuanus Heller, 1928: 107. (Lophocheirus)

DisTRIBUTION: New Guinea.

wollastoni Marshall, 1915: 525. (Lophocheirus)

DistRIBUTION: West Papua: Utakwa River.

MENECTETORUS Faust, 1894: 284

TYPE-SPECIES: Menectetorus luctuosus Faust, 1894; monobasic. KeY: to Pseudapries: Lea, 1910b: 607.

femoralis (Heller), 1937a: 277. (Pseudapries)

Distribution: Papua New Guinea: Bongu.

gronopoides (Pascoe), 1885: 278. (Chatectetorus)

Distribution: Papua New Guinea: Ighibirei, Yule Island. Notes: Treated in Pseudapries in Lea, 1910b: 611.

NAUPHAEUS Pascoe, 1871a: 221

TYPE-SPECIES: Nauphaeus miliaris Pascoe, 1871a; monobasic. NOTES: Transferred to Gasterocercini: Lyal et al., 2006: 241.

miliaris Pascoe, 1871a: 222. (Nauphaeus)

Distribution: Papua New Guinea: Fly River, Paumomu River; West Papua: Mysol Isand; Waigeo Island. 
nebulosus Heller, 1908b: 179. (Nauphaeus)

DISTRIBUTION: Solomon Islands.

NeDYMORA Pascoe, 1871c: 209

TYPE-SPECIES: Nedymora ventricosa Pascoe, 1871c; monobasic. Notes: Transferred to Gasterocercini: Lyal et al., 2006: 241.

ventricosa Pascoe, 1871c: 209. (Nedymora)

DisTRIBUTION: Aru Islands.

ODOSYLLIS Pascoe, 1874: 40

TYPE-SPECIES: Odosyllis congesta Pascoe, 1874; subsequent designation by Pascoe, 1885: 277.

irrorata Pascoe, 1874: 42. (Odosyllis)

DistRIBUTION: West Papua: Saylee.

vitiosa Pascoe, 1874: 41. (Odosyllis)

DisTRIBUTION: New Guinea; Waigeo Island; Saylee.

OROCHLESIS Pascoe, 1871c: 194

TYPE-SPECIES: Orochlesis annularis Pascoe, 1871c; subsequent designation by Lea, 1913: 327. Diagnosis of Papuan species: Zimmerman, 1936: 17.

annularis Pascoe, 1871c: 195. (Orochlesis)

DistRIBUTION: Papua New Guinea: Yule Island; West Papua: Dorey.

flesina Pascoe, 1871c: 195. (Orochlesis)

DisTRIBUTION: Aru Islands.

maculosa Pascoe, 1874: 40. (Orochlesis)

DisTRIBUTION: Salawatti Island.

\section{PSEPHOLACINI}

DYSPEITHES Kirsch, 1877: 153

TYPE-SPECIES: Dyspeithes dentifer Kirsch, 1877; monobasic. KeY: Faust, 1899: 54.

dentifer Kirsch, 1877: 153. (Dyspeithes)

DistRIBUTION: West Papua: Geelvink Bay, Mafor Island.

gestroi (Pascoe), 1885: 278. (Amadus)

Distribution: Papua New Guinea: Haveri, Moroka; West Papua: Hatam, Ramoi; Aru Island.

nechyroides Faust, 1893: 7. (Dyspeithes)

DISTRIBUTION: New Guinea.

pascoei Hartmann, 1901: 287. (Dyspeithes)

DisTRIBUTION: Papua New Guinea: Bongu.

suspiciosus Faust, 1899: 54. (Dyspeithes)

DistRIBUTION: Papua New Guinea: Moroka.

MAGARIS Pascoe, 1885: 249 
TYPE-SPECIES: Magaris variabilis Pascoe, 1885; monobasic.

variabilis Pascoe, 1885: 250. (Magaris)

DistRIBUTION: Papua New Guinea: Fly River.

OSSETERIS Pascoe, 1872d: 479

TYPE-SPECIES: Osseteris scutellaris Pascoe, 1872d; monobasic.

scutellaris Pascoe, 1872d: 480. (Osseteris)

DisTRIBUTION: West Papua: Dorey.

tristis Voss, 1960: 318. (Osseteris)

DistRIBUTION: Papua New Guinea: Huon Gulf.

Polyzelus Pascoe, 1885: 248

TYPE-SPECIES: Polyzelus crassicollis Pascoe, 1885; monobasic.

crassicollis Pascoe, 1885: 248. (Polyzelus)

DistRIBUTION: Papua New Guinea: Fly River.

\section{CURCULIONINAE}

\section{ACALYPTINI}

NISEIDA Pascoe, 1885: 236

TYPE-SPECIES: Niseida virginea Pascoe, 1885; monobasic.

virginea Pascoe, 1885: 236. (Niseida)

DiSTRIBUTION: Aru Islands.

EUDELODES Zimmerman, 1994a: 665

TYPE-SPECIES: Amorphoidea bicolor Faust, 1899: 44; original designation. NOTES: Transferred to Acalyptini: Kojima \& Morimoto, 2005: 78.

bicolor (Faust), 1899: 44. (Amorphoidea)

DistriBution: Papua New Guinea: Kapakapa, Rigo. Notes: Transferred: Zimmerman, 1994a: 665.

discedens (Faust), 1899: 45. (Amorphoidea)

DistRIBUTION: Papua New Guinea: Kapakapa.

\section{CENCHRENINI}

Cenchrena Pascoe, 1874: 24

TYPE-SPECIES: Cenchrena fasciata Pascoe, 1874; present designation. 
dubia Voss, 1960: 345. (Cenchrena)

Distribution: Papua New Guinea: Sattelberg.

fasciata Pascoe, 1874: 24. (Cenchrena)

DISTRIBUTION: West Papua: Waigeo Island; Aru Island.

variabilis Heller, 1941a: 213. (Cenchrena)

DISTRIBUTION: Bismarck Archipelago: New Britain.

\section{CERATOPODINI}

STELECHODES Faust, 1899: 42

TYPE-SPECIES: Stelechodes loriai Faust, 1899: 42; monobasic.

loriai Faust, 1899: 42. (Stelechodes)

DISTRIBUTION: Papua New Guinea: Ighibirei.

\section{CURCULIONINI}

\section{CURCULIONINA}

CurCulio Linnaeus, 1758: 337

TYPE-SPECIES: Curculio nucum Linnaeus, 1758; subsequent designation by Latreille, 1810: 430.

bellus (Heller), 1927b: 262. (Balaninus)

DisTRIBUTION: Mysol Island.

c-album Fabricius, 1798: 170. (Curculio)

DisTRIBUTION: West Papua: Fly River; West Papua: Waigeo Island.

cerbereus (Heller), 1925c: 284. (Balaninus)

DISTRIBUTION: New Guinea.

epimeralis (Heller), 1927b: 263. (Balaninus)

DISTRIBUTION: New Guinea.

flavodorsalis (Heller), 1927b: 263. (Balaninus)

DistRIBUTION: Papua New Guinea: Aroa River.

galbula (Pascoe), 1883: 92. (Balaninus)

DisTRIBUTION: West Papua: Dorey

luctuosus (Pascoe), 1883: 91. (Balaninus)

Distribution: West Papua: Dorey

missionis (Heller), 1941a: 214. (Balaninus)

DistRIBUTION: Bismarck Archipelago: New Britain, Gazelle Peninsula.

nivatus (Heller), 1927b: 264. (Balaninus)

DisTRIBUTION: New Guinea; Bismarck Archipelago: New Britain.

nivosus (Faust), 1892b: 205. (Balaninus)

DisTRIBUTION: New Guinea.

pluto (Faust), 1892b: 206. (Balaninus)

DISTRIBUTION: New Guinea.

plutonius (Heller), 1925c: 284. (Balaninus)

DISTRIBUTION: New Guinea. 
quadriguttatus (Heller), 1927b: 265. (Balaninus)

Distribution: Papua New Guinea: Huon Peninsula, Sattelburg.

simillimus (Heller), 1927b: 266. (Balaninus)

DISTRIBUTION: New Guinea.

stramineoplagiatus (Heller), 1925c: 286. (Balaninus)

DiSTRIBUTION: New Guinea.

tenuistratus (Heller), 1942: 211. (Balaninus)

DisTRIBUTION: West Papua.

tersus (Pascoe), 1885: 235. (Balaninus)

Distribution: Papua New Guinea: Fly River.

\section{PSEUDOBALANININA}

CarPoninus Heller, 1924a: 187

TYPE-SPECIES: Balaninus axillaris Faust, 1895a; original designation.

solarii Heller, 1925b: 131. (Carponinus)

DisTRIBUTION: West Papua: Andai.

stramineopictus (Heller), 1925c: 285. (Balaninus)

DISTRIBUTION: New Guinea.

\section{RHAMPHINI}

\section{RHAMPHINA}

RHYNCHAENOPHAENUS Voss, 1956: 127

TYPE-SPECIES: Rhynchaenophaenus prodromus Voss, 1956; original designation.

prodromus Voss, 1956: 127. (Rhynchaenophaenus)

DisTRIBUTION: Papua New Guinea: Sattelberg.

\section{STOREINI}

IMATHIA Pascoe, 1885: 251

TYPE-SPECIES: Imathia bella Pascoe,1885: 251; subsequent designation by Zimmerman, 1967: 192.

bella Pascoe, 1885: 251. (Imathia)

DisTRIBUTION: West Papua: Arfak Mountains, Hatam.

brevis Faust, 1899: 51. (Imathia)

DisTRIBUTION: Papua New Guinea: Moroka.

unicolor Pascoe, 1885: 251. (Imathia)

DisTRIBUTION: West Papua: Arfak Mountains, Hatam.

Melanterus Erichson, 1842: 209

TYPE-SPECIES: Melanterus porcatus Erichson, 1842; subsequent designation by Lea, 1899b: 206. 
papuensis Lea, 1931c: 377. (Melanterus)

DISTRIBUTION: New Guinea.

NEOMELANTERIUS Lea, 1899: 247

TYPE-SPECIES: Melanterus carinicollis Pascoe, 1875: 63; present designation.

interruptus Lea, 1913: 195. (Neomelanterius)

DISTRIBUTION: New Guinea; [Australia].

TYCHIINI

DEMIMAEINA

Demimaea Pascoe, 1870c: 440

TYPE-SPECIES: Demimaea luctuosa Pascoe, 1870c; monobasic. KeY: Voss, 1937b: 451.

fasciata Voss, 1937b: 458. (Demimaea)

DISTRIBUTION: New Guinea.

\section{OCHYROMERINA}

NEOCHYROMERA Heller, 1910a: 27

TYPE-SPECIES: Neochyromera turbans Heller, 1910a; monobasic.

turbans Heller, 1910a: 27. (Neochyromera)

DisTRIBUTION: New Guinea.

\section{TYCHIINA}

SibinIELLA Voss, 1956: 129

TYPE-SPECIES: Sibiniella ravilla Voss, 1956; original designation.

ravilla Voss, 1956: 129. (Sibiniella)

Distribution: Papua New Guinea: Sattelberg.

\section{CYCLOMINAE}

\section{ATERPINI}

AADES Schönherr, 1823: 1138

TYPE-SPECIES: Curculio cultratus Fabricius, 1775; subsequent designation by Schönherr, 1833.

franklini (Heller), 1925c: 283. comb. nov. (Aterpus) sedis mutabilis

DisTRIBUTION: Papua New Guinea. 
DeXAGIA Pascoe, 1871c: 166

TYPE-SPECIES: Dexagia superciliaris Pascoe, 1871c; monobasic.

superciliaris Pascoe, 1871c: 166. (Dexagia)

DISTRIBUTION: Aru Islands.

JULIETIELLA Lyal \& Alonso-Zarazaga, 1999: 140

TYPE-SPECIES: Hypermetra analis Pascoe, 1871c; monobasic.

analis (Pascoe), 1871c: 167. (Hypermetra)

DistRIBUtion: West Papua: Mysol Island. NOTES: Transferred: Alonso-Zarazaga \& Lyal, 1999: 140.

RHINARIA Kirby, 1819: 430

TYPE-SPECIES: Rhinaria cristata Kirby, 1819; original designation.

variegata Boisduval, 1835: 411. (Rhinaria)

DisTRIBUTION: [Australia]; New Guinea (?).

\section{ENTIMINAE}

\section{CELEUTHETINI}

ACOPTORRHYNCHUS Heller, 1933b: 149

TYPE-SPECIES: Coptorhynchus sus Faust, 1897b; monobasic.

sus (Faust), 1897b: 282. (Coptorhynchus)

DisTRIBUTION: Papua New Guinea.

ALBERTISIUS Thompson, 1977: 253

TYPE-SPECIES: Apirocalus gestroi Pascoe, 1885; original designation. KeY: Thompson, 1977: 256.

excellens (Faust), 1899: 27. (Idiopsis)

Distribution: Papua New Guinea: Astrolabe Mountains, Mt. Obree. Notes: Transferred: Thompson, 1977: 253.

gestroi (Pascoe), 1885: 209. (Apirocalus)

Distribution: Papua New Guinea: Yule Island. NOTES: Thompson, 1991 described the female of this species.

APIEZONOTUS Heller, 1941c: 153

TYPE-SPECIES: Piezonotus albosignatus Heller, 1935c; original designation.

albosignatus (Heller), 1935: 166. (Piezonotus? [sic]) 
Distribution: Papua New Guinea: Eddie Creek, Huon Gulf.

APIROCALus Pascoe, 1881: 590

TYPE-SPECIES: Apirocalus cornutus Pascoe, 1881; monobasic. KeYs: Marshall, 1956: 17; Thompson, 1977: 199.

acutus Thompson, 1977: 248. (Apirocalus)

Distribution: Papua New Guinea: Mt. Kaindi, Wau.

anatinus Thompson, 1977: 253. (Apirocalus)

DisTRIBUTION: Papua New Guinea: Aiyura, Eastern Highlands.

asper Marshall, 1956: 17. (Apirocalus)

DisTRIBUTION: Papua New Guinea: Mafulu.

ater Thompson, 1977: 231. (Apirocalus)

DISTRIBUTION: D’Entrecasteaux Islands: Goodenough Island.

atrigenua Thompson, 1977: 227. (Apirocalus)

DistRIBUTION: Papua New Guinea: Finisterre Range, Teptep.

avus avus Thompson, 1977: 218. (Apirocalus)

DistRibution: Papua New Guinea: Aiyura, Kassem Pass, Lifu, Okapa, Purosa, Wanatabe Valley.

avus finisterrae Thompson, 1977: 221. (Apirocalus)

DistRibution: Papua New Guinea: Finisterre Range, Komba, Matoko, Wantoat.

avus intermedius Thompson, 1977: 220. (Apirocalus)

Distribution: Papua New Guinea: Dimifa SE. of Mt. Giluwe, Mt. Hagen, Pangia, Rumpi, Tambul,

Tomba, Yaibos.

avus karimuicus Thompson, 1977: 220. (Apirocalus)

DisTRIBUTION: Papua New Guinea: Karimui.

avus marawakanus Thompson, 1977: 221. (Apirocalus)

DisTRIBUTION: Papua New Guinea: Marawaka, Mt. Piora (?).

avus tarii Thompson, 2005: 123. (Apirocalus)

DisTRIBUTION: Papua New Guinea: between Tari and Koroba.

bacchusi Thompson, 1977: 228. (Apirocalus)

DisTRIBUTION: Papua New Guinea: Finisterre Range.

canus Thompson, 1977: 224. (Apirocalus)

Distribution: Papua New Guinea: Bome, Giolala, Iongai, Kokoda, Mafulu, Mondo, Mt. St. Mary.

carinirostris Thompson, 2005: 126. (Apirocalus)

DisTRIBUTION: Papua New Guinea: Mt. Bosavi.

cornutus cornutus Pascoe, 1881: 590. (Apirocalus)

Distribution: Papua New Guinea: Aroa River, Astrolabe Range, Bisianumu, Eriama, Ihu, Kerema,

Kikori, Mt. Epa, Popondetta? [possibly introduced], Pt. Moresby, Redscar Bay, Vanapa River, Yule Island; [Australia].

cornutus tenuiscapus Thompson, 1977: 237. (Apirocalus)

Distribution: Papua New Guinea: Bapiti, Bome, Diene, Kamulai, Loloipa, Mafulu, Mondo, Mt. Tafa, Tororo.

cornutus virescens Thompson, 1977: 238. (Apirocalus)

Distribution: Papua New Guinea: Lake Kutubu, Mt. Hagen, Nipa, Olsobip, Purosa, Tauri; West Papua:

Star Mountains.

cornutus paradoxus Thompson, 1977: 239. (Apirocalus paradoxus)

Distribution: Papua New Guinea: Fore, Karimui, Paniga. 
cornutus bosavii Thompson, 2005: 128. (Apirocalus)

DistRibution: Papua New Guinea: Mt. Bosavi.

ebrius ebrius Faust, 1892b: 189. (Apirocalus cornutus v. ebrius)

Distribution: Papua New Guinea: Aiyura, Aseki, Astrolabe Bay, Bogia, Bulolo, Finisterre Range, Finschhafen, Garaina, Goroka, Kassem Pass, Lae, Madang, Maprik, Markham River, Milne Bay, Mt. Kaindi, Mt. Missim, Nadzab, Okapa, Oro Bay, Popondetta, Sattelberg, Sepik River, Torricelli Mountains, Umboi Island, Upper Jimmi Valley, Wau; Admiralty Islands: Manus Island; Bismarck Archipelago: New Britain; West Papua: Angguruk, Archbold Lake, Bewani Mountains, Bokondini, Cyclops Mountains, Dafo, Humboldt Bay, Maffin Bay, Nemfor Island, Sentani, Swart Valley, Wamena. NotES: Status changed to full species: Thompson, 1977: 241.

ebrius angustus Thompson, 1977: 246. (Apirocalus)

Distribution: Papua New Guinea: Bismarck Mountain, Fore, Lufa, Mt. Michael, Omkalai near Wahgi River?

ebrius wagneri Thompson, 1977: 245. (Apirocalus)

Distribution: Papua New Guinea: Cromwell Range, Finisterre Range, Finschhafen, Funyende, Huon Peninsula, Komba, Matoko, Saruwaged Range, Wareo.

fallax Thompson, 1977: 214. (Apirocalus)

Distribution: Papua New Guinea: Bome, Garina, Giolala, Mt. Missim, Wau.

granulicollis Thompson, 1977: 225. (Apirocalus)

DistRiBution: Papua New Guinea: Saruwaged Range.

grossus Thompson, 2005: 131. (Apirocalus)

Distribution: Papua New Guinea: between Aseki and Menyamya.

hornabrooki Thompson, 1977: 246. (Apirocalus)

DisTRIBUTION: Papua New Guinea: Karkar Island.

hydrographicus Marshall, 1956: 18. (Apirocalus)

Distribution: Papua New Guinea: Hydrographer Mountains, Ishurava, Kokoda, Mt. Lammington, Orrori, Popondetta.

inornatus Thompson, 1977: 233. (Apirocalus)

DistRIBUTION: Papua New Guinea: Moroka.

insperatus Thompson, 1977: 216. (Apirocalus)

Distribution: Papua New Guinea: Mt. Kaindi, Wau.

mus Thompson, 1977: 240. (Apirocalus)

Distribution: Papua New Guinea: Bulolo, Bulum River E. of Lae, Kassam Pass E. of Kainantu, Markham Valley, Nadzab, Singuawa River, Wau.

nivosus Thompson, 1977: 226. (Apirocalus)

Distribution: Papua New Guinea: Huon Peninsula, Kalalo, Mt. Piora, Saruwaged Range.

olivaceus Thompson, 1977: 251. (Apirocalus)

Distribution: Papua New Guinea: Asaro Valley, Daulo Pass, Okapa, Marifuanga, Mt. Giluwe, Mt. Michael, Mt. Wilhelm.

orientalis Thompson, 1977: 231. (Apirocalus)

Distribution: Papua New Guinea: Komania, Milne Bay, Mt. Mura.

perturbans Thompson, 2005: 122. (Apirocalus)

DisTRIBUTION: Papua New Guinea: Engabena.

riedeli Thompson, 2005: 119. (Apirocalus)

Distribution: Papua New Guinea: between Aseki and Menyamya.

sedlaceki sedlaceki Thompson, 1977: 221. (Apirocalus)

Distribution: Papua New Guinea: Aseki, Bulolo, Mt. Kaindi, Mt. Missim, Wau. 
sedlaceki laminifer Thompson, 2005: 124. (Apirocalus)

Distribution: Papua New Guinea: Aseki, Mt. Amingwiwa, Oiwa.

specillifer Thompson, 2005: 132. (Apirocalus)

DisTRIBUTION: Papua New Guinea: between Aseki and Menyamya.

stellifer Thompson, 1977: 250. (Apirocalus)

DistRIBUTION: Papua New Guinea: Kainantu, Okapa, Purosa, Wau.

strigifrons Thompson, 1977: 247. (Apirocalus)

DistRIBUTION: Papua New Guinea: Bulldog Road, Wau.

subcostatus Thompson, 1977: 217. (Apirocalus)

Distribution: Papua New Guinea: Aiyura, Asaro Valley, Goroka, Mt. Otto, Okapa.

suppuratus Thompson, 1977: 223. (Apirocalus)

DistRIBUTION: Papua New Guinea: Goilala, Woitape.

tenebricosus Thompson, 1977: 253. (Apirocalus)

DistRIBUTION: Papua New Guinea: Asaro Valley, Mt. Otto.

verrucosus Thompson, 2005: 118. (Apirocalus)

DisTRIBUTION: Papua New Guinea: Aseki.

vexillarius Marshall, 1956: 19. (Apirocalus)

DistRIBUtION: Papua New Guinea: Goilala, Owgarra near Bereina?, Woitape.

ApIrocalus (MolobriUM) Thompson, 1977: 207

TYPE-SPECIES: Apirocalus io Thompson, 1977; original designation.

celeatus Thompson, 1977: 210. (Apirocalus (Molobrium))

DistRIBUTION: Papua New Guinea: Kassam Pass E. of Kainantu.

fordi Thompson, 2005: 112. (Apirocalus (Molobrium))

DistRIBUTION: Papua New Guinea: Saruwaged Range.

gracilis Thompson, 1977: 207. ((Apirocalus (Molobrium))

Distribution: Papua New Guinea: Finisterre Range, Funyende, Matoko.

io Thompson, 1977: 209. (Apirocalus (Molobrium))

DisTRIBUTION: Papua New Guinea: Bulolo, Wau.

occultator Thompson, 1977: 211. (Apirocalus (Molobrium))

DistRibution: Papua New Guinea: Butemu, Damanti, Finiestrre Range, Moro.

scaber Thompson, 2005: 114. (Apirocalus (Molobrium))

Distribution: Papua New Guinea: Huon Peninsula.

stibicki Thompson, 1977: 209. (Apirocalus (Molobrium))

DisTRIBUTION: Papua New Guinea: Lae.

terrestris terrestris Thompson, 1977: 212. (Apirocalus (Molobrium))

Distribution: Papua New Guinea: Goroka, Kainantu, Lake Sirunki, Mendi, Mt. Hagen, Okapa, Wabag, Tauri Valley, Wanatabe Valley;

terrestris dissidens Thompson, 1977: 213. (Apirocalus (Molobrium))

Distribution: Papua New Guinea: Eliptamin Valley, Feramin; West Papua: Beta Bip, Kampong, Star

Mountains.

AUlACOPHRYs Marshall, 1956: 86

TYPE-SPECIES: Aulacophrys nodifer Marshall, 1956; original designation. 
facialis Marshall, 1956: 88. (Aulacophrys)

DisTRIBUTION: New Guinea: Aiyura.

nodifer Marshall, 1956: 86. (Aulacophrys)

DisTRIBUTION: New Guinea: Komba.

BEHRENSIELLUS Berg, 1898: 17

TYPE-SPECIES: Cataphractus aethiops Behrens, 1887 [=Siteytes glabratus Pascoe, 1871c]; monobasic. KEYS: Heller, 1908a: 13; Heller, 1914a: 648; Heller, 1935: 163.

difficilis Heller, 1914a: 649. (Behrensiellus)

DisTRIBUTION: Papua New Guinea: Sattelberg.

elongatus (Heller), 1901: 11. (Cataphractus)

DistRIBUTION: Papua New Guinea: Aroa River.

glabratus glabratus (Pascoe), 1871c: 157. (Siteytes)

Distribution: Papua New Guinea: Fly River, Paumomu River; West Papua: Saylee, Siwi.

Cataphractus aethiops Behrens, 1887: 233.

glabratus subvirens Heller, 1914a: 648. (Behrensiellus)

DISTRIBUTION: West Papua: Lorentz River.

substriatus Heller, 1908a: 12. (Behrensiellus)

DISTRIBUTION: Bismarck Archipelago: New Britain.

BRACHYNEDUS Marshall, 1956: 77

TYPE-SPECIES: Brachynedus cheesmanae Marshall, 1956; original designation. KEYS: Marshall, 1956: 78; Faust, 1899: 17 (separates B. pectoralis and B. posticus).

cheesmanae Marshall, 1956: 78. (Brachynedus)

Distribution: Papua New Guinea: Kokoda, Orori, Ishurava, Oquqli, Diene.

crinipes Marshall, 1956: 81. (Brachynedus)

DisTRIBUTION: Papua New Guinea: Kokoda.

hebes Marshall, 1956: 80. (Brachynedus)

DistRiBution: Papua New Guinea: Finschhafen, Huon Gulf, Manumbo, Madang.

pectoralis (Faust), 1897b: 283. (Coptorrhynchus)

DISTRIBUTION:

posticus (Faust), 1899: 18. (Coptorrhynchus)

DISTRIBUTION: D'Entrecasteaux Islands: Goodenough Island.

remissus Marshall, 1956: 80. (Brachynedus)

DisTRIBUTION: Papua New Guinea: Kokoda.

CeLeuthetes Schönherr, 1842: 250

TYPE-SPECIES: Sphaeromus australis Gyllenhal in Schönherr, 1834 [=Curculio echinatus Fabricius, 1801]; original designation.

boisduvali Faust, 1897b: 262. (Celeuthetes)

DistRIBUTION: [Australia]; Papua New Guinea: Goodenough Island.

Otiorrhynchus australis Boisduval, 1835: 298.

cinerascens Blanchard, 1853: 231. (Celeuthetes) 
DistRIBUTION: Papua New Guinea: Yule Island; Bismarck Archipelago; Aru Island.

echinatus (Fabricius), 1801: 525. (Curculio)

DistRIBUTION: Papua New Guinea: Fly River; West Papua: Dorey; Aru Islands; Salawatti Islands; [Australia (?)].

Sphaeromus australis Gyllenhal in Schönherr, 1834: 539.

granulosus Faust, 1897b: 263. (Celeuthetes)

DistRIBUTION: Papua New Guinea; D’Entrecasteaux Islands: Fergusson Island, Goodenough Island. Celeuthetes granosus Faust, 1897b: 263.

paganus Gyllenhal in Schönherr, 1834: 539. (Celeuthetes)

DISTRIBUTION: North Solomon Islands: Bougainville. NOTES: Range extension: Tanner, 1969: 10.

ChOERORHAMPHUS Faust, 1899: 24

TYPE-SPECIES: Choerorhamphus setiger Faust, 1899; monobasic.

setiger Faust, 1899: 25. (Choerorhamphus)

DistRIBUTION: Papua New Guinea: Dilo, Ighibirei.

ColPomus Marshall, 1956: 26

TYPE-SPECIES: Colpomus striatus Marshall, 1956; original designation.

porosus Marshall, 1956: 28. (Colpomus)

DISTRIBUTION: Louisaide Archipelago: Misima Island.

striatus Marshall, 1956: 27. (Colpomus)

DISTRIBUTION: Woodlark Islands.

CyPHOPus Marshall, 1956: 110

TYPE-SPECIES: Cyphopus cheesmanae Marshall, 1956; original designation. KEY: Marshall, 1956: 111.

bicristatus (Faust), 1897b: 276. (Ectemnomerus)

DisTRIBUTION: D'Entrecasteaux Islands: Fergusson Island.

cheesmanae Marshall, 1956: 113. (Cyphopus)

DisTRIBUTION: Papua New Guinea: Kokoda, Orrori, Ishurava.

pennatus Marshall, 1956: 111. (Cyphopus)

Distribution: Papua New Guinea: Kokoda, Orrori, Oquali, Mondo.

ECTEMNOMERUS Faust, 1897b: 274

TYPE-SPECIES: Ectemnomerus elegans Faust, 1897b; original designation.

elegans Faust, 1897b: 275. (Ectemnomerus)

Distribution: Papua New Guinea: Moroka.

nigroplagiatus Faust, 1899: 23. (Ectemnomerus)

DistRibution: Papua New Guinea: Astrolabe Mountains, Dilo, Ighibirei, Kapakapa, Paumomu River. pertusicollis Faust, 1899: 24. (Ectemnomerus) 
DisTRIBUTION: Papua New Guinea: Haveri, Moroka.

setiger (Pascoe), 1885: 210. (Celeuthetes)

DisTRIBUTION: Papua New Guinea: Yule Island.

sus Heller, 1926a: 277. (Ectemnomerus)

DisTRIBUTION: Papua New Guinea.

viridanus Faust, 1899: 23. (Ectemnomerus)

DisTRIBUTION: Papua New Guinea: Astrolabe Mountains.

ELYTROCHEILUS Faust, 1891: 182

TYPE-SPECIES: Siteytes graniger Pascoe, 1881; subsequent designation by Faust, 1897b: 233. KeYs: Faust, 1897b: 248;

Heller, 1905a: 70.

albovittatus Heller, 1910b: 183. (Elytrocheilus)

DisTRIBUTION: Papua New Guinea.

anthonyi Faust, 1897b: 253. (Elytrocheilus)

DistRIBUTION: Papua New Guinea: Astrolabe Mountains, Haveri, Moroka.

boviei Heller, 1905a: 69. (Elytrocheilus)

DISTRIBUTION: New Guinea.

brevicollis Heller, 1935: 163. (Elytrocheilus)

DISTRIBUTION: Papua New Guinea.

breviusculus Heller, 1905a: 70. (Elytrocheilus)

DisTRIBUTION: Papua New Guinea: Sattelberg.

coeruleatus (Pascoe), 1881: 594. (Siteytes caruleatus)

Distribution: Papua New Guinea: Paumomu River, Yule Island.

confinis confinis Faust, 1897b: 251. (Elytrocheilus)

DistRIBUTION: Papua New Guinea: Paumomu River.

confinis novobritannicus Heller, 1937a: 260. (Elytrocheilus)

DISTRIBUTION: Bismarck Archipelago: New Britain.

crassicornis Faust, 1899: 14. (Elytrocheilus)

DISTRIBUTION: Bara-Bara.

cyphoides (Pascoe), 1881: 588. (Eupholus)

DISTRIBUTION: Aneitem.

depressior Faust, 1897b: 253. (Elytrocheilus)

DisTRIBUTION: D’Entrecasteaux Islands: Fergusson Island.

expansus Faust, 1897b: 254. (Elytrocheilus)

Distribution: Papua New Guinea: Moroka.

Elytrocheilus expansus v. nigripes Faust, 1899: 16. DisTRIBUTION: Papua New Guinea: Paumomu River.

falsoconfinis Heller, 1935: 164. (Elytrocheilus)

DISTRIBUTION: Papua New Guinea.

graniger (Pascoe), 1881: 593. (Siteytes)

DistRIBUTION: Papua New Guinea: Yule Island.

helenae Faust, 1891: 183. (Elytrocheilus)

DistRIBUTION: Papua New Guinea: Paumomu River.

intermedius Faust, 1897b: 252. (Elytrocheilus)

Distribution: Papua New Guinea: Milne Bay, Moroka, Paumomu River. 
laevigatus Heller, 1937a: 259. (Elytrocheilus)

DISTRIBUTION: West Papua: Idenburg River.

oxygaster (Montrouzier), 1855: 49. (Otiorhynchus)

DISTRIBUTION: Woodlark Islands.

Elytrocheilus validus Heller, 1910b: 186.

proximus Faust, 1899: 14. (Elytrocheilus)

DistriBution: Papua New Guinea: Astrolabe Mountains, Haveri, Kelesi, Milne Bay, Moroka.

rhinoceros Heller, 1910b: 185. (Elytrocheilus)

DISTRIBUTION: Louisiade Archipelago.

ruficollis Faust, 1899: 16. (Elytrocheilus)

Distribution: Papua New Guinea: Paumomu River.

rufipes Faust, 1899: 15. (Elytrocheilus)

Distribution: Papua New Guinea: Astrolabe Mountains, Haveri, Kelesi, Milne Bay, Port Moresby.

subcoeruleatus Heller, 1910b: 184. (Elytrocheilus)

DisTRIBUTION: Louisiade Archipelago.

validus Heller, 1910b: 186. (Elytrocheilus)

DISTRIBUTION: Woodlark Islands.

ENAPTOMIAS Faust, 1897b: 266

TYPE-SPECIES: Enaptomias rothschildi Faust, 1897b; original designation.

immaculatus Faust, 1899: 17. (Enaptomias)

DiSTRIBUTION: D'Entrecasteaux Islands: Goodenough Island.

incisus (Montrouzier), 1855: 50. (Otiorrhynchus)

DisTRIBUTION: Woodlark Islands. NOTES: Transferred: Günther, 1943.

oculatus Faust, 1897b: 268. (Enaptomias)

DisTRIBUTION: Papua New Guinea.

rothschildi Faust, 1897b: 266. (Enaptomias)

DisTRIBUTION: D'Entrecasteaux Islands: Fergusson Island.

GUINEOBIUS Osella, 1983: 114

TYPE-SPECIES: Guineobius viduus Osella, 1983; original designation. KEY: Riedel \& Schönitizer, 1996: 455. Notes: Placement in Celeuthetini follows Riedel, 1996.

baliemensis Riedel, 1996: 467. (Guineobius)

DistRIBUTION: West Papua: Jiwika, Wamena.

deharvengei Osella, 1983: 115. (Guineobius)

DistRIBUTION: Papua New Guinea: Port Moresby.

depressus Riedel, 1996: 466. (Guineobius)

DistRIBUTION: West Papua: Jiwika, Wamena.

niger Riedel, 1996: 456. (Guineobius)

DisTRIBUTION: West Papua: Jiwika, Wamena.

minutus Riedel, 1996: 462. (Guineobius)

DisTRIBUTION: West Papua: Diuremna, Jiwika.

viduus Osella, 1983: 114. (Guineobius)

Distribution: Papua New Guinea: Chuave. 
HELLERRHINUS Marshall, 1938: 95

TYPE-SPECIES: Hellerrhinus furculus Marsall, 1938; original designation. KeYS: Marshall: 1938: 96; Rheinheimer: 2004b: 440.

bispinosus Marshall, 1956: 20. (Hellerrhinus)

Distribution: Papua New Guinea: Kokoda.

cheesmanae Marshall, 1938: 100. (Hellerrhinus)

DistRibUtion: Papua New Guinea: Mt. Tafa, North West of Mt. Albert Edward.

conifer Marshall, 1938: 101. (Hellerrhinus)

DistRIBUTION: Papua New Guinea: Mt. Tafa, North West of Mt. Albert Edward.

furculus Marshall, 1938: 97. (Hellerrhinus)

Distribution: Papua New Guinea: Mt. Tafa, North West of Mt. Albert Edward.

humeralis Marshall, 1938: 98. (Hellerrhinus)

DistRibution: Papua New Guinea: Mt. Tafa, North West of Mt. Albert Edward.

papuanus (Heller), 1935: 162. (Elytrurus? [sic])

DistRIBUTION: Papua New Guinea: Eddie Creek, Huon Gulf.

platypterus Rheinheimer, 2004b: 435. (Hellerrhinus)

DISTRIBUTION: New Guinea.

sursumcauda Marshall, 1938: 99. (Hellerrhinus)

Distribution: Papua New Guinea: Mt. Tafa, North West of Mt. Albert Edward.

ventralis Marshall, 1938: 98. (Hellerrhinus)

DistRiBUtion: Papua New Guinea: Mt. Tafa, North West of Mt. Albert Edward.

viklundi Rheinheimer, 2004b: 439. (Hellerrhinus)

DistRIBUTION: Papua New Guinea: Murray Pass.

HYPOTACTUS Marshall, 1956: 66

TYPE-SPECIES: Platyacus papillatus Faust, 1897b; original designation.

papillatus (Faust), 1897b: 274. (Platyacus)

DistRIBUTION: Bismarck Archipelago: New Britain. Notes: Transferred:Marshall, 1956: 66.

ruralis (Faust), 1897b: 273. (Platyacus)

DISTRIBUTION: Bismarck Archipelago: New Britain. NOTES: Transferred:Marshall, 1956: 66.

IDIOPSODES Thompson, 1977: 256

TYPE-SPECIES: Idiopsis griseus Faust, 1897b; original designation.

coeruleus (Faust), 1897b: 295. comb. nov. (Idiopsis coerulea)

DisTRIBUTION: Papua New Guinea: Haveri.

griseus (Faust), 1897b: 294. (Idiopsis)

Distribution: Papua New Guinea: Astrolabe Mountains, Port Moresby. NoTES: Transferred: Thompson, 1977: 256.

kochi (Heller), 1914a: 651. comb. nov. (Idiopsis koki)

DistRibution: West Papua: Etna Bay.

maculosus (Faust), 1899: 26. comb. nov. (Idiopsis maculosa)

Distribution: Papua New Guinea: Ighibirei, Paumomu River. 
ornatus (Faust), 1897b: 296. comb. nov. (Idiopsis)

DisTRIBUTION: New Guinea.

perplexus (Faust), 1897b: 287. comb. nov. (Coptorrhynchus)

DisTRIBUTION: West Papua: Humboldt Bay.

quinarius (Pascoe), 1885: 214. comb. nov. (Coptorrhynchus)

DisTRIBUTION: West Papua: Geelvink Bay.

KIETANA Heller, 1910b: 195

TYPE-SPECIES: Kietana episomoides Heller, 1910b; monobasic. KeYs: Marshall, 1956: 38; Tanner, 1969: 7. aluensis Marshall, 1956: 38. (Kietana)

DISTRIBUTION: North Solomon Islands: Shortland Island.

episomoides Heller, 1910b: 196. (Kietana)

DISTRIBUTION: North Solomon Islands: Bougainville, Kieta.

KOKODANUS Marshall, 1956: 21

TYPE-SPECIES: Kokodanus binodosus Marshall, 1956; original designation.

binodosus Marshall, 1956: 22. (Kokodanus)

Distribution: Papua New Guinea: Kokoda.

LOPHOTHETES Marshall, 1956: 75

TYPE-SPECIES: Trigonops penicilligera Heller, 1934; original designation.

biroi Voss, 1958b: 213. (Lophothetes)

DISTRIBUTION: Deslacs Islands.

gazellae (Heller), 1941a: 213. (Trigonops)

DISTRIBUTION: Bismarck Archipelago: New Britain.

incultus Voss, 1958b: 212. (Lophothetes)

DistRIBUTION: Papua New Guinea: Huon Gulf, Simbang.

penicilliger (Heller), 1934: 17. (Trigonops)

DisTRIBUTION: Bismarck Archipelago: New Britain.

Trigonops penicilliger $\mathrm{v}$. deslacsensis (Voss), 1958b: 212. DISTRIBUTION: Deslacs Islands.

sexmaculatus (Heller), 1935: 167. (Trigonops sexmaculata)

DISTRIBUTION: Papua New Guinea.

variabilis Faust, 1897b: 268. (Trigonops)

DisTRIBUTION: D’Entrecasteaux Islands: Fergusson Island, Trobriand Island.

LOPHOTHETES (ACELEUTHETES) Voss, 1958b: 215

TYPE-SPECIES: Lophothetes (Aceleuthetes) trigiduanus Voss, 1958b: 215; original designation.

trigiduanus Voss, 1958b: 215. (Lophothetes (Aceleuthetes))

DisTRIBUTION: Papua New Guinea: Huon Gulf.

MACHAERostylus Heller, 1934: 13

TYPE-SPECIES: Machaerostylus insulanus Heller, 1934; original designation. KEY: Marshall, 1956: 73. 
insulanus Heller, 1934: 14. (Machaerostylus)

DISTRIBUTION: Bismarck Archipelago: New Britain.

laqueus (Heller), 1937a: 258. (Pseudosphaeropterus? [sic])

DistRIBUTION: Bismarck Archipelago: New Britain.

simulans (Heller), 1934: 14. (Pseudosphaeropterus)

DISTRIBUTION: Bismarck Archipelago: New Ireland.

MiCROTHETES Marshall, 1956: 102

TYPE-SPECIES: Microthetes biligatus Marsall, 1956; original designation.

biligatus Marshall, 1956: 102. (Microthetes)

DisTRIBUTION: West Papua: Humboldt Bay.

stictus Marshall, 1956: 103. (Microthetes)

DISTRIBUTION: Aru Islands.

MoLucCoBIUS Marshall, 1956: 40

TYPE-SPECIES: Moluccobius wallacei Marshall, 1956; original designation. KeY: Marshall, 1956: 41.

brevicornis Marshall, 1956: 44. (Moluccobius)

DisTRIBUTION: Aru Islands; Kei Island.

ligatus (Pascoe), 1885: 212. (Coptorhynchus)

DistRIBUTION: West Papua: Dorey, Ramoi, Sorong, Saylee.

terrenus (Marshall), 1935: 41. (Coptorrhynchus)

DistRIBUtion: West Papua: Mysol Island, Weeim Island. Notes: Marshall, 1956: 91 lists this species in the genus Moluccobius, however it is omitted from the generic treatment and key from the same paper.

MUTILLARIUS Marshall, 1956: 81

TYPE-SPECIES: Mutillarius albonotatus Marshall, 1956; original designation.

albonotatus Marshall, 1956: 82. (Mutillarius)

DistRIBUTION: Papua New Guinea: Mt. Tafa, North West of Mt. Albert Edward.

OPTERUS Marshall, 1956: 91

TYPE-SPECIES: Coptorhynchus griseus Pascoe, 1885; original designation. KEY: Marshall, 1956: 92.

cretaceus Marshall, 1956: 94. (Opterus)

DistRIBUTION: Papua New Guinea; Bismarck Archipelago: Kavieng, New Ireland.

costifer Marshall, 1956: 95. (Opterus)

DistriBution: West Papua: Geelvink Bay, Mt. Baduri, Yepen Island.

gibber Marshall, 1956: 95. (Opterus)

DistriBution: West Papua: Geelvink Bay, Mt. Baduri, Yepen Island.

moerens (Pascoe), 1885: 212. (Coptorhynchus)

DiSTRIBUTION: West Papua: Korido. Notes: Transferred: Marshall, 1956: 91.

tutus Marshall, 1956: 98. (Opterus)

DISTRIBUTION: Waigeo Island. 
waigeensis Marshall, 1956: 93. (Opterus)

DisTRIBUTION: West Papua: Mt. Nok, Waigeo Island.

ORIBIUS Marshall, 1956: 88

TYPE-SPECIES: Coptorrhynchus generosus Faust, 1897b; original designation. KEYS: Faust, 1899: 17 (separates O. discretus and O. indiscretus); Heller, 1900b (Coptorhynchus).

ambiguus (Faust), 1897b: 282. (Coptorrhynchus)

DISTRIBUTION: New Guinea.

blanchardi (Faust), 1897b: 282. (Coptorrhynchus)

DISTRIBUTION: New Guinea.

bombicollis (Macleay), 1887: 185. (Coptorhynchus)

DisTRIBUTION: Papua New Guinea: Fly River.

bombylius (Guérin-Méneville), 1841b: 192. (Coptorhynchus)

DistRIBUTION: Papua New Guinea: Fly River, Triton Bay; Aru Island.

caudatus (Guérin-Méneville), 1841b: 192. (Coptorhynchus)

Distribution: West Papua: Dorey.

cinereus Marshall, 1959b: 44. (Oribius)

DistRIBUTION: Papua New Guinea: Windiluk Village near Saidor.

crassirostris (Pascoe), 1885: 213. (Coptorhynchus)

DisTRIBUTION: West Papua: Sorong; Salawatti Island.

cruceatoides Voss, 1958b: 216. (Oribius)

DISTRIBUTION: New Guinea.

cruciatus (Faust), 1897b: 280. (Coptorrhynchus)

DistRIBUTION: Papua New Guinea: Paumomu River, Port Moresby.

demeijerei (Heller), 1914a: 650. (Coptorhynchus)

DISTRIBUTION: Biak Island.

destructor Marshall, 1957: 3. (Oribius)

Distribution: Papua New Guinea: Asaro Valley, Mt. Otto, Goroka, Upper Jimmi Valley, Tsenga, Mt. Hagen.

discretus (Faust), 1899: 18. (Coptorrhynchus)

DisTRIBUTION: West Papua: Haveri.

distans (Faust), 1899: 18. (Coptorrhynchus)

Distribution: Papua New Guinea: Paumomu River.

elegans (Guérin-Méneville), 1841b: 191. (Sphaeropterus)

DistRIBUTION: Papua New Guinea: Yule Island.

ellipticus (Faust), 1897b: 293. (Coptorrhynchus)

DISTRIBUTION: New Guinea.

fraterculus (Faust), 1899: 20. (Coptorrhynchus)

Distribution: Papua New Guinea: Paumomu River.

fuscipes (Faust), 1897b: 285. (Coptorhynchus)

DistRIBUTION: Papua New Guinea: Moroka.

generosus (Faust), 1897b: 290. (Coptorrhynchus)

DistRIBUTION: Papua New Guinea: Dilo, Ighibirei, Kelesi.

gratus (Faust), 1897b: 279. (Coptorrhynchus)

Distribution: Papua New Guinea: Milne Bay.

guineensis (Lona), 1936: 296. (Coptorrhynchus) 
DisTRIBUTION: New Guinea. NotES: Replacement name for Coptorrhynchus improvidus Heller, 1935: 166, non Marshall, 1915.

guttatus (Pascoe), 1885: 214. (Coptorhynchus)

DisTRIBUTION: Papua New Guinea: Yule Island.

guttiger (Blanchard), 1853: 222. (Isomerinthus)

DISTRIBUTION: New Guinea.

hellerianus (Lona), 1936: 297. (Coptorhynchus)

Distribution: Papua New Guinea: Mt. Kani. Notes: Replacement name for Coptorrhynchus leucostictus Heller, 1935: 164, non Pascoe 1885.

hospes (Faust), 1899: 19. (Coptorhynchus)

DistRIBUTION: Papua New Guinea: Paumomu River.

hostis (Heller), 1935: 165. (Coptorrhynchus)

DisTRIBUTION: Papua New Guinea.

humilis (Faust), 1897b: 285. (Coptorrhynchus)

DisTRIBUTION: Papua New Guinea: Mailu Island.

hypocritus (Faust), 1897b: 289. (Coptorrhynchus)

DisTRIBUTION: D’Entrecasteaux Islands: Fergusson Island.

immitis (Pascoe), 1885: 212. (Coptorhynchus)

DisTRIBUTION: West Papua: Hatam, Korido.

improvidus (Marshall), 1915: 510. (Coptorrhynchus)

DistRIBUTION: West Papua: Mimika River.

indiscretus (Faust), 1899: 18. (Coptorrhynchus)

Distribution: Papua New Guinea: Paumomou River.

inimicus Marshall, 1959: 45. (Oribius)

DistRIBUtion: Papua New Guinea: Wabag, Asaro Valley, Upper Jimmi Valley. Notes: Replacement name for O. hostis Marshall, 1957: 4, non Heller, 1935.

lepidus (Boisduval), 1835: 397. (Otiorrhynchus)

DisTRIBUTION: Bismarck Archipelago: New Ireland.

leucopleurus (Faust), 1897b: 277. (Coptorrhynchus)

DistRIBUTION: Papua New Guinea: Moroka.

leucostictus (Pascoe), 1885: 214. (Coptorhynchus)

DisTRIBUTION: West Papua: Sorong.

nudus (Macleay), 1887: 185. (Coptorhynchus)

DistRIBUTION: Papua New Guinea: Fly River.

pellax (Faust), 1899: 21. (Coptorrhynchus)

DistRIBUTION: Papua New Guinea: Paumomu River.

perornatus (Heller), 1908a: 13. (Coptorhynchus)

Distribution: West Papua.

plagiatus (Blanchard), 1853: 299. (Psomeles)

DisTRIBUTION: Papua New Guinea.

puncticollis (Faust), 1897b: 286. (Coptorrhynchus)

DisTRIBUTION: West Papua: Humboldt Bay.

quadruplex (Heller), 1914a: 649. (Coptorhynchus)

DisTRIBUTION: West Papua: Lorertz River; Biak Island.

quattuordecimmaculatus (Chevrolat), 1877: 189. (Coptorrhynchus 14-maculatus)

DISTRIBUTION: New Guinea.

servilis (Pascoe), 1885: 211. (Coptorhynchus) 
Distribution: Papua New Guinea: Fly River, Paumomu River.

speculatus (Macleay), 1887: 185. (Coptorhynchus)

DistRIBUTION: Papua New Guinea: Fly River.

subcylindricus (Faust), 1897b: 291. (Coptorrhynchus)

DISTRIBUTION: New Guinea.

subligatus (Marshall), 1935: 39. (Coptorrhynchus)

DistRIBUTION: West Papua: Kamakahwalla Lake, Lomira, Sekatwa River; Aroe Island.

tessellatus (Blanchard), 1853: 223. (Isomerinthus)

DistRIBUTION: Papua New Guinea: Fly River; Aru Island (?).

unifasciatus (Faust), 1897b: 292. (Coptorrhynchus)

DISTRIBUTION: Papua New Guinea.

valens (Heller), 1908a: 14. (Coptorhynchus)

Distribution: Papua New Guinea: Astrolabe Mountains; Bismarck Archipelago: New Britain. vittaticollis (Heller), 1914a: 650. (Coptorhynchus)

DISTRIBUTION: West Papua: Lorertz River; Biak Island.

PaChYRhYNChIDIUS Faust, 1897b: 239

TYPE-SPECIES: Pachyrhynchidius quadripustulatus Faust, 1897b; original designation.

quadripustulatus Faust, 1897b: 240. (Pachyrhynchidius)

DISTRIBUTION: Papua New Guinea: Moroka.

Parasphenogaster Heller, 1901: 15

TYPE-SPECIES: Parasphenogaster gracilicollis Heller, 1901; original designation.

gracilicollis Heller, 1901: 15. (Parasphenogaster)

DisTRIBUTION: Papua New Guinea: Aroa River.

ottonis Heller, 1910a: 26. (Parasphenogaster)

DISTRIBUTION: New Guinea.

Paratactus Heller, 1934: 11

TYPE-SPECIES: Paratactus seriatogranosus Heller, 1934; original designation.

albidoplagiatus (Fairmaire), 1883: 35. (Sphaeropterus)

DisTRIBUTION: Bismarck Archipelago: Duke of York Island, New Britain, New Ireland. NoTES: Transferred: Marshall, 1956: 66.

carbunculus (Heller), 1908a: 14. (Coptorhynchus)

DISTRIBUTION: Bismarck Archipelago: New Britain. NOTES: Transferred: Marshall, 1956: 66.

confluentinus Voss, 1958b: 210. (Paratactus)

DisTRIBUTION: Bismarck Archipelago: New Britain.

lihirensis Marshall, 1957: 1. (Paratactus)

DisTRIBUTION: Bismarck Archipelago: Lihir Island.

seriatogranosus Heller, 1934: 12. (Paratactus)

DisTRIBUTION: Bismarck Archipelago: New Ireland.

virescens Heller, 1934: 12. (Paratactus)

DISTRIBUTION: North Solomon Islands: Bougainville Island. 


\section{Paratactus (Mesotactus) Voss, 1958b: 211}

TYPE-SPECIES: Paratactus inflatipes Voss, 1958b; original designation.

inflatipes Voss, 1958b: 211. (Paratactus (Mesotactus))

DistRIBUtion: Papua New Guinea: Erima, Stephansort, Astrolabe Bay, Sattelberg, Simbang, Huon Gulf.

\section{Peteinus Marshall, 1956: 113}

TYPE-SPECIES: Apirocalus thomsoni Waterhouse, 1889; original designation.

thomsoni (Waterhouse), 1889: 364. (Apirocalus)

DisTRIBUTION: Louisiade Archipelago: St. Aignan Island, Misima Island.

PhraOtes Pascoe, 1885: 208

TYPE-SPECIES: Phraotes tuberculatus Pascoe, 1885; monobasic. KeY: Marshall, 1956: 25.

mammillatus Heller, 1910a: 25. (Phraotes)

DisTRIBUTION: Papua New Guinea: Bongu.

torvus Marshall, 1956: 24. (Phraotes)

Distribution: Papua New Guinea: Hydrographer Mountains, Bubu River, Upper Waria River.

tuberculatus Pascoe, 1885: 209. (Phraotes)

Distribution: Papua New Guinea: Fly River, Haveri.

sellatus Marshall, 1956: 23. (Phraotes)

DisTRIBUTION: Bismarck Archipelago: New Britain, Talesea.

Platyacus Faust, 1897b: 270

TYPE-SPECIES: Platyacus websteri Faust, 1897b; original designation. KeYs: Heller, 1910b: 191 [Colposternum]; Marshall, 1956: 67; Tanner, 1969: 11.

laticollis (Heller), 1910b: 193. (Colposternum laticolle)

DISTRIBUTION: North Solomon Islands: Bougainville.

malachiticus (Heller), 1910b: 192. (Colposternum malachiticum)

DISTRIBUTION: North Solomon Islands: Bougainville.

Hoplotrigonops chlorizans Heller, 1934: 23.

minor Marshall, 1956: 71. (Platyacus)

DisTRIBUTION: North Solomon Islands: Bougainville.

narinus Marshall, 1956: 71. (Platyacus)

DISTRIBUTION: North Solomon Islands: Bougainville.

nigrocristatus (Heller), 1934: 22. (Hoplotrigonops nigrocristata)

DISTRIBUTION: North Solomon Islands: Bougainville.

subalatus (Heller), 1910b: 194. (Colposternum subalatum)

DISTRIBUTION: North Solomon Islands: Bougainville.

websteri Faust, 1897b: 270. (Platyacus)

DisTRIBUTION: North Solomon Islands: Alu Island, Buka Island, Shortland Island. NotES: Erroneously listed from Aru Island in the Coleopterorum Catalogus (Lona, 1937). 
SPHAEROPTERUS Guérin-Méneville, 1838: 122

TYPE-SPECIES: Sphaeropterus albolineatus Guérin-Méneville, 1838; monobasic.

albolineatus albolineatus Guérin-Méneville, 1838: 123. (Sphaeropterus)

Distribution: West Papua: Dorey, Hatam, Andai, Moemi, Siwi, Sakoemi.

Otiorrhynchus luctuosus Boisduval, 1835: 396.

Sphaeropterus albolineatus v. scaposus (Pascoe), 1881: 592. (Isomerinthus scaposus). DistRIBUTION: West

Papua: Dorey.

albolineatus interruptus Heller, 1935: 164. (Sphaeropterus)

DISTRIBUTION: New Guinea.

bituberculatus Kirsch, 1877: 152. (Sphaeropterus)

DistRIBUTION: West Papua: Yapen Island.

SPHENOGASTER Faust, 1897b: 241

TYPE-SPECIES: Sphenogaster bipustulata Faust, 1897b; original designation. KEY: Marshall, 1956: 26.

bipustulata Faust, 1897b: 242. (Sphenogaster)

DisTRIBUTION: New Guinea.

carinrostris Heller, 1910a: 26. (Sphenogaster)

DisTRIBUTION: New Guinea.

gyrosicollis Marshall, 1956: 25. (Sphenogaster)

DisTRIBUTION: Papua New Guinea: Orror Island.

nitidula Faust, 1897b: 243. (Sphenogaster)

DistRIBUTION: Papua New Guinea: Haveri, Paumomu River, Yule Island.

STROTUS Marshall, 1956: 35

TYPE-SPECIES: Otiorrhynchus bicristatus Montrouzier, 1855; original designation.

bicristatus (Montrouzier), 1855: 49. (Otiorrhynchus)

DisTRIBUTION: New Guinea; D’Entrecasteaux Islands: Fergusson Island; Woodlark Island.

TrigONOPS Guérin-Méneville, 1841a: 128

TYPE-SPECIES: Trigonops rugosus Guérin-Méneville, 1841a; monobasic. KEYS: Heller, 1934: 21; Tanner, 1961: 21.

carinithorax Heller, 1934: 20. (Trigonops)

DisTRIBUTION: North Solomon Islands: Bougainville.

bougainvillensis Tanner, 1969: 38. (Trigonops)

DISTRIBUTION: North Solomon Islands: Bougainville.

gloriosa Tanner, 1969: 45. (Trigonops)

DISTRIBUTION: North Solomon Islands: Bougainville.

gressiti Tanner, 1969: 44. (Trigonops)

DISTRIBUTION: North Solomon Islands: Bougainville.

irrorata Heller, 1934: 18. (Trigonops)

DisTRIBUTION: North Solomon Islands: Bougainville. 
minuta Tanner, 1969: 26. (Trigonops)

DISTRIBUTION: North Solomon Islands: Bougainville, Shortland Island.

salomonis Heller, 1934: 18. (Trigonops)

DISTRIBUTION: North Solomon Islands: Bougainville.

vitticollis Fairmaire, 1883: 34. (Trigonops)

DISTRIBUTION: Bismarck Archipelago: Duke of York Island.

TrigonOSPARTUS Faust, 1897b: 245

TYPE-SPECIES: Trigonospartus rufidorsum Faust, 1897b; original designation.

cheesmanae Marshall, 1956: 85. (Trigonospartus)

Distribution: Papua New Guinea: Mafulu, Mondo.

rufidorsum Faust, 1897b: 245. (Trigonospartus)

Distribution: Papua New Guinea: Haveri, Moroka.

ZEUGORRHINUS Marshall, 1956: 29

TYPE-SPECIES: Zeugorrhinus granulatus Marshall, 1956; original designation.

granulatus Marshall, 1956: 29. (Zeugorrhinus)

DISTRIBUTION: North Solomon Islands: Bougainville, Kieta.

\section{CYPHICERINI}

\section{CYPHICERINA}

CYPHICERUS Schönherr, 1823: 1144

TYPE-SPECIES: Curculio orbitalis Wiedemann, 1819 [=Curculio novemlineatus Olivier, 1807]; original designation.

praecanus Faust, 1892b: 193. (Cyphicerus)

DistribUtion: Papua New Guinea: Hula, Paumomu River, Rigo.

\section{ELYTRURINI}

GYNARIA Pascoe, 1883: 89

TYPE-SPECIES: Gynaria nasuta Pascoe, 1883; monobasic.

nasuta Pascoe, 1883: 89. (Gynaria)

DISTRIBUTION: Aru Islands.

\section{EUPHOLINI}

CELEBIA Thomson, 1857a: 287

TYPE-SPECIES: Celebia azureipes Thomson, 1857a [=Geonemus arrogans Boisduval, 1835]; monobasic. KEYs: Faust, 1891: 181; Heller, 1897a. 
arrogans (Boisduval), 1835: 358. (Geonemus)

DistRIBUTION: New Guinea. NOTES: Heller, 1933b: 146 transferred this species from Rhinoscapha to Celebia.

EPISOMELLUS Kirsch, 1877: 151

TYPE-SPECIES: Episomellus papuanaus Kirsch, 1877; monobasic.

papuanaus Kirsch, 1877: 151. (Episomellus)

DISTRIBUTION: West Papua: Rubi.

EUPHOLUS Boisduval, 1835: 363

TYPE-SPECIES: Geonemus geoffroyi Guérin-Méneville, 1831; subsequent designation by Alonso-Zarazaga \& Lyal, 1999: 157. KEYS: Thomson, 1857b: 439; Heller, 1908a: 7; Heller, 1915: 515 (separates subspecies of E. schoenherri); Heller, 1923: 149.

albofasciatus Heller, 1910b: 182. (Eupholus)

DisTRIBUTION: Papua New Guinea: Garina, Waria Valley.

alternans Kirsch, 1877: 149. (Eupholus)

DisTRIBUTION: West Papua: Rubi.

amaliae Gestro, 1875: 1004. (Eupholus)

DisTRIBUTION: West Papua: Ramoi.

Eupholus amaliae v. arfaki Chevrolat, 1880b: 333.

amalulu (Porion), 1993: 13. comb. nov. (Eupholus Guérin-Méneville, non Boisduval)

Distribution: Papua New Guinea: Baga, Popondetta.

astrolabensis Heller, 1937a: 265. (Eupholus)

DisTRIBUTION: Papua New Guinea: Astrolabe Mountains.

azureus Macleay, 1885: 704. (Eupholus)

Distribution: Papua New Guinea: Finschhafen, Haia, Wareo.

Eupholus azureus v. bilineellus Heller, 1908a: 8. DistRIBUTION: New Guinea.

beccarii Gestro, 1875: 1005. (Eupholus)

DisTRIBUTION: West Papua: Dorey, Rubi.

Eupholus admirandus Kirsch, 1877: 150.

bennetti Gestro, 1876: 387. (Eupholus bennettii)

DistRIBUTION: Papua New Guinea: Aseki, Kanabea, Menyamya.

Eupholus bennetti v. apicalis Heller, 1923: 153. Distribution: Papua New Guinea: Aroa River.

Eupholus bennetti v. bicolor Faust, 1899: 12. DistRIBUTION: Papua New Guinea: Kapakapa, Paumomu River.

bennigseni Heller, 1908a: 8. (Eupholus)

DisTRIBUTION: Papua New Guinea: Siassi Island; Bismarck Archipelago: New Britain.

browni Bates, 1877: 155. (Eupholus)

DISTRIBUTION: Bismarck Archipelago: Duke of York Island, New Britain, New Ireland.

bruijnii Gestro, 1875: 1007. (Eupholus)

DISTRIBUTION: West Papua: Arfak, Hatam.

chaminadei (Porion), 2000: 6. (Eupholus Guérin-Méneville, non Boisduval)

DisTRIBUTION: West Papua: Sorong.

chevrolatii (Guérin-Méneville), 1838: 117. (Eupholus Guérin-Méneville, non Boisduval)

DisTRIBUTION: New Guinea; Aru Islands. 
cinnamomeus Pascoe, 1888: 410. (Eupholus)

Distribution: Papua New Guinea: Astrolabe Range, Mailu, Moroka, Mt. Alexander - Mt. Nisbet, Pt. Moresby.

Eupholus cinnamomeus v. caesius Faust, 1899: 12. DistribUtion: Papua New Guinea: Astrolabe Mountains, Haveri, Port Moresby.

Eupholus cinnamomeus v. fasciatus Heller, 1937a: 265. Distribution: Papua New Guinea: Moroka.

clarki (Porion), 1993: 19. comb. nov. (Eupholus Guérin-Méneville, non Boisduval)

Distribution: Papua New Guinea: Lumi.

compositus Faust, 1892b: 188. (Eupholus)

DisTRIBUTION: Papua New Guinea: Finschhafen, Wasu.

cuvierii (Guérin-Méneville), 1838: 118. (Eupholus Guérin-Méneville, non Boisduval)

Distribution: West Papua: Andai, Arfak Range, Dorey, Hatam, Humboldt Bay. Notes: Synonymy:

Riedel, 2002b: 2.

detanii (Limoges \& Porion), 2004: 4. comb. nov. (Eupholus Guérin-Méneville, non Boisduval) DistRIBUTION: West Papua: Mapia.

dhuyi (Porion), 1993: 11. (Eupholus Guérin-Méneville, non Boisduval) Distribution: Papua New Guinea: Karimui, Kerowagi, Lufa, Tari.

ducopeaui (Porion), 2000: 5. (Eupholus Guérin-Méneville, non Boisduval) DistRIBUTION: Papua New Guinea: Jimmi Valley.

euphrosyne (Porion), 1993: 12. (Eupholus Guérin-Méneville, non Boisduval) DistRIBUTION: Papua New Guinea: Aseki

fleurenti (Porion), 1993: 11. comb. nov. (Eupholus Guérin-Méneville, non Boisduval)

DistRIBUTION: Papua New Guinea: Okapa, Upper Jimmi Valley, Wapenamanda, Yanderra.

geoffroyi geoffroyi (Guérin-Méneville), 1831: 115. (Geonemus)

Distribution: Papua New Guinea: Bogia, Lumi, Madang, Vanimo; West Papua: Andai, Dorey, Humboldt Bay, Triton Bay.

Eupholus mirabilis Boisduval, 1832: 35.

Eupholus intermedius Ritsema, 1881: 87.

geoffroyi chrysites Heller, 1915: 514. (Eupholus)

DistRIBUTION: Papua New Guinea: Monumbo.

Eupholus geoffroyi v. tupinieri (Guérin-Méneville), 1838: 65. DisTRIBUTION: Djawur Island.

helleri (Porion), 1993: 12. comb. nov. (Eupholus Guérin-Méneville, non Boisduval)

DisTRIBUTION: Papua New Guinea: Marawaka.

hephaistos (Porion), 1993: 20. comb. nov. (Eupholus Guérin-Méneville, non Boisduval)

DistRIBUTION: Papua New Guinea: Kainantu, Kerowagi, Okapa, Wau.

hudsoni (Porion), 2000: 3. comb. nov. (Eupholus Guérin-Méneville, non Boisduval)

DistRIBUTION: Papua New Guinea: Yaningya.

humeralis Heller, 1908a: 9. (Eupholus)

DistRIBUTION: Papua New Guinea: Daru, Mt. Bosavi.

humeridens Heller, 1895b: 12. (Eupholus)

Distribution: Papua New Guinea: Finschhafen, Mt. Bosavi, Sattelberg.

labbei (Porion), 2000: 6. (Eupholus Guérin-Méneville, non Boisduval)

Distribution: West Papua: Bokondini.

kotaseaoi (Porion), 2000: 3. (Eupholus Guérin-Méneville, non Boisduval)

Distribution: Papua New Guinea: Philimu. Notes: Transferred: Riedel, 2002b: 2.

kuntzmannorum (Limoges \& Porion), 2004: 4. comb. nov. (Eupholus Guérin-Méneville, non Boisduval)

DistRIBUTION: Papua New Guinea: Eastern Highlands, Lifu. 
lachaumei (Porion), 1993: 21. comb. nov. (Eupholus Guérin-Méneville, non Boisduval)

Distribution: Papua New Guinea: Lufa.

lacordairei (Limoges \& Porion), 2004: 3. comb. nov. (Eupholus Guérin-Méneville, non Boisduval)

DisTRIBUTION: West Papua: Kebar.

leblanci (Limoges \& Porion), 2004: 3. comb. nov. (Eupholus Guérin-Méneville, non Boisduval)

DisTRIBUTION: Bismarck Archipelago: Bainings Mountains, New Britain, Raunsepna.

linnei Thomson, 1857b: 442. (Eupholus)

DISTRIBUTION: Aru Islands; Banda Island.

Eupholus bandanus Snellen van Vollenhoven, 1871: 102.

Eupholus thomsoni Chevrolat, 1880: XC.

loriai Gestro, 1902: 48. (Eupholus)

Distribution: Papua New Guinea: Aseki, Marawaka, Tekadu,Watut, Wau.

Eupholus modestus Heller, 1908a: 10. NotES: Synonymy: Porion, 1993: 21.

magnificus Kirsch, 1877: 148. (Eupholus)

DisTRIBUTION: West Papua: Ansus, Korido, Mafor Island.

Eupholus raffrayi Chevrolat, 1880: XVI.

Eupholus magnificus v. concolor Heller, 1923: 150.

malotrus (Porion), 2000: 4. (Eupholus Guérin-Méneville, non Boisduval)

DistRIBUtion: Papua New Guinea: Madang, Yanderra, Jimmi Valley.

mamberamonis Heller, 1942: 210. (Eupholus)

Distribution: Papua New Guinea: Mamberamo River, Mt. Bosavi.

messagieri (Porion), 1993: 13. comb. nov. (Eupholus Guérin-Méneville, non Boisduval)

Distribution: Papua New Guinea: Bulolo, Okapa.

nagaii (Porion), 1993: 19. (Eupholus Guérin-Méneville, non Boisduval)

DistRIBUTION: Papua New Guinea: Maprik.

nickerli Heller, 1913: 46. (Eupholus)

DistRIBUTION: Papua New Guinea: Goroka, Kainantu, Lae, Okapa, Watut.

prasinus Heller, 1910a: 25. (Eupholus humeridens ab. prasinus)

Distribution: Papua New Guinea: Goroka, Kani (Kandt?), Lufa, Torricelli Mountains; West Papua:

Cyclops Mountains. NotES: Status changed to full species: Porion, 1993: 21.

Eupholus humeridens prasinus Heller, 1915: 517. NoTES: Synonymy with E. humeridens ab. prasinus: Heller,

1921: 196.

quadrimaculatus Kirsch, 1877: 149. (Eupholus)

DisTRIBUTION: West Papua: Arfak Mountains.

Eupholus quadrimaculatus v. jugatus Ritsema, 1881: 87. DistRIBUTION: West Papua: Andai.

quinitaenia Heller, 1915: 516. (Eupholus)

DisTRIBUTION: Papua New Guinea: Marprik, Wewak.

rigouti (Porion), 1993: 16. comb. nov. (Eupholus Guérin-Méneville, non Boisduval)

DisTRIBUTION: Papua New Guinea: Goroka, Kainantu, Okapa.

saugrenus (Porion), 1993: 20. comb. nov. (Eupholus Guérin-Méneville, non Boisduval)

DisTRIBUTION: Papua New Guinea: Kainantu, Okapa.

schneideri Riedel, 2002b: 6. (Eupholus)

DisTRIBUTION: West Papua: Membahan near Kosarek.

schoenherrii schoenherrii (Guérin-Méneville), 1838: 116. (Eupholus Guérin-Méneville, non Boisduval)

Distribution: Papua New Guinea: Lumi, Maprik, Wewak; West Papua: Andai, Arfak Range, Dorey,

Korido Island, Salawatti Island.

Eupholus celebesus Chevrolat, 1880b: 333. 
Eupholus desmaresti Chevrolat, 1880a: XVII.

Eupholus salawattensis Snellen van Vollenhoven, 1871: 103.

schoenherrii petiti Guérin-Méneville, 1841c: 216. (Eupholus)

Distribution: West Papua: Dorey, Triton Bay, Waigeo; Aru Island; Mysol Island.

Eupholus latreillei Kirsch, 1877: 148. NotES: Synonymy: Porion, 1993: 15.

Eupholus guerini Chevrolat, 1880a: XVI.

Eupholus schoenherrii v. arfaki Chevrolat, 1880: 333. NotES: Synonymy: Porion, 1993: 15.

schoenherrii mimikanus Heller, 1923: 151. (Eupholus)

DistRIBUTION: West Papua: Mimika River.

schoenherrii semicoeruleus Heller, 1915: 515. (Eupholus)

DistRIBUTION: Papua New Guinea: Dallmannhafen.

sofia (Porion), 2000: 5. (Eupholus Guérin-Méneville, non Boisduval)

DisTRIBUTION: Papua New Guinea: Kayapit, Mangiang.

suhandai (Porion), 2000: 5. (Eupholus Guérin-Méneville, non Boisduval)

DISTRIBUTION: West Papua: Sorong.

sulcicollis Heller, 1915: 517. (Eupholus)

DisTRIBUTION: Papua New Guinea: Wewak.

vlasimskii Balke \& Riedel in Riedel, 2002b: 4. (Eupholus)

DistRIBUTION: West Papua: Wondiwoi Mountains, Yeretua.

vethi Heller, 1914d: 306. (Eupholus)

DisTRIBUTION: Papua New Guinea: Bulolo, Finschhafen.

waigeuensis (Limoges \& Porion), 2004: 5. comb. nov. (Eupholus Guérin-Méneville, non Boisduval)

DISTRIBUTION: Waigeo Island.

GYMNOPHOLUS Heller, 1901: 8

TYPE-SPECIES: Gymnopholus weiskei Heller, 1901; monobasic. KeYs: Marshall, 1959a: 71; Gressitt, 1966a: 226; Gressitt \& Sedlacek, 1967: 483.

ajax Gressitt, 1966a: 237. (Gymnopholus)

DistRIBUTION: Papua New Guinea: Mt. Yelia near Menyamya.

angustus Marshall, 1959a: 80. (Gymnopholus)

Distribution: Papua New Guinea: Mondo, Mt. Tafa, between Murry Pass and Woitape.

biformis Gressitt \& Sedlacek, 1970: 757. (Gymnopholus)

Distribution: Papua New Guinea: Garaina, Mt. Strong, Owen Stanley Range.

brandti Gressitt, 1966a: 245. (Gymnopholus)

DisTRIBUTION: Papua New Guinea: Bome, Goilala.

carinatus Heller, 1937a: 265. (Gymnopholus)

DisTRIBUTION: Papua New Guinea: Finschhafen.

colmani Gressitt \& Sedlacek, 1970: 762. (Gymnopholus)

DISTRIBUTION: Papua New Guinea: Mt. Shungol near Lae.

cyphothorax (Heller), 1901: 9. (Aroaphila)

Distribution: Papua New Guinea: Aroa River, Bome, Goilala, Mt. St. Mary, Wau.

divaricatus Gressitt, 1966a: 246. (Gymnopholus)

Distribution: Papua New Guinea: Daulo Pass, Asaro-Chimbu divide, Wagai Valley.

ellynae Gressitt \& Sedlacek, 1967: 485. (Gymnopholus)

DisTRIBUTION: Papua New Guinea: Schrader Range near Simbai.

engabenae Gressitt, 1977: 188. (Gymnopholus) 
Distribution: Papua New Guinea: Aseki, Engabena, Watut Valley.

fulvospretus (Heller), 1926a: 276. (Aroaphila fulvospreta)

Distribution: Papua New Guinea: Finisterre Range, Huon Peninsula, Saruwaged Range.

gemmifer Gressitt, 1966a: 240. (Gymnopholus)

DistRIBUTION: Papua New Guinea: Mt. Michael near Goroka.

glochidionis Gressitt, 1966a: 247. (Gymnopholus)

DistRIBUTION: Papua New Guinea: Aiyura, Daulo Pass, Okapa South of Kainantu.

gressitti Marshall, 1959a: 77. (Gymnopholus)

Distribution: Papua New Guinea: Daulo Pass, Asaro-Chimbu divide, Mt. Otto.

harti Gressitt, 1977: 189. (Gymnopholus)

DisTRIBUTION: Papua New Guinea: Aseki, Watut Valley.

hornabrooki Gressitt, 1966a: 252. (Gymnopholus)

DistRIBUTION: Papua New Guinea: Mt. Michael near Goroka.

howcrofti Gressitt, 1977: 186. (Gymnopholus)

DistRIBUTION: Papua New Guinea: Marafunga East of Goroka.

interpres Heller, 1935: 180. (Gymnopholus (Aroaphila))

DistriBution: Papua New Guinea: Bulldog Road, Eddie Creek, Huon Gulf, Mt. Kaindi, Wau. integrirostris (Heller), 1913: 43. (Aroaphila)

Distribution: Papua New Guinea: Huon Peninsula, Sattelberg.

ludificator Gressitt, 1966a: 248. (Gymnopholus)

DistRIBUtion: Papua New Guinea: Huon Peninsula, Saruwaged Range.

magister Gressitt, 1966a: 252. (Gymnopholus)

DistRiBution: Papua New Guinea: Daulo Pass, Finisterre Range, Freyberg Pass.

mammifer Gressitt, 1966a: 235. (Gymnopholus)

DisTRIBUTION: Papua New Guinea: Mt. Shungol near Lae.

marquardti Heller, 1935: 179. (Gymnopholus)

Distribution: Papua New Guinea: Bulldog Road, Eddie Creek, Huon Gulf (?), Mt. Kaindi.

marshalli Gressitt, 1966a: 243. (Gymnopholus)

Distribution: Papua New Guinea: Bome, Goilala.

muscosus Gressitt, 1966a: 235. (Gymnopholus)

Distribution: Papua New Guinea: Mt. Obree near Pt. Moresby.

nothofagi Gressitt, 1966a: 231. (Gymnopholus)

DistRIBUTION: Papua New Guinea: Lake Sirunki near Wabag.

perspicax Gressitt, 1966a: 241. (Gymnopholus)

DistRIBUTION: Papua New Guinea: Mt. Yelia near Menyamya.

pulcher Gressitt \& Sedlacek, 1970: 756. (Gymnopholus)

Distribution: Papua New Guinea: Mt. Strong, Owen Stanley Range.

regalis Gressitt, 1966a: 250. (Gymnopholus)

Distribution: Papua New Guinea: Finisterre Range, Huon Peninsula.

rennii Gressitt, 1977: 182. (Gymnopholus)

Distribution: Papua New Guinea: between Watut and Aseki.

rostralis Gressitt \& Sedlacek, 1967: 490. (Gymnopholus)

Distribution: West Papua.

rubi Gressitt, 1977: 185. (Gymnopholus)

DisTRIBUTION: Papua New Guinea: South of Aseki.

sedlaceki Gressitt, 1966a: 251. (Gymnopholus)

Distribution: Papua New Guinea: Aiyurop near Mendi, Karimui, Tomba West of Mt. Hagen. 
seriatus Gressitt \& Sedlacek, 1967: 493. (Gymnopholus)

DisTRIBUTION: West Papua.

setosus Gressitt \& Sedlacek, 1967: 488. (Gymnopholus)

DISTRIBUTION: West Papua: Arboebivak.

splendidus Gressitt, 1966a: 238. (Gymnopholus)

DisTRIBUTION: Papua New Guinea: Mt. Karimui near Goroka.

subnacreus Gressitt \& Sedlacek, 1967: 489. (Gymnopholus)

Distribution: West Papua.

suturalis suturalis (Heller), 1910b: 181. (Aroaphila)

Distribution: Papua New Guinea: Mt. Kani.

suturalis angularis Gressitt, 1977: 187. (Gymnopholus)

DistRIBUTION: Papua New Guinea: Aseki, Watut Valley.

szentivanyi Gressitt, 1966a: 253. (Gymnopholus)

Distribution: Papua New Guinea: Daulo Pass, Asaro-Chimbu divide.

toxopei Gressitt \& Sedlacek, 1967: 486. (Gymnopholus)

DisTRIBUTION: West Papua.

tricolor Gressitt \& Sedlacek, 1970: 757. (Gymnopholus)

DistRIBUTION: Papua New Guinea: Mt. St. Mary, Owen Stanley Range.

urticivorax Gressitt, 1966a: 233. (Gymnopholus)

Distribution: Papua New Guinea: Mt. Missim, Wau.

vetustus Gressitt \& Sedlacek, 1967: 491. (Gymnopholus)

DistRibUtion: Papua New Guinea: Mt. Piora.

weiskei Heller, 1901: 8. (Gymnopholus)

Distribution: Papua New Guinea: Aroa River, Aseki, Bulldog Road, Bulolo, Daulo Pass, Eddie Creek,

Kainantu, Wau.

Gymnopholus forticornis Heller, 1935: 179. DistRIBUTION: Papua New Guinea: Huon Gulf, Eddie Creek.

GYMNOPHOLUS (SYMBIOPHOLUS) Gressitt, 1966a: 254

TYPE-SPECIES: Gymnopholus reticulatus Marshall, 1959a; original designation.

acarifer Gressitt, 1966a: 270. (Gymnopholus (Symbiopholus))

Distribution: Papua New Guinea: Bulldog Road, Eddie Creek, Mt. Amingwina near Wau, Mt. Kaindi,

Mt. Shungol near Lae.

algifer Gressitt, 1966a: 259. (Gymnopholus (Symbiopholus))

Distribution: Papua New Guinea: Bome, Goilala, Mt. St. Mary, Murray Pass to Woitape near Mt. Albert

Edward.

audax Gressitt, 1966a: 254. (Gymnopholus (Symbiopholus))

DisTRIBUTION: West Papua: Araboebivak, Itouda near Wissel Lakes, Panini.

botanicus Gressitt, 1966a: 263. (Gymnopholus (Symbiopholus))

Distribution: Papua New Guinea: Mt. Wilhelm.

carolynae Gressitt \& Sedlacek, 1967: 496. (Gymnopholus (Symbiopholus))

DistRIBUTION: Papua New Guinea: Kubor Range, Western Highlands.

cheesemanae Marshall, 1959a: 78. (Gymnopholus)

Distribution: Papua New Guinea: Mondo, Mt. Tafa, Murray Pass to Woitape near Mt. Albert Edward.

didiman Gressitt, 1977: 190. (Gymnopholus (Symbiopholus))

Distribution: Papua New Guinea: Mt. Missim, Snake River, Wau.

euryae Gressitt, 1977: 194. (Gymnopholus (Symbiopholus)) 
Distribution: Papua New Guinea: Mt. Amde between Aseki and Kaintiba, Renni.

fallax Gressitt, 1966a: 257. (Gymnopholus (Symbiopholus))

DisTRIBUTION: Papua New Guinea: Iongai near Mt. Albert Edward.

fungifer Gressitt, 1966a: 266. (Gymnopholus (Symbiopholus))

DistRIBUTION: Papua New Guinea: Finisterre Range, Freyberg Pass, Saruwaged Range.

hepaticus Gressitt, 1966a: 274. (Gymnopholus (Symbiopholus))

DistriBution: Papua New Guinea: Murray Pass near Mt. Albert Edward.

herbarius herbarius Gressitt, 1966a: 269. (Gymnopholus (Symbiopholus))

Distribution: Papua New Guinea: Murray Pass to Woitape near Mt. Albert Edward, Tapini.

herbarius oribatifer Gressitt, 1966a: 270. (Gymnopholus (Symbiopholus))

DisTRIBUTION: Papua New Guinea: Iongai to Murray Pass near Mt. Albert Edward, Tapini, Woitape.

huttoni Gressitt, 1977: 192. (Gymnopholus (Symbiopholus))

DisTRIBUTION: Papua New Guinea: Garaina.

kokodae Marshall, 1959a: 73. (Gymnopholus)

Distribution: Papua New Guinea: Kokoda.

lichenifer Gressitt, 1966a: 267. (Gymnopholus (Symbiopholus))

Distribution: Papua New Guinea: Bulldog Road, Eddie Creek, Mt. Kaindi, Wau. Notes: Listed as vulnerable in IUCN red data book: Wells et. al. 1983.

magnus Gressitt, 1977: 194. (Gymnopholus (Symbiopholus))

Distribution: Papua New Guinea: Mt. Missim.

nitidus Gressitt \& Sedlacek, 1967: 494. (Gymnopholus (Symbiopholus))

DisTRIBUTION: West Papua.

nodifer Gressitt, 1977: 192. (Gymnopholus (Symbiopholus))

DistRIBUTION: Papua New Guinea: Aseki to Watut, between Menyamya and Bulolo.

piorae Gressitt, 1977: 190. (Gymnopholus (Symbiopholus))

DisTRIBUTION: Papua New Guinea: Mt. Piora.

praecox Gressitt, 1966a: 256. (Gymnopholus (Symbiopholus))

Distribution: Papua New Guinea: Lake Sirunki, Western Highlands.

rebeccae Gressitt \& Sedlacek, 1967: 496. (Gymnopholus (Symbiopholus))

Distribution: Papua New Guinea: Kratka Range, Mt. Piora.

reticulatus Marshall, 1959a: 75. (Gymnopholus)

Distribution: Papua New Guinea: Daulo Pass, Asaro-Chimbu divide.

rugicollis (Heller), 1913: 44. (Aroaphila)

DistRIBUTION: Papua New Guinea: Sattelberg.

schefflerae Gressitt \& Sedlacek, 1970: 761. (Gymnopholus (Symbiopholus))

DisTRIBUTION: Papua New Guinea: Mt. Shungol near Lae.

schuurmannsiae Gressitt \& Sedlacek, 1967: 497. (Gymnopholus (Symbiopholus))

DistRIBUTION: Papua New Guinea: Kratka Range, Mt. Piora.

senex Gressitt, 1966a: 272. (Gymnopholus (Symbiopholus))

DistRIBUTION: Papua New Guinea: Mt. Yelia near Menyamya.

shungolus Gressitt \& Sedlacek, 1970: 759. (Gymnopholus (Symbiopholus))

DistRIBUTION: Papua New Guinea: Mt. Shungol near Lae.

symbioticus Gressitt, 1966a: 260. (Gymnopholus (Symbiopholus))

DisTRIBUTION: Papua New Guinea: Mt. Strong, Owen Stanley Range, Tapini.

vegetatus Gressitt, 1966a: 264. (Gymnopholus (Symbiopholus))

Distribution: Papua New Guinea: Mt. Michael near Goroka.

zoarkes Gressitt, 1966a: 261. (Gymnopholus (Symbiopholus)) 
DistRIBUTION: Papua New Guinea: Murray Pass, Sibibamu near Woitape, Tapini.

PenthosCaPha Heller, 1914a: 646

TYPE-SPECIES: Penthoscapha lorentzi Heller, 1914a; monobasic.

lorentzi Heller, 1914a: 647. (Penthoscapha)

DisTRIBUTION: New Guinea: Hellwig Mountains.

RHINOSCAPHA Montrouzier, 1855: 47

TYPE-SPECIES: Rhinoscapha bicincta Montrouzier, 1855; monobasic. KEYS: Faust, 1897a: 163 (R. richteri-group); Faust, 1897a: 171; Heller, 1908a: 4; Heller, 1935: 173 (R. tricolor-group); Heller, 1937a: 262 (separates the three aberrations of $R$. doriae Pascoe).

albaria Faust, 1899: 8. (Rhinoscapha)

DisTRIBUTION: Papua New Guinea: Hula, Irupara, Kelesi.

aequata Heller, 1935: 170. (Rhinoscapha)

DISTRIBUTION: North Solomon Islands: Bougainville.

albertisi Pascoe, 1885: 204. (Rhinoscapha albertisii)

DisTRIBUTION: West Papua: Sorong.

albipennis albipennis Pascoe, 1885: 205. (Rhinoscapha)

DisTRIBUTION: Papua New Guinea: Yule Island.

albipennis cinnamomea Faust, 1897a: 157.

DistRIBUTION: Papua New Guinea: Dilo, Haveri, Ighibirei, Moroka.

alboguttata Heller, 1935: 169. (Rhinoscapha)

DISTRIBUTION: Papua New Guinea.

angustata Blanchard, 1853: 218. (Rhinoscapha)

DisTRIBUTION: North Solomon Islands. NOTES: Erroneously listed from Aru in the Coleopterorum Catalogus (Schenkling \& Marshall, 1931: 62); locality corrected in Heller, 1933b: 147.

aspersa Heller, 1913: 42. (Rhinoscapha)

DISTRIBUTION: New Guinea.

axillaris Faust, 1897a: 161. (Rhinoscapha)

DISTRIBUTION: Papua New Guinea: Mailu Island.

azureipes azureipes (Blanchard), 1853: 219. (Geonemus)

DISTRIBUTION: New Guinea; Aru Islands; Faor Island.

Geonemus zonata Blanchard, 1853: table 13 no.16.

Rhinoscapha alma Pascoe, 1874: 3. NOTES: Transferred: Marshall, 1952: 265.

azureipes chrysochlora Heller, 1935: 169. (Rhinoscapha)

DisTRIBUTION: West Papua: Korrido, Roon Island.

azureipes foveolata Heller, 1914a: 644. (Rhinoscapha)

DISTRIBUTION: West Papua: Lorentz River; Biak Island.

basilica Pascoe, 1874: 1. (Rhinoscapha)

DistRIBUTION: West Papua: Dorey.

beccarii Pascoe, 1885: 206. (Rhinoscapha)

DisTRIBUTION: West Papua: Ramoi.

bicincta Montrouzier, 1855: 48. (Rhinoscapha)

DISTRIBUTION: Woodlark Islands. 
bifasciata Chevrolat, 1881a: 494. (Rhinoscapha)

DistRIBUTION: Papua New Guinea: Fly River, Mailu, Najabui, (Nijenburg), Paumomu River.

Rhinoscapha cruenta Faust, 1897a: 157.

biundulata Heller, 1897b: 2. (Rhinoscapha)

DISTRIBUTION: New Guinea.

bucana Heller, 1935: 171. (Rhinoscapha)

DISTRIBUTION: North Solomon Islands: Bougainville, Buka.

Rhinoscapha bucana v. pulverulenta Heller, 1935: 171. DISTRIBUTION: North Solomon Islands: Bougainville.

canescens Faust, 1897a: 160. (Rhinoscapha)

DISTRIBUTION: D'Entrecasteaux Islands: Fergusson Island.

chlora Heller, 1913: 41. (Rhinoscapha)

DiSTRIBUTION: Louisiade Archipelago.

chloropunctata Heller, 1901: 9. (Rhinoscapha)

DistRIBUTION: Papua New Guinea: Astrolabe Mountains.

cincta Faust, 1897a: 156. (Rhinoscapha)

DISTRIBUTION: Isabel Island.

colbaltina Heller, 1914d: 307. (Rhinoscapha cobaltinata)

DistRIBUTION: Darnley Island, Torres Strait Islands. NoteS: Erroneously listed from New Guinea in the

Coleopterorum Catalogus (Schenkling \& Marshall, 1931); locality corrected by Heller in 1933b: 147.

Rhinoscapha darnleyensis Lea, 1917: 720. NOTES: Synonoymy: Marshall, 1952: 265.

consueta (Chevrolat), 1880b: 333. (Ladoice)

DISTRIBUTION: New Guinea.

Rhinoscapha consueta ab. imperfecta Faust, 1897a: 172. DisTRIBUTION: Papua New Guinea: Rigo.

Rhinoscapha consueta v. $x$-album Faust, 1897a: 172. Distribution: Papua New Guinea: Ighibirei, Port Moresby.

demissa Marshall, 1915: 509. (Rhinoscapha)

DISTRIBUTION: West Papua: Utakwa River.

diluta Heller, 1935: 172. (Rhinoscapha)

DistRIBUTION: Papua New Guinea: Finnesterre Mountains.

dolosa Heller, 1913: 35. (Rhinoscapha)

DISTRIBUTION: New Guinea.

doriae doriae Pascoe, 1885: 206. (Rhinoscapha)

DistRIBUTION: Papua New Guinea: Fly River, Kapakapa.

Rhinoscapha doriae ab. viridiaurea Heller, 1937a: 263.

doriae heurni Heller, 1937a: 261. (Rhinoscapha)

DistRIBUTION: West Papua: Idenburg River.

Rhinoscapha doriae heurni ab. bizonata Heller, 1937a: 262.

Rhinoscapha doriae heurni ab. dives Heller, 1937a: 263.

Rhinoscapha doriae heurni ab. reducta Heller, 1937a: 263.

egregia Heller, 1913: 36. (Rhinoscapha)

DISTRIBUTION: Bismarck Archipelago: New Britain.

eluta Heller, 1913: 37. (Rhinoscapha)

DISTRIBUTION: New Guinea.

evanida Faust, 1897a: 174. (Rhinoscapha)

DistRIBUTION: Papua New Guinea: Haveri.

fabricii (Thomson), 1857b: 443. (Geonemus)

DisTRIBUTION: Aru Islands; Kei Island. 
fausti Heller, 1897b: 3. (Rhinoscapha)

DISTRIBUTION: New Guinea.

fenestrata Heller, 1926a: 277. (Rhinoscapha)

DISTRIBUTION: New Guinea.

funebris (Chevrolat), 1880c: CIII. (Laodice)

DistrIBUTION: Papua New Guinea: Fly River, Port Moresby, Yule Island; Darnley Island.

gagatina Heller, 1913: 33. (Rhinoscapha)

DISTRIBUTION: New Guinea.

ganglbaueri Heller, 1908a: 3. (Rhinoscapha)

DISTRIBUTION: New Guinea.

gebehiana Heller, 1913: 39. (Rhinoscapha)

DISTRIBUTION: Gebeh Island.

generosa Pascoe, 1885: 204. (Rhinoscapha)

DistRIBUTION: West Papua: Dorey.

gestroi Faust, 1899: 9. (Rhinoscapha)

DisTRIBUTION: Papua New Guinea: Dilo, Ighibirei.

hasterti Heller, 1935: 171. (Rhinoscapha)

DISTRIBUTION: North Solomon Islands: Bougainville, Kieta.

humboldtiana Heller, 1908a: 4. (Rhinoscapha)

DistRIBUTION: West Papua: Humboldt Bay.

immaculata Faust, 1897a: 176. (Rhinoscapha)

DisTRIBUTION: Papua New Guinea: Haveri.

impexa Heller, 1901: 10. (Rhinoscapha)

DistRIBUTION: Papua New Guinea: Gulf region.

integrirostris Heller, 1925c: 282. (Rhinoscapha)

DISTRIBUTION: New Guinea.

jordani Faust, 1897a: 175. (Rhinoscapha)

DisTRIBUTION: Papua New Guinea: Haveri.

laevior Faust, 1897a: 173. (Rhinoscapha)

Distribution: Papua New Guinea: Astrolabe Mountains, Dilo.

lamasonga Heller, 1913: 38. (Rhinoscapha)

DisTRIBUTION: Bismarck Archipelago: New Britain.

lameerei Heller, 1905a: 68. (Rhinoscapha)

DisTRIBUTION: Papua New Guinea: Sattelberg.

leochroma Heller, 1935: 172. (Rhinoscapha)

DISTRIBUTION: Admiralty Islands: Manus Island.

litoralis Faust, 1900: 625. (Rhinoscapha)

DISTRIBUTION: New Guinea.

loriai Faust, 1899: 10. (Rhinoscapha)

DisTRIBUTION: Papua New Guinea: Rigo.

maclayi Macleay, 1885: 705. (Rhinoscapha)

DisTRIBUTION: Papua New Guinea: Madang.

margaritifera Faust, 1897a: 164. (Rhinoscapha)

Distribution: Papua New Guinea: Moroka.

miliaris Pascoe, 1874: 5. (Rhinoscapha)

DistRIBUTION: West Papua: Mysol Island.

nitidifrons Heller, 1913: 34. (Rhinoscapha) 
DISTRIBUTION: New Guinea.

obsidiana Heller, 1913: 32. (Rhinoscapha)

DISTRIBUTION: New Guinea.

opalescens Pascoe, 1874: 3. (Rhinoscapha)

DISTRIBUTION: West Papua: Waigeo Island, Mysol, Dorey.

pauperula Heller, 1913: 40. (Rhinoscapha)

DisTRIBUTION: New Guinea.

perfecta Faust, 1900: 624. (Rhinoscapha)

DisTRIBUTION: New Guinea.

perversa Pascoe, 1885: 205. (Rhinoscapha)

DisTRIBUTION: West Papua: Dorey.

plagiata Blanchard, 1853: 216. (Rhinoscapha)

DisTRIBUTION: North Solomon Islands. Erroneously listed from Aru in the Coleopterorum Catalogus

(Schenkling and Marshall, 1931: 62); locality corrected in Heller, 1933b: 147.

plicata Faust, 1897a: 166. (Rhinoscapha)

DisTRIBUTION: Papua New Guinea: Moroka.

ribbei Heller, 1935: 171. (Rhinoscapha)

DISTRIBUTION: North Solomon Islands: Shortland Island.

richteri Faust, 1891: 176. (Rhinoscapha)

DISTRIBUTION: New Guinea.

rothschildi Faust, 1897a: 176. (Rhinoscapha)

DisTRIBUTION: New Guinea.

Rhinoscapha rothschildi ab. rufithorax Faust, 1897a: 178.

scalaris Pascoe, 1885: 205. (Rhinoscapha)

DisTRIBUTION: New Guinea; Halmahera.

Rhinoscapha heydeni Faust, 1895a: 93. NOTES: Synonymy: Heller, 1933b: 147.

schmeltzi schmeltzi Fairmaire, 1877: 185. (Rhinoscapha)

DisTRIBUTION: Bismarck Archipelago: Duke of York Island, New Britain.

schmeltzi hedigeri Heller, 1935: 168. (Rhinoscapha)

DistRIBUTION: Papua New Guinea: Umboi Island.

schmeltzi intermedia Heller, 1935: 168. (Rhinoscapha)

DistRIBUTION: Papua New Guinea: Stephansort; Bismarck Archipelago: New Britain.

schmeltzi papei Heller, 1913: 37. (Rhinoscapha)

DisTRIBUTION: Bismarck Archipelago: New Britain.

sellata Pascoe, 1874: 4. (Rhinoscapha)

DisTRIBUTION: New Guinea: Faor Island.

simplex Heller, 1935: 170. (Rhinoscapha)

DistRIBUTION: Papua New Guinea: Astrolabe Bay, Stephansort.

sordida Blanchard, 1853: 217. (Rhinoscapha)

DisTRIBUTION: Aru Islands.

staintoni Pascoe, 1874: 2. (Rhinoscapha)

DisTRIBUTION: West Papua: Dorey, Sorong, Saylee.

staudingeri Faust, 1897a: 169. (Rhinoscapha)

DistRIBUTION: Papua New Guinea: Milne Bay; Fergusson Island.

Rhinoscapha staudingeri v. sanguinicollis Faust, 1897a: 169.

stolifera Pascoe, 1874: 4. (Rhinoscapha)

DISTRIBUTION: Waigeo Island. 
striatopunctata (Guérin-Méneville), 1830: 113. (Geonemus striato-punctata)

DistRIBUTION: West Papua: Andai, Dorey, Rubi.

stridulatoria Heller, 1913: 40. (Rhinoscapha)

DISTRIBUTION: Gebeh Island.

subhumeralis Heller, 1937a: 264. (Rhinoscapha)

DISTRIBUTION: West Papua: Mamberamo River.

thomsoni Waterhouse, 1889: 364. (Rhinoscapha)

DISTRIBUTION: Louisiade Archipelago: Goodenough Island; Trobriand Islands.

Rinoscapha dubia Faust, 1897a: 162. NOTES: Synonymized in Heller, 1912b: 364; treated as a seperate species in the Coleopterorum Catalogus (Schenkling and Marshall, 1931) and synonymized again in Heller, 1933b: 147 and Marshall, 1952: 265.

tricolor Faust, 1891: 175. (Rhinoscapha)

DISTRIBUTION: New Guinea.

tuberculata Faust, 1897a: 160. (Rhinoscapha)

Distribution: Papua New Guinea: Paumomu River.

usta Faust, 1897a: 158. (Rhinoscapha)

DisTRIBUTION: Papua New Guinea: Moroka.

vibrans Heller, 1914a: 644. (Rhinoscapha)

DisTRIBUTION: New Guinea.

viridans Heller, 1937a: 263. (Rhinoscapha)

DisTRIBUTION: West Papua: Idenburg River.

viridula Kirsch, 1877: 147. (Rhinoscapha)

DistRIBUTION: West Papua: Jobi, Yapen Island, Geelvink Bay.

vollenhoveni Heller, 1913: 31. (Rhinoscapha)

DISTRIBUTION: Roon Island.

vormanni Heller, 1914d: 307. (Rhinoscapha)

Distribution: Papua New Guinea: Manumbo, Madang.

Rhinoscapha primitiva Heller, 1914d: 305.

RHINOSCAPHA (NIPHETOSCAPHA) Heller, 1914a: 645

TYPE-SPECIES: Rhinoscapha wichmanni Heller, 1914a; monobasic.

wichmanni Heller, 1914a: 645. (Rhinoscapha (Niphetoscapha))

DisTRIBUTION: West Papua: Hellwig Mountain.

\section{NAUPACTINI}

EXOPHTHALMIDA Faust, 1892b: 187

TYPE-SPECIES: Exophthalmida glauca Faust, 1892b; monobasic. KeY: Marshall, 1941a: 51.

glauca Faust, 1892b: 188. (Exophthalmida)

DISTRIBUTION: Admiralty Islands; Bismarck Archipelago: New Britain; Solomon Islands.

laticollis Heller, 1915: 513. (Exophthalmida)

Distribution: Papua New Guinea. 
namatanai Marshall, 1941a: 53. (Exophthalmida)

DISTRIBUTION: Bismarck Archipelago: Namatana, New Ireland.

planirostris Heller, 1910b: 180. (Exophthalmida)

DISTRIBUTION: North Solomon Islands: Bougainville.

Parexophthalmus Heller, 1908a: 2

TYPE-SPECIES: Parexophthalmus semiotus Heller, 1908a; original designation.

semiotus Heller, 1908a: 2. (Parexophthalmus)

DisTRIBUTION: Bismarck Archipelago: New Britain.

\section{OTTISTIRINI}

Atrotitis Heller, 1925a: 58

TYPE-SPECIES: Atrotitis albopicta Heller, 1925a; monobasic.

albopicta Heller, 1925a: 87. (Atrotitis)

DisTRIBUTION: Papua New Guinea: Torricelli Mountains.

EUPHOLOCIS Lea, 1930: 464

TYPE-SPECIES: Eupholocis dentipes Lea, 1930; original designation.

maculatus Lea, 1930: 465. (Eupholocis)

DISTRIBUTION: Bismarck Archipelago: New Britain.

EUTINOPHAEA Pascoe, 1870a: 181

TYPE-SPECIES: Eutinophaea nana Pascoe, 1870a; monobasic. KeY: Heller, 1925a: 82.

flavovirens Heller, 1925a: 84. (Eutinophaea)

DiSTRIBUTION: New Guinea.

laeta (Faust), 1899: 31. (Ottistira)

DistribUtion: Papua New Guinea: Paumomu River.

papuensis Lea, 1930: 462. (Eutinophaea)

DISTRIBUTION:

viridis (Faust), 1899: 30. (Ottistira)

Distribution: Papua New Guinea: Paumomu River.

ITTOSTIRA Heller, 1925a: 70

TYPE-SPECIES: Ittostira elongatus Heller, 1925a: 73; present designation. KeY: Heller, $1925 \mathrm{a}: 70$.

moluccana Heller, 1925a: 81. (Ittostira)

Distribution: West Papua: Mysol Island.

nasalis Heller, 1925a: 74. (Ittostira)

DistRIBUTION: West Papua: Geelvink Bay, Roon Island; Aru Island. 
papuana Heller, 1925a: 75. (Ittostira)

DisTRIBUTION: New Guinea.

OTTISTIRA Pascoe, 1872d: 440

TYPE-SPECIES: Ottistira bispinosa Pascoe, 1872d; subsequent designation by Heller, 1925a: 55. KeYs: Heller, 1925a: 63;

Riedel \& O'Brien, 1995: 250 (O. longispina-species group).

arfakensis Riedel, 1995: 253. (Ottistira)

DistRIBUTION: West Papua: Anggi, Arfak Mountains.

ascendens Riedel, 1995: 257. (Ottistira)

Distribution: West Papua: Diuremna, Eipomek-Langda in Oranje Mountains, Mt. Mandala, Yem Yem

Valley.

bicornis Pascoe, 1872d: 441. (Ottistira)

DistRIBUTION: West Papua: Ramoi.

bispinosa Pascoe, 1872d: 440. (Ottistira)

DistRIBUTION: West Papua: Dorey, Ramoi; Aru Island; Mysol Island; Waigeo Island.

dani Riedel, 1995: 251. (Ottistira)

DisTRIBUTION: West Papua: Jiwika, Kiroma, Wamena.

fasciata Macleay, 1887: 184. (Ottistira)

DisTRIBUTION: Papua New Guinea: Fly River.

gibbicollis Heller, 1925a: 66. (Ottistira)

DisTRIBUTION: West Papua: Andai.

iniquicollis Heller, 1925a: 67. (Ottistira)

DisTRIBUTION: West Papua: Mysol Island.

irrorata Pascoe, 1885: 202. (Ottistira)

DisTRIBUTION: Salawatti Island.

longispina Riedel, 1995: 267. (Ottistira)

Distribution: West Papua: Diuremna, Nalca, Pass Valley, Wamena, Wanyok.

mixta Pascoe, 1885: 203. (Ottistira)

DISTRIBUTION: Aru Islands.

obrienorum Riedel, 1995: 273. (Ottistira)

Distribution: West Papua: Bommela, Mt. Goliath.

paniaiensis Riedel, 1995: 271. (Ottistira)

DistRIBUTION: West Papua: Enarotadi, Itouda, Nabire, Okaitadi, Paniai Lakes.

planiuscula Heller, 1925a: 67. (Ottistira)

DisTRIBUTION: Papua New Guinea: Torricelli Mountains.

robusta Riedel, 1995: 255. (Ottistira)

Distribution: West Papua: Borme, Emdoman, Kosarek, Membahan, Wamena.

stiracromia Heller, 1908a: 12. (Ottistira)

DistRIBUTION: West Papua.

sulcicollis Faust, 1899: 33. (Ottistira)

DistRIBUTION: Papua New Guinea: Moroka, Paumomu River.

telefominensis Riedel, 1995: 263. (Ottistira)

Distribution: Papua New Guinea: Telefomin.

tumidirostris Riedel, 1995: 265. (Ottistira)

Distribution: Papua New Guinea: Aiyurop and Anga Gorge near Mendi. 
TistorTiA Heller, 1925a: 57

TYPE-SPECIES: Tistortia aroana Heller, 1925a; monobasic.

aroana Heller, 1925a: 61. (Tistortia)

DISTRIBUTION: Papua New Guinea.

TistortiElla Heller, 1925a: 57

TYPE-SPECIES: Ottistira nitida Faust 1899; monobasic.

nitida (Faust), 1899: 32. (Ottistira)

DistRIBUtion: Papua New Guinea: Moroka, Paumomu River.

\section{PACHYRHYNCHINI}

Pantorhytes Faust, 1892b: 193

TYPE-SPECIES: Pachyrhynchus chrysomelas Mountrouzier, 1855 [= P. stanleyanus (White), 1852: 388]; original designation. KeYs: Heller, 1901: 14; Heller, 1905b: 305; Heller, 1935: 160; Voss, 1956: 126 (P. quadripustulatus-group); Gressitt, 1966b: 920; Stibick, 1978: 116 (Division A); Stibick, 1979: 104 (Division B).

admiralis Stibick, 1979: 105. (Pantorhytes)

DistRIBUTION: Admiralty Islands: Manus.

albopunctulatus Heller, 1901: 13. (Pantorhytes)

DisTRIBUTION: Papua New Guinea: Aroa River.

algifer Gressitt, 1966b: 957. (Pantorhytes)

Distribution: Papua New Guinea: Mt. Guilwe, Mt. Hagen, Webag.

araneus Gressitt, 1966b: 944. (Pantorhytes)

DisTRIBUTION: Papua New Guinea: Eastern Highlands, Okapa.

arcuatus Gressitt, 1966b: 948. (Pantorhytes)

Distribution: Papua New Guinea: Liyi Valley and Werr Valley near Menyamya.

bakeri Stibick, 1978: 129. (Pantorhytes)

DisTRIBUTION: D'Entrecasteaux Islands: Goodenough Island.

batesi Faust, 1892b: 194. (Pantorhytes)

Distribution: Papua New Guinea: Bubia, Finschhafen, Huon Gulf, Lae, Wau.

biakensis Gressitt, 1966b: 938. (Pantorhytes)

Distribution: West Papua: Biak Island.

biplagiatus (Guérin-Méneville), 1841c: 216. (Pachyrhynchus)

DISTRIBUTION: North Solomon Islands: Bougainville.

Pantorhytes salomonis Heller, 1903: 13.

biroi Voss, 1956: 125. (Pantorhytes)

DistribUtion: Papua New Guinea: Madang, Manumbo, Sattelburg.

bourkei Stibick, 1978: 127. (Pantorhytes)

Distribution: Papua New Guinea: Garaina.

brandti Gressitt, 1966b: 937. (Pantorhytes)

DistRIBUTION: Papua New Guinea: Fly River, Kiunga.

carbonarius Heller, 1926a: 276. (Pantorhytes) 
DisTRIBUTION: Papua New Guinea.

chimbuensis Gressitt, 1966b: 960. (Pantorhytes)

Distribution: Papua New Guinea: Chimbu Valley, Daulo Pass, Mt. Wilhelm.

corallifer Heller, 1901: 12. (Pantorhytes)

DistRIBUTION: Papua New Guinea: Aroa River.

cyclopei Stibick, 1979: 107. (Pantorhytes)

Distribution: West Papua: Cyclops Mountains.

decempustulatus (Gestro), 1879: 562. (Pachyrrhynchus)

Distribution: Papua New Guinea: Astrolabe Range, Fly River, Haveri, Kiunga, Moroka.

decemverrucosus Heller, 1935: 159. (Pantorhytes)

Distribution: West Papua.

fenneri Stibick, 1978: 124. (Pantorhytes)

Distribution: Papua New Guinea: Mt. Suckling.

fictabidi Stibick, 1979: 109. (Pantorhytes)

DisTRIBUTION: Papua New Guinea: Mt. Bosavi.

fictacarbonarius Stibick, 1979: 110. (Pantorhytes)

DisTRIBUTION: West Papua: Humblodt Bay; Yapen Island.

granulatus Heller, 1905b: 307. (Pantorhytes)

DISTRIBUTION: Woodlark Islands. NOTES: Gressitt 1966b provisionally placed this species as a synomym

of $P$. stanleyanus (White).

goilalae Gressitt, 1966b: 952. (Pantorhytes)

Distribution: Papua New Guinea: Goilala, Owen Stanley Mountains.

gracilis Gressitt, 1966b: 944. (Pantorhytes)

DisTRIBUTION: West Papua: Baliem Valley, Wamena.

gravis Heller, 1914a: 647. (Pantorhytes)

DistRibution: West Papua: Lorenz River, Mimika River, Utakawa River; Biak Island.

hagenensis Gressitt, 1966b: 963. (Pantorhytes)

Distribution: Papua New Guinea: Mt. Hagen.

healyi Gressitt, 1966b: 928. (Pantorhytes)

Distribution: Papua New Guinea: Biagi Valley near Kokoda.

hornabrooki Gressitt, 1966b: 961. (Pantorhytes)

DistRIBUTION: Papua New Guinea: Eastern Highlands, Mt. Michael.

horni Stibick, 1978: 120. (Pantorhytes)

DistRIBUTION: Papua New Guinea: Poho.

huonaris Stibick, 1979: 112. (Pantorhytes)

Distribution: Papua New Guinea: Finischhafen, Toricelli Mountains, Wareo.

interruptus Gressitt, 1966b: 951. (Pantorhytes)

Distribution: Papua New Guinea: Finisterre Range, Huon Gulf, Saidor.

irregularis Gressitt, 1966b: 946. (Pantorhytes)

DisTRIBUTION: West Papua: Bomberi near Fakfak.

jimmiensis Gressitt, 1966b: 948. (Pantorhytes)

Distribution: Papua New Guinea: Upper Jimmi Valey.

lamingtona Gressitt, 1966b: 929. (Pantorhytes)

Distribution: Papua New Guinea: Mt. Lamington.

lichenifer Gressitt, 1966b: 956. (Pantorhytes)

DisTRIBUTION: Papua New Guinea.

maai Gressitt, 1966b: 956. (Pantorhytes) 
DistRIBUTION: Papua New Guinea: Eastern Highlands, Okapa.

maccawi Stibick, 1979: 115. (Pantorhytes)

DistRIBUTION: West Papua: Hoofdbivak, Kobokma, Zanona.

manni Gressitt, 1966b: 947. (Pantorhytes)

DistRibution: Papua New Guinea: Murua near Kerema.

melanostictus Heller, 1935: 157. (Pantorhytes batesi melanostictus)

DistRIBUTION: Papua New Guinea: Sattelberg. NOTES: Status changed to full species in Stibik, 1978: 135.

minor Gressitt, 1966b: 942. (Pantorhytes)

DistRIBUTION: Papua New Guinea: Eastern Highlands, Karimui near Goroka.

montanus Gressitt, 1966b: 941. (Pantorhytes)

DistRIBUTION: Papua New Guinea: Eastern Highlands, Karanka.

multipustulosus Heller, 1935: 157. (Pantorhytes)

Distribution: Papua New Guinea: Finnesterre Mountains.

obliquus Gressitt, 1966b: 949. (Pantorhytes)

DistRIBUTION: Papua New Guinea: Eastern Highlands, Karimui near Goroka, Okapa.

octopustulatus Heller, 1935: 158. (Pantorhytes)

DistRIBUTION: Papua New Guinea: Finnesterre Mountains.

opacus Faust, 1899: 29. (Pantorhytes)

Distribution: Papua New Guinea: Astrolabe Mountains, Haveri, Musgrave Range near Pt. Moresby.

pallidus Gressitt, 1966b: 943. (Pantorhytes)

DisTRIBUTION: West Papua: Star Mountains.

papillosus Heller, 1901: 11. (Pantorhytes)

Distribution: Papua New Guinea: Aroa River.

papuanus Gestro, 1923: 63. (Pantorhytes)

DistRIBUTION: Papua New Guinea: Purari River.

pilosus Heller, 1935: 159. (Pantorhytes)

Distribution: Papua New Guinea: Bulldog Road, Eddie Creek, Huon Gulf, Mt. Kaindi.

plutus (Oberthür), 1883: XXV. (Pachyrrhynchus)

DisTRIBUTION: Bismarck Archipelago: Duke of York Island, New Britain, New Ireland.

Pachyrrhynchus biplagiatus Bates, 1877: 154.

polynodus Gressitt, 1966b: 953. (Pantorhytes)

Distribution: Papua New Guinea: Finisterre Range, Huon Gulf, Saidor.

proximus Faust, 1899: 29. (Pantorhytes)

Distribution: Papua New Guinea: Moroka, Paumomu River. NotEs: Heller, 1912b: 364 placed this spe-

cies as a synonym of P. stanleyanus (White); Returned to full species status: Gressitt, 1966b: 931.

pseudoabidi Stibick, 1979: 119. (Pantorhytes)

Distribution: West Papua: Star Mountains.

pseudocarbonaris Stibick, 1979: 121. (Pantorhytes)

DistRIBUTION: Papua New Guinea: Aitape, Angoram, Sepik River.

quadripustulatus (Gestro), 1875: 1008. (Pachyrhynchus IV-pustulatus)

Distribution: Papua New Guinea; Bismarck Archipelago: Duke of York Island, New Britain (in error);

West Papua: Geelvink Bay, Korido; Misori Islands.

quatei Stibick, 1979: 123. (Pantorhytes)

DisTRIBUTION: West Papua: Star Mountains.

rarus Heller, 1901: 12. (Pantorhytes)

DistRIBUTION: Papua New Guinea: Papuan Gulf.

rubroverucatus (Tryon), 1890: 72. (Pachyrhynchus) 
Distribution: Papua New Guinea: Paumomu River, St. Joseph River, Yule Island.

rufescens Gressitt, 1966b: 940. (Pantorhytes)

DisTRIBUTION: Papua New Guinea: Upper Jimmi Valley.

rugosus Gressitt, 1966b: 959. (Pantorhytes)

DistribUtion: Papua New Guinea: Mt. Giluwe.

samuelsoni Gressitt, 1966b: 958. (Pantorhytes)

DisTRIBUTION: Papua New Guinea: Huon Peninsula.

sedlaceki Gressitt, 1966b: 927. (Pantorhytes)

Distribution: Papua New Guinea: Iongai near Mt. Albert Edward, Owen Stanley Range.

sexpustulatus Heller, 1912a: 309. (Pantorhytes VI-pustulatus)

DistRIBUTION: Papua New Guinea: Finisterre Mountains, Kani Mountains.

squamistriatus Gressitt, 1966b: 954. (Pantorhytes)

DisTRIBUTION: Papua New Guinea: Eastern Highlands, Lifu.

stanleyanus (White), 1852: 388. (Pachyrhynchus)

Distribution: Papua New Guiena: Fly River, Yule Island; D’Entrecasteaux Islands: Normanby Island;

Louisiade Archipelago: Misima Island, Sudest Island; Trobriand Islands; Woodlark Island.

Pantorhytes chrysomelas (Montrouzier), 1855: 46. (Pachyrhynchus) NoTES: Synonymy: Stibick, 1978: 134.

Pantorhytes australasiae Boheman, 1859: 119. (Pachyrhynchus) Notes: Synonymy: Gressitt, 1966b: 931.

Pantorhytes subcostatus Heller, 1905b: 308. Notes: Synonymy: Stibick, 1978: 134.

Pantorhytes fraudus Janczyk, 1959a: 434. Distribution: Papua New Guinea: Yule Island. Notes: Synonymy: Stibick, 1978: 134.

swartus Stibick, 1979: 125. (Pantorhytes)

DisTRIBUTION: West Papua: Geelvink Bay, Swart Valley.

szentivanyi Marshall, 1957: 6. (Pantorhytes)

Distribution: Papua New Guinea: Popondetta.

telefominarius Stibick, 1979: 128. (Pantorhytes)

Distribution: Papua New Guinea: Telefomin.

torricellianus Heller, 1935: 159. (Pantorhytes)

Distribution: Papua New Guinea: Torricelli Mountains.

truncatus Gressitt, 1966b: 962. (Pantorhytes)

Distribution: Papua New Guinea: Eastern Highlands, Okapa.

varinodis Gressitt, 1966b: 958. (Pantorhytes)

Distribution: Papua New Guinea: Asaro Valley, Daulo Pass, Eastern Highlands, Mt. Wilhelm.

verrucatus (Bates), 1877: 154. (Pachyrrhynchus)

DisTRIBUTION: Bismarck Archipelago: New Britain, New Ireland. Notes: Macleay, 1887: 183 considered

this species a synonym of P. quadripustulatus Gestro. Rank changed to subspecies of P. quadripustulatus

Gestro in Heller, 1905b: 310 and full species was status resumed in Gressitt, 1966 b: 936.

vibicifer Heller, 1901: 13. (Pantorhytes)

DistRIBUtion: Papua New Guinea: Aroa River, Goilala, Owen Stanley Mountains.

waghianus Gressitt, 1966b: 961. (Pantorhytes)

DistRIBUtion: Papua New Guinea: Waghi Valley.

SPHENOMORPHA Behrens, 1887: 222

TYPE-SPECIES: Sphenomorpha suturalis Behrens, 1887; present designation. KEYS: Behrens, 1887: 224; Heller, 1908a: 11.

aenea (Fabricius), 1801: 508. (Pachyrrhynchus) 
DISTRIBUTION:

atra Behrens, 1887: 226. (Sphenomorpha)

DiSTRIBUTION: Waigeo Island, Gebeh Island.

dohertyi Heller, 1908a: 12. (Sphenomorpha)

DistRIBUTION: West Papua: Andai, Hattam.

froggatti (Macleay), 1887: 183. (Apocyrtus)

DisTRIBUTION: Papua New Guinea: Fly River.

impressa (Chevrolat), 1879: 133. (Apocyrtus impressus)

DistRIBUTION: West Papua: Dorey.

nitidula (Pascoe), 1871c: 157. (Apocyrtus nitidulus)

DistriBution: West Papua: Waigeo Island.

pulchra Behrens, 1887: 231. (Sphenomorpha)

DistRibution: West Papua: Dorey.

roelofsi (Ancey), 1880: 205. (Apocyrtus)

DistRibution: West Papua: Hatam.

rufipes Marshall, 1935: 43. (Sphenomorpha)

Distribution: New Guinea: Angi Gita.

spectabilis Hartmann, 1903: 24. (Sphenomorpha)

DisTRIBUTION: New Guinea.

suturalis Behrens, 1887: 224. (Sphenomorpha)

DISTRIBUTION: New Guinea.

viridis (Chevrolat), 1879: 134. (Apocyrtus)

DisTRIBUTION: West Papua: Dorey.

wallacei fasciolata Heller, 1908a: 11. (Sphenomorpha)

DisTRIBUTION: New Guinea.

\section{TANYMECINI}

\section{PIAZOMIINA}

HYPOMECES Schönherr, 1823: 1141

TYPE-SPECIES: Curculio squamosus Fabricius, 1792 [=Curculio pulviger Herbst, 1795]; original designation.

inflatus Chevrolat, 1877: 189. (Hypomeces)

DisTRIBUTION: New Guinea.

\section{TROPIPHORINI}

Peripagis Pascoe, 1870a: 187

TYPE-SPECIES: Periagis rufipes Pascoe, 1870a; monobasic.

limbatus Pascoe, 1871d: 94. (Catastygnus)

DISTRIBUTION: Bismarck Archipelago: Duke of York Island. 


\section{HYPERINAE}

\section{HYPERINI}

LAMPROHYPERA Heller, 1908a: 15

TYPE-SPECIES: Lamprohypera colbaltina Heller, 1908a; monobasic.

colbaltina Heller, 1908a: 15. (Lamprohypera)

DiSTRIBUTION: Bismarck Archipelago: Duke of York Island; New Ireland.

\section{CEPURINI}

SAgINESIS Pascoe, 1872d: 452

TYPE-SPECIES: Saginesis latipennis Pascoe, 1872d; monobasic.

latipennis Pascoe, 1872d: 452. (Saginesis)

DISTRIBUTION: Aru Islands.

\section{LIXINAE}

\section{LIXINI}

GASTEROCLISUS Desbrochers des Loges, 1904: 103

TYPE-SPECIES: Lixus augurius Boheman in Schönherr, 1836; monobasic. NOTES: This genus and Hypolixus Desbrochers des Loges are treated as synonyms in the Coleoterorum Catalogus (Ciski, 1934: 94). They are treated as separate in Alonso-Zarazaga \& Lyal, 1999, however the proper allocation of the species within these two genera remains unclear. KEY: Petri, 1912: 343.

duponti (Schönherr), 1842: 478. (Lixus)

DISTRIBUTION: New Guinea.

Lixus farinosus Boisduval, 1835: 406.

papilifer Petri, 1912: 367. (Gasteroclisus)

DisTRIBUTION: New Guinea.

HyPOLIXUS Desbrochers des Loges, 1898: 54

TYPE-SPECIES: Lixus nubilosus Boheman in Schönherr, 1836; original designation. NoTES: See discussion with Gasteroclisus above.

australis (Boisduval), 1835: 404. (Lixus)

DisTRIBUTION: New Guinea.

ritsemae (Pascoe), 1883: 87. (Lixus)

Distribution: Papua New Guinea: Port Moresby, Rigo; West Papua: Andai.

Hypolixus ritsemae v. coriaceus (Petri), 1914: 22. (Paralixis ritsemae v. coriaceus) DISTRIBUTION: New Guinea.

truncatulus (Fabricius), 1798: 167. (Lixus)

DisTRIBUTION: New Guinea. 
LIXUS Fabricius, 1801: 498

TYPE-SPECIES: Curculio paraplecticus Linnaeus, 1758; subsequent designation by Latreille, 1810: 430.

binodulus Boheman in Schönherr, 1835: 52. (Lixus)

DisTRIBUTION: Papua New Guinea: Haveri, Rigo.

bonguensis Hartmann, 1901: 273. (Lixus)

DisTRIBUTION: Papua New Guinea: Bongu.

conformatus Csiki, 1934b: 130. (Lixus conformis)

Distribution: West Papua: Hatam. NOTES: Replacement name for Lixus conformis Pascoe 1885: 223, non Capiomont.

confusus Faust, 1896: 144. (Lixus)

DisTRIBUTION: Aru Island. NOTES: Range extension: Heyden, 1911: 361.

mimicanus Marshall, 1915: 511. (Lixus)

Distribution: New Guinea: Mimika River, Sakoemi, Siwi.

monticola Kirsch, 1877: 152. (Lixus)

Distribution: Papua New Guinea: Astrolabe Mountains, Haveri, Paumomu River, Port Moresby.

\section{MESOPTILIINAE}

\section{LAEMOSACCINI}

NEOLAEMOSACCUS Hustache, 1937: 201

TYPE-SPECIES: Laemosaccus subsignatus Boheman in Schönherr, 1844; original designation.

australis (Boisduval), 1835: 426. (Lamosaccus)

DisTRIBUTION: New Guinea; [Tasmania in error.]

insularis (Pascoe), 1885: 238. (Lamosaccus)

DistRIBUTION: Papua New Guinea: Yule Island.

longiceps (Pascoe), 1873a: 281. (Lamosaccus)

Distribution: Papua New Guinea: Fly River, Rigo; Aru Island.

petulans (Pascoe), 1885: 238. (Lamosaccus)

Distribution: Papua New Guinea: Fly River, Paumomu River; D’Entrecasteaux Islands: Goodenough

Island.

\section{MOLYTINAE}

\section{AMINYOPINI}

NIPHADES Pascoe, 1871c: 174

TYPE-SPECIES: Niphades paradalotus Pascoe, 1871c; subsequent designation by Voss, 1958a: 47.

costatus Pascoe, 1871c: 174. (Niphades)

DISTRIBUTION: Aru Islands. 
interruptus Faust, 1899: 34. (Niphades)

Distribution: Papua New Guinea: Moroka.

\section{CLEOGONINI}

Pantoxystus Pascoe, 1881: 600

TYPE-SPECIES: Cleogonus rubricollis Boisduval, 1835; original designation.

=Neplaxa Casey, 1922: 163 syn. $\mathbf{n}$.

TYPE-SPECIES: Neplaxa illustris Casey, 1922; original designation.

cyaneus Macleay, 1887: 189. (Pantoxystus)

DistRIBUTION: Papua New Guinea: Astrolabe Bay, Fly River, Haveri.

rubricollis (Boisduval), 1835: 442. (Cleogonus)

Distribution: Papua New Guinea: Fly River, Paumomu River; West Papua: Dorey, Sakoemi.

Neplaxa illustris Casey, 1922: 163. syn. n. Notes: Casey described this species as a Brazilian Baridinae;

Alonso-Zarazaga \& Lyal, 1999: 99 reported the correct locality as New Guinea.

rubripennis (Chevrolat), 1881b: LXIX. (Cleogonus)

Distribution: Papua New Guinea: Fly River.

schneideri (Heller), 1941a: 218. (Pseudeurhinus)

DISTRIBUTION: Bismarck Archipelago: New Britain, Gazelle Peninsula.

\section{HYLOBIINI}

\section{HYLOBIINA}

ACLEES Schönherr, 1835: 238

TYPE-SPECIES: Aclees cribratus Gyllenhal in Schönherr, 1835; original designation. Species diagnosis: Marshall, 1915: 513.

cribratus Gyllenhal in Schönherr, 1835: 239. (Aclees)

Distribution: Papua New Guinea: Haveri, Moroka, Paumomu River.

granulosus Faust, 1899: 36. (Aclees)

Distribution: Papua New Guinea: Paumomu River.

gyllenhali Pascoe, 1871c: 172. (Aclees gyllenhallii)

DistRIBUTION: West Papua: Waigeo Island.

indignus Marshall, 1915: 512. (Aclees)

Distribution: West Papua: Mimika River.

porosus Pascoe, 1871c: 172. (Aclees)

Distribution: Papua New Guinea: Ighibirei, Paumomu River; West Papua: Dorey, Saylee, Kaiou, Bouru;

Aru Island.

HeSYCHOBIUS Marshall, 1931: 263

TYPE-SPECIES: Hesychobius nebulosus Marshall, 1931; original designation. 
nebulosus Marshall, 1931: 264. (Hesychobius)

DistribUtion: Papua New Guinea: Houn Gulf.

PseudaClees Faust, 1892a: 50

TYPE-SPECIES: Hylobius fasciatus Pascoe, 1871c; monobasic.

acalloides (Pascoe), 1885: 224. (Hylobius)

DistRIBUTION: Papua New Guinea: Fly River, Moroka.

crassirostris (Pascoe), 1885: 225. (Hylobius)

DistRIBUTION: Papua New Guinea: Haveri.

\section{ITHYPORINI}

\section{ITHYPORINA}

Diomia Pascoe, 1885: 285

TYPE-SPECIES: Diomia tetragramma Pascoe, 1885; monobasic.

tetragramma Pascoe, 1885: 285. (Diomia)

DistRIBUTION: Papua New Guinea: Fly River.

ECTATORHINUS Lacordaire, 1866: 53

TYPE-SPECIES: Ectatorhinus wallacei Lacordaire, 1866; monobasic.

magicus (Gerstaecker), 1860: 389. (Colobodes)

DistRIBUTION: Papua New Guinea; Bismarck Archipelago: New Britain, Duke of York Island.

Ectatorhinus godeffroyi Fairmaire, 1881c: 389.

trinarius (Pascoe), 1882b: 453. (Aryptacus)

DisTRIBUTION: West Papua: Dorey

Ithyporus nanus Heller, 1908b: 176.

\section{COLOBODINA}

APRIES Pascoe, 1871c: 196

TYPE-SPECIES: Apries eremita Pascoe, 1871c; subsequent designation by Heller, 1922b: 559.

palliatus Pascoe, 1871c: 196. (Apries)

Distribution: West Papua: Saylee. Notes: Returned to Apries from Deretiosus in Zimmerman, 1941: 195.

eremita Pascoe, 1871c: 196. (Apries)

DistRIBUtIOn: New Guinea. Notes: Returned to Apries from Deretiosus in Zimmerman, 1941: 194.

COLOBODES Schönherr, 1837: 465

TYPE-SPECIES: Colobodes billbergii Boheman in Schönherr, 1837; original designation.

cavisquamus Marshall, 1915: 517. (Colobodes) 
DistRIBUTION: West Papua: Mimika River.

longirostris Faust, 1898a: 141. (Colobodes)

DistRIBUTION: Papua New Guinea: Ighibirei, Mailu Island.

DERETIODES Marshall, 1931: 292

TYPE-SPECIES: Deretiodes swezeyi Marshall, 1931; original designation.

amoenus Voss, 1960: 320. (Deretiodes)

Distribution: Papua New Guinea: Astrolabe Bay, Sattelberg, Simbang, Stephansort.

Deretiosus Pascoe, 1871c: 186

TYPE-SPECIES: Deretiosus aridus Pascoe, 1871c; monobasic. KEYS: Lea, 1909: 709; Zimmerman, 1941: 197.

aridus Pascoe, 1871c: 185. (Deretiosus)

Distribution: Papua New Guinea: Dilo, Finschhafen, Mt. Lamington, Paumomu River; Bismarck Archipelago: New Britain (?); West Papua: Dorey, Saylee.

Deretiosus blandus Lea, 1909: 710. NOTES: Synonymy: Zimmerman, 1941.

Deretiosus zopherus Lea, 1913: 283.

carinirostris Lea, 1931c: 388. (Deretiosus)

DisTRIBUTION: Papua New Guinea: Mt. Lamington.

collaris Lea, 1931c: 389. (Deretiosus)

DistriBution: Papua New Guinea: Mt. Lamington.

erimanus Voss, 1960: 319. (Deretiosus)

Distribution: Papua New Guinea: Erima, Astrolabe Bay.

latus Lea, 1931c: 388. (Deretiosus)

DistriBution: Papua New Guinea: Mt. Lamington; West Papua: Dorey.

subaridus Lea, 1928a: 70. (Deretiosus)

Distribution: Papua New Guinea: Manumbo, Fly River.

squamipennis Lea, 1931c: 390. (Deretiosus)

DisTRIBUTION: Papua New Guinea: Mt. Lamington.

turbatus (Faust), 1892b: 210. (Colobodes)

DistRIBUTION: New Guinea; D'Entrecasteaux Islands: Fergusson Island. NotES: Transferred: Zimmerman, 1941: 199.

Deretiosus amplipennis Lea, 1931c: 387. NoTES: Synonymy: Zimmerman, 1941: 180.

verrucifer Faust, 1899: 52. (Deretiosus)

Distribution: Papua New Guinea: Finschhafen, Wareo; [Australia: Cooktown]. Deretiosus pustulosus Lea, 1931c: 390. NoTES: Synonymy: Zimmerman, 1941: 181.

OCOBLODES Heller, 1922: 569

TYPE-SPECIES: Ocoblodes lineola Heller, 1922; original designation.

parvus (Lea), 1931c: 392. (Deretiosus)

Distribution: Papua New Guinea: Mt. Lamington. Notes: Transferred: Zimmerman, 1941: 195. 
TYPE-SPECIES: Perrhaebius ephippiger Pascoe, 1874; monobasic.

ephippiger Pascoe, 1874: 34. (Perrhaebius)

DistRIBUTION: West Papua: Dorey; Aru Islands.

fasciculatus Hartmann, 1901: 282. (Perrhaebius)

Distribution: Papua New Guinea: Bongu.

tibialis (Lea), 1928a: 73. (Deretiosus)

DisTRIBUTION: New Guinea. NOTES: Transferred: Zimmerman, 1941: 196.

v-album Faust, 1898a: 142. (Perrhaebius)

DistRIBUTION: Papua New Guinea: Mailu Island; D’Entrecasteaux Islands: Fergusson Island.

\section{LITHININI}

\section{LITHININA}

SELEUCA Pascoe, 1871c: 173

TYPE-SPECIES: Seleuca amicta Pascoe, 1871c; subsequent designation by Voss, 1958a: 47.

turbatus (Pascoe), 1885: 219. (Ergias)

DisTRIBUTION: Papua New Guinea: Fly River.

\section{MECYSOLOBINI}

\section{ALCIDODES Marshall, 1939a: 582}

TYPE-SPECIES: Lixus trilobus Fabricius, 1801 [for Alcides Schönherr, 1825]; original designation. NoTES: This is not a natural group as listed herein and the genera and species-groups that comprise the following species list need to be revised. I follow Haaf (1963) in retaining Alcidodes auct. until the problematic Mecysolobini classification is better resolved. KEYS: Heller, 1929: 114; Haaf, 1963: 88.

abbreviatus (Heller), 1942: 211. (Alcides)

Distribution: Papua New Guinea: Bongu.

albocinctus Blanchard, 1853: 246. (Alcides)

Distribution: West Papua: Bintoeni Bay, MacCluer Gulf, Ramoi, Sorong; Salawatti Island; Waigeo Island.

Alcides bicinctus Faust, 1895a: 97.

albolituratus Blanchard, 1853: 245. (Alcides)

DistRIBUTION: Papua New Guinea; Aru Islands.

Alcidodes elegans v. albolituratus Blanchard, 1853: 245.

amoenus Pascoe, 1882b-448. (Alcides)

DisTRIBUTION: Bacan Island; West Papua: Saylee.

Alcides bellus Pascoe, 1882b-448. Notes: Synonymy: Haaf, 1963-112.

apicalis (Heller), 1914a: 653. (Alcides)

DisTRIBUTION: West Papua: Lorentz River; Biak Island.

asperatus Haaf, 1963: 141. (Alcidodes) 
DisTRIBUTION: West Papua: Noord River.

australis australis (Boisduval), 1835: 423. (Alcides)

Distribution: Papua New Guinea; West Papua; Molluccas; Solomon Islands.

Alcides profluens Pascoe, 1885: 239.

australis pervicax Faust, 1892b: 207. (Alcides)

Distribution: Papua New Guinea; Bismarck Archipelago: New Britain; West Papua: Cyclops Mountains, Humboldt Bay.

australis platysomus Haaf, 1963: 132. (Alcidodes)

DisTRIBUTION: West Papua: Biak Island, Schouten Island.

australis ramosus Haaf, 1963: 132. (Alcidodes)

DISTRIBUTION: West Papua: Paniai, Araboebivak.

australis segnis Faust, 1897a: 180. (Alcides)

DistRIBUTION: Papua New Guinea: Astrolabe Bay, Moroka, HaverIsland.

australis septemaculatus Faust, 1897a: 181. (Alcides 7-maculatus)

DistRIBUTION: Papua New Guinea: Mailu Island.

blandus Haaf, 1963: 116. (Alcidodes)

DistRIBUTION: West Papua: Paninai.

bonguensis (Hartmann), 1901: 276. (Alcides)

DisTRIBUTION: Papua New Guinea: Bongu; Lorentz Island.

brevicollis brevicollis Pascoe, 1885: 243. (Alcides)

DistRIBUTION: Papua New Guinea: Katau River; Mysol Island.

brevicollis reductus Faust, 1892b: 208. (Alcides)

Distribution: Papua New Guinea: Alexishafen, Astrolabe Bay, Bogia, Monumbo, Madang; West Papua:

Idenburg River.

cervinus Haaf, 1963: 123. (Alcidodes)

DISTRIBUTION: Aru Islands; Kei Island.

confusus Haaf, 1963: 153. (Alcidodes)

Distribution: West Papua.

elegans Guérin-Méneville, 1838: 124. (Alcides)

Distribution: Papua New Guinea: April River, Aroa River, Astrolabe Bay, Bongu, Finschhafen,

Kokoda, Mafulu, Monumbo, Sepik, Torricelli Mountains, Tumleo, Wareo, Yule Island. West Papua: Arfak

Mountains, Idenburg River, Sorong, Takar; Aru Island; Biak Island; Yepen Island; Salawatti Island;

Waigeo Island.

Alcides elegans v. atrocretosus Fairmaire, 1883: 37. DiSTRIBUTION: Bismarck Archipelago: Duke of York Island.

eremitus Haaf, 1963: 99. (Alcidodes)

Distribution: Papua New Guinea: Wareo.

exornatus Chevrolat, 1880b: 333. (Alcides)

Distribution: Papua New Guinea: Fly River, Monumbo, Paumomu River, Yule Island.

Alcides gestroi Pascoe, 1885: 242.

ferrugineus (Hartmann), 1901: 274. (Alcides)

DisTRIBUTION: Papua New Guinea: Bongu.

firmus Haaf, 1963: 167. (Alcidodes)

DisTRIBUTION: Papua New Guinea: Kokoda, Simbang.

frater (Hartmann), 1901: 276. (Alcides)

Distribution: Papua New Guinea: Bongu, Sattelberg, Tumleo.

freudei Haaf, 1963: 159. (Alcidodes) 
DISTRIBUTION: North Solomon Islands: Bougainville.

gallus Pascoe, 1887: 354. (Alcides galliarius)

DISTRIBUTION: West Papua: Saylee.

geniculatus (Faust), 1897a: 182. (Alcides)

Distribution: Papua New Guinea: Huon Gulf, Mailu, Mope, Moroka, Rigo, Yule Island; Bismarck

Archipelago: New Britain, Gazelle Peninsula, New Laudenburg Island, Trobriand Islands, Fergusson Island, Solomon Islands.

herteli Haaf, 1963: 140. (Alcidodes)

DiSTRIBUTION: Bismarck Archipelago: New Britain.

idenburgi (Heller), 1942: 212. (Alcides)

DisTRIBUTION: West Papua: Idenburg River.

indigaceus Pascoe, 1882b: 447. (Alcides)

DistRIBUTION: West Papua: Mysol Island, Saylee Island.

Alcides parilis Pascoe, 1882b: 448.

lascivus Haaf, 1963: 144. (Alcidodes)

DistRIBUTION: Papua New Guinea: Bogia, Bongu.

lemniscatus Haaf, 1964: 293. (Alcidodes)

Distribution: Papua New Guinea: Finschhafen, Wareo.

lepidus Haaf, 1963: 122. (Alcidodes)

Distribution: Papua New Guinea: Port Moresby, Ighibirei.

magister (Pascoe), 1871c: 181. (Alcides)

Distribution: Papua New Guinea: Bongu, Fly River, Torricelli Mountains, Yule Island; West Papua:

Dorey, Noord River; Aru Island.

nitidus Pascoe, 1887: 356. (Alcides)

DISTRIBUTION: Waigeo Island; Bacan Island.

novellus Haaf, 1963: 157. (Alcidodes)

Distribution: Papua New Guinea: Sattelberg; West Papua.

ochraceus Haaf, 1963: 155. (Alcidodes)

DISTRIBUTION: Admiralty Islands: Lorengau, Manus Island.

parentheticus Marshall, 1915: 515. (Alcides)

DistRIBUTION: West Papua: Utakwa River.

pentastictus Ancey, 1881: 372. (Alcides)

DistRIBUTION: West Papua: Aru Islands; [Australia].

perditor Haaf, 1963: 137. (Alcidodes)

DistRIBUTION: West Papua: Alkmaar, Jayapura, Maffin Bay, Noord River; Biak Island.

praeustus (Guérin-Méneville), 1838: 123. (Alcides)

Distribution: Papua New Guinea: Astrolabe Bay, Alexishafen, Bongu, Dallmannshafen, Fly River,

Monoumbo, Moroka, Paumomu River, Stephansport, Tumleo. West Papua: Andai, Arfak, Maffin Bay,

Lorentz Island, Sekroe, Mamberamo River, Sorong, Fakfak, Biak Island, Aru Island, Kei Island, Waigeo

Island, Salawatti Island.

praevius Haaf, 1963: 156. (Alcidodes)

DisTRIBUTION: Admiralty Islands: Los Negros

pretiosus Haaf, 1963: 162. (Alcidodes)

DISTRIBUTION: Waigeo Island.

prolixus Haaf, 1963: 124. (Alcidodes)

DisTRIBUTION: West Papua: Dorey; Waigeo Island.

pusillus Pascoe, 1885: 243. (Alcides) 
DistRIBUTION: Papua New Guinea: Fly River, Katau River; [Australia: Somerset].

reductus (Faust), 1892b: 208. (Alcides)

DISTRIBUTION: New Guinea.

richteri (Faust), 1892b: 207. (Alcides)

Distribution: Papua New Guinea: Alexishafen, Bogia, Bongu, Monumbo.

rostratus Pascoe, 1885: 241. (Alcides)

DistRIBUTION: Papua New Guinea: Fly River.

scitus Haaf, 1963: 147. (Alcidodes)

Distribution: Papua New Guinea: Tumleo.

segnis (Faust), 1897a: 180. (Alcides)

DistRIBUTION: Papua New Guinea: Moroka; Kei Island.

semiochraceus (Faust), 1897a: 179. (Alcides)

Distribution: Papua New Guinea: Mt. Alexander to Mt. Nisbet; West Papua.

septemmaculatus (Faust), 1897a: 181. (Alcides)

Distribution: Papua New Guinea: Mailu Island.

speculator Haaf, 1963: 148. (Alcidodes)

DisTRIBUTION: West Papua.

sturnus Haaf, 1963: 152. (Alcidodes)

Distribution: Papua New Guinea: Astrolabe Mountains.

Alcides blanchardi Faust, 1899: 45.

tenuistria (Heller), 1914a: 654. (Alcides)

DistRIBUTION: West Papua: Lorentz River; Biak Island.

Alcidodes incomptus Marshall, 1915: 516. Distribution: West Papua: Mimika River, Lorentz Island; Bivak Island.

terrosus Haaf, 1963: 149. (Alcidodes)

DisTRIBUTION: Bismarck Archipelago: New Hannover.

tetanicus Pascoe, 1887: 355. (Alcides)

DistRIBUtion: Papua New Guinea: Mailu Island; West Papua: Saylee.

umbrifer Haaf, 1963: 120. (Alcidodes)

Distribution: Papua New Guinea: Hauptlager bei Malu; West Papua: Mamberamo River.

Alcidodes aspericollis Heller, 1929: 114. DisTRIBUTION: [Buru Island].

waltoni Boheman in Schönherr, 1844: 58. (Alcides)

DistRIBUTION: New Guinea; [Celebes].

Alcidodes albolineatus Roelofs, 1875: CVII.

Alcidodes roelofsi Lewis, 1879: 23.

Alcidodes sexvittatus Faust, 1894: 243.

\section{METATYGINI}

OMOPHORUS Schönherr, 1835: 479

TYPE-SPECIES: Omophorus stomachosus Boheman in Schönherr, 1835; original designation. 
TYPE-SPECIES: Omophorus (Pangomorphus) biroi Voss, 1960; original designation.

biroi Voss, 1960: 344. (Omophorus (Pangomorphus))

DISTRIBUTION: Papua New Guinea: Sattelberg.

\section{MOLYTINI}

\section{MOLYTINA}

Carbonomassula Heller, 1908a: 15

TYPE-SPECIES: Carbonomassula glaberrima Heller, 1908a; monobasic.

glaberrima Heller, 1908a: 16. (Carbonomassula)

DisTRIBUTION: Papua New Guinea: Aroa River.

\section{PAIPALESOMINI}

PERIBLEPTUS Schönherr, 1843: 192

TYPE-SPECIES: Peribleptus scalptus Boheman in Schönherr, 1843; original designation. KEY: Marshall, 1944: 656.

dealbatus dealbatus (Boisduval), 1835: 425. (Alcides)

Distribution: Papua New Guinea: Astrolabe Mountains, Dilo, Paumomu River; West Papua: Aru Island. dealbatus zonatus (Pascoe), 1871c: 168. (Papalosomus zonatus)

DisTRIBUTION: Papua New Guinea.

\section{PISSODINI}

\section{ORTHORHININA}

\section{ORTHORHINUS Schönherr, 1825: 582}

TYPE-SPECIES: Orthorhinus cylindrirostris Fabricius, 1775; original designation.

albosparsus Faust, 1892b: 204. (Orthorhinus)

DisTRIBUTION: West Papua: Mimika River.

arrogans Pascoe, 1874: 23. (Orthorhinus)

DisTRIBUTION: New Guinea: Bouru.

ilex Faust, 1899: 37. (Orthorhinus)

DISTRIBUTION:

insularis Voss, 1960: 314. (Orthorhinus)

DistRIBUTION: New Guinea; Salomon Islands.

klugi Boheman in Schönherr, 1835: 246. (Orthorhinus)

DisTRIBUTION: Papua New Guinea: Yule Island.

perversus Pascoe, 1885: 226. (Orthorhinus) 
Distribution: Papua New Guinea: Fly River; West Papua: Ansus, Yapen Island; Waigeo Island; Aru Island.

postoculatus Marshall, 1915: 513. (Orthorhinus)

DistRIBUTION: West Papua: Utakwa River.

rugosus Montrouzier, 1855: 50. (Orthorhinus)

DISTRIBUTION: Woodlark Islands.

ORTHORHINUS (HOMORTHORRHINUS) Voss, 1960: 313

TYPE-SPECIES: Orthorhinus brachypus Pascoe, 1885; original designation.

brachypus brachypus Pascoe, 1885: 226. (Orthorhinus)

Distribution: Papua New Guinea: Fly River, Ighibirei, Moroka, Yule Island; Aru Island.

brachypus tibialis Voss, 1960: 313. (Orthorhinus (Homorthorrhinus))

DistRIBUTION: Papua New Guinea: Sattelberg, Simbang.

cylindrirostris (Fabricius), 1775: 137. (Curculio)

DisTRIBUTION: Papua New Guinea: Yule Island.

patruelis Pascoe, 1885: 225. (Orthorhinus)

Distribution: Papua New Guinea: Astrolabe Mountains, Fly River, Ighibirei, Mimika River, Paumomu

River, Port Moresby, Rigo, Utakwa River, Yule Island; West Papua: Waigeo, Aru Island.

\section{PISSODINA}

VANAPA Pouillaude, 1915: 101

TYPE-SPECIES: Vanapa oberthuri Pouillaude, 1915; monobasic.

oberthuri Pouillaude, 1915: 102. (Vanapa)

Distribution: Papua New Guinea: Angi Lakes, Bulolo, Wau; West Papua: Arfak Mountains. Angianus pratti Sharp, 1919: 152.

\section{TRACHODINI}

\section{ACICNEMIS Fairmaire, 1849b: 511}

TYPE-SPECIES: Acicnemis variegata Fairmaire, 1849b; subsequent designation by Fairmaire, 1881e: 299. Key: Hubenthal, 1917a: 101.

angustula Pascoe, 1885: 247. (Acicnemis)

DisTRIBUTION: Aru Islands.

clavigera Hubenthal, 1917a: 113. (Acicnemis)

Distribution: Papua New Guinea: Milne Bay, Sattelberg; West Papua: Mysol Island.

clypeifera Pascoe, 1885: 246. (Acicnemis)

Distribution: Papua New Guinea: Bongu; West Papua: Ansus; Aru Islands.

decussata Hubenthal, 1917a: 149. (Acicnemis)

DISTRIBUTION: New Guinea.

deridicula Hubenthal, 1917a: 141. (Acicnemis)

Distribution: Papua New Guinea: Milne Bay.

distinguenda Voss, 1960: 315. (Acicnemis) 
DistriBution: Papua New Guinea: Stephansort, Astrolabe Bay, Sattelberg, Huon Gulf.

exclusa Faust, 1899: 49. (Acicnemis)

Distribution: Papua New Guinea: Ighibirei, Paumomu River.

Acicnemis exclusa v. persona Faust, 1899: 49.

fausti Hubenthal, 1917a: 152. (Acicnemis)

DistRIBUTION: Papua New Guinea: Aroa River.

figurata Hartmann, 1901: 281. (Acicnemis)

DisTRIBUTION: Papua New Guinea: Bongu; Solomon Islands.

gestroi Pascoe, 1885: 245. (Acicnemis)

Distribution: West Papua: Ansus.

laqueata Faust, 1899: 49. (Acicnemis)

DistRIBUTION: Papua New Guinea: Ighibirei, Yule Island.

laticollis Pascoe, 1885: 245. (Acicnemis)

Distribution: Papua New Guinea: Astrolabe Bay, Fly River, Ighibirei, Yule Island.

latiuscula Hubenthal, 1917a: 156. (Acicnemis)

Distribution: Papua New Guinea: Astrolabe Bay.

linea Pascoe, 1885: 245. (Acicnemis)

Distribution: Papua New Guinea: Yule Island; Aru Island.

lobicollis Maceay, 1887: 188. (Acicnemis)

DistRIBUTION: Papua New Guinea: Fly River; Bismarck Archipelago: New Britain.

longirostris Pascoe, 1885: 244. (Acicnemis)

DistRIBUTION: Papua New Guinea: Fly River.

meriones Pascoe, 1872d: 462. (Acicnemis)

DistRIBUTION: Papua New Guinea: Yule Island.

modesta Faust, 1899: 47. (Acicnemis)

Distribution: Papua New Guinea: Ighibirei, Milne Bay; West Papua: Andai; Kei Island.

ornata Macleay, 1887: 187. (Acicnemis)

DistRIBUtion: Papua New Guinea: Fly River; Bismarck Archipelago: New Britain.

perfecta Hubenthal, 1917: 122. (Acicnemis)

DISTRIBUTION: New Guinea.

praeculta Faust, 1899: 48. (Acicnemis)

DisTRIBUTION: Papua New Guinea: Ighibirei.

quadrifasciculata Voss, 1960: 316. (Acicnemis)

DistRIBUTION: Papua New Guinea: Sattelberg.

sannio (Pascoe), 1872d: 463. (Berethia)

Distribution: Papua New Guinea: Astrolabe Mountains, Kapakapa, Mailu Island; [Australia: Somerset]. sororia Pascoe, 1885: 247. (Acicnemis)

DISTRIBUTION: [Australia: Somerset]; New Guinea (?).

spilonota Pascoe, 1885: 247. (Acicnemis)

DistRIBUTION: Papua New Guinea: Kapakapa, Yule Island.

spinipennis Heller, 1905a: 73. (Acicnemis)

Distribution: Papua New Guinea: Sattelberg.

testacea Kleine, 1924: 156. (Acicnemis)

DISTRIBUTION: Bismarck Archipelago: New Britain.

zelivira Hubenthal, 1917a: 152. (Acicnemis)

DisTRIBUTION: West Papua: Andai. 


\section{TRIGONOCOLINI}

MENECHIRUS Hartmann, 1901: 278

TYPE-SPECIES: Menechirus oculatus Hartmann, 1901; original designation.

oculatus Hartmann, 1901: 279. (Menechirus)

DistRIBUTION: Papua New Guinea: Bongu.

PyCNOCHIRUS Berg, 1898: 18

TYPE-SPECIES: Megachirus fuscovarius Faust, 1859; monobasic.

fuscovarius (Faust), 1895b: 213. (Megachirus)

DistRIBUTION: Papua New Guinea: Redscar Bay.

\section{TRYPETIDINI}

TYRPETES Heller, 1908a: 30

TYPE-SPECIES: Tyrpetes ottonis Heller, 1908a; monobasic.

ottonis Heller, 1908a: 31. (Tyrpetes)

DISTRIBUTION: North Solomon Islands: Bougainville.

\section{PLATYPODINAE}

\section{PLATYPODINI}

\section{PLATYPODINA}

BAIOCIS Browne, 1962: 651

TYPE-SPECIES: Crossotarsus pernanulus Schedl, 1935d; original designation.

annularis (Schedl), 1975d: 379. (Platypus)

DistRIBUTION: Papua New Guinea: Bulolo.

imitatrix Schedl, 1973b: 94. (Baiocis)

DistriBUTION: Papua New Guinea: Bulolo.

inimicus (Schedl), 1935d: 482. (Crossotarsus)

DISTRIBUTION: Bismarck Archipelago: New Britain.

Platypus inimicus Schedl, 1958: 152.

nubilosus Roberts \& Morimoto, 1986: 162. (Baiocis)

DistRIBUTION: Papua New Guinea: Bulolo.

pernanulus (Schedl), 1935d: 482. (Crossotarsus)

DISTRIBUTION: New Guinea.

unispinosus Roberts, 1986: 39. (Baiocis)

DistRIBUTION: Papua New Guinea: Bulolo. 
TYPE-SPECIES: Platypus wallacei Thomson, 1857c; subsequent designation by Hopkins, 1914: 119.

aruensis Beeson, 1937: 54. (Crossotarsus)

DISTRIBUTION: Aru Islands.

aureipilus Roberts, 1989a: 88. (Crossotarsus)

DisTRIBUTION: Papua New Guinea: Morobe Province.

barbatus Chapuis, 1865: 66. (Crossotarsus)

DiSTRIBUTION: New Guinea.

biconcavus Schedl, 1962a: 76. (Crossotarsus)

DisTRIBUTION: New Guinea; Bismarck Archipelago: New Ireland.

caliginosus Roberts, 1979: 81. (Crossotarsus)

Distribution: Papua New Guinea: Mt. Giluwe.

chalcographus Schedl, 1972c: 64. (Crossotarsus)

DistRIBUTION: Papua New Guinea: Wau.

cheesmani Schedl, 1935b: 275. (Crossotarsus)

Distribution: Papua New Guinea: Mt. Tafa, North West of Mt. Albert Edward.

corrugatus Roberts, 1989a: 88. (Crossotarsus)

DiSTRIBUTION: Papua New Guinea: Bulolo.

coxalis Schedl, 1970a: 129. (Crossotarsus)

DisTRIBUTION: Papua New Guinea: Porotop.

denticulus Browne, 1983b: 57. (Crossotarsus)

DistRIBUTION: Papua New Guinea: Wau.

denturus Browne, 1983b: 55. (Crossotarsus)

Distribution: Papua New Guinea: Mt. Kaindi.

dolus Schedl, 1975d: 373. (Crossotarsus)

DisTRIBUTION: Papua New Guinea: Bulolo.

externedentatus (Fairmaire), 1849b: 78. (Platypus)

DistRIBUTION: Bismarck Archipelago.

fluminalis Beeson, 1937: 57. (Crossotarsus)

DisTRIBUTION: New Guinea.

forcipes Schedl, 1975d: 374. (Crossotarsus)

DisTRIBUTION: Papua New Guinea: Bulolo.

fractus Sampson, 1912: 249. (Crossotarsus)

DISTRIBUTION: Bismarck Archipelago.

gressitti Schedl, 1972b: 53. (Crossotarsus)

DisTRIBUTION: Papua New Guinea: Oriomo.

intermedius Chapuis, 1865: 69. (Crossotarsus)

DiSTRIBUTION: New Guinea.

javanus Beeson, 1937: 51. (Crossotarsus)

DisTRIBUTION: New Guinea.

keyensis Strohmeyer, 1913: 163. (Crossotarsus)

DistRIBUTION: New Guinea; Kei Island.

kuntzeni (Schedl), 1937a: 40. (Platypus)

Distribution: West Papua: Humboldt Bay, Dorey. Notes: Transferred to Baiocis: Browne, $1983 \mathrm{a}$ : 555.

Transfered from Baiocis: Wood, 1993: 275. 
lacordairei Chapuis, 1865: 85. (Crossotarsus)

DisTRIBUTION: New Guinea; Bismarck Archipelago, New Britain, New Ireland; Aru Island.

longicornis (Schedl), 1942b: 196. (Platypus)

DISTRIBUTION: New Guinea.

majusculus Sampson, 1924: 89. (Crossotarsus)

DistRIBUTION: New Guinea: Mamberamo River; West Papua: Geelvink Bay.

Crossotarsus abdominalis Schedl, 1969b: 220. Notes: Synonymy: Schedl, 1972a: 112.

minor (Browne), 1980b: 388. (Platypus)

DistRIBUTION: Papua New Guinea: Vanimo.

minusculus Chapuis, 1865: 68. (Crossotarsus)

Distribution: New Guinea; Bismarck Archipelago, New Britain, New Ireland; North Solomon Islands:

Bougainville Island.

Crossotarsus minusculus grandis Schedl, 1975d: 374.

Crossotarsus inornatus Chapuis, 1865: 68. NOTES: Synonymy: Browne, 1955: 365.

Crossotarsus novaeguineensis Roberts, 1989a: 90.

mniszechi Chapuis, 1865: 62. (Crossotarsus)

DistRIBUtion: New Guinea; Bismarck Archipelago, New Britain, New Ireland; D’Entrecasteaux Islands:

Fergusson Island; Aru Island.

moutui Schedl, 1973a: 76. (Crossotarsus)

DistRIBUTION: Papua New Guinea: Mt. Wilhelm.

nakazawai Browne, 1986b: 342. (Crossotarsus)

DisTRIBUTION: West Papua: Fakfak.

oligodontus Roberts, 1979: 82. (Crossotarsus)

DistRIBUTION: Papua New Guinea: Mt. Giluwe.

paucidentatus Roberts, 1989a: 90. (Crossotarsus)

Distribution: Papua New Guinea.

pectinatus Browne, 1966: 240. (Crossotarsus)

DistriBution: Bismarck Archipelago: Gazelle Peninsula, New Britain.

piceus Chapuis, 1865: 56. (Crossotarsus)

DISTRIBUTION: New Guinea.

porcatus Roberts, 1988: 91. (Crossotarsus)

DisTRIBUTION: Papua New Guinea: Bulolo.

prociduus Schedl, 1975c: 222. (Crossotarsus)

DistRIBUTION: West Papua: Geevlink Bay.

rasilis Roberts, 1979: 83. (Crossotarsus)

Distribution: Papua New Guinea: Mt. Giluwe.

scorpius (Schedl), 1942b: 195. (Crossotarsus)

DisTRIBUTION: New Guinea. NOTES: Transferred: Browne, 1962: 643.

semicinctus Schedl, 1973b: 93. (Crossotarsus)

DiSTRIBUTION: Bismarck Archipelago: Hoskins, New Britain.

siporanus Schedl, 1936c: 44. (Crossotarsus)

DISTRIBUTION: New Guinea.

spectrum Schedl, 1975d: 376. (Crossotarsus)

DistRIBUTION: Papua New Guinea: Bulolo.

spinipennis Schedl, 1972b: 54. (Crossotarsus)

DISTRIBUTION: North Solomon Islands: Bougainville.

squamulatus Chapuis, 1865: 87. (Crossotarsus) 
DISTRIBUTION: Bismarck Archipelago.

subopacus Schedl, 1969b: 222. (Crossotarsus)

DistRIBUTION: Papua New Guinea: Watut Valley.

subpellucidus Lea, 1910a: 134. (Crossotarsus)

DISTRIBUTION: New Guinea.

terminatus Chapuis, 1865: 83. (Crossotarsus)

DisTRIBUTION: Papua New Guinea: Wau; West Papua.

Crossotarsus terminatus sedulus Schedl, 1972c: 66. NOTES: Rank change to subspecies: Schedl, 1972a: 107.

toralis Schedl, 1975d: 376. (Crossotarsus)

DistRIBUTION: Papua New Guinea: Bulolo.

tumidus Schedl, 1975d: 377. (Crossotarsus)

Distribution: Papua New Guinea: Bulolo.

vafer Schedl, 1972c: 66. (Crossotarsus)

DISTRIBUTION: North Solomon Islands: Bougainville.

ventricornis Schedl, 1972c: 67. (Crossotarsus)

DisTRIBUTION: Bismarck Archipelago: New Britain.

ventrispinis Schedl, 1969b: 222. (Crossotarsus)

DisTRIBUTION: Papua New Guinea: Mt. Hagen.

ventrosus Schedl, 1973b: 94. (Crossotarsus)

Distribution: Papua New Guinea: Bulolo.

vicinus Schedl, 1975: 378. (Crossotarsus)

DistRIBUTION: Papua New Guinea: Karamui.

wallacei (Thomson), 1857c: 343. (Platypus)

DisTRIBUTION: Borneo; New Guinea (?).

DiNOPLATYPUS Wood, 1993: 273

TYPE-SPECIES: Dinoplatypus cupulatulus Chapuis, 1865; original designation.

chevrolati (Chapuis), 1865: 281. (Platypus)

DistRIBUTION: West Papua: Waigeo Island. Notes: Transfered: Wood, 1993: 273.

cupulatulus (Schedl), 1941b: 420. (Platypus)

DisTRIBUTION: New Guinea. NoTES: Transfered: Wood, 1993: 273.

cupulatus (Chapuis), 1865: 278. (Platypus)

DistRIBUTION: New Guinea. NOTES: Transfered: Wood, 1993: 273.

forficula (Chapuis), 1865: 283. (Platypus)

DistRIBUTION: New Guinea; Bismarck Archipelago: New Britain, New Ireland. Notes: Transfered:

Wood, 1993: 273.

lepidus (Chapuis), 1865: 282. (Platypus)

DistRIBUTION: New Guinea. Notes: Transfered: Wood, 1993: 273.

luniger (Motschulsky), 1863: 510. (Platypus)

DistRIBUTION: New Guinea. Notes: Transfered: Wood, 1993: 273.

pallidus (Chapuis), 1865: 284. (Platypus)

DistRibUtion: West Papua: Dorey. Notes: Transfered: Wood, 1993: 273.

Platypus pallidus sabroni Schedl, 1940: 433.

pseudocupulatus (Schedl), 1935e: 635. (Platypus)

DistRIBUtion: Papua New Guinea: Finschhafen, Kokoda, Mafulu, Mondo; West Papua: Cyclops Moun- 
tains; Aru Islands. NoTES: Transfered: Wood, 1993: 273.

Platypus pseudocupulatus artecavus Schedl, 1941b: 422.

Peroplatypus Wood, 1993: 272

TYPE-SPECIES: Peroplatypus truncatipennis Schedl, 1964d; original designation.

capito (Browne), 1983b: 60. (Platypus)

Distribution: Papua New Guinea: Mt. Kaindi.

obliquecaudatus (Schedl), 1936c: 59. (Platypus)

DistRIBUtion: Papua New Guinea: Moroka. Notes: Transfered: Wood, 1993: 273.

Platypus obliquecaudatus Schedl, 1942c: 210.

semisulcatus (Schedl), 1969b: 231. (Platypus)

DistRIBUTION: Papua New Guinea: Kum near Mt. Hagen. Notes: Transfered: Wood, 1993: 273.

truncaticauda (Schedl), 1968b: 536. (Platypus)

DistRIBUTION: New Guinea: Bodem. NoTES: Transfered: Wood, 1993: 273.

truncatigranosus (Schedl), 1970a: 132. (Platypus)

DistRIBUTION: Papua New Guinea: Porotop. Notes: Transfered: Wood, 1993: 273.

truncatipennis (Schedl), 1964d: 302. (Platypus)

Distribution: Papua New Guinea: Madang, Gogol, Oriomo; West Papua: Humboldt Bay. Notes: Trans-

fered: Wood, 1993: 273.

Platypus diversiporus Schedl, 1972b: 56. NOTES: Synonymy: Roberts, 1989b: 285.

Platypus multifoveolatus Schedl, 1975d: 389. NOTES: Synonymy: Roberts, 1989b: 285.

PlatyPus Herbst, 1793: 128

TYPE-SPECIES: Bostrichus cylindrus Fabricius, 1792; monobasic.

abnormis Schedl, 1975d: 378. (Platypus)

Distribution: Papua New Guinea: Bulolo.

abruptifer Wood, 1992b: 90. (Platypus)

DisTRIBUTION: New Guinea: Adi Island.

Platypus abruptus Browne, 1986b: 337.

abruptulus Browne, 1984a: 158. (Platypus)

DisTRIBUTION: Bismarck Archipelago: New Britain.

acetabuliformis Roberts, 1986: 40. (Platypus)

Distribution: Papua New Guinea: Bulolo, Popondetta.

Platypus acetabuliformis comalis Roberts, 1989b: 263.

acuticornifer Wood, 1981: 121. (Platypus)

DisTRIBUTION: Papua New Guinea: Porotop.

Platypus acuticornis Schedl, 1975c: 223.

Platypus longicornis Browne, 1980e: 215.

acuticornis Schedl, 1973a: 77. (Platypus)

DistRIBUTION: Papua New Guinea: Mt. Dayman.

advena Schedl, 1972b: 54. (Platypus)

DISTRIBUTION: New Guinea.

aequalis Schedl, 1975c: 223. (Platypus)

DistRIBUtion: Papua New Guinea: Porotop. 
aequilaterus Roberts, 1986: 40. (Platypus)

DistRIBUTION: Papua New Guinea: Bulolo.

ambiguus Roberts, 1987: 163. (Platypus)

DistribUtion: Papua New Guinea: Bulolo, Gumi.

anguis Browne, 1983b: 59. (Platypus)

DistRIBUTION: Papua New Guinea: Bulolo.

angusticollis Schedl, 1942b: 197. (Platypus)

DISTRIBUTION: New Guinea.

angustior Schedl, 1942b: 196. (Platypus)

DISTRIBUTION: New Guinea.

anomalus Schedl, 1975c: 224. (Platypus)

DISTRIBUTION: New Guinea.

anoplus Schedl, 1975d: 380. (Platypus)

DistRiBution: Papua New Guinea: Bulolo.

apertulus Schedl, 1975d: 380. (Platypus)

DistRIBUTION: Papua New Guinea: Bulolo.

apertus (Schedl), 1979b: 119. (Diapus)

Distribution: Papua New Guinea: Bulolo.

apicatulus Schedl, 1975d: 381. (Platypus)

Distribution: Papua New Guinea: Bulolo.

apicatus Schedl, 1974: 466. (Platypus)

DistRibUtion: Papua New Guinea: Watut Valley.

artetruncatus Schedl, 1979b: 109. (Platypus)

DistRIBUTION: Papua New Guinea: Bulolo.

aspicis Roberts \& Morimoto, 1986: 163. (Platypus)

Distribution: Papua New Guinea: Bulolo, Gumi.

associatus Schedl, 1973b: 95. (Platypus)

DISTRIBUTION: Bismarck Archipelago: New Britain.

atrans Roberts, 1989b: 264. (Platypus)

Distribution: Papua New Guinea: Mt. Kaindi.

bifurcus (Schedl), 1938b: 422. (Crossotarsus)

DisTRIBUTION: New Guinea. NOTES: Originally described as a subspecies of Platypus shoreanus.

bigranulatus Schedl, 1980: 122. (Platypus)

DistRIBUTION: Papua New Guinea: Wau.

Platypus bituberculatus Schedl, 1975c: 224.

Platypus bituberculifer Wood, 1981: 121.

Platypus jeffersi Browne, 1980e: 215.

bipyramidus Roberts \& Morimoto, 1986: 165. (Platypus)

DistRIBUTION: Papua New Guinea: Bulolo.

bispinatus Browne, 1984c: 457. (Platypus)

DisTRIBUTION: West Papua: Usau.

caelestis Roberts \& Morimoto, 1986: 165. (Platypus)

Distribution: Papua New Guinea: Bulolo, Gumi.

caledoniae Schedl, 1974: 466. (Platypus)

DISTRIBUTION: New Guinea.

capitilanatus Roberts \& Morimoto, 1986: 166. (Platypus)

DistRIBUTION: Papua New Guinea: Bulolo, Gumi. 
castigatus (Schedl), 1936c: 47. (Crossotarsus)

DistRIBUTION: Papua New Guinea: Moroka. NOTES: Transferred: Schedl, 1972a: 237.

celsus Roberts, 1979: 84. (Platypus)

Distribution: Papua New Guinea: Mt. Giluwe.

chimbui Schedl, 1972a: 207. (Platypus)

DisTRIBUTION: New Guinea.

cicatricosus Roberts, 1989b: 264. (Platypus)

DistRiBUTION: Papua New Guinea: Bulolo, Gumi.

circularicepes Schedl, 1975: 382. (Platypus)

DisTRIBUTION: Papua New Guinea: Bulolo.

circularis Chapuis, 1865: 285. (Platypus)

DisTRIBUTION: New Guinea.

coadunatus Schedl, 1975c: 224. (Platypus)

DisTRIBUTION: Papua New Guinea: Wau.

cognatus Roberts, 1989b: 266. (Platypus)

Distribution: Papua New Guinea: Bulolo.

collaris Schedl, 1979b: 110. (Platypus)

Distribution: Papua New Guinea: Bulolo.

conclavifrons Schedl, 1975d: 383. (Platypus)

DistRIBUTION: Papua New Guinea: Karamui.

concentriporus Roberts, 1979: 85. (Platypus)

Distribution: Papua New Guinea: Mt. Giluwe.

crassicornis Schedl, 1975d: 384. (Platypus)

Distribution: Papua New Guinea: Mt. Kaindi.

Platypus inversus Roberts, 1979: 88. DistriBUTION: Papua New Guinea: Mt. Giluwe.

crassus Strohmeyer, 1913: 162. (Platypus)

DistRIBUTION: Papua New Guinea: Aroa River.

cymbiformis Roberts, 1979: 86. (Platypus)

Distribution: Papua New Guinea: Mt. Giluwe.

daedalus Roberts, 1986: 41. (Platypus)

DistRIBUTION: Papua New Guinea: Bulolo.

denticollis Browne, 1983b: 61. (Platypus)

DistRIBUTION: Papua New Guinea: Mt. Kaindi.

deperditus Schedl, 1975d: 384. (Platypus)

DistRIBUTION: Papua New Guinea: Bulolo.

Crossotarsus deperditus Schedl, 1975d: 375. NOTES: Synonymy: Roberts, 1989b: 285.

depressus Schedl, 1979b: 110. (Platypus)

Distribution: Papua New Guinea: Bulolo.

dibrachiatus Roberts, 1989b: 267. (Platypus)

DisTRIBUTION: Papua New Guinea: Bulolo.

diffinis Schedl, 1972b: 55. (Platypus)

DistRIBUTION: Papua New Guinea: Wau; Bismarck Archipelago: New Britain.

digestus Schedl, 1975c: 255. (Platypus)

DistRIBUTION: Papua New Guinea: Porotop.

discipennis Schedl, 1975c: 256. (Platypus)

DISTRIBUTION: New Guinea.

distinctipes Schedl, 1972b: 55. (Platypus) 
Distribution: Papua New Guinea: Bulolo, Wau.

Platypus ponamae Schedl, 1975d: 392. NOTES: Synonymy: Roberts, 1989: 285.

dobunabae Roberts \& Morimoto, 1986: 167. (Platypus)

DistRIBUTION: Papua New Guinea: Bulolo, Gumi.

dolus Schedl, 1975d: 385. (Platypus)

DistRiBUTION: Papua New Guinea: Bulolo.

dorsatus Schedl, 1975c: 226. (Platypus)

DISTRIBUTION: New Guinea.

duplosignatus Schedl, 1975d: 386. (Platypus)

DistRIBUTION: Papua New Guinea: Bulolo.

echinatus Roberts \& Morimoto, 1986: 167. (Platypus)

DistRIBUTION: Papua New Guinea: Bulolo, Gumi.

elaboratus Schedl, 1975c: 227. (Platypus)

Distribution: Papua New Guinea: Wau.

emdeni Schedl, 1935a: 174. (Platypus)

DisTRIBUTION: New Guinea.

enormis Schedl, 1970a: 130. (Platypus)

DisTRIBUTION: Papua New Guinea: Eastern Highlands.

evanidinervius Roberts \& Morimoto, 1986: 169. (Platypus)

Distribution: Papua New Guinea: Bulolo.

evanidus Roberts, 1986: 42. (Platypus)

DistribUtion: Papua New Guinea: Bulolo.

excedens Chapuis, 1865: 276. (Platypus)

Distribution: Papua New Guinea; Bismarck Archipelago; West Papua: Dorey.

excellens Schedl, 1973b: 95. (Platypus)

Distribution: Papua New Guinea: Huon Gulf, Sattelberg.

extensus Schedl, 1979b: 111. (Platypus)

DistRIBUTION: Papua New Guinea: Bulolo.

fenestrallatus Roberts, 1989b: 268. (Platypus)

DISTRIBUTION: New Guinea.

filipennis Schedl, 1979b: 111. (Platypus)

DistRIBUTION: Papua New Guinea: Bulolo.

firmis Schedl, 1975c: 227. (Platypus)

Distribution: Papua New Guinea: Sepik River.

fracticinctus Schedl, 1975d: 386. (Platypus)

Distribution: Papua New Guinea: Bulolo.

fracticornis (Schedl), 1936c: 49. (Crossotarsus)

DistRIBUTION: Papua New Guinea: Moroka.

fracticostis Schedl, 1972b: 57. (Platypus)

DistRIBUTION: Papua New Guinea: Wau.

frontebulbifer Schedl, 1979b: 112. (Platypus)

DistRIBUTION: Papua New Guinea: Bulolo.

fugax Schedl, 1975c: 228. (Platypus)

DistRIBUTION: Papua New Guinea: Wau.

fulgidus Browne, 1986b: 340. (Platypus)

DisTRIBUTION: West Papua: Fakfak.

gabensis Schedl, 1974: 467. (Platypus) 
Distribution: Papua New Guinea: Morobe Province.

galbulimimae Roberts \& Morimoto, 1986: 169. (Platypus)

Distribution: Papua New Guinea: Bulolo, Gumi.

gemellus (Schedl), 1936c: 48. (Crossotarsus)

Distribution: Papua New Guinea: Moroka.

geminatus Chapuis, 1865: 239. (Platypus)

DistRIBUTION: New Guinea; North Solomon Islands: Bougainville Island.

gerstaeckeri Chapuis, 1865: 240. (Platypus)

DisTRIBUTION: New Guinea.

giluwei Roberts, 1979: 87. (Platypus)

DistRIBUTION: Papua New Guinea: Mt. Giluwe.

globicollis Schedl, 1979b: 112. (Platypus)

DistribUtion: Papua New Guinea: Bulolo.

glochideus Roberts \& Morimoto, 1986: 170. (Platypus)

Distribution: Papua New Guinea: Bulolo, Gumi.

goilalae Schedl, 1975d: 387. (Platypus)

DisTRIBUTION: Papua New Guinea: Bulolo.

gongylodes Roberts \& Morimoto, 1986: 170. (Platypus)

Distribution: Papua New Guinea: Bulolo, Gumi.

gracilicornis (Schedl), 1936c: 49. (Crossotarsus)

DistRIBUTION: Papua New Guinea: Moroka.

gracilior Browne, 1983b: 63. (Platypus)

DistRIBUTION: Papua New Guinea: Mt. Kaindi.

gracilis Broun, 1893: 1254. (Platypus)

DISTRIBUTION: New Guinea.

granosus Browne, 1986b: 341. (Platypus)

Distribution: West Papua: Fakfak.

granulosus Browne, 1984c: 455. (Platypus)

DisTRIBUTION: West Papua: Manokwari.

grayi Schedl, 1972b: 59. (Platypus)

DISTRIBUTION: Bismarck Archipelago: New Ireland; North Solomon Islands: Bougainville Island.

Platypus grayi immersus Schedl, 1972b: 60.

grumosus Roberts \& Morimoto, 1986: 171. (Platypus)

Distribution: Papua New Guinea: Bulolo, Gumi.

gumiensis Roberts \& Morimoto, 1986: 170. (Platypus)

Distribution: Papua New Guinea: Bulolo, Gumi.

hashimotoi Browne, 1986b: 338. (Platypus)

DisTRIBUTION: West Papua: Fakfak.

hebetatus Roberts, 1986: 43. (Platypus)

Distribution: Papua New Guinea: Bulolo, Gumi.

hippocrepicus Roberts, 1986: 44. (Platypus)

Distribution: Papua New Guinea: Popondetta.

hirtus Schedl, 1955: 310. (Platypus)

DistRiBution: West Papua: Maffin Bay.

Platypus solidus hirtus Schedl, 1955: 310. NOTES: Raised to full species by Browne, 1966: 235.

hybridus Schedl, 1935c: 395. (Platypus)

DisTRIBUTION: New Guinea. 
imberbis Roberts, 1989b: 271. (Platypus)

DistRiBution: Papua New Guinea: Bulolo.

imparidens Browne, 1981b: 604. (Platypus)

DisTRIBUTION: West Papua: Kimi.

incertus Schedl, 1970a: 130. (Platypus)

DisTRIBUTION: Papua New Guinea: Wabag.

Platypus heteromorphus Schedl, 1975d: 388. NOTES: Synonymy: Roberts, 1989b: 285.

iriani Browne, 1980a: 377. (Platypus)

DisTRIBUTION: West Papua: Kimi.

ivagaii Roberts \& Morimoto, 1986: 171. (Platypus)

DistRIBUTION: Papua New Guinea: Bulolo, Gumi.

jansoni Chapuis, 1865: 244. (Platypus)

DisTRIBUTION: New Guinea; Bismarck Archipelago; Admiralty Islands.

juvencus Schedl, 1972c: 68. (Platypus)

Distribution: Papua New Guinea: Gogol.

katoi Browne, 1983a: 568. (Platypus)

DisTRIBUTION: West Papua: Wapoga.

kokodaensis (Schedl), 1935b: 274. (Crossotarsus)

Distribution: Papua New Guinea: Kokoda. Notes: Male described in Schedl, 1941a: 152 from

Geelvink Bay.

lablabiae Schedl, 1979b: 113. (Platypus)

DisTRIBUTION: Papua New Guinea: Lab Lab, Umboi Island.

labrifrons Schedl, 1974: 467. (Platypus)

DistribUTION: Papua New Guinea: Bulolo.

laevis Browne, 1980a: 376. (Platypus)

DISTRIBUTION: Bismarck Archipelago: New Britain.

latelobatus Browne, 1983b: 62. (Platypus)

DistRIBUTION: Papua New Guinea: Mt. Kaindi.

leprosus Browne, 1980e: 215. (Platypus)

DistRIBUTION: Papua New Guinea: Bulolo.

Platypus neotruncatus Schedl, 1979b: 114.

lesiniformis Roberts, 1989b: 273. (Platypus)

Distribution: Papua New Guinea: Bulolo.

longicalcaratus Roberts, 1989b: 274. (Platypus)

DistRIBUTION: Papua New Guinea: Mt. Giluwe.

longissimus Roberts, 1979: 90. (Platypus)

Distribution: Papua New Guinea: Mt. Giluwe.

loriae (Schedl), 1936c: 44. (Crossotarsus)

DistRIBUTION: Papua New Guinea: Moroka.

lucaris Schedl, 1979b: 114. (Platypus)

DistRIBUTION: Papua New Guinea: Bulolo.

lucasi Chapuis, 1865: 243. (Platypus)

Distribution: West Papua: Dorey.

lunatellus Browne, 1983a: 569. (Platypus)

DisTRIBUTION: West Papua: Usau.

margaritaceus Roberts \& Morimoto, 1986: 173. (Platypus)

DistRIBUTION: Papua New Guinea: Wau. 
matoae Browne, 1986b: 337. (Platypus)

DISTRIBUTION: West Papua: Fakfak. micrographus Schedl, 1979b: 114. (Platypus)

DISTRIBUTION: New Guinea.

mirandus Schedl, 1969b: 227. (Platypus)

DistRIBUTION: Papua New Guinea: Karamui.

misoolensis Browne, 1984c: 453. (Platypus)

DisTRIBUTION: Mysol Island.

morigerus Schedl, 1970a: 131. (Platypus)

DisTRIBUTION: Papua New Guinea: Eastern Highlands, Awande.

morobeensis Browne, 1983b: 65. (Platypus)

DistRIBUTION: Papua New Guinea: Mt. Kaindi.

morosus Schedl, 1975d: 388. (Platypus)

Distribution: Papua New Guinea: Mt. Hagen, Kum.

muricatus Roberts, 1989b: 274. (Platypus)

Distribution: Papua New Guinea: Bulolo.

negatus Schedl, 1973b: 96. (Platypus)

DistRIBUTION: Papua New Guinea: Gogol.

nemorosus Roberts, 1986: 45. (Platypus)

DistRIBUTION: Papua New Guinea: Popondetta.

neopartibilis Roberts, 1989b: 276. (Platypus)

DistRIBUTION: Papua New Guinea: Bulolo, Gumi.

nitens Browne, 1980a: 379. (Platypus)

DistRIBUTION: Bismarck Archipelago: Ulamona, New Britain.

nothofagus Roberts, 1979: 91. (Platypus)

DistRIBUTION: Papua New Guinea: Mt. Giluwe.

novaeguineensis Roberts, 1989b: 277. (Platypus)

DistRIBUTION: Papua New Guinea: Bulolo.

nudiusculus Roberts, 1986: 46. (Platypus)

Distribution: Papua New Guinea: Bulolo.

obliquesectus Schedl, 1973a: 77. (Platypus)

DistRIBUTION: Papua New Guinea: Mt. Wilhelm.

obliquetruncatus Schedl, 1972c: 69. (Platypus)

DistRIBUTION: Papua New Guinea: Bulolo.

obliquus Wood, 1981: 121. (Platypus)

Distribution: Papua New Guinea: Mt. Wilhelm.

Platypus obliquesectus Schedl, 1975c: 229.

omissus Schedl, 1968a: 267. (Platypus)

DisTRIBUTION: Papua New Guinea: Eastern Highlands, Afafiningetu.

omnivorus (Lea), 1904: 104. (Crossotarsus)

DISTRIBUTION: New Guinea.

opacicauda Browne, 1983a: 565. (Platypus)

DistRibution: West Papua: Usau.

opacicaudus Browne, 1983a: 566. (Platypus)

DISTRIBUTION: West Papua: Usau.

opacideclivis Schedl, 1969b: 227. (Platypus)

DistRIBUTION: Papua New Guinea: Tari. 
opacifrons Schedl, 1936e: 515. (Platypus)

DISTRIBUTION: New Guinea; North Solomon Islands: Bougainville Island.

opacipennis Schedl, 1975c: 230. (Platypus)

DistRIBUTION: Papua New Guinea: Wau.

opacurus Browne, 1980b: 387. (Platypus)

DisTRIBUTION: West Papua: Jayapura.

opimus Schedl, 1975d: 390. (Platypus)

Distribution: Papua New Guinea: Bulolo.

ornatifrons Schedl, 1942c: 198. (Platypus)

DISTRIBUTION: New Guinea.

othiodes Schedl, 1974: 469. (Platypus)

DistRiBUtion: Papua New Guinea: Bulolo.

panduriformis Roberts, 1989b: 278. (Platypus)

Distribution: Papua New Guinea: Bulolo, Gumi.

papuanus Roberts, 1979: 92. (Platypus)

Distribution: Papua New Guinea: Mt. Giluwe.

papulosus Roberts, 1989b: 279. (Platypus)

Distribution: Papua New Guinea: Bulolo, Gumi.

paradoxus Schedl, 1979b: 115. (Platypus)

DisTRIBUTION: Papua New Guinea: Bulolo.

paralleliventrus Roberts, 1989b: 280. (Platypus)

DistribUtion: Papua New Guinea: Bulolo.

parapetax Roberts \& Morimoto, 1986: 174. (Platypus)

Distribution: Papua New Guinea: Bulolo, Gumi.

partibilis Roberts \& Morimoto, 1986: 174. (Platypus)

Distribution: Papua New Guinea: Bulolo, Gumi. patens Roberts, 1986: 46. (Platypus)

Distribution: Papua New Guinea: Popondetta.

pectinatus Roberts, 1986: 47. (Platypus)

Distribution: Papua New Guinea: Bulolo.

pedellus Schedl, 1975c: 230. (Platypus)

DistRIBUTION: Papua New Guinea: Wau.

perditus Schedl, 1975d: 391. (Platypus)

DistRIBUTION: Papua New Guinea: Bulolo.

petalinus Roberts \& Morimoto, 1986: 175. (Platypus)

Distribution: Papua New Guinea: Bulolo, Gumi.

petaloideus Roberts, 1979: 93. (Platypus)

DistribUtion: Papua New Guinea: Mt. Giluwe.

petax Schedl, 1974: 469. (Platypus)

DisTRIBUTION: Papua New Guinea: Bulolo.

pilidens Schedl, 1955: 309. (Platypus)

DistRIBUTION: Papua New Guinea: Berlinhafen.

plumatus Schedl, 1979b: 116. (Platypus)

Distribution: Papua New Guinea: Bulolo.

porcellinus Schedl, 1975d: 392. (Platypus)

DistRIBUTION: Papua New Guinea: Bulolo.

porcellus Schedl, 1942c: 209. (Platypus) 
DISTRIBUTION: New Guinea.

proicollis Schedl, 1950: 147. (Platypus)

DisTRIBUTION: New Guinea.

Platypus porosus Schedl, 1942b: 193.

porosulus Browne, 1983a: 564. (Platypus)

DistRIBUTION: West Papua: Teminaban.

praealtus Schedl, 1975d: 393. (Platypus)

DistribUtion: Papua New Guinea: Bulolo.

praecellens Schedl, 1969b: 228. (Platypus)

Distribution: Papua New Guinea: Western Highlands, Porotop.

praepositus Schedl, 1969b: 229. (Platypus)

DistRIBUTION: Papua New Guinea: Watut Valley.

praeteritus Schedl, 1969b: 230. (Platypus)

Distribution: Papua New Guinea: Western Highlands, Porotop.

praetermissus Schedl, 1979b: 117. (Platypus)

Distribution: Papua New Guinea: Bulolo.

pseudoporus Schedl, 1979b: 117. (Platypus)

Distribution: Papua New Guinea: Bulolo.

pseudoselysi Schedl, 1972c: 71. (Platypus)

DisTRIBUTION: New Guinea.

pseudosindorae Browne, 1983a: 570. (Platypus)

Distribution: West Papua: Wapoga.

pulvinatus Roberts \& Morimoto, 1986: 175. (Platypus)

DisTRIBUTION: Papua New Guinea: Bulolo.

Platypus quadricornutus Roberts, 1989b: 281.

quadrinotatus Schedl, 1975c: 231. (Platypus)

DisTRIBUTION: New Guinea.

quadrispinis Browne, 1980d: 496. (Platypus)

DistRIBUTION: West Papua: Manokwari.

quercivorus Murayama, 1925: 229. (Platypus)

DisTRIBUTION: New Guinea.

ramosissimus Roberts \& Morimoto, 1986: 176. (Platypus)

Distribution: Papua New Guinea: Bulolo, Gumi.

ramulosus Roberts \& Morimoto, 1986: 177. (Platypus)

Distribution: Papua New Guinea: Bulolo, Gumi.

rasilis Schedl, 1979b: 118. (Platypus)

Distribution: Papua New Guinea: Bulolo.

refractus Roberts \& Morimoto, 1986: 178. (Platypus)

Distribution: Papua New Guinea: Bulolo, Gumi.

runcinatus Roberts, 1986: 52. (Platypus)

Distribution: Papua New Guinea: Mt. Giluwe.

scalptor Schedl, 1975d: 394. (Platypus)

DisTRIBUTION: Papua New Guinea: Bulolo.

scrobicularis Roberts, 1979: 95. (Platypus)

Distribution: Papua New Guinea: Mt. Giluwe.

sellaeformis Roberts \& Morimoto, 1986: 178. (Platypus)

Distribution: Papua New Guinea: Bulolo, Gumi. 
selysi Chapuis, 1865: 215. (Platypus)

DistRIBUTION: West Papua: Dorey.

semiopacus Strohmeyer, 1913: 163. (Platypus)

DisTRIBUTION: Papua New Guinea: Aroa River.

sepaloideus Roberts, 1989b: 281. (Platypus)

DistRIBUTION: Papua New Guinea: Bulolo.

setaceus Chapuis, 1865: 234. (Platypus)

DisTRIBUTION: New Guinea.

shoreanus (Beeson), 1937: 98. (Crossotarsus)

Distribution: Papua New Guinea: Morobe Province. Notes: Transferred: Schedl, 1972a: 224.

Platypus shoreanus tersus Schedl, 1972c: 71.

singularis Schedl, 1975d: 394. (Platypus)

DistRIBUTION: Papua New Guinea: Bulolo.

solicitatulus Schedl, 1975d: 395. (Platypus)

Distribution: Papua New Guinea: Bulolo.

solidus Walker, 1859: 261. (Platypus)

Distribution: New Guinea; Admiralty Islands; Aru Island.

spiniventris Schedl, 1969b: 232. (Platypus)

Distribution: Papua New Guinea: Mt. Hagen, Kum.

strenuus Schedl, 1969b: 233. (Platypus)

DistRIBUTION: Papua New Guinea: Western Highlands, Togoba.

striatodeclivis Schedl, 1975d: 396. (Platypus)

DistRIBUTION: Papua New Guinea: Bulolo.

subcuratus Browne, 1983a: 563. (Platypus)

DistRIBUTION: West Papua: Usau.

subpraeteritus Schedl, 1975c: 231. (Platypus)

DistRIBUTION: Papua New Guinea: Western Highlands, Porotop.

subpronus Schedl, 1969b: 233. (Platypus)

Distribution: Papua New Guinea: Watut Valley.

sulcaticeps Schedl, 1979b: 118. (Platypus)

DistRIBUTION: Papua New Guinea: Bulolo.

sulcinodis Schedl, 1975d: 397. (Platypus)

DisTRIBUTION: Papua New Guinea: Mt. Kaindi.

takeharai Browne, 1983a: 563. (Platypus)

DISTRIBUTION: West Papua: Usau.

taxicornis Schedl, 1968b: 537. (Platypus)

Distribution: Papua New Guinea: Wau. Notes: Transferred to Treptoplatypus in Schedl, 1972a: 245

and returned in Wood, 1993: 272.

tenuisculptus Roberts, 1979: 95. (Platypus)

Distribution: Papua New Guinea: Mt. Giluwe.

ternus Roberts, 1986: 48. (Platypus)

DistRIBUTION: Papua New Guinea: Bulolo.

torulosus Roberts \& Morimoto, 1986: 179. (Platypus)

DistRIBUTION: Papua New Guinea: Bulolo.

transversus Schedl, 1979b: 119. (Platypus)

DistRIBUTION: Papua New Guinea: Bulolo.

tristis Roberts, 1986: 49. (Platypus) 
DistRIBUTION: Papua New Guinea: Bulolo.

turcicus Schedl, 1970b: 366. (Platypus)

DistRIBUTION: Papua New Guinea: Vanimo.

turriformis Roberts \& Morimoto, 1986: 180. (Platypus)

DistRIBUTION: Papua New Guinea: Bulolo.

umbrinus Roberts, 1989b: 283. (Platypus)

Distribution: Papua New Guinea: Bulolo, Gumi.

uniformis Schedl, 1969b: 234. (Platypus)

Distribution: Papua New Guinea: Western Highlands, Porotop.

ustus Schedl, 1968a: 268. (Platypus)

DistRIBUTION: Papua New Guinea: Eastern Highlands, Okapa.

uter Schedl, 1969b: 236. (Platypus)

DistRIBUTION: Papua New Guinea: Karamui.

uvarius Roberts, 1989b: 284. (Platypus)

DistRIBUTION: Papua New Guinea: Bulolo, Gumi, Watut.

vaginervis Roberts, 1986: 51. (Platypus)

Distribution: Papua New Guinea: Bulolo.

valdephlebius Roberts, 1979: 96. (Platypus)

DistribUtion: Papua New Guinea: Mt. Giluwe.

validus Schedl, 1973b: 97. (Platypus)

DistRIBUTION: Papua New Guinea: Bulolo.

varipennis Schedl, 1968a: 269. (Platypus)

DistRIBUtion: Papua New Guinea: Eastern Highlands, Afafiningetu.

vesculus Schedl, 1972b: 58. (Platypus)

Distribution: Papua New Guinea: Karamui.

viaticus Schedl, 1974: 470. (Platypus)

Distribution: Papua New Guinea: Bulolo.

vicarius Schedl, 1975c: 232. (Platypus)

DistRIBUTION: Papua New Guinea: Wau.

volaticus Schedl, 1936b: 35. (Platypus)

Distribution: New Guinea; Bismarck Archipelago: New Britain, New Ireland; North Solomon Islands:

Bougainville Island.

watutensis Roberts, 1986: 53. (Platypus)

DistRIBUTION: Papua New Guinea: Bulolo.

TrePtoplatyPuS Schedl, 1939b: 401

TYPE-SPECIES: Crossotarsus trepanatus Chapuis, 1865; subsequent designation by Wood, 1992b: 91.

micrurus (Schedl), 1951: 69. (Platypus)

DistRIBUTION: Bismarck Archipelago: New Britain, Rabaul. NotES: Transfered: Wood, 1993: 272.

Platypus longecaudatus Nunberg, 1961: 623. NOTES: Synonymy: Schedl, 1972: 198.

multiporus Schedl, 1968a: 270. (Treptoplatypus)

Distribution: Papua New Guinea: Eastern Highlands, Okapa.

Platypus fastuosus Schedl, 1969: 226.

solidus (Walker), 1859: 261. (Platypus)

DistRIBUTION: New Guinea; Aru Islands. NotEs: Transfered: Wood, 1993: 272. 


\section{TESSEROCERINI}

\section{DIAPODINA}

\section{DIAPUS Chapuis, 1865: 329}

TYPE-SPECIES: Diapus quadrispinatus Chapuis, 1865; subsequent designation by Hopkins, 1914: 121. KeY: Roberts, 1993: 5.

amblylaminatus Roberts, 1993: 8. (Diapus)

DistRIBUTION: Papua New Guinea: Morobe Province.

angustidontus Roberts, 1993: 8. (Diapus)

DistRIBUTION: Papua New Guinea: Gumi.

bilunatus Schedl, 1975d: 399. (Diapus)

Distribution: Papua New Guinea: Mt. Missim.

bispinus Schedl, 1974: 471. (Diapus)

DistRIBUTION: Papua New Guinea: Watut Valley.

elongatus Schedl, 1969b: 225. (Diapus)

DistRIBUTION: New Guinea: Marafunga.

kuperi Roberts, 1993: 12. (Diapus)

DistRIBUTION: Papua New Guinea: Kuper Range.

latespinis Schedl, 1979b: 120. (Diapus)

Distribution: Papua New Guinea: Bulolo.

nanodontus Roberts, 1993: 13. (Diapus)

Distribution: Papua New Guinea: Morobe Province.

nanus Schedl, 1969b: 225. (Diapus)

DISTRIBUTION: Papua New Guinea.

nebulosus Roberts, 1993: 15. (Diapus)

DistRIBUTION: Papua New Guinea: Gumi.

oomsis Roberts, 1993: 16. (Diapus)

DistRIBUtion: Papua New Guinea: Morobe Province.

oreogenus Roberts, 1993: 17. (Diapus)

DisTRIBUTION: Papua New Guinea: Morobe Province.

papuanus Schedl, 1968a: 269. (Diapus)

DistRIBUTION: Papua New Guinea: Eastern Highlands, Afafiningetu.

perpygmaeus Roberts, 1993: 20. (Diapus)

DistRIBUTION: Papua New Guinea: Morobe Province.

pusillimus Chapuis, 1865: 335. (Diapus)

Distribution: Papua New Guinea: Angorum, Vanimo, Lake Kutubu, Karkar Island, Mt. Guiluwe,

Bulolo; D'Entrecasteaux Islands: Fergusson Island; Bismarck Archipelago: New Britain; West Papua:

Dorey.

Crossotarsus grevilleae Lea, 1914: 226.

quinquespinatus Chapuis, 1865: 334. (Diapus)

DisTRIBUTION: New Guinea.

robustus Schedl, 1969b: 225. (Diapus)

DistRIBUtion: Papua New Guinea: Kum near Mt. Hagen.

spinifer Schedl, 1969b: 224. (Diapus) 
DistRIBUTION: Papua New Guinea: Porotop.

turgidus Roberts, 1993: 24. (Diapus)

DistRIBUTION: Papua New Guinea: Morobe Province.

unispineus Roberts, 1993: 25. (Diapus)

DistRIBUTION: Papua New Guinea: Kuper Range, Wau.

GENYOCERUS Motschulsky, 1858: 68

TYPE-SPECIES: Genyocerus albipennis Motschulsky, 1858; monobasic. KEY: Roberts, 1993: 27.

papuanus Roberts, 1993: 27. (Genyocerus)

DisTRIBUTION: Papua New Guinea: Wawoi: Guavi.

puer (Schedl), 1969b: 223. (Diapus)

DistRIBUTION: Papua New Guinea: Milne Bay.

Diacavus trispinatus Schedl, 1974: 471. NOTES: Synonymy: Roberts, 1993: 28.

TESSEROCERINA

NotoplatyPuS Lea, 1910a: 135

TYPE-SPECIES: Notoplatypus elongatus Lea, 1910a; monobasic.

elongatus Lea, 1910a: 135. (Notoplatypus)

DistRIBUTION: [Australia]; New Guinea (?).

SPATHIDICERUS Chapuis, 1865: 42

TYPE-SPECIES: Spathidicerus thomsoni Chapuis, 1865; subsequent designation by Hopkins, 1914: 129.

nobilis Chapuis, 1865: 315. (Spathidicerus)

DISTRIBUTION: New Guinea.

Spathidicerus intermedius Schedl, 1936e: 519. DistRIBUTION: Papua New Guinea: Mt. Lamington. Notes:

Synonymy: Schedl, 1972a: 258.

papuanus Schedl, 1935b: 276. (Spathidicerus)

DistRIBUTION: Papua New Guinea: Kokoda.

thomsoni Chapuis, 1865: 314. (Spathidicerus)

DisTRIBUTION: New Guinea.

\section{SCOLYTIDINAE}

\section{HYLESININI}

\section{DIAMERINA}

ACACACIS Lea, 1910a: 149

TYPE-SPECIES: Acacacis abundans Lea, 1910a; monobasic.

bicornis Wood, 1988a: 34. (Acacacis)

Distribution: Papua New Guinea: Bulolo. 
TYPE-SPECIES: Hylesinus hispidus Klug, 1833; monobasic.

brevicollis Eggers, 1923: 131. (Diamerus)

DisTRIBUTION: Papua New Guinea: Moroka.

curvifer (Walker), 1859: 261. (Hylesinus)

DISTRIBUTION: New Guinea.

granifer Browne, 1984d: 87. (Diamerus)

DistRIBUTION: Papua New Guinea: Popondetta.

interstitialis (Lea), 1910a: 145. (Hylesinus)

DisTRIBUTION: New Guinea.

\section{HYLESININA}

DACTYLIPALPUS Chapuis, 1869: 12

TYPE-SPECIES: Dactylipalpus transversus Chapuis, 1869; subsequent designation by Hopkins, 1914: 120.

transversus Chapuis, 1869: 12. (Dactylipalpus)

DistRIBUTION: Papua New Guinea; Goodenough Island.

FICICIS Lea, 1910a: 147

TYPE-SPECIES: Ficicis varians Lea, 1910a; subsequent designation by Hopkins, 1914: 122.

brevipilosus (Schedl), 1942b: 167. (Hylesinus)

DISTRIBUTION: New Guinea.

despectus (Walker), 1859: 261. (Hylesinus)

DISTRIBUTION: New Guinea.

maculipennis (Schedl), 1975c: 217. (Hylesinus)

DistRIBUTION: Papua New Guinea: Porotop.

pertinax (Schedl), 1975c: 217. (Hylesinus)

DISTRIBUTION: West Papua: Vogelkop.

philippinensis (Eggers), 1923: 137. (Hylesinus)

DISTRIBUTION: New Guinea.

sulcinodis (Schedl), 1974: 458. (Hylesinus)

DistRIBUTION: Papua New Guinea: Watut Valley.

wallacei (Blandford), 1896b: 197. (Hylesinus)

DisTRIBUTION: Mysol Island.

HYLESINUS Fabricius, 1801: 390

TYPE-SPECIES: Bostrichus crenatus Fabricius, 1787; subsequent designation by Westwood, 1838: 39.

cordipennis Lea, 1910a: 144. (Hylesinus)

DisTRIBUTION: Papua New Guinea: Yule Island. 
Hylesinus papuanus Eggers, 1923: 133. NOTES: Synonymy: Wood, 1985: 268.

nanulus Schedl, 1940: 433. (Hylesinus)

Distribution: Papua New Guinea: Kokoda.

niligrinus Eggers, 1923: 133. (Hylesinus)

DisTRIBUTION: New Guinea.

regius (Schedl), 1942b: 166. (Leperisinus)

DisTRIBUTION: New Guinea.

\section{HYORRHYNCHINA}

SuEUS Murayama, 1951: 1

TYPE-SPECIES: Sueus sphareotrypoides Murayama, 1951 [=Hyorrhynchus niisimai Eggers, 1926]; original designation.

niisimai (Eggers), 1926: 133. (Hyorrhynchus)

DisTRIBUTION: New Guinea.

\section{PHLOEOSININA}

Cladoctonus Strohmeyer, 1911: 17

TYPE-SPECIES: Cladoctonus affinis Strohmeyer, 1911; monobasic.

contractus Schedl, 1975d: 340. (Cladoctonus)

DisTRIBUTION: Papua New Guinea: Bulolo.

HYLEDIUS Sampson, 1921: 35

TYPE-SPECIES: Hylesius asper Sampson, 1921 [=Olonthogaster nitidicollis Motschulsky, 1866: 401]; monobasic.

nitidicollis (Motschulsky), 1866: 401. (Olonthogaster)

DisTRIBUTION: Papua New Guinea: Yule Island.

Phloeosinus vagans Eggers, 1923: 139. NotES: Synonymy: Schedl, 1958: 152.

papuanus (Schedl), 1936e: 521. (Phloeosinus)

DistRIBUTION: Papua New Guinea: Mt. Lamington.

PhlOeOSINOPSIOIDES Schedl, 1964c: 317

TYPE-SPECIES: Phloeosinopsis triseriatus Schedl, 1964d; original designation.

pumilus Wood, 1985: 274. (Phloeosinopsioides)

DisTRIBUTION: Papua New Guinea: Bulolo.

triseriatus (Schedl), 1964d: 297. (Phloeosinopsis)

DisTRIBUTION: Papua New Guinea: Bulolo.

Xylechinus papuanus Schedl, 1970a: 128. Distribution: Papua New Guinea: Long Island, Bulolo.

Phloeosinus Chapuis, 1869: 37

TYPE-SPECIES: Hylesinus thujae Perris, 1855; subsequent designation by Hopkins, 1914: 126. 
granulipennis Schedl, 1975c: 218. (Phloeosinus)

DistRIBUTION: Papua New Guinea: Wau.

\section{PHLOEOTRIBINA}

\section{ARICERUS Blandford, 1894b: 133}

TYPE-SPECIES: Aricerus chapuisi Blandford, 1894b: 134; subsequent designation by Hopkins, 1914: 117.

analis Schedl, 1975c: 216. (Aricerus)

DISTRIBUTION: Bismarck Archipelago: New Britain.

eichhoffi Blandford, 1894b: 135. (Aricerus)

DISTRIBUTION: New Guinea.

\section{TOMICINA}

Chaetoptelius Fuchs, 1913: 43

TYPE-SPECIES: Hylesinus vestitus Mulsant \& Rey, 1860; Automatic.

impar (Schedl), 1975c: 216. (Hylesinus)

Distribution: Papua New Guinea: Porotop.

opimus (Wood), 1985: 270. (Acrantus)

DISTRIBUTION: West Papua: Vogelkop.

HYLURDRECTONUS Schedl, 1938a: 40

TYPE-SPECIES: Hylurdrectonus piniarius Schedl, 1938a; monobasic.

araucariae Schedl, 1964b: 213. (Hylurdrectonus)

DisTRIBUTION: Papua New Guinea: Wau.

corticinus Wood, 1980: 94. (Hylurdrectonus)

Distribution: Papua New Guinea: Bulolo.

Xylogpinus araucaria Schedl, 1972c: 64. NotES: Synonymy: Wood, 1980: 94.

nanus Browne, 1984d: 87. (Hylurdrectonus)

Distribution: Papua New Guinea.

piniarius Schedl, 1938: 40. (Hylurdrectonus)

DISTRIBUTION: New Guinea.

\section{PACHYCOTES Sharp, 1877: 10}

TYPE-SPECIES: Pachycotes ventralis Sharp, 1877 [=Hylastes peregrinus Chapuis, 1869]; monobasic.

araucariae Schedl, 1975d: 340. (Pachycotes)

DistRIBUTION: Papua New Guinea: Bulolo. 


\section{SCOLYTINI}

\section{CORTHYLINA}

Mimiocurus Schedl, 1957: 72

TYPE-SPECIES: Mimiocurus acuminatus Schedl, 1957; monobasic.

orientalis (Schedl), 1972b: 51. (Mimiophthorus)

DisTRIBUTION: New Guinea.

rosseli (Schedl), 1973b: 88. (Taphroterus)

Distribution: Papua New Guinea: Rossel Island.

\section{CRYPHALINA}

COSMODERES Eichhoff, 1878: 495

TYPE-SPECIES: Cosmoderes monilicollis Eichhoff, 1878; monobasic.

anticus (Schedl), 1975d: 343. (Erioschidias)

DisTRIBUTION: Papua New Guinea: Bulolo.

elegans Schedl, 1975d: 342. (Cosmoderes)

DiSTRIBUTION: Papua New Guinea: Bulolo.

papuanus Schedl, 1975d: 341. (Cosmoderes)

DiSTRIBUTION: New Guinea: Kwargira River.

CryPhalus Erichson, 1836: 61

TYPE-SPECIES: Bostrichus asperatus Gyllenhal, 1813; subsequent designation by Thomson,1859: 147.

araucariae Schedl, 1969b: 214. (Cryphalus)

DistRIBUTION: Papua New Guinea: Bulolo.

armatus Schedl, 1974: 459. (Cryphalus)

DistRIBUtion: Papua New Guinea: Wau.

ater Browne, 1984d: 91. (Cryphalus)

Distribution: Papua New Guinea: Gumi.

bicolor (Browne), 1983b: 69. (Cryphalomorphus)

DistRIBUTION: Papua New Guinea: Wau.

brevipilosus Schedl, 1942b: 173. (Cryphalus)

DISTRIBUTION: New Guinea.

brunneus Browne, 1984d: 93. (Cryphalus)

DistRIBUTION: Papua New Guinea.

buloloensis Browne, 1983b: 67. (Cryphalus)

DistRIBUTION: Papua New Guinea: Bulolo.

cylindricus Browne, 1984d: 92. (Cryphalus)

DistRIBUTION: Papua New Guinea: Bulolo.

diversicolor Browne, 1984d: 90. (Cryphalus)

DISTRIBUTION: Papua New Guinea. 
erraticum Schedl, 1979b: 96. (Cryphalus)

DisTRIBUTION: Papua New Guinea: Bulolo.

fugax Schedl, 1973b: 87. (Cryphalus)

DisTRIBUTION: Louisaide Archipelago: Sudest Island.

gigas Schedl, 1979b: 218. (Cryphalus)

DistRIBUTION: Papua New Guinea: Wau.

kolbei (Hagedorn), 1912: 355. (Allarthrum)

DISTRIBUTION: New Guinea.

laticollis Browne, 1984b: 288. (Cryphalus)

DistRIBUTION: New Guinea; Bismarck Archipelago: New Britain.

longior Browne, 1984d: 91. (Cryphalus)

DistRIBUTION: New Guinea: Kaisenik.

nitidipennis Browne, 1984d: 89. (Cryphalus)

DisTRIBUTION: Papua New Guinea.

nothofagi Browne, 1983b: 68. (Cryphalus)

DistRIBUTION: Papua New Guinea: Mt. Kaindi.

pilosulus Browne, 1980b: 385. (Cryphalus)

DISTRIBUTION: New Guinea.

punctatus Browne, 1983b: 66. (Cryphalus)

DisTRIBUTION: Papua New Guinea: Wau.

pusillimus Schedl, 1942b: 171. (Cryphalus)

DisTRIBUTION: New Guinea.

securus (Schedl), 1940: 436. (Ericryphalus)

DistRIBUTION: West Papua: Cyclops Mountains.

striatulus Browne, 1981: 127. (Cryphalus)

DISTRIBUTION: Bismarck Archipelago: New Britain.

subtuberculatus Schedl, 1942b: 168. (Cryphalus)

DisTRIBUTION: Papua New Guinea: Mt. Lamington.

terminaliae Browne, 1980b: 384. (Cryphalus)

Distribution: Papua New Guinea; Bismarck Archipelago: New Britain.

tetricus (Schedl), 1940: 437. (Ericryphalus)

Distribution: Papua New Guinea: Bulolo, Mt. Lamington; West Papua: Dorey, Cyclops Mountains.

Cryphalus papuanus Schedl, 1942b: 170. NotES: Synonymy: Schedl, 1979b: 95.

Cryphalus grayi Schedl, 1968a: 265. NOTES: Synonymy: Schedl, 1979b: 95.

Ptilopodius brevis Browne, 1970: 556. NOTES: Synonymy: Schedl, 1979b: 95.

wapleri Eichhoff, 1872: 131. (Cryphalus)

Distribution: Papua New Guinea: Milne Bay Province.

Cryphalus mekeoi Schedl, 1972c: 61. NOTES: Synonymy: Schedl, 1979c: 126.

EIDOPHELUS Eichhoff, 1875: 200

TYPE-SPECIES: Eidophelus imitans Eichhoff, 1875; monobasic.

gracilis Browne, 1984a: 152. (Eidophelus)

DISTRIBUTION: Bismarck Archipelago: New Britain. 
TYPE-SPECIES: Bostrichus tiliae Panzer, 1793; original designation.

antennarius Schedl, 1974: 461. (Ernoporus)

DisTRIBUTION: Papua New Guinea: Bulolo.

Euptilius papuanus Browne, 1983b: 70. NOTES: Synonymy: Wood, 1989: 172.

dispar (Schedl), 1972b: 49. (Cryphalops)

DISTRIBUTION: Bismarck Archipelago: New Ireland: Karbil.

papuanus (Schedl), 1973b: 87. (Margadillius)

DistRIBUTION: Papua New Guinea: Bulolo.

HYPOCRYPHALUS Hopkins, 1915a: 8

TYPE-SPECIES: Hypocryphalus rotundus Hopkins, 1915a; original designation.

laevis Browne, 1980a: 374. (Hypocryphalus)

DISTRIBUTION: Batanta Island.

longipilis Browne, 1981a: 128. (Hypocryphalus)

DISTRIBUTION: Bismarck Archipelago: New Britain.

montanus Schedl, 1974: 460. (Hypocryphalus)

Distribution: Papua New Guinea: Mt. Kaindi.

nitidicollis Schedl, 1975c: 219. (Hypocryphalus)

DistRIBUTION: New Guinea; Bismarck Archipelago: New Britain.

perminimus (Schedl), 1942a: 13. (Cryphalus)

DisTRIBUTION: New Guinea; Bismarck Archipelago: New Britain.

Cryphalus constrictus Schedl, 1942b: 174. Notes: Synonymy: Kalshoven, 1958: 164.

Hypocryphalus froggatti Nunberg, 1961: 611. NOTES: Synonymy: Schedl, 1962c: 697.

pilifer Schedl, 1979b: 98. (Hypocryphalus)

Distribution: Papua New Guinea: Mt. Kaindi, Wau.

reflexus Browne, 1980b: 383. (Hypocryphalus)

DISTRIBUTION: Bismarck Archipelago: New Britain.

triangularis Schedl, 1975: 351. (Hypocryphalus)

Distribution: Papua New Guinea: Bulolo.

HYPOTHENEMUS Westwood, 1836: 34

TYPE-SPECIES: Hypothenemus eruditus Westwood, 1836; monobasic.

birmanus (Eichhoff), 1878: 486. (Triarmocerus)

DistRIBUTION: West Papua: Andai.

Stephanoderes uter Eggers, 1923: 219. NOTES: Synonymy: Browne, 1970: 556.

inermis Browne, 1984d: 94. (Hypothenemus)

DistRIBUTION: New Guinea: Kaisenik.

parvulus Browne, 1984d: 93. (Hypothenemus)

DisTRIBUTION: Papua New Guinea.

spinosus (Schedl), 1979b: 98. (Lepiceroides)

DistRIBUTION: Papua New Guinea: Bulolo. 
MARGADILLIUS Hopkins, 1915a: 8

TYPE-SPECIES: Margadillius margadilaonis Hopkins, 1915a; original designation.

carinatus Browne, 1980b: 382. (Margadillius)

DisTRIBUTION: Bismarck Archipelago: New Britain.

concentralis Schedl, 1975d: 344. (Margadillius)

DisTRIBUTION: Papua New Guinea: Bulolo.

fulvus Browne, 1984b: 289. (Margadillius)

DISTRIBUTION: West Papua: Fakfak.

magnus Browne, 1984d: 88. (Margadillius)

Distribution: Papua New Guinea: Mt. Giluwe.

PTILOPODIUS Hopkins, 1915a: 7

TYPE-SPECIES: Ptilopodius stephegynis Hopkins, 1915a; original designation.

bambusae Browne, 1983b: 69. (Ptilopodius)

DISTRIBUTION: Papua New Guinea: Wau.

SCOLYTOGENES Eichhoff, 1878: 475

TYPE-SPECIES: Scolytogenes darwini, Erichhoff, 1878; monobasic.

absonus (Schedl), 1975d: 344. (Cryphalomorphus)

Distribution: Papua New Guinea: Bulolo.

alternans (Schedl), 1975d: 345. (Cryphalomorphus)

DistRIBUTION: Papua New Guinea: Bulolo.

ankius (Schedl), 1979b: 96. (Cryphalomorphus)

Distribution: Papua New Guinea: Bulolo.

approximatus (Schedl), 1975d: 346. (Cryphalomorphus)

DistRIBUTION: Papua New Guinea: Bulolo.

cicatricosus (Schedl), 1942b: 176. (Lepicerinus)

DISTRIBUTION: New Guinea.

concentralis (Schedl), 1975d: 342. (Cryphalomorphus)

DisTRIBUTION: Papua New Guinea: Bulolo.

creber (Schedl), 1975d: 346. (Cryphalomorphus)

DistRIBUTION: Papua New Guinea: Bulolo.

darwini Eichhoff, 1878: 497. (Scolytogenes)

DisTRIBUTION: New Guinea.

excellens (Schedl), 1979b: 97. (Cryphalomorphus)

Distribution: Papua New Guinea.

fugax (Schedl), 1975d: 347. (Cryphalomorphus)

Distribution: Papua New Guinea: Bulolo.

fulgens (Schedl), 1975d: 348. (Cryphalomorphus)

Distribution: Papua New Guinea: Bulolo.

fulgidus (Schedl), 1975d: 348. (Cryphalomorphus) 
DistRIBUTION: Papua New Guinea: Bulolo.

hylesinopsis (Schedl), 1975d: 349. (Cryphalomorphus)

DisTRIBUTION: Papua New Guinea: Wau.

mus (Schedl), 1975d: 349. (Cryphalomorphus)

DistRIBUTION: Papua New Guinea: Wau.

papuanus (Schedl), 1974: 459. (Cryphalomorphus)

Distribution: Papua New Guinea: Morobe Province.

papuensis Wood, 1989: 179. (Scolytogenes)

Distribution: Papua New Guinea: Bulolo.

Xylocryptus papuanus Schedl, 1975d: 352.

paradoxus Wood, 1992a: 80. (Scolytogenes)

Distribution: Papua New Guinea.

Cryphalomorphus papuanus Schedl, 1979b: 97.

quadridens (Browne), 1983b: 71. (Hypothenemus)

DistRIBUTION: Papua New Guinea: Wau.

splendens (Schedl), 1975d: 350. (Cryphalomorphus)

DisTRIBUTION: Papua New Guinea: Bulolo.

squamosus (Schedl), 1942b: 175. (Lepicerinus)

DisTRIBUTION: New Guinea.

varius (Schedl), 1975: 350. (Cryphalomorphus)

DisTRIBUTION: Papua New Guinea: Bulolo.

\section{DRYOCOETINA}

COCCOTRYPES Eichhoff, 1878: 308

TYPE-SPECIES: Bostrichus dactyliperda Fabricius, 1801; subsequent designation by Hopkins, 1914: 118.

abruptulus (Schedl), 1975e: 347. (Poecilips)

Distribution: Papua New Guinea: Kiunga.

abruptus (Schedl), 1979b: 99. (Poecilips)

DistRIBUtion: Papua New Guinea: Kiunga.

aciculatus Schedl, 1952: 360. (Coccotrypes)

DisTRIBUTION: New Guinea.

advena Blandford, 1894a: 100. (Coccotrypes)

DistRIBUTION: New Guinea.

chimbui (Schedl), 1975d: 354. (Poecilips)

Distribution: Papua New Guinea: Huon Gulf, Sattelberg, Bulolo.

creber (Schedl), 1955: 290. (Poecilips)

Distribution: Papua New Guinea: Huon Gulf, Sattelberg.

dactyliperda (Fabricius), 1801: 387. (Bostrichus)

DisTRIBUTION: New Guinea.

distinctus (Motschulsky), 1866: 403. (Anodius)

DistRIBUTION: New Guinea.

fulgens (Schedl), 1979b: 99. (Poecilips)

DisTRIBUTION: Papua New Guinea: Bulolo.

longicollis (Eggers), 1927b: 397. (Thamnurgides) 
Distribution: West Papua.

minimus (Schedl), 1955: 291. (Poecilips)

Distribution: Papua New Guinea: Astrolabe Bay.

minutissimus (Schedl), 1955: 292. (Poecilips)

Distribution: Papua New Guinea: Astrolabe Bay, Erima Bay.

minutus Schedl, 1975e: 347. (Coccotrypes)

DistRIBUTION: Bismarck Archipelago: New Britain, Rabaul.

morokensis (Eggers), 1923: 151. (Dendrurgus)

DisTRIBUTION: Papua New Guinea: Moroka.

myristicae (Roepke), 1919: 23. (Thamnurgides)

DISTRIBUTION: New Guinea.

nitidus (Eggers), 1923: 147. (Dendrurgus)

DiSTRIBUTION: New Guinea.

omissus Schedl, 1979b: 101. (Coccotrypes)

DistRiBUtion: Papua New Guinea: Bulolo.

papuanus (Eggers), 1923: 148. (Dendrurgus)

DistRIBUTION: West Papua: Andai.

Thamnurgides glandis (Beeson), 1939: 287. NOTES: Synonoymy: Schedl, 1960: 105.

pterydophytae (Schedl), 1968a: 266. (Poecilips)

Distribution: Papua New Guinea: Bulolo.

queenslandi (Schedl), 1942b: 180. (Poecilips)

DisTRIBUTION: New Guinea.

regularis (Schedl), 1955: 292. (Poecilips)

Distribution: Papua New Guinea: Sattelberg.

rhizophorae (Hopkins), 1915a: 48. (Spermatoplex)

DistRIBUTION: New Guinea. NOTES: Range extention: Bright \& Skidmore, 1997: 132.

robustulus Wood, 1989: 178. (Poecilips robustus)

DisTRIBUTION: New Guinea.

Poecilips robustus Schedl, 1972d: 227.

similis (Eggers), 1923: 148. (Dendrurgus)

Distribution: West Papua.

sparserugosus (Schedl), 1972c: 64. (Poecilips)

DistRIBUTION: Papua New Guinea: Sattelberg.

spinipennis (Schedl), 1955: 293. (Poecilips)

DisTRIBUTION: Papua New Guinea: Astrolabe Bay.

squamifer (Schedl), 1975d: 355. (Poecilips)

DisTRIBUTION: D'Entrecasteaux Islands: Normanby Island.

suaui (Schedl), 1973a: 70. (Poecilips)

Distribution: Papua New Guinea: Milne Bay Province.

subacuminatus (Eggers), 1927b: 399. (Poecilips)

DISTRIBUTION: New Guinea.

subvulgaris (Browne), 1966: 246. (Poecilips)

DisTRIBUTION: Admiralty Islands: Manus Island.

vulgaris (Eggers), 1923: 151. (Dendrurgus)

DistriBUtion: Papua New Guinea; Bismarck Archipelago: New Britain. 
TYPE-SPECIES: Cyrtogenius bicolor Strohmeyer, 1910; monobasic.

acuminatus (Schedl), 1975c: 221. (Eidophelus)

Distribution: Papua New Guinea: Mt. Missim, Wau.

aequalis (Schedl), 1979b: 100. (Ozodendron)

DistRIBUTION: Papua New Guinea: Bulolo.

affinis (Schedl), 1940: 439. (Pelicerus)

DistRIBUTION: Papua New Guinea: Kokoda.

alternantes Schedl, 1975d: 353. (Cyrtogenius)

Distribution: Papua New Guinea: Bulolo.

anisopterae (Browne), 1985b: 291. (Ozodendron)

Distribution: West Papua.

aries (Schedl), 1969a: 158. (Artepityophthorus)

DistRIBUTION: Papua New Guinea: Wau.

brevior (Eggers), 1927a: 86. (Pelicerus)

DistRIBUtIOn: New Guinea. Notes: Range extension: Bright \& Skidmore, 1997: 124.

curtus (Schedl), 1973a: 71. (Ozodendron)

DisTRIBUTION: D'Entrecasteaux Islands: Normanby Island, Waikaiuna.

cyclopus (Schedl), 1940: 439. (Pelicerus)

DistRIBUTION: West Papua: Cyclops Mountains.

declivis (Schedl), 1955: 294. (Carposinus)

DistRIBUTION: Papua New Guinea: Friedrich: Wilhelmshafen.

elongatulus Wood, 1988b: 197. (Cyrtogenius)

Distribution: Papua New Guinea.

festivus Schedl, 1975c: 220. (Cyrtogenius)

DistRIBUTION: Papua New Guinea: Mt. Wilhelm.

gracilis (Schedl), 1974: 462. (Ozodendron)

Distribution: Papua New Guinea: Bulolo.

gracillimus Wood, 1988b: 197. (Cyrtogenius)

DISTRIBUTION: New Guinea.

granistriatus (Schedl), 1942b: 180. (Orosiotes)

DISTRIBUTION: New Guinea.

hirtus (Eggers), 1923: 162. (Dryocoetes)

DisTRIBUTION: West Papua.

hornus (Schedl), 1975d: 356. (Eidophelus)

Distribution: Papua New Guinea: Bulolo. Notes: Transferred: Wood, 1983: 15.

inermis Browne, 1983b: 72. (Cyrtogenius)

Distribution: Papua New Guinea: Wau.

laevis (Browne), 1984b: 289. (Ozodendron)

DisTRIBUTION: West Papua: Fakfak.

lineatopunctatus (Eggers), 1927b: 401. (Xyleborus)

DisTRIBUTION: New Guinea. NOTES: Transferred: Wood, 1989: 172.

minor (Eggers), 1923: 218. (Pelicerus)

DistRIBUTION: West Papua: Cyclops Mountains.

Pelicerus minor robustus Schedl, 1940: 438. 
nitidus (Hagedorn), 1910b: 1. (Lepicerus)

DisTRIBUTION:

Pelicerus nitidus orientalis Eggers, 1923: 217. DisTRIBUTION: Salawatti Island.

papuae Wood, 1988b: 197. (Pelicerus papuanus)

DisTRIBUTION: West Papua.

Pelicerus papuanus Eggers, 1923: 217.

papuanus (Eggers), 1923: 162. (Dryocoetes)

DisTRIBUTION: West Papua: Ansus.

papuensis Wood, 1988b: 197. (Eidophelus papuanus)

DistRIBUTION: Papua New Guinea: Mt. Dayman.

Cyrtogenius papuanus (Schedl), 1973: 71. NOTES: Transferred: Wood, 1983: 15.

parvus Browne, 1980d: 495. (Cyrtogenius)

DisTRIBUTION: West Papua.

peregrinus Schedl, 1972b: 50. (Cyrtogenius)

Distribution: West Papua.

preparvus Browne, 1986c: 664. (Cyrtogenius)

DISTRIBUTION: New Guinea.

scabricollis Browne, 1980b: 385. (Cyrtogenius)

DISTRIBUTION: Bismarck Archipelago: New Britain.

sedlaceki (Schedl), 1975d: 353. (Ozodendron)

DisTRIBUTION: West Papua.

subgranosus (Schedl), 1973a: 72. (Eidophelus)

DisTRIBUTION: D’Entrecasteaux Islands: Normanby Island. NOTES: Transferred: Wood, 1983: 15.

Eidophelus subgranosus affinis Schedl, 1973a: 73. NOTES: Transferred: Wood, 1983: 15.

subsulcatus (Schedl), 1979b: 103. (Eidophelus)

Distribution: Papua New Guinea: Central Province, Baipaa.

sulcatus (Schedl), 1979b: 103. (Eidophelus)

DisTRIBUTION: Papua New Guinea: Bulolo.

tuberculatus (Schedl), 1973a: 72. (Eidophelus)

DistRibution: Papua New Guinea: Mt. Dayman.

unicus (Schedl), 1975d: 355. (Eidophelus)

DisTRIBUTION: Papua New Guinea: Bulolo.

DRYOCOETIOPS Schedl, 1957: 13

TYPE-SPECIES: Ozopemon laevis Strohmeyer, 1911; original designation.

coffeae (Eggers), 1923: 161. (Dryocoetes)

DISTRIBUTION: New Guinea.

inopinatus (Schedl), 1955: 294. (Dryocoetes)

DISTRIBUTION: New Guinea.

nitidus (Schedl), 1942c: 179. (Dryocoetes)

DISTRIBUTION: New Guinea.

OZOPEMON Hagedorn, 1908: 382

TYPE-SPECIES: Ozopemon regius Hagedorn, 1908; monobasic. 
augustae Eggers, 1923: 159. (Ozopemon)

Distribution: Papua New Guinea; Bismarck Archipelago: New Britain; West Papua.

giganteus Schedl, 1934: 38. (Ozopemon)

DISTRIBUTION: New Guinea.

granulatus Schedl, 1936e: 526. (Ozopemon)

DistRIBUTION: Papua New Guinea: Finschhafen.

grossepunctatus Eggers, 1923: 155. (Ozopemon)

DISTRIBUTION: New Guinea.

obanus Hagedorn, 1910b: 3. (Ozopemon)

DistRIBUTION: Papua New Guinea; Bismarck Archipelago: New Britain.

papuanus Eggers, 1923: 157. (Ozopemon)

DisTRIBUTION: New Guinea.

uniseriatus Eggers, 1923: 158. (Ozopemon)

DisTRIBUTION: Papua New Guinea: Moroka.

Peridryocoetes Wood, 1984: 230

TYPE-SPECIES: Ozodendron nitens Schedl, 1964a; original designation.

squamipennis Schedl, 1979b: 101. (Peridryocoetes)

DistRIBUTION: Papua New Guinea: Kiunga.

IPINA

ACANTHOTOMICUS Blandford, 1894a: 89

TYPE-SPECIES: Acanthotomicus spinosus Blandford, 1894a; monobasic.

celtis (Schedl), 1955: 295. (Ips)

DISTRIBUTION: New Guinea.

craterigerus (Nunberg), 1961: 621. (Orthotomicus)

DISTRIBUTION: Bismarck Archipelago: New Ireland: Namatanai.

eximius (Schedl), 1955: 296. (Ips)

DISTRIBUTION: New Guinea.

fici Browne, 1984a: 154. (Acanthotomicus)

Distribution: Papua New Guinea.

grandis Browne, 1986c: 664. (Acanthotomicus)

DisTRIBUTION: New Guinea.

inclinans (Schedl), 1972b: 51. (Ips)

DisTRIBUTION: New Guinea: Bodem.

perexiguus (Blandford), 1896b: 201. (Tomicus)

DisTRIBUTION: Papua New Guinea.

Acanthotomicus denticulus Eggers, 1923: 163. NoTES: Synonoymy: Schedl, 1969c: 101.

\section{SCOLYTOPLATYPODINA}

SCOLYTOPLATYPUS Schaufuss, 1891: 31

TYPE-SPECIES: Scolytoplatypus permirus Schaufuss, 1891; monobasic. 
papuanus Eggers, 1923: 165. (Scolytoplatypus)

DisTRIBUTION: New Guinea.

Scolytoplatypus setosus Schedl, 1942b: 192. NoteS: Synonymy: Schedl, 1975: 217.

\section{XYLEBORINA}

Amasa Lea, 1893: 322

TYPE-SPECIES: Amasa thoracica Lea, 1893 [=Tomicus truncatus Erichson, 1842: 212]; monobasic.

aglaiae (Browne), 1984a: 156. (Xyleborus)

DisTRIBUTION: Bismarck Archipelago: New Britain. NoTEs: Transferred: Beaver, 1995: 202

anomalus (Schedl), 1955: 298. (Xyleborus)

DISTRIBUTION: New Guinea.

cylindriformis (Schedl), 1942b: 190. (Xyleborus)

DISTRIBUTION: New Guinea.

fulgens (Schedl), 1975: 365. (Xyleborus)

DistRIBUTION: Papua New Guinea: Bulolo.

mixtus (Schedl), 1979b: 108. (Xyleborus)

DistRIBUTION: Papua New Guinea: Bulolo.

nakazawai (Browne), 1984a: 156. (Xyleborus)

DisTRIBUTION: Bismarck Archipelago: New Britain.

sirambeanus (Eggers), 1923: 169. (Xyleborus)

Distribution: New Guinea. Notes: Range extension: Bright \& Skidmore, 1997: 148.

tereticollis (Schedl), 1951: 82. (Xyleborus)

DiSTRIBUTION: New Guinea.

truncatiformis (Eggers), 1923: 170. (Xyleborus)

DistRIBUTION: Papua New Guinea: Moroka.

umbratulus (Schedl), 1975c: 221. (Xyleborus)

DISTRIBUTION: Bismarck Archipelago: New Britain.

\section{AMBrosiodmus Hopkins, 1915a: 55}

TYPE-SPECIES: Xyleborus tachygraphus Zimmermann, 1868; original designation.

colossus (Blandford), 1896b: 207. (Xyleborus)

Distribution: Papua New Guinea: Central Province; West Papua: Humboldt Bay.

Ambrosiodmus szentivanyi Schedl, 1968a: 267.

declivispinatus (Schedl), 1969b: 216. (Xyleborus)

Distribution: Papua New Guinea: Karamui.

Xyleborus tectus Schedl, 1972c: 63. NoTES: Synonymy: Wood, 1989: 169.

funereus (Lea), 1910a: 139. (Xyleborus)

DisTRIBUTION: New Guinea.

funestus (Schedl), 1979b: 107. (Xyleborus)

DisTRIBUTION: Papua New Guinea.

incertus (Schedl), 1969b: 217. (Xyleborus)

Distribution: Papua New Guinea: Jimmi Valley.

semicarinatus (Schedl), 1942b: 191. (Xyleborus)

DISTRIBUTION: New Guinea. 
TYPE-SPECIES: Arixyleborus rugosipes Hopkins, 1915a; original designation.

abruptus Schedl, 1975d: 358. (Arixyleborus)

DisTRIBUTION: Papua New Guinea: Bulolo.

canaliculatus (Eggers), 1923: 216. (Xyleboricus)

DisTRIBUTION: Papua New Guinea; Bismarck Archipelago: New Britain.

Arixyleborus subsimilis Schedl, 1970b: 362.

cariniceps Schedl, 1975d: 358. (Arixyleborus)

DisTRIBUTION: Papua New Guinea: Wewak.

deceptus Schedl, 1979b: 103. (Arixyleborus)

DistRIBUTION: Papua New Guinea: Wau.

granulicauda Schedl, 1975d: 359. (Arixyleborus)

DisTRIBUTION: Papua New Guinea: Bulolo.

guttifer (Schedl), 1955: 297. (Xyleboricus)

DisTRIBUTION: New Guinea.

iriani Browne, 1983a: 560. (Arixyleborus)

Distribution: West Papua: Mysol Island.

minor (Eggers), 1940: 134. (Xyleboricus)

DISTRIBUTION: New Guinea.

morio (Eggers), 1923: 207. (Xyleborus)

DISTRIBUTION: New Guinea.

scabripennis (Blandford), 1896b: 216. (Xyleborus)

DistRIBUTION: New Guinea. Notes: Range extension: Bright \& Skidmore, 1997: 145.

sus (Schedl), 1973b: 93. (Xyleborus)

DistRIBUTION: Papua New Guinea: Bulolo, Moroka.

Arixyleborus varicus Schedl, 1975d: 360.

CNeStus Sampson, 1911: 383

TYPE-SPECIES: Cnestus magnus Sampson, 1911; monobasic.

aterrimus (Eggers), 1927b: 400. (Xyleborus)

DISTRIBUTION: New Guinea.

bimaculatus (Eggers), 1927a: 88. (Xyleborus)

DisTRIBUTION: New Guinea.

rotundatus Schedl, 1975d: 357. (Cnestus)

DistRIBUTION: Papua New Guinea: Morobe Province.

triangularis (Schedl), 1975d: 370. (Xyleborus)

Distribution: Papua New Guinea: Bulolo.

COPTOBORUS Hopkins, 1915a: 53

TYPE-SPECIES: Coptoborus emarginatus Hopkins, 1915a [=Xyleborus vespatocranus Schedl, 1931: 342]; original designation.

bispinus (Schedl), 1979b: 104. (Xyleborus)

DisTRIBUTION: Papua New Guinea: Bulolo. 
longispinis (Browne), 1986a: 91. (Streptocranus)

DisTRIBUTION: West Papua: Fakfak.

superbulus (Schedl), 1958: 148. (Xyleborus superbus)

Distribution: New Guinea.

Xyleborus superbus Schedl, 1951: 95.

superbus (Schedl), 1942b: 188. (Xyleborus)

DISTRIBUTION: New Guinea.

COPTODRYAS Hopkins, 1915a: 10

TYPE-SPECIES: Coptodryas confusa Hopkins, 1915a; original designation.

amphicauda (Browne), 1986c: 666. (Xyleborus)

DisTRIBUTION: West Papua.

atava (Schedl), 1979b: 104. (Xyleborus)

DisTRIBUTION: Papua New Guinea: Bulolo.

chimbui (Schedl), 1973a: 74. (Xyleborus)

DisTRIBUTION: Papua New Guinea: Kwagira River.

Xyleborus chimbui Schedl, 1973b: 90.

diversicolor (Eggers), 1923: 202. (Xyleborus)

DisTRIBUTION: New Guinea.

docta (Schedl), 1975d: 364. (Xyleborus)

Distribution: Papua New Guinea: Bulolo.

extensa (Schedl), 1955: 301. (Xyleborus)

DISTRIBUTION: New Guinea.

intermedius (Eggers), 1923: 210. (Xyleborus)

DisTRIBUTION: New Guinea; Bismarck Archipelago: New Britain.

libra (Eggers), 1923: 202. (Xyleborus)

DisTRIBUTION: Bismarck Archipelago: New Britain.

Xyleborus bismarcensis Browne, 1966: 255. NOTES: Synonymy: Schedl, 1971a: 155.

nitella (Browne), 1984d: 99. (Xyleborus)

DisTRIBUTION: New Guinea.

popondettae (Browne), 1970: 573. (Xyleborus)

Distribution: Papua New Guinea: Popondetta.

recidens (Sampson), 1923: 287. (Xyleborus)

DisTRIBUTION: New Guinea.

rosseli (Schedl), 1975a: 34. (Xyleborus)

DisTRIBUTION: Papua New Guinea: Rossel Island.

CryPtoXyleborus Schedl, 1937b: 550

TYPE-SPECIES: Cryptoxyleborus naevus Schedl, 1937b; subsequent designation by Schedl, 1962b: 103.

vestigator (Schedl), 1973b: 93. (Xyleborus)

Distribution: Papua New Guinea: Bulolo. 
TYPE-SPECIES: Cyclorhipidion pelliculosus Hagedorn, 1912; monobasic.

agnatum (Eggers), 1923: 197. (Xyleborus)

DisTRIBUTION: New Guinea; Bismarck Archipelago.

anoplum (Schedl), 1975d: 362. (Xyleborus)

DisTRIBUTION: Papua New Guinea: Bulolo.

apicipenne (Schedl), 1974: 462. (Xyleborus)

DistRIBUTION: Papua New Guinea: Bulolo.

bituberculatum (Eggers), 1923: 183. (Xyleborus)

Distribution: West Papua.

brevius (Eggers), 1923: 183. (Xyleborus)

Distribution: West Papua.

canarii (Browne), 1984a: 155. (Xyleborus)

DistRIBUTION: New Guinea; Bismarck Archipelago: New Britain. NoTES: Transferred, Beaver, 1995: 202.

delicatum (Schedl), 1955: 300. (Xyleborus)

DisTRIBUTION: New Guinea.

hastatum (Schedl), 1942a: 39. (Xyleborus)

DISTRIBUTION: New Guinea.

indigens (Schedl), 1955: 303. (Xyleborus)

DisTRIBUTION: New Guinea.

longius (Eggers), 1923: 171. (Xyleborus)

DISTRIBUTION: New Guinea.

multipunctatum (Browne), 1980b: 386. (Xyleborus)

DiSTRIBUTION: New Guinea (?).

punctatopilosum (Schedl), 1936e: 532. (Xyleborus)

DiSTRIBUTION: New Guinea.

revocabile (Schedl), 1942b: 186. (Xyleborus)

DISTRIBUTION: New Guinea.

subagnatum Wood, 1992a: 85. (Cyclorhipidion)

DISTRIBUTION: New Guinea.

Xyleborus subagnatum Schedl, 1979a: 239.

sulcinoides (Schedl), 1974: 463. (Xyleborus)

DistRIBUTION: Papua New Guinea: Morobe Province.

tuberculifer (Eggers), 1923: 195. (Xyleborus)

DISTRIBUTION: New Guinea.

ECCOPTOPTERUS Motschulsky, 1863: 515

TYPE-SPECIES: Eccoptopterus sexspinosus Motschulsky, 1863 [=Scolytus spinosus Olivier, 1795: 9]; monobasic.

gracilipes (Eichhoff), 1886: 25. (Platydactylus)

DISTRIBUTION: New Guinea.

spinosus (Olivier), 1795: 9. (Scolytus)

Distribution: New Guinea. 
TYPE-SPECIES: Xyleborus wallacei Blandford, 1896b; original designation.

andamanensis (Blandford), 1896b: 222. (Xyleborus)

DISTRIBUTION: New Guinea.

barbatus (Hagedorn), 1910b: 11. (Xyleborus)

DisTRIBUTION: New Guinea.

destruens (Blandford), 1896b: 221. (Xyleborus)

DISTRIBUTION: New Guinea.

filiformis (Schedl), 1975d: 364. (Xyleborus)

DisTRIBUTION: Papua New Guinea: Bulolo.

fornicatus (Eichhoff), 1868: 151. (Xyleborus)

DiSTRIBUTION: New Guinea; Bismarck Archipelago: New Britain.

kersianus (Browne), 1981a: 132. (Xyleborus)

DiSTRIBUTION: Bismarck Archipelago: New Britain.

laevis (Eggers), 1923: 201. (Xyleborus)

DISTRIBUTION: New Guinea

limatus (Schedl), 1936d: 65. (Xyleborus)

DisTRIBUTION: New Guinea.

piceus (Motschulsky), 1863: 512. (Xyleborus)

DisTRIBUTION: New Guinea.

procerrimus (Schedl), 1969b: 214. (Xyleborus)

DisTRIBUTION: Bismarck Archipelago: New Britain.

russulus (Schedl), 1942b: 187. (Xyleborus)

DISTRIBUTION: New Guinea.

talumalai (Browne), 1966: 248. (Xyleborus)

DISTRIBUTION: Bismarck Archipelago.

tumidus (Schedl), 1975d: 371. (Xyleborus)

Distribution: Papua New Guinea: Bulolo.

wallacei (Blandford), 1896b: 220. (Xyleborus)

Distribution: Papua New Guinea; Bismarck Archipelago; West Papua: Dorey.

Xyleborus confinis Eggers, 1923: 200. NoTES: Synonymy: Wood, 1989: 173.

HADRODEMIUS Wood, 1980: 94

TYPE-SPECIES: Xyleborus globus Blandford, 1896b; original designation.

globus (Blandford), 1896b: 208. (Xyleborus)

DisTRIBUTION: West Papua: Dorey.

LEPTOXYLEBORUS Wood, 1980: 94

TYPE-SPECIES: Phloeotrogus sordicauda Motschulsky, 1863; original designation.

puer (Eggers), 1923: 191. (Xyleoborus)

DISTRIBUTION: New Guinea.

sordicauda (Motschulsky), 1863: 514. (Phloeotrogus)

DisTRIBUTION: New Guinea. 
TYPE-SPECIES: Xyleborus sumatrana Hagedorn, 1908; original designation. KeY: Bright, 1980: 371.

brownei Bright, 1980: 369. (Schedlia)

DisTRIBUTION: Papua New Guinea: Fly River.

convexa Bright, 1980: 370. (Schedlia)

DISTRIBUTION: Louisaide Archipelago: Sudest Island.

paraconvexa Bright, 1980: 370. (Schedlia)

DisTRIBUTION: Papua New Guinea; Rossel Island.

praeusta (Eggers), 1923: 167. (Xyleborus)

DisTRIBUTION: West Papua.

sumatrana (Hagedorn), 1908: 381. (Xyleborus)

DISTRIBUTION: New Guinea.

usitata (Schedl), 1942b: 188. (Xyleborus)

DisTRIBUTION: New Guinea.

TAPHRODASUS Wood, 1980: 95

TYPE-SPECIES: Xyleborus percorthylus Schedl, 1935b; original designation.

cuspidus (Schedl), 1975d: 363. (Xyleborus)

Distribution: Papua New Guinea: Bulolo.

WeBBIA Hopkins, 1915b: 222

TYPE-SPECIES: Webbia dipterocarpi Hopkins, 1915b; original designation.

acutus (Schedl), 1975d: 361. (Xyleborus)

DistRIBUTION: Papua New Guinea: Bulolo.

armifer (Schedl), 1942b: 185. (Xyleborus)

DisTRIBUTION: New Guinea.

Xyleborus spinachius Schedl, 1955: 306. DistRIBUTION: New Guinea.

circumcisus Schedl, 1975d: 373. (Webbia)

DISTRIBUTION: New Guinea.

denticulatus Browne, 1983a: 561. (Webbia)

DistRIBUTION: West Papua: Terminaban.

picicauda Schedl, 1979c: 129. (Webbia)

DISTRIBUTION: Papua New Guinea.

quadricinctus Schedl, 1972c: 62. (Webbia)

DistRIBUTION: Papua New Guinea: Wau.

similis (Eggers), 1923: 213. (Xyleboricus)

DisTRIBUTION: New Guinea.

squamatilis (Schedl), 1955: 307. (Xyleborus)

DisTRIBUTION: New Guinea.

XYLEbORINUS Reitter, 1913: 79

TYPE-SPECIES: Bostrichus saxeseni Ratzeburg, 1837; subsequent designation by Swaine, 1918a: 50. andrewesi (Bland- 
ford), 1896b: 227. (Xyleborus)

DiSTRIBUTION: Papua New Guinea: Long Island. Cryptoxyleborus gracilior Browne, 1984d: 101.

Xyleborus persphenos Schedl, 1969b: 219. NOTES: Synonymy: Beaver and Brown, 1978: 603.

ankius (Schedl), 1975d: 361. (Xyleborus)

DistriBUTiOn: Papua New Guinea: Bulolo.

artestriatus (Eichhoff), 1878: 507. (Xyleborus)

DISTRIBUTION: New Guinea; Bismarck Archipelago.

exiguus (Walker), 1859: 260. (Bostrichus exiguus)

DISTRIBUTION: New Guinea.

perexiguus (Schedl), 1971b: 381. (Xyleborus)

DiSTRIBUTION: New Guinea; Bismarck Archipelago: New Britain.

saxeseni (Ratzeburg), 1837: 167. (Bostrichus saxeseni)

DisTRIBUTION: New Guinea.

XYLEBORUS Eichhoff, 1864: 37

TYPE-SPECIES: Bostrichus monographus Fabricius, 1792; subsequent designation by Lacordaire, 1886: 381.

abbreviatipennis Sched1, 1973b: 88. (Xyleborus)

DISTRIBUTION: New Guinea; Bismarck Archipelago.

anisopterae Browne, 1983a: 558. (Xyleborus)

DistRIBUTION: West Papua: Usau.

annexus Schedl, 1973b: 89. (Xyleborus)

Distribution: Papua New Guinea: Central Province.

approximatus Schedl, 1951: 77. (Xyleborus)

DisTRIBUTION: West Papua: Star Range.

Xyleborus potens Schedl, 1964d: 298. NoTES: Synonymy: Wood, 1989: 176.

aries Schedl, 1969b: 215. (Xyleborus)

DistRIBUTION: Papua New Guinea: Eastern Highlands.

bidentatus (Motschulsky), 1863: 514. (Phloeotrogus)

DISTRIBUTION: New Guinea.

biuncus Browne, 1984d: 96. (Xyleborus)

DISTRIBUTION: New Guinea.

brevicollis Browne, 1984d: 97. (Xyleborus)

DiSTRIBUTION: New Guinea.

canarivorus Browne, 1986a: 96. (Xyleborus)

DISTRIBUTION: West Papua; Bismarck Archipelago.

ciliatoformis Schedl, 1953: 81. (Xyleborus)

DisTRIBUTION: New Guinea.

cinctipes Schedl, 1979b: 105. (Xyleborus)

DistRIBUTION: Papua New Guinea: Bulolo.

circumspinosus Schedl, 1972b: 52. (Xyleborus)

DisTRIBUTION: Papua New Guinea: Umboi Island.

cognatus Blandford, 1896a: 19. (Xyleborus)

DISTRIBUTION: New Guinea.

cruciatus Schedl, 1973b: 90. (Xyleborus)

DisTRIBUTION: Papua New Guinea: Vanapa River. 
curvatus Browne, 1986a: 98. (Xyleborus)

Distribution: West Papua.

cyclopus Schedl, 1940: 440. (Xyleborus)

Distribution: West Papua: Cyclops Mountains.

densatus Schedl, 1979b: 106. (Xyleborus)

DisTRIBUTION: Papua New Guinea: Bulolo.

dentatulus Browne, 1981a: 131. (Xyleborus)

DisTRIBUTION: Bismarck Archipelago: New Ireland.

depressurus Browne, 1985a: 192. (Xyleborus)

DISTRIBUTION: Bismarck Archipelago: New Britain.

devius Schedl, 1979b: 106. (Xyleborus)

DistRIBUTION: Papua New Guinea: Bulolo.

duodecimspinatus Schedl, 1936e: 531. (Xyleborus 12-spinatus)

DistRIBUTION: Papua New Guinea: Mt. Lamington.

emarginatus Eichhoff, 1878: 510. (Xyleborus)

DisTRIBUTION: Papua New Guinea.

Xyleborus emarginatus semicircularis Schedl, 1973b: 92. NoTES: Synonymy: Wood, 1989: 176.

falcarius Schedl, 1942b: 183. (Xyleborus)

DisTRIBUTION: New Guinea.

fallax Eichhoff, 1878: 508. (Xyleborus)

DISTRIBUTION: New Guinea.

fastigatus Schedl, 1935c: 402. (Xyleborus)

DisTRIBUTION: New Guinea.

ferrugineus (Fabricius), 1801: 388. (Bostrichus)

DisTRIBUTION: New Guinea.

flavipennis Schedl, 1979b: 107. (Xyleborus)

DisTRIBUTION: New Guinea.

flavopilosus Schedl, 1936e: 533. (Xyleborus)

DISTRIBUTION: New Guinea.

fuyugei Schedl, 1973a: 74. (Xyleborus)

DistribUtion: Papua New Guinea: Rossel Island.

gorggae Schedl, 1973b: 91. (Xyleborus)

DISTRIBUTION: Bismarck Archipelago: New Ireland.

graniger Schedl, 1955: 303. (Xyleborus)

DisTRIBUTION: Papua New Guinea: Aitape.

granulipes Schedl, 1973a: 91. (Xyleborus)

DISTRIBUTION: D'Entrecasteaux Islands: Goodenough Island.

gratiosus Schedl, 1975d: 366. (Xyleborus)

DistRIBUTION: Papua New Guinea: Western Highlands.

hashimotoi Browne, 1986a: 95. (Xyleborus)

DisTRIBUTION: West Papua; Bismarck Archipelago.

hopeae Browne, 1986a: 96. (Xyleborus)

Distribution: West Papua.

immersus Schedl, 1972b: 52. (Xyleborus)

DisTRIBUTION: Bismarck Archipelago: New Britain.

immitatrix Schedl, 1975d: 367. (Xyleborus)

DistRIBUTION: Papua New Guinea: Bulolo. 
impexus Schedl, 1942b: 184. (Xyleborus)

DISTRIBUTION: New Guinea.

insulindicus Eggers, 1923: 177. (Xyleborus)

DiSTRIBUTION: New Guinea.

Xyleborus glaberrimus Schedl, 1942b: 184. NOTES: Synonymy: Browne, 1961: 173.

ipidia Schedl, 1972c: 63. (Xyleborus)

DISTRIBUTION: Bismarck Archipelago: New Ireland.

mesoleiulus Schedl, 1979b: 108. (Xyleborus)

DisTRIBUTION: Papua New Guinea: Bulolo.

mucronatoides Schedl, 1975d: 368. (Xyleborus)

DisTRIBUTION: West Papua: Vokelkop.

multipunctulus Browne, 1984d: 98. (Xyleborus)

Distribution: Papua New Guinea: Gumi.

nitens Browne, 1984d: 97. (Xyleborus)

Distribution: Papua New Guinea: Popondetta.

operosus Schedl, 1973b: 91. (Xyleborus)

DistRIBUTION: Papua New Guinea: Wau.

opulentus Schedl, 1975d: 369. (Xyleborus)

Distribution: Papua New Guinea: Mt. Dayman.

papatrae Schedl, 1972b: 53. (Xyleborus)

DisTRIBUTION: Bismarck Archipelago: New Ireland.

papuanus Blandford, 1896b: 209. (Xyleborus)

DisTRIBUTION: New Guinea.

partitus Browne, 1974: 69. (Xyleborus)

DistRIBUTION: Papua New Guinea: Bulolo.

perforans (Wollaston), 1857: 96. (Tomicus)

DISTRIBUTION: New Guinea.

Xyleborus apertus Schedl, 1939a: 355. NOTES: Synonymy: Bright \& Skidmore, 1997: 154.

Xyleborus minimus Schedl, 1955: 305. NoTES: Synonymy: Bright \& Skidmore, 1997: 161.

perplexus Schedl, 1969b: 218. (Xyleborus)

DisTRIBUTION: Bismarck Archipelago: New Britain.

pileatulus Schedl, 1975d: 369. (Xyleborus)

DisTRIBUTION: Papua New Guinea: Bulolo.

pilifer Eggers, 1923: 178. (Xyleborus)

DistRIBUTION: West Papua.

platyurus Browne, 1983b: 74. (Xyleborus)

Distribution: Papua New Guinea: Mt. Kaindi.

protii Browne, 1984d: 101. (Xyleborus)

DISTRIBUTION: New Guinea.

pumilus Eggers, 1923: 209. (Xyleborus)

DisTRIBUTION: New Guinea; Bismarck Archipelago.

putputensis Browne, 1986a: 97. (Xyleborus)

DisTRIBUTION: Bismarck Archipelago: New Britain.

quadrispinosulus Eggers, 1923: 189. (Xyleborus)

DISTRIBUTION: New Guinea.

rapandus Schedl, 1942b: 186. (Xyleborus)

DISTRIBUTION: New Guinea. 
rotundicollis Browne, 1983b: 73. (Xyleborus)

DistRIBUTION: Papua New Guinea: Mt. Kaindi.

scabricollis (Schedl), 1975c: 220. (Ozopemon)

DisTRIBUTION: Papua New Guinea: Brown River.

similis Ferrari, 1867: 23. (Xyleborus)

DisTRIBUTION: New Guinea.

Xyleborus submarginatus Blandford, 1896b: 223. NOTES: Synonymy: Eggers, 1929: 48.

Xyleborus novaguineanus Schedl, 1936e: 530. NotES: Synonymy: Wood, 1989: 177.

spinicornis Schedl, 1975d: 370. (Xyleborus)

Distribution: Papua New Guinea: Biniguni, Gwariu River.

striatulus Browne, 1980c: 487. (Xyleborus)

Distribution: Papua New Guinea: Vanimo.

suaui Schedl, 1973a: 75. (Xyleborus)

Distribution: Papua New Guinea: Biniguni, Gwariu River.

subdentatulus Browne, 1986a: 97. (Xyleborus)

DISTRIBUTION: New Guinea.

takeharai Browne, 1983a: 557. (Xyleborus)

DistRIBUTION: West Papua: Teminaban.

teninabani Browne, 1986c: 668. (Xyleborus)

DisTRIBUTION: West Papua: Teminaban.

timidus Schedl, 1973a: 76. (Xyleborus)

DistRIBUTION: Papua New Guinea: Kwagira River.

truncaticauda Browne, 1984d: 100. (Xyleborus)

DISTRIBUTION: Papua New Guinea: Gumi.

venustulus Schedl, 1969b: 219. (Xyleborus)

DistRIBUTION: Papua New Guinea: Jimmi Valley, Western Highlands.

vernaculus Schedl, 1975d: 372. (Xyleborus)

DistRIBUTION: Admiralty Islands: Manus Island, Nuwok.

viaticus Schedl, 1974: 464. (Xyleborus)

Distribution: Papua New Guinea: Bulolo.

XYLOSANDRUS Reitter, 1913: 80

TYPE-SPECIES: Xyleborus morigerus Blandford, 1894c; monobasic.

crassiusculus (Motschulsky), 1866: 403. (Phloeotrogus)

DISTRIBUTION: New Guinea.

morigerus (Blandford), 1894c: 264. (Xyleborus)

DistRIBUTION: New Guinea; Bismarck Archipelago: New Britain.

ursa (Eggers), 1923: 172. (Xyleborus)

DisTRIBUTION: West Papua: Haveri.

\section{XYLOCTONINA}

SCOLYTOMimus Blandford, 1895: 319

TYPE-SPECIES: Scolytomimus dilutus Blandford, 1895; monobasic. 
baloghi Schedl, 1969a: 157. (Scolytomimus)

DisTRIBUTION: Papua New Guinea: Mt. Kaindi.

maculatus Beeson, 1929: 223. (Scolytomimus)

DistriBution: West Papua. Notes: Range extension: Browne, 1984a.

pusillus (Eggers), 1927a: 88. (Neoxyloctonus)

DisTRIBUTION: Papua New Guinea; West Papua.

quadridens Wood, 1988b: 200. (Scolytomimus)

DisTRIBUTION: Papua New Guinea: Finschhafen.

\section{REFERENCES}

Agassiz, L. (1846) Nomina systematica generum Coleopterum, tam viventium quam fossilium, secundum ordinem alphabeticum disposita, adjectis auctoribus, libris in quibus reperiuntur, anno editionis, etymologica et familiisad quas pertinent. Pp. xi + 170. In: Agassiz, L. 1842-1846, Nomenclator Zoologicus, continens nomina systematica generum animalium tam viventium quam fossilium, secundum ordinem alphabeticum disposita, adjectis auctoribus, libris, in quibis reperiunitur, amno editionis, etymologica et familiis, ad quas pertinent, in singulis classibus. Fasc. 11. Jent et Gassman, Soloduri.

Alonso-Zarazaga, M.A. \& Lyal, C.H.C. (1999) A World Catalogue of Families and Genera of Curculionoidea (Insecta: Coleoptera) (Excepting Scolytidae and Platypodidae). Entomopraxis, $315 \mathrm{pp}$.

Alonso-Zarazaga, M.A. \& Lyal, C.H.C. (2002) Addenda and corrigenda to 'A World Catalogue of Families and Genera of Curculionoidea (Insecta: Coleoptera)'. Zootaxa, 63, 1-37.

Ancey, C.M.F. (1880) Description de Coléoptéres nouveaux. Le Naturaliste, 2(26), 205-206.

Ancey, C.M.F. (1881) Diagnoses de Curculionides Nouveaux. Le Naturaliste, 3(47), 372.

Anderson, R.S. (1993) Weevils and plants: phylogenetic versus ecological mediation of evolution of host plant association in Curculioninae (Coleoptera: Curculionidae). Memoirs of the Entomological Society of Canada, 165, $197-232$.

Anderson, R.S. (1995) An evolutionary perspective on diversity in Curculionoidea. Memoir of the Entomological Society of Washington (14), 103-114.

Arnett, R.H., G.A. Samuelson \& Nishida, G.M. (1993) The Insect and Spider Collections of the World. Flora \& Fauna Handbook No. 11. Sandhill Crane Press, Gainsville, ii-vi +310 pp.

Arrow, G.J. (1940) A note on certain genera of Brenthid Coleoptera and description of a new species. Annals and Magazine of Natural History, 6(33), 318-320.

Basset, Y. \& Novotny, V. (1999) Species richness of insect herbivore communities on Ficus in Papua New Guinea. Biological Journal of the Linnean Society, 67, 477-499.

Bates, H.W. (1877) On the Coleoptera collected by the Rev. G. Brown, C.M.Z.C. on Duke York Island, New Ireland and New Britain. Proceedings of the Zoological Society of London, 1877, 151-159.

Beaver, R.A. (1995) New synonymy and taxonomic changes in Oriental and Australasian Scolytidae and Platypodidae (Insecta: Coleoptera). Annalen des Naturhistorischen Museums in Wien, 97B, 197-204.

Beaver, R.A. (1998) New synonymy, new combinations and taxonomic notes on Scolytidae and Platypodidae (Insecta: Coleoptera). Annalen des Naturhistorischen Museums in Wien, 100B, 179-192.

Beaver, R.A. \& Browne, F.G. (1978) The Scolytidae and Platypodidae (Coleoptera) of Penang, Malaysia. Oriental Insects, 12(4), 575-624.

Bedel, L. (1882) Faune des Coléoptères du Bassin de la Seine. Vol. VI. Rhynchophora. Annales de la Société entomologique de France, (6)2(3), 1-16.

Beeson, C.F.C. (1929) Platypodidae and Scolytidae. British Museum (Natural History). Insects of Samoa Part 4. Coleoptera, 4, 217-248.

Beeson, C.F.C. (1937) New Crossotarsus (Platypodidae, Col.). The Indian Forest Records (Entomology Series), 3(3), 47-103.

Beeson, C.F.C. (1939) New species and biology of Coccotrypes and Thamnurgides (Scolytidae, Col.). The Indian Forest Records (N.S.) 279-308.

Behrens, W. (1887) Materialien zu einer Monographie der Curculionengruppe Pachyrrhynchidae. Stettiner Entomologische Zeitung, 48(7-9), 211-257.

Berg, C. (1898) Substitución de nombres genéricos. Comunicaciones del Museo Nacional de Buenos Aires, 1(1), 16-19.

Berthold, A.A. (1827) Latreille's Natürliche Familien des Tierreichs aus dem Französischen mit Anmerkungen und Zusätzen. Landes-Industrie Comptoirs, Weimar, $\mathrm{x}+606 \mathrm{pp}$.

Bigger, M. \& Schofieldn P. (1983) Checklist of Cerambycidae, Curculionidae, Attelabidae, Scolytidae and Platypodidae 
of Melanesia. Overseas Development Administration Miscellaneous Publication No. 60, London, United Kingdom, $62 \mathrm{pp}$.

Billberg, G.J. (1820) Enumeratio Insectorum in Musaeo Gust. Joh. Billberg. Typis Gadelianis, Stockholm, 138 pp.

Blackburn, T. (1900) Further notes on Australian Coleoptera, with descriptions of new genera and species. XXVII. Transactions of the Royal Society of South Australia, 24(2), 113-169.

Blackburn, T. \& Sharp, D. (1885) Memoirs on the Coleoptera of the Hawaiian Islands. Scientific Transactions of the Royal Dublin Society, 2(3), 119-300 + pl. IV, V.

Blanchard, E. (1849) Note sur les Coléoptères du genre Eurhinus, de la famille des Curculioniens. Annales des Sciences Naturelles, 3(10), 143-144.

Blanchard, E. (1853) Description des Insectes. In: Hombron, J.B. \& Jacquinot, H. Voyage au Pôle Sud et dans l'Océanie sur les corvettes l'Astrolabe et la Zélée; exécuté par ordre du Roi pendant les années 1837-1838-1839-1840 sous le commandement de M.J. Dumont-d'Urville, Capitaine de vasseau. Baudry, Paris, 4(1), pp. 1-422.

Blandford, W.F.H. (1894a) The Rhynchophorous Coleoptera of Japan. Part III. Scolytidae. Transactions of the Royal Entomological Society of London, 1894, 53-141.

Blandford, W.F.H. (1894b) Description d'un nouveau genre de Scolytides: Aricerus. Annales de la Société entomologique de Belgique, 38, 133-136.

Blandford, W.F.H. (1894c) Notes on Scolytidae and their food-plants. Insect Life, 6, 260-265.

Blandford, W.F.H. (1895) A list of the Scolytidae collected in Ceylon by Mr. George Lewis, with descriptions of new species. Annals and Magazine of Natural History, 6(15), 315-328.

Blandford, W.F.H. (1896a) Contributions a la Faune indochinoise 16e Memoire (1). Scolytidae. Annales de la Société entomologique de France, 16, 19-22.

Blandford, W.F.H. (1896b) Descriptions of new Scolytidae from the Indo-Malayan and Austro-Malayan regions. Transactions of the Royal Entomological Society of London, 1896, 191-228.

Boheman, C.H. (1859) Coleoptera. Kongliga Svenska Fregatten Eugenies Resa omkring jorden under befäl af C.A. Virgin Ahren 1851-1853. Vetenskapliga iakttagelser pa H.M. Konung Oscar Den Förstes befallning. P.A. Norstedt \& Söner. 1858-68, K Svenska Vetenskaps Akademian. Stockholm, 617 pp. + pl. IX.

Boisduval, J.B.A.D. (1835a) Voyage de découvertes de L'Astrolabe exécuté par ordre du Roi, pendant les années 18261827-1828-1829, sous le commandement de M. J. Durmont d'Urville. Faune Entomologique de l'Océan Pacifique, avec l'illustration des insectes nouveaux recueillis pendant le voyage, par le Docteur Boisduval, Deuxième partie. Coléoptères et autres ordres. J. Tastu, Paris, vii +716 pp.

Boisduval, J.B.A.D. (1835b) Faune entomologique de l'Océanie, comprenant les Coléoptères, les Hémiptères, les Néuroptères, les Hyménoptères et les Diptères. Librarie Encyclopédique de Roret, Paris, 705 pp.

Bolkay, S. (1911) Descriptions of some new Brenthidae. Annales Historico-Naturales Musei Nationalis Hungarici, 9(1), 263-265.

Bovie, A. (1908) Notes sur les Curculionides. Troisième partie. Annales de la Société Entomologique de Belgique, 52, 43-44.

Bright, D.E. (1980) Studies on the Xyleborini 1. Three new species of Schedlia from New Guinea (Coleoptera: Scolytidae). Coleopterists Bulletin, 34(4), 369-372.

Bright, D.E. \& Skidmore, R.E. (1997) A Catalog of Scolytidae and Platypodidae (Coleoptera), Supplement 1 (19901994). A Publication of the National Research Council of Canada, Monograph Publishing Program, Ottawa, vii + $368 \mathrm{pp}$.

Bright, D.E. \& Skidmore, R.E. (2002) A Catalog of Scolytidae and Platypodidae (Coleoptera), Supplement 2 (19951999). A Publication of the National Research Council of Canada, Monograph Publishing Program, Ottawa, 523 pp.

Broun, T. (1893) Manual of New Zealand Coleoptera. Parts V, VI, VII. Colonial Museum and Geological Survey Department. Wellington. xvii + pp. 975-1504.

Browne, F.G. (1950) New Scolytidae and Platypodidae (Coleoptera) from Malaya. Annals and Magazine of Natural History, 12(3), 641-650.

Browne, F.G. (1962) Taxonomic notes on Platypodidae (Coleoptera). Annals and Magazine of Natural History, (13)4, 641-656.

Browne, F.G. (1966) Some Platypodidae and Scolytidae (Coleoptera) from the Philippine, Bismarck and Solomon Islands. Noona Dan No. 21. Entomologische Meddelelser, 34(3), 233-257.

Browne, F.G. (1968) Scolytidae (Coleoptera) of Rennell Island. In: T. Wolff (Ed.), The Natural History of Rennell Island, British Solomon Islands. Danish Science Press, Ltd., Copenhagen, 5, pp. 111-113.

Browne, F.G. (1970) Some Scolytidae and Platypodidae (Coleoptera) in the collection of the British Museum. Journal of Natural History, 4, 539-583.

Browne, F.G. (1974) A summary of the scolytid fauna (Coleoptera) of Fiji, with some new species. Commonwealth Forestry Review, 53(1), 63-71.

Browne, F.G. (1980a) Bark beetles and ambrosia beetles (Coleoptera, Scolytidae, and Platypodidae) intercepted at Japanese ports, with descriptions of new species, I. Kontyu, 48(3), 370-379. 
Browne, F.G. (1980b) Bark beetles and ambrosia beetles (Coleoptera, Scolytidae, and Platypodidae) intercepted at Japanese ports, with descriptions of new species, II. Kontyu, 48(3), 380-389.

Browne, F.G. (1980c) Bark beetles and ambrosia beetles (Coleoptera, Scolytidae, and Platypodidae) intercepted at Japanese ports, with descriptions of new species, III. Kontyu, 48(4), 482-489.

Browne, F.G. (1980d) Bark beetles and ambrosia beetles (Coleoptera, Scolytidae, and Platypodidae) intercepted at Japanese ports, with descriptions of new species, IV. Kontyu, 48(4), 490-500.

Browne, F.G. (1980e) Three new specific names in the genus Platypus Herbst (Col., Platypodidae). Entomologist's Monthly Magazine, 116, 215.

Browne, F.G. (1981a) Bark beetles and ambrosia beetles (Coleoptera, Scolytidae, and Platypodidae) intercepted at Japanese ports, with descriptions of new species, V. Kontyu, 49(1), 125-136.

Browne, F.G. (1981b) Bark beetles and ambrosia beetles (Coleoptera, Scolytidae, and Platypodidae) intercepted at Japanese ports, with descriptions of new species, VI. Kontyu, 49(4), 597-606.

Browne, F.G. (1983a) Bark beetles and ambrosia beetles (Coleoptera, Scolytidae, and Platypodidae) intercepted at Japanese ports, with descriptions of new species, VII. Kontyu, 51(4), 554-572.

Browne, F.G. (1983b) Some new species of Platypodidae and Scolytidae (Coleoptera) from Papua New Guinea. South Pacific Journal of Natural Sciences, 4, 55-75.

Browne, F.G. (1984a) Bark beetles and ambrosia beetles (Coleoptera, Scolytidae, and Platypodidae) intercepted at Japanese ports, with descriptions of new species, VIII. Kontyu, 52(1), 150-158.

Browne, F.G. (1984b) Bark beetles and ambrosia beetles (Coleoptera, Scolytidae, and Platypodidae) intercepted at Japanese ports, with descriptions of new species, IX. Kontyu, 52(2), 286-292.

Browne, F.G. (1984c) Bark beetles and ambrosia beetles (Coleoptera, Scolytidae, and Platypodidae) intercepted at Japanese ports, with descriptions of new species, X. Kontyu, 52(3), 448-457.

Browne, F.G. (1984d) More new species of Scolytidae (Coleoptera) from Papua New Guinea. South Pacific Journal of Natural Sciences, 6, 86-102.

Browne, F.G. (1985a) Bark beetles and ambrosia beetles (Coleoptera, Scolytidae, and Platypodidae) intercepted at Japanese ports, with descriptions of new species, XI. Kontyu, 53(1), 190-198.

Browne, F.G. (1985b) Bark beetles and ambrosia beetles (Coleoptera, Scolytidae, and Platypodidae) intercepted at Japanese ports, with descriptions of new species, XII. Kontyu, 53(2), 290-296.

Browne, F.G. (1986a) Bark beetles and ambrosia beetles (Coleoptera, Scolytidae and Platypodidae) intercepted at Japanese ports, with descriptions of new species, XIII. Kontyu, 54, 89-99.

Browne, F.G. (1986b) Bark beetles and ambrosia beetles (Coleoptera, Scolytidae and Platypodidae) intercepted at Japanese ports, with descriptions of new species, XIII. Kontyu, 54, 333-343.

Browne, F.G. (1986c) Bark beetles and ambrosia beetles (Coleoptera, Scolytidae and Platypodidae) intercepted at Japanese ports, with descriptions of new species, XVI. Kontyu, 54, 661-671.

Calabresi, E. (1922) Sopra due nuove specie di Brentidi esistenti nella collezione Fleutiaux. Bollettino della Società entomologica italiana, 54, 107-111.

Calder, A.A. \& Sands, D.P.A. (1985) A new Brazilian Crytobagous Hustache (Coleoptera: Curculionidae) introduced into Australia to control salvinia. Journal of the Australian Entomolgical Society, 24, 57-64.

Capiomont, G. (1867) Révision de la Tribu des Hypérides, Lacordaire, et en particulier des genres Hypera Germ., Limobius, Schönh. et Coniatus (Germ.) Schönh. renfermant la description de plusieurs genres nouveaux et de 85 espèces nouvelles. Annales de la Société entomologique de France, (4 )7(3), 417-456 + pl.11-12.

Casey, T.L. (1922) Studies in the Rhynchophorous Subfamily Barinae of the Brazilian Fauna. Memoirs on the Coleoptera, 10, 1-520.

Champion, G.C. (1909) Insecta. Coleoptera. Rhynchophora. Curculionidae. Curculioninae (concluded) and Calandrinae. Pp. 49-78. In: Champion, G.C. 1909-1910. Biologia Centralia-Americana, 4, part 7, vi + 221 pp. +9 pl.

Chapuis, F. (1865) Monographie des Platypides. H, Dessain, Leige, 344 pp.

Chapuis, F. (1869) Synopsis des Scolytides. J. Desoer, Liege, 56 pp.

Chevrolat, L.A.A. (1833) Nouveau Genre de Curculionites, ordre des orthocères, division des Cylades. Annales de la Société entomologique de France, 2, 357-360 + pl. XV.

Chevrolat, L.A.A. (1877) Diagnoses de Curculionides exotiques. Petites Nouvelles Entomologiques, 2(186), 189.

Chevrolat, L.A.A. (1879) Diagnoses de Curculionides nouveaux des genres Psalidium et Apocyrtus. Naturaliste, 1(17), 133-134.

Chevrolat, L.A.A. (1880a) Description de trois Eupholus nouveaux Coléoptères de la famille des Curculionides, tribu des Aterpides. Annales de la Société Entomologique de France, (5)10, XVI-XVII.

Chevrolat, L.A.A. (1880b) Diagnoses de Curculionides. Le Naturaliste, 1(42), 333.

Chevrolat, L.A.A. (1880c) Descriptions de deux Curculionides nouveaux. Annales de la Société entomologique de France, (5)10(3), CII-CIII.

Chevrolat, L.A.A. (1881a) Diagnoses de Curculionides exotiques. Le Naturaliste, 1(62), 494-495.

Chevrolat, L.A.A. (1881b) Descriptions d'espèces de Curculionides de la Nouvelle-Guinée et d'Australie, dont l'un est le 
type d'un genre nouveau. Annales de la Société entomologique de France, (6)1(2), LXVIII-LXIX.

Chevrolat, L.A.A. (1882) Note synonymique relative aux Coléoptères Curculionites de la tribu des Calandrides. Annales de la Société entomologique de France, (6)2(3), CXXXVII-CXL.

Chevrolat, L.A.A. (1883) Calandrides. Nouveaux genres et nouvelles espèces, observations, synonymies, doubles emplois de noms de genres et d'espèces. Lière partie. Annales de la Société entomologique de France, (6)2(4), 555582 .

Chevrolat, L.A.A. (1885) Calandrides. Nouveaux genres et nouvelles espèces, observations, synonymies, doubles emplois de noms de genres et d'espèces, etc. 3e partie. Annales de la Société entomologique de France, 6(5), 275292.

Clairville, J.P. (1798) Entomologie helvétique ou catalogue des Insectes de la Suisse rangés d'aprés une nouvelle méthode. Helvetische Entomologie oder Verzeichniss der Schweizer Insecten, nach einer neuen Methode geordnet mit Beschreibungen und Abbildungen. Vol. 1. Orell, Füssli \& Co., Zürich, 149 pp. + 16 pl.

Colonnelli, E. (1979) Un nouveau Mescysmoderes de la Nouvelle Guineé (Coleoptera: Curculionidae). Folia Entomologicia Hungarica, 32(2), 2-29.

Colonnelli, E. (1992) Notes on the Ceutorhynchinae tribe Mecysmoderini Wagner, 1938 (Coleoptera, Cuculionidae). Entomologica Basiliensia, 15, 395-422.

Csiki, E. (1934a) Curculionidae: Subfam. Hyperinae. In: S. Schenkling (Ed.), Coleopterorum Catalogus. W. Junk, Berlin, 137, pp. 1-66.

Csiki, E. (1934b) Curculionidae: Subfam. Cleoninae. In: S. Schenkling (Ed.), Coleopterorum Catalogus. W. Junk, Berlin, 134, pp. 1-152.

Csiki, E. (1936a) Curculionidae: Rhynchophorinae. In: S. Schenkling (Ed.), Coleopterorum Catalogus. W. Junk, 's Gravenhage, 149, pp. 1-104.

Csiki, E. (1936b) Curculionidae: Cossoninae. In: S. Schenkling (Ed.), Coleopterorum Catalogus. W. Junk, 's Gravenhage, 149, pp. 105-212.

Curtis, J. (1839) British Entomology; being illustrations and descriptions of the genera of insects found in Great Britain and Ireland: containing coloured figures from Nature of the most rare and beautiful species, and in many instances of the plants upon which they are found. Author, London, Vol. 16. (Parts 181-192: plates 722-769).

Dalla Torre, K.W. \& Emden, F.v. (1931) Curculionidae: Brachyderinae: Pachyrrhynchini. In: S. Schenkling (Ed.), Coleopterorum Catalogus. W. Junk, Berlin, 119, pp. 1-44.

Dalla Torre, K.W. \& Emden, F.v. (1939) Curculionidae: Brachyderinae III. In: S. Schenkling (Ed.), Coleopterorum Catalogus. W. Junk, 's Gravenhage, 164, pp. 197-327.

Dalla Torre, K.W. \& Schenkling, S. (1932) Curculionidae: Subfam. Curculioninae. In: S. Schenkling (Ed.), Coleopterorum Catalogus. W. Junk, Berlin, 123, pp. 1-46.

Dalla Torre, K.W., Schenkling, S. \& Marshall, G.A.K. (1932a) Curculionidae. Subfam. Hylobiinae. In: S. Schenkling (Ed.), Coleopterorum Catalogus. W. Junk, Berlin, 122, pp. 1-112.

Dalla Torre, K.W., Schenkling, S. \& Marshall, G.A.K. (1932b) Curculionidae. Subfam. Pissodinae. In: S. Schenkling (Ed.), Coleopterorum Catalogus. W. Junk, Berlin, 125, pp. 1-29.

Dalla Torre, K.W. \& Voss, E. (1930) Curculionidae: Archolabinae, Attelabinae, Apoderinae. In: S. Schenkling (Ed.), Coleopterorum Catalogus. W. Junk, Berlin, 110, pp. 1-42.

Dalla Torre, K.W. \& Voss, E. (1935) Curculionidae. Subfam. Belinae. In: S. Schenkling (Ed.), Coleopterorum Catalogus. W. Junk, Berlin, 144, pp. 1-14.

Dalla Torre, K.W. \& Voss, E. (1937) Curculionidae. Subfam. Rhynchitinae I. In: S. Schenkling (Ed.), Coleopterorum Catalogus. W. Junk, Berlin, 158, pp. 3-56.

Dalla Torre, K.W. \& Voss, E. (1939) Curculionidae. Subfam. Rhynchitinae II. In: S. Schenkling (Ed.), Coleopterorum Catalogus. W. Junk, Berlin, 167, pp. 57-127.

Damoiseau, R. (1962) Nouveaux Brentidae. Annali del Museo Civico di Storia Naturale di Genova, 73, 109-120.

Damoiseau, R. (1963) Contribution à la connaissance des Brentidae (Coleoptera-Phytophagoidea) 14.-Le groupe Eterozemus (Première Partie). Bulletin et Annales de la Société Royale d'Entomologie de Belgique, 39(29), 1-44.

Damoiseau, R. (1964a) Contribution à la connaissance des Brentides (Coleoptera-Phytophagoidea). 16.-Le groupe Eterozemus (Deuxième partie). Bulletin et Annales de la Société Royale d'Entomologie de Belgique, 100(31), 385418.

Damoiseau, R. (1964b) Contribution à la connaissance des Brentidae (Coleoptera-Phytophagoidea). 18.-Genres Calodromus Guérin et Cyphagogus Parry: notes synonymiques et description d'espèces nouvelles. Bulletin et Annales de la Société Royale d'Entomologie de Belgique, 100(36), 453-500.

Damoiseau, R. (1965) Contribution à la connaissance des Brentidae (Coleoptera-Curculionoidea). 22.-Révision des Calodrominae palaeotropicaux et description d'espèces nouvelles. Bulletin de l'Institut Royal des Sciences Naturelles de Belgique, 41(34), 1-28.

Damoiseau, R. (1966a) A propos des Coléoptères de Woodlark et de Nouvelle-Calédonie déscrits par Montrouzier et Perroud (1855-1864). Bulletin de l'Institut Royal des Sciences Naturelles de Belgique, 42(142), 1-27. 
Damoiseau, R. (1966b) Nouveaux Brentidae de l'Ancien Monde (Coleoptera-Curculionoidea). Bulletin et Annales de la Société Royale d'Entomologie de Belgique, 102(9), 139-154.

Damoiseau, R. (1966c) Nouveaux Brentidae du Muséum Frey (Coleoptera-Phytophagoidea). Entomologische Arbeiten aus dem Museum G. Frey, 17, 7-24.

Damoiseau, R. (1966d) Brentidae (Col., Curculionoidea) recoltés par l'expedition danoise du Noona Dan aux Philippines et aux Îles Bismark et Salomon. Entomologische Meddelelser, 34, 421-455.

Damoiseau, R. (1972) Contribution à la connaissance des Brentidae (Coleoptera) de la région néo-guinéenne. Annales Historico-Naturales Musei Nationalis Hungarici, 64, 271-276.

Damoiseau, R. (1980) Les Amorphocephalini (Coleoptera-Brentidae). 2-Cordus Schoenherr et les genres voisins. Bulletin de l'Institut Royal des Sciences Naturelles de Belgique, Entomologie, 52(21), 1-32 + pl. I-III.

Damoiseau, R. (1987) Contribution à la systématique et corrections à la nomenclature des Calodrominae (ColeopteraBrentidae) II. Tribus des Hoplopisthiini, Atopobrentini et Stereodermini. Bulletin de l'Institut Royal des Sciences Naturelles de Belgique, Entomologie, 57, 31-91.

Damoiseau, R. (1989a) Contribution à la systématique et corrections à la nomenclature des Calodrominae (ColeopteraBrentidae) III. Tribu des Calodromini (complément posthume). Bulletin de l'Institut Royal des Sciences Naturelles de Belgique, Entomologie, 58, 101-173.

Damoiseau, R. (1989b) Contribution à la systématique et corrections à la nomenclature des Brentinae et Ceocephalinae (Coleoptera-Brentidae). Bulletin de l'Institut Royal des Sciences Naturelles de Belgique, Entomologie, 59, 43-98.

Decelle, J. \& Voss, E. (1972) La Faune terrestre de l'île de Sainte-Helène (Deuxième partie). II. Insectes. 9. Coleoptera. 35. Fam. Curculionidae. Annales du Musée Royal de l'Afrique Centrale, Tervuren, in-8o, Sciences Zoologiques, 192, $306-515$.

DeGeer, C. (1775) Memoires pour servir à l'historie des insectes. Stockholm, Tome cinquième, vii + 448 pp.

Dejean, P.F.M.A. (1834) Catalogue des Coléoptères de la collection de M. le Compte Dejean. Méquignon-Marvis, Paris, $443 \mathrm{pp}$.

Dejean, P.F.M.A. (1835) Catalogue de la collection de Coléoptères de M. le Baron Dejean. Méquignon-Marvis, Ed. 2. Paris, Part 4: 257-360.

Desbrochers, J. (1869) Monographie des Rhinomacérides d'Europe et des pays limitrophes, comprenant les genres Rhynchites, Auletes, Auletobius (N. G.), Diodyrynchus, Rhinomacer \& Nemonyx. L'Abeille, 5, 317-428.

Desbrochers, J. (1890) Description de Curculionides et de Brenthides inédits faisant partie des collections du Musée Indien de Calcutta. Journal of the Asiatic Society of Bengal (II Natural Sciences), 3, 211-224.

Desbrochers, J. (1892) Description d'un Brenthide nouveau des Nouvelles-Hébrides. Le Frélon, 1(12), 109-110.

Desbrochers, J. (1898) Description d'un nouveau genre de la Tribu des Cleonidae avec tableau et description des espèces connues de la faune de l'Ancien Monde appartenant à ce genre. Le Frélon, 7(4), 54-63.

Desbrochers, J. (1904) Etudes sur les Curculionides de la faune européenne et des bassins de la Méditerranée, en Afrique et en Asie, suivies de tableaux synoptiques. Le Frélon, 12(5): 65-80, (6): 81-96, (7): 97-104.

Draeseke, J. (1957) Dr. K. M. Heller. Abhandlungen und Berichte aus dem Staatliches Museum für Tierkunde in Dresden/Abhandlungen und Berichte aus dem Staatliches Museum für Tierkunde in Dresden, 23, 257-268.

Duncan, J. (1843) Entomology. Beetles. Natural history of Coleopterous insects. The Naturalist's Library. W.H. Lizars, Edinburgh, 2, $269 \mathrm{pp}$.

Eggers, H. (1923) Neue Indomalayische Borkenkäfer (Ipidae). Zoologische Mededeelingen, 7, 129-220.

Eggers, H. (1926) Japanische Borkenkäfer, I. Entomologische Blätter, 22, 133-138.

Eggers, H. (1927a) Neue Indo-malayische Borkenkäfer (Ipidae). II. Nachtrag. Philippine Journal of Science, 33(1), 67108.

Eggers, H. (1927b) Neue Indomalayische Borkenkäfer (Ipidae). I. Nachtrag. Treubia, 9(4), 390-408.

Eggers, H. (1940) Neue Indomalayische Borkenkäfer (Ipidae). III. Nachtrag (Forstsetzung). Tijdschrift voor Entomologie, $83,132-154$.

Eichhoff, W.J. (1864) Ueber die Mundtheile und die Fuhlerbildung der europaischen Xylophagi sens. strict. Berliner Entomologische Zeitschrift, 8, 17-46 + pl. 1 .

Eichhoff, W.J. (1868) Neue amerikanische Borkenkäfer-Gattungen und Arten. Berliner Entomologische Zeitschrift, 12, $145-152$.

Eichhoff, W.J. (1872) Neue exotische Tomiciden-Arten. Berliner Entomologische Zeitschrift, 15, 131-136.

Eichhoff, W.J. (1875) Pages 200-203 in Felicien Chapuis et W. Eichhoff, Scolytides recueillis au Japan par M.C. Lewis. Annales de la Société entomologique de Belgique, 18, 195-203.

Eichhoff, W.J. (1878) Ratio, descriptio, emendatio eorum Tomicinorum qui sunt in Dr. Medin. Chapuisi et autoris ipsius collectionibus et quos praeterea recognovit. Mémoires de la Société Entomologique de Liege, (2)8, $1+\mathrm{iv}+531+5$ pl.

Eichhoff, W.J. (1886) Zwei neue ost-indische Scolytiden-Gattungen. Leiden Museum Notes, 8, $24-26$.

Erichson, W.F. (1836) Systematische Auseinandersetzung der Familie der Borkenkäfer (Bostrichidae). Archiv für Naturgeschichte, 2(1), 45-65. 
Erichson, W.F. (1842) Beitrag zur Insecten-fauna von Vandiemensland, mit besonderer Berücksichtigung der geographischen Verbreitung der Insecten. Archiv für Naturgeschichte, 8(1), 83-287 + pl. IV-V.

Fabricius, J.C. (1775) Systema entomologiae, sistens insectorum classes, ordines, genera, species adiectis synonimis, locis, descriptionibus, observationibus. Flensburgi, Lipsiae, $832 \mathrm{pp}$.

Fabricius, J.C. (1781) Species insectorum exhibentes eorum differentias specificas, synonyma, auctorum, loca natalia, metamorphosin adiectis observationibus, descriptionibus. Bohn, Hamburgi \& Kilonii, viii + 552 pp.

Fabricius, J.C. (1787) Mantissa insectorum sistens eorum species nuper detectas adiectis characteribus genericis, differentiis, specificis, emendationibus, observationibus. C.G. Proft, Hafniae, $\mathrm{xx}+348$ pp.

Fabricius, J.C. (1792) Entomologia systematica emendata et aucta. Secundum classes, ordines, genera, species adiectis synonymis, locis, observationibus, descriptionibus. Proft, Hafniae, 1, 538 pp.

Fabricius, J.C. (1798) Supplementum entomologiae systematicae. Proft \& Storch, Hafniae, 172 pp.

Fabricius, J.C. (1801) Systema eleutheratorum secundum ordines, genera, species: adiectis synonimis, locis, observationibus, descriptionibus. Bibliopoli Academici Novi, Kiliae, 2, 687 pp.

Fairmaire, L. (1849a) Insectes de Taïti Marquises et des îles voisines. Revue et Magasin de Zoologie pure et appliquée, (2) $1,34-36$.

Fairmaire, L. (1849b) Essai sur les Coléoptères de la Polynésie. Revue et Magasin de Zoologie pure et appliquée, (2)1, 277-291, 352-365, 410-422, 445-460, 504-516, 550-559.

Fairmaire, L. (1877) Diagnoses de Coléoptères de la Nouvelle-Bretagne. Petites Nouvelles Entomologiques, 2(185), 185-186.

Fairmaire, L. (1878) Diagnoses de Coléoptères des îles Viti, Samoa, etc. Petites Nouvelles Entomologiques, 2 (209), 282.

Fairmaire, L. (1881a) Diagnoses de Coléoptères de la Mélanésie. Le Naturaliste, 1(44), 348-349.

Fairmaire, L. (1881b) Diagnoses de Coléoptères de la Mélanésie. Le Naturaliste, 3(47), 372-373.

Fairmaire, L. (1881c) Diagnoses de Coléoptères de la Mélanésie. Le Naturaliste, 1(49), 389.

Fairmaire, L. (1881d) Diagnoses de Coléoptères nouveaux. Le Naturaliste, 1(53), 421-422.

Fairmaire, L. (1881e) Essai sur laes Coléoptères des îles Viti (Fidgi). Annales de la Société Entomologique de France, (6)1(2): 243-256; (3): 257-318.

Fairmaire, L. (1883) Essai sur les Coléoptères de l'Archipel de la Nouvelle-Bretagne. Annales de la Société Entomologique de Belgique, 27(2), 1-58.

Farrell, B.D. (1998) "Inordinate Fondness" Explained: Why are There so Many Beetles? Science, 281, 555-559.

Faust, J. (1890) Rüsselkäfer von S. Asien und den Sundainseln. Stettiner Entomologische Zeitung, 51(4-6), 65-82.

Faust, J. (1891) Neue Rüsselkäfer aller Länder. (Fortsetzung). Stettiner Entomologische Zeitung, 51(7-12), 165-195.

Faust, J. (1892a) Notizen über Rüsselkäfer. Stettiner Entomologische Zeitung, 53(1-3), 44-52.

Faust, J. (1892b) Curculioniden aus dem Malayischen Archipel. Stettiner Entomologische Zeitung, 53(7-9), 184-228.

Faust, J. (1893) Zwei neue Dyspeithes-Arten. Deutsche Entomologische Zeitschrift, 37, 7-8.

Faust, J. (1894) Viaggio di Leonardo Fea in Birmania e regioni vicine. LX. Curculionidae. Annali del Museo Civico di Storia Naturale di Genova, 34, 153-370.

Faust, J. (1895a) Rüsselkäfer aus dem Malayischen Archipel. Stettiner Entomologische Zeitung, 56(1-6), 81-114.

Faust, J. (1895b) Sechs neue Curculioniden-Gattungen und ein neuer Glochinorhinus. Stettiner Entomologische Zeitung, $56(7-9), 212-228$.

Faust, J. (1897a) Curculioniden aus dem Malayischen und Polynesischen Inselgebiet I. Stettiner Entomologische Zeitung, 58(4-6), 155-183.

Faust, J. (1897b) Neue Gattungen und Arten in der Celeuthetiden-Gruppe. Stettiner Entomologische Zeitung, 58(7-12), 229-298.

Faust, J. (1898a) Curculioniden aus dem Malayischen und Polynesischen Inselgebiet II. Stettiner Entomologische Zeitung, 59(1-6), 140-213.

Faust, J. (1898b) Beschreibung neuer Coleopteren von Vorder-und Hinterindien aus der Sammlung des Hrn. Andrewes in London. Curculionidae. Deutsche Entomologische Zeitschrift, 1898(2), 273-333.

Faust, J. (1899) Viaggo di Lamberto Loria nella Papuasia orientale. XXIII. Curculionidae. Annali del Museo Civico di Storia Naturale di Genova, 40, 1-130.

Faust, J. (1900) In Heller, Systematische Aufzählung der Coleopteren mit Neubeschreibungen von Arten von E. Brenske und J. Faust. In R. Semon, Zoologische Forschungsreisen in Australien und dem Malayischen Archipel, 5, 616-626.

Fauvel, A. (1862) Coléoptères de la Nouvelle-Calédonie, recueillis par M.E. Déplanche, chirurgien de la marine impériale (1858-59-60). Notices Entomologiques, 1, 1-68 + pl. IX-Xbis. [see Lyal \& Alonso-Zarazaga, 2006 for notes on this troublesome publication]

Ferrari, J.A. (1867) Die Forst-und Baumzuchtschadlichen Borkenkäfer (Tomicides Lac.) aus der Familie der Holzverderber (Scolytides Lac.). Carl Gerold's Sohn, Wien, 96 pp.

Frieser, R. (1983) Zur kenntnis der Anthribidenfauna Neuguineas (Coleoptera: Anthribidae). Folia Entomologica Hungarica, 44(1), 47-62.

Frieser, R. (1985) Neue Anthribiden, meist aus Fernost (Coleoptera, Anthribidae). Mitteilungen der Münchner Entomol- 
ogischen Gesellschaft, 75, 83-101.

Frieser, R. (1989) Ein weiterer Bericht zur Anthribidenfauna Papua-Neuguineas (Coleoptera: Anthribidae). Beiträge zur Entomologie, 39, 105-123.

Frieser, R. (1992) Die von A. Riedel in Irian-Jaya gesammelten Anthribiden (Coleoptera: Anthribidae). Acta Coleopterologica, 8(1), 22-45.

Frieser, R. (1993) Ein weiterer Beitrag zur Kenntnis der Anthribiden (Coleoptera: Anthribidae). Acta Coleopterologica, 9(1), 1-14.

Frieser, R. (1995a) Neue Anthribiden von Neu Guinea (Coleoptera Anthribidae). Acta Coleopterologica, 11(1), 3-12.

Frieser, R. (1995b) Neue Anthribiden von Neu Guinea und der indomalaiischen Region (Coleoptera Anthribidae). Acta Coleopterologica, 11(2), 3-35.

Frieser, R. (1996) Neue und bemerkenswerte Anthribidenfunde aus der orientalischen Region und von Neu Guinea (Coleoptera: Anthribidae). Acta Coleopterologica, 12(3), 29-39.

Frieser, R. (1997) Neue und bemerkenswerte Anthribiden aus der indomalaiischen Region und von Neu Guinea (Coleoptera Anthribidae). Acta Coleopterologica, 13(2), 7-72.

Frieser, R. (1999) Ein neuer Beitrag zur Kenntnis der Anthribiden (Coleoptera: Anthribidae). Acta Coleopterologica, 15, 21-39.

Frieser, R. (2000) Neue Anthribiden von Madagaskar, Neu Guinea und aus der orientalischen Region (Coleoptera: Anthribidae). Acta Coleopterologica, 16(2), 23-40.

Frieser, R. (2001a) Beitrag zur Kenntnis der Anthribiden (Coleoptera: Anthribidae). Acta Coleopterlogica, 17(4), 15-32.

Frieser, R. (2001b) Neue Anthribiden von Australien, NeuGuinea und aus der indomalaiischen Region (Coleoptera Anthribidae). Acta Coleopterologica, 17(2), 39-67.

Frieser, R. (2004) Einige neue Arten aus der Gattung Basitropis Jekel (1855) (Coleoptera: Anthribidae). Acta Coleopterologica, 20(2), 3-11.

Froden, D.G. \& Gressitt, J.L. (1982) Biological exploration in New Guinea. In: J.L. Gressitt, Biogeography and ecology of New Guinea. W. Junk Publishers, The Hague, Monographiae Biologicae 42, pp. 87-130.

Gemminger, M. \& Harold, E.v. (1871) Familia LXII. Curculionidae. In: Catalogus Coleopterorum hucusque descriptorum synonimicus et systematicus. E.H. Gummi (G. Beck), Monachii, Munich, 8, 2181-2668 pp.

Gemminger, M. \& Harold, E.v. (1872) Familia LXIV. Brenthidae. In: Catalogus Coleopterorum hucusque descriptorum synonimicus et systematicus. IX Scolytidae, Brenthidae, Anthribidae, Cerambycidae. E.H. Gummi (G. Beck), Monachii, Munich, IX, 2703-2721 pp.

Germar, E.F. (1817) Miscellen und Correspondenz-Nachrichten. Magazin der Entomologie, 2, 339-341.

Germar, E.F. (1824) Insectorum species novae aut minus cognitae, descriptionibus illustratae. Vol. 1. Coleoptera. J.C. Hendelii et filii., Halae, xxiv +624 pp. +2 pl.

Gerstäecker, C.E.A. (1860) Beiträge zur Kenntnis der Curculionen. No. II. Stettiner Entomologische Zeitung, 21(10-12), 376-398.

Gestro, R. (1875) Descrizione di un nuovo genera e di alcune nuove specie di Coleotteri Papuani. Annali del Museo Civico di Storia Naturale di Genova, 7, 993-1027.

Gestro, R. (1876) Diagnosi di alcune nuove specie di Coleotteri raccolte nella regione Austro-Malese dai Signori Dott. O. Beccari, L.M. D'Albertis e A.A. Bruijn. Annali del Museo Civico di Storia Natrale di Genova, 8, 516-524.

Gestro, R. (1879) Descrizioni di nuove specie di Coleotteri raccolte nella regoine Austro-Malese dai signori L. M. D'Albertis. Annali del Museo Civico di Storia Naturale di Genova, XIV, 552-565.

Gestro, R. (1902) Frammenti entomologici. Bollettino della Società Entomologica Italiana, 34(1-2), 33-49.

Gestro, R. (1923) Cenni su alcuni Pantorhytes (Col.) con descrizione di una nuova specie. Estratto dalle Memorie della Società Entomologica Italiana, LV(4), 61-64.

Gestro, R. \& D'Albertis, L.M. (1876) Descrizione di una nuova specie di Eupholus. Annali del Museo Civico di Storia Naturale di Genova, VIII, 387-388.

Gistel, J. (1856) Die Mysterien der europäischen Insectenwelt. Dannheimer, Kempten, $12+532$ pp.

Gistel, J. (1857) Vacuna oder die Geheimnisse aus der organischen und leblosen Welt. Straubing, Schorner, 2, 1031 pp.

Goossens, J. (2005) Contribution à la connaissance des Brentidae des régions australienne et orientale: description d' espèces nouvelles et notes taxonomiques (Coleoptera Curculionoidea). Bulletin de la Societe Royale Belge d'Entomologie, 141, 45-55.

Gray, G.R. (1832) New species of insects of all the orders. In: Griffith, E., The Animal Kingdom arranged in conformity with its organization, by the Baron Cuvier, with supplementary additions to each order. Whittaker, Treacher and Co., London, 15, pp. $796+87$ pl.

Gressitt, J.L. (1966a) Epizoic symbiosis: the Papuan weevil genus Gymnopholus (Leptopiinae) symbiotic with cryptogamic plants, oribatid mites, rotifers and nematodes. Pacific Insects, 8(1), 221-280.

Gressitt, J.L. (1966b) The weevil genus Pantorhytes (Coleoptera), involving cacao pests and Epizoic Symbiosis with Cryptogamic plants and microfauna. Pacific Insects, 8(4), 915-965.

Gressitt, J.L. (1977) Papuan weevil genus Gymnopholus: third supplement and further studies in Epizoic Symbiosis. 
Pacific Insects, 17(2-3), 179-195.

Gressitt, J.L. \& Sedlacek, J. (1967) Papuan weevil genus Gymnopholus: supplement and further studies in Epizoic Symbiosis. Pacific Insects, 9(3), 481-500.

Gressitt, J.L. \& Sedlacek, J. (1970) Papuan weevil genus Gymnopholus: second supplement and further studies in Epizoic Symbiosis. Pacific Insects, 12(4), 753-762.

Gressitt, J.L. \& Szent-Ivany, J.J.H. (1968) Bibliography of New Guinea Entomology. Pacific Insects, 18, 1-674.

Guérin-Méneville, F.E. (1831) Histoire naturelle, Zoologie. Atlas. In: Duperrey, L.I., Voyage autour du Monde exécuté par ordre du Roi, sur la Corvette de Sa Majesté La Coquille, pendant les années 1822, 1823, 1824 et 1825 . Arthus Bertrand, Paris, pp. 1-21 + 14bis.

Guérin-Méneville, F.E. (1833) Insectes. In: Iconographie du Règne Animal de G. Cuvier, ou représentation d'après nature de l'une des espèces les plus remarquables, et souvent non encore figurées, de chaque genre d'animaux. Avec un texte descriptif mis au courant de la science. Ouvrage pouvant servir d'atlas à tous les traités de Zoologie. Tome II (1829-1844). J. B. Baillière, Paris, Vol. 7, 1829-1838, 110 pl.

Guérin-Méneville, F.E. (1838) Histoire Naturelle des Crustacés, Arachnides et Insectes, recueillis dans le voyage autour du Monde de la Corvette de Sa Majesté, La Coquille, exécuté pendant les années 1822, 1823, 1824 et 1825 sous le commandement du capitaine Duperrey. In: Duperrey, L.I., Voyage autour du Monde exécuté par ordre du Roi, sur la Corvette de Sa Majesté La Coquille, pendant les années 1822, 1823, 1824 et 1825. Arthus Bertrand, Paris, 2(2), Première Division, pp. i-xii + 9-319.

Guérin-Méneville, F.E. (1841a) Description de quelques coléoptères nouveaux provenant de la Tasmanie, du port Otago (Nouvelle Zélande), d'Esington-Bay (Australie septentrionale), de Triton-Bay (Nouvelle-Guinée) et des îles Vavao, Salomon, Ternate, Borneo, Aukland, etc. Revue Zoologique par la Société Cuvierienne, 4(4), 120-128.

Guérin-Méneville, F.E. (1841b) Description de quelques Coléoptères nouveaux, provenant de la Tasmanie, des iles Vavao et Ternate, de Triton Bay, à la Nouvelle-Guinée, et du port Famine, dans le détroit de Magellan. Revue Zoologique par la Société Cuvierienne, 4(6), 186-193.

Guérin-Méneville, F.E. (1841c) Descriptions de quelques Coléoptères nouveax provenant des îles Aukland, de Triton Bay, des îles Salomon et du Port-Famine, dans le détroit de Magellan. Revue Zoologique par la Société Cuvierienne, 4, 213-217.

Günther, K. (1934) Ueber südostasiatische und papuanische Calandriden (Col. Curcul.). Internationale Entomologische Zeitschrift Guben, 28(35-36), 441-444, 454-456.

Günther, K. (1935) Neue Calandriden aus dem Museo Civico di Storia Naturale, Genova (Col., Curcul.). Annali del Museo Civico di Storia Naturale Genova, 57, 212-218.

Günther, K. (1938) Revision der gattung Otidognathus Lac. (Coleoptera, Curculionidae, Calandrinae). Temminckia, 3, 45-108.

Günther, K. (1941) Ergänzungen und Berichtigungen zu Coleopterorum Catalogus, pars 149: Rhynchophorinae (Curcul.) mit einigen Neubeschreibungen. Deutsche Entomologische Zeitschrift, 1941, 24-53.

Günther, K. \& Zumpt, F. (1933) Curculionidae: Subfam. Tanymecinae. In: S. Schenkling (Ed.), Coleopterorum Catalogus. W. Junk, Berlin, 131, pp. 1-131.

Gyllenhal, L. (1813) Insecta svecica descripta, Clasis I, Coleoptera sive Eleuterata. F.J. Leverentz, Scaris, 1(3), 730 pp.

Györffy, E. (1917) Analecta ad cognitionem Apioniarum. I. Species quatuor novae generis Chalcocybebus Voll. Annales Musei Nationalis Hungarici, 15, 276-82.

Haaf, E. (1963) Die Alcidodes Melanesiens (Coleoptera, Curculionidae). Entomologische Abhandlungen und Berichte aus dem Staatl. Museum für Tierkunde in Dresden, 28(4), 85-176.

Haaf, E. (1964) Neue Alcidodes-Arten aus Ostafrika und Neu-Guinea (Col. Curc.). Entomologische Arbeiten aus dem Museum G. Frey, 15, 291-295.

Haedo Rossi, J.A. (1955) Notas brentidológicas I (Coleopt.). Neotropica, 1(4), 61-64.

Hagedorn, J.M. (1908) Diagnosen bisher unbeschriebener Borkenkäfer. Deutsche Entomologische Zeitschrift, 1908(3), $369-382+29$ figs.

Hagedorn, J.M. (1910a) Ipidae. In: S. Schenkling (Ed.), Coleopterorum Catalogus. W. Junk, Berlin, 4, pp. 1-134.

Hagedorn, J.M. (1910b) Diagnosen bisher unbeschriebener Borkenkäfer (Col.). Deutsche Entomologische Zeitschrift, (2) $1910(1), 1-13$.

Hagedorn, J.M. (1912) Neue Borkenkäfergattungen und arten aus Afrika (Col.). Deutsche Entomologische Zeitschrift, 1912, 351-356, pl. 6-7.

Hallett, R.H., Crespi, B.J. \& Bordens, J.H. (2004) Synonymy of Rhynchophorus ferrugineus (Olivier), 1790 and R. vulneratus (Panzer), 1798 (Coleoptera: Rhynchophorinae). Journal of Natural History, 38, 2863-2882.

Hartmann, F. (1901) Neue Rüsselkäfer aus Kaiser Wilhelms-Land. Deutsche Entomologische Zeitschrift, 1900(2), 273295.

Hartmann, F. (1903) Neue exotische Rüsselkäfer. Wiener Entomologische Zeitung, 22(1), 23-30.

Hedicke, H. (1923) Nomina nova. II. Deutsche Entomologische Zeitschrift, 1923(1), 72.

Heller, K.M. (1894a) Zygopiden-Studien mit besonderer Berücksichtigung der Gattung Mecopus. Abhandlungen und 
Berichte des Königlichen Zoologischen und Anthropologisch-Etnographischen Museums zu Dresden, 4(2), 1-48 + 1 $\mathrm{pl}$.

Heller, K.M. (1894b) Neue Zygopiden, Isorhynchiden und Sympiezopiden, nebst ergänzenden Bemerkungen zu bekannten Arten. Tijdschrift voor Entomologie, 37, 33-66.

Heller, K.M. (1895a) Zygopiden-Studien II, mit besonder Berücksichtigung der gattung Copturus. Abhandlungen und Berichte des Königlichen Zoologischen und Anthropologisch-Etnographischen Museums zu Dresden, 1894/95,(11), $1-70+1 \mathrm{pl}$.

Heller, K.M. (1895b) Erster Beitrag zur Papuaniscen Käferfauna. Abhandlungen und Berichte des Königlichen Zoologischen und Anthropologisch-Etnographischen Museums zu Dresden, 16, 1-17.

Heller, K.M. (1896) Remarks on the curculionid genus Chalcocybebus. Notes from the Leyden Museum, $18,19-24$.

Heller, K.M. (1897a) Neue Käfer von Celebes II. Abhandlungen und Berichte des Königlichen Zoologischen und Anthropologisch-Etnographischen Museums zu Dresden, 6(10), 1-36.

Heller, K.M. (1897b) Zweiter beitrag Papuanischen Käferfauna. Abhandlungen und Berichte des Königlichen Zoologischen und Anthropologisch-Etnographischen Museums zu Dresden, 6(11), 1-7.

Heller, K.M. (1900) Neue Käfer von Celebes IV. Abhandlungen und Berichte des Königlichen Zoologischen und Anthropologisch-Etnographischen Museums zu Dresden, 9(5), 1-46 + 1 pl.

Heller, K.M. (1901) Dritter Beitrag zur Papuanischen Käferfauna. Abhandlungen und Berichte des Königlichen Zoologischen und Anthropologisch-Ethnographischen Museums zu Dresden, 10(2), 1-20.

Heller, K.M. (1903) Sechs neue Käfer aus Deutsch Neu-Guinea. Deutsche Entomologische Zeitschrift, 1903(2), 305314.

Heller, K.M. (1905a) Zehn neue Käfer aus Neu-Guinea. Deutsche Entomologische Zeitschrift, 49(1), 65-76.

Heller, K.M. (1905b) Die Arten der Gattung Pantorhytes Faust. Wiener Entomologische Zeitung, 24(9,10), 305-310.

Heller, K.M. (1908a) Vierter Beitrag zur Papuanischen Käferfauna. Abhandlungen und Berichte des Königlichen Zoologischen und Anthropologisch-Etnographischen Museums zu Dresden, 12(1), 1-34 + 1 pl.

Heller, K.M. (1908b) Neue indomalayische Rüsselkäfer, vorwiegend aus Madras und Borneo. Stettiner Entomologische Zeitung, 69(1), 122-194 + pl. 1.

Heller, K.M. (1910a) Fünfter Beitrag zur Papuanischen Käferfauna hauptsächlich auf Grund der Ausbeute von Dr. Schlaginhaufen. Abhandlungen und Berichte des Königlichen Zoologischen und Anthropologisch-Ethnographischen Museums zu Dresden, 13(3), 1-42 + 1 pl.

Heller, K.M. (1910b) Neue Rüsselkäfer aus dem Papuanischen Faunengebiete. Wiener Entomologische Zeitung, 29(5-6), 179-197.

Heller, K.M. (1912a) Neuheiten aus Herrn R.v. Bennigsen's Sammlung von Coleopteren aus den deutschen Kolonien. Curculionidae: Pachyrrhynchinae. Entomologische Mitteilungen, 1(10), 309-310.

Heller, K.M. (1912b) Faunistische und systematische Notizen über Rüsselkäfer. Entomologische Mitteilungen, 1(11), 364-365.

Heller, K.M. (1913) Neue papuanische Leptosiden (Curc.). Archiv für Naturgeschichte, 79, 31-47.

Heller, K.M. (1914a) Coleoptera. Nova Guinea. Resultats des expédition scientifique Néerlandaise a la Nouvelle-Guinée en 1907 et 1909 sous les auspices du Dr H. A. Lorentz, 9, 615-666.

Heller, K.M. (1914b) Übersicht über die Gattung Barystethus (Coleopt., Curcul.). Entomologische Mitteilungen, 3(5), $137-143$.

Heller, K.M. (1914c) Coleoptera von Seran (Ceram) und Bali. (Zoologische Ergebnisse der II. Freiburger MoluckkenExpedition.). Entomologische Mitteilungen, 3(10/12), 293-315.

Heller, K.M. (1914d) Neue papuanische Käfer. Deutsche Entomologische Zeitschrift, Berlin, 3, 305-318 + pl. XII.

Heller, K.M. (1915) Neue papuanische Rüsselkafer. Deutsche Entomologische Zeitschrift, 1915(5), 513-528.

Heller, K.M. (1916) Die Käfer von Neu-Caledonien und den benachbarten Inselgruppen. In: Sarasin, F. \& Roux, J. (Eds.), Nova Caledonia. A. Zoologie, 2(3), 229-364.

Heller, K.M. (1918) Die philippinischen Arten der Rüsselkäfergattung Alcides Schönh. Stettiner Entomologische Zeitung, 78(2)[1917], 209-245.

Heller, K.M. (1921) Systematische und faunistische Notizen über Käfer. Entomologische Mitteilungen, 10(6), 195-198.

Heller, K.M. (1922) New Philippine Coleoptera. Philippine Journal of Science, 19(5), 523-627 + pl. I-III.

Heller, K.M. (1923) Bestimmungsschlüssel aussereuropäischer Käfer. Curculionidae: Genus Eupholus Guér. Koleopterologische Rundschau, 10(4), 146-154.

Heller, K.M. (1924a) Neue philippinische Ruesselkaefer aus der Sammlung des Herrn Prof. C.F. Baker und Herrn Angelo Solari in Genova. Memorie della Società Entomologica Italiana, 3, 181-200 + 1 pl.

Heller, K.M. (1924b) New Philippine Zygopinae, Calandrinae, and Cryptoderminae (Curculionidae, Coleoptera). Philippine Journal of Science, 25(3), 287-309 + 1 pl.

Heller, K.M. (1925a) Bestimmungsschlüssel aussereuropäischer Käfer. Curculionidae, Tribus n.: Ottistirini. Wiener Entomologische Zeitung, 42(4-10), 55-91 + pl. I.

Heller, K.M. (1925b) Studien zur Systematik der altweltlichen Balanini (Coleoptera: Curculionidae). Stettiner Entomolo- 
gische Zeitung, 86(2), 86-134.

Heller, K.M. (1925c) Wissenschaftliche Ergebnisse der Bearbeitung der Coleopteren-Sammlung von Franklin Müller. (Beitrag III) Papuanische Curculionidae. Entomologische Mitteilungen, 14(3/4), 281-290.

Heller, K.M. (1926a) Coleoptera: Curculionidae. Nova Guinea. Résultats des expédition scientifique Néerlandaise a la Nouvelle-Guinée, 15, 275-291.

Heller, K.M. (1926b) Bestimmungsschlüssel aussereuropäischer Käfer: Gattung Mecocorynus und Ectatorrhinus (Curculionidae). Wiener Entomologische Zeitung, 43(3-4), 147-154.

Heller, K.M. (1927a) Bestimmungsschlüssel aussereuropäischer Käfer: Calandrini spurii (Laogenia etc.) und Verwandte. Entomologische Blätter, 28(1), 1-14.

Heller, K.M. (1927b) Studien zur Systematik der altweltlichen Balanini II. Stettiner Entomologische Zeitung, 88(2), 175$287+$ pl. $1-2$.

Heller, K.M. (1928) 7. Bestimmungschlüssel aussereuropäischer Käfer: Gattung Lophochirus G. A. K. Marshall (Curculionidae). Entomologisches Blätter, 24(3), 103-111.

Heller, K.M. (1929) Fauna Buruana. Coleoptera, Fam. Curculionidae. Treubia, 7(3), 105-138 + pl. III.

Heller, K.M. (1932) Die Gattung Diatassa Pasc. und ihre Arten (Coleopt., Curculioninae, Sophrorrhinini). Wiener Entomologische Zeitung, 49(2), 106-108.

Heller, K.M. (1933a) Beitrag zur Kenntnis der papuanischen Rüsselkäfergattung: Arachnopus Guér. (Zygopini). Entomologische Blätter, 29(1), 18-25.

Heller, K.M. (1933b) Faunistische und systematische Notizen über Käfer, nebst Beschreibung einer neuen Gattung und einer neuen Art. (241). Entomologische Blätter, 29(4), 145-150.

Heller, K.M. (1934) Käfer aus dem Bismarck und Salomo-Archipel. Verhandlungen der Naturforschenden Gesellschaft in Basel, 45, 1-34 + pl. 1.

Heller, K.M. (1935) Coleoptera Curculionidae. Nova Guinea. Résultats des Expeditions Scientifiques à la Nouvelle Guinée, 17(2), 155-202 + 1 pl.

Heller, K.M. (1937a) Neue papuanische Rüsselkäfer. Arbeiten über morphologische und taxonomische Entomologie aus Berlin-Dahlem, 4(4), 257-282 + pl. I.

Heller, K.M. (1937b) Coleoptera Javana, mit Berücksichtigung der ihnen nahestehenden Formen anderer Herkunft. Stettiner Entomologische Zeitung, 98(1), 51-78.

Heller, K.M. (1941a) Neue Rüsselkäfer von der Gazelle-Halbinsel, gesammelt vom Missionar P. Josef Schneider auf Neu-Pommern. Entomologische Blätter, 37(5/6), 213-218.

Heller, K.M. (1941b) Peribleptus Schonh. und Carcilia Roelofs. (Coleoptera Curculionidae, Hylobiinae). Entomologische Blätter, 37(2), 78-83.

Heller, K.M. (1941c) Neues über die Unterfamilien der Otiorrhynchinae und Calandrinae, besonders von Piezonotus u. Nassophasis (Coleoptera: Curculionidae). Arbeiten über morphologische und taxonomische Entomologie aus Berlin-Dahlem, 8(3), 149-161.

Heller, K.M. (1942) Neue Papuanische Russelkäfer aus der Sammlung des Deutschen Entomologischen Institutes (Coleoptera: Curculionoidea). Arbeiten über morphologische und taxonomische Entomologie aus Berlin-Dahlem, 9(4), 209-214.

Herbst, J.F.W. (1793) Natursystem aller bekannten in-und auslandischen Insekten, als eine Fortsetzung der von Büffonschen Naturgeschichte. Der Käfer. Pauli, Berlin, 5, 392 pp. + 16 pl.

Herbst, J.F.W. (1795) Natursystem aller bekannten in-und auslandischen Insekten, als eine Fortsetzung der von Büffonschen Naturgeschichte. Der Käfer. Pauli, Berlin, 6, xxiv + 520 pp. + pl. LX-XCV.

Herbst, J.F.W. (1797) Natursystem aller bekannten in-und auslandischen Insekten, als eine Fortsetzung der von Büffonschen Naturgeschichte. Der Käfer. Pauli, Berlin, 7, xi + 346 pp. + pl. XCVI-CXVI.

Heyden, L.v. (1911) Coleopteren der Aru-und Kei-Inseln (Ergebnisse e, zool. Forschungsreise v. H. Merton). Abhandlungen herausgegeben von der Senckenbergischen naturforschenden Gesellschaft (Frankfurt au Main), 33, 347372.

Holloway, B.A. (1982) Anthribidae (Insecta: Coleoptera). Fauna of New Zealand 3. DSIR, Wellington, 269 pp.

Hopkins, A.D. (1914) List of generic names and their type-species in the coleopterous superfamily Scolytoidea. Proceedings of the United States National Museum, 48(2066), 115-136.

Hopkins, A.D. (1915a) Classification of the Cryphalinae with descriptions of new genera and species. United States Department of Agriculture, Report 99, 1-75 + pl. 1-4

Hopkins, A.D. (1915b) Contributions toward a monograph of the scolytid beetles, Part II. Preliminary classification of the superfamily Scolytoidea. United States Department of Agriculture, Bureau of Entomology, Technical Bulletin, 17(2), 165-23 + pl. 9-15.

Horn, W. \& Kahle, I. (1935-1937) Ueber entomologische sammlungen. Entomologische Beihefte aus Berlin-Dahlem, 2$4, \mathrm{vi}+536$ pp. + pl. I-XXXVIII.

Horn, W. \& Schenkling, S. (1928-1929) Index Literature Entomologicae. W. Horn, Berlin, Band I-IV, xxi + 1426 pp.

Hubenthal, W. (1917a) Beiträge zur kenntnis der Curculioniden-Gattung Acicnemis Lacordaire (Col.). Archiv für 
Naturgeschichte, 83, (A) 8, 92-160; 9, 53-155.

Hubenthal, W. (1917b) Die indomalaiischen Arten der Curculionidengattung Desmidophorus Schönherr. Entomologische Blätter, 13(4-6), 103-123.

Hustache, A. (1925) Synopsis des Curculionides de Madagascar. Bullétin de l'Académie Malgache, Nouvelle Série, [1924], 7, 1-582.

Hustache, A. (1929) Nouveaux Curculionides de I'Amérique du Sud. Revista de la Sociedad Entomológica Argentina, 2(5), 227-232.

Hustache, A. (1934) Curculionidae: Zygopinae. In: S. Schenkling (Ed.), Coleopterorum Catalogus. W. Junk, Berlin, 136, pp. $1-96$.

Hustache, A. (1936) Curculionidae: Cryptorrhynchinae. In: S. Schenkling (Ed.), Coleopterorum Catalogus. W. Junk, Berlin, 151, pp. 1-317.

Hustache, A. (1937) Magdalinae (Coleoptera: Curculionidae) de l'Amérique méridionale. Annals and Magazine of Natural History, (10) 19(110), 198-248.

Hustache, A. (1938) Curculionidae: Barinae. In: S. Schenkling (Ed.), Coleopterorum Catalogus. W. Junk, 's Gravenhage, 163, pp. 1-219.

International Commission on Zoological Nomenclature (1991) Opinion 1655. Curculio viridicollis Fabricius, 1792 (currently Phyllobius viridicollis; Insecta, Coleoptera): specific name conserved, and Rhyncolus Germar, 1817: Curculio ater Linnaeus, 1758 designated as the type species. Bulletin of Zoological Nomenclature, 48(3), 268-269.

International Commission on Zoological Nomenclature (1999) International Code of Zoological Nomenclature, Fourth Edition. International Trust for Zoological Nomenclature, London, $306 \mathrm{pp}$.

Janczyk, F. (1959) Neue Curculioniden der Zoologische Abteilung des Naturhistorischen Museum (2. Beitrag zur Kenntnis des Curculionidae). Annalen des Naturhistorischen Museums in Wien, 63, 430-435.

Jekel, H. (1855) Coleoptera. Fam. Curculionides. In: Saunders, W.W., Insecta Saundersiana; or characters of undescribed insects in the collection of William Wilson Saunders, Esq. John van Voorst, London, 2(1), pp. 1-153 + 2 pl.

Jekel, H. (1860) Coleoptera. Fam. Curculionides. In: Saunders, W.W., Insecta Saundersiana: or characters of undescribed insects in the collection of William Wilson Saunders, Esq. John van Voorst, London, 2(2), pp. 155-244 +1 table $+1 \mathrm{pl}$.

Jekel, H. (1865) Recherches sur la classification naturelle des Curculionides. 1re partie. Annales de la Société Entomologique de France, (4) 4(3)[1864], 537-566.

Jordan, K. (1894) On Anthribidae in the Museum of the Honorable Walter Rothschild. Novitates Zoologicae, 1(4), 591651.

Jordan, K. (1895a) Beitrag zur Kenntniss der Anthribidae. Stettiner Entomologische Zeitung, 56(1-6), $122-204$.

Jordan, K. (1895b) Zur Kenntniss der Anthribidae. III. Stettiner Entomologische Zeitung, 56(7-9), 245-265.

Jordan, K. (1895c) Einige neue Käfer der Indo-Australischen region in der Sammlung des Tring-Museums. Stettiner Entomologische Zeitung, 56, 266-271.

Jordan, K. (1895d) Zur Kenntniss der Anthribidae. IV. Stettiner Entomologische Zeitung, 56, 369-401.

Jordan, K. (1897) Some new Anthribidae in the Tring Museum. Novitates Zoologicae, 4(1), 173-177.

Jordan, K. (1898) New Anthribidae. Novitates Zoologicae, 5(3), 359-373.

Jordan, K. (1903) New oriental Anthribidae. Novitates Zoologicae, 10(3), 415-434.

Jordan, K. (1904) Some new Oriental Anthribidae. Annali del Museo Civico di Storia Naturale di Genova, 41, 80-91.

Jordan, K. (1906) Two new Xenocerus (Anthribidae) in the collection of R. von Bennigsen. Novitates Zoologicae, 13(2), 410.

Jordan, K. (1908) Anthribidae. Résultats de l'expédition scientifique néerlandaise à la Nouvelle Guinée. Nova Guinea, $5(2), 351-352$.

Jordan, K. (1911) New Anthribidae. Novitates Zoologicae, 18(1), 92-116.

Jordan, K. (1913) The Oriental Anthribidae of the Van de Poll collection. Novitates Zoologicae, 20(2), $257-277$.

Jordan, K. (1914) Reports of the Percy Sladen Trust expedition to the Indian Ocean in 1905, under the leadership of Mr. J. Stanley Gardiner, M.A. Vol. 5. XIII. Coleoptera: Anthribidae. Transactions of the Linnean Society of London, (2) $16(3), 247-267+$ pl. 15 .

Jordan, K. (1916) On the oriental anthribid genus Apolecta. Novitates Zoologicae, 23, 342-349.

Jordan, K. (1923a) New Eastern Anthribidae. Novitates Zoologicae, 30(1), 167-185.

Jordan, K. (1923b) New Anthribidae from the Eastern hemisphere. Novitates Zoologicae, 30(216-221).

Jordan, K. (1924a) New Anthribidae. Novitates Zoologicae, 31(2), 231-255.

Jordan, K. (1924b) New Anthribidae allied to Choragus. Annals and Magazine of Natural History, (9) 13(78), 606-620.

Jordan, K. (1925a) Anthribidae from the Eastern Hemisphere. Novitates Zoologicae, 32(2), 242-257.

Jordan, K. (1925b) New Anthribidae collected by J.C. Corporaal on Sumatra. Annals and Magazine of Natural History, (9) 16(92), 257-264.

Jordan, K. (1928a) New Anthribidae from the Old World. Novitates Zoologicae, 34(2), 105-128.

Jordan, K. (1928b) Anthribidae. Insects of Samoa and other Samoan terrestrial Arthropoda, Part IV, Coleoptera, (2), 
$161-172$.

Jordan, K. (1932) New Oriental Anthribidae (Coleoptera). Novitates Zoologicae, 38(2), 362-383.

Jordan, K. (1936) Descriptions and records of Oriental Anthribidae. Novitates Zoologicae, 39(4), 311-325.

Jordan, K. (1937) Some Old World Anthribidae. Novitates Zoologicae, 40(2), 199-207.

Jordan, K. (1939a) Descriptions of Anthribidae, mostly from Fiji. Novitates Zoologicae, 41(4), 423-432.

Jordan, K. (1939b) On some Anthribidae from Africa and the Solomon Islands. Novitates Zoologicae, 41, 437-442.

Jordan, K. (1945) On the Oriental Anthribid genus Xenocerus Germar, 1833, with descriptions of new species and subspecies (Coleoptera). Proceedings of the Royal Entomological Society of London, (B) 14(1-2), 10-20.

Kalshoven, L.G.E. (1958) Studies on the biology of Indonesian Scolytoidea. 4. Data on the habits of Scolytidae. First part. Tijdschrift voor Entomologie, 101(3/4), 157-180 + 7 pl.

Kirby, W. (1819) A Century of Insects, including several new Genera described from his Cabinet. Transactions of the Linnean Society of London [1818], 12(2), 375-453 + pl. XXI-XXII.

Kirby, W. (1837) Part the fourth and last. The Insects. In: Richarson, J., Fauna Boreali-Americana; or the zoology of the northern parts of British America: containing descriptions of the objects of natural history collected on the late Northern Land Expeditions, under command of Captain Sir John Franklin, R. N., Norwich, xxxix +325 pp. +8 pl.

Kirsch, T. (1875) Neue Käfer Aus Malacca. Mitteilungen aus dem Koeniglichen Zoologischen Museum zu Dresden, 1 , $27-58$.

Kirsch, T. (1877) Beitrag zur Kenntniss der Coleopteren-fauna von Neu Guinea. Mittelungen aus dem Koeniglichen Zoologischen Museum zu Dresden, 2, 135-161.

Kirsch, T. (1879) Zwei neue Coleopteren-Arten aus Neu-Guinea. Annali del Museo Civico di Storia Naturale di Genova, $14,18-20$.

Kissinger, D.G. (1968) Curculionidae subfamily Apioninae of North and Central America with reviews of the World genera of Apioninae and World subgenera of Apion Herbst (Coleoptera). Taxonomic Publications, South Lancaster, Massachusetts, vii + 559 pp.

Kleine, R. (1914) Neue Brenthiden aus dem Stettiner naturhist. Museum. Stettiner Entomologische Zeitung, 75(1), 159183.

Kleine, R. (1915) Über die Gattung Ectocemus Pascoe und die systematische Stellung von E. pogonocerus Fairm. Stettiner Entomologische Zeitung, 75(2), 233-239.

Kleine, R. (1916a) Die Gattung Gyalostoma Kleine und ihr Verwandtschaftskreis. Stettiner Entomologische Zeitung, $77(1), 175-186$.

Kleine, R. (1916b) Die Gattung Prophthalmus Lac. und ihr Verwandtschaftskreis. Stettiner Entomologische Zeitung, 77(2), 217-286.

Kleine, R. (1916c) Neue Taphroderini (Brenthidae, Col.). Entomologische Mitteilungen, 5(1-4), 1-92 + pl. I-II.

Kleine, R. (1916d) Die Gattung Baryrrhynchus und ihr Verwandtschaftskreis (Fortsetzung). Entomologische Blätter, 12(7/9), 150-190.

Kleine, R. (1916e) Die Gattung Baryrrhynchus und ihr Verwandtschaftskreis. Entomologische Blätter, 12(4/6), 121-137.

Kleine, R. (1917a) Die Gattung Eupsalis und ihr Verwandtschaftskreis. Archiv für Naturgeschichte, 82, A, (4), 55-150.

Kleine, R. (1917b) Beiträge zur Kenntis der Amorphocephalus Verwandtschaft. Zoologische Mededeelingen Leiden, 3, $275-278$.

Kleine, R. (1917c) Neue Miolispa aus dem Leidener Museum. Zoologische Mededeelingen Leiden, 3(4), $289-294$.

Kleine, R. (1918) Die Gattung Hypomiolispa Kleine. Entomologische Blätter, 14(1-3), 76-84.

Kleine, R. (1919a) Über die Gattung Elytracantha Kleine. Archiv für Naturgeschichte, 83, A, (1), 37-42.

Kleine, R. (1919b) Die Gattung Ithystenus Pascoe. Archiv für Naturgeschichte, 83, A, (7), 40-136.

Kleine, R. (1919c) Die Gattung Miolispa Pascoe. Ein Versuch zu ihrer Rekonstruierung. Stettiner Entomologische Zeitung, 80(1), 176-329.

Kleine, R. (1920a) Die Gattung Jonthocerus Lacordaire. Archiv für Naturgeschichte, 85, A, (8), 30-50.

Kleine, R. (1920b) Neue und wenig bekannte Brenthiden aus dem Schwedischen Reichsmuseum. Arkiv för Zoologi, 13(13), 1-9.

Kleine, R. (1920c) Neue Brenthidae (Trachelizini) in Leidens Museum. Zoologische Mededeelingen Leiden, 5(4), 236247.

Kleine, R. (1920d) Neue Brenthiden der papuanischen Fauna aus dem Museum von "Natura artis magistra" zu Amsterdam. Tijdschrift voor Entomologie, 62(1-2), 43-51.

Kleine, R. (1921) Die Gattung Orychodes Pascoe und ihr Verwandtschaftskreis. Archiv für Naturgeschichte, 86, A, (9), 62-102.

Kleine, R. (1922a) Beitrag zur Kenntnis der Brenthidenfauna der Philippinen. Archiv für Naturgeschichte, 87, A, (10), 24-37.

Kleine, R. (1922b) Neue Brenthiden. Archiv für Naturgeschichte, 88, A, (7), 172-174.

Kleine, R. (1922c) Neue Brenthiden. Deutsche Entomologische Zeitschrift, 1922(1), 130-147.

Kleine, R. (1922d) Brenthidenstudien. II. Folge. Archiv für Naturgeschichte, 88, A, (3), 201-222. 
Kleine, R. (1922e) Neue Brenthiden aus dem Dahlemer Museum. Archiv für Naturgeschichte, 88, A, (3), $223-228$.

Kleine, R. (1923) Neue Brenthiden (Col.) aus Niederlaendisch-Indien. Treubia, 3(3-4), 405-410.

Kleine, R. (1924) Drei neue Brenthiden aus der Hauserschen Sammlung. Societas Entomologica, 39(9), $33-34$.

Kleine, R. (1925) Fauna Buruana. Coleoptera, Fam. Brenthidae. Treubia, 7(1), 14-30.

Kleine, R. (1926a) Die Brenthiden der Niederländischen Ost-Indischen Kolonieen. Capita Zoologica, 2(4), 1-86 + 3 pl.

Kleine, R. (1926b) Die Brenthiden des papuanischen Gebietes. Résultats de l'expédition scientifique Néerlandaise à la Nouvelle Guinée. Nova Guinea, 15(2), 214-274.

Kleine, R. (1926c) Neue Gattungen und Arten aus der Familie Brenthidae. Stettiner Entomologische Zeitung, 87(2), 354373.

Kleine, R. (1927) Brenthidae. In: S. Schenkling (Ed.), Coleopterorum Catalogus. W. Junk, Berlin, 89, pp. 1-94.

Kleine, R. (1928) Bestimmungstabelle der Brenthidae. Entomologische Zeitschrift, 41, 76-80, 90-94, 157-160, 296299, 325-329, 444-445, 459-462.

Kleine, R. (1929) Bestimmungstabelle der Brenthidae. Entmologische Zeitschrift, 42, 41-42, 66-68, 267-69, $291-93$.

Kleine, R. (1930) 1 Neue Brenthide und 2 Neue Lyciden aus Neu-Guinea. Nova Guinea, 15(4), 409-410.

Kleine, R. (1931) Mitteilungen über Brenthiden. Entomologische Rundschau, 48(9-11), 93-118.

Kleine, R. (1935) Bericht über die von Miss Cheesman in Britisch Neu-Guinea gesammelten Brenthiden und Lyciden. Nova Guinea. Nova Guinea. Résultats des Expeditions Scientifiques à la Nouvelle Guinée, 17(2), 303-322.

Kleine, R. (1937) Bestimmungstabelle der Brenthidae. Entomologisches Nachrichtenblatt, Troppau, 11(1): 17-29, (2): 91-103.

Kleine, R. (1938) Coleoptera. Fam. Brenthidae. In: Wytsman, P., Genera Insectorum, 207, 1-297 + 6 pl.

Kleine, R. (1939) Neue Brenthiden und Lyciden die Miss Cheesman 1936 in Holländisch Neu-Guinea gesammelt hat. Nova Guinea, 3, 106-112.

Kleine, R. (1941) Zwei neue Brenthiden den Neu-Hebriden aus dem Pariser Museum nebst einigen faunistischen Bemerkungen. Revue Française d'Entomologie, 8, 130-131.

Kleine, R. (1943) Neue Brenthiden aus dem Hungarischen National-Museum (Coleopt.). Annales Hist-Nat. Musei Nationalis Hungarici (pars Zoologica), 36, 138-144.

Kleine, R. (1944) Neue Brenthiden des Pariser Museums (Coleoptera). Revue française d'Entomologie, 10(4), 149-158.

Kleine, R., F. Spaeth \& Moser, J. (1925) Wissenschaftliche Ergebnisse der Bearbeitung der Coleopteren-Sammlungen von Franklin Müller. Entomologische Mitteilungen, 14(1), 52-55.

Klima, A. (1934a) Curculionidae. Subfam. Gymnetrinae. In: S. Schenkling (Ed.), Coleopterorum Catalogus. W. Junk, Berlin, 135, pp. 3-68.

Klima, A. (1934b) Curculionidae. Subfam. Erirrhininae. In: S. Schenkling (Ed.), Coleopterorum Catalogus. W. Junk, Berlin, 140, pp. 1-167.

Klima, A. (1935a) Curculionidae. Subfam. Ceratopinae. In: S. Schenkling (Ed.), Coleopterorum Catalogus. W. Junk, Berlin, 145, pp. 1-3.

Klima, A. (1935b) Curculionidae. Subfam. Trigonocolinae. In: S. Schenkling (Ed.), Coleopterorum Catalogus. W. Junk, Berlin, 145, pp. 1-3.

Klima, A. (1935c) Curculionidae. Subfam. Acicnemidinae. In: S. Schenkling (Ed.), Coleopterorum Catalogus. W. Junk, Berlin, 145, pp. 1-10.

Klug, C.F. (1833) Bericht über eine auf Madagascar veranstaltete Sammlung von Insecten aus der Ordnung Coleoptera. Physikalische Abhandlungen der Königlichen Akademie der Wissenschaften zu Berlin, Aus dem Jahre 1832. Berlin, 91-223 + pl. I-V.

Kojima, H. \& Morimoto, K. (2005) Weevils of the tribe Acalyptini (Coleoptera: Curculionidae: Curculioninae): redefinition and a taxonomic treatment of Japanese, Korean, and Taiwanese species. Esakia, 45, 69-115.

Kolbe, H.J. (1892) Beiträg zur Kenntniss der Brenthiden. Stettiner Entomologische Zeitung, 53(4-6), 162-175.

Kolbe, H.J. (1895) Coleopteren aus Afrika. Stettiner Entomologische Zeitung, 55(10-12), 361-397.

Kolenati, F.A. (1858) Ein neues österreichisches Curculioniden-Genus, entdeckt von Grafen von Khuenburg. Verhandlungen der kaiserlich-königlichen zoologisch-botanischen Gesellschaft in Wien, 8, 343-344 + pl. VI.

Korotyaev, B.A. (1987) Novye vidy dolgonosikov podsemejstva Apioninae (Coleoptera, Apionidae) iz tropicheskikh i subtropicheskikh rajonov Azii. In: Medvedev, L.N., Entomofauna Vietnama, 94-120.

Kuschel, G. (1995) A phylogenetic classification of Curculionoidea to families and subfamilies. Memoirs of the Entomological Society of Washington. 14, 5-33.

Kuschel, G. (1998) Comments on some Anthribidae described by Montrouzier (Coleoptera). Bulletin de l'Institute Royal des Sciences Naturelles de Belgique Entomologie, 68, 193-195.

Kuschel, G., Leschen, R.A.B. \& Zimmerman, E.C. (2000) Platypodidae under scrutiny. Invertebrate Taxonomy, 14, 771805.

Labram, D. \& Imhoff, L. (1838) Singulorum generum Curculionidum unam alteramve speciem additis iconibus. Die Gattungen der Rüsselkäfer erläutert durch bildliche Darstellung einzelner Arten. Verlag der Schweighauser'schen Buchhandlung, Basel, 1, fasc. 1-4, 8 plates + text in each fascicle, unnumbered. 
Lacordaire, T. (1863) Histoire Naturelle des Insectes. Genera des Coléoptères ou exposé méthodique et critique de tous les genres proposés jusqu 'ici dans cet ordre d'insectes. Roret, Paris, 6, 637 pp.

Lacordaire, T. (1866) Histoire Naturelle des Insectes. Genera des Coléoptères ou exposé méthodique et critique de tous les genres proposés jusqu 'ici dans cet ordre d'insectes. Roret, Paris, 7, 620 pp.

Latreille, P.A. (1802) Histoire Naturelle, Générale et Particulière, des Crustacés et des Insectes. Ouvrage faisant suite aux Oeuvres de Leclercq de Buffon, et partie du Cours complet d'Histoire naturelle rédigé par C. S. Sonnini, membre de plusieurs Sociétés Savantes. Dufart, Paris, 3, xii + 467 pp.

Latreille, P.A. (1810) Considérations genérales sur l'ordre naturel des animaux composant les classes des Crustacés, des Arachnides et des Insectes; avec un tableau méthodique de leurs genres, disposés en familles. F. Schoell, Paris, 444 pp.

Lea, A.M. (1893) Descriptions of new species of Bostrychidae. The Proceedings of the Linnean Society of New South Wales, 8, 317-323.

Lea, A.M. (1898a) Revision of the Australian Curculionidae belonging to the subfamily Cryptorhynchides. Part II. Proceedings of the Linnean Society of New South Wales, 23(2), 178-217.

Lea, A.M. (1898b) Descriptions of new species of Australian Coleoptera. Part V. Proceedings of the Linnean Society of New South Wales, 23, 521-645.

Lea, A.M. (1899) Revision of the Australian Curculionidae belonging to the subfamily Cryptorhynchides. Part III. Proceedings of the Linnean Society of New South Wales, 24(2), 200-270.

Lea, A.M. (1903) Revision of the Australian Curculionidae belonging to the Subfamily Cryptorhynchides. Part VI. Proceedings of the Linnean Society of New South Wales, 28(3), 643-679.

Lea, A.M. (1904) Descriptions of new species of Australian Coleoptera. Part VII. The Proceedings of the Linnean Society of New South Wales, 29, 60-107.

Lea, A.M. (1905) Revision of the Australian Curculionidae belonging to the Subfamily Cryptorhynchides. Part VII. Proceedings of the Linnean Society of New South Wales, 30(2), 235-258.

Lea, A.M. (1906) Descriptions of Australian Curculionidae, with notes on previously described species. Part IV. Transactions and Proceedings and Report of the Royal Society of South Australia, 30, 71-103.

Lea, A.M. (1907) Revision of the Australian Curculionidae belonging to the subfamily Cryptorhynchides. Part VIII. Proceedings of the Linnean Society of New South Wales, 32(2), 400-430.

Lea, A.M. (1908a) Notes on Australian Curculionidae in the Belgian Museum with descriptions of new species. Part 1. Mémoires de la Société entomologique de Belgique, 16, 127-186.

Lea, A.M. (1908b) The Coleoptera of King Island, Bass Strait. Proceedings of the Royal Society of Victoria, (New Series), 20(2), 143-207.

Lea, A.M. (1909) Revision of the Australian Curculionidae belonging to the Subfamily Cryptorhynchides. Part IX. Proceedings of the Linnean Society of New South Wales, 33(4), 701-732.

Lea, A.M. (1910a) On Australian and Tasmanian Coleoptera, with descriptions of new species. Part I. Proceedings of the Royal Society of Victoria, 22, 113-152 + pl. 30.

Lea, A.M. (1910b) Revision of the Australian Curculionidae belonging to the subfamily Cryptorhynchides. Part X. Proceedings of the Linnean Society of New South Wales, 32(2), 593-635.

Lea, A.M. (1912) Descriptions of Australian Curculionidae, with notes on previously described species. Part X. Transactions and Proceedings of the Royal Society of South Australia, 36, 76-139.

Lea, A.M. (1913) Notes on Cryptorhynchides (Coleoptera, Curculionidae) in the South Australian Museum, with descriptions of new species. Transactions of the Royal Society of South Australia, 37, 182-300.

Lea, A.M. (1914) On Australian and Tasmanian Coleoptera, with descriptions of new species. Part II. Proceedings of the Royal Society of Victoria, 26, 211-227.

Lea, A.M. (1915) On some Australian Malacodermidae and Curculionidae collected by Mr. G.E. Bryant. Annals and Magazine of Natural History, (8) 15(89), 452-481.

Lea, A.M. (1917) Descriptions of Australian Coleoptera. Part XII. Proceedings of the Linnean Society of New South Wales, 41, 720-745.

Lea, A.M. (1926) On some Australian Curculionidae. Proceedings of the Linnean Society of New South Wales, 51(3), $327-362$.

Lea, A.M. (1928ab) Cryptorhynchides (Curculionidae) mostly from Australia. Records of the South Australian Museum, 4(1), 49-90.

Lea, A.M. (1928b) Australian Curculionidae of the subfamilies Haplonycides and Cryptorhynchides. Transactions and Proceedings of the Royal Society of South Australia, 52, 95-164 + pl. VII-IX.

Lea, A.M. (1930) Descriptions of new species of Australian Coleoptera. XXI. Proceedings of the Linnean Society of New South Wales, 55(4), 451-467.

Lea, A.M. (1931a) Notes on some miscellaneous Coleoptera, with descriptions of new species. Part VIII. Transactions and Proceedings of the Royal Society of South Australia, 55, 39-54.

Lea, A.M. (1931b) On Baridiinae (Curculionidae), mostly from New Guinea. Proceedings of the Linnean Society of New 
South Wales, 56(3), 139-171.

Lea, A.M. (1931c) Australasian Coleoptera. Records of the South Australian Museum, 4(3), 365-408.

Leconte, J.L. \& Horn, G.H. (1876) The Rhynchophora of America North of Mexico. Proceedings of the American Philosophical Society, 15(96), i-xvi + 1-455.

Legalov, A.A. (2003) Taxonomy, classification and phylogeny of the leaf-rolling weevils (Coleoptera: Rhynchitidae: Attelabidae) of the world fauna. Novosibirsk. CD-R. 641 MB. 733 pp.

Lewis, G. (1879) A catalogue of Coleoptera, from the Japanese Archipelago. Taylor and Francis, London, $31 \mathrm{pp.}$

Lewis, G. (1883) On Japan Brenthidae, and Notes of their Habits. Journal of the Linnaean Society of London, Zoology, 17(101), 295-302 + pl. XII.

Limoges, R. \& Porion, T. (2004) Volume 19 Supplement 2: Eupholus-Nouveaux Eupholus de Nouvelle Guinee. Les Coleopteres du Monde. Hillside Books, Canterbury, U.K., 19, 7 pp.

Linnaeus, C. (1758) Systema Naturae per regna tria naturae secundum classes, ordines, genera, species, cum characteribus, differentiis, synonymis, locis. Holmiae, Laurentii Salvii, 1, 824 pp.

Linnaeus, C. (1763) Centura insectorum rariorum. Amoenitates Acadenicae, 6, 384-415.

Lona, C. (1936) Curculionidae: Otiorrhynchinae I. In: S. Schenkling (Ed.), Coleopterorum Catalogus. W. Junk, 's Gravenhage, 148, pp. 1-226.

Lona, C. (1937) Curculionidae: Otiorrhynchinae II. In: S. Schenkling (Ed.), Coleopterorum Catalogus. W. Junk, 's Gravenhage, 160, pp. 227-412.

Lona, C. (1938) Curculionidae: Otiorrhynchinae III. In: S. Schenkling (Ed.), Coleopterorum Catalogus. W. Junk, 's Gravenhage, 162, pp. 413-600.

Lucas, R. (1920) Catalogus alphabeticus generum et subgenerum Coleopterum orbis terrarum totius (famil., trib., subtrib., sect. incl.). Pars I. Archiv für Naturgeschichte, 84, A(1-5), XXXI + 696 pp.

Lund, N.T. (1800) Nogle Arten af slaegten Brentus. Skrivter af Naturhistorie-Selskabet, 5(2), 54-91.

Lyal, C.H.C. \& Alonso-Zarazaga, M.A. (2006) Addenda and corrigenda to 'A World Catalogue of Families and Genera of Curculionoidea (Insecta: Coleoptera)'. 2. Zootaxa, 1202, 21-31.

Lyal, C.H.C., Douglas, D.A. \& Hine, S.J. (2006) Morphology and systematic significance of sclerolepidia in the weevils (Coleoptera: Curculionoidea). Systematics and Biodiversity, 4(2), 203-241.

Macleay, W.J. (1885) The insects of the Maclay-coast, New Guinea. The Proceedings of the Linnean Society of New South Wales, 9.

Macleay, W.J. (1887) The insects of the Fly River, New Guinea, "Coleoptera.". The Proceedings of the Linnean Society of New South Wales, 1(2), 136-157, 183-204.

Mantilleri, A. (2005) Phylogénie des Stereodermini Sharp, 1895, et description d'un nouveau genre afrotropical (Coleoptera, Brentidae). Bulletin de la Société Entomologique de France, 110(4/5), 415-426.

Marseul, S.A.d. (1863) Catalogue des Coléoptères d'Europe et du Bassin de la Méditérranée en Afrique et en Asie. Imprimerie de Mary-Beauchêne, Laval, 300 pp.

Marshall, G.A.K. (1915) Part II. Curculionidae. In: Arrow, G.J. Marshall, G.A.K. Gahan, C.J. \& Blair, K.G. Report on the Coleoptera collected by the British Ornithologists' Union Expedition and the Wollaston Expedition in Dutch New Guinea. Transactions of the Zoological Society of London, 20(17), 497-543 + pl. 39.

Marshall, G.A.K. (1916) Coleoptera. Rhynchophora: Curculionidae. In: Shipley, A.E. (Ed.), The Fauna of British India, including Ceylon and Burma. Taylor \& Francis, London. $\mathrm{xv}+367 \mathrm{pp}$.

Marshall, G.A.K. (1921) On the Curculionidae of the Samoan Islands (Coleoptera). Proceedings of the Hawaiian Entomological Society, 4(3), 585-600.

Marshall, G.A.K. (1930) New Curculionidae, with notes on synonymy. Annals and Magazine of Natural History, 10(6), 551-577.

Marshall, G.A.K. (1931) Curculionidae. Insects of Samoa and other Samoan terrestrial Arthropoda. Part IV. Coleoptera, $5,249-346$.

Marshall, G.A.K. (1932) Notes on the Hylobiinae (Col., Curc.). Annals and Magazine of Natural History, (10) 9, 341355.

Marshall, G.A.K. (1935) Contributions à l'étude de la faune du Mozambique. Voyage de M.P. Lesne (1928-1929). 21. Coleoptera: Curculionidae. Memorias e Estudos do Museo Zoologico da Universidade de Coimbra, (1) 81, 1-53.

Marshall, G.A.K. (1938) On Elytrurus (Col. Curcul.) and an allied new genus. Transactions of the Entomological Society of London, 87(3), 69-101.

Marshall, G.A.K. (1939a) New tropical African Curculionidae (Col.). Annals and Magazine of Natural History, (11) 3, 561-583.

Marshall, G.A.K. (1939b) On the Curculionid tribe Coryssomerini (Coleopt.). Annals and Magazine of Natural History, (11) $4,1-32$.

Marshall, G.A.K. (1941a) On Exophthalmida and Parexophthalmus (Coleoptera: Curculionidae). Proceedings of the Royal Entomological Society of London, 10(4), 51-55.

Marshall, G.A.K. (1941b) New Indian Curculionidae (Col.). Annals and Magazine of Natural History, (11) 7, 184-195. 
Marshall, G.A.K. (1943) New Indian Curculionidae (Col.). Annals and Magazine of Natural History, (11) 10, $105-119$.

Marshall, G.A.K. (1944) On the genera of the tribe Cyphicerini (Col., Curc.). II. Annals and Magazine of Natural History, (11) 11, 433-462.

Marshall, G.A.K. (1946) Taxonomic notes on Curculionidae (Col.). Annals and Magazine of Natural History, (11) 13, 93-98.

Marshall, G.A.K. (1952) Taxonomic notes on Curculionidae (Col.). Annals and Magazine of Natural History, (12) 5, 261-270.

Marshall, G.A.K. (1954) On the genus Pseudocossonus Woll. (Col., Curculionidae). Entomologist's Monthly Magazine, 90(10), 232-233.

Marshall, G.A.K. (1956) The Otiorrhynchine Curculionidae of the tribe Celeuthetini (Col.). Trustees of the British Museum, London, $134 \mathrm{pp}$.

Marshall, G.A.K. (1957) Some injurious Curculionidae (Coleoptera) from New Guinea. Commonweatlth Institute of Entomology. Bulletin of Entomological Research, 48(1), 1-7.

Marshall, G.A.K. (1958) On some Indonesian Cossoninae (Coleoptera, Curculionidae). Tijdschrift voor Entomologie, 101(2), 93-99.

Marshall, G.A.K. (1959a) Curculionidae genus Gymnopholus (Coleoptera). Occasional Papers of the Bernice P. Bishop Museum, 22(7), 71-81.

Marshall, G.A.K. (1959b) Two weevil pests of coffee in New Guinea (Coleoptera). Papua and New Guinea Agriculture Journal, 12(1), 44-46.

Marvaldi, A.E., A.S. Sequeira, C.W. O'Brien \& B. Farrell (2002) Molecular and morphologcal phylogenetics of weevils (Coleoptera: Curculionoidea): Do nice shifts acompany diversification? Systematic Biology, 51(5), 761-785.

Masters, G. (1888) Catalogue of the known Coleoptera of New Guinea, including the islands of New Ireland, New Britain, Duke of York, Aru, Mysol, Waigiou, Salwatty, Key, and Jobie. Part II. Family Curculionidae. Subfamily Brachyderides. Proceedings of the Linnean Society of New South Wales, 3, 925-959.

Miller, S.E. (2004) Entomological bibliographies of New Guinea including the Solomon Islands. Smithsonian National Museum of Natural History, Department of Systematic Biology Entomology, Washington, D.C. Available from http://entomology.si.edu/Entomology/NewGuineaBib/search.lasso (accessed 13 July 2006).

Montrouzier, X. (1855) Essai sur la faune de l'lle de Woodlark ou Moiou. Annales des Sciences Physiques et Naturelles, d'Agriculture et d'Industrie, publiées par La Société impériale d'Agriculture, etc., de Lyon, 7(1), 1-114.

Montrouzier, X. (1861) Essai sur la faune entomologique de la Nouvelle-Calédonie (Balade) et des îles des Pins, Art, Lifu, etc. Annales de la Société Entomologique de France, (3)8(4), 867-916 + pl. 7.

Morimoto, K. (1962) Key to families, subfamilies, tribes and genera of the superfamily Curculionoidea of Japan excluding Scolytidae, Platypodidae and Cossoninae. (Comparative morphology, phylogeny and systematics of the superfamily Curculionoidea of Japan. III). Journal of the Faculty of Agriculture, Kyushu University, 12, 21-66.

Morimoto, K. (1972) A key to the genera of Oriental Anthribidae (Coleoptera). Bulletin of the Government Forest Experiment Station, 246, 35-54.

Morimoto, K. (1978) The family Anthribidae of Japan (Coleoptera). Part 1. Esakia, 12, 17-47.

Morimoto, K. \& Yoshihara, K. (1996) On the genera of Oriental Baridinae (Coleoptera, Curculionidae). Esakia, 36, 159.

Motschulsky, V.d. (1854) Diagnoses de Coléoptères nouveaux, trouvés par M.M. Tatarinoff et Gaschkéwitsch aux environs de Pékin. Ètudes Entomologiques, 2, 44-51.

Motschulsky, V.d. (1858) Insectes des Indes orientales. Ètudes Entomologiques, 7, 20-122.

Motschulsky, V.d. (1863) Essai d'un catalogue des insectes de l'ile Ceylon. Moskov Obshch Isp. Prirody Biol. Biul. Bulletin de la Societe Imperiale des Naturalistes de Moscou, 36, 509-517.

Motschulsky, V.d. (1866) Neue Borkenkäfer de Ceylon. Moskov Obshch Isp. Prirody Biol. Biul. Bulletin de la Societe Imperiale des Naturalistes de Moscou, 39, 401-404.

Motschulsky, V.d. (1875) Enumération des nouvelles espèces de coléoptères rapportés de ses voyages. 14ième Article. Bulletin de la Société Impériale des Naturalistes de Moscou, 48(4), 226-242.

Mulsant, M.E. \& Rey, C. (1860) Description de quelques coleopteres nouveaux ou peu connus. Annales de la Société Linnéenne de Lyon, 7, 300-345.

Murayama, J.J. (1925) Supplementary notes on the Platypodidae of Formosa. Hokkaido Imperial University, College of Agriculture, Journal, 15, 229-236.

Murayama, J.J. (1951) New genius and species of Scolytidae (Coleoptera) from Ohshima and Shionomisaki, Wakayama prefecture. Yamaguti University, Faculty of Agriculture, Bulletin, 2, 1-7.

Nunberg, M. (1961) Zur kenntnis der Malayischen und Aethiopischen Borken-und Kernkaferfauna (Col. Scolytidae und Platypodidae). Annals and Magazine of Natural History, (13) 3(34), 609-632.

Oberprieler, R.G. (2000) The larvae of the weevil tribe Eurhynchini and the phylogeny of the Brentidae (Coleoptera, Curculionoidea). Invertebrate Taxonomy, 14, 755-770.

Oberprieler, R.G. (2004) Eurhynchinae Lacordaire, 1863 (Coleoptera, Curculionoidea). In: A. Sforzi \& L. Bartolozzi, 
Brentidae of the World (Coleoptera, Curculionoidea), Torino, Spain, 39, (873-903) 976 pp.

Oberthür, R. (1883) Description de deux espèces nouvelles de Curculionides appartenant aus genre Pachyrhynchus. Annales de la Société Entomologique de France, (6) 3, XXV.

O'Brien, C.W. (1995) Curculionidae, premiere biocontrol agents (Coleoptera: Curculionidae). Memoirs of the Entomological Society of Washington, (14)5, 119-128.

O'Brien, C.W. \& Wibmer, G.J. (1989) Revision of the Neotropical genus Neohydronomus Hustache (Coleoptera: Curculionidae). Coleopterists Bulletin, 43, 291-304.

Olivier, A.G. (1790) Encyclopedie Méthodique. Histoire Naturelle. Insectes. Panckouchke, Paris, 5, 793 pp.

Olivier, A.G. (1795) Entomologie, ou Histoire Naturelle des Insectes, avec leurs caractères génériques et spécifiques, leur description, leur synonymie, et leur figure enluminée. Coléoptères. Lanneau, Paris, 4, parts 66-80, 519 pp. + 72 pl.

Olivier, A.G. (1807) Entomologie, ou Histoire Naturelle des Insectes, avec leurs caractères génériques et spécifiques, leur description, leur synonymie, et leur figure enluminée. Coléoptères. Desray, Paris, 5, 612 pp. +59 pl.

Olliff, A.S. (1889) The insect fauna of Lord Howe Island. Memoirs of the Australian Museum, 2, 75-98 + pl. VI.

Omar, Y.M., Zhang, R. \& Davis, S.R. (2006) Descriptions of two new species of Pseudocossonus Wollaston (Coleoptera: Curculionidae: Cossoninae) from mainland China with a key to the world species. Zootaxa, 1375, 59-68.

Osella, G. (1976) Eduard Voss (1884-1974) Museo Civico di Storia Naturale di Verona, 223-224.

Osella, G. (1983) Nuove specie di Curculionidi delle Regioni Orientale ed Australiana. Revue suisse de Zoologie, 90(1), 111-126.

Panzer, G.W.F. (1793) Faunae insectorum germanicae initia oder Deutschlands Insecten, Nürnberg, in der Felseckerschen Buchhandlung. Heften 8.

Parry, J.F.S. (1849) Descriptions of some new species of Coleoptera. Transactions of the Entomological Society of London, 5(7), 179-185.

Parsons, M. (1999) The Butterflies of Papua New Guinea: Their Systematics and Biology. Academic Press, London, xvi +736 pp. + pl. I-XXVI.

Pascoe, F.P. (1859a) On some new Anthribidae. Annals and Magazine of Natural History, (3) 4(23), $327-333$.

Pascoe, F.P. (1859b) On some new Anthribidae. Annals and Magazine of Natural History, (3) 4(24), 431-439.

Pascoe, F.P. (1860a) On some new Anthribidae. Annals and Magazine of Natural History, (3) 5(25), 35-48.

Pascoe, F.P. (1860b) Notices of new or little-known genera and species of Coleoptera. Part I. Journal of Entomology, 1(1), 36-64 + pl. 2-3.

Pascoe, F.P. (1862a) Notices of new or little-known genera and species of Coleoptera. Part III. Journal of Entomology, London, 1(5), 319-370 + pl. XVI-XVII.

Pascoe, F.P. (1862b) Notes on the Brenthidae. Journal of Entomology, London, 1(5), 388-394.

Pascoe, F.P. (1864) Sitting of October 3, 1864. Journal of Proceedings of the Entomological Society of London, 1864, $44-46$.

Pascoe, F.P. (1865) On some new genera of Curculionidae. Part I. Journal of Entomology, London, 2(13), 413-432 + pl. XVII.

Pascoe, F.P. (1870a) Descriptions of some genera and species of Australian Curculionidae. Transactions of the Entomological Society of London, 1870(2), 181-209 + pl. V.

Pascoe, F.P. (1870b) Descriptions of some genera and species of Australian Curculionidae. Transactions of the Entomological Society, 4, 445-484 + pl. VII.

Pascoe, F.P. (1870c) Contributions towards a knowledge of the Curculionidae. Part I. Journal of the Linnaean Society of London, 10(47), 434-458 + pl. XVII.

Pascoe, F.P. (1870d) Contributions towards a knowledge of the Curculionidae. Part I. (Continued). Journal of the Linnaean Society of London, 10(48), 459-493 + pl. XVIII-XIX.

Pascoe, F.P. (1871a) Catalogue of Zygopinae, a subfamily of Curculionidae, found by Mr Wallace in the Eastern Archipelago. Annals and Magazine of Natural History, (4) 7(39), 198-222, 258-266 + pl. XV, XVI.

Pascoe, F.P. (1871b) Notes on Coleoptera, with descriptions of new genera and species. Part I. Annals and Magazine of Natural History, (4) 8(47), 345-361 + pl. XIV.

Pascoe, F.P. (1871c) Contributions towards a knowledge of the Curculionidae. Part II. Journal of the Linnaean Society of London, Zoology, 11(51), 154-218 + pl. VI-IX.

Pascoe, F.P. (1871d) Additions to the Australian Curculionidae. Part I. Annals and Magazine of Natural History, (4) 8(44), 89-99.

Pascoe, F.P. (1872a) Additions to the Australian Curculionidae. Part II. Annals and Magazine of Natural History, (4) $9(50), 132-142$.

Pascoe, F.P. (1872b) Additions to the Australian Curculionidae. Part III. Annals and Magazine of Natural History, (4) $10(56), 84-101+$ pl. I.

Pascoe, F.P. (1872c) Notes on Coleoptera, with descriptions of new genera and species. Part II. Annals and Magazine of Natural History, (4) 10(59), 317-326 + pl. XV. 
Pascoe, F.P. (1872d) Contributions towards a knowledge of the Curculionidae. Part III. Journal of the Linnean Society of London, Zoology, 11(55), 440-492 + pl. X-XIII.

Pascoe, F.P. (1873) Additions to the Australian Curculionidae. Part V. Annals and Magazine of Natural History, (4) 12(70), 278-286.

Pascoe, F.P. (1873b) Additions to the Australian Curculionidae. Part IV. Annals and Magazine of Natural History, (4) 11, $178-199$.

Pascoe, F.P. (1874) Contributions towards a knowledge of the Curculionidae. Part IV. Journal of the Linnean Society of London, Zoology, 12, 1-99 + pl. I-IV.

Pascoe, F.P. (1875) Additions to the Australian Curculionidae. Part VIII. Annals and Magazine of Natural History, (4) $16(91), 55-67+1 \mathrm{pl}$.

Pascoe, F.P. (1881) Descriptions of Curculionidae. Part I. Cistula Entomologica, 2(24), 587-601.

Pascoe, F.P. (1882a) Notes on Coleoptera, with descriptions of new genera and species. Part IV. Annals and Magazine of Natural History, (5) 9, 25-37.

Pascoe, F.P. (1882b) Descriptions of some new genera and species of Curculionidae, mostly Asiatic. Annals and Magazine of Natural History, (5) 10(60), 443-455 + pl. XVIII.

Pascoe, F.P. (1883) Descriptions of some new genera and species of Curculionidae, mostly Asiatic. Part II. Annals and Magazine of Natural History, 12(68), 88-101.

Pascoe, F.P. (1885) List of the Curculionidae of the Malay Archipelago collected by Dr. Odoardo Beccari, L. M. d'Albertis, and others. Annali del Museo Civico di Storia Naturale di Genova, 22, 201-332 + pl. I-III.

Pascoe, F.P. (1887) Descriptions of some new genera and species of Curculionidae, mostly Asiatic. Part IV. Annals and Magazine of Natural History, (5) 20(119), 348-361.

Pascoe, F.P. (1888) Descriptions of some new genera and species of Curculionidae, mostly Asiatic. Part V. Annals and Magazine of Natural History, (6) 2(11), 409-418.

Paykull, G. (1792) Monographia Curculionum Sueciae. J. Erdman, Upasliae, pp. 1-4 + 1-151.

Perris, E. (1855) Description de sept Coléoptères nouveaux pris dans le Department des Landes. Annales de la Société Entomologique de France, 3(3), 77-79.

Perroud, B.P. (1853) Descriptions de quelques Coléoptères nouveaux ou peu connues. Annales de la Société Linnéenne de Lyon (N.S.), 1, 389-528.

Perroud, B.P. \& Montrouzier, X. (1865) Essai sur la faune entomologique de Kanala (Nouvelle-Calédonie) et description de quelques espèces nouvelles ou peu connues. Annales de la Société Linnéenne de Lyon (N.S.), 11 (1864), 46-257 + pl. I.

Petri, K. (1912) Die Gattung Gasteroclisus Desbr. Annales Musei Nationalis Hungarici (pars Zoologica) Hist-Nat, 10, 340-374.

Petri, K. (1914) Beiträge zur Käferfauna. Verhandlungen und Mitteilungen des Siebenbürgisches Vereins Naturwissenschaften, 65, 1-23.

Pierce, W.D. (1916) Studies on weevils (Rhynchophora) with descriptions of new genera and species. Proceedings of the United States National Museum, 51, 461-473.

Porion, T. (1993) Eupholus. Les Coléoptères Du Monde. Sciences Naturelles, Vennette, 19, 112 pp.

Porion, T. (2000) Eupholus.Nouveaux Eupholus de Nouvelle Guinee. Les Coléoptères Du Monde. Hillside Books, Canterbury, Volume 19, Supplement 1, 11 pp.

Pouillaude, I. (1915) Vanapa Oberthüri nouveau genre et nouvelle espèce de Curculionide (Col.). Insecta, Rennes, 5(5557), 101-105.

Power, G. (1879) Diagnoses de nouvelles espèces de Brenthides. Petites Nouvelles Entomologiques, 2(213), 297-298.

Prior, R.N.B. (1983) Introducing a weevil from Africa to pollinate oil palm in Papua New Guinea. Harvest, 9(2), 53-57.

Quoy, J.R.C. \& Gaimard, J.P. (1824-1825) Des Arachnides et des Insectes. In: L. de Freycinet (Ed.), Voyage autour du monde executé sur les corvettes de SM "L'Uranie" et "La Physicienne" pendant les années 1817-1820, Zoologie, pp. $\mathrm{i}-\mathrm{iv}+1-712$.

Rafinesque, C.S. (1815) Analyse de la Nature ou tableau de l'Univers et des corps organisés. Auteur, Palerme, 224 pp.

Ramamurthy, V.V., Poorani, J., \& Devakumar, C. (1998) Cuticular hydrocarbons and biosystematics of entimine weevil genera Lepropus Schoenherr and Brachyaspistes Fahraeus (Curculionidae: Coleoptera). Entomon, 23(4), $251-257$.

Ratzeburg, J.T.C. (1837) Die Forst-insekten oder Abbildung und Beschreibung der in den Waldern Preussens und der Nachbarstaaten als schadlich oder nutzlich bekannt gewordenen Insekten. Nicolai, Berlin, 1, x + 202 pp.

Redtenbacher, L. (1868) Coleopteren. Reise der Oesterreichischen Fregatte Novara um die Erde, in den Jahren 1857-59, unter den Befehln des Commodore B. von Wüllerstorf-Urbair. 1864-1875. Zoologischer Theil. 1867., 2(1), Wien. iv +249 pp. +5 pl.

Reitter, E. (1913a) Bestimmungs-tabelle der Borkenkäfer (Scolytidae) aus Europa und den angrenzenden Landern. Wiener Entomologische Zeitung, 32, 1-116.

Reitter, E. (1913b) Coleopterologische Notizen. Wiener Entomologische Zeitung, 32, 190.

Reitter, E. (1916) Fauna Germanica. Die Käfer des Deutschen Reiches. Nachder analytischen Methode bearbeitet. K.G. 
Lutz' Verlag, Stuttgart, 5, 343 pp. + pl. 153-168.

Rheinheimer, J. (2002) Die gattungen Exonotus und Gloeodema aus Südostasien (Coleoptera: Curculionidae: Cossoninae). Koleopterologische Rundschau, 72, 197-203.

Rheinheimer, J. (2004a) Illustrierter katalog und bibliographie der Anthribidae der Welt (Insecta: Coleoptera). Mitteilungen des Entomologischen Vereins Stuttgart, 39(1/2), 1-244.

Rheinheimer, J. (2004b) Neue arten der gattung Hellerrhinus Marshall aus Neuguinea. Koleopterologische Rundschau, $74,435-441$.

Riedel, A. (1998) Catalogue and bibliography of the genus Euops Schoenherr (Insecta, Coleoptera, Curculionoidea, Attelabidae). Spixiana, 21(2), 97-124.

Riedel, A. (1999) The spinosus-group of Euops Schoenherr (Coleoptera: Curculionoidea, Attelabidae), weevils with humeral spines from New Guinea. Entomologica scandinavica, 30, 75-117.

Riedel, A. (2001a) Revision of the simulans-group of Euops Schoenherr (Coleoptera: Curculionidae, Attelabidae) from the Papuan region. Deutsche Entomologische Zeitschrift, 48(2), 139-221.

Riedel, A. (2001b) Revision of the Euops quadrifasciatus-group (Coleoptera: Curculionoidea: Attelabidae) from the Australian region, with a discussion of shifts between Nothofagus and Eucalyptus host plants. Inveterbrate Taxonomy, 15, 551-587.

Riedel, A. (2001c) The pygmaeus-group of Euops Schoenherr (Coleoptera, Curculionoidea, Attelabidae), weevils associated with Nothofagus in New Guinea. Journal of Natural History, 35, 1173-1237.

Riedel, A. (2002a) Revision of the subgenus Neosynaptops Voss of Euops Schoenherr (Coleoptera, Curculionoidea, Attelabidae) from the Papuan region. Zoologica Scripta, 31(2), 135-165.

Riedel, A. (2002b) Two new species of Eupholus Boisduval (Coleoptera, Curculionidae, Entiminae) from West New Guinea, a discussion of their taxonomic characters, and notes on nomenclature. Zootaxa, 90, 1-16.

Riedel, A. (2002c) Taxonomy, phylogeny, and zoogeography of the weevil genus Euops (Insecta: Coleoptera, Curculionoidea) in the Papuan region. Dissertation zur Erlangung des Doktorgrades der Fakultät für Biologie der LudwigMaximilians-Universität, München, Teil 1, 216 pp.

Riedel, A. (2006) Revision of the subgenus Metaeuops Legalov of Euops Schoenherr (Coleoptera, Curculionoidea, Attelibidae) from the Papuan region. Zootaxa, 1181, 1-102.

Riedel, A. \& O'Brien, C.W. (1995) A new species-group of Ottistira Pascoe from New Guinea (Coleoptera: Curculionidae: Entiminae: Ottistirini). Invertbrate Taxonomy, 9, 247-277.

Riedel, A. \& Schönitzer, K. (1996) Revision of the soil-weevil genus Guineobius Osella from New Guinea (Coleoptera, Curculionoidea, Entiminae, Celeuthetini). Revue Suisse de Zoologie, 103(2), 453-473.

Ritsema, C. (1885) Remarks on Hymenoptera and Coleoptera. Notes from the Leyden Museum, 7(1), 54.

Roberts, H. (1979) New Platypodidae (Coleoptera) from Mt. Giluwe, Papua New Guinea. Journal of Natural History, 13(1), 81-98.

Roberts, H. (1986) New Platypodidae (Coleoptera) from the rain forests of Papua New Guinea. Esakia, $24,37-58$.

Roberts, H. (1987) Entomology bulletin: No. 46. Forest insect pests of Papua New Guinea. 2. Pin-hole borers (shot hole borers) attacks on logs, lumber and living trees. Harvest, 12(3), 91-96.

Roberts, H. (1989a) New Crossotarsus from Papua New Guinea (Platypodidae, Coleoptera). Koleopterologische Rundschau, 59, 87-93.

Roberts, H. (1989b) More new Platypodidae (Coleoptera) from Papua New Guinea. Linzer Biologische Beitrage 21, 1, 261-289.

Roberts, H. (1993) The tribe Diapodini (Coleoptera: Platypodidae) of Papua New Guinea. Occasional Papers of the Bernice P. Bishop Museum, 35, 1-32 + pl. 33-39.

Roberts, H. \& Morimoto, K. (1986) New Platypodidae (Coleoptera) from the mountain forests of Papua New Guinea. Esakia, 25, 161-181.

Roelofs, W. (1875) Lecture de la note suivante, en faisant voir à l'Assemblée l'insecte dont il y est question. Annales de la Société Entomologique de Belgique, 18, CVI-CVII.

Roelofs, W. (1879) Diagnoses de nouvelles espèces de Curculionides, Brenthides, Anthribides et Bruchides du Japon. Annales de la Société Entomologique de Belgique, 22, Comptes-Rendus, LIII-LV.

Roelofs, W. (1880) Description de deux nouvelles espèces de Cholides et de deux nouvelles espèces de Cryptorhynchides. Annales de la Société Entomologique de Belgique, 23 Comptes-Rendus, XXXIX-XLV.

Roelofs, W. (1892) Deux nouveaux genres et deux nouvelles espèces du groupe des Rhynchophorides. Notes from the Leyden Museum, 14(3/4), 207-212.

Roepke, W. (1919) Thamnurgides myristicae, eine neue javanische Ipide (Coleoptera: Scolytidae) aus Muskat-Nussen. Treubia, 1, 23-29.

Rossi, P. (1790) Fauna Etrusca sistens insecta quae in provinciis Florentina et Pisana praesertim collegit. T. Masu \& Chanda Sociorum, Liburni, Tomus primus, xii $+272 \mathrm{pp}$.

Samouelle, G. (1819) The Entomologist's Useful Compendium; or an introduction to the knowledge of British insects, comprising the best means of obtaining and preserving them, and a description of the apparatus generally used; 
together with the genera of Linné, and the Modern Method of arranging the classes Crustacea, Myriapoda, Spiders, Mites, and Insects, from their affinities and structure, according to the views of Dr. Leach. Also an explanation of the terms used in Entomology; a calendar of the times of appearance and usual situations of near 3,000 species of British Insects; with instructions for collecting and fitting up objects for the microscope. Thomas Boys, London, $496 \mathrm{pp}$. $+12 \mathrm{pl}$.

Sampson, F.W. (1911) On two new woodboring beetles (Ipidae). Annals and Magazine of Natural History, 8(8), 381383.

Sampson, F.W. (1912) Some new species of Ipidae and Platypodidae in the British Museum. Annals and Magazine of Natural History, 8(10), 245-250.

Sampson, F.W. (1914) Coleoptera; Platypodidae and Ipidae from the Seychelles Islands. Zoological Journal of the Linnean Society of London, (2) Zoology 16, 379-391.

Sampson, F.W. (1919) Notes on Platypodidae and Scolytidae collected by Mr. G. E. Bryant and others. Annals and Magazine of Natural History, 4(21), 105-114.

Sampson, F.W. (1921) Further notes on Platypodidae and Scolytidae collected by Mr. G. E. Bryant and others. Annals and Magazine of Natural History, 9(7), 25-37.

Sampson, F.W. (1923) Previously undescribed Scolytidae and Platypodidae from the Indian area, Part II. Annals and Magazine of Natural History, 9(11), 285-289.

Sampson, F.W. (1924) A new species of Platypodidae, genus Crossotarsus, from New Guinea. Nova Guinea, 15(1), 8990.

Sawada, Y. (1993) A systematic study of the family Rhynchitidae of Japan (Coleoptera, Curculionoidea). Humans and Nature, 2, 1-93.

Schaufuss, C.F.C. (1891) Beitrag zur Kaferfauna Madagascar's II. Tijdschrift voor Entomologie, 34, 1-35.

Schaufuss, L.W. (1864) Neue Coleopteren aus Neu-Guinea. Sitzungs-berichte der Naturwissenschaftlichen Gesellschaft Isis zu Dresden, 1864(1-3), 19-23.

Schaufuss, L.W. (1882) Descriptions de Coléoptères nouveaux. Annales de la Société Entomologique de France, (6) 2(1), 43-45.

Schedl, K.E. (1931) Notes on the genus Xyleborus Eichh. Annals and Magazine of Natural History, (10) 8, $339-347$.

Schedl, K.E. (1934) Neue Borkenkäfer. Entomologische Blätter, 30(1), 37-39.

Schedl, K.E. (1935a) Neue Platypodiden aus Afrika, Neuguinea, Zentral-und Südamerika. Entomologische Nachrichten, 9(3), 149-154, 174-175.

Schedl, K.E. (1935b) New barkbeetles and ambrosia-beetles (Col.). Stylops, 4(12), 270-276.

Schedl, K.E. (1935c) Fauna Philippinensis (Platypodidae et Scolytidae), III. Philippine Journal of Science, 56(3), 395403.

Schedl, K.E. (1935d) Scolytidae and Platypodidae: new species from the Philippine Islands and Formosa. Philippine Journal of Science, 57(4), 479-489.

Schedl, K.E. (1935e) Some new Platypodidae from Borneo and Malaya. Journal of the Federated Malay States Museums, 17(4), 632-642.

Schedl, K.E. (1936a) Notes on Malayan Scolytidae and Platypodidae and descriptions of some new species. Journal of the Federated Malay States Museums, 18(1), 1-18.

Schedl, K.E. (1936b) Some new Scolytidae and Platypodidae from the Malay Peninsula. Journal of the Federated Malay States Museums, 18(1), 19-35, 1 fig.

Schedl, K.E. (1936c) Platypodidae des Museo Civico di Storia Naturale di Genova. Annali del Museo Civico di Storia Naturale di Genova, 59, 43-62.

Schedl, K.E. (1936d) Scolytidae and Platypodidae: Fauna Philippinensis, IV. Philippine Journal of Science, 60(1), 5967.

Schedl, K.E. (1936e) Scolytidae and Platypodidae. Contribution 35. The collection of the South Australian Museum. Records of the South Australian Museum, 5(4), 513-535.

Schedl, K.E. (1937a) Platypodidae des Berliner Zoologischen Museums. Entomologische Blätter, 33(1), 33-44.

Schedl, K.E. (1937b) Scolytidae und Platypodidae. 34 Contribution. Fauna Borneensis, Part 1. Sarawak Museum Journal, Kuching, 4(4), 543-552.

Schedl, K.E. (1938a) Scolytidae und Platypodidae. Contribution 49. New species from Australia and the Fiji Islands with some revisional notes. Transactions of Royal Society of South Australia, 62, 34-52.

Schedl, K.E. (1938b) Scolytidae and Platypodidae: Fauna Philippinensis, V. Philippine Journal of Science, 67(4), 421429.

Schedl, K.E. (1939a) Malaysian Scolytidae and Platypodidae (IV). 57 Contribution. Journal of the Federated Malay States Museums, 18(3), 327-364.

Schedl, K.E. (1939b) Die Einteilung und geographische Verbreitung der Platypodidae. Verhandllungen VII Internationaler Kongre $\beta$ von Entomologie, 7(1), 377-410.

Schedl, K.E. (1940) Scolytidae and Platypodidae. 61 Contribution. Annals and Magazine of Natural History, (11) 5(29), 
433-442.

Schedl, K.E. (1941a) Platypodiden und Scolytiden. 43 Beitrag. Revue Française d'Entomologie, 7, $152-157$.

Schedl, K.E. (1941b) Die Variations-Breite in den Platypi cupulati Chap. 73 Beitrag. Archiv für Naturgeschichte, 10(3), 416-426.

Schedl, K.E. (1942a) Neue Scolytidae aus Java. 76 Beitrag. Tijdschrift voor Entomologie, 85, 1-49.

Schedl, K.E. (1942b) Interessante und neue Scolytiden und Platypodiden aus der australischen Region. 79 Beitrag. Mitteilungen der Münchener Entomologischen Gesellschaft, 32, 162-201.

Schedl, K.E. (1942c) Forschungs-Berichte zur Scolytoiden-Fauna der malayischen Halbinsel. V. 80 Beitrag. Kolonialforstliche Mitteilungen Neudamm und Berlin, 5(2-3), 169-218.

Schedl, K.E. (1950) Neotropical Scolytoidea, II. 107 Contribution. Dusenia, 1(3), 145-180.

Schedl, K.E. (1951) Fauna Indomalayaensis, I. Tijdschrift voor Entomologie, 93, 41-98.

Schedl, K.E. (1952) Neotropische Scolytoidea, III. 110 Beitrag. Dusenia, 3(5), 343-366.

Schedl, K.E. (1953) New Scolytoidea. Queensland Museum Memoirs, 13, 80-83.

Schedl, K.E. (1955) Borken-und Ambrosiakäfer aus dem Pazifischen Raum. 150 Beitrag. Entomologische Arbeiten aus dem Museum G. Frey, 6, 277-310.

Schedl, K.E. (1957) Scolytoidea nouveaux du Congo Belge, II. Mission R. Mayne-K.E. Schedl 1952. Annales du Musee Royale du Congo Belge Tervuren (Belgique), Ser. 8, Sciences Zoologiques, 56, 1-162.

Schedl, K.E. (1958a) Zur Synonymie der Borkenkäfer II. 159 Beitrag. Tijdschrift voor Entomologie, 101(3-4), 141-155.

Schedl, K.E. (1960) Zur Synonymie der Borkenkäfer V. 181 Beitrag. Entomologische Blätter, 56(2), $103-112$.

Schedl, K.E. (1962a) Scolytidae und Platypodidae aus dem australisch-polynesischen Raum. 206 Beitrag zur Mophologie und Systematik der Scolytoidea. Entomologische Arbeiten aus dem Museum G. Frey, 13(1), 72-78.

Schedl, K.E. (1962b) Zur Synonymie der Borkenkäfer, X. 213 Beitrag. Mitteilungen der Münchener Entomologischen Gesellschaft, 52, 85-107.

Schedl, K.E. (1962c) Synonymies of bark beetles, VII. 204 Contribution. Annals and Magazine of Natural History, (13) 4, 697-699.

Schedl, K.E. (1964a) Scolytoidea from Borneo, III. 185 Contribution. Reichenbachia, 4, 241-254.

Schedl, K.E. (1964b) On some Coleoptera of economic importance from New Guinea and Australia. Pacific Insects, $6(1), 211-214$.

Schedl, K.E. (1964c) Zur Synonymie der Borkenkäfer, XV. 228 Beitrag. Reichenbachia, 3, 303-317.

Schedl, K.E. (1964d) Neue und interessante Scolytoidea von den Sunda-Inseln, Neue Guinea und Australien. 202 Beitrag. Tijdschrift voor Entomologie, 107(5), 297-306.

Schedl, K.E. (1965) Scolytidae und Platypodidae aus dem Naturhistorika Riksmuseum in Stockholm. 235 Beitrag. Arkiv foÌr Zoologi, (2) 18(3), 17-31.

Schedl, K.E. (1966a) Pin-hole borers and bark-beetles (Scolytidae and Platypodidae) intercepted from imported logs in Japanese ports. 241 Contribution to the morphology and taxonomy of the Scolytoidea. Kontyû, 34, $29-43$.

Schedl, K.E. (1966b) Etwas über die Borkenkäfer der Araucarien. 239 Beitrag. Anzeiger für Schädlingskunde, 39(3), 4245.

Schedl, K.E. (1968a) On some Scolytidae and Platypodidae of economic importance from the Territory of Papua and New Guinea. 250 Contribution. Pacific Insects, 10(2), 261-270.

Schedl, K.E. (1968b) Some interesting and new Platypodidae (Coleoptera) from New Guinea. 257 Contribution. Pacific Insects, 10, 535-537.

Schedl, K.E. (1969a) Scolytidae und Platypodidae aus Neu-Guinea (Coleoptera). 263 Beitrag. Opuscula Zoologica Budapest, 9(1), 155-158.

Schedl, K.E. (1969b) Further new Scolytoidea from the territory of Papua and Guinea. 267 Contribution. The Proceedings of the Linnean Society of New South Wales, 94(3), 214-236.

Schedl, K.E. (1969c) Zur Synonymie der Borkenkäfer, XVIII. 253 Beitrag. Entomologische Arbeiten aus dem Museum G. Frey, 20, 79-105.

Schedl, K.E. (1970a) Another collection of Scolytidae and Platypodidae of economic importance from the Territory of Papua New Guinea, 254 Contribution to the morphology and taxonomy of Scolytoidea. Proceedings of the Linnean Society, New South Wales, 94(2), 128-132.

Schedl, K.E. (1970b) Bark beetles and pin-hole borers (Scolytidae and Platypodidae) intercepted from imported logs in Japanese ports, IV. 274 Contribution. Kontyû, 38, 353-370.

Schedl, K.E. (1971a) Scolytidae und Platypodidae aus dem Zoologischen Museum der Universitat in Kopenhagen (Insecta, Coleoptera). Steenstrupia, 1, 145-156.

Schedl, K.E. (1971b) Indomalayan bark and timber beetles. 276 Contribution. Oriental Insects, 5(3), 361-399.

Schedl, K.E. (1972a) Monographie der Familie Platypodidae Coleoptera. W. Junk, Den Haag, v + 322 pp.

Schedl, K.E. (1972b) New Scolytidae and Platypodidae from the Papuan subregion and New Caledonia, I. 271 Beitrag. Papua New Guinea Agriculture Journal, 23(3-4), 49-60.

Schedl, K.E. (1972c) Scolytidae and Platypodidae from the Papuan subregion and Australia. 279 Beitrag. Papua New 
Guinea Agriculture Journal, 23(3-4), 61-72.

Schedl, K.E. (1972d) Scolytidae of Ceylon. 287 Contribution. Mitteilungen der Schweizerischen Entomologischen Gesellschaft, 45(1-3), 221-229.

Schedl, K.E. (1972e) Bark and timber beetles of the Pacific Islands. 282 Beitrag. New Zealand Journal of Science, 15(3), 265-272.

Schedl, K.E. (1973a) Scolytidae and Platypodidae of the Archbold Expeditions to New Guinea. 280 Contribution. Papua New Guinea Agricultural Journal, 24(2), 70-77.

Schedl, K.E. (1973b) New Scolytidae and Platypodidae from the Papuan subregion. 299 Contribution to morphology and taxonomy of Scolytoidea. Papua New Guinea Agricultural Journal, 24(3), 87-97.

Schedl, K.E. (1974) New Scolytidae and Platypodidae from the Papuan subregion and New Caledonia, III. 302 Contribution. Annalen des Naturhistorischen Museums in Wien, 79, 457-472.

Schedl, K.E. (1975a) Zur Synonymie der Borkenkäfer, XXVI. 318 Beitrag. Zeitschrift der Arbeitsgemeinschaft Osterreich Entomologen, 27(1/2), 33-38.

Schedl, K.E. (1975b) Die Unterfamilie Scolytoplatypinae (Coleoptera, Scolytoidea). 307 Beitrag zur Morphologie und Systematik der Scolytoidea. Entomologische Abhandlungen Staatliches Museum für Tierkunde in Dresden, 40(7), 199-267.

Schedl, K.E. (1975c) New Scolytidae and Platypodidae from Papua New Guinea (Coleoptera). 315 Contribution to the Morphology and Taxonomy of the Scolytoidea. Reichenbachia, 15, 215-232.

Schedl, K.E. (1975d) New Scolytidae and Platypodidae from Papua New Guinea, IV. 317 Contribution. Annalen des Naturhistorischen Museums in Wien, 79, 337-399.

Schedl, K.E. (1975e) Scolytidae und Platypodidae (Coleoptera) aus Papua-Neu-Guinea. Folia Entomologica Hungarica, 28(2), 345-348.

Schedl, K.E. (1978) Die Typen der Sammlung Schedl Familie Platypodidae (Coleoptera). Kataloge der wissenschaftlichen Sammlungen des Naturhistorisches Museum Wien, Entomologie, 1, 1-82.

Schedl, K.E. (1979a) Die Typen der Sammlung Schedl Familie Scolytidae (Coleoptera). Kataloge der wissenschaftlichen Sammlungen des Naturhistorisches Museum Wien, Entomologie, 3(2), 1-286.

Schedl, K.E. (1979b) New Scolytidae and Platypodidae from Papua New Guinea V. (Coleoptera). 311 Contribution. Faunistische Abhandlungen, 7, 95-120.

Schedl, K.E. (1979c) Zur Synonymie der Borkenkäfer, XXIX. 345 Beitrag. Entomologischen Arbeiten aus dem Museum G. Frey, 28, 119-132.

Schedl, K.E. (1979d) Scolytidae aus West Irian. 338 Beitrag zur Morphologie und Systematik der Scolytoidea. Entomologische Blätter, 74(3), 158.

Schedl, K.E. (1980) Zur Synonymie der Borkenkäfer, 28. 339 Beitrag. Zeitschrift der Arbeitsgemeinschaft Osterreich Entomologen, 31(3/4), 117-124.

Schenkling, S. (1941) Curculionidae: Subfam. Magdalinae. In: S. Schenkling (Ed.), Coleopterorum Catalogus. W. Junk, Berlin, 141, pp. 1-31.

Schenkling, S. \& Marshall, G.A.K. (1931) Curculionidae: Subfam. Leptopiinae. In: S. Schenkling (Ed.), Coleopterorum Catalogus. W. Junk, Berlin, 114, pp. 1-83.

Schenkling, S. \& Marshall, G.A.K. (1934) Curculionidae: Subfam. Laemosaccinae. In: S. Schenkling (Ed.), Coleopterorum Catalogus. W. Junk, Berlin, 139, pp. 1-8.

Schenkling, S. \& Marshall, G.A.K. (1936a) Curculionidae: Subfam. Aterpinae. In: S. Schenkling (Ed.), Coleopterorum Catalogus. W. Junk, Berlin, 150, pp. 1-9.

Schenkling, S. \& Marshall, G.A.K. (1936b) Curculionidae: Subfam. Prionomerinae. In: S. Schenkling (Ed.), Coleopterorum Catalogus. W. Junk, Berlin, 150, pp. 3-11.

Schneider, D.H. (1791) Nachrichten von neu angenommenen Gattungen (Generibus) im entomologischen System. Neuestes Magazin für die Liebhaber der Entomologie, 1(1), 11-89.

Schneider, M.F. (1999) Entomology, A Textbook for Students, Agriculturalists and Foresters in Papua New Guinea. Training Manual no. 19. Bulolo University College, Port Moresby, viii $+312 \mathrm{pp}$.

Schönfeldt, H.v. (1906) Brenthidae. Nova Guinea. Résultants de l'expédition scientifique néerlandaise à la NouvelleGuinée en 1903 sous les auspices de Arthur Wichmann, 5(1), 35-36.

Schönherr, C.J. (1823) Curculionides [Tabula synoptica familiae Curculionidum]. Isis von Oken, 1823(10), 1132-1146.

Schönherr, C.J. (1825) Continuatio Tabulae synopticae Familiae Curculionidum. Isis von Oken, 1825(5), 581-588.

Schönherr, C.J. (1826) Curculionidum dispositio methodica cum generum characteribus, descriptionibus atque observationibus variis seu Prodromus ad Synonymiae Insectorum, partem IV. Fleischer, Lipsiae, x + 338 pp.

Schönherr, C.J. (1833) Genera et species curculionidum, cum synonymia hujus familiae. Species novae aut hactenus minus cognitae, descriptionibus a Dom. Leonardo Gyllenhal, C.H. Boheman, et entomologis aliis illustratae. Roret, Paris, 1 (1), i-Xv + 1-381 pp.

Schönherr, C.J. (1834) Genera et species curculionidum, cum synonymia hujus familiae. Species novae aut hactenus minus cognitae, descriptionibus a Dom. Leonardo Gyllenhal, C.H. Boheman, et entomologis aliis illustratae. Roret, 
Paris, 2 (2), pp. 329-669.

Schönherr, C.J. (1835) Genera et species curculionidum, cum synonymia hujus familiae. Species novae aut hactenus minus cognitae, descriptionibus a Dom. Leonardo Gyllenhal, C.H. Boheman, et entomologis aliis illustratae. Roret, Paris, 3 (1), pp. 1-505.

Schönherr, C.J. (1836) Genera et species curculionidum, cum synonymia hujus familiae. Species novae aut hactenus minus cognitae, descriptionibus a Dom. Leonardo Gyllenhal, C.H. Boheman, et entomologis aliis illustratae. Roret, Paris, 3 (2), pp. 506-858.

Schönherr, C.J. (1837) Genera et species curculionidum, cum synonymia hujus familiae. Species novae aut hactenus minus cognitae, descriptionibus a Dom. Leonardo Gyllenhal, C.H. Boheman, et entomologis aliis illustratae. Roret, Paris, 4 (1), pp. 1-600.

Schönherr, C.J. (1838) Genera et species curculionidum, cum synonymia hujus familiae. Species novae aut hactenus minus cognitae, descriptionibus a Dom. Leonardo Gyllenhal, C.H. Boheman, et entomologis aliis illustratae. Roret, Paris, 4 (2), pp. 601-1121.

Schönherr, C.J. (1839) Genera et species curculionidum, cum synonymia hujus familiae. Species novae aut hactenus minus cognitae, descriptionibus a Dom. Leonardo Gyllenhal, C.H. Boheman, et entomologis aliis illustratae. Roret, Paris, 5 (1), pp. 1-456.

Schönherr, C.J. (1840) Genera et species curculionidum, cum synonymia hujus familiae. Species novae aut hactenus minus cognitae, descriptionibus a Dom. Leonardo Gyllenhal, C.H. Boheman, et entomologis aliis illustratae. Roret, Paris, 5 (2), pp. 465-970.

Schönherr, C.J. (1842) Genera et species curculionidum, cum synonymia hujus familiae. Species novae aut hactenus minus cognitae, descriptionibus a Dom. Leonardo Gyllenhal, C.H. Boheman, et entomologis aliis illustratae. Roret, Paris, 7 (1) (1843), pp. 1-479.

Schönherr, C.J. (1843) Genera et species curculionidum, cum synonymia hujus familiae. Species novae aut hactenus minus cognitae, descriptionibus a Dom. Leonardo Gyllenhal, C.H. Boheman, et entomologis aliis illustratae. Roret, Paris, 7 (2), pp. 1-461.

Schönherr, C.J. (1844) Genera et species curculionidum, cum synonymia hujus familiae. Species novae aut hactenus minus cognitae, descriptionibus a Dom. Leonardo Gyllenhal, C.H. Boheman, et entomologis aliis illustratae. Roret, Paris, 8 (1), pp. 1-442.

Schönherr, C.J. (1845) Genera et species curculionidum, cum synonymia hujus familiae. Species novae aut hactenus minus cognitae, descriptionibus a Dom. Leonardo Gyllenhal, C.H. Boheman, et entomologis aliis illustratae. Roret, Paris, 8 (2), pp. 1-504.

Schönherr, C.J. (1847) Mantissa secunda familiae curculionidum seu descriptiones novorum quorundam generum curculionidum. Holmiae, 1-86 pp.

Schrank, F.P. (1798) Fauna Boica. Durchgedachte Geschichte der in Baiern einheimischen und zahmen Thiere. Steinsche Buchhandlung, Nürnburg, 1, xii +720.

Semenov-Tian-Shanskij, A. (1892) De Brenthidarum genere novo palaearctico. Horae Societatis Entomologicae Rossicae, 26(3/4), 438-443.

Senna, A. (1892a) Contributions to the knowledge of the family Brenthidae. VIII. Enumeration of the species known as yet from Java. Notes from the Leyden Museum, 14(3/4), 161-186.

Senna, A. (1892b) Viaggio di Leonardo Fea in Birmania e regioni vicine. XLV. Brentidi. Annali del Museo Civico di Storia Naturale di Genova, (2) 12, 429-494.

Senna, A. (1892c) Contribuzioni allo studio dei Brentidi. Nota X. Aggiunte, correzioni e note critche ai cataloghi dei Brentidi. Bollettino della Società entomologica italiana, 24, 38-63.

Senna, A. (1893a) Contribuzioni allo studio dei Brentidi. Nota XIII. Diagnosi di alcune nuove specie del genere Hoplopisthius. Bollettino della Società entomologica italiana, 24, 253-256.

Senna, A. (1893b) Contribuzioni allo studio dei Brentidi. Nota XVI. Brentidi raccolti nell'Isola di Engano dal Dott. Elio Modigliani. Annali del Museo Civico di Storia Naturale di Genova, (2) 13, 256-284.

Senna, A. (1893c) Contribuzioni allo studio dei Brentidi. Nota XXII. Su alcuni Brentidi importati col tabacco secco di Sumatra. Bullettino della Società Entomologica Italiana, 25, 288-335 + pl. II-IV.

Senna, A. (1893d) Voyage de M.E. Simon au Venezuela. (Decembre 1887-Avril 1888). 22 Mémoire. Brenthidae. Note XI. Annales de la Société entomologique de France, 62, 51-56.

Senna, A. (1894a) Viaggio di Lamberto Loria nella Papuasia orientale. Annali del Museo Civico di Storia Naturale di Genova, 2(14), 554-564.

Senna, A. (1894b) Diagnosi di due nuove specie di Brentidi. Società entomologica italiana. Resoconti di Adunanze e Bullettino bibliografico, 26, 11-13.

Senna, A. (1895a) On some new Brenthidae from Java and Sumatra. Notes from the Leyden Museum, 16(1/2), 179-196.

Senna, A. (1895b) Description of two new Brenthidae. Notes from the Leyden Museum, 17(1/3), 57-62.

Senna, A. (1895c) Quelques espèces de Miolipsa Pasc. du Musée Royal de Belgique, du Musée Rothschild à Tring et de ma collection. Annales de la Société entomologique de Belgique, 39, 358-367. 
Senna, A. (1896) On the genus Cerobates Schh. and description of some new species. Notes from the Leyden Museum, 17(4), 209-224.

Senna, A. (1897) Quelques espèces nouvelles de Brenthides du Musée Royal de Belgique et de ma collection et notes diverses. Annales de la Société entomologique de Belgique, 41, 227-236.

Senna, A. (1898a) On a new genus and several new species of Brenthidae. Notes from the Leyden Museum, 20(1), 65-76.

Senna, A. (1898b) Viaggio del Dott. Elio Modigliani nelle Isole Mentawei. Nota sui Brentidi. Annali del Museo Civico di Storia Naturale di Genova, (2) 19, 221-239.

Senna, A. (1899) Aggiunte alla fauna brentidologica di Celebes. Bollettino della Società entomologica italiana, 31, 300311.

Senna, A. (1902) Coleoptera: Rhynchophora, Brenthidae. In: Shelford R. Observations on some Mimetic Insects and Spiders from Borneo and Singapore. Proceedings of the Zoological Society of London, 2, 279-281.

Senna, A. (1903) Brentidi delle regioni dei Batacchi indipendenti (Sumatra). Bullettino della Società Entomologica Italiana, 34, 152-178.

Senna, A. (1909) Note sulle specie di Diuruse descrizione del nuovo genere Eterodiurus. Bullettino della Società Entomologica Italiana, 41, 28-53.

Senna, A. \& Calabresi, E. (1919) Contribuzione allo studio dei Brentidi. Revisione del gruppo Hoplopisthi. Bullettino della Società Entomologica Italiana, 50, 63-77.

Senoh, T. (1984) A revision of the genus Deropygus Sharp in Japan, with descriptions of a new genus and five new species (Coleoptera: Anthribidae). Transactions of the Shikoku Entomological Society, 16(3), 25-40.

Sforzi, A. \& Bartolozzi, L. (2004) Brentidae of the world (Coleoptera, Curculionoidea). Monografie. Museo Regionale di Science Naturali, Torino, 39, 976 pp.

Sharp, D. (1876) On the Anthribidae of New Zealand. Annals and Magazine of Natural History, 4(17), 422-439.

Sharp, D. (1877) Descriptions of some new species, and indications of new genera of Coleoptera from New Zealand. Entomologist's Monthly Magazine, 14, 7-10.

Sharp, D. (1889) The Rhynchophorous Coleoptera of Japan. Part I. Attelabidae and Rhynchitidae. Transactions of the Entomological Society of London, 1889(1), 41-74.

Sharp, D. (1891) The Rhynchophorous Coleoptera of Japan. Part II. Apionidae and Anthribidae. Transactions of the Entomological Society of London, 1891(2), 293-328.

Sharp, D. (1900) On the Insects from New Britain. Zoological results based on material from New Britain, New Guinea, Loyalty Islands and elsewhere, collected during the years 1895, 1896 and 1897. University Press, Cambridge, 4, 381-394 pp. + pl. XXXV.

Sharp, D. (1919) Studies in Rhynchophora. 4. An aberrant new genus and tribe from New Guinea. Entomologist's Monthly Magazine, 55, 151-153.

Shaw, S. (1953) Brenthidae (Col.) from Sumba and Flores. Verhandlungen der Naturforschenden Gessellschaft in Basel, $64,328-332$.

Snellen van Vollenhoven, M. (1864) Description de quelques espèces nouvelles de coléopteres. Tijdschrift voor Entomologie, $7,145-170+3$ pls.

Snellen van Vollenhoven, M. (1866) Beschrijving van eenige niewe soorten van Coleoptera uit Oost-Indie. Tijdschrift voor Entomologie, (3) 9(1), 222 -229.

Snellen van Vollenhoven, M. (1871) Quelques espèces nouvelles de curculionites et de longicornes. Tijdschrift voor Entomologie, 14, 101-112 + pl. 4-5.

Stibick, J.N. (1978) The genus Pantorhytes (Coleoptera: Curculionidae) Division A.I. Additions and changes to the common and major cacao species. Pacific Insects, 18(3-4), 115-136.

Stibick, J.N. (1979) The genus Pantorhytes (Coleoptera: Curculionidae) II. Additions to the four-spotted species of studded weevils of New Guinea. Pacific Insects, 20(1), 101-131.

Strand, E. (1918) Anmerkung bei der Korrektur. Archiv für Naturgeschichte, (A) 82(12), 167.

Strohmeyer, H. (1910) Neue Borkenkäfer aus Abessynien, Madagaskar, Indien und Tasmania. Entomologische Blätter, 6, 126-132.

Strohmeyer, H. (1911) Borkenkäfer der Philippinen. Philippine Journal of Science, D. General Biology, (D) 6(1), 17-29.

Strohmeyer, H. (1912) Platypodidae. In: S. Schenkling (Ed.), Coleopterorum Catalogus. W. Junk, Berlin, 44, 1-26.

Strohmeyer, H. (1913) Neue Platypodiden. Entomologische Blätter, 9(7-8), 161-165.

Swaine, J.M. (1918) Canadian bark-beetles, Part. 2. A preliminary classification with an account of the habits and means of control. Dominion of Canada Department of Agriculture, Entomological Branch, Technical Bulletin, 14(2), 1$143+31$ pls.

Tanner, V.M. (1969) A Study of the weevil tribe Celeuthetini of the Solomon Islands (Coleoptera: Curculionidae). Brigham Young University Science Bulletin, X(3), 1-46.

Thompson, R.T. (1977) A revision of the New Guinea weevil genus Apirocalus Pascoe (Coleoptera: Curculionidae). Bulletin of the British Museum (Natural History), Entomology series, 36(5), 193-280.

Thompson, R.T. (1984) A taxonomic review of the genus Isoleptus (=Nothoballus) (Coleoptera: Curculionidae: Crypto- 
rhynchinae). International Journal of Entomology, 26(3), 206-221.

Thompson, R.T. (1991) The "missing" females of Albertisius gestroi (Pascoe) (Coleoptera: Curculionidae), an enigma of natural history, with a note on Dr. H. James. Proceedings of the Entomological Society of Washington, 93(2), 240243.

Thompson, R.T. (1992) Observations on the morphology and classification of weevils (Coleoptera, Curculionidae) with a key to major groups. Journal of Natural History, 26, 835-891.

Thompson, R.T. (1996) The species of Phaenomerus Schönherr (Coleoptera:Curculionidae: Zygopinae) of the Australian Region. Invertebrate Taxonomy, 10, 937-993.

Thompson, R.T. (2005) New species and records of Apirocalus Pascoe (Coleoptera: Curculionidae: Entiminae: Celeuthetini) from New Guinea. Australian Entomologist, 32(3), 111-133.

Thomson, C.G. (1859) Skandinaviens Coleoptera, synoptiskt bearbetade. Vol. 1. Berlingska Boktryckeriet, Lund, 290 pp.

Thomson, J. (1857a) Description de treize Coléoptères. Archives Entomologiques, 1, 281-290.

Thomson, J. (1857b) Wallace. Voyage dans L'Asie orientale. Fragments entomologiques renfermant la description de Coléoptères nouveaux ou rares. Archives Entomologiques, 1, 425-460.

Thomson, J. (1857c) Description de trois espèces de Coléoptères. Archives Entomologiques, 1, 341-344.

Toxopeus, L.J. (1940) Entomologische notities uit Nieuw-Guinea. Entomologische Mededeelingen van NederlandschIndie, 6(2), 17-21, 37-43.

Valentine, B.D. (1971) Notes on Anthribid weevils. I. Some Indo-Australian genera related to Eucornyus. Proceedings of he Biological Society of Washington, 84(54), 459-466.

Valentine, B.D. (1989) The Anthribidae of the Seychelles and Mascarene Islands: taxonomy, keys and a bibliographic catalogue (Coleoptera). Insecta Mundi, 3(3), 233-246.

Valentine, B.D. (1998) A review of Nearctic and some related Anthribidae (Coleoptera). Insecta Mundi, 12, $251-296$.

Vaurie, P. (1971) Weevils of the tribe Sipalini (Coleoptera, Curculionidae, Rhynchophorinae) Part 3. The genus Sipalinus. American Museum Novitates, 246, 1-43.

Voss, E. (1922a) Monographische Bearbeitung der Unterfamilie Rhynchitinae (Curc.). I. Teil: Nemonychini-Auletini. Archiv für Naturgeschichte, (A) 88(8), 1-113.

Voss, E. (1922b) Indo-Malayische Rhynchitinen (Curculionidae). I. Philippine Journal of Science, 21(4), 385-415.

Voss, E. (1924) Die Gattung Euops Schh. (Col. Curc.). (11. Beitrag zur Kenntnis der Curculioniden.). Deutsche entomologische Zeitschrift, 1924(1), 33-64.

Voss, E. (1925) Die Unterfamilien Attelabinae und Apoderinae (Col. Curc.). (18. Beitrag zur Kenntnis der Curculioniden). Stettiner Entomologische Zeitung, 85, (1): 1-78, (2): 191-304 + pl. I-III.

Voss, E. (1929a) Die Unterfamilien Attelabinae und Apoderinae. (Col. Curc.). (18. Beitrag zur Kenntnis der Curculioniden). Stettiner Entomologische Zeitung, 90(1), 90-159.

Voss, E. (1929b) Die Unterfamilien Attelabinae und Apoderinae. (Col. Curc.). (18. Beitrag zur Kenntnis der Curculioniden). (Fortsetzung). Stettiner Entomologische Zeitung, 90(2), 161-242.

Voss, E. (1930) Attelabiden der Hauserschen Sammlung (Col. Curc.). Wiener entomologische Zeitung, 47(2), 65-88.

Voss, E. (1932) Monographie der Rhynchitinen-Tribus Rhinomacerini und Rhinorhynchini. Entomologische Blätter, 28 (1): 11-18, (2): 67-73, (3): 100-108.

Voss, E. (1933) Monographie der Rhynchitinen-Tribus Auletini. III. Teil der Monographie der Rhynchitinae-Pterocolinae. Stettiner Entomologische Zeitung, 94, (1), 108-136, (2), 273-286.

Voss, E. (1934a) Die Cossoninen Afrikas und Madagaskars des Deutschen Entomologischen der Kaiser-Wilhelm-Gesellschaft. Arbeiten über morphologische und taxonomische Entomologie, Berlin-Dahlem, 1(2), 118-123.

Voss, E. (1934b) Monographie der Rhynchitinen-Tribus Auletini. III. Teil der Monographie der Rhynchitinae-Pterocolinae. Stettiner Entomologische Zeitung, 95, (1): 109-135, (2): 330-344.

Voss, E. (1937a) Über Arten und gattungen der Unterfamilien Belinae, Rhynchitinae und Attelabinae (Curc., Col.). (69. Beitrag zur Kenntnis der Curculioniden). Stettiner Entomologische Zeitung, 98(2), 199-209.

Voss, E. (1937b) Die Gattung Demimaea (Col., Curc.). Entomologische Blätter, 33(6), 448-463.

Voss, E. (1938) Monographie der Rhynchitinen-Tribus Deporaini sowie der Unterfamilien Pterocolinae-Oxycorininae (Allocorynini). VII. Teil der Monographie der Rhynchitinae-Pterocolinae. Stettiner Entomologische Zeitung, 99(1), 59-117.

Voss, E. (1940) Über Rüsselkäfer der Indomalayischen Subregion, vorwiegend von Java (Col., Curc.) Teil I. Tijdschrift voor Entomologie, 83(1/2), 17-93.

Voss, E. (1941) Monographie der Rhynchitinen-Tribus Deporaini sowie der Unterfamilien Pterocolinae-Oxycoryninae (Allocorynini). VII. Teil der Monographie der Rhynchitinae-Pterocolinae (Fortsetzung). Stettiner Entomologische Zeitung, 102(1), 132-141.

Voss, E. (1942) Monographie der Rhynchitinen-Tribus Deporaini sowie der Unterfamilien Pterocolinae-Oxycorininae (Allocorynini). VII. Teil der Monographie der Rhynchitinae-Pterocolinae. Stettiner Entomologische Zeitung, 103(1), $129-155$.

Voss, E. (1951) Über einige Cossoninen-Gattungen des Indo-Australischen Faunengebietes (Coleoptera: Curculionidae). 
Beiträge zur Entomologie, 1(1), 77-92.

Voss, E. (1952) Curculionidae: Mesoptiliinae, Rhynchitinae I et II, Allocoryninae, Pterocolinae. In: W. D. Hinks (Ed.), Coleopterorum Catalogus Supplementa. W. Junk, 's Gravenhage, 158/167, pp. 1-32.

Voss, E. (1953) Curculionidae: Oxycoryninae, Belinae, Archolabinae, Attelabinae, Apoderinae. In: W. D. Hincks (Ed.), Coleopterorum Catalogus Supplementa. W. Junk, 144: pp. 1-19 + 110: pp. 1-34.

Voss, E. (1955) Contributions à l'étude de la faune entomologique du Ruanda-Urundi (Mission P. Basilewsky 1953). XXIV. Coleoptera Curculionidae Apoderinae. (128. Beitrag zur Kenntnis der Curculioniden). Annales du Musée du Congo, Tervuren, in-8 , Zoologie, 36, 252-256.

Voss, E. (1956) Die von Biró auf Neu Guinea aufgefundenen Rüsselkäfer (Col.). I. Annales Historico-Naturales Musei Nationalis Hungarici, (S.N.), 7, 121-142.

Voss, E. (1957) Neue und bekannte, vorwiegend Indonesische Curculioniden (Coleoptera). Treubia, 24(1), 7-63.

Voss, E. (1958a) Ein Beitrag zur Kenntnis der Curculioniden im Grenzgebiet der Orientalischen zur Paläarktischen Region (Col., Curc.). Die von J. Klapperich und Tschung Sen in der Provinz Fukien gesammelten Rüsselkäfer. Decheniana Beihefte, 5, 1-139.

Voss, E. (1958b) Die von Biró auf Neu Guinea aufgefundenen Rüsselkäfer, II (Coleoptera, Curculionidae). Annals Historico-Naturales Musei Nationalis Hungarici, 50, 209-222.

Voss, E. (1960) Die von Biró auf Neu Guinea aufgefundenen Rüsselkäfer, III. (Coleoptera, Curculionidae). Annales Historico-Naturales Musei Nationalis Hungarici, pars zool., 52, 313-346.

Voss, E. (1971) Beschreibung von vier Cossoninen, nebst je einer Gattung und Untergattung (Col., Curc.). (Beitrag 209 zur Kenntnis der Curculioniden). Entomologische Mitteilungen aus dem Zoologischen Museum Hamburg, 4(74), 195-200.

Voss, E. (1974) Coleoptera: Curculionidae partim. South African Animal Life, 15, 395-479.

Wagner, H. (1910) Curculionidae: Subfam. Apionidae. In: S. Schenkling (Ed.), Coleopterorum Catalogus. W. Junk, Berlin, 6, pp. 1-81.

Wagner, H. (1912) Neuheiten aus Herrn R. v. Bennigsen's Sammlung von Coleopteren aus den Deutschen Kolonien. Curculionidae: Apioninae. Entomologische Mitteilungen, 1(10), 310-318.

Wagner, H. (1938) Monographie der paläarktischen Ceuthorrhynchinae (Curcul.). Entomologische Blätter, 34(4), 145172.

Walker, F. (1859) Characters of some apparently undescribed Ceylon insects. Annals and Magazine of Natural History, (3) 3: 258-265, 4: 217-244.

Wanat, M. (1990) Studies on Oriental Apionidae (Coleoptera). 1. New genera and species from Thailand, Sumatra, Moluccas and New Caledonia. Annales Zoologici, 43(17), 347-361.

Wanat, M. (2001) Genera of Australo-Pacific Rhadincybinae and Myrmacicelinae, with biogeography of the Apionidae (Coleoptera: Curculionoidea) and phylogeny of the Brentidae (s. lato). Mantis, Olsztyn, Poland, 432 pp.

Waterhouse, C.O. (1889) Descriptions of two new rhynchophorous Coleoptera from the Louisiade Archipelago. Annals and Magazine of Natural History, (6) 4, 363-365.

Wattanapongsiri, A. 1966. A revision of the genera Rhynchophorus and Dynamis (Coleoptera: Curculionidae). Department of Agriculture Science Bangkok, 1(1), 1-328.

Weber, F. (1801) Observationes entomologicae, continentes novorum quae condidit generum characteres, et nuper detectarum specierum descriptiones. Bibliopolii Academici Novi, Kiliae, 116 pp.

Weidner, H. (1976) Die Entomologischen Sammlungen des Zoologischen Instituts und Zoologischen Museums der Universität Hamburg. IX Teil. Insecta VI. Mitteilungen aus dem Hamburgischen Zoologischen Museum und Institut, 73, 87-264.

Weidner, H. (1979) Die Entomologischen Sammlungen des Zoologischen Institut und Zoologischen Museums der Universität Hamburg, Nachtrag zum IX Teil, Insecta VI (erschienen in dieser Zeitschrift Band 73, S. 87-264. Mitteilungen aus dem Hamburgischen Zoologischen Museum und Institut, 76, 395-468.

Wells, S.M., Pyle, R.M. \& Collins, N.M. (1983) The IUCN Invetebrate Red Data Book. IUCN, Gland, Switzerland, $1+$ $632 \mathrm{pp}$.

Westwood, J.O. (1836) Description of a minute coleopterous insect, forming the type of a new subgenus allied to Tomicus, with some observations upon the affinities of the Xylophaga. Transactions of the Royal Entomological Society of London, 1(1), 34-36 + pl. VII.

Westwood, J.O. (1838) Synopsis of the genera of the British Insects. In: Westwood, J.O. 1838-40. An introduction to the modern classification of insects, $587 \mathrm{pp}$.

Westwood, J.O. (1848) The Cabinet of Oriental Entomology; being a selection of some of the rarer and more beautiful species of insects, natives of India and the adjacent islands, the greater portion of which are now for the first time described and figured, London, 88 pp. +42 pls.

White, A. (1852) Descriptions of some apparently new species of Annulosa (collected by Mr. MacGillivray during the voyage of H.M.S. Rattlesnake). In: John MacGillivray's narrative of the voyage of H. M. S. Rattlesnake commanded by the late Captain Owen Stanley, R. N., F. R. S. etc. during the years 1846-50, etc., Vol. 2 (Appendix 6), pp. 387- 
395.

Wibmer, G.J. \& O'Brien, C.W. (1986) Annotated checklist of the weevils (Curculionidae sensu lato) of South America (Coleoptera: Curculionoidea). Memoirs of the American Entomological Institute, 39, i-xvi + 1-563.

Wiedemann, C.R.W. (1819) Neue Käfer aus Bengalen und Java. Zoologische Magazin, 1(3), 157-183.

Wiedemann, C.R.W. (1823) Nöthige Berichtigungen und Zusätze zu den Beschreibungen der Käfer aus Ostindien und vom Cap, im dritten Stückes dieses und im vierten Bande des Germarischens Magazins. Zoologische Magazin, 2(1), $162-164$.

Wolfrum, P. (1925) Wissenschaftliche Ergebnisse der Bearbeitung der Coleopteren-Sammlung von Franklin Müller. Beitrag II. Anthribidae. Entomologische Mitteilungen, 14(2), 165-166.

Wolfrum, P. (1929) Anthribidae. In: S. Schenkling (Ed.), Coleopterorum Catalogus. W. Junk, Berlin, 102, pp. 1-145.

Wolfrum, P. (1938) Beitrag zur Kenntnis der Anthribiden. Entomologische Blätter, 34, 67-76.

Wolfrum, P. (1949) Neue Anthribidae aus China. Entomologische Blätter, 41-44, 133-148.

Wolfrum, P. (1953) Anthribidae. In: W. D. Hincks (Ed.), Coleopterorum Catalogus Supplementa. W. Junk, 102 , pp. 1-62.

Wolfrum, P. (1956) Neue Anthribiden aus der Sammlung des Ungarischen Naturwissenschaftlichen Museums (Coleoptera). Annales Hisorico-Naturales Musei Nationalis Hungarici, (Series Nova), 7, 109-119.

Wolfrum, P. (1957) Neue Anthribiden aus dem Zoologisch Museum in Amsterdam unter Hinzufügung einer Neubeschreibung aus dem Koninklijk Instituut voor de Tropen. Entomologische berichten, 17(6), 101-6.

Wollaston, T.V. (1854) Description of a new genus and species of British Curculionidae. Annals and Magazine of Natural History, (2) 14(80), 129-131.

Wollaston, T.V. (1857) Catalogue of the Coleopterous insects of Madeira in the collection of the British Museum. Taylor and Francis, London, $234 \mathrm{pp}+1 \mathrm{pl}$.

Wollaston, T.V. (1873a) On the Cossonidae of Japan. Transactions of the Entomological Society of London, 1873(1), 543.

Wollaston, T.V. (1873b) On the genera of the Cossonidae. Transactions of the Entomological Society of London, 1873(4), 427-657.

Wollaston, T.V. (1877) Coleoptera Sanctae-Helenae. J. van Voorst, London, xxv + 256 pp.

Wood, S.L. (1960) Coleoptera. Platypodidae and Scolytidae. Insects of Micronesia, 18(1), 1-73.

Wood, S.L. (1973) On the taxonomic status of Platypodidae and Scolytidae (Coleoptera). Great Basin Naturalist, 33(2), 77-90.

Wood, S.L. (1980) New genera and new generic synonymy in Scolytidae (Coleoptera). Great Basin Naturalist, 40(1), 89-97.

Wood, S.L. (1981) Nomenclatural changes and new species in Platypodidae and Scolytidae (Coleoptera). Great Basin Naturalist, 41, 121-128.

Wood, S.L. (1984) New generic synonymy and new genera of Scolytidae (Coleoptera). Great Basin Naturalist, 44(2), 223-230.

Wood, S.L. (1985) New synonymy and new species of bark beetles (Coleoptera: Scolytidae). Great Basin Naturalist, 45(2), 266-275.

Wood, S.L. (1986) A reclassification of the genera of Scolytidae (Coleoptera). Great Basin Naturalist Memoirs, 10, 1126.

Wood, S.L. (1988a) Nomenclatural changes and new species of Scolytidae (Coleoptera). Great Basin Naturalist, 48, 3138.

Wood, S.L. (1988b) Nomenclatural changes and new species of Scolytidae (Coleoptera), part III. Great Basin Naturalist, 48, 196-201.

Wood, S.L. (1989) Nomenclatural changes and new species of Scolytidae (Coleoptera), part IV. Great Basin Naturalist, 48, 167-185.

Wood, S.L. (1992a) Nomenclatural changes in Scolytidae and Platypodidae (Coleoptera), Part II. Great Basin Naturalist, 52(1), 78-88.

Wood, S.L. (1992b) Nomenclatural changes in Scolytidae and Platypodidae (Coleoptera). Great Basin Naturalist, 52 , 89-92.

Wood, S.L. (1993) Revision of the genera of Platypodidae (Coleoptera). Great Basin Naturalist, 53(3), $259-281$.

Wood, S.L. \& Bright, D.E. (1987) A Catalog of Scolytidae and Platypodidae (Coleoptera), Part 1: Bibliography. Great Basin Naturalist Memoirs, 11, 1-685.

Wood, S.L. \& Bright, D.E. (1992) A Catalog of Scolytidae and Platypodidae (Coleoptera), Part 2: Taxonomic Index. Great Basin Naturalist Memoirs, 13, 1-1553. (Vol. A: 1-833; Vol. B: 834-1553).

Zerikhin, V.V. \& Gratshev, V.G. (1995) A comparative study of the venation of the superfamily Curculionoidea, with phylogenetic implications. In: J. Pakaluk \& S.A. Elipiñski (Eds.), Biology, Phylogeny, and Classification of Coleoptera: Papers Celebrating the $80^{\text {th }}$ Birthday of Roy A. Crowson. Muzeum i Instytut Zoologii PAN, Warsaw, pp. 633-777.

Zimmerman, E.C. (1936) Review of the genus Orochlesis (Coleoptera: Curculionidae). Occassional Papers of the Ber- 
nice P. Bishop Museum, 12(1), 1-19.

Zimmerman, E.C. (1938) Idotasia in New Ireland (Coleoptera: Curculionidae). Proceedings of the Hawaiian Entomological Society, 10(1), 148-150.

Zimmerman, E.C. (1939) Blepiarda of Fiji (Coleoptera: Curculionidae). Proceedings of the Hawaiian Entomological Society, 10(2), 325-328.

Zimmerman, E.C. (1940a) Synopsis of the genera of Hawaiian Cossoninae with notes on their origin and DISTRIBUTION (Coleoptera, Curculionidae). Occasional Papers of the Bernice P. Bishop Museum, 15(25), 271-293.

Zimmerman, E.C. (1940b) A new Philippine cucurbit-boring barid (Coleoptera, Curculionidae). Philippine Journal of Science, 73, 313-319.

Zimmerman, E.C. (1941) A revision of the genus Deretiosus (Coleoptera, Curculionidae). Occasional Papers of the Bernice P. Bishop Museum, 16(8), 177-214.

Zimmerman, E.C. (1944a) Douttia, a new genus of New Hebridean Curculionidae (Coleoptera: Cryptorhynchinae). Annals of the Entomological Society of America, 37(2), 193-197.

Zimmerman, E.C. (1944b) Pembertonia, a new genus of papuan Cossoninae (Coleoptera, Curculionidae). Proceedings of the Hawaiian Entomological Society, 12(1), 200-205.

Zimmerman, E.C. (1956) Notes on Conarthrus, Eutornicus and Macrancylus weevils (Coleoptera: Curculionidae: Cossoninae). The Entomologist, 89, 56-64.

Zimmerman, E.C. (1957) Formosan Cossonine weevils of bamboo (Coleoptera: Curculionidae: Cossoninae). Proceedings of the United States National Museum, 107, 13-23.

Zimmerman, E.C. (1967) Imathia and Amblycnemus (Coleoptera: Curculionidae: Cryptorhynchinae). Pacific Insects, 9(2), 187-196.

Zimmerman, E.C. (1968) The Cosmopolites banana weevils (Coleoptera: Curculionidae: Rhynchophorinae). Pacific Insects, 10(2), 295-299.

Zimmerman, E.C. (1974) The Papuan Erebaces weevils (Coleoptera: Curculionidae: Cryptorhynchinae). Journal of the Australian Entomological Society, 13, 61-64.

Zimmerman, E.C. (1991) Colour Plates 1-304. Australian Weevils (Coleoptera: Curclionoidae). CSIRO Publications, Melbourne, 5, $\mathrm{x}+633$ pp.

Zimmerman, E.C. (1992) Colour Plates 305-632. Australian Weevils (Coleoptera: Curclionoidae). CSIRO Publications, Melbourne, 6, viii +707 pp.

Zimmerman, E.C. (1993) Nanophylidae, Rhynchophoridae, Erirhinidae, Curculionidae: Amycterinae, Literature Consulted. Australian Weevils (Coleoptera: Curclionoidea). CSIRO Publications, Melbourne, 3, x + 854 pp.

Zimmerman, E.C. (1994a) Orthoceri, Anthribidae to Attelabidae, The Primitive Weevils. Australian Weevils (Coleoptera: Curclionoidae). CSIRO Publications, Melbourne, 1, xxxii + $741 \mathrm{pp}$.

Zimmerman, E.C. (1994b) Brentidae, Eurhynchidae, Apionidae and a chapter on Immature Stages by Brenda May. Australian Weevils (Coleoptera: Curclionoidea). CSIRO Publications, Melbourne, 2, $\mathrm{x}+755 \mathrm{pp}$.

Zimmermann, C. (1868) Synopsis of the Scolytidae of America north of Mexico. Transactions of the American Entomological Society, 2, 141-149. 


\section{Index}

Aades 132

abbreviatipennis (Xyleborus) 216

abbreviatus (Alcidodes, Alcides) 174

abdominalis (Crossotarsus) 183

aberrans (Barystethus) 76

aberrans (Exillis) 29

abnormis (Platypus) 185

\section{Abrachius 74}

abruptifer (Platypus) 185

abruptulus (Coccotrypes, Poecilips) 205

abruptulus (Platypus) 185

abruptus (Arixyleborus) 211

abruptus (Coccotrypes, Poecilips) 205

abruptus (Platypus) 185

absonus (Scolytogenes, Cryphalomorphus) 204

Acacacis 197

Acalles 111

acalloides (Pseudaclees, Hylobius) 172

\section{ACALYPTINI 129}

Acanthotomicus 209

acarifer (Symbiopholus) 155

Aceleuthetes 142

acetabuliformis (Platypus) 185

acetabuliformis comalis (Platypus) 185

Achirozetes 91

Achoragus 38

ACICNEMIDINI 179

aciculatus (Coccotrypes) 205

Aclees 171

Acoptorrhynchus 133

Acorynus 32

aculeatus (Riedelinius) 50

acuminatus (Arachnobas, Arachnopus) 86

acuminatus (Cyrtogenius, Eidophelus) 207

acuticollis (Garyus) 37

acuticornifer (Platypus) 185

acuticornis (Platypus) 185

acutipennis (Arachnobas, Arachnopus) 86

acutus (Apirocalus) 134

acutus (Deropygus) 40

acutus (Webbia, Xyleborus) 215

Acythopeus 83

adamsoni (Lepropus) 15

admiralis (Pantorhytes) 164

admirandus (Eupholus) 150

adoptivus (Ithystenus) 60

adspersus (Meroleptus) 115

advena (Coccotrypes) 205

advena (Platypus) 185

adumbratus (Cyamobolus) 106

adustus (Cerobates) 57

AEDEMONINI 101

aenea (Sphenomorpha, Pachyrrhynchus) 167

aeneicollis (Miolispa) 66

aeneus (Apionocybus, Rhadinocyba) 69

aequabilis (Hypseus) 23

aequalis (Cerobates) 57 aequalis (Cyrtogenius, Ozodendron) 207

aequalis (Misthosima) 41

aequalis (Platypus) 185

aequata (Rhinoscapha) 157

aequilaterus (Platypus) 186

aeruginosa (Agametis) 91

aeruginosus (Exillis) 29

aethiops (Araecerus) 38

aethiops (Cataphractus) 137

affinis (Arachnobas, Arachnopus) 86

affinis (Cyrtogenius, Pelicerus) 207

affinis (Mechistocerus, Isotocerus) 102

affinis (Miolispa) 66

affinis (Stereoborus) 97

affinis (Telaugia) 122

afflictus (Poropterus) 120

Agametis 91

aglaiae (Amasa, Xyleborus) 210

agnatum (Cyclorhipidion, Xyleborus) 213

ajax (Gymnopholus) 153

alatus (Ithystenus) 60

albaria (Rhinoscapha) 157

albatus (Apterorrhinus) 64

albertisi (Rhinoscapha) 157

albertisii (Eudyasmus) 107

Albertisius 133

albicollis (Pseudoperissops) 109

albidoplagiatus (Paratactus, Sphaeropterus) 146

albifrons (Asytesta) 104

albipennis (Rhinoscapha) 157

albipennis cinnamomea (Rhinoscapha) 157

albocinctus (Alcidodes, Alcides) 174

albofasciatus (Eupholus) 150

alboguttata (Aporhina, Chalcocybebus) 58

alboguttata (Rhinoscapha) 157

alboguttatus (Amalthus) 110

alboguttatus (Hypsophorus, Protopalus) 111

albolineatus (Alcides) 177

albolineatus (Sphaeropterus) 148

albolineatus (Xenocerus, Anthribus) 34

albolineatus interruptus (Sphaeropterus) 148

albolineatus v. scaposus (Sphaeropterus) 148

albolituratus (Alcidodes, Alcides) 174

albonotatus (Mutillarius) 143

albopicta (Atrotitis) 162

albopicta (Pioenidia) 31

albopictum (Apion) 71

albopictus (Elassophilus) 94

albopunctulatus (Pantorhytes) 164

alboscapulatus (Arachnobas, Arachnopus) 86

albosignatus (Apiezonotus, Piezonotus) 134

albosparsus (Dysopirhinus) 107

albosparsus (Orthorhinus) 178

albosparsus v. ochreatus (Dysopirhinus) 107

albovarius (Mechistocerus, Isotocerus) 102

albovertex (Chaetoctesius) 106 
albovittatus (Elytrocheilus) 139

\section{Alcidodes 174}

algifer (Pantorhytes) 164

algifer (Symbiopholus) 155

alienus (Deropygus) 40

alienus (Tracheloschizus, Schizotrachelus) 67

Alissapion 68

Allaeometrus 54

Allodapinus 65

Allonotapion 69

alma (Rhinoscapha) 157

alternans (Eupholus) 150

alternans (Scolytogenes, Cryphalomorphus) 204

alternantes (Cyrtogenius) 207

altilis (Tracheloschizus, Schizotrachelus) 68

Altipectus 29

altipetax (Platycybus) 70

aluensis (Kietana) 142

amabilis (Dysmorphorhynchus) 56

amabilis (Mecocerinopis, Acorynus) 33

amabilis nigriventris (Mecocerinopis) 33

amabilis tarsalis (Mecocerinopis) 33

amaliae (Eupholus) 150

amaliae v. arfaki (Eupholus) 150

Amalthus 110

amalulu (Eupholus) 150

Amasa 210

ambiguus (Oribius, Coptorrhynchus) 144

ambiguus (Platypus) 186

amblylaminatus (Diapus) 196

Ambonodiras 112

Ambrosiodmus 210

AMINYOPINI 170

amitinus (Trigonopterus, Idotasia) 123

amoena (Ganae) 73

amoenus (Alcidodes, Alcides) 174

amoenus (Deretiodes) 173

amoenus (Diathetes) 76

amoenus (Guiomatus) 94

Amorphorhynchus 96

amphicauda (Coptodryas, Xyleborus) 212

ampliata (Telaugia) 122

ampliatus (Trigonopterus, Idotasia) 123

amplicollis (Anaballus) 122

amplicollis (Hypseus) 23

amplicollis (Tamphilus, Acalles) 122

amplipennis (Deretiosus) 173

anaballoides (Pachytragopus, Tragopus) 118

analis (Aricerus) 200

analis (Julietiella, Hypermetra) 133

Anathymus 75

anatinus (Apirocalus) 134

Anchithyrus 112

Anchyptolycus 112

Ancylotropis 25

Ancyttalia 14

andamanensis (Euwallacea, Xyleborus) 214

andrewesi (Xyleborinus, Xyleborus) 216

anggiensis (Euops) 49 anguis (Platypus) 186

angulicollis (Apolecta) 38

angulicollis (Poteriophorus) 78

angulithorax (Guineoeuops) 46

angustata (Rhinoscapha) 157

angustatus (Ithystenus, Brenthus) 60

angusticollis (Platypus) 186

angustidontus (Diapus) 196

angustifrons (Homalotrogus) 96

angustior (Platypus) 186

angustipennis (Cerobates) 57

angustula (Acicnemis) 179

angustus (Gymnopholus) 153

anisopterae (Cyrtogenius, Ozodendron) 207

anisopterae (Xyleborus) 216

ankius (Scolytogenes, Cryphalomorphus) 204

ankius (Xyleborinus, Xyleborus) 216

Ankleineella 53

annae (Miolispa) 66

annexus (Xyleborus) 216

annularis (Baiocis, Platypus) 181

annularis (Orochlesis) 128

anomalus (Amasa, Xyleborus) 210

anomalus (Platypus) 186

anoplum (Cyclorhipidion, Xyleborus) 213

anoplus (Platypus) 186

antennalis (Baris) 80

antennarius (Ernoporus) 203

anthonyi (Arachnobas, Arachnopus) 86

anthonyi (Elytrocheilus) 139

anthracinus (Trigonopterus, Idotasia) 123

ANTHRIBIDAE 19

ANTHRIBINAE 19

anthriboides continens (Xenocerus) 34

anticus (Cosmoderes, Erioschidias) 201

Antioxenus 20

anxius (Cossonus) 95

Apatenia 21

aper (Dinema, Proscopus) 26

apertulus (Platypus) 186

apertus (Platypus, Diapus) 186

apertus (Xyleborus) 218

Aphanocorynes 99

Aphyoda 101

apicalis (Alcidodes, Alcides) 174

apicalis (Perissops) 109

apicallis (Lophocheirus, Odosyllis) 127

apicallis v. divisa (Lophocheirus) 127

apicatulus (Platypus) 186

apicatus (Platypus) 186

apicenotatus (Hypseus) 23

apicepilosa (Miolispa) 66

apicipenne (Cyclorhipidion, Xyleborus) 213

Apiezonotus 133

apina (Metanthia) 84

APIONIDAE 68

APIONINAE 68

Apionocybus 69

Apirocalus 134 
Apolecta 38

APOLECTINI 38

Aporhina 58

Aporonotus 113

Aporophemus 75

appendiculatus (Ithystenus) 60

approximatus (Scolytogenes, Cryphalomorphus) 204

approximatus (Xyleborus) 216

Apries 172

Arachnobas 86

ARACHNOPODINI 86

arachnopus (Asytesta) 104

ARAECERINI 38

Araecerus 38

Araeocorynus 40

Aragomacer 19

Araiorrhinus 62

araneus (Pantorhytes) 164

araucaria (Xylogpinus) 200

araucariae (Cryphalus) 201

araucariae (Hylurdrectonus) 200

araucariae (Pachycotes) 200

archaicus (Pseudoporopterus, Poropterus) 120

archboldi (Cordus) 53

Archeuops 45

arciferus (Anthribus) 34

arciferus (Dendropemon, Xenocerus) 25

arcuatus (Pantorhytes) 164

arduus (Papuatorhaenas) 37

arfakensis (Metaeuops) 47

arfakensis (Ottistira) 163

Aricerus 200

aridus (Deretiosus) 173

aries (Cyrtogenius, Artepityophthorus) 207

aries (Xyleborus) 216

Aristoxenus 101

Arixyleborus 211

armaticeps (Cordus) 53

armatipennis (Armeuops) 45

armatus (Araiorrhinus) 62

armatus (Cryphalus) 201

armatus (Riedelinius) 50

Armeuops 11, 45

armifer (Webbia, Xyleborus) 215

aroana (Tistortia) 164

aroanus (Poropteropis) 119

ARRHENODINI 51

arrogans (Celebia, Geonemus) 150

arrogans (Orthorhinus) 178

artestriatus (Xyleborinus, Xyleborus) 216

artetruncatus (Platypus) 186

aruensis (Crossotarsus) 182

aruensis (Lophocheirus) 127

aruensis (Miolispa) 66

aruensis (Xylinada, Xylinades) 36

ascendens (Ottistira) 163

asekianus (Guineoeuops) 46

asekianus (Myrmecocybus) 70

asignatus (Arachnobas, Arachnopus) 86 asper (Apirocalus) 134

asper (Diathryptus) 114

asperatus (Alcidodes) 174

aspericollis (Alcides) 177

aspericollis (Aporhina, Chalcocybebus) 58

aspersa (Rhinoscapha) 157

aspicis (Platypus) 186

assilimis (Mallorrhynchus) 36

assimilis (Aporhina, Chalcocybebus) 58

assimilis (Telaugia) 122

associatus (Platypus) 186

asterias (Rhaphitrophis) 36

astrolabensis (Eupholus) 150

Asytesta 104

atava (Coptodryas, Xyleborus) 212

Atenophthalmus 63

ater (Acalles, Tylodes) 111

ater (Apirocalus) 134

ater (Barystethus) 76

ater (Cryphalus) 201

ater (Erebaces) 114

ater (Nechyrus) 117

ater (Phaenomerus) 89

ater v. basalis (Barystethus) 76

ater v. dispar (Barystethus, Dialtates) 76

ater v. parvulus (Barystethus) 76

ater v. puncticollis (Barystethus) 76

ater v. rufus (Barystethus) 76

ATERPINI 132

aterrimus (Cnestus, Xyleborus) 211

Athanasius 105

atomaria (Eczesaris) 28

atomaria aureomaculata (Eczesaris) 28

atomaria cyclopis (Eczesaris) 28

atomaria fuscata (Eczesaris) 28

atomosparsus (Cyambolus) 103

atra (Sphenomorpha) 168

atramentarius (Araecerus) 38

atramentarius latior (Araecerus) 38

atrans (Platypus) 186

atrigenua (Apirocalus) 134

atronitens (Melanopsacus) 42

atronitidus (Mechistocerus) 102

atropolita (Baris) 80

Atrotitis 162

ATTELABIDAE 45

ATTELABINAE 45

attenuatum (Oxydema) 96

aucta (Asytesta) 104

audax (Symbiopholus) 155

aversandus (Tyrtaeosus) 125

auguralis (Chirozetes) 92

augustae (Ozopemon) 209

Aulacobaridia 80

Aulacocnemus 113

Aulacophrys 136

Auletanus 44

AULETINA 43

AULETINI 43 


\section{Auletobius 43 \\ AULETORHININI 44}

aureipilus (Crossotarsus) 182

auricomus (Dysnos) 42

auricomus (Phaenomerus) 89

australasiae (Iphthimorhinus) 77

australasiae (Pantorhytes) 167

australiae (Mitrastethus) 108

australiana (Miolispa) 66

australis (Alcidodes, Alcides) 175

australis (Coeliosomus, Mecysmoderes) 86

australis (Hypolixus, Lixus) 169

australis (Neolaemosaccus, Lmosaccus) 170

australis (Otiorrhynchus) 137

australis (Sphaeromus) 138

australis pervicax (Alcidodes, Alcides) 175

australis platysomus (Alcidodes) 175

australis ramosus (Alcidodes) 175

australis segnis (Alcidodes, Alcides) 175

australis septemaculatus (Alcidodes, Alcides) 175

Australoeuops 46

Autillia 105

avus (Apirocalus) 134

avus (Arachnobas, Arachnopus) 86

avus finisterrae (Apirocalus) 134

avus intermedius (Apirocalus) 134

avus karimuicus (Apirocalus) 134

avus marawakanus (Apirocalus) 134

avus tarii (Apirocalus) 134

axilaris (Hypseus) 23

axillaris (Rhinoscapha) 157

axillaris major (Hypseus) 23

azureipes (Rhinoscapha, Geonemus) 157

azureipes chrysochlora (Rhinoscapha) 157

azureipes foveolata (Rhinoscapha) 157

azureus (Eupholus) 150

azureus v. bilineellus (Eupholus) 150

bacatus (Meroleptus) 116

bacchusi (Apirocalus) 134

bacchusi (Nesidiobrentus) 56

bacillifer (Blepiarda, Trichogonus) 111

Baiocis 181

bakeri (Pantorhytes) 164

balanophorus (Conomalthus) 106

baliemensis (Guineobius) 140

balkei (Guineoeuops) 46

baloghi (Scolytomimus) 220

bambusae (Ptilopodius) 204

bandanus (Eupholus) 152

barbatus (Crossotarsus) 182

barbatus (Euwallacea, Xyleborus) 214

barbatus (Myctides) 85

barbicornis (Xenocerus) 34

BARIDINA 80

BARIDINAE 80

BARIDINI 80

Barinogyna 83

Baris 80
Bartolozziella 110

Baryrhynchus 51

Barystethus 76

basalis (Arachnobas, Arachnopus) 87

basalis (Cossonus) 95

basilica (Rhinoscapha) 157

Basiliogeus 15

basimaculata (Douttia) 114

basiplaga (Rhaphitrophis) 36

basiplaga manca (Rhaphitrophis) 36

\section{BASITROPIDINI 19}

\section{Basitropis 19}

batantae (Guineoeuops) 46

batantae (Hypseus) 23

batantae (Protaedus) 26

batantae salwattii (Protaedus) 26

batesi (Pantorhytes) 164

beccarii (Dryophthoroides, Elatticus) 79

beccarii (Erebaces) 114

beccarii (Eupholus) 150

beccarii (Rhinoscapha) 157

beccarii (Sphenophorus) 78

Behrensiellus 137

BELIDAE 43

BELINAE 43

bella (Imathia) 131

bellus (Alcides) 174

bellus (Curculio, Balaninus) 130

bennetti (Eupholus) 150

bennetti v. apicalis (Eupholus) 150

bennetti v. bicolor (Eupholus) 150

bennigseni (Antioxenus) 20

bennigseni (Apionocybus, Rhadinocyba) 69

bennigseni (Aporhina, Chalcocybebus) 58

bennigseni (Eothaumas) 34

bennigseni (Eupholus) 150

Berosicus 105

bhamoensis (Microtrachelizus, Trachelizus) 62

biakensis (Misthosima) 41

biakensis (Pantorhytes) 164

biakianus (Microsebus, Eusebus) 56

bialbivittata (Baris) 80

bicaudatus (Tracheloschizus) 68

bicincta (Rhinoscapha) 157

bicinctus (Alcides) 174

bicolor (Cryphalus, Cryphalomorphus) 201

bicolor (Eudelodes, Amorphoidae) 129

bicolor (Euops) 49

bicolor (Idopelma) 93

bicolor (Ithystenus) 60

bicolor (Phocylides, Brenthus) 65

bicolor (Xenopsilus) 86

biconcavus (Crossotarsus) 182

biconifer (Poropteropis) 119

bicornis (Acacacis) 197

bicornis (Ottistira) 163

bicristatus (Cyphopus, Ectemnomerus) 138

bicristatus (Strotus, Otiorrhynchus, Trigonops) 148

bidentatus (Xyleborus, Phloeotrogus) 216 
bifasciata (Dystropicus, Theoclia) 102

bifasciata (Rhinoscapha) 158

biformis (Gymnopholus) 153

bifurcus (Platypus, Crossotarsus) 186

bigeminatus (Acythopeus) 83

bigranulatus (Platypus) 186

biguttatus (Arachnobas, Arachnopus) 87

biligatus (Microthetes) 143

bilineatus (Cossonus, Isotrogus) 95

bilineatus (Rhynchophorus, Calandra) 75

bilocalis (Coeliapion) 69

bilogayensis (Ithyocybus) 70

bilunatus (Diapus) 196

bimaculatus (Allaeometrus) 54

bimaculatus (Cnestus, Xyleborus) 211

bimaculatus (Cyphagogus) 54

bimaculibasis (Baris) 81

binodifrons (Stereoborus) 97

binodifrons v. testaceiventris (Stereoborus) 97

binodosus (Kokodanus) 142

binodulus (Lixus) 170

binotatus (Acythopeus) 83

binotatus (Arachnobas, Arachnopus) 87

binotatus (Trigonopterus) 123

biplagiatus (Arachnopus) 87

biplagiatus (Pachyrrhynchus) 166

biplagiatus (Pantorhytes, Pachyrhynchus) 164

bipunctatus (Ozotomerus, Oedecerus) 29

bipustulata (Sphenogaster) 148

bipyramidus (Platypus) 186

birmanus (Hypothenemus, Triarmocerus) 203

biroi (Aporhina, Chalcocybebus) 58

biroi (Lophothetes) 142

biroi (Omophorus) 178

biroi (Pantorhytes) 164

biroi (Plintheria) 32

biroi (Rhinotia, Belus) 43

biru (Metaeuops) 47

bismarcensis (Xyleborus) 212

bispinatus (Platypus) 186

bispinosa (Aporhina, Chalcocybebus) 58

bispinosa (Ottistira) 163

bispinosa aruensis (Aporhina, Chalcocybebus) 58

bispinosa guttifera (Aporhina, Chalcocybebus) 58

bispinosa intermedia (Aporhina, Chalcocybebus) 58

bispinosa macrospilotus (Aporhina, Chalcocybebus) 58

bispinosus (Hellerrhinus) 141

bispinosus (Mecopus, Rhynchaenus) 92

bispinosus moluccarum (Mecopus) 92

bispinus (Coptoborus, Xyleborus) 211

bispinus (Diapus) 196

bistriatus (Ithystenus) 60

bisulcatus (Trachelizus) 67

bituberculata (Dinomelaena) 22

bituberculatum (Cyclorhipidion, Xyleborus) 213

bituberculatus (Platypus) 186

bituberculatus (Sphaeropterus) 148

bituberculifer (Platypus) 186

bituberosus (Poropterus) 120 bivirgata (Asytesta) 104

bivittatus (Pseudocholus) 84

bivittatus (Pseudoporopterus, Poropterus) 120

bivittipennis (Mechistocerus) 102

biuncus (Xyleborus) 216

biundulata (Rhinoscapha) 158

blanchardi (Alcides) 177

blanchardi (Oribius, Coptorrhynchus) 144

blandicaudatus (Araecerus) 39

blandus (Alcidodes) 175

blandus (Deretiosus) 173

Blepiarda 111

Bobandersonia 111

boisduvali (Celeuthetes) 137

boisduvalii (Copurus, Zygops) 95

bolana (Eczesaris) 28

bombicollis (Oribius, Coptorhynchus) 144

bombylius (Oribius, Coptorhynchus) 144

bommelense (Allonotapion) 69

bonguensis (Alcidodes, Alcides) 175

bonguensis (Lixus) 170

botanicus (Symbiopholus) 155

Bothriorhinus 65

bougainvillei (Kleineella) 53

bougainvillensis (Trigonops) 148

boviei (Elytrocheilus) 139

bourkei (Pantorhytes) 164

BRACHYCERIDAE 72

Brachycybus 69

Brachynedus 137

Brachypezichus 105

brachypus (Homorthorrhinus, Orthorhinus) 179

brachypus tibialis (Homorthorrhinus) 179

brachyurus (Araeocorynus) 40

brachyurus reductus (Araeocorynus) 40

brachyurus variabilis (Araeocorynus) 40

brandti (Gymnopholus) 153

brandti (Pantorhytes) 164

brenthoides (Aphyoda) 101

BRENTIDAE 51

BRENTINAE 51

brevicepes (Allaeometrus) 54

brevicollis (Alcidodes, Alcides) 175

brevicollis (Diamerus) 198

brevicollis (Elytrocheilus) 139

brevicollis (Xyleborus) 216

brevicollis reductus (Alcidodes, Alcides) 175

brevicornis (Moluccobius) 143

brevior (Apatenia) 21

brevior (Cyrtogenius) 207

brevipennis (Asytesta) 104

brevipennis (Isoleptus) 115

brevipilosus (Cryphalus) 201

brevipilosus (Ficicis, Hylesinus) 198

brevis (Eczesaris, Idiopus) 28

brevis (Imathia) 131

brevis (Ptilopodius) 202

brevispinosus (Eutyrhinus, Euthyrhinus) 126

brevisulcatus (Microtrachelizus) 62 
brevitibia (Microtrachelizus, Trachelizus) 63 brevius (Cyclorhipidion, Xyleborus) 213 breviusculus (Elytrocheilus) 139 breyniae (Guineoeuops) 46 brownei (Schedlia) 215 browni (Eupholus) 150 bruchi (Neochetina) 16 bruijnii (Eupholus) 150 brunneovaria (Plesiobasis) 20 brunneus (Cryphalus) 201 bucana (Rhinoscapha) 158 bucana v. pulverulenta (Rhinoscapha) 158 buergersi (Guineoeuops) 46 buloloensis (Cryphalus) 201

\section{Cacephatus 22}

Cacoschizus 64

caelestis (Platypus) 186

Caenochira 89

c-album (Curculio) 130

caledoniae (Platypus) 186

calidris (Dystropicus, Berosiris) 102

caliginosus (Crossotarsus) 182

Callanthribus 31

callima (Misthosima) 41

Callipareius 54

callosiventris (Hypseus) 23

Calyptulus 64

cameratus (Tracheloschizus, Schizotrachelus) 68

Camia 113

CAMPTORHININI 103

Camptorhinus 103

\section{CAMPYLOSCELINI 89}

canaliculatus (Arixyleborus, Xyleboricus) 211

canaliculatus (Cossonus) 95

canalipectus (Phaenomerus) 89

canarii (Cyclorhipidion, Xyleborus) 213

canarivorus (Xyleborus) 216

canescens (Rhinoscapha) 158

canus (Apirocalus) 134

capitilanatus (Platypus) 186

capito (Peroplatypus, Platypus) 185

carbonaria (Thyestetha) 122

carbonarius (Pantorhytes) 164

Carbonomassula 178

carbunculus (Paratactus, Coptorhynchus) 146

Carcinopisthius 56

CARIDAE 15

CARINAE 15

carinatus (Gymnopholus) 153

carinatus (Margadillius) 204

carinatus (Ptolycus) 121

cariniceps (Arixyleborus) 211

carinicollis (Ipsichora) 84

carinifrons (Garyus) 37

carinirostris (Apirocalus) 134

carinirostris (Deretiosus) 173

carinirostris (Ptolycus) 121

carinithorax (Trigonops) 148 carinrostris (Sphenogaster) 148

carinulatus (Cacephatus) 22

carinulatus (Notocryptorhynchus) 108

Carodes 15

carolynae (Symbiopholus) 155

Carponinus 131

castaneicornis (Baris) 81

castaneipennis (Rhyncolus) 100

castaneus (Degis) 83

castigatus (Platypus, Crossotarsus) 187

castigatus (Stereodermus, Metatrachelizus) 58

casuarinae (Solenobaris) 82

caudatus (Arachnobas, Arachnopus) 87

caudatus (Ithystenus) 60

caudatus (Oribius, Coptorhynchus) 144

cavicaudatus (Ithystenus) 60

cavicollis (Mucronianus) 33

cavipectus (Phaenomerus) 89

cavisquamus (Colobodes) 172

cavus (Eubactrus) 64

celeatus (Molobrium) 136

celebesus (Eupholus) 152

Celebia 149

Celeuthetes 137

CELEUTHETINI 133

celsus (Platypus) 187

celtis (Acanthotomicus, Ips) 209

Cenchrena 129

CENCHRENINI 129

Centrinopsis 85

Ceocephalus 59

CEPURINI 169

CERATOPODINI 130

cerbera 51

cerbereus (Curculio, Balaninus) 130

cercidoptera (Pseudaphioda) 97

Cerobates 57

cervinus (Alcidodes) 175

CEUTHORHYNCHINAE 86

Chaetectetorus 126

Chaetoctesius 106

Chaetoptelius 200

chalcographus (Crossotarsus) 182

Chalybdicus 64

chaminadei (Eupholus) 150

cheesemanae (Symbiopholus) 155

cheesmanae (Brachynedus) 137

cheesmanae (Cordus) 53

cheesmanae (Cyphagogus) 54

cheesmanae (Cyphopus) 138

cheesmanae (Hellerrhinus) 141

cheesmanae (Trigonospartus) 149

cheesmani (Crossotarsus) 182

cheesmani (Schizotrachelus, Hypomiolispa) 65

chevrolati (Barystethus) 76

chevrolati (Dinoplatypus, Platypus) 184

chevrolatii (Eupholus) 150

chevrolatii (Ithystenus, Brenthus) 60

chimbuensis (Pantorhytes) 165 
chimbui (Coccotrypes, Poecilips) 205

chimbui (Coptodryas, Xyleborus) 212

chimbui (Platypus) 187

chimbui (Xyleborus) 212

Chirogonia 113

Chirozetes 91

chlora (Rhinoscapha) 158

chlorizans (Hoplotrigonops) 147

chloropunctata (Rhinoscapha) 158

Choerorhamphus 138

CHORAGINAE 38

CHORAGINI 42

chrysomelas (Pantorhytes) 167

cicatricosus (Platypus) 187

cicatricosus (Scolytogenes, Lepicerinus) 204

ciliatoformis (Xyleborus) 216

cincta (Rhinoscapha) 158

cinctipes (Xyleborus) 216

cinctor (Meroleptus) 116

cinctus (Acalles, Tylodes) 111

cinctus (Pseudocholus) 84

cinerascens (Celeuthetes) 137

cinereus (Chaetectetorus) 126

cinereus (Oribius) 144

cingulatus (Ionthocerus, Cerobates) 57

cinnamomeus (Eupholus) 151

cinnamomeus v. caesius (Eupholus) 151

cinnamomeus v. fasciatus (Eupholus) 151

circularicepes (Platypus) 187

circularis (Platypus) 187

circulifera (Asytesta) 104

circumcisus (Webbia) 215

circumscriptus (Lophocheirus) 127

circumspinosus (Xyleborus) 216

citius (Araecerus) 39

Cladoctonus 199

clarki (Eupholus) 151

clarus (Pseudophocylides) 62

clavigera (Acicnemis) 179

Cleodus 113

CLEOGONINI 171

cletusi (Barystethus) 76

clypeifera (Acicnemis) 179

Cnestus 211

coadunatus (Platypus) 187

Coccotrypes 205

coelestinus (Metaeuops) 47

coeletais (Ipsichora) 84

Coeliapion 69

Coeliosomus 86

coeloderes (Cossonus) 95

coelosternoides (Tyrtaeosus) 125

coerulans (Miolispa) 66

coeruleatus (Elytrocheilus, Siteytes) 139

coeruleus (Idiopsodes, Idiopsis) 141

coeruleus (Metaeuops) 47

coffeae (Dryocoetiops, Dryocoetes) 208

cognatus (Platypus) 187

cognatus (Xyleborus) 216 colbaltina (Lamprohypera) 169

colbaltina (Rhinoscapha) 158

collare (Cryptoderma, Oxyrhynchus) 72

collaris (Deretiosus) 173

collaris (Mormosintes) 117

collaris (Phocylides) 65

collaris (Platypus) 187

colligens papuanus (Dendrotrogus) 25

colmani (Gymnopholus) 153

Colobodes 172

COLOBODINA 172

colorata (Mauia) 26

colossus (Ambrosiodmus, Xyleborus) 210

Colpomus 138

combinatus (Pseudocholus) 85

comma (Sclerodontus) 82

comoda (Misthosima) 41

compositus (Baryrhynchus) 51

compositus (Eupholus) 151

compositus (Mechistocerus) 102

compressicornis (Kleineella) 53

compressipes (Asytesta, Arachnopus) 104

compressitarsis 64

comptus (Mechistocerus) 102

Conarthrus 99

concentralis (Margadillius) 204

concentralis (Scolytogenes, Cryphalomorphus) 204

concentriporus (Platypus) 187

conclavifrons (Platypus) 187

concolor (Heterophasis) 95

concretus (Oxymelus) 109

concretus (Pseudoporopterus, Poropterus) 120

concussus (Nechyrus) 117

confinis (Elytrocheilus) 139

confinis (Xyleborus) 214

confinis novobritannicus (Elytrocheilus) 139

confluens (Ithystenus) 60

confluens (Synommatus) 79

confluentinus (Paratactus) 146

conformatus (Lixus) 170

conformis (Araecerus) 39

confragosus (Stereodermus, Metatrachelizus) 58

confusa (Pempherulus, Phylaitis) 93

confusus (Alcidodes) 175

confusus (Lixus) 170

conifer (Hellerrhinus) 141

conjuncta (Miolispa) 66

conjunctus (Xenocerus) 34

\section{CONODERINAE 86}

Conomalthus 106

consobrinus (Araecerus) 39

constans (Stereodermus, Metatrachelizus) 58

constrictus (Cryphalus) 203

consueta (Rhinoscapha, Ladoice) 158

consueta ab. imperfecta (Rhinoscapha) 158

consueta v. $x$-album (Rhinoscapha) 158

continua (Rhaphitrophis) 37

continus (Deropygus) 40

contractus (Cladoctonus) 199 
contrarius (Phaenomerus) 90

conturbator (Phaenomerus) 90

convergens (Baris) 81

convexa (Schedlia) 215

convexus (Euops) 49

convexus (Trigonopterus, Idotasia) 123

Coptoborus 211

Coptodryas 212

Coptonus 101

Coptus 100

corae (Xenocerus) 34

corae austrinus (Xenocerus) 35

corae diversemaculatus (Xenocerus) 35

corae singularis (Xenocerus) 35

corae ypsilon (Xenocerus) 35

corallifer (Pantorhytes) 165

corallimera (Rhinotia, Belus) 43

cordiformis (Miolispa) 66

cordipennis (Baris) 81

cordipennis (Hylesinus) 198

Cordus 53

corismus (Araeocorynus) 40

cornutus (Apirocalus) 134

cornutus bosavii (Apirocalus) 135

cornutus paradoxus (Apirocalus) 134

cornutus tenuiscapus (Apirocalus) 134

cornutus virescens (Apirocalus) 134

corpulenta (Telaugia) 122

corpulentus (Arachnobas, Arachnopus) 87

CORRHECERINI 20

corrugatus (Crossotarsus) 182

CORTHYLINA 201

corticinus (Hylurdrectonus) 200

corysmus fuscopustulosus (Araeocorynus) 40

corysmus ornatipennis (Araeocorynus) 40

Coryssoglymma 113

CORYSSOMERINI 91

Cosmoderes 201

Cosmopolites 76

COSSONINAE 95

COSSONINI 95

Cossonus 95

costatus (Dysopirhinus) 107

costatus (Niphades) 170

costatus (Psilodryophthorus) 72

costifer (Opterus) 143

costulatus (Arachnobas, Arachnopus) 87

costulipennis (Bothriorhinus) 65

coxalis (Crossotarsus) 182

coxalis (Degis) 83

crassicollis (Cyphagogus) 54

crassicollis (Polyzelus) 129

crassicornis (Elytrocheilus) 139

crassicornis (Eucorynus, Anthribus) 25

crassicornis (Platypus) 187

crassifemoralis (Miolispa) 66

crassirostris (Oribius, Coptorhynchus) 144

crassirostris (Pseudaclees, Hylobius) 172

crassiusculus (Xylosandrus, Phloeotrogus) 219 crassus (Anaballus) 115

crassus (Platypus) 187

craterigerus (Acanthotomicus, Orthotomicus) 209

CRATOPARINI 24

creber (Coccotrypes, Poecilips) 205

creber (Scolytogenes, Cryphalomorphus) 204

credula (Miolispa) 66

cretaceus (Opterus) 143

cribratus (Aclees) 171

cribratus (Trigonopterus, Idotasia) 123

cribricollis (Sphenophorus) 76

cribricollis (Trigonopterus, Idotasia) 123

cribrosus (Sitophilus, Calandra) 74

crinipes (Brachynedus) 137

cristulatus (Nechyrus) 117

Critomerus 106

croceicornis (Centrinopsis) 86

croceodiscus (Semiathe) 122

Crossotarsus 182

cruceatoides (Oribius) 144

cruceicollis (Mucronianus) 34

cruciatus (Oribius, Coptorrhynchus) 144

cruciatus (Xyleborus) 216

crucifera (Misthosima) 41

cruciger v. fuscotriangularis (Lophocheirus) 127

crucigera (Lophocheirus, Odosyllis) 127

cruenta (Rhinoscapha) 158

cruralis angustefasciatus (Acorynus) 32

CRYPHALINA 201

Cryphalus 201

Cryptoderma 72

CRYPTODERMATINAE 72

CRYPTORHYNCHINA 104

CRYPTORHYNCHINAE 101

CRYPTORHYNCHINI 104

Cryptorhynchus 110

Cryptoxyleborus 212

Ctylindra 80

cucullatus (Glyptoporopterus, Poropterus) 114

cultellatus (Ithystenus) 60

cummingii (Araeocorynus) 40

cuneatus (Trigonopterus, Idotasia) 123

cupido (Ipsichora) 84

cupreosplendens (Neosynaptops) 49

cupreus (Ithystenus) 60

cuprifer (Eusintor) 31

cupronitens (Chalybdicus) 64

cupulatulus (Dinoplatypus, Platypus) 184

cupulatus (Dinoplatypus, Platypus) 184

Curculio 130

CURCULIONIDAE 80

CURCULIONINA 130

CURCULIONINAE 129

CURCULIONINI 130

curtus (Cyrtogenius, Ozodendron) 207

curtus (Trigonopterus, Idotasia) 123

curvatus (Xyleborus) 217

curvidens (Ithystenus, Leptorynchus) 60

curvifer (Diamerus, Hylesinus) 198 
curvipes (Metaeuops) 48

curvipes (Microporopterus) 116

curvirostris (Stenotrupis) 97

cuspidus (Taphrodasus, Xyleborus) 215

cuverii (Eupholus) 151

Cyamobolus 106

Cyamotrox 107

cyanea (Metanthia) 84

cyaneiventris (Ithystenus) 60

cyaneus (Pantoxystus) 171

cyanoptera (Panigena) 94

CYCLOMINAE 132

cyclopei (Pantorhytes) 165

cyclopensis (Guineoeuops) 46

cyclops (Apatenia) 21

cyclopus (Cyrtogenius, Pelicerus) 207

cyclopus (Xyleborus) 217

Cyclorhipidion 213

CYLADINAE 54

Cylas 54

cylindricus (Cryphalus) 201

cylindriformis (Amasa, Xyleborus) 210

cylindrirostris (Homorthorrhinus, Curculio) 179

cymbiformis (Platypus) 187

cynethioides (Zena) 85

CYPHAGOGINAE 54

CYPHAGOGINI 54

Cyphagogus 54

CYPHICERINA 149

CYPHICERINI 149

Cyphicerus 149

cyphoides (Elytrocheilus, Eupholus) 139

Cyphopus 138

cyphothorax (Gymnopholus, Aroaphila) 153

Cyrtogenius 207

cyrtus (Araecerus) 39

\section{Dactylipalpus 198}

dactyliperda (Coccotrypes, Bostrichus) 205

daedalus (Platypus) 187

Daedania 92

dani (Ottistira) 163

darnleyensis (Rhinoscapha) 158

darwini (Scolytogenes) 204

dealbatus (Peribleptus, Alcides) 178

dealbatus zonatus (Peribleptus, Ppalosomus) 178

debilifrons (Heterocybus) 70

debilis (Ithystenus) 61

decapitatus (Poropterus) 120

decemmaculatus (Ectocemus, Megacerus) 51

decempustulatus (Pantorhytes, Pachyrrhynchus) 165

decemverrucosus (Pantorhytes) 165

deceptus (Arixyleborus) 211

deceptus (Guineoeuops) 46

decipiens (Pseudocholus) 85

decisus (Nechyrus) 117

declivis (Araecerus) 39

declivis (Cyrtogenius, Carposinus) 207

declivispinatus (Ambrosiodmus, Xyleborus) 210 decorus (Ithystenus) 61

decussata (Acicnemis) 179

Degis 83

deharvengei (Guineobius) 140

deleta (Agametis) 91

delicatum (Cyclorhipidion, Xyleborus) 213

delicatus (Cyphagogus) 54

demeijerei (Oribius, Coptorhynchus) 144

Demimaea 132

DEMIMAEINA 132

demissa (Rhinoscapha) 158

demissus (Poropteropis) 119

Dendropemon 25

densatus (Trigonopterus, Idotasia) 123

densatus (Xyleborus) 217

densepunctatus (Ithystenus) 61

densleonis (Phaenomerus) 90

dentatulus (Xyleborus) 217

dentatus (Schizotrachelus) 68

denticollis (Platypus) 187

denticollis (Telephae) 93

denticulata (Solenobaris) 82

denticulatus (Webbia) 215

denticulus (Acanthotomicus) 209

denticulus (Crossotarsus) 182

dentifer (Dyspeithes) 128

dentifer (Protaedus) 26

denturus (Crossotarsus) 182

deperditus (Crossotarsus) 187

deperditus (Platypus) 187

deplanata (Nessiara) 34

deplanatus (Hypseus) 23

DEPORAINI 44

depressicollis (Stenotrupis, Dioedimorpha) 97 depressior (Elytrocheilus) 139

depressurus (Xyleborus) 217

depressus (Guineobius) 140

depressus (Platypus) 187

Deretiodes 173

Deretiosus 173

deridicula (Acicnemis) 179

Deropygus 40

desmaresti (Eupholus) 153

DESMIDOPHORINI 72

Desmidophorus 72

despectus (Ficicis, Hylesinus) 198

destructor (Oribius) 144

destruens (Euwallacea, Xyleborus) 214

detanii (Eupholus) 151

devius (Xyleborus) 217

Dexagia 133

deyrollei (Gyalostoma, Baryrrhynchus) 52

dhuyi (Eupholus) 151

DIAMERINA 197

Diamerus 198

DIAPODINA 196

Diapus 196

Diatassa 101

Diathetes 76 


\section{Diathryptus 113}

dibrachiatus (Platypus) 187

dicyphus (Poropteropis) 119

didiman (Symbiopholus) 155

difficilis (Behrensiellus) 137

difficilis (Trigonopterus, Idotasia) 123

diffinis (Platypus) 187

digestus (Platypus) 187

digramma (Orychodes, Arrhenodes) 52

dilaticollis (Trigonopterus, Idotasia) 123

diluta (Basitropis) 20

diluta (Rhinoscapha) 158

diluta rostralis (Basitropis) 20

diluta ventralis (Basitropis) 20

dimidiata (Telaugia) 122

dimidiatus (Pseudocossonus) 97

Dinema 26

Dinomelaena 22

Dinoplatypus 184

dintelmanni (Metaeuops) 48

Diocalandra 73

DIOCALANDRINI 73

Diomia 172

discedens (Eudelodes, Amorphoidae) 129

discipennis (Platypus) 187

discolor (Baryrhynchus) 51

discors (Cryptoderma, Calandra) 72

\section{DISCOTENINI 25}

discrepans (Tyrtaeosus) 125

discretus (Oribius, Coptorrhynchus) 144

dispar (Barystethus, Dialtates) 76

dispar (Ernoporus, Cryphalops) 203

dispar (Hyparinus) 108

dispar (Melanopsacus) 42

dispar (Syrichius) 110

dispar basalis (Barystethus) 76

disparatus (Auletanus) 44

disparilis (Arachnobas, Arachnopus) 87

disparilis (Orthotemnus) 100

dissimilis (Hypomiolispa) 66

Dissostomus 101

distans (Exillis) 29

distans (Oribius, Coptorrhynchus) 144

distinctipes (Platypus) 187

distinctus (Coccotrypes, Anodius) 205

distinguenda (Acicnemis) 179

divaricatus (Gymnopholus) 153

diversicolor (Coptodryas, Xyleborus) 212

diversicolor (Cryphalus) 201

diversicolor (Ithystenus) 61

diversiporus (Platypus) 185

divisa (Diatassa) 101

divisa (Rhinotia, Belus) 43

divisus (Euopsidius) 46

diura (Aphyoda) 101

dobunabae (Platypus) 188

docta (Coptodryas, Xyleborus) 212

Doeothena 29

doertheae (Neosynaptops) 49 dohertyi (Sphenomorpha) 168

dohertyi (Xenocerus) 35

Dolichocera 28

dolosa (Basitropis) 20

dolosa (Rhinoscapha) 158

dolosa quadrispinosus (Basitropis) 20

dolosa tenuiclava (Basitropis) 20

dolus (Crossotarsus) 182

dolus (Platypus) 188

doriae (Caenochira) 89

doriae (Camptorhinus) 103

doriae (Carcinopisthius, Hoplopisthius) 57

doriae (Rhinoscapha) 158

doriae (Zygara, Asytesta) 126

doriae ab. viridiaurea (Rhinoscapha) 158

doriae heurni (Rhinoscapha) 158

doriae heurni ab. bizonata (Rhinoscapha) 158

doriae heurni ab. dives (Rhinoscapha) 158

doriae heurni ab. reducta (Rhinoscapha) 158

doriai (Stereonotus) 98

dorsalis (Asytesta) 104

dorsalis (Camptorhinus) 103

dorsalis (Eutyrhinus, Euthyrhinus) 126

dorsatus (Platypus) 188

doryphorus (Mecopus) 92

Doticus 41

douei (Uropteroides, Brenthus) 65

Douttia 114

draco (Trigonosomus) 125

draco papuanus (Trigonosomus) 125

driesi (Misthosima) 41

DRYOCOETINA 205

Dryocoetiops 208

DRYOPHTHORIDAE 72

DRYOPHTHORINAE 72

Dryophthoroides 79

Dryophthorus 72

DRYOTRIBINI 98

dubia (Cenchrena) 130

dubia (Rhinoscapha) 161

dubius (Microtrachelizus) 63

ducopeaui (Eupholus) 151

duodecimspinatus (Xyleborus) 217

duplicatus (Cyamobolus) 106

duplosignatus (Platypus) 188

duponti (Gasteroclisus, Lixus) 169

Dyscamptorhinus 103

Dysnos 42

Dysopirhinus 107

Dyspeithes 128

Dystropicus 102

ebenina (Barinogyna) 83

ebenina (Baris) 81

ebenina (Metanthia) 84

ebenina (Miolispa) 66

ebenius (Phocylides) 65

ebriosus (Trigonopterus, Idotasia) 123

ebrius (Apirocalus) 135 
ebrius angustus (Apirocalus) 135

ebrius wagneri (Apirocalus) 135

Eccoptopterus 213

ECELONERINI 25

echinatus (Celeuthetes, Curculio) 138

echinatus (Platypus) 188

echymis (Poropterus) 120

Ectatocyba 114

Ectatorhinus 172

Ectemnomerus 138

Ectocemus 51

Eczesaris 28

edentata (Baris) 81

edentula (Pioenidia) 31

effigies (Arachnobas, Arachnopus) 87

effusa (Bartolozziella, Endymia) 111

egregia (Rhinoscapha) 158

eichhoffi (Aricerus) 200

eichhorni (Cyphagogus) 54

eichhorni (Xenocerus) 35

eichhorniae (Neochetina) 16

Eidophelus 202

elaboratus (Platypus) 188

Elassophilus 94

elegans (Alcidodes, Alcides) 175

elegans (Cosmoderes) 201

elegans (Ectemnomerus) 138

elegans (Eusintor) 31

elegans (Gyalostoma) 52

elegans (Oribius, Coptorhynchus) 144

elegans v. albolituratus (Alcides) 174

elegans v. atrocretosus (Alcides) 175

ellipticus (Oribius, Coptorrhynchus) 144

ellynae (Gymnopholus) 153

elongata (Oxydema) 96

elongatulus (Cyrtogenius) 207

elongatus (Behrensiellus, Cataphractus) 137

elongatus (Cyphagogus) 54

elongatus (Diapus) 196

elongatus (Notoplatypus) 197

eluta (Rhinoscapha) 158

Elytracantha 51

Elytrocheilus 139

elytrostriatus (Cordus) 53

ELYTRURINI 149

emarginatus (Xyleborus) 217

emarginatus semicircularis (Xyleborus) 217

emblematicus (Critomerus) 106

emdeni (Platypus) 188

Emydica 107

Enaptomias 140

engabenae (Gymnopholus) 153

enodata (Coryssoglymma) 113

enormis (Platypus) 188

ENTIMINAE 133

Eothaumas 34

ephippiatus (Trigonopterus, Idotasia) 124

ephippiger (Cossonus) 95

ephippiger (Perrhaebius) 174 ephippiger centromaculatus (Cossonus) 95

epimeralis (Curculio, Balaninus) 130

epipona (Basitropis) 20

Episomellus 150

episomoides (Kietana) 142

episternalis (Glyptoporopterus) 114

equestris (Xenocerus) 35

equestris ancorninus (Xenocerus) 35

equestris olivaceus (Xenocerus) 35

equestris suadus (Xenocerus) 35

Erebaces 114

eremita (Apries) 172

eremitus (Alcidodes) 175

EREMOXENINI 53

erimanus (Deretiosus) 173

ERIRHINIDAE 80

ERIRHININAE 80

erirhinoides (Tadius) 80

Ernoporus 203

erraticum (Cryphalus) 202

Esocus 32

Eterozemus 55

evanida (Rhinoscapha) 158

evanidinervius (Platypus) 188

evanidus (Platypus) 188

Eubactrus 64

Eucorynus 25

Eucryptorhynchus 107

Eudelodes 129

Eudyasmodes 114

eudyasmoides (Asytesta) 104

Eudyasmus 107

EUGNOMINI 14

EUOPINI 45

Euops 45

Euopsidius 46

Euparius 24

EUPHOLINI 149

Eupholocis 162

Eupholus 150

euphrosyne (Eupholus) 151

Eupsalomimus 51

EURHYNCHINAE 58

europus (Rhaphitrophis) 37

eurous (Acorynus, Hucus) 32

euryae (Symbiopholus) 155

eurysterna (Baris) 81

Eusintor 31

Eutinophaea 162

Eutyrhinus 126

Euwallacea 214

exarata (Hypomiolispa, Miolispa) 66

exarata (Myocalandra, Sitophilus) 73

exarmata (Aporhina, Chalcocybebus) 59

excedens (Platypus) 188

excellens (Albertisius, Idiopsis) 133

excellens (Platypus) 188

excellens (Scolytogenes, Cryphalomorphus) 204

excelsior (Phaenomerus) 90 
excisus (Deropygus) 40

excisus (Protaedus) 27

exclusa (Acicnemis) 180

exclusa v. persona (Acicnemis) 180

exiguus (Mechistocerus, Isotocerus) 102

exiguus (Xyleborinus, Bostrichus) 216

exilis (Phaenomerus) 90

exilis (Stenotrupis) 100

Exillis 29

eximius (Acanthotomicus, Ips) 209

Exophthalmida 161

exornatus (Alcidodes) 175

expansus (Elytrocheilus) 139

expansus v. nigripes (Elytrocheilus) 139

exportatus (Araiorrhinus) 62

extensa (Coptodryas, Xyleborus) 212

extensus (Orthotemnus, Psilotrogus) 100

extensus (Platypus) 188

extensus (Psilotrogus) 97

extorris (Poropteropis) 119

fabricii (Rhinoscapha, Geonemus) 158

facialis (Aulacophrys) 137

falcarius (Xyleborus) 217

fallax (Apirocalus) 135

fallax (Mechistocerus, Isotocerus) 102

fallax (Symbiopholus) 156

fallax (Xyleborus) 217

falsoconfinis (Elytrocheilus) 139

farinosus (Lixus) 169

fasciata (Cenchrena) 130

fasciata (Demimaea) 132

fasciata (Ottistira) 163

fasciatum (Altipectus) 29

fasciatus (Litocerus) 33

fasciculatus (Araecerus, Curculio) 39

fasciculatus (Perrhaebius) 174

fasciger (Exillis) 30

fastigatus (Xyleborus) 217

fastuosus (Platypus) 195

fastuosus (Xenocerus) 35

fastuosus albosignatus (Xenocerus) 35

faunus (Poropteropis) 119

fausti (Acicnemis) 180

fausti (Rhinoscapha) 159

femoralis (Ithystenomorphus) 60

femoralis (Lijudmilinius) 47

femoralis (Menectetorus, Pseudapries) 127

femoralis (Trigonopterus, Idotasia) 124

femorata (Ipsichora) 84

fenestrallatus (Platypus) 188

fenestrata (Rhinoscapha) 159

fenestratus (Arachnobas, Arachnopus) 87

fenestratus v. demissus (Arachnopus) 87

fenneri (Pantorhytes) 165

ferox (Poropteropis, Poropterus) 119

ferrugineus (Alcidodes, Alcides) 175

ferrugineus (Conarthrus, Eutornus) 99

ferrugineus (Rhynchophorus, Curculio) 75 ferrugineus (Xyleborus, Bostrichus) 217

ferus (Arachnobas, Arachnopus) 87

festiva (Agametis) 91

festiva (Apatenia) 21

festivus (Cyrtogenius) 207

fici (Acanthotomicus) 209

Ficicis 198

fictabidi (Pantorhytes) 165

fictacarbonarius (Pantorhytes) 165

ficus (Cryptorhynchus) 110

figurata (Acicnemis) 180

filiformis (Euwallacea, Xyleborus) 214

filipennis (Platypus) 188

filirostris (Myctides) 85

fimbriatus (Arachnobas, Arachnopus) 87

finschi (Arachnobas, Arachnopus) 87

firmis (Platypus) 188

firmus (Alcidodes) 175

flavipennis (Xyleborus) 217

flavirostris (Osphilia) 91

flavoangulatus (Protaedus) 27

flavodorsalis (Curculio, Balaninus) 130

flavolineatus (Callipareius) 54

flavomaculatus (Milichacalles) 112

flavomaculatus (Trigonopterus, Idotasia) 124

flavopilosus (Xyleborus) 217

flavosignatus (Allodapinus) 65

flavovirens (Eutinophaea) 162

flavus (Protaedus) 27

flesina (Orochlesis) 128

fleurenti (Eupholus) 151

flexuosocarinatus (Dyscamptorhinus) 103

flimbritarsis (Pseudocholus) 85

fluminalis (Crossotarsus) 182

flyensis (Guineoeuops) 46

foersteri (Otidognathus) 74

forcipes (Crossotarsus) 182

forcipitiger (Carcinopisthius) 57

fordi (Molobrium) 136

forficula (Dinoplatypus, Platypus) 184

forficulatus (Ithystenus) 61

formicarius (Attelabus) 54

formicarius (Cylas) 54

formidatus (Hypseus) 23

fornicatus (Euwallacea, Xyleborus) 214

forticatus (Pseudotaphroderes) 57

forticornis (Gymnopholus) 155

forticula (Stratiopisthius) 57

fortis (Arachnobas, Arachnopus) 87

fortis (Tracheloschizus, Schizotrachelus) 68

fossulatus (Arachnobas, Arachnopus) 87

fossulatus nigrofimbriatus (Arachnobas, Arachnopus) 87

foveifrons (Meroleptus) 116

fracticinctus (Platypus) 188

fracticornis (Platypus, Crossotarsus) 188

fracticostis (Platypus) 188

fractus (Crossotarsus) 182

francoisi (Ithystenus) 61

franklini (Aades, Aterpus) 132 
franklini (Ithystenus) 61

franklini-muelleri (Eczesaris, Idiopus) 28

franklinmuelleri (Ithystenus) 61

frater (Alcidodes, Alcides) 175

frater (Ptolycus) 121

frater (Rhaphitrophis) 37

fraterculus (Euops) 49

fraterculus (Oribius, Coptorrhynchus) 144

fraterna (Kleineella) 53

fraudatrix (Miolispa) 66

fraudus (Pantorhytes) 167

frenatus (Arachnobas, Arachnopus) 87

freudei (Alcidodes) 175

freudei (Misthosima) 41

frivola (Miolispa) 66

froggatti (Hypocryphalus) 203

froggatti (Sphenomorpha, Apocyrtus) 168

frontalis (Eutyrhinus) 126

frontalis (Ithystenus) 61

frontebulbifer (Platypus) 188

fronto (Stereoderus) 98

frugalis (Cyphagogus) 55

fruhstorferi (Carcinopisthius) 57

frumenti (Diocalandra, Clandra) 73

frumenti v. subfasciata (Sitophilus) 73

fucatus (Eterozemus) 55

fucatus (Xenocerus) 35

fugax (Cryphalus) 202

fugax (Platypus) 188

fugax (Scolytogenes, Cryphalomorphus) 204

fulgens (Amasa, Xyleborus) 210

fulgens (Coccotrypes, Poecilips) 205

fulgens (Scolytogenes, Cryphalomorphus) 204

fulgidus (Platypus) 188

fulgidus (Scolytogenes, Cryphalomorphus) 204

fuligineus (Ptolycus) 121

fuliginosa (Microspathe, Calandra) 74

fuliginosus (Phaenomerus) 90

fulvicornis (Baris) 81

fulvospretus (Gymnopholus, Aroaphila) 154

fulvus (Margadillius) 204

funebris (Desmidophorus, Desmidophorinus) 72

funebris (Rhinoscapha, Laodice) 159

funebris fulvopilosus (Desmidophorus) 72

funereus (Ambrosiodmus, Xyleborus) 210

funereus (Cyamobolus) 106

funestus (Ambrosiodmus, Xyleborus) 210

fungifer (Symbiopholus) 156

furcillifer (Arachnobas, Arachnopus) 87

furculus (Hellerrhinus) 141

fuscatus (Protaedus) 27

fuscicornis (Xenopsilus) 86

fuscipes (Oribius, Coptorhynchus) 144

fuscovarius (Pycnochirus, Megachirus) 181

fusiformis (Xenotrupis) 100

fusirostre (Amorphorhynchus) 97

fuyugei (Xyleborus) 217

gabensis (Platypus) 188 gagatina (Metanthia) 84

gagatina (Rhinoscapha) 159

gagatinum (Apion, Conapion) 71

galbula (Curculio, Balaninus) 130

galbulimimae (Platypus) 189

gallus (Alcidodes) 176

Ganae 73

ganglbaueri (Rhinoscapha) 159

Ganonotapion 69

garnotii (Hylopemon, Anthribus) 26

Garyus 37

GASTEROCERCINI 126

Gasteroclisus 169

Gasterosaga 108

gazella (Arachnobas) 87

gazella (Asytesta, Rhynchaenus) 104

gazella v. vitticollis (Arachnopus) 87

gazellae (Apatenia) 21

gazellae (Lophothetes, Trigonops) 142

gebehiana (Rhinoscapha) 159

gemellus (Platypus, Crossotarsus) 189

geminata (Laogenia) 73

geminatus (Platypus) 189

gemmatus (Cleodus, Cleobis) 113

gemmatus (Lophocheirus, Odosyllis) 127

gemmatus (Meroleptus) 116

gemmifer (Gymnopholus) 154

gemmifer (Pseudoporopterus, Poropterus) 120

generosa (Rhinoscapha) 159

generosus (Oribius, Coptorrhynchus) 144

Genethila 25

geniculatus (Alcidodes, Alcides) 176

geniculatus (Nechyrus) 117

Genyocerus 197

geoffroyi (Eupholus) 151

geoffroyi chrysites (Eupholus) 151

geoffroyi v. tupinieri (Eupholus) 151

germari (Pantiala) 119

gerstackeri (Platypus) 189

gestroi (Acicnemis) 180

gestroi (Albertisius, Apirocalus) 133

gestroi (Alcides) 175

gestroi (Anchithyrus) 112

gestroi (Apion) 71

gestroi (Asytesta) 104

gestroi (Dysopirhinus) 107

gestroi (Dyspeithes, Amadus) 128

gestroi (Rhinoscapha) 159

gestroi (Uropteroides, Uropterus) 65

gestroi v. aeruginosus (Dysopirhinus) 107

gibber (Opterus) 143

gibbicollis (Baris) 81

gibbicollis (Ottistira) 163

gibbirostris (Trigonopterus, Idotasia) 124

gibbosa (Ectatocyba) 114

gibbus (Ptyctonotus) 82

giganteus (Ozopemon) 209

gigas (Cryphalus) 202

gigas (Phloeobius, Anthribus) 30 
gigas granulatus (Sipalinus, Calandra) 73 giluwei (Platypus) 189

glaberrima (Carbonomassula) 178

glaberrimus (Xyleborus) 218

glabratus (Behrensiellus, Siteytes) 137

glabratus subvirens (Behrensiellus) 137

gladiator (Neosynaptops) 49

glandis (Thamnurgides) 206

glanis (Poropterus) 120

glauca (Exophthalmida) 161

globatus (Trigonopterus, Idotasia) 124

globicollis (Platypus) 189

globithorax (Barystethus) 76

globus (Hadrodemius, Xyleborus) 214

glochideus (Platypus) 189

glochidionis (Gymnopholus) 154

Gloedema 95

gloriosa (Trigonops) 148

Glyptoporopterus 114

godeffroyi (Ectatorhinus) 172

goilala (Euops) 50

goilalae (Pantorhytes) 165

goilalae (Platypus) 189

gongylodes (Platypus) 189

gorggae (Xyleborus) 217

gracilicollis (Parasphenogaster) 146

gracilicornis (Platypus, Crossotarsus) 189

gracilior (Cryptoxyleborus) 216

gracilior (Platypus) 189

gracilipes (Eccoptopterus, Platydactylus) 213

gracilipes (Misthosima) 41

gracilis (Apatenia) 21

gracilis (Cyrtogenius, Ozodendron) 207

gracilis (Eidophelus) 202

gracilis (Molobrium) 136

gracilis (Pantorhytes) 165

gracilis (Platypus) 189

gracillimus (Cyrtogenius) 207

grammicus (Chirozetes) 92

granarius (Sitophilus, Curculio) 74

grandis (Acanthotomicus) 209

grandis (Orphanistes) 118

grandis (Protaedus) 27

granibasis (Mechistocerus) 102

granifer (Diamerus) 198

granifrons (Meroleptus) 116

graniger (Elytrocheilus, Siteytes) 139

graniger (Xyleborus) 217

granistriatus (Cyrtogenius, Orosiotes) 207

granosispina (Aporhina, Chalcocybebus) 59

granosus (Celeuthetes) 138

granosus (Platypus) 189

granulatus (Ozopemon) 209

granulatus (Pantorhytes) 165

granulatus (Salcus) 121

granulatus (Zeugorrhinus) 149

granulicauda (Arixyleborus) 211

granulicollis (Apirocalus) 135

granulifera (Asytesta) 104 granulipennis (Arachnobas, Arachnopus) 87

granulipennis (Phloeosinus) 200

granulipes (Metanthia) 84

granulipes (Xyleborus) 217

granulosus (Aclees) 171

granulosus (Celeuthetes) 138

granulosus (Platypus) 189

gratiosus (Xyleborus) 217

gratus (Oribius, Coptorrhynchus) 144

gravis (Pantorhytes) 165

grayi (Aragomacer) 19

grayi (Cryphalus) 202

grayi (Platypus) 189

grayi immersus (Platypus) 189

gressiti (Trigonops) 148

gressitti (Crossotarsus) 182

gressitti (Gymnopholus) 154

gressitti (Riedelinius) 50

grevilleae (Crossotarsus) 196

griseovaria (Misthosima) 41

griseus (Acalles, Tylodes) 112

griseus (Anthribus) 30

griseus (Ceocephalus, Diurus) 59

griseus (Idiopsodes, Idiopsis) 141

gronopoides (Menectetorus, Chtectetorus) 127

grootaerti (Diurus) 59

grossepunctatus (Ozopemon) 209

grossus (Apirocalus) 135

grumosa (Apatenia) 21

grumosus (Platypus) 189

guerini (Cryptorhynchus) 110

guerini (Eupholus) 153

guerinii (Syggenithystenus, Leptorhynchus) 62

Guineauletes 44

GUINEAULETINA 44

guineensis (Oribius, Coptorrhynchus) 144

Guineobius 140

Guineoeuops 46

Guiomatus 94

gularis (Apatenia) 21

gumiensis (Platypus) 189

guttatus (Oribius, Coptorhynchus) 145

guttifer (Arixyleborus, Xyleboricus) 211

guttiger (Oribius, Isomerinthus) 145

guttulifer (Arachnobas, Arachnopus) 87

Gyalostoma 52

Gygaeus 115

gyllenhali (Cryptorhynchus) 110

gyllenhalli (Aclees) 171

Gymnopholus 153

Gynaria 149

gyrosicollis (Sphenogaster) 148

Habrissus 23

Hadrodemius 214

hagenensis (Pantorhytes) 165

hamata (Solenobaris) 82

harti (Gymnopholus) 154

hashimotoi (Platypus) 189 
hashimotoi (Xyleborus) 217

hastatum (Cyclorhipidion, Xyleborus) 213

hasterti (Rhinoscapha) 159

hauseri (Cyphagogus) 54

healyi (Pantorhytes) 165

hebes (Brachynedus) 137

hebetatus (Platypus) 189

helenae (Elytrocheilus) 139

helleri (Aporhina, Chalcocybebus) 59

helleri (Eupholus) 151

helleri (Mecopus) 92

hellerianus (Oribius, Coptorrhynchus) 145

Hellerrhinus 141

Henorychodes 52

hepaticus (Symbiopholus) 156

hephaistos (Eupholus) 151

herbarius (Symbiopholus) 156

herbarius oribatifer (Symbiopholus) 156

hercules (Deropygus) 40

herteli (Alcidodes) 176

Hesychobius 171

Heterocybus 69

heteromorphus (Platypus) 190

Heterophaseolus 96

Heterophasis 95

heurni (Eudyasmodes) 114

heydeni (Prodioctes) 78

heydeni (Rhinoscapha) 160

Higonius 62

Higonodes 62

hilaris (Mallorrhynchus) 36

Himatium 100

hippocrepicus (Platypus) 189

hirtellus (Garyus) 37

hirtus (Cyrtogenius, Dryocoetes) 207

hirtus (Platypus) 189

histrio (Chaetectetorus) 126

histrio fluviatilis (Litocerus) 33

hollandiae (Ithystenus, Brenthus) 61

Homalotrogus 96

Homorthorrhinus 179

hopeae (Xyleborus) 217

\section{HOPLOPISTHIINI 56}

Hormocerus 64

hornabrooki (Apirocalus) 135

hornabrooki (Cyphagogus) 55

hornabrooki (Dissostomus) 101

hornabrooki (Ganonotapion) 69

hornabrooki (Gymnopholus) 154

hornabrooki (Pantorhytes) 165

hornabrooki (Phaenomerus) 90

horni (Pantorhytes) 165

hornus (Cyrtogenius, Eidophelus) 207

horrendus (Mormosintes) 117

horridipes (Autillia) 105

hospes (Oribius, Coptorrhynchus) 145

hospes (Stereoderus) 98

hospiton (Allodapinus) 65

hostis (Oribius, Coptorrhynchus) 145 howcrofti (Gymnopholus) 154

Hucus 32

hudsoni (Eupholus) 151

humboldti (Metopum) 43

humboldti (Neozemioses) 56

humboldtiana (Rhinoscapha) 159

humeralis (Basitropis) 20

humeralis (Eupholus) 151

humeralis (Hellerrhinus) 141

humeralis (Nechyrus) 117

humeralis (Protaedus) 27

humeralis (Xenocerus) 35

humeridens (Eupholus) 151

humeridens prasinus (Eupholus) 152

humilis (Oribius, Coptorrhynchus) 145

huonaris (Pantorhytes) 165

huttoni (Symbiopholus) 156

Hybicus 115

hybridus (Platypus) 189

hybus (Araecerus) 39

hydrographicus (Apirocalus) 135

Hyledius 199

HYLESININA 198

HYLESININI 197

hylesinopsis (Scolytogenes, Cryphalomorphus) 205

Hylesinus 198

HYLOBIINA 171

HYLOBIINI 171

Hylopemon 26

Hylurdrectonus 200

HYORRHYNCHINA 199

Hyparinus 108

HYPERINAE 169

HYPERINI 169

hypocrita (Dendropemon, Dendrotrogus) 25

hypocrita levis (Dendropemon, Dendrotrogus) 25

hypocritus (Oribius, Coptorrhynchus) 145

Hypocryphalus 203

Hypolixus 169

Hypomeces 168

Hypomiolispa 66

Hypotactus 141

Hypothenemus 203

Hypseus 23

Hypsophorus 111

ibaense (Alissapion) 68

ibelensis (Euops) 49

iconicus (Eutyrhinus, Euthyrhinus) 126

idenburgi (Alcidodes, Alcides) 176

Idiopsodes 141

Idopelma 93

ilex (Orthorhinus) 178

iliacus (Critomerus, Perissops) 106

illegalovi (Metaeuops) 48

illex (Trigonopterus, Idotasia) 124

illigeri (Cossonus) 95

illitus (Trigonopterus, Idotasia) 124

illusa (Pantiala) 119 
illustris (Neplaxa) 171

Imathia 131

imberbis (Platypus) 190

imhoffi (Acalles, Tylodes) 112

imitator (Arachnobas, Arachnopus) 87

imitator (Degis) 83

imitatrix (Baiocis) 181

immaculata (Dinomelaena, Apatenia) 22

immaculata (Rhinoscapha) 159

immaculata diversicollis (Dinomelaena) 22

immaculatus (Enaptomias) 140

immersus (Xyleborus) 217

immitatrix (Xyleborus) 217

immitis (Oribius, Coptorhynchus) 145

impar (Chaetoptelius, Hylesinus) 200

impar (Ithystenus) 61

impar (Trigonopterus, Idotasia) 124

imparatus (Barystethus, Diathetes) 76

imparidens (Platypus) 190

imperalis (Barystethus) 76

imperialis (Melanopsacus) 42

impexa (Rhinoscapha) 159

impexus (Xyleborus) 218

impressa (Sphenomorpha, Apocyrtus) 168

impressicollis (Papuatorhaenas) 38

impressifrons (Dinomelaena) 22

impressus (Exillis) 30

impressus discoidalis (Exillis) 30

imprima (Apatenia) 21

improvidus (Oribius, Coptorrhynchus) 145

incanus (Arachnobas, Arachnopus) 88

incertus (Ambrosiodmus, Xyleborus) 210

incertus (Dystropicus, Berosiris) 102

incertus (Microtrachelizus) 63

incertus (Platypus) 190

incisus (Cossonus) 95

incisus (Enaptomias, Otiorrhynchus) 140

incisus (Microtrachelizus) 63

inclinans (Acanthotomicus, Ips) 209

inclusus (Trigonopterus, Idotasia) 124

incomptus (Alcides) 177

inculta (Phaulimia) 24

incultus (Lophothetes) 142

indecorata (Apatenia) 21

indigaceus (Alcidodes, Alcides) 176

indigens (Cyclorhipidion, Xyleborus) 213

indignus (Aclees) 171

indignus (Nechyrus) 118

indiscretus (Oribius, Coptorrhynchus) 145

indocilis (Baryrhynchus) 51

inepta (Penestica) 30

inermis (Aporhina, Chalcocybebus) 59

inermis (Cyrtogenius) 207

inermis (Hypothenemus) 203

inermis (Solenobaris) 82

infaustum (Apion) 71

inflatipennis (Isoleptus) 115

inflatipes (Mesotactus) 147

inflatus (Hypomeces) 168 ingens (Lophocheirus, Odosyllis) 127

ingens (Tyrtaeosus) 125

ingentus (Pioenia, Tropidobasis) 30

ingenuus (Mechistocerus) 102

inimicus (Baiocis, Crossotarsus) 181

inimicus (Platypus) 181

iniquicollis (Ottistira) 163

iniquus (Melanopsacus) 42

inopinatus (Dryocoetiops, Dryocoetes) 208

inornata (Rhinotia, Belus) 43

inornatus (Apirocalus) 135

inornatus (Crossotarsus) 183

insignicollis (Glyptoporopterus) 114

insignirostris (Solenobaris) 82

insignis (Acythopeus) 83

insignis (Aporhina, Chalcocybebus) 59

insperatus (Apirocalus) 135

instructa (Genethila) 25

insulanus (Machaerostylus) 143

insularis (Abrachius) 74

insularis (Auletobius) 43

insularis (Neolaemosaccus, Lmosaccus) 170

insularis (Orthorhinus) 178

insularis (Pseudophocylides) 62

insulindicus (Xyleborus) 218

integrirostris (Gymnopholus, Aroaphila) 154

integrirostris (Rhinoscapha) 159

intermedius (Conomalthus) 106

intermedius (Coptodryas, Xyleborus) 212

intermedius (Crossotarsus) 182

intermedius (Elytrocheilus) 139

intermedius (Eupholus) 151

intermedius (Parapisthius) 63

intermedius (Spathidicerus) 197

interpositus (Trigonopterus, Idotasia) 124

interpres (Gymnopholus, Aroaphila) 154

interrupticosta (Carcinopisthius) 57

interruptocostatus (Sphenophorus) 78

interruptus (Arachnobas, Arachnopus) 88

interruptus (Microporopterus) 116

interruptus (Neomelanterius) 132

interruptus (Niphades) 171

interruptus (Pantorhytes) 165

interruptus ornatus (Microporopterus) 116

interstitialis (Diamerus, Hylesinus) 198

interstitialis (Rhabdoscelus, Sphenophorus) 78

intrusus (Diathetes) 77

inversus (Platypus) 187

invisitata (Hypomiolispa) 66

inusiata (Baris) 81

io (Molobrium) 136

Ionthocerus 57

Iphthimorhinus 77

ipidia (Xyleborus) 218

IPINA 209

Ipsichora 83

iriani (Arixyleborus) 211

iriani (Platypus) 190

irregularis (Pantorhytes) 165 
irritans (Calyptulus) 64

irrorata (Apatenia) 21

irrorata (Odosyllis) 128

irrorata (Ottistira) 163

irrorata (Trigonops) 148

irroratus (Eutyrhinus, Euthyrhinus) 126

Ischnomerus 60

Isoleptus 115

Isomorphus 55

Ithyocybus 70

ITHYPORINA 172

ITHYPORINI 172

ITHYSTENINI 59

Ithystenomorphus 60

Ithystenus 60

Ittostira 162

ivagaii (Platypus) 190

IXAPIINI 68

jansoni (Platypus) 190

japensis (Guineoeuops) 46

javanus (Crossotarsus) 182

jeffersi (Platypus) 186

jekeli (Archeuops) 45

jimmiensis (Pantorhytes) 165

jordani (Rhinoscapha) 159

\section{JORDANTHRIBINI 26}

jucundum (Gyalostoma) 52

judithae (Metaeuops) 48

Julietiella 133

junix (Chirozetes) 92

juvencus (Platypus) 190

karnyi (Pseudoporopterus) 120

kaszabi (Maculinvolvulus, Involvulus) 45

katoi (Platypus) 190

kaupi (Rhynchophorus) 75

kersianus (Euwallacea, Xyleborus) 214

keyensis (Crossotarsus) 182

keysseri (Telaugia) 122

Kietana 142

Kleinella 53

klugi (Orthorhinus) 178

kochi (Idiopsodes, Idiopsis) 141

kokodae (Symbiopholus) 156

kokodaensis (Platypus, Crossotarsus) 190

Kokodanus 142

kolbei (Carcinopisthius) 57

kolbei (Cryphalus, Allarthrum) 202

kolbei (Pseudodeporaus) 45

kotaseaoi (Eupholus) 151

kukenthali (Diathetes) 77

kukukuku (Guineoeuops) 46

kuntzeni (Crossotarsus, Platypus) 182

kuntzmannorum (Eupholus) 151

kuperi (Diapus) 196

kurulu (Euops) 49

kutubu (Guineoeuops) 47 labbei (Eupholus) 151

lablabiae (Platypus) 190

labrifrons (Platypus) 190

lacerta (Arachnobas, Arachnopus) 88

lachaumei (Eupholus) 152

lacordairei (Crossotarsus) 183

lacordairei (Eupholus) 152

lacordairei (Pseudocholus) 85

lacrymans (Xenocerus) 35

lacrymans herbertus (Xenocerus) 35

lacteomaculata (Baris) 81

lactospretus (Lophocheirus) 127

LAEMOSACCINI 170

laeta (Eutinophaea, Ottistira) 162

laevicollis (Garyus) 37

laevicollis (Pantorhaenas) 24

laevigatus (Elytrocheilus) 140

laevior (Rhinoscapha) 159

laevipennis (Cacoschizus) 64

laevipennis (Cerobates) 57

laevis (Cyrtogenius, Ozodendron) 207

laevis (Euwallacea, Xyleborus) 214

laevis (Hypocryphalus) 203

laevis (Platypus) 190

laevissima (Baris) 81

lakekamuensis (Euops) 50

lamasonga (Rhinoscapha) 159

lameerei (Rhinoscapha) 159

lamingtona (Pantorhytes) 165

lamingtoni (Carcinopisthius) 57

laminifer (Phaenomerus) 90

Lamprohypera 169

lanceolatus (Arachnobas, Arachnopus) 88

languidus (Mechistocerus) 103

lani (Guineoeuops) 47

Laogenia 73

laqueata (Acicnemis) 180

laqueus (Machaerostylus, Pseudosphaeropterus) 143

larvata (Xychusa) 126

lascivus (Alcidodes) 176

lata (Misthosima) 41

latelobatus (Platypus) 190

lateripennis (Aristoxenus, Deretiosus) 101

lateripictus (Euparius, Caccorhinus) 24

lateritius (Pseudacalles) 120

laterosignatus (Meroleptus) 116

latespinis (Diapus) 196

laticollis (Acicnemis) 180

laticollis (Araeocorynus) 40

laticollis (Cryphalus) 202

laticollis (Exophthalmida) 161

laticollis (Garyus) 37

laticollis (Nechyrus) 118

laticollis (Platyacus, Colposternum) 147

latifrons (Mucronianus) 34

latipennis (Saginesis) 169

latirostris (Baryrhynchus) 51

latreillei (Eupholus) 153

latus (Deretiosus) 173 
leai (Aporhina, Chalcocybebus) 59

leai (Pseudoporopterus) 121

leblanci (Eupholus) 152

lectus (Orphanyasmus) 108

lemienensis (Stenotrupis) 97

lemniscatus (Alcidodes) 176

leochroma (Rhinoscapha) 159

lepidus (Alcidodes) 176

lepidus (Dinoplatypus, Platypus) 184

lepidus (Oribius, Otiorrhynchus) 145

Lepropus 15

leprosus (Platypus) 190

leptacinus (Protaedus) 27

LEPTOSCHOININA 83

Leptotapion 71

Leptoxyleborus 214

lesiniformis (Platypus) 190

leucomelas (Acythopeus) 83

leucomelas (Protaedus) 27

leucopleurus (Oribius, Coptorrhynchus) 145

leucospila (Baris) 81

leucostictus (Oribius, Coptorhynchus) 145

levigata (Aporhina, Chalcocybebus) 59

libra (Coptodryas, Xyleborus) 212

lichenifer (Pantorhytes) 165

lichenifer (Symbiopholus) 156

ligatus (Moluccobius, Coptorhynchus) 143

lihirensis (Paratactus) 146

Lijudmilinius 47

limatus (Euwallacea, Xyleborus) 214

limbalis papuensis (Rhaphitrophis) 37

limbatus (Periagis, Catastygnus) 168

linea (Acicnemis) 180

linearis (Ithystenus) 61

lineata (Pempherulus, Phylaitis) 93

lineatopunctatus (Cyrtogenius, Xyleborus) 207

lineatus (Pachyonyx) 104

lineatus (Phaenomerus) 90

linecollis kleinei (Baryrhynchus) 51

lineicollis (Baryrhynchus) 51

linnei (Eupholus) 152

linnei (Semiathe) 122

liosoma (Baris) 81

Lissapion 15

Lissopis 96

Listrodiathes 77

LITHININA 174

LITHININI 174

litigiosus (Acorynus, Litocerus) 32

Litocerus 33

litoralis (Rhinoscapha) 159

LITOSOMINI 73

litturatus (Protaedus) 27

LIXINAE 169

LIXINI 169

Lixus 170

lobicollis (Acicnemis) 180

lobipes (Guineoeuops) 47

LOBOTRACHELINI 91 longecaudatus (Platypus) 195

longicalcaratus (Platypus) 190

longiceps (Neolaemosaccus, Lmosaccus) 170

longicollidis (Baris) 81

longicollis (Coccotrypes, Thamnurgides) 205

longicornis (Anthribus) 30

longicornis (Crossotarsus, Platypus) 183

longicornis (Platypus) 185

longicornis (Sympaector) 29

longimanus (Pseudocholus) 85

longior (Cryphalus) 202

longipennis (Stereoderus) 98

longipes (Ipsichora) 84

longipilis (Hypocryphalus) 203

longipilis (Trichacalles) 112

longirostris (Acicnemis) 180

longirostris (Colobodes) 173

longirostris (Neohigonius) 63

longispina (Ottistira) 163

longispinis (Coptoborus, Streptocranus) 212

longissimus (Platypus) 190

longithorax (Allodapinus) 65

longius (Cyclorhipidion, Xyleborus) 213

Lophocheirus 127

lophonotus (Conomalthus) 106

Lophothetes 142

lorentzi (Penthoscapha) 157

loriae (Carcinopisthius) 57

loriae (Eusintor) 31

loriae (Microsebus) 56

loriae (Platypus) 190

loriai (Arachnobas, Arachnopus) 88

loriai (Eupholus) 152

loriai (Rhinoscapha) 159

loriai (Stelechodes) 130

lucaris (Platypus) 190

lucasi (Platypus) 190

luctificus (Xenocerus) 35

luctuosa (Plintheria) 32

luctuosus (Curculio, Balaninus) 130

luctuosus (Otiorrhynchus) 148

ludificator (Gymnopholus) 154

ludiosus (Cyamobolus) 106

ludovici (Mecopus) 92

lugubris (Asytesta) 105

lugubris bidentatus (Asytesta) 105

lumbaris (Oemethylus) 118

lunatellus (Platypus) 190

luniger (Dinoplatypus, Platypus) 184

luteicornis (Oebrius, brius) 94

luteopictus (Chirozetes) 92

lypriformus (Microlarinus) 16

maai (Pantorhytes) 165

maccawi (Pantorhytes) 166

macer (Cossonus) 95

Machaerostylus 142

macilentus (Barystethus) 76

maclayi (Rhinoscapha) 159 
Macroauletes 44

macrophtalmus (Litocerus, Stenocerus) 33

macropus (Talanthia) 93

maculatus (Eupholocis) 162

maculatus (Mesoderes) 56

maculatus (Metasynaptops) 48

maculatus (Scolytomimus) 220

maculibasis (Mechistocerus) 102

Maculinvolvulus 45

maculipennis (Ficicis, Hylesinus) 198

maculipes (Tropidobasis) 31

maculiventris (Hypseus) 23

maculosa (Orochlesis) 128

maculosus (Diathetes) 77

maculosus (Idiopsodes, Idiopsis) 141

maculosus (Mechistocerus) 102

maculosus (Perissops) 109

madangensis (Athanasius, Neomystocis) 105

MADARINI 83

MADOPTERINA 85

MADOPTERINI 85

maestus (Rhadinomerus) 103

Magaris 129

magdalenae (Aporhina, Chalcocybebus) 59

magicus (Ectatorhinus,) 172

magicus (Poropteropis) 119

magister (Alcidodes, Alcides) 176

magister (Gymnopholus) 154

magnicolle (Micronotapion) 70

magnificus (Eupholus) 152

magnificus v. concolor (Eupholus) 152

magnum (Leptotapion) 71

magnus (Margadillius) 204

magnus (Symbiopholus) 156

maior (Lophocheirus, Odosyllis) 127

maior ab. lateralis (Lophocheirus) 127

majusculus (Crossotarsus) 183

malachiticus (Platyacus, Colposternum) 147

Mallorrhynchus 36

malotrus (Eupholus) 152

mamberamonis (Eupholus) 152

mammifer (Gymnopholus) 154

mammillatus (Phraotes) 147

Manilabaris 15

manni (Pantorhytes) 166

Margadillius 204

margaritaceus (Platypus) 190

margaritifera (Rhinoscapha) 159

marginalis (Anchyptolycus) 112

marmorata (Blepiarda) 111

marmoratus (Dendropemon, Eucorynus) 25

marmoreus (Chirozetes) 92

marquardti (Gymnopholus) 154

marshalli (Gymnopholus) 154

massutei (Aporhina, Chalcocybebus) 59

massutei interrupta (Aporhina, Chalcocybebus) 59

matoae (Platypus) 191

Mauia 26

MAUIINI 26
Mechistocerus 102

MECISTOSTYLINA 110

Mecocerina 33

MECOCERINI 27

Mecocerinopis 33

MECOMACERINI 19

MECOPINI 91

Mecopus 92

Mecotropus 28

MECYSMODERINI 86

MECYSOLOBINI 174

mediocra (Misthosima) 41

meditabundus (Eutyrhinus, Curculio) 126

Megadiathetes 77

megalops (Baris) 81

megaphola (Zena) 85

megapoda (Acalles, Tylodes) 112

Megax 28

mejai (Hypseus) 23

mekeoi (Cryphalus) 202

Melanopsacus 42

melanospilus (Arachnobas, Arachnopus) 88

melanostictus (Pantorhytes) 166

Melanterus 131

melas (Acythopeus) 83

melas (Trigonopterus, Idotasia) 124

meleagris (Daedania) 92

meleagris v. papuana (Daedania) 92

mellerborgii (Polytus, Sitophilus) 74

Menechirus 181

Menectetorus 127

MENEMACHINI 93

meriones (Acicnemis) 180

Meroleptus 115

mesites (Xenocerus) 35

Mesoderes 56

mesoleiulus (Xyleborus) 218

mesoleuca (Daedania) 92

mesopila (Aporhina, Chalcocybebus) 59

MESOPTILIINAE 170

mesosternalis (Baris) 81

Mesotactus 147

messagieri (Eupholus) 152

Metaeuops 47

Metanthia 84

Metasynaptops 48

METATYGINI 177

Metetra 91

Metialma 91

Metopum 43

Microcossonus 99

micrographus (Platypus) 191

Microgymnapterus 116

Micronotapion 70

Microporopterus 116

micros (Euops) 50

Microsebus 56

Microspathe 74

Microthetes 143 
MICROTRACHELIZINI 62

Microtrachelizus 62

micrurus (Treptoplatypus, Platypus) 195

miliaris (Nauphaeus) 127

miliaris (Rhinoscapha) 159

Milichacalles 112

milnei (Apatenia) 21

milnei subgibbosa (Apatenia) 21

mimicanus (Lixus) 170

mimicus (Cyamobolus) 106

Mimidotasia 117

Mimiocurus 201

minimus (Coccotrypes, Poecilips) 206

minimus (Xyleborus) 218

minor (Apatenia) 21

minor (Arixyleborus, Xyleboricus) 211

minor (Coptus) 100

minor (Crossotarsus, Platypus) 183

minor (Cyrtogenius, Pelicerus) 207

minor (Pantorhytes) 166

minor (Platyacus) 147

minor (Solenobaris) 82

minor nigripennis (Apatenia) 21

minor robustus (Pelicerus) 207

minor signaticollis (Apatenia) 21

minusculus (Crossotarsus) 183

minusculus grandis (Crossotarsus) 183

minuta (Trigonops) 149

minutissimus (Coccotrypes, Poecilips) 206

minutus (Coccotrypes) 206

minutus (Guineobius) 140

minutus (Microgymnapterus) 116

minutus (Trigonopterus, Idotasia) 124

Miocalles 117

Miolispa 66

Miotus 108

mirabilis (Eupholus) 151

mirabilis (Guineauletes) 44

mirabilis (Stratiorrhina) 53

mirandus (Platypus) 191

misoolensis (Platypus) 191

misoriensis (Arachnobas, Arachnopus) 88

missai (Platycybus) 70

missionis (Curculio, Balaninus) 130

Misthosima 41

Mitrastethus 108

mitratus (Pseudoporopterus, Poropterus) 121

mixta (Berosiris) 102

mixta (Ottistira) 163

mixtus (Amasa, Xyleborus) 210

mniszechi (Crossotarsus) 183

modesta (Acicnemis) 180

modestus (Eupholus) 152

modiglianii (Cyphagogus) 55

moerens (Opterus, Coptorhynchus) 143

molitor (Dendropemon, Ecelonerus) 25

Molobrium 136

moluccana (Ittostira) 162

Moluccobius 143
MOLYTINA 178

MOLYTINAE 170

MOLYTINI 178

monachus (Cryptorhynchus) 126

monstruosus (Riedelinius) 50

montanus (Araecerus) 39

montanus (Hypocryphalus) 203

montanus (Pantorhytes) 166

monticola (Lixus) 170

montrouzieri (Rhynchophorus) 75

mopsus (Araecerus) 39

morigerus (Platypus) 191

morigerus (Xylosandrus, Xyleborus) 219

morio (Arixyleborus, Xyleboricus) 211

morio (Diathetes) 77

Mormosintes 117

morobeensis (Platypus) 191

morokana (Plintheria) 32

morokensis (Coccotrypes, Dendrurgus) 206

morokensis (Trigonopterus, Idotasia) 124

morosus (Platypus) 191

Morrisella 117

moutui (Crossotarsus) 183

mucronatoides (Xyleborus) 218

Mucronianus 33

muelleri (Ithystenus) 61

multatus (Araeocorynus) 40

multatus centralis (Araeocorynus) 40

multatus nigroapicatus (Araeocorynus) 40

multicollis (Telephae) 93

multifoveolatus (Platypus) 185

multigranulatus (Ptolycus) 121

multiguttata (Plesiobasis) 20

multimaculatus (Mechistocerus) 102

multiporus (Treptoplatypus) 195

multipunctatum (Cyclorhipidion, Xyleborus) 213

multipunctatus (Tracheloschizus) 68

multipunctulus (Xyleborus) 218

multipustulosus (Pantorhytes) 166

mundus (Cyphagogus) 55

munus (Aragomacer) 19

muricatus (Platypus) 191

mus (Apirocalus) 135

mus (Meroleptus) 116

mus (Scolytogenes, Cryphalomorphus) 205

muscosus (Gymnopholus) 154

mutica (Aporhina, Chalcocybebus) 59

Mutillarius 143

MYCTEINI 29

Myctides 85

Myocalandra 73

myristicae (Coccotrypes, Thamnurgides) 206

MYRMACICELINAE 15

Myrmacicelus 15

Myrmacyba 15

Myrmecocybus 70

mysolensis (Guineoeuops) 47

mysolensis (Pseudodeporaus) 45 
nagaii (Eupholus) 152

nakazawai (Amasa, Xyleborus) 210

nakazawai (Crossotarsus) 183

namatanai (Exophthalmida) 162

nanodontus (Diapus) 196

Nanomyrmacyba 15

nanulus (Hylesinus) 199

nanus (Diapus) 196

nanus (Hylurdrectonus) 200

nanus (Ithyporus) 172

narinus (Platyacus) 147

nasalis (Ittostira) 162

naso (Oxydema) 96

nasuta (Gynaria) 149

nasutus (Trigonopterus, Idotasia) 124

NAUPACTINI 161

Nauphaeus 127

Neauletes 44

nebulosa (Sphenophorus) 79

nebulosus (Diapus) 196

nebulosus (Hesychobius) 172

nebulosus (Nauphaeus) 128

nechyroides (Dyspeithes) 128

Nechyrus 117

Nedymora 128

negatus (Platypus) 191

neglectus (Trigonopterus, Idotasia) 124

NEMONYCHIDAE 19

nemorosus (Platypus) 191

Neochyromera 132

neohibernicus (Tracheloschizus) 68

Neohigonius 63

Neolaemosaccus 170

Neomelanterius 132

neopartibilis (Platypus) 191

neophyta (Blepiarda) 111

Neosynaptops 48

Neosynaptopsis 48

neotruncatus (Platypus) 190

Neozemioses 56

Neplaxa 171

nepos (Arachnobas, Arachnopus) 88

Nesidiobrentus 56

Nessiara 24

nickerli (Eupholus) 152

niger (Guineobius) 140

nigerrimus (Orychodes, Caenorhychodes) 52

nigraustralis (Cyphagogus) 55

nigriceps (Hypseus) 23

nigricollis (Apionocybus, Rhadinocyba) 69

nigritarsis (Paleticus, Petosiris) 119

nigrocristatus (Platyacus, Hoplotrigonops) 147

nigroflava (Apatenia) 21

nigromarginatus (Protaedus) 27

nigronitens (Ithystenus) 62

nigropictus dissimilis (Mecocerina) 33

nigroplagiatus (Ectemnomerus) 138

nigrorufus (Eusintor) 32

nigrosuturatus (Hypseus) 24 nigrothorax (Ionthocerus, Jonthocerus) 57

niisimai (Sueus, Hyorrhynchus) 199

niligrinus (Hylesinus) 199

Niphades 170

Niphetoscapha 161

Niseida 129

nitella (Coptodryas, Xyleborus) 212

nitens (Aporhina, Chalcocybebus) 59

nitens (Platypus) 191

nitens (Xyleborus) 218

nitens papuana (Aporhina, Chalcocybebus) 59

nitida (Thyestetha) 123

nitida (Tistortiella, Ottistira) 164

nitidfrons (Rhinoscapha) 159

nitidicollis (Hyledius, Olonthogaster) 199

nitidicollis (Hypocryphalus) 203

nitidipennis (Cryphalus) 202

nitidula (Sphenogaster) 148

nitidula (Sphenomorpha, Apocyrtus) 168

nitidulus (Myctides) 85

nitidus (Alcidodes, Alcides) 176

nitidus (Coccotrypes, Dendrurgus) 206

nitidus (Cyrtogenius, Lepicerus) 208

nitidus (Dryocoetiops, Dryocoetes) 208

nitidus (Symbiopholus) 156

nitidus orientalis (Pelicerus) 208

nivatus (Curculio, Balaninus) 130

niveinasus (Macrotrichus) 25

niveofasciatus (Xenocerus) 35

nivosus (Apirocalus) 135

nivosus (Curculio, Balaninus) 130

nobilis (Spathidicerus) 197

nodifer (Aulacophrys) 137

nodifer (Symbiopholus) 156

nodosus (Ptolycus) 121

nodulosus (Brachypezichus) 105

nodusus (Mormosintes) 117

nonfriedi (Miolispa) 66

noonadani (Ithystenus) 61

notabilis (Xola) 110

Notapion 70

NOTAPIONINI 68

notatus (Miocalles) 117

notatus (Nechyrus) 118

notatus (Phaenomerus) 90

nothofagi (Cryphalus) 202

nothofagi (Euops) 49

nothofagi (Gymnopholus) 154

nothofagus (Platypus) 191

Notocryptorhynchus 108

Notocybus 69

Notoplatypus 197

novaeguineae (Kleineella, Amorphocephalus) 53

novaeguineae (Lophocheirus) 127

novaeguineensis (Crossotarsus) 183

novaeguineensis (Platypus) 191

novaguineanus (Xyleborus) 219

novaguineensis (Cossonus) 95

novaguineensis (Miolispa) 66 
novaguineensis pumilla (Miolispa, Cephalobarbus) 67

novaguineensis v. antennata (Miolispa) 67

novata (Metialma) 91

novellus (Alcidodes) 176

novenarius (Higonodes, Higonius) 62

nubilosus (Baiocis) 181

nudicollis (Sphenophorus) 78

nudiusculus (Platypus) 191

nudus (Oribius, Coptorhynchus) 145

obanus (Ozopemon) 209

oberthuri (Vanapa) 179

obesus (Acalles) 115

obesus (Anchithyrus) 112

obesus (Isoleptus, Tylodes) 115

obesus (Tylodes) 115

obliquatus (Cyamotrox, Cyamobolus) 107

obliquecaudatus (Peroplatypus, Platypus) 185

obliquecaudatus (Platypus) 185

obliquesectus (Platypus) 191

obliquetruncatus (Platypus) 191

obliquus (Pantorhytes) 166

obliquus (Platypus) 191

oblongus (Trigonopterus, Idotasia) 124

obnixus (Trigonopterus, Idotasia) 124

obreinorum (Ottistira) 163

obscuripes (Baris) 81

obscurus (Cacephatus) 22

obscurus (Cyamobolus) 106

obscurus (Rhabdoscelus, Calandra) 78

obsidiana (Rhinoscapha) 160

obsoletus (Microporopterus) 116

obtusispina (Aporhina, Chalcocybebus) 59

occultator (Molobrium) 136

occultus (Microtrachelizus) 62

occultus (Phaenomerus) 90

ochra (Mauia) 26

ochraceus (Alcidodes) 176

ochraceus (Gyalostoma) 52

ochraceus (Opisthenoxys) 56

Ochronanus 98

OCHYROMERINA 132

OCLADIINAE 72

Ocoblodes 173

octopustulatus (Pantorhytes) 166

ocularis (Carcinopisthius) 57

oculatus (Enaptomias) 140

oculatus (Menechirus) 181

odewahni (Cyphagogus) 55

odiosa (Coryssoglymma) 113

Odosyllis 128

Oebrius 93

oedothorax (Acalles, Tylodes) 112

Oemethylus 118

okapicus (Phaenomerus) 90

oligodontus (Crossotarsus) 183

olivacea (Apatenia) 21

olivaceus (Apirocalus) 135

olivieri (Arachnobas, Arachnopus) 88 omissus (Coccotrypes) 206

omissus (Platypus) 191

Ommatocybus 70

omnivorus (Platypus, Crossotarsus) 191

Omophorus 177

onca (Daedania, Mecopus) 92

ONYCHOLIPINI 98

oomsis (Diapus) 196

oops (Metaeuops) 48

opacicauda (Platypus) 191

opacicaudus (Platypus) 191

opacideclivis (Platypus) 191

opacifrons (Platypus) 192

opacipennis (Platypus) 192

opacurus (Platypus) 192

opacus (Pantorhytes) 166

opalescens (Rhinoscapha) 160

opatroidea (Chirogonia) 113

operosus (Xyleborus) 218

ophiopsis (Ithystenus) 61

ophthalmica (Semiathe) 122

ophthalmicus (Ionthocerus) 58

opimus (Chaetoptelius, Acrantus) 200

opimus (Platypus) 192

Opisthenoxys 56

oppositus (Lophocheirus, Odosyllis) 127

Opterus 143

opulentus (Allodapinus) 65

opulentus (Schizotrachelus) 65

opulentus (Xyleborus) 218

ordinarius (Poropterus) 120

oreogenus (Diapus) 196

Orfilaia 52

Oribius 144

orientalis (Apirocalus) 135

orientalis (Mimocurus, Mimiophthorus) 201

ornata (Acicnemis) 180

ornatifrons (Platypus) 192

ornatus (Idiopsodes, Idiopsis) 142

Orochlesis 128

Orphanistes 118

Orphanyasmus 108

ORTHOGNATHINAE 72

ORTHOGNATHINI 73

ORTHORHININA 178

Orthorhinus 178

orthorrhina (Solenobaris) 82

Orthotemnus 99

ortyx (Agametis) 91

Orychodes 52

oryzae (Sitophilus, Curculio) 74

Osphilia 91

Osseteris 129

othiodes (Platypus) 192

Othippia 94

OTHIPPIINI 93

Otidognathus 74

Ottistira 163

OTTISTIRINI 162 
ottonis (Mormosintes) 117

ottonis (Parasphenogaster) 146

ottonis (Tyrpetes) 181

ovalis (Mauia) 26

ovatulus (Microporopterus) 116

Oxyconus 42

Oxydema 96

oxygaster (Elytrocheilus, Otiorhynchus) 140

Oxymelus 109

oxystomoides (Heterocybus) 70

Ozopemon 208

OZOTOMERINI 29

Ozotomerus 29

Pachycotes 200

Pachyonyx 104

Pachyrhynchidius 146

PACHYRHYNCHINI 164

Pachytragopus 118

paganus (Celeuthetes) 138

PAIPALESOMINI 178

Paleticus 118

pallens (Acalles) 115

palliatus (Apries) 172

pallidicepes (Apatenia) 21

pallidus (Dinoplatypus, Platypus) 184

pallidus (Pantorhytes) 166

pallidus (Protaedus) 27

pallidus sabroni (Platypus) 184

palmarum (Sphenophorus) 75

pan (Poropteropis) 119

panduriformis (Platypus) 192

Pangomorphus 178

paniaiensis (Ottistira) 163

paniaiensis (Riedelinius) 50

Panigena 94

pantherinus (Mecotropus) 28

Pantiala 119

Pantorhaenas 24

Pantorhytes 164

Pantoxystus 171

papatrae (Xyleborus) 218

papilifer (Gasteroclisus) 169

papillatus (Hypotactus, Platyacus) 141

papillosus (Pantorhytes) 166

papillosus (Poropteropis, Poropterus) 119

papua (Arachnobas, Arachnopus) 88

papua (Archeuops) 45

papuae (Aragomacer) 19

papuae (Cyrtogenius, Pelicerus) 208

papuana (Apolecta) 38

papuana (Ittostira) 163

papuana (Miolispa) 67

papuana (Talanthia) 93

papuana virgata (Apolecta) 38

papuanaus (Episomellus) 150

papuanus (Achoragus) 38

papuanus (Allodapinus) 65

papuanus (Carcinopisthius) 57 papuanus (Coccotrypes, Dendrurgus) 206

papuanus (Coptonus) 101

papuanus (Cosmoderes) 201

papuanus (Cossonus) 95

papuanus (Cryphalomorphus) 205

papuanus (Cryphalus) 202

papuanus (Cyphagogus) 55

papuanus (Cyrtogenius) 208

papuanus (Cyrtogenius, Dryocoetes) 208

papuanus (Diapus) 196

papuanus (Ernoporus, Margadillius) 203

papuanus (Eterozemus) 55

papuanus (Eucryptorhynchus, Cryptorhynchus) 107

papuanus (Euptilius) 203

papuanus (Genyocerus) 197

papuanus (Hellerrhinus, Elytrurus) 141

papuanus (Hyledius, Phloeosinus) 199

papuanus (Hylesinus) 199

papuanus (Isomorphus) 55

papuanus (Lophocheirus) 127

papuanus (Otidognathus) 74

papuanus (Ozopemon) 209

papuanus (Pantorhytes) 166

papuanus (Pelicerus) 208

papuanus (Phloeobius) 30

papuanus (Platypus) 192

papuanus (Pseudotaphroderes) 57

papuanus (Scolytogenes, Cryphalomorphus) 205

papuanus (Scolytoplatypus) 210

papuanus (Spathidicerus) 197

papuanus (Xestothorax) 56

papuanus (Xyleborus) 218

papuanus (Xylechinus) 199

papuanus (Xylocryptus) 205

Papuapion 68

Papuatorhaenas 37

papuensis (Basitropis) 20

papuensis (Cyrtogenius, Eidophelus) 208

papuensis (Eutinophaea) 162

papuensis (Exillis) 30

papuensis (Ionthocerus, Jonthocerus) 58

papuensis (Melanterus) 132

papuensis (Scolytogenes) 205

papuensis subcarinatus (Exillis) 30

papulosus (Platypus) 192

paraconvexa (Schedlia) 215

paraconvexus (Euops) 49

paradoxus (Platypus) 192

paradoxus (Scolytogenes) 205

Paraeuops 49

Paraeuopsis 50

paralleliventrus (Platypus) 192

parangulithorax (Guineoeuops) 47

parapetax (Platypus) 192

Parapisthius 63

Parasphenogaster 146

paraspinosus (Riedelinius) 50

Parasynommatus 79

Paratactus 146 
paratibialis (Metaeuops) 48

paraviridiceps (Neosynaptops) 49

Parendymia 103

parentheticus (Alcidodes, Alcides) 176

Parexophthalmus 162

parilis (Alcides) 176

partibilis (Platypus) 192

partitus (Xyleborus) 218

parvicollis (Mechistocerus) 103

parvidentipes (Baris) 81

parvula (Apolecta, Mecocerus) 38

parvulus (Hucus, Litocerus) 32

parvulus (Hypothenemus) 203

parvus (Cyrtogenius) 208

parvus (Euops) 49

parvus (Ocoblodes, Deretiosus) 173

pascha (Rhynchophorus) 75

pascha v. papuanus (Rhynchophorus) 75

pascoei (Dyspeithes) 128

pascoei (Phocylides) 65

passer (Tropidobasis) 31

patens (Platypus) 192

patronoides (Arachnobas, Arachnopus) 88

patronoides v. bicolor (Arachnopus) 88

patronus (Arachnobas, Arachnopus) 88

patruelis (Homorthorrhinus, Orthorhinus) 179

patruelis (Psilodryophthorus) 72

paucidentatus (Crossotarsus) 183

paucis (Plintheria) 32

pavonius (Perissops) 109

pauperula (Rhinoscapha) 160

pauxillus (Arachnobas, Arachnopus) 88

pectinatus (Crossotarsus) 183

pectinatus (Platypus) 192

pectoralis (Brachynedus, Coptorrhynchus) 137

pectoralis (Parasynommatus) 79

pedalis (Misthosima) 41

pedellus (Platypus) 192

pedestris (Panigena) 94

Pelephicus 109

pellax (Oribius, Coptorrhynchus) 145

pembertoni (Trigonopterus, Idotasia) 124

Pempherulus 93

Penestica 30

penicilliger (Lophothetes, Trigonops) 142

penicilliger $\mathrm{v}$. deslacsensis (Trigonops) 142

penicillus (Eczesaris, Idiopus) 28

penicillus (Hypseus) 24

pennatus (Cyphopus) 138

PENTARTHRINI 99

Pentarthrum 99

pentastictus (Alcidodes, Alcides) 176

Penthoscapha 157

perditor (Alcidodes) 176

perditus (Platypus) 192

peregrinator (Phaenomerus) 90

peregrinator salomonicus (Phaenomerus) 90

peregrinus (Cyrtogenius) 208

perexiguus (Acanthotomicus, Tomicus) 209 perexiguus (Xyleborinus, Xyleborus) 216

perfecta (Rhinoscapha) 160

perforans (Xyleborus, Tomicus) 218

perfossus (Xenocerus) 35

Peribleptus 178

Perichius 119

Peridryocoetes 209

Peripagis 168

Perissops 109

perlongus (Ithystenus) 61

perminimus (Hypocryphalus, Cryphalus) 203

permutata (Ectatocyba) 114

pernanulus (Baiocis, Crossotarsus) 181

Peroplatypus 185

perornatus (Oribius, Coptorrhynchus) 145

perplexus (Idiopsodes, Idiopsis) 142

perplexus (Litocerus) 33

perplexus (Xenocerus) 35

perplexus (Xyleborus) 218

perplexus syndetus (Xenocerus) 35

perpygmaeus (Diapus) 196

Perrhaebius 174

persona (Arachnobas, Arachnopus) 88

persona (Berosicus) 105

persphenos (Xyleborus) 216

perspicax (Gymnopholus) 154

pertenuis (Phaenomerus) 90

pertinax (Ficicis, Hylesinus) 198

pertinax (Pseudoporopterus, Poropterus) 121

perturbans (Apirocalus) 135

pertusicollis (Ectemnomerus) 138

perversa (Rhinoscapha) 160

perversus (Orthorhinus) 178

pervicax (Poropterus) 120

petalinus (Platypus) 192

petaloideus (Platypus) 192

petax (Mechistocerus, Isotocerus) 103

petax (Platypus) 192

Peteinus 147

petulans (Neolaemosaccus, Lmosaccus) 170

PHAENOMERINA 89

Phaenomerus 89

phaeura (Apatenia) 21

phalerata (Diatassa) 101

phaleratus (Arachnobas, Arachnopus) 88

phaleratus v. frater (Arachnopus) 88

Phaulimia 24

philippinensis (Ficicis, Hylesinus) 198

philippinicus (Ceocephalus, Diurus) 59

Phloeobius 30

PHLOEOPHAGINA 100

Phloeophagosoma 96

Phloeops 34

PHLOEOSININA 199

Phloeosinopsioides 199

Phloeosinus 199

PHLOEOTRIBINA 200

Phocylides 64

Phraotes 147 
Phylaitis 93

PIAZOMIINA 168

PIAZURINI 94

piazuroides (Bobandersonia, Eurhopala) 111

piceus (Crossotarsus) 183

piceus (Euwallacea, Xyleborus) 214

piceus (Metaeuops) 48

picicauda (Webbia) 215

picipes (Euops) 47

picta (Diatassa) 101

picticornis (Macroauletes, Auletes) 44

pictipennis (Aulacobaridia, Aulacobaris) 80

picturata (Misthosima) 41

pictus (Diathetes) 77

pileatulus (Xyleborus) 218

pilicornis (Habrissus) 23

pilidens (Platypus) 192

pilifer (Hypocryphalus) 203

pilifer (Xyleborus) 218

pilipes (Parendymia) 103

piliventris (Ipsichora) 84

pilosulus (Cryphalus) 202

pilosus (Cyphagogus) 55

pilosus (Himatium, Pholidonotus) 100

pilosus (Pantorhytes) 166

piniarius (Hylurdrectonus) 200

Pioenia 30

Pioenidia 31

piorae (Symbiopholus) 156

PISSODINA 179

PISSODINI 178

pithekis (Mauia) 26

plagiata (Rhinoscapha) 160

plagiatus (Oribius, Psomeles) 145

plagifer (Apatenia) 21

planata (Nessiara) 34

planatus (Arachnobas, Arachnopus) 88

planatus (Araeocorynus, Doticus) 40

planatus (Doticus) 41

planidorsis (Eudyasmus) 108

planipennis (Prophthalmus) 52

planirostris (Exophthalmida) 162

planitarsus (Callipareius, Diastrophus) 54

planiventris (Meroleptus) 116

planiuscula (Ottistira) 163

planus (Diathetes) 77

Platyacus 147

Platycybus 70

platynota (Emydica) 107

platypennis (Phloeops, Stenocerus) 34

platypoda (Doeothena) 29

PLATYPODINA 181

PLATYPODINAE 181

PLATYPODINI 181

platypterus (Hellerrhinus) 141

Platypus 185

platyrostris (Euops) 49

PLATYSTOMINI 29

Platystomos 31

\section{Platytenes 109}

platyurus (Xyleborus) 218

plebejus (Tyrtaeosus) 125

Plesiobasis 20

plicata (Rhinoscapha) 160

Plintheria 32

plumatus (Platypus) 192

pluto (Curculio, Balaninus) 130

plutonius (Curculio, Balaninus) 130

plutus (Pantorhytes, Pachyrrhynchus) 166

podagrica (Othippia) 94

poecila (Apatenia) 21

pogonocera (Elytracantha, Belopherus) 51

politus (Degis) 83

politus (Trigonopterus, Idotasia) 124

polynodus (Pantorhytes) 166

POLYTINI 74

Polytus 74

Polyzelus 129

ponamae (Platypus) 188

popondettae (Coptodryas, Xyleborus) 212

porcatus (Crossotarsus) 183

porcellinus (Platypus) 192

porcellus (Platypus) 192

Poropteropis 119

Poropterus 120

porosterna (Baris) 81

porosulus (Platypus) 193

porosus (Aclees) 171

porosus (Colpomus) 138

porosus (Platypus) 193

porulosus (Euops) 50

postica (Basitropis) 20

posticus (Brachynedus, Coptorrhynchus) 137

postoculatus (Orthorhinus) 179

potens (Xyleborus) 216

Poteriophorus 78

praealtus (Platypus) 193

praecanus (Cyphicerus) 149

praecellens (Platypus) 193

praecipuus (Cyphagogus) 55

praecox (Eudyasmus) 108

praecox (Symbiopholus) 156

praeculta (Acicnemis) 180

praepositus (Platypus) 193

praeteritus (Platypus) 193

praetermissus (Platypus) 193

praevius (Alcidodes) 176

praevius (Arachnobas, Arachnopus) 88

praeusta (Schedlia, Xyleborus) 215

praeustus (Alcidodes, Alcides) 176

praeustus (Conarthrus) 99

prasinus (Eupholus) 152

pratti (Angianus) 179

preparvus (Cyrtogenius) 208

pretiosus (Alcidodes) 176

pretiosus (Henorychodes) 52

primitiva (Rhinoscapha) 161

procerrimus (Euwallacea, Xyleborus) 214 
procerus (Mucronianus) 34

prociduus (Crossotarsus) 183

prodigus (Gygaeus) 115

Prodioctes 78

prodromus (Rhynchaenophaenus) 131

PROECINI 99

profluens (Alcides) 175

proicollis (Platypus) 193

prolixus (Alcidodes) 176

Promicrocossonus 99

promissa (Schizoeupsalis, Eupsalis) 53

promissa (Sphenophorus) 78

Prophthalmus 52

propinqua (Asytesta) 105

Protaedus 26

protii (Xyleborus) 218

Protoplatypus 100

proximaus (Trigonopterus, Idotasia) 124

proximus (Elytrocheilus) 140

proximus (Pantorhytes) 166

Pseniclea 94

\section{PSEPHOLACINI 128}

Pseudacalles 120

Pseudaclees 172

Pseudaphioda 97

pseudoabidi (Pantorhytes) 166

PSEUDOBALANININA 131

pseudocarbonaris (Pantorhytes) 166

PSEUDOCEOCEPHALINI 63

Pseudocholus 84

Pseudocossonus 97

pseudocupulatus (Dinoplatypus, Platypus) 184

pseudocupulatus artecavus (Platypus) 185

Pseudodeporaus 44

Pseudohigonius 63

pseudomonstruosus (Riedelinius) 50

Pseudoperissops 109

Pseudophocylides 62

Pseudoporopterus 120

pseudoporus (Platypus) 193

pseudoselysi (Platypus) 193

pseudosindorae (Platypus) 193

Pseudostenoscelis 98

Pseudosynaptos 48

Psilodryophthorus 72

Psilotrogus 97

pterydophytae (Coccotrypes, Poecilips) 206

pterygorhinus (Ectocemus) 51

Ptilopodius 204

Ptolycus 121

Ptyctonotus 82

puellaris (Pseniclea) 94

puer (Genyocerus, Diapus) 197

puer (Leptoxyleborus, Xyleoborus) 214

pulchella (Ganae) 73

pulchella (Ipsichora) 84

pulchellus (Diathetes) 77

pulchellus (Megacerus) 51

pulchellus (Trigonopterus, Idotasia) 124 pulcher (Gymnopholus) 154

pulchra (Sphenomorpha) 168

pulicaris (Trigonopterus, Idotasia) 125

pullus (Trigonopterus, Idotasia) 125

pulverulentus (Isoleptus, Tylodes) 115

pulvinatus (Platypus) 193

pumilus (Ithystenus, Brenthus) 61

pumilus (Phloeosinopsioides) 199

pumilus (Xyleborus) 218

punctata (Phaulimia, Stenocerus) 24

punctaticeps (Neosynaptops) 49

punctatocarinulatus (Notocryptorhynchus) 108

punctatopilosum (Cyclorhipidion, Xyleborus) 213

punctatus (Atenophthalmus, Arrhenodes) 63

punctatus (Cryphalus) 202

puncticollis (Brenthus) 67

puncticollis (Habrissus) 23

puncticollis (Nechyrus) 118

puncticollis (Oribius, Coptorrhynchus) 145

puncticollis (Semiathe) 122

punctifrons (Ithystenus) 61

punctiger (Auletobius) 44

punctigera (Calandra) 73

punctimedia (Baris) 81

punctirostris (Stereoborus) 97

punctirostris v. obliteratus (Stereoborus) 98

punctivaria (Baris) 81

pupa (Dolichocera, Meganthribus) 28

pupa papuanus (Dolichocera, Meganthribus) 28

purpuratus (Otidognathus) 74

pusillimus (Cryphalus) 202

pusillimus (Diapus) 196

pusillus (Alcidodes, Alcides) 176

pusillus (Scolytomimus, Neoxyloctonus) 220

pusillus (Trigonopterus, Idotasia) 125

pustulifer (Hypseus) 24

pustulosus (Deretiosus) 173

putputensis (Xyleborus) 218

Pycnochirus 181

pygmaea (Miolispa) 67

pygmaeus (Euops) 50

pygmaeus (Ochronanus) 98

pyritosa (Metanthia) 84

quadraticollis (Arachnobas, Arachnopus) 88

quadricinctus (Webbia) 215

quadricornutus (Platypus) 193

quadridens (Scolytogenes, Hypothenemus) 205

quadridens (Scolytomimus) 220

quadrifasciculata (Acicnemis) 180

quadriguttatus (Curculio, Balaninus) 131

quadrimaculatus (Eupholus) 152

quadrimaculatus v. jugatus (Eupholus) 152

quadrinodosus (Guiomatus) 94

quadrinotatus (Platypus) 193

quadripunctatus (Anchithyrus) 112

quadripustulatus (Pachyrhynchidius) 146

quadripustulatus (Pantorhytes, Pachyrrhynchus) 166

quadrispinis (Platypus) 193 
quadrispinosulus (Xyleborus) 218

quadrituberculata (Dinomelaena, Stenocerus) 22

quadruplex (Oribius, Coptorhynchus) 145

quaesitus (Trachelizus) 67

quatei (Pantorhytes) 166

quattuordecimmaculatus (Oribius, Coptorhynchus) 145

queenslandi (Coccotrypes, Poecilips) 206

quercivorus (Platypus) 193

querulus (Pseudocholus) 85

quinarius (Idiopsodes, Coptorrhynchus) 142

quinitaenia (Eupholus) 152

quinquespinatus (Diapus) 196

raffrayi (Eupholus) 152

ramosissimus (Platypus) 193

ramulosus (Platypus) 193

raniceps (Apatenia) 22

raniceps rectangula (Apatenia) 22

raniceps rufovariegata (Apatenia) 22

rapandus (Xyleborus) 218

raphatus (Uncifer) 37

rara (Basitropis) 20

rarus (Pantorhytes) 166

rasilis (Crossotarsus) 183

rasilis (Platypus) 193

rata (Asytesta) 105

ratcliffei (Metaeuops) 48

ravilla (Sibiniella) 132

rebeccae (Symbiopholus) 156

recidens (Coptodryas, Xyleborus) 212

rectangulus (Exillis) 30

reductus (Alcidodes, Alcides) 177

reductus (Tetracoptus) 98

reflexus (Hypocryphalus) 203

reflexus (Orthotemnus) 100

refractus (Platypus) 193

regalis (Gymnopholus) 154

regius (Hylesinus, Leperisinus) 199

regularis (Coccotrypes, Poecilips) 206

regularis (Metanthia) 84

relicta (Basitropis) 20

relictus (Neauletes) 44

remissus (Brachynedus) 137

rennii (Gymnopholus) 154

residuus (Degis) 83

restrictus (Nechyrus) 118

reticulatus (Guineoeuops) 47

reticulatus (Hormocerus, Brentus) 64

reticulatus (Symbiopholus) 156

revocabile (Cyclorhipidion, Xyleborus) 213

Rhabdoscelus 78

RHADINOCYBINAE 68

Rhadinomerus 103

RHAMPHINA 131

RHAMPHINI 131

Rhaphitrophis 36

Rhinaria 133

rhinoceros (Elytrocheilus) 140

RHINORHYNCHINAE 19

\section{RHINORHYNCHINI 15 \\ Rhinoscapha 157 \\ Rhinotia 43}

rhizophorae (Coccotrypes, Spermatoplex) 206

rhombicus (Litocerus) 33

rhomboidea (Ctylindra) 80

Rhynchaenophaenus 131

RHYNCHITIDAE 43

RHYNCHITINAE 43

RHYNCHITINI 45

RHYNCHOPHORINAE 73

RHYNCHOPHORINI 74

Rhynchophorus 75

RHYNCOLINA 100

RHYNCOLINI 100

Rhyncolus 100

ribbei (Rhinoscapha) 160

richteri (Alcidodes, Alcides) 177

richteri (Aporhina, Chalcocybebus) 59

richteri (Rhinoscapha) 160

riedeli (Apirocalus) 135

riedeli (Hypseus) 24

riedeli (Notapion) 70

riedeli (Papuatorhaenas) 37, 38

riedeli (Phaenomerus) 90

Riedelinius 50

rigouti (Eupholus) 152

rigua (Misthosima) 41

ritsemae (Hypolixus, Lixus) 169

ritsemae v. coriaceus (Hypolixus) 169

robusta (Ottistira) 163

robusticornis (Protaedus) 27

robustulus (Coccotrypes, Poecilips) 206

robustus (Diapus) 196

robustus (Poecilips) 206

robustus (Stereoborus) 98

roelofsi (Alcides) 177

roelofsi (Sphenomorpha, Apocyrtus) 168

roppeli (Misthosima) 41

rosseli (Coptodryas, Xyleborus) 212

rosseli (Mimocurus, Taphroterus) 201

rostralis (Gymnopholus) 154

rostratus (Alcidodes, Alcides) 177

rostratus (Calyptulus) 64

rothschildi (Enaptomias) 140

rothschildi (Rhinoscapha) 160

rothschildi ab. rufithorax (Rhinoscapha) 160

rotunda (Mauia) 26

rotundatus (Cnestus) 211

rotundatus (Hybicus) 115

rotundicolle (Amorphorhynchus) 97

rotundicollis (Xyleborus) 219

rotundipennis (Arachnopus) 104

rubi (Gymnopholus) 154

rubigineus (Isoleptus, Anaballus) 115

rubricollis (Pantoxystus, Cleogonus) 171

rubripennis (Pantoxystus, Cleogonus) 171

rubrocinctus (Rhynchophorus) 75

rubroverucatus (Pantorhytes, Pachyrrhynchus) 166 
rufescens (Otidognathus) 75

rufescens (Pantorhytes) 167

rufficollis (Diathetes) 77

rufficollis v. buergersi (Diathetes) 77

rufficollis v. eremothocus (Diathetes) 77

ruficauda (Ectocemus) 51

ruficollis (Elytrocheilus) 140

ruficollis (Gloedema) 96

ruficollis (Heterophasis) 95

ruficornis (Metaeuops) 48

rufidorsum (Trigonospartus) 149

rufipennis (Semiathe) 122

rufipennis (Trigonopterus, Idotasia) 125

rufipes (Elytrocheilus) 140

rufipes (Mucronianus rufipes) 34

rufipes (Sphenomorpha) 168

rufomaculatus (Eterozemus) 55

rugaticollis (Cyphagogus) 55

rugiceps (Melanopsacus) 42

rugicollis (Symbiopholus) 156

rugiventrus (Araecerus) 39

rugosisculpturatus (Psudohigonius) 63

rugosulus (Sitophilus, Calandra) 74

rugosum (Pentarthrum) 99

rugosus (Arachnobas, Arachnopus) 88

rugosus (Orthorhinus) 179

rugosus (Pantorhytes) 167

rugulosa (Tropidobasis) 31

runcinatus (Platypus) 193

ruralis (Hypotactus, Platyacus) 141

russulus (Euwallacea, Xyleborus) 214

rusticus (Syrichius) 110

rutilicollis (Pseudocholus) 85

sabulosus (Ithystenus) 61

sagax (Perissops) 109

Saginesis 169

salawattensis (Eupholus) 153

salawattii (Hypseus) 24

salawattii robustior (Hypseus) 24

Salcus 121

salomonensis (Miolispa) 67

salomonis (Plintheria) 32

salomonis (Trigonops) 149

salubris (Trigonopterus, Idotasia) 125

salviniae (Cyrtobagous) 16

samuelsoni (Pantorhytes) 167

samuelsoni (Penestica) 30

sanguinosus (Diathetes) 77

sannio (Acicnemis, Berethia) 180

sannio (Arachnobas, Arachnopus) 88

sannio (Diathetes) 77

sarasini (Cyphagogus) 55

satanus (Nechyrus) 118

saugrenus (Eupholus) 152

saxeseni (Xyleborinus, Bostrichus) 216

scaber (Molobrium) 136

scabiosus (Arachnobas, Arachnopus) 88

scabratus (Ancylotropis, Macrotrichus) 25 scabricollis (Cyrtogenius) 208

scabricollis (Xyleborus, Ozopemon) 219

scabripennis (Arixyleborus, Xyleboricus) 211

scabrosus (Anaballus) 122

scalaris (Rhinoscapha) 160

scalptor (Platypus) 193

scapha (Ambonodiras) 112

scaphioides (Trigonopterus, Idotasia) 125

scelesta (Dinomelaena, Nessiara) 22

scelesta angulicollis (Dinomelaena, Apatenia) 22

scelesta innotata (Dinomelaena) 23

schedli (Promicrocossonus) 99

Schedlia 215

schefflerae (Symbiopholus) 156

Schismataion 71

Schizoeupsalis 53

Schizotrachelus 65

Schlaginhaufenia 78

schmeltzi (Rhinoscapha) 160

schmeltzi hedigeri (Rhinoscapha) 160

schmeltzi intermedia (Rhinoscapha) 160

schmeltzi papei (Rhinoscapha) 160

schmeltzii (Cacoschizus, Schizotrahelus) 64

schneideri (Eupholus) 152

schneideri (Gasterosaga) 108

schneideri (Pantoxystus, Pseudeurhinus) 171

schoeherrii (Eupholus) 152

schoeherrii mimikanus (Eupholus) 153

schoeherrii semicoeruleus (Eupholus) 153

schoeherrii v. arfaki (Eupholus) 153

schoenherri (Megadiathetes, Sphenophorus) 77

schoenherri (Megax, Eugigas) 29

schuurmannsiae (Symbiopholus) 156

scitus (Alcidodes) 177

sciureus (Poropterus) 120

Sclerodontus 82

SCOLYTIDINAE 197

SCOLYTINI 201

Scolytogenes 204

Scolytomimus 219

SCOLYTOPLATYPODINA 209

Scolytoplatypus 209

scorpius (Crossotarsus) 183

scripta (Dinomelaena) 23

scrobicularis (Platypus) 193

sculptipennis (Cacotrachelus) 67

sculpturatum (Apion, Conapion) 71

sculpturatum (Papuapion, Conapion) 68

sculpturatus (Guineauletes) 44

scutellaris (Cyamobolus) 106

scutellaris (Metanthia) 84

scutellaris (Osseteris) 129

scutellatus (Nechyrus) 118

seclusus (Trigonopterus, Idotasia) 125

sectator (Arachnobas, Arachnopus) 88

securus (Cryphalus, Ericryphalus) 202

sedaceki (Pantorhytes) 167

sedlaceki (Apirocalus) 135

sedlaceki (Basitropis) 20 
sedlaceki (Cyrtogenius, Ozodendron) 208

sedlaceki (Dinomelaena) 23

sedlaceki (Euops) 50

sedlaceki (Gymnopholus) 154

sedlaceki laminifer (Apirocalus) 136

seftoni (Dryophthoroides, Pembertonia) 79

segnis (Alcidodes, Alcides) 177

sejuncta (Asytesta) 105

sejunctus (Trigonopterus, Idotasia) 125

Seleuca 174

sellaeformis (Platypus) 193

sellata (Rhinoscapha) 160

sellatus (Phraotes) 147

sellatus (Trigonopterus, Idotasia) 125

selysi (Platypus) 194

Semiathe 121

semicarinatus (Ambrosiodmus, Xyleborus) 210

semicinctus (Crossotarsus) 183

semiochraceus (Alcidodes, Alcides) 177

semiopaca (Camia) 113

semiopacus (Platypus) 194

semiotus (Parexophthalmus) 162

semipunctata (Baris) 81

semiruber (Arachnobas, Arachnopus) 89

semirufa (Semiathe) 122

semistriatus (Microtrachelizus) 63

semisulcatus (Peroplatypus, Platypus) 185

senex (Symbiopholus) 156

sentus (Doticus) 41

sentus subornatus (Doticus) 41

sepaloideus (Platypus) 194

septemmaculatus (Alcidodes, Alcides) 177

seramis griseolus (Protaedus) 27

seriatogranosus (Paratactus) 146

seriatus (Gymnopholus) 155

serrirostris (Mecopus) 92

servilis (Oribius, Coptorhynchus) 145

servulus (Syrichius) 110

setaceus (Platypus) 194

setiger (Choerorhamphus) 138

setiger (Ectemnomerus, Celeuthetes) 139

setiger (Mechistocerus, Isotocerus) 103

setipes (Asytesta) 105

setosus (Allaeometrus) 54

setosus (Gymnopholus) 155

setosus (Microporopterus) 116

setosus (Scolytoplatypus) 210

sexcarinatus (Exillis) 30

sexmaculatus (Lophothetes, Trigonops) 142

sexnotatus (Mesoderes) 56

sexpustulatus (Pantorhytes) 167

sexsulcatus (Cerobates) 57

sexvittatus (Alcides) 177

sharpi (Glyptoporopterus, Poropterus) 115

shoreanus (Platypus, Crossotarsus) 194

shoreanus tersus (Platypus) 194

shungolus (Symbiopholus) 156

Sibiniella 132

signata (Asytesta) 105 signatellus (Protaedus) 27

signaticollis (Listrodiathes, Diathetes) 77

signatifrons (Misthosima) 41

signatus (Protaedus) 27

silenus (Poropteropis) 119

silex (Araecerus) 39

silvanus (Cyphagogus) 55

silvaticus (Brachycybus) 69

similis (Coccotrypes, Dendrurgus) 206

similis (Ithystenus) 61

similis (Nechyrus) 118

similis (Neosynaptops) 49

similis (Trigonopterus, Idotasia) 125

similis (Webbia, Xyleboricus) 215

similis (Xyleborus) 219

simillimus (Curculio, Balaninus) 131

simius (Arachnobas, Arachnopus) 89

simplex (Aporonotus) 113

simplex (Eudyasmus) 108

simplex (Ommatocybus) 70

simplex (Rhinoscapha) 160

simplicipennis (Baris) 82

simulans (Guineoeuops) 47

simulans (Machaerostylus, Pseudosphaeropterus) 143

simulans (Miolispa) 67

simulator (Blepiarda) 111

sinakiensis (Tapinocybus) 71

singularis (Anathymus) 75

singularis (Cyrtobagous) 16

singularis (Euops) 50

singularis (Platypus) 194

SINTORINI 31

sinuaticollis (Heterophaseolus, Phloeophagosoma) 96

Sipalinus 73

siporanus (Crossotarsus) 183

sirambeanus (Amasa, Xyleborus) 210

Sitophilus 74

slomonis (Pantorhytes) 164

sobrinus (Perissops) 109

socius (Protaedus) 27

socius (Pseudoporopterus, Poropterus) 121

socius obtusus (Protaedus) 27

sofia (Eupholus) 153

solarii (Carponinus) 131

solarii (Cyamotrox) 107

Solenobaris 82

solicitatulus (Platypus) 194

solidus (Baryrhynchus) 51

solidus (Platypus) 194

solidus (Poropteropis, Poropterus) 119

solidus (Treptoplatypus, Platypus) 195

solidus (Trigonopterus, Idotasia) 125

solidus hirtus (Platypus) 189

solidus prodigiosus (Poropteropis) 119

solitarius (Aulacocnemus) 113

solus (Pseudoporopterus) 121

sordicauda (Leptoxyleborus, Phloeotrogus) 214

sordida (Rhinoscapha) 160

sordidus (Cosmopolites, Calandra) 76 
sordidus (Mechistocerus) 103

sororia (Acicnemis) 180

sororia (Baris) 82

sororia (Miolispa) 67

Sparganobasis 78

sparserugosus (Coccotrypes, Poecilips) 206

sparsutus (Achoragus) 38

Spathidicerus 197

spatula (Gloedema) 95, 96

spatula v. bispatulata (Gloedema) 96

spatula v. dimidiata (Gloedema) 96

spatula $\mathrm{v}$. penicillata (Gloedema) 96

specillifer (Apirocalus) 136

spectabilis (Sphenomorpha) 168

spectrum (Crossotarsus) 183

speculator (Alcidodes) 177

speculatus (Oribius, Coptorhynchus) 146

speculifrons (Lissopis) 96

speracerus (Xenocerus) 35

speracerus mancus (Xenocerus) 36

speracerus rosseliensis (Xenocerus) 36

speracerus sudestensis (Xenocerus) 36

SPHADASMINI 94

Sphaeropterus 148

Sphenogaster 148

Sphenomorpha 167

\section{SPHENOPHORINI 75}

Sphenophorus 79

spilonota (Acicnemis) 180

spinachius (Xyleborus) 215

spinicornis (Xyleborus) 219

spinifer (Diapus) 196

spinipennis (Acicnemis) 180

spinipennis (Coccotrypes, Poecilips) 206

spinipennis (Crossotarsus) 183

spinipennis (Ectocemus) 52

spiniventris (Platypus) 194

spinosa (Aporhina) 58

spinosus (Eccoptopterus, Scolytus) 213

spinosus (Hypothenemus, Lepiceroides) 203

spinosus (Ithystenus) 61

spinosus (Riedelinius) 50

spissicornis (Eubactrus) 64

splendens (Cyphagogus) 55

splendens (Scolytogenes, Cryphalomorphus) 205

splendida (Miolispa) 67

splendidum (Titanapion, Rhadinocyba) 71

splendidus (Eurhinus) 58

splendidus (Gymnopholus) 155

squalens (Mauia) 26

squalidus (Dystropicus) 102

squalidus (Meroleptus) 116

squamatilis (Webbia, Xyleborus) 215

squamifer (Coccotrypes, Poecilips) 206

squamipennis (Deretiosus) 173

squamipennis (Peridryocoetes) 209

squamistriatus (Pantorhytes) 167

squamosus (Scolytogenes, Lepicerinus) 205

squamulatus (Crossotarsus) 183 staintoni (Rhinoscapha) 160

stanleyanus (Pantorhytes, Pachyrrhynchus) 167

staudingeri (Rhinoscapha) 160

staudingeri $\mathrm{v}$. sanguinicollis (Rhinoscapha) 160

steinwehri (Pachytragopus) 118

Stelechodes 130

stellata (Perissops) 109

stellifer (Apirocalus) 136

STENOCERINI 32

Stenotrupis 97

stephana (Mauia) 26

stephanus (Rhaphitrophis) 37

stephanus gazellae (Rhaphitrophis) 37

Stereoborus 97

STEREODERMINI 57

Stereodermus 58

Stereoderus 98

Stereonotus 98

stevensi (Eucorynus) 25

stibicki (Molobrium) 136

Stictauletes 43

stictoptera (Baris) 82

stictus (Microthetes) 143

stigmaticollis (Sitophilus) 73

stigmaticus (Pelephicus) 109

stigmatus (Arachnobas, Arachnopus) 89

stipinus (Oxyconus) 42

stiracromia (Ottistira) 163

stolifera (Rhinoscapha) 160

STOREINI 131

stramineopictus (Carponinus, Balaninus) 131

stramineoplagiatus (Curculio, Balaninus) 131

strandi (Miolispa) 67

strangulatus (Trochorhopalus, Sphenophorus) 79

Stratiorrhina 53

strenuous (Diathetes) 77

strenuus (Platypus) 194

striatodeclivis (Platypus) 194

striatopunctata (Rhinoscapha, Geonemus) 161

striatulus (Cryphalus) 202

striatulus (Xyleborus) 219

striatus (Colpomus) 138

striatus (Litocerus) 33

striatus inflexus (Litocerus) 33

stridulatoria (Rhinoscapha) 161

striga (Arachnobas, Arachnopus) 89

striga (Eczesaris, Idiopus) 28

striga denutatus (Arachnobas, Arachnopus) 89

strigifer (Hypseus) 24

strigifrons (Apirocalus) 136

STROMBOSCERINAE 79

Strotus 148

sturnus (Alcidodes) 177

stypholoides (Miotus) 108

swartensis (Metaeuops) 48

swartus (Pantorhytes) 167

suaui (Coccotrypes, Poecilips) 206

suaui (Xyleborus) 219

subacuminatus (Coccotrypes, Poecilips) 206 
subagnatum (Cyclorhipidion) 213

subagnatum (Xyleborus) 213

subalatus (Platyacus, Colposternum) 147

Subaphanocorynus 99

subaridus (Deretiosus) 173

subbicristatus (Cyamobolus, Cyambolus) 106

subcarinatus (Papuatorhaenas) 38

subcoeruleatus (Elytrocheilus) 140

subconvexus (Mucronianus, Litocerus) 34

subcostatus (Apirocalus) 136

subcostatus (Arachnobas, Arachnopus) 89

subcostatus (Pantorhytes) 167

subcruciata (Sparganobasis) 78

subcuratus (Platypus) 194

subcylindricus (Oribius, Coptorrhynchus) 146

subdentatulus (Xyleborus) 219

subfasciatus (Arachnobas, Arachnopus) 89

subgranosus (Cyrtogenius, Eidophelus) 208

subgranosus affinis (Eidophelus) 208

subhumeralis (Rhinoscapha) 161

subligatus (Oribius, Coptorrhynchus) 146

submarginatus (Xyleborus) 219

submetallica (Mimidotasia) 117

subnacreus (Gymnopholus) 155

subnodosus (Ptolycus) 121

subopacus (Crossotarsus) 184

subparallelus (Exillis) 30

subpellucidus (Crossotarsus) 184

subpraeteritus (Platypus) 194

subpronus (Platypus) 194

subreflexus (Ochronanus) 98

subrubra (Misthosima) 42

subsellatus (Cyamobolus) 106

subsellatus humeralis (Cyamobolus, Cyambolus) 107

subsignata (Misthosima) 42

subsignata striolata (Misthosima) 42

subsimilis (Arixyleborus) 211

substriatus (Behrensiellus) 137

subsulcatus (Cyrtogenius, Eidophelus) 208

subtilis (Telaugia) 122

subtuberculatus (Cryphalus) 202

subvittigera (Apatenia) 22

subvittigera suturata (Apatenia) 22

subvulgaris (Coccotrypes, Poecilips) 206

Sueus 199

suhandai (Eupholus) 153

sulcaticeps (Platypus) 194

sulcatus (Cyrtogenius, Eidophelus) 208

sulcicollis (Ankleineella, Amorphocephalus) 53

sulcicollis (Eupholus) 153

sulcicollis (Miolispa) 67

sulcicollis (Ottistira) 163

sulcicollis (Pseudoporopterus) 121

sulcifrons (Amalthus) 110

sulcinodis (Ficicis, Hylesinus) 198

sulcinodis (Platypus) 194

sulcinoides (Cyclorhipidion, Xyleborus) 213

sumatrana (Schedlia, Xyleborus) 215

superbulus (Coptoborus, Xyleborus) 212 superbus (Coptoborus, Xyleborus) 212

superbus (Eurhynchus) 58

superbus (Xyleborus) 212

superciliaris (Camia) 113

superciliaris (Dexagia) 133

suppuratus (Apirocalus) 136

sursumcauda (Hellerrhinus) 141

sus (Acoptorrhynchus, Coptorhynchus) 133

sus (Arixyleborus, Xyleboricus) 211

sus (Ectemnomerus) 139

suspensus (Tracheloschizus, Schizotrachelus) 68

suspiciosus (Dyspeithes) 128

suturalis (Australoeuops) 46

suturalis (Gymnopholus, Aroaphila) 155

suturalis (Metetra) 91

suturalis (Sphenomorpha) 168

suturalis (Tyrtaeosus) 126

suturalis (Xenocerus) 36

suturalis angularis (Gymnopholus) 155

suturalis tombarus (Xenocerus) 36

Sybulus 15

Syggenithystenus 62

Symbiopholus 155

symbioticus (Symbiopholus) 156

symbolum (Apion) 71

Sympaector 29

Synommatus 79

Syrichius 110

szentivanyi (Ambrosiodmus) 210

szentivanyi (Gymnopholus) 155

szentivanyi (Pantorhytes) 167

tabaci (Microtrachelizus) 63

TADIINAE 80

TADIINI 80

Tadius 80

takeharai (Platypus) 194

takeharai (Xyleborus) 219

Talanthia 93

talumalai (Euwallacea, Xyleborus) 214

Tamphilus 122

tangens (Oxyconus) 42

TANYMECINI 168

Taphrodasus 215

Tapinocybus 71

targionii (Microtrachelizus) 63

taxicornis (Platypus) 194

tectus (Subaphanocorynus) 99

tectus (Xyleborus) 210

tegminalis (Notocybus) 69

Telaugia 122

telefominarius (Pantorhytes) 167

telefominensis (Ottistira) 163

Telephae 93

temporalis (Microtrachelizus) 63

tenebricosus (Apirocalus) 136

teninabani (Xyleborus) 219

tenuicornis (Baris) 82

tenuicostatus (Atenophthalmus) 63 
tenuiflagellaris (Metaeuops) 48

tenuipes (Baris) 82

tenuipes (Mechistocerus, Isotocerus) 103

tenuipes (Mecopus) 92

tenuisculptus (Platypus) 194

tenuisignatus (Arachnobas, Arachnopus) 89

tenuistratus (Curculio, Balaninus) 131

tenuistria (Alcidodes, Alcides) 177

tereticollis (Amasa, Xyleborus) 210

teretirostris (Phaenomerus) 90

terminaliae (Cryphalus) 202

terminatus (Crossotarsus) 184

terminatus sedulus (Crossotarsus) 184

ternus (Platypus) 194

terrenus (Moluccobius, Coptorrhynchus) 143

terrestris (Molobrium) 136

terrestris dissidens (Molobrium) 136

terrosus (Alcidodes) 177

tersus (Curculio, Balaninus) 131

tessellatus (Eutyrhinus, Euthyrhinus) 126

tessellatus (Oribius, Isomerinthus) 146

TESSEROCERINA 197

TESSEROCERINI 196

testacea (Acicnemis) 180

testacea (Dinomelaena) 23

testacea (Miolispa) 67

testacea (Orfilaia) 52

testaceus (Euopsidius) 46

tetahoense (Schismataion) 71

tetanicus (Alcidodes, Alcides) 177

Tetracoptus 98

tetragramma (Diomia) 172

tetricus (Cryphalus, Ericryphalus) 202

thomsoni (Eupholus) 152

thomsoni (Peteinus, Apirocalus) 147

thomsoni (Rhinoscapha) 161

thomsoni (Spathidicerus) 197

Thyestetha 122

tibialis (Metaeuops) 48

tibialis (Perrhaebius, Deretiosus) 174

tibilalis (Ipsichora) 84

tibioclaratus (Mucronianus) 34

timidus (Xyleborus) 219

Tistortia 164

Tistortiella 164

Titanapion 71

TOMICINA 200

Tomicoproctus 15

TONESIINA 85

toralis (Crossotarsus) 184

torricellianus (Pantorhytes) 167

torricellianus (Poropteropis) 120

torricelliensis (Metaeuops) 48

torrida (Sphenophorus) 79

torulosus (Platypus) 194

torvus (Phraotes) 147

toxopei (Gymnopholus) 155

TRACHELIZINAE 59

TRACHELIZINI 65
Trachelizus 67

Tracheloschizus 67

trachypterus (Ptolycus) 121

Tragopus 123

transversa (Apolecta, Macrocephalus) 38

transversa (Nessiara) 24

transversus (Dactylipalpus) 198

transversus (Platypus) 194

Treptoplatypus 195

triangularis (Cnestus, Xyleborus) 211

triangularis (Hypocryphalus) 203

Trichacalles 112

trichocnemis (Baris) 82

Trichoxymus 123

tricolor (Arachnobas, Arachnopus) 89

tricolor (Gymnopholus) 155

tricolor (Rhabdoscelus) 78

tricolor (Rhaphitrophis) 37

tricolor (Rhinoscapha) 161

tricuspis (Dryophthorus) 72

tridentatus (Prophthalmus, Brentus) 52

trigeminatus (Metasynaptops) 48

trigiduanus (Aceleuthetes) 142

trigiduanus (Lophothetes) 142

TRIGONOCOLINI 181

Trigonops 148

Trigonopterus 123

trigonopterus (Degis) 83

Trigonosomus 125

Trigonospartus 149

trilineatus (Mecopus) 92

trimastus (Ptolycus, Anchithyrus) 121

trinarius (Ectatorhinus, Aryptacus) 172

triseriatus (Phloeosinopsioides, Phloeosinopsis) 199

trispinatus (Diacavus) 197

tristiculus (Arachnobas, Arachnopus) 89

tristis (Acythopeus) 83

tristis (Arachnobas, Arachnopus) 89

tristis (Dystropicus, Berosiris) 102

tristis (Ischnomerus, Cediocera) 60

tristis (Osseteris) 129

tristis (Platypus) 194

tristis (Poropteropis) 120

tristriatus (Cerobates, Brenthus) 57

tristriatus (Cyphagogus) 55

trisulcatus (Higonius) 62

trisulcatus (Protaedus) 27

trivittata (Asytesta, Cyamobolus) 104

Trochorhopalus 79

tropicus (Barystethus) 76

TROPIDERINI 32

Tropidobasis 31

TROPIPHORINI 168

truncalis (Basitropis) 20

truncaticauda (Peroplatypus, Platypus) 185

truncaticauda (Xyleborus) 219

truncatiformis (Amasa, Xyleborus) 210

truncatigranosus (Peroplatypus, Platypus) 185

truncatipennis (Peroplatypus, Platypus) 185 
truncatulus (Hypolixus, Lixus) 169

truncatus (Anchithyrus) 112

truncatus (Pantorhytes) 167

\section{TRYPETIDINI 181}

tuberculata (Rhinoscapha) 161

tuberculatus (Cyrtogenius, Eidophelus) 208

tuberculatus (Phraotes) 147

tuberculifer (Cyclorhipidion, Xyleborus) 213

tuberculosa (Dinomelaena, Apatenia) 23

tuberculosus (Meroleptus) 116

tuberosa (Ectatocyba) 114

tuberosus (Orphanyasmus) 109

tumidirostris (Ottistira) 163

tumidus (Crossotarsus) 184

tumidus (Euwallacea, Xyleborus) 214

turbans (Neochyromera) 132

turbatus (Anaballus) 115

turbatus (Deretiosus, Colobodes) 173

turbatus (Seleuca, Ergias) 174

turcicus (Platypus) 195

turgidus (Diapus) 197

turpis (Trichoxymus) 123

turriformis (Platypus) 195

tutus (Opterus) 143

TYCHIINA 132

TYCHIINI 132

TYLODINA 111

Tyrpetes 181

Tyrtaeosus 125

umbratulus (Amasa, Xyleborus) 210

umbrifer (Alcidodes) 177

umbrinus (Platypus) 195

Uncifer 37

unicolor (Idopelma) 93

unicolor (Imathia) 131

unicolor (Ithystenus) 61

unicolor (Tracheloschizus, Arrhenodes) 68

unicolor (Tragopus, Acalles) 123

unicus (Cyrtogenius, Eidophelus) 208

unifasciata (Misthosima) 42

unifasciatus (Oribius, Coptorrhynchus) 146

uniformis (Anaballus) 115

uniformis (Camptorhinus) 103

uniformis (Mechistocerus) 103

uniformis (Platypus) 195

unilineata (Miolispa) 67

uniseriatus (Ozopemon) 209

unispineus (Diapus) 197

unispinosus (Baiocis) 181

unituberculata (Apatenia) 22

Uropteroides 65

ursa (Xylosandrus, Xyleborus) 219

ursinus (Otidognathus, Litorhynchus) 75

ursinus squamulosus (Otidognathus) 75

ursus (Araeocorynus) 40

urticivorax (Gymnopholus) 155

usitata (Schedlia, Xyleborus) 215

usta (Rhinoscapha) 161 ustus (Platypus) 195

uter (Platypus) 195

uter (Stephanoderes) 203

uvarius (Platypus) 195

vafer (Crossotarsus) 184

vagans (Phloeosinus) 199

vaginervis (Platypus) 195

v-alba (Phylaitis) 93

v-album (Perrhaebius) 174

valdephlebius (Platypus) 195

valens (Oribius, Coptorrhynchus) 146

valida (Schlaginhaufenia) 78

validipes (Achirozetes, Chirozetes) 91

validus (Araecerus) 39

validus (Elytrocheilus) 140

validus (Platypus) 195

validus capucinus (Araecerus) 39

validus tripartitus (Araecerus) 39

Vanapa 179

vanus (Trigonopterus, Idotasia) 125

variabilis (Cenchrena) 130

variabilis (Lophothetes, Trigonops) 142

variabilis (Magaris) 129

variabilis (Miolispa) 67

varicus (Arixyleborus) 211

variegata (Rhinaria) 133

variegatus (Isoleptus) 115

variegatus (Litocerus) 33

varinodis (Pantorhytes) 167

variolosus (Melanopsacus) 42

varipennis (Platypus) 195

varius (Platytenes) 109

varius (Scolytogenes, Cryphalomorphus) 205

vegetatus (Symbiopholus) 156

vellanum (Dinema, Proscopus) 26

velutinus (Meroleptus) 116

velutinus (Otidognathus) 75

velutinus (Rhynchophorus) 75

velutinus (Xenocerus) 36

velutinus signifer (Xenocerus) 36

venosa (Diatassa) 101

venosus (Araecerus) 39

ventralis (Hellerrhinus) 141

ventralis (Nechyrus) 118

ventricornis (Crossotarsus) 184

ventricosa (Nedymora) 128

ventrispinis (Crossotarsus) 184

ventrosus (Crossotarsus) 184

venustulus (Xyleborus) 219

verecunda (Asytesta) 105

vernaculus (Xyleborus) 219

verruca (Eczesaris, Idiopus) 28

verruca circumornata (Eczesaris) 28

verrucatus (Pantorhytes, Pachyrrhynchus) 167

verrucifer (Deretiosus) 173

verrucosa (Ectatocyba) 114

verrucosus (Apirocalus) 136

verrucosus (Perichius) 119 
versuta (Asytesta) 105

vesculus (Platypus) 195

vestigator (Cryptoxyleborus, Xyleborus) 212

vethi (Eupholus) 153

vetulus (Protoplatypus) 100

vetustus (Gymnopholus) 155

vexillarius (Apirocalus) 136

viaticus (Platypus) 195

viaticus (Xyleborus) 219

vibicifer (Pantorhytes) 167

vibrans (Rhinoscapha) 161

vicarius (Platypus) 195

vicarius (Pseudoporopterus, Poropterus) 121

vicinus (Crossotarsus) 184

vicinus (Ochronanus) 98

viduus (Guineobius) 140

vigilans (Baris) 82

viklundi (Hellerrhinus) 141

violaceipes (Morrisella, Pyroderes) 117

vipio (Bartolozziella, Endymia) 111

virescens (Paratactus) 146

virgata (Zena, Baris) 85

virgatus (Hucus) 32

virginea (Niseida) 129

viridans (Rhinoscapha) 161

viridanus (Ectemnomerus) 139

viridescens (Tracheloschizus, Schizotrachelus) 68

viridiceps (Neosynaptops) 49

viridimettalica (Rhinotia, Belus) 43

viridis (Eutinophaea, Ottistira) 162

viridis (Sphenomorpha, Apocyrtus) 168

viridula (Rhinoscapha) 161

vitiata (Blepiarda) 111

vitiosa (Odosyllis) 128

vittata (Asytesta) 105

vittaticollis (Oribius, Coptorhynchus) 146

vitticollis (Trigonops) 149

vittiger (Esocus) 32

vittipennis (Zena) 85

vlasimskii (Eupholus) 153

volaticus (Platypus) 195

vormanni (Rhinoscapha) 161

vollenhoveni (Rhinoscapha) 161

voluta (Blepiarda) 111

vulgaris (Coccotrypes, Dendrurgus) 206

vulgaris (Guineoeuops) 47

vulneratus (Rhynchophorus, Curculio) 75

waghianus (Pantorhytes) 167

wahnesi (Barystethus) 76

waigeensis (Opterus) 144

waigeoensis (Neosynaptops) 49

waigeoi (Exillis) 30

waigeoi (Protaedus) 27

waigeuensis (Eupholus) 153

wallacei (Crossotarsus) 184

wallacei (Ectocemus) 51

wallacei (Euwallacea, Xyleborus) 214

wallacei (Ficicis, Hylesinus) 198

wallacei (Guineoeuops) 47

wallacei (Ithystenus) 61

wallacei (Microcossonus) 99

wallacei (Platystomos, Anthribus) 31

wallacei (Rhinotia, Belus) 43 wallacei fasciolata (Sphenomorpha) 168

waltoni (Alcidodes, Alcides) 177

wapleri (Cryphalus) 202

wapogae (Neosynaptops) 49

waterhousei (Ancylotropis) 25

waterhousei (Ozotomerus) 29

watutensis (Platypus) 195

Webbia 215

websteri (Platyacus) 147

websteri (Xenocerus) 36

wei (Guineoeuops) 47

weidenbachi (Perissops) 109

weigeli (Protaedus) 27

weigeli (Tropidobasis) 31

weiskei (Aporophemus) 76

weiskei (Gymnopholus) 155

weiskei (Iphthimorhinus, Flamingorhynchus) 78

wichmanni (Rhinoscapha) 161

wollastoni (Lophocheirus) 127

woodlarkiana (Plintheria) 32

woodlarkianus (Cryptorhynchus) 110

woodlarkianus (Exillis) 30

xanthomelas (Callanthribus) 31

XENOCERINI 34

xenoceroides (Mucronianus) 34

Xenocerus 34

Xenopsilus 86

Xenotrupis 100

Xestothorax 56

Xola 110

Xychusa 126

XYLEBORINA 210

Xyleborinus 215

Xyleborus 216

Xylinada 36

XYLINADINI 36

XYLOCTONINA 219

Xylosandrus 219

yali (Riedelinius) 50

ypsilon (Asytesta) 105

yuliensis (Cyamotrox) 107

zazae (Cerobates) 57

zelivira (Acicnemis) 180

zelusus (Araecerus) 39

Zena 85

Zeugorrhinus 149

zimmeranni (Riedelinius) 50

zimmii (Guineoeuops) 47

zoarkes (Symbiopholus) 156

zonata (Geonemus) 157

zopherus (Deretiosus) 173

ZYGAENODINI 36

Zygara 126 


\section{APPENDIX 1.}

Summary of historical entomological expeditions and past weevil collectors that assembled significant weevil collections from the Papuan region. Expedition information from 1818-1980 is modified from Haaf, 1963, Frodin \& Gressitt, 1982, and Parsons, 1999. Repository information refers only to weevil material and was gleaned from the literature or from Horn \& Kahle, 1935 and Arnett et al., 1993. This list is not exhaustive.

\begin{tabular}{llll}
\hline Collector(s) \& collections & Year(s) & Collection localities & Repository \\
\hline J.R.C. Quoy \& G. J. P. Gaimard & $1818-1819$ & West Papua: Waigeo Island & MNHN
\end{tabular}

(in the Uranie and Physicienne)

D. d'Urville (in the Coquille)

$1822-1824$

Solomon Islands; Bismarck Archipelago:

MNHN, INSB

New Ireland; West Papua: Dorey Bay

D. d'Urville, J. R. C. Quoy \& G. 1826-1829

West Papua: Dorey Bay; Waigeo Island; Bis- MNHN

J.P. Gaimard (in the Astrolabe)

marck Archipelago: New Ireland

D. d'Urville (in the Astrolabe) $\quad 1837-1840$

South East coast of Papua New Guinea; Lou- MNHN

isiaide and d'Entrecasteaux Islands

J. MacGillivary (in the H. M.S. $\quad 1849-1850$

costal areas of New Guinea

BMNH

Rattlesnake)

P.J.X. Montrousier

$1847-1852$

Woodlark Islands

MNHN, INSB

A.R. Wallace

1858

West Papua: Dorey Bay; Aru Islands;

BMNH

Waigeo Island; (assistants collected in the

Arfak Mountains, Sorong (West Papua) and

on Mysol Island)

N.N. de Miklucho Maclay

$1871,1876-$

Papua New Guinea: Astrolabe Bay, Samarai

AMSA, MAMU

1877,1878 ,

Island (1880)

1880,1883

L.M. d'Albertis and O. Beccari

1872-1873

West Papua: Andai, Arfak Mountains, Dorey

MCSN

Bay, Ramoi

L.M. d'Albertis

1875,1876

Papua New Guinea: Fly River, Yule Island

MCSN

1877

1885

Papua New Guinea: Fly River, Yule Island

AMSA

L. Loria

1889

Papua New Guinea: Astrolabe Mountains,

? MCSN, SMTD

Milne Bay, Pt. Moresby area, Rigo

W. Doherty

1892-1893

West Papua: Geevlink Bay, Vokelkop; Yapen

BMNH via Tring Island

to be continued. 
APPENDIX 1. (continued)

\begin{tabular}{llll}
\hline Collector(s) \& collections & Year(s) & Collection localities & Repository \\
\hline A.S Anthony & $1895-1896$ & $\begin{array}{l}\text { South East Papua New Guinea, Mailu, Owen } \\
\text { Stanley Range. }\end{array}$ & $\begin{array}{l}\text { BMNH, MNHN, } \\
\text { SMTD }\end{array}$
\end{tabular}

A.S. Meek

1894-1916

E. Weiske

$1897-1898$

1895-1901

1897-1909

1899-1901

R. von Bennigsen

Dutch New Guinea Expeditions (with H. A. Lorentz \& L.F. de Beaufort)

A.E. Pratt (see Parsons 1999 for 1904 discussion of other the Pratts that collected in New Guinea)

Freiberg Expedition I

1907-1908

Aru Islands

SMTD

O. Schlanginhaufen

1909

1909-1910

Mimika, Utakwa Rivers

Papua New Guinea: Mt. Bougainville, Paup

(E. of Aitape),Torricelli Mountains

British Ornithologists Union Expedition (with A. R. F. Wollaston)

Freiberg Expedition II

1911

Mysol Island

SMTD

A.R.F. Wollaston Expedition 
APPENDIX 1. (continued)

\begin{tabular}{llll}
\hline Collector(s) \& collections & Year(s) & Collection localities & Repository \\
\hline W.C. von Huern & 1920 & $\begin{array}{l}\text { West Papua: Geelvink Bay; Idenburg and } \\
\text { Mamberambo Rivers }\end{array}$ & SMTD
\end{tabular}

L. Wagner

C.T. McNamara

Archbold Expedition I-VI (with

L.E. Cheesman on the second and L. J. Toxopeus on the third)

L.E. Cheesman

W.W. Brandt

Bishop Museum biological research station at Wau, became the Wau Ecology Institute (WEI) in 1971

J.J.H. Szent-Ivany

J.L. Gressitt,

1955-?

1961-?

1929 1964

1954-?
$1928-1930$

Papua New Guinea: Mt. Lamington

between 1933- Papua New Guinea: Fly River, South East;

$1932-1939$

1949-1963

1961-in decline since the late $1980 \mathrm{~s}$ West Papua: Cyclops Mountains, Jayapura, Snow Mountains, etc.

New Guinea and the surrounding islands; New Guinea: Kokoda, Mondo, Mt. Tafa (North West of Mt. Albert Edward), Torricelli Range, Wiotpe, Vanimo to Aitape, West Papua: Cyclops Mountains, Geelvink Bay, Waigeo Islands

Papua New Guinea: Finisterre Mountains, Fly River, Owen-Stanley Range, Woodlark Island, Bismarck Archipelago

\section{Papua New Guinea at Wau. Served as the} base for many collecting trips and expeditions into much of the region, especially the Highlands and Wau-Bulolo area. Associated researchers: A. Allison, W. Gagné, J.L. Gressitt, R.W. Hornabrook, G.A. Samuelson, J. Sedlacek, and J.J.H. Szent-Ivany.

Papua New Guinea: Asaro, Chimbu, Daulo Pass, around Wau (WEI)

Many localities in Papua New Guinea and West Papua

Papua New Guinea: Brown River, Bena Bena, Kainantu, Kassam Pass, Markham Valley, Mt. Albert Edward, Mt. Hagen, Mt. Giluwe, Popondetta, Warrongoi River, Wau, Woitape Bismarck Archipelago: New Britain
AMSA

AMNH, RMNH

AMSA, BMNH, BPBM

BPBM

Primarily BPBM, WEIC; some Gressitt types also in USNM

BPBM, KONE

BPBM

BPBM

ZMUC 
APPENDIX 1. (continued)

\begin{tabular}{|c|c|c|c|}
\hline Collector(s) \& collections & Year(s) & Collection localities & Repository \\
\hline H. Roberts & $1962,1984-?$ & $\begin{array}{l}\text { Papua New Guinea: Wau-Bulolo region, } \\
\text { Lae, Pt. Moresby, etc. Bismarck Archipel- } \\
\text { ago: New Britain }\end{array}$ & FICB/BPBM \\
\hline $\begin{array}{l}\text { Bishop Museum-National Geo- } \\
\text { graphic Society Expedition (with } \\
\text { J.L. Gressitt) }\end{array}$ & 1969 & $\begin{array}{l}\text { Papua New Guinea: Angoram, Bulldog } \\
\text { Road, Mt. Wilhelm, etc. }\end{array}$ & BPBM \\
\hline J.E. Tobler & $1969-1970$ & $\begin{array}{l}\text { Papua New Guinea: Wau; Bismarck Archi- } \\
\text { pelago: New Britain, New Ireland; North } \\
\text { Solomon Islands: Bougainville }\end{array}$ & CASC \\
\hline O. Missa & 1993-1996 & Papua New Guinea: Madang, Batiteta & INSB \\
\hline K. Morimoto and H. Roberts & 1984 & $\begin{array}{l}\text { Papua New Guinea: Lae, Madang, Pt. } \\
\text { Moresby, Bismarck Archipelago: New Brit- } \\
\text { ain }\end{array}$ & KUEC, FICB \\
\hline A. Riedel ${ }^{1}$ & 1990-present & $\begin{array}{l}\text { Many localities in West Papua; Moluccas, } \\
\text { and Papua New Guinea }\end{array}$ & $\begin{array}{l}\text { ARC in SMNK; } \\
\text { ZSMC, SMNS, } \\
\text { MBBJ }\end{array}$ \\
\hline $\begin{array}{l}\text { Christensen Research Institute } \\
\text { became The Parataonomist } \\
\text { Training Center (PTC) changed } \\
\text { to the New Guinea Binatang } \\
\text { research Center (BRC) }\end{array}$ & 1994-present & $\begin{array}{l}\text { Papua New Guinea: Chimbu, (based in } \\
\text { Madang) Madang area, Wau. Associated } \\
\text { researchers: A. Allison, Y. Basset, J. Hulcr, } \\
\text { S.E. Miller, V. Novotny, G.A. Samuelson, } \\
\text { G.P. Setliff }\end{array}$ & BPBM \\
\hline G. P. Setliff ${ }^{2}$ & $1999-2003$ & $\begin{array}{l}\text { Papua New Guinea: Bogia, Finsiterre Range, } \\
\text { Madang area, Mt. Hagen, Sepik area. Loui- } \\
\text { saide Archipelago: Misima Island. }\end{array}$ & GPS in USNM \\
\hline J. Hulcr ${ }^{3}$ & 2002-present & $\begin{array}{l}\text { Papua New Guinea: Madang area, Chimbu, } \\
\text { Sandaun, and Oro Provinces }\end{array}$ & MSUC \\
\hline
\end{tabular}

\footnotetext{
${ }^{1}$ ARC =Alexander Riedel collection; on indeterminate loan in SMNK. Repositories for holo- and allotypes from ARC are as follows: Euops simulans-group types are in SMNS; Guineobius types are deposited in MBBJ. Types of Indonesian species of Euops (Metaeuops) are deposited in MBBJ and SMNK and Papua New Guinean species are in SMNK. The remaining type material for Euops, Ottistira, Phaenomerus (Described in Thompson, 1996), and Apirocalus (described in Thompson, 2005) reported as deposited in ARC are now deposited in ZSMC (Riedel, in litt.).

${ }^{2}$ GPS =Gregory P. Setliff collection; on indeterminate loan in USNM.

${ }^{3}$ Jiri Hulcr's collection of bark and ambrosia beetles (focus on Xyleborina and Platypodinae) is deposited in MSUC. Duplicates will be deposited in the USNH, BMNH, and NMW (Hulcr, pers. comm.).
} 


\section{APPENDIX 2.}

Parts of the Coleopterorum Catologus that contain Papuan weevils. Taxa listed represents the families and subfamilies treated in each work. The taxa listed here have not been updated to follow more recent classifications or reflect current nomenclature.

\begin{tabular}{|c|c|c|}
\hline Catalog citation & Taxa & Volume \\
\hline Hagedorn, 1910, part 4 & Ipidae & XXVI \\
\hline Wagner, 1910, part 6 & Apionidae & XXVIII \\
\hline Strohmeyer, 1912, part 44 & Platypodidae & XXVI \\
\hline Kleine, 1927, part 89 & Brenthidae & XXVI \\
\hline Wolfrum, 1929, part 102 & Anthribidae & XXVI \\
\hline Dalla Torre \& Voss, 1930, part 110 & Archolabinae, Attelabinae, Apoderinae & XXIX \\
\hline Dalla Torre \& van Emden, 1931, part 119 & Brachyderinae; Pachyrrhynchini & XXVII \\
\hline Schenkling \& Marshall, 1931, part 114 & Eremninae; Leptopiinae & XXVIII \\
\hline Dalla Torre et al., 1932a, part 122 & Hylobiinae & XXVIII \\
\hline Dalla Torre \& Schenkling, 1932, part 123 & Curculioninae & XXIX \\
\hline Dalla Torre et al., 1932b, part 125 & Pissodinae & XXVIII \\
\hline Günther, \& Zumpt, 1933, part 131 & Tanymecinae & XXVII \\
\hline Csiki, 1934b, part 134 & Cleoninae & XXVIII \\
\hline Hustache, 1934, part 136 & Zygopinae & $\mathrm{XXX}$ \\
\hline Csiki, 1934a, part 137 & Hyperinae & XXVIII \\
\hline Klima, 1934a, part 135 & Gymnetrinae & XXIX \\
\hline Schenkling \& Marshall, 1934, part 139 & Laemosaccinae & XXIX \\
\hline Klima, 1934b, part 140 & Erirrhininae & XXVIII \\
\hline Dalla Torre \& Voss, 1935, part 144 & Belinae & XXVIII \\
\hline Klima, 1935a, part 145 & Ceratopinae & XXIX \\
\hline Klima, 1935b, part 145 & Trigonocolinae & XXIX \\
\hline Klima, 1935c, part 145 & Acicnemidinae & XXIX \\
\hline Csiki, 1936a, part 149 & Rhynchophorinae & $\mathrm{XXX}$ \\
\hline Csiki, 1936b, part 149 & Cossoninae & $\mathrm{XXX}$ \\
\hline Schenkling \& Marshall, 1936a, part 150 & Aterpinae & XXVIII \\
\hline Schenkling \& Marshall, 1936b, part 150 & Prionomerinae & XXIX \\
\hline Hustache, 1936, part 151 & Cryptorrhynchinae & XXIX \\
\hline Dalla Torre \& Voss, 1937, part 158 & Rhynchitinae I & XXIX \\
\hline Lona, 1937, part 160 & Otiorrhynchinae II & XXVII \\
\hline Lona, 1938, part 162 & Otiorrhynchinae III & XXVII \\
\hline Hustache, 1938, part 163 & Barinae & $\mathrm{XXX}$ \\
\hline Dalla Torre \& van Emden, 1939, part 164 & Brachyderinae III & XXVII \\
\hline Dalla Torre \& Voss, 1939, part 167 & Rhynchitinae II & XXIX \\
\hline Voss, 1952 , supplement to parts $158 ; 167$ & $\begin{array}{l}\text { Mesoptiliinae; Rhynchitinae; Alloc- } \\
\text { oryninae; Pterocolinae }\end{array}$ & $\mathrm{n} / \mathrm{a}$ \\
\hline Voss, 1953 , supplement to parts $144 ; 110$ & $\begin{array}{l}\text { Oxycoryninae; Belinae; Archolabinae; } \\
\text { Apoderinae }\end{array}$ & $\mathrm{n} / \mathrm{a}$ \\
\hline Wolfrum, 1953, supplement to part 102 & Anthribidae & $\mathrm{n} / \mathrm{a}$ \\
\hline
\end{tabular}




\section{APPENDIX 3.}

Major Taxonomic works treating Papuan weevils published since the Coleopterorum Catalogus (weevil parts 19101953) arranged by family (subfamily within Curculionidae).

\begin{tabular}{ll}
\hline Taxa & Citation \\
\hline Anthribidae & Frieser, 1983; 1985; 1989; 1992; 1993; 1995a; 1995b; 1996; 1997; 1999; 2000; \\
& $2001 \mathrm{a} ; 2001 \mathrm{~b} ;$ Kuschel, Rheinheimer 2004a; 1998; Wolfrum, 1956; Zimmerman, \\
& 1994a \\
& \\
& Korotyaev, 1987; Wanat, 2001; Zimmerman, 1994b \\
& \\
Apionidae & Riedel, 1998; 1999; 2001a; 2001b; 2001c; 2002a, 2002c; 2006; Legalov, 2003; Voss, \\
& 1956 \\
Attelibidae & Sforzi \& Bartolozzi, 2004; Damoiseau, 1963; 1964a; 1964b; 1966a; 1966b; 1966c; \\
& $1966 d ; 1972 ; 1980 ; 1987 ; 1989 \mathrm{a} ; 1989 \mathrm{~b} ;$ Goosens, 2005; Györffy, 1917; Kleine, \\
& 1930; 1935; 1939; 1943; Kleine et al., 1925; Mantilleri, 2005; Oberprieler, 2004; \\
Brentidae & Wagner, 1912; Zimmerman, 1994b
\end{tabular}

Curculionidae: Ceutorhynchinae Colonnelli, 1979

Curculionidae: Conoderinae

Curculiondae: Cossoninae

Curculionidae: Cryptorhynchinae

Curculionidae: Curculioninae

Curculionidae:Entiminae

Curculionidae: Molytinae

Curculionidae: Platypodinae
Heller, 1937a; 1942; Marshall, 1941b; Thompson, 1996

Kuschel, 2000; Marshall, 1954; 1958; Rheinheimer, 2002; Voss 1956; 1971; Zimmerman, 1944b

Heller, 1937a; 1941a; 1942; Marshall, 1959b, Thompson, 1984; Zimmerman, 1941; 1967, 1974, 1994a; Voss, 1958b; 1960

Heller, 1941a; 1942; Kojima \& Morimoto, 2005; Zimmerman, 1994a

Gressitt, 1966a; 1966b; 1967; 1970; 1977; Gressitt \& Sedlacek, 1967; 1970; Heller, 1937a; 1941c; 1942; Limoges \& Porion, 2004; Marshall, 1938; 1941a; 1956; 1957; 1959a; 1959b; Osella, 1983; Porion 1993; 2000; Rheinheimer, 2004a; Riedel 2002; Riedel \& O'Brien, 1995; Riedel \& Schönitzer, 1996; Stibick, 1978; 1979; Tanner, 1969; Thompson, 1977; 2005; Voss, 1958b

Haaf, 1963; 1964; Heller, 1942; Voss, 1958b; 1960; Zimmerman, 1941

Beaver, 1995; Beeson, 1937; Browne, 1966; 1980a; 1980b; 1980c; 1980d; 1981a; 1981b; 1983a; 1983b; 1984a; 1984b; 1984c; 1984d; 1985a; 1985b; 1986a; 1986b; 1986c; Kuschel et al., 2000; Murayama, 1925; Nunberg, 1961; Roberts, 1979; 1986; 1987; 1988a; 1989b; 1993; Roberts \& Morimoto; 1986; Schedl, 1935; 1936c; 1936e; 1937a; 1942b; 1968a; 1968b; 1969b; 1970a; 1972b; 1972c; 1973a; 1973b; 1974; 1975c; 1975d; 1979b; 1979d Sampson, 1912; 1924 
APPENDIX 3. (continued)

\begin{tabular}{ll}
\hline Taxa & Citation \\
\hline Curculionidae: Scolytinae & Beeson, 1929; 1939; Bright, 1980; Browne, 1966; 1980a; 1980b; 1980c; 1980d; \\
& 1981a; 1981b; 1983a; 1983b; 1984a; 1984b; 1984c; 1984d; 1985a; 1985b; 1986a; \\
& 1986b; 1986c; Eggers, 1923; 1926; 1927a; 1927b; 1940; Hagedorn, 1912; \\
& Murayama, 1925; Nunberg, 1961; Roepke, 1919; Sampson, 1923; Schedl, 1940; \\
& $1942 \mathrm{~b} ; 1942 \mathrm{c} ; 1951 ; 1955 ; 1964 \mathrm{~b} ; 1964 \mathrm{~d} ; 1968 \mathrm{a} ; 1969 \mathrm{~b} ; 1972 \mathrm{~b} ; 1972 \mathrm{c} ; 1973 \mathrm{a} ;$ \\
& $1973 \mathrm{~b} ; 1974 ; 1975 \mathrm{c} ; 1975 \mathrm{~d} ; 1979 \mathrm{~b} ; 1979 \mathrm{~d}$ \\
& \\
Gryophthoridae & 1994b \\
& \\
Kuschel in Zimmerman 2004a & \\
&
\end{tabular}




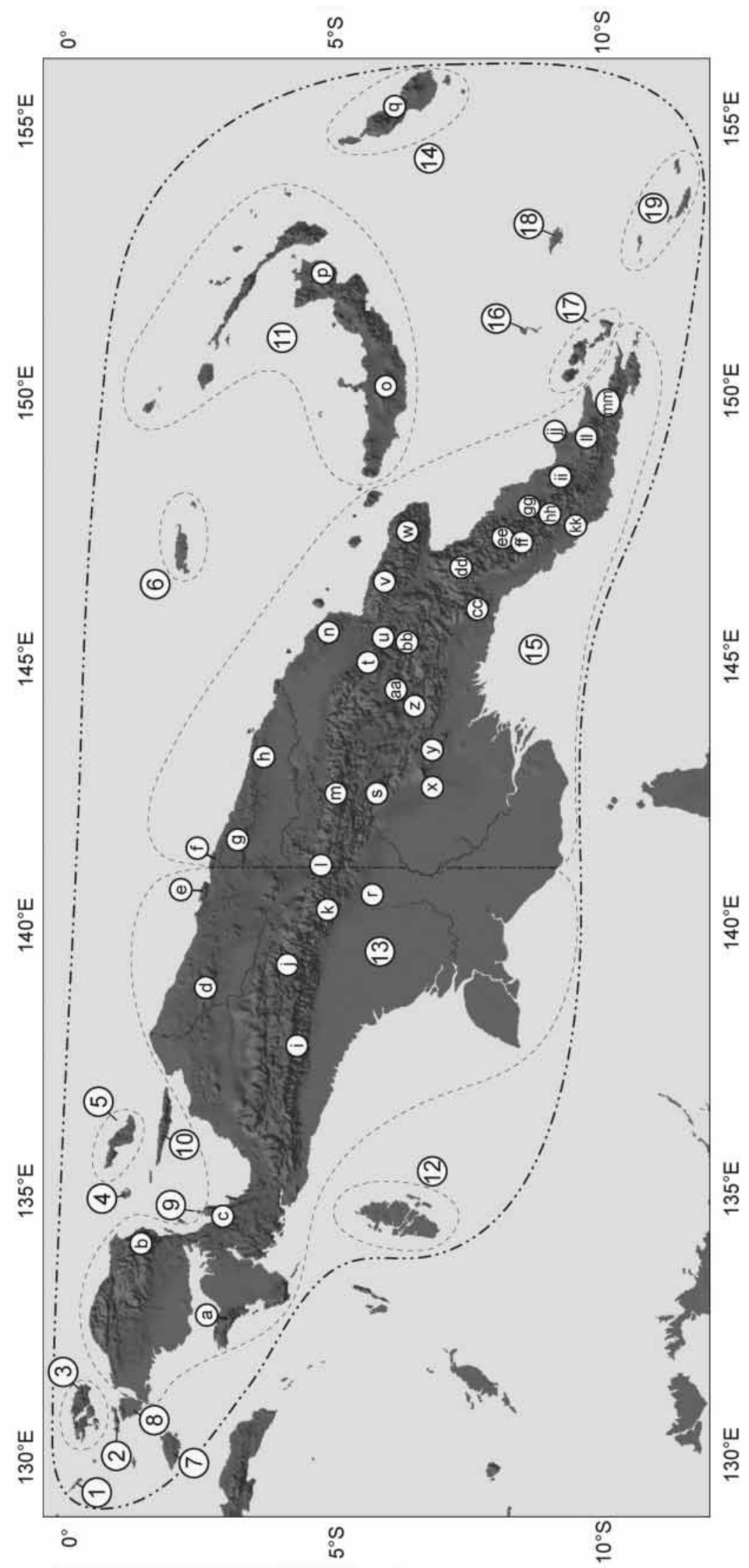

FIGURE 1. Map of Papuan region showing the region covered in this checklist; indicated by a bold, broken and dotted line. Numbers indicate the subdivisions of the region used in the checklist; light broken lines delineate island groups and show the subdivision of mainland New Guinea along its political border. Letters indicate mountains or mountain ranges that are important collection localities. Numbers and letters are arranged left to right from top to bottom. 
FIGURE 1. Indexes.

\section{Subdivisions}

Index of the subdivisions of the Papuan region used in the checklist.
1. Gebeh Island
2. Batanta Island
3. Waigeo Islands
4. Numfor Island
5. Biak Island; Korido
6. Admiralty Islands
7. Mysol Island
8. Salawatti Island
9. Roon Island
10. Yapen Island
11. Bismarck Archipelago
12. Aru Islands
13. West Papua
14. North Solomon Islands
15. Papua New Guinea
16. Trobriand Islands
17. D'Entrecasteaux Islands
18. Woodlark Island
19. Louisaide Archipelago

\section{Mountains and Mountain Ranges}

Index of major mountains and mountain ranges that represent important collection localities. Alternative locality names follow in square brackets; $\dagger$ denotes names no longer in use; names preceded by $=$ are alternative place names currently in use, but not used in this checklist.

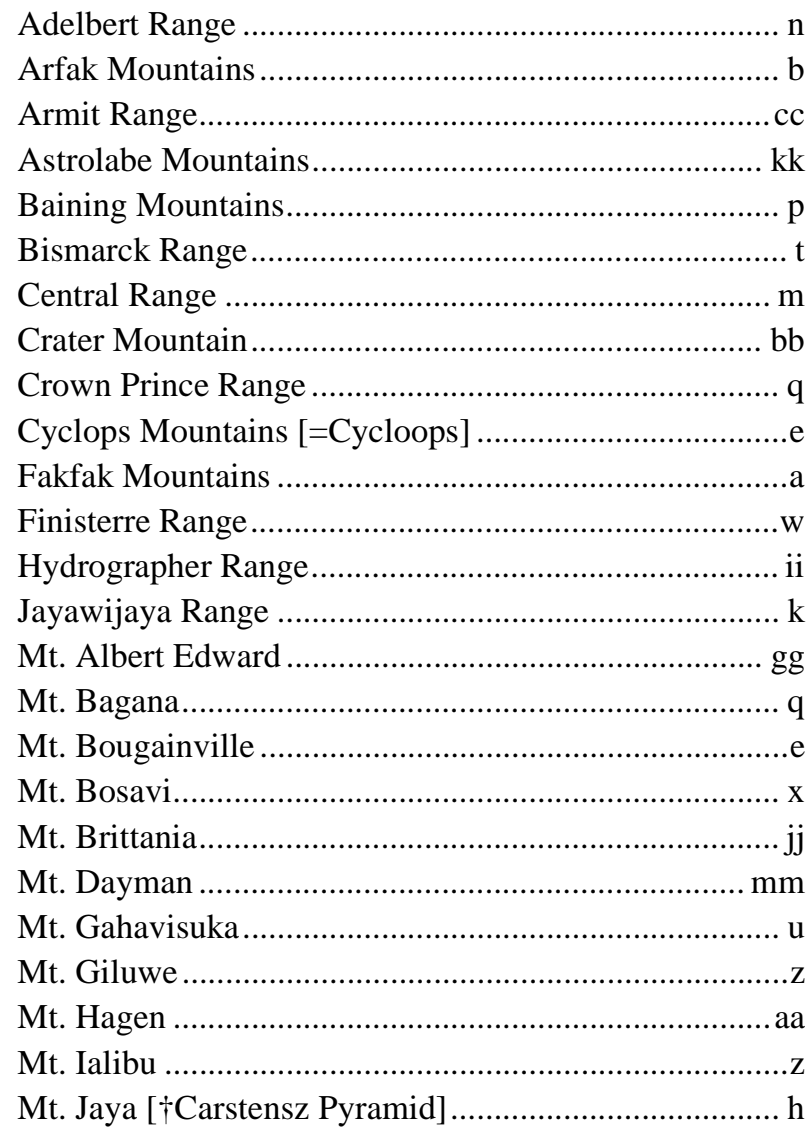

Mt. Kaindi ............................................................ dd

Mt. Lamington ......................................................... ii

Mt. Mandala [†Mt. Juliana] .................................... r

Mt. Michael.............................................................. bb

Mt. Missim........................................................... dd

Mt. Otto............................................................... u

Mt. Saint Mary ….........................................................ee

Mt. Simpson......................................................... mm

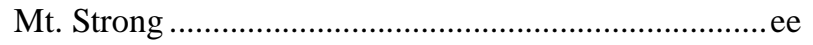

Mt. Suckling …............................................................ 11

Mt. Victoria ............................................................. hh

Mt. Victory ...........................................................

Mt. Wilhelm ................................................................... $\mathrm{t}$

Mt. Yule .....................................................................ff

Muller Range ........................................................... $\mathrm{s}$

Murray Range …...................................................... y

Prince Alexander Mountains ......................................... $\mathrm{g}$

Rouffaer Mountains ...................................................... d

Saruwaged Range [†Salawakit Range]..........................w

Snow Mountains [=Maoke Mountains] ..................... i-k

Star Mountains ............................................................. 1

Torricelli Range ......................................................... f

Whiteman Range ...................................................... $\mathrm{o}$ 


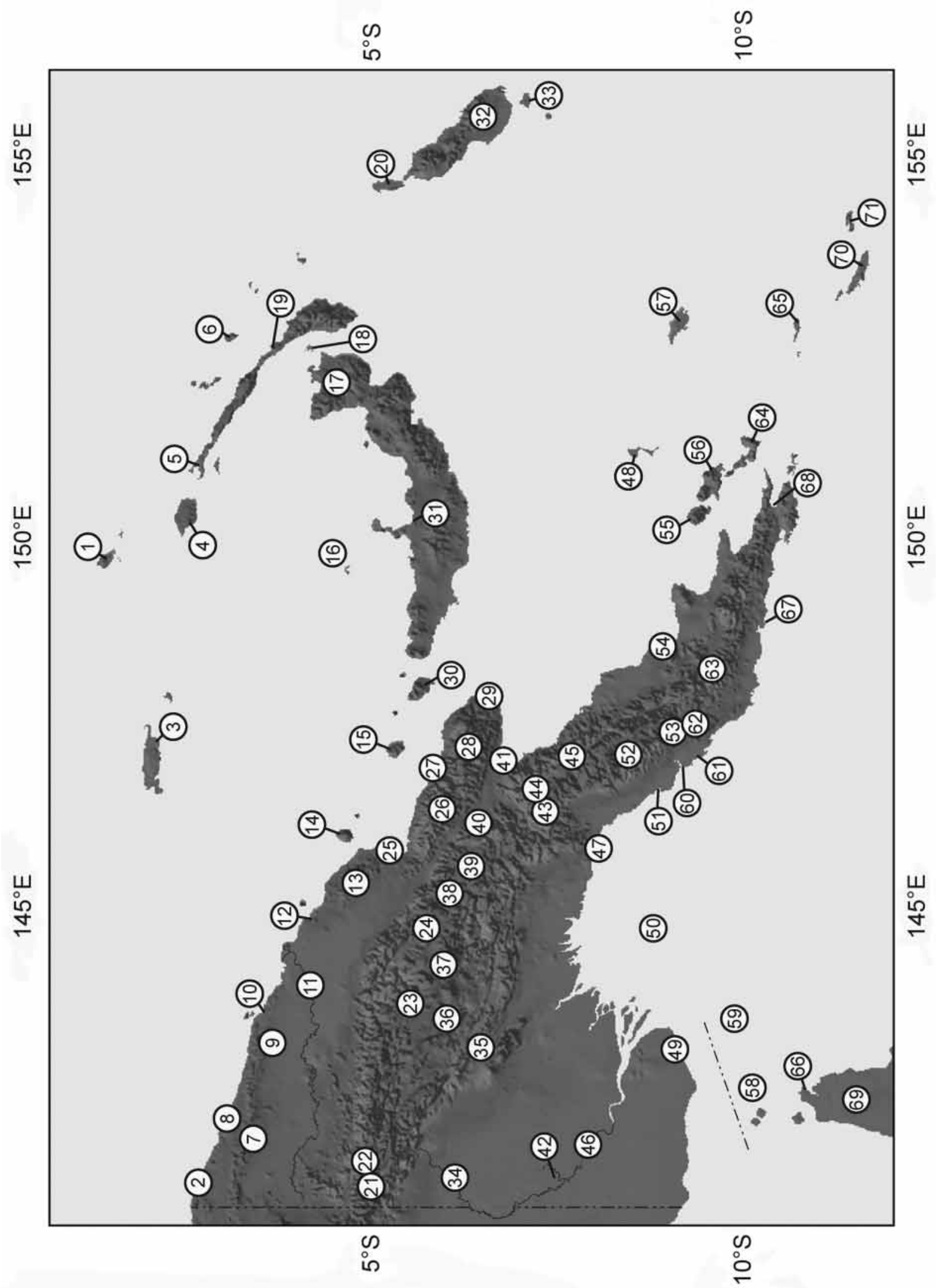

FIGURE 2. Map of Papua New Guinea and satellite archipelagoes. Numbers indicate island groups including major islands, towns, villages, and some geographical features that are important collection localities. Numbers are arranged left to right from top to bottom. Australia and the Torres Straights Islands (South of the dashed line) are not included in the region covered by this checklist, however, these localities are commonly included in works on the Papuan region. 
FIGURE 2. Index.

Alphabetical index of place names arranged by major regional subdivisions used in the checklist (see Fig. 1). Alternative locality names follow in square brackets; $\dagger$ denotes names no longer in use; names preceded by $=$ are alternative place names currently in use, but not used in this checklist.

Papua New Guinea [†Kaiser-Wilhelms Land, †North-East \& †South-East New Guinea, †Deutsch (German) New Guinea $\& \nmid$ British New Guinea, †Territory of Papua \& New Guinea]

\begin{tabular}{|c|c|}
\hline 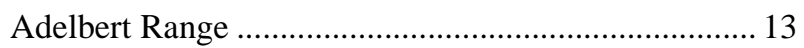 & Mt. Hagen [town] \\
\hline Aitape $[\dagger$ Berlinhafen $] .. .$. & Mt. Wilhelm.. \\
\hline Alexander Mountains... & Nadzab... \\
\hline Alotau.......... & Nagada Bay.................. \\
\hline Angoram... & Owen Stanley Range... \\
\hline Aroa River.. & Popondetta .. \\
\hline Asaro .... & Port Moresby ....... \\
\hline Asek ....................... & 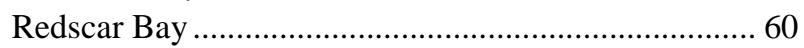 \\
\hline Astrolabe Bay.............. & Rigo............................. \\
\hline Astrolabe Mountains ....... & Sattelberg ................ \\
\hline Bogia ................ & Saruwaged Range $[\uparrow$ Salawakit Range $] \ldots \ldots . .$. \\
\hline Bongu ........ & Sepik River $[\dagger$ Kaiserin-August River $] ..$ \\
\hline Brown River. & Tabubil .. \\
\hline Bulldog trail .. & Telefomin .................... \\
\hline Bulolo................... & 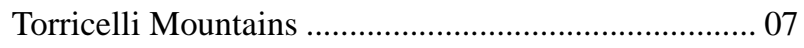 \\
\hline Daru................. & Umboi Island $[\dagger$ Rook Island $] \ldots . . .$. \\
\hline Eddie Creek... & Vanapa River.............. \\
\hline Erima Harbor.......... & Vanimo .............. \\
\hline Finisterre Mountain..... & Wabag .......................... \\
\hline Finschhafen ............... & Wau ............. \\
\hline Fly River ................ & Wareo ............................ \\
\hline Garaina .................... & Wewak $[\dagger$ Dallmannhafen $]$............ \\
\hline Goroka............... & 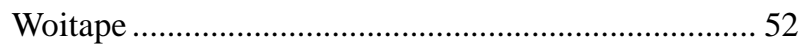 \\
\hline Gulf of Papua ............. & Yule Island ..... \\
\hline Huon Gulf ........... & \\
\hline Huon Peninsula ...... & Admiralty Islands \\
\hline Ighibirei............. & Manus Island.......... \\
\hline Jimmi Valley ......... & \\
\hline Kainantu.................. & Bismark A \\
\hline Kar Kar Island............. & 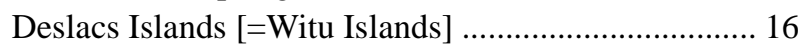 \\
\hline Kerema................ & Duke of York [†New Laudenburg Island] ................... 18 \\
\hline Kiunga.................. & 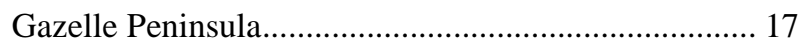 \\
\hline Kokoda trail.. & Kimbe ...................... \\
\hline Lae...................... & Namatanai ................................................. \\
\hline Lake Kutubu............ & 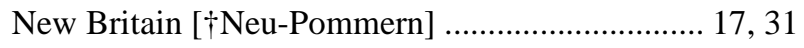 \\
\hline Lake Murray............. & New Hanover [=Lavongai Island] ............. \\
\hline 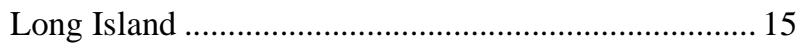 & New Ireland $[\dagger$ Neu-Mecklenburg] ....................... 05, 19 \\
\hline 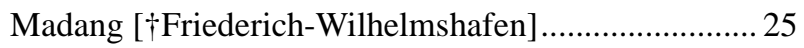 & Rabaul [destroyed by volcanic eruption] ..................... 17 \\
\hline 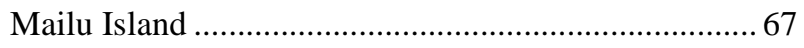 & St. Matthias Islands [=Mussau Islands] ......................... 01 \\
\hline 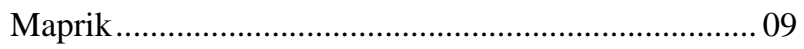 & \\
\hline Markham Valley.......... & D’Entrecasteaux Islands \\
\hline Mendi ....................... & Fergusson Island ............ \\
\hline 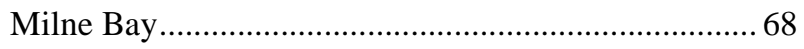 & 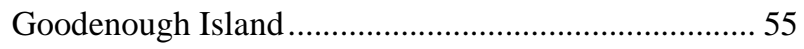 \\
\hline 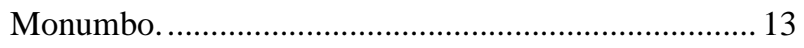 & Normanby Island ........ \\
\hline
\end{tabular}


Louisaide Archipelago

Misima Island 65

Sudest Island [=Tagula Islands]

.70

Yela Island [=Rossel Island].....

North Solomon Islands

Bougainville Island.

Buka Island.

Shortland Island.

20

33

Trobriand Islands.
Woodlark Island [=Muyua Islands] ...........................57

[Localities not considered in the checklist]

Australia [ $†$ New Hollandia]

Cape York

69

Darnley Island ............................................................5

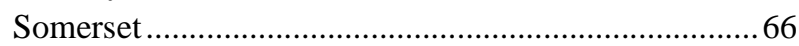

Torre Straits Islands.................................................. 58 


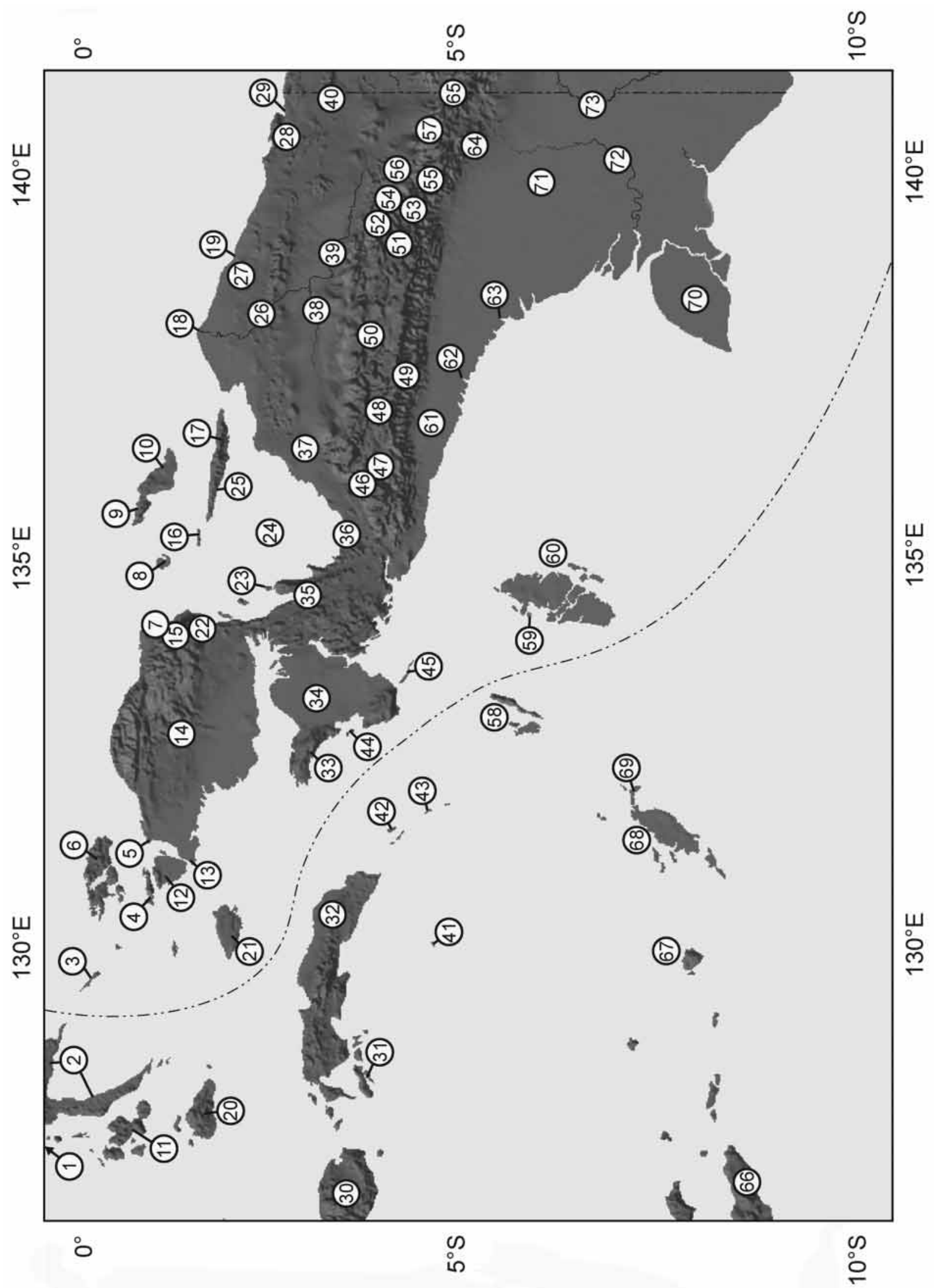

FIGURE 3. Map of West Papua and satellite archipelagoes. Numbers indicate island groups including major islands, towns, villages, and some geographical features that are important collection localities. Numbers are arranged left to right from top to bottom. Islands to the West of the dashed line are not included in the region covered by this checklist, however, these localities are commonly included in works on the Papuan region. 


\section{FIGURE 3. INDEX}

Alphabetical index of place names arranged by major regional subdivisions used in the checklist (see Fig. 1). Alternative locality names follow in square brackets; $\dagger$ denotes names no longer in use; names preceded by $=$ are alternative place names currently in use, but not used in this checklist.

Indonesia: West Papua [†Dutch New Guinea, †Irain Jaya, †West New Guinea; =Papua Barat]

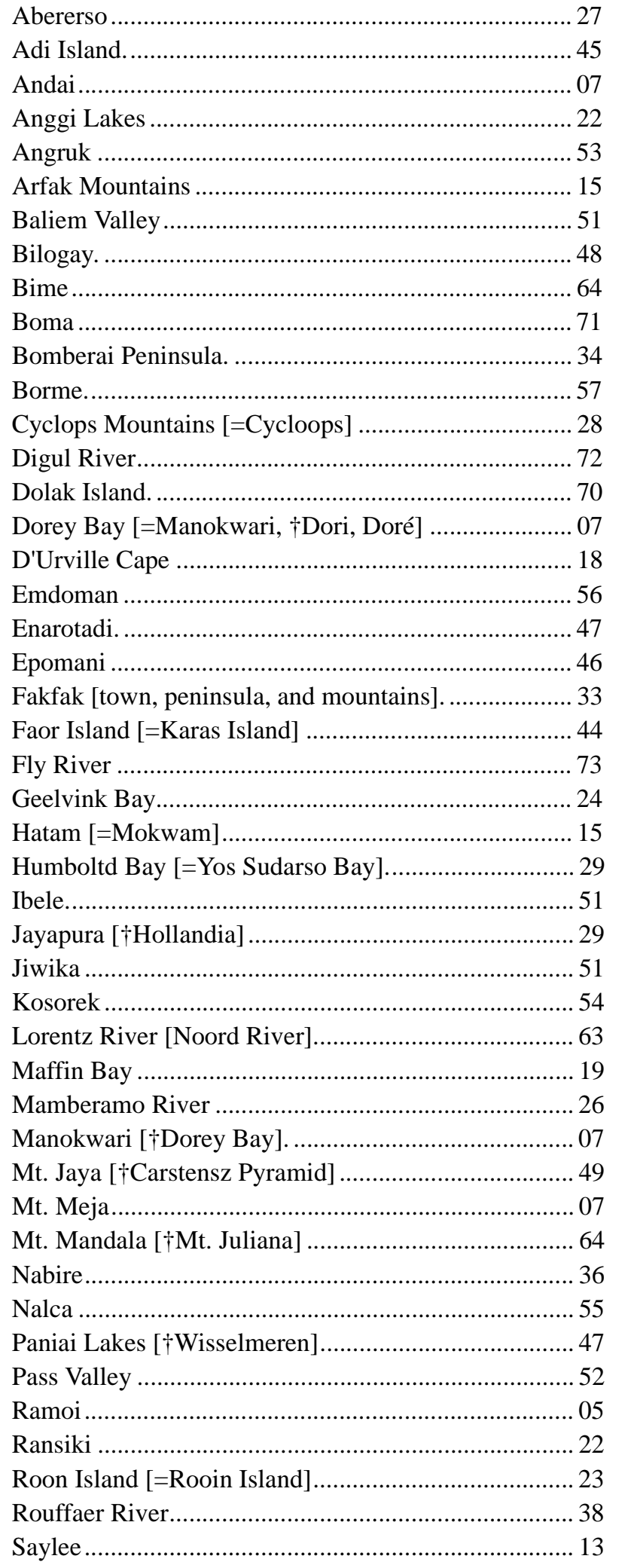

Sentani............................................................... 28

Sinak …............................................................... 50

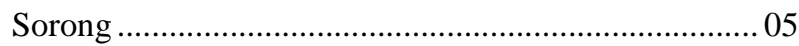

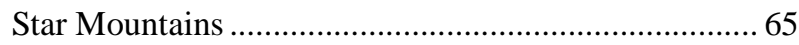

Taritatu River [†Idenburg River] ................................ 39

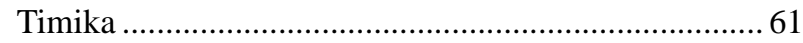

Utakwa River [=Otakwa River] ................................. 62

Vogelkop Peninsula [=Doberai Peninsula] .................. 14

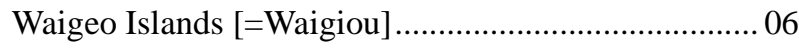

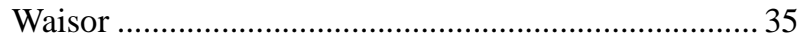

Wamena................................................................... 51

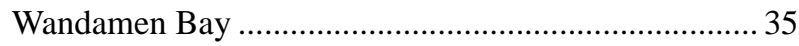

Waris ............................................................................. 40

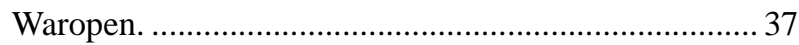

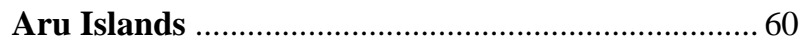

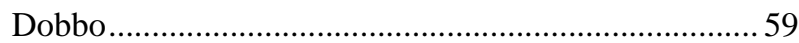

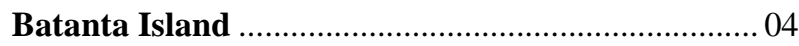

Gebeh Island ...................................................... 03

$†$ Misori Islands [ $†$ Schouten Islands]

Biak Island [†Bivak Island] ...................................... 10

Korido [=Supiori Island] ....................................... 09

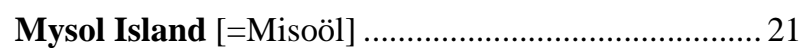

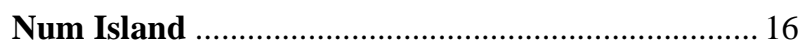

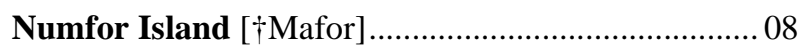

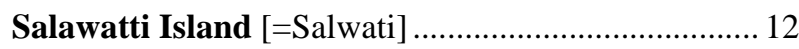

Yapen Island [†Japen Island; †Jobi Island] ............... 17

Ansus......................................................................... 25

[Localities not considered in the checklist]

Kei Islands [=Kai, †Key Islands] ............................. 58

Moluccas [ $†$ Spice Islands]

Ambon.................................................................. 31

Bacan Island [ $†$ Batcian, $†$ Batjan, etc.] ......................... 11

Banda Island................................................................. 41

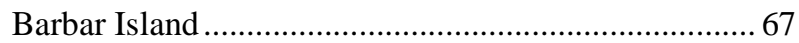


Buru. 30

Goram Island [=Gorong Island]. ................................. 42

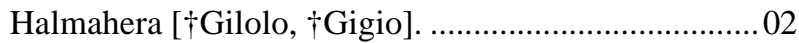

Matebella Island [=Watubela Archipelago] .................44

Obi Island .............................................................. 20

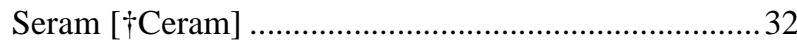

Ternate .01
Tanimbar Islands 68

Larat Island 69

West Timor. 66

\section{Notes added in proof.}

1. Cosmopolites Chevrolat, 1885: 289 was transferred to Dryophthoridae: Litosomini from Dryophthoridae: Sphenophorini by Anderson (2002).

2. Oebrius Pascoe, 1874: 54 was transferred to Curculioninae: Storeini from Conoderinae: Othippiini by Kojima \& Lyal (2002).

3. Panigena Pascoe, 1874: 52 and Pseniclea Pascoe, 1874: 51 were transferred to Conoderinae: incertae sedis from Conoderinae: Othippiini by Kojima \& Lyal, 2002.

4. Mitrastethus australiae Lea, 1908a: 177 (Curculionidae: Cryptorhynchinae: Cryptorhynchina) was synonymized with M. baridioides Redtenbacher, 1868: 168 by Zimmerman (1994b).

5. Wylie \& Shanahan (1973) reported the following species from Araucaria and Eucalyptus plantations in Bulolo: Aesiotes notabilis Pascoe, Hylesinus porcatus Chapius, Illacuris laticollis (Pascoe), Sympiezoscelus sp. nr. spencei Waterhouse, Xyleborinus artestriatus (Eichhoff), and Webbia pabo Sampson.

\section{Additional Literature Cited}

Anderson, R.S. (2002) The Dryophthoridae of Costa Rica and Panama: checklist with keys, new synonymy and descriptions of new species of Cactophagus, Mesocordylus, Metamasius and Rhodobaenus (Coleoptera; Curculionoidea). Zootaxa, 80, 1-94.

Kojima, H. \& C.H.C. Lyal (2002). New Oriental and Australian Conoderinae, with taxonomic notes on the tribe Othippiini (Coleoptera: Curculionidae). Esakia, 42, 161-174.

Wylie, F.R. \& P.J. Shanahan (1973). Insect attack in fire-damaged plantation trees at Bulolo in Papua New Guinea. Journal of the Australian Entomological Society, 14, 371-382. 